\title{
Plug\&Play Places:
}

Lifeworlds of multilocal creative knowledge workers

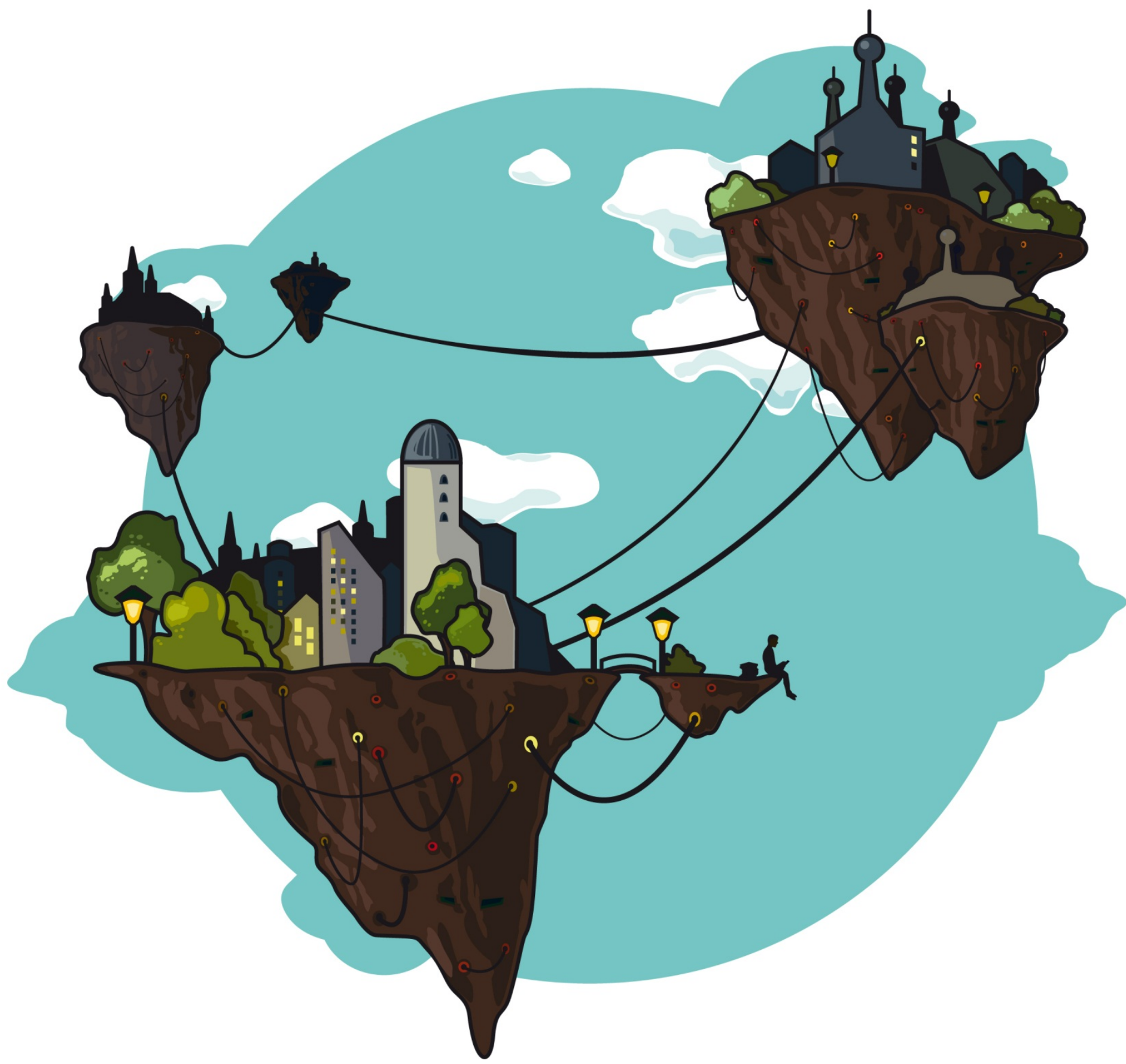

Robert Nadler 



\section{Plug\&Play Places:}

\section{Lifeworlds of multilocal creative knowledge workers}

\section{Robert Nadler}

PhD Thesis

Submitted 31 January 2013

in the frame of the

European Doctoral Programme 'Urban and Local European Studies' (URBEUR)

University of Milano-Bicocca, Italy

Supervised by Prof. Matteo Colleoni, Department of Sociology and Social Research, University of Milano-Bicocca, Italy 


\section{Table of contents}

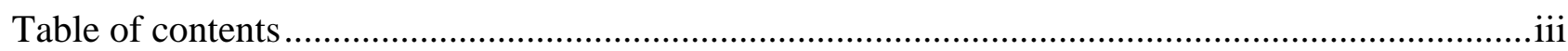

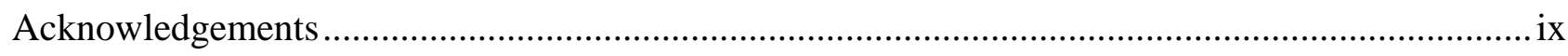

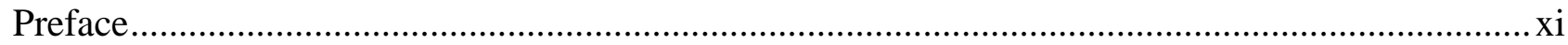

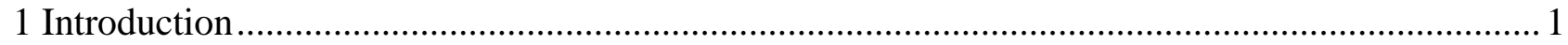

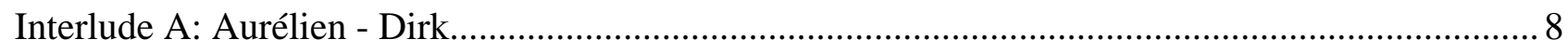

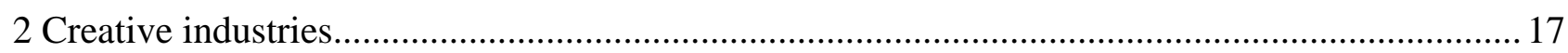

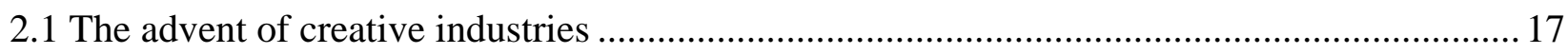

2.1.1 The information society and the creative industries .................................................... 18

2.1.2 The knowledge economy and the creative industries .................................................... 22

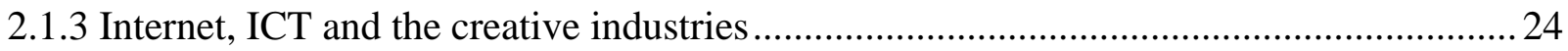

2.1.4 Information, knowledge, and creativity .................................................................. 27

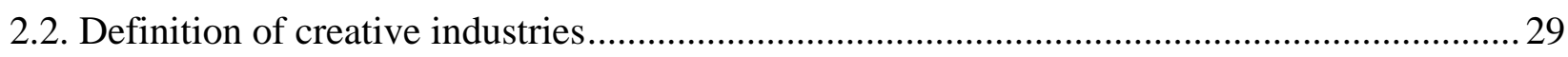

2.2.1 Soft definitions: the qualitative dimension of creative industries................................... 30

2.2.2 Hard definitions: the quantitative dimension of creative industries ................................ 35

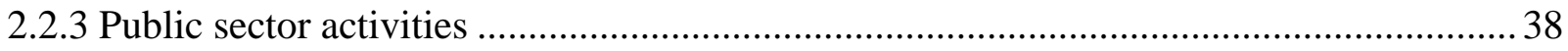

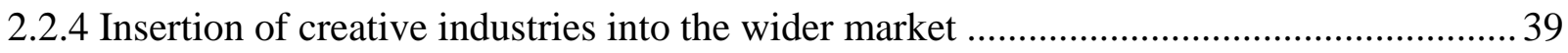

2.3 The creative knowledge workers and their work environment............................................ 43

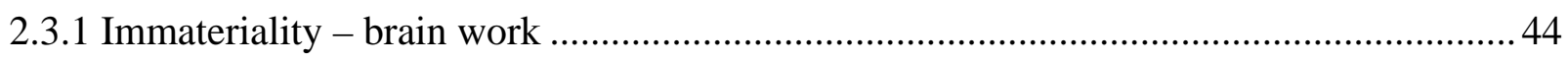

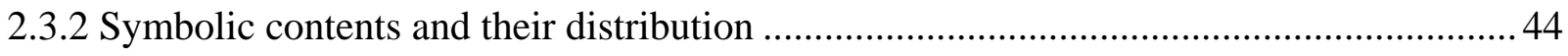

2.3.3 Project based work and irregular time schedules............................................................ 45

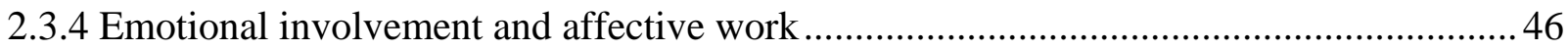

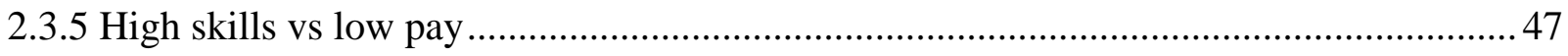

2.3.6 Missing regulation and liberal labour markets......................................................... 48

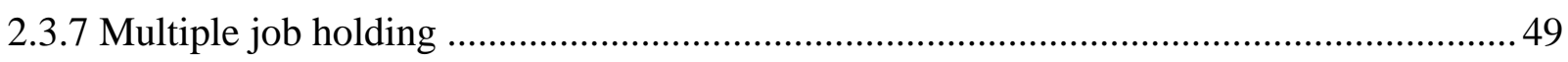

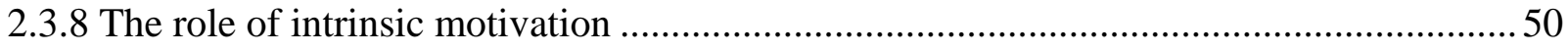

2.3.9 Gender aspects and compatibility of family and work life ........................................... 51

2.3.10 Synthesis: Creative knowledge work in dilemma......................................................... 51

2.4 The creative knowledge worker between arts and science: my own definition...................... 53

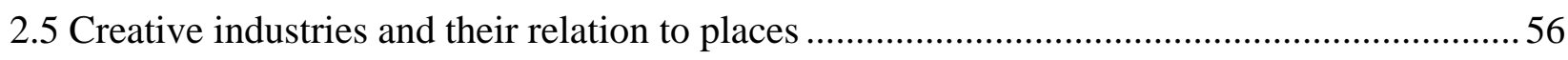

2.5.1 Urban diversity, open-mindedness and tolerance ....................................................... 57 
2.5.2 Third places, networking and information flow............................................................ 58

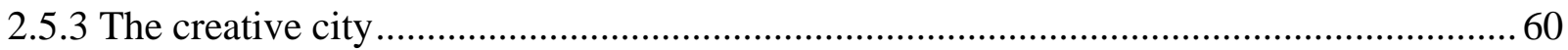

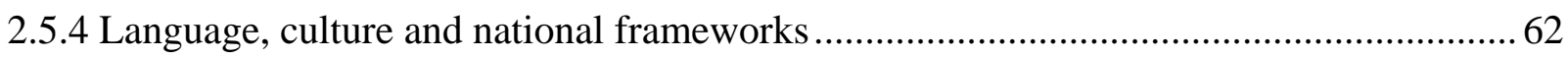

2.5.5 The role of geographic proximity: Creative industries and local networks .....................63

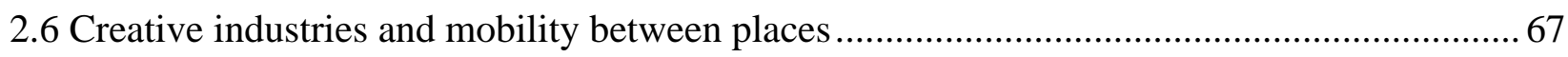

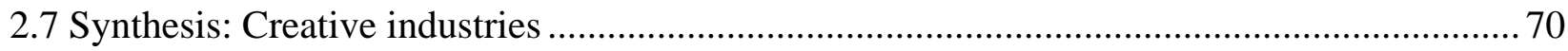

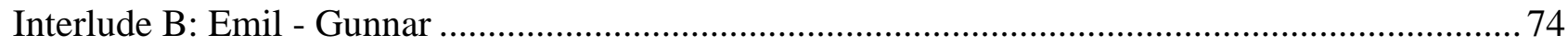

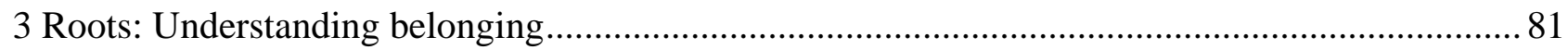

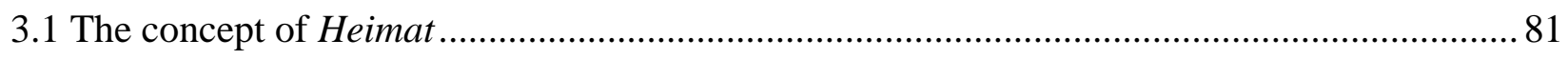

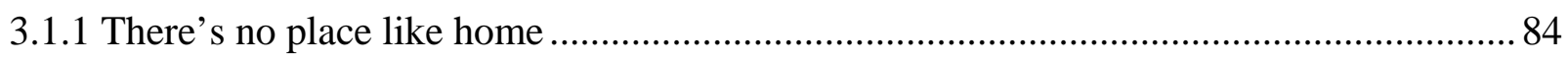

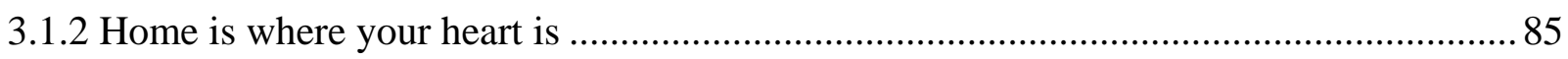

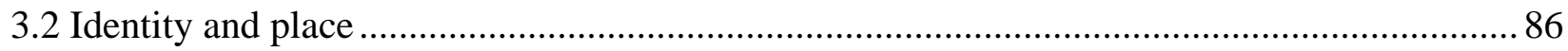

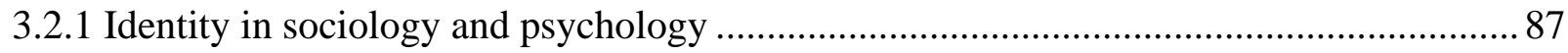

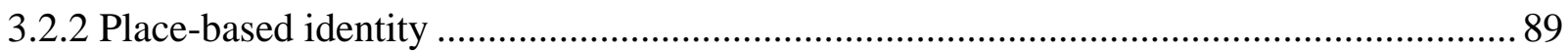

3.2.3 Place-based identity in the European Union: some empirical insights........................... 91

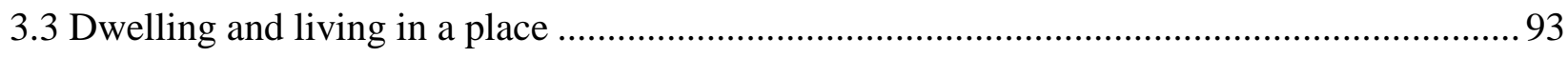

3.4 Jung's concept of psycho-social sedentariness ................................................................. 96

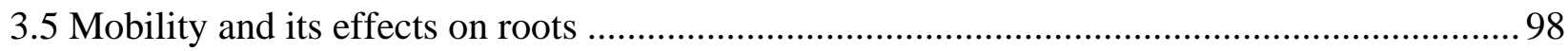

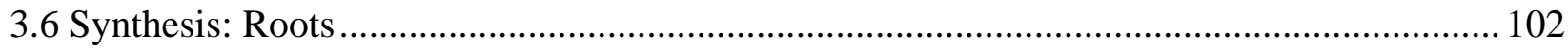

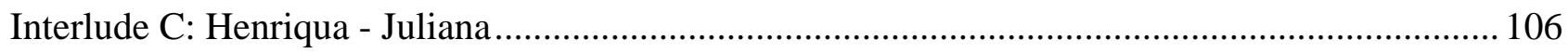

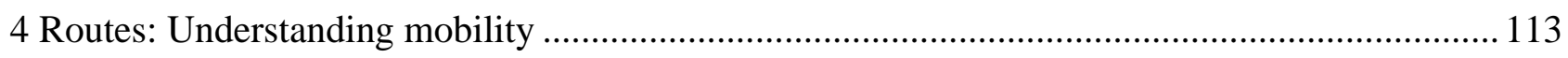

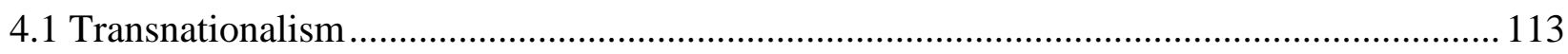

4.1.1 Transnational social spaces \& transnational social fields ........................................... 116

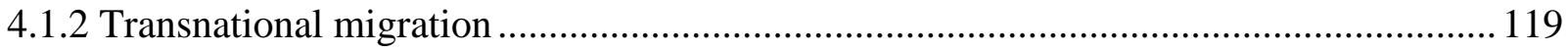

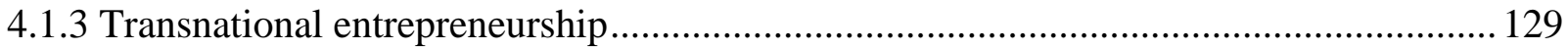

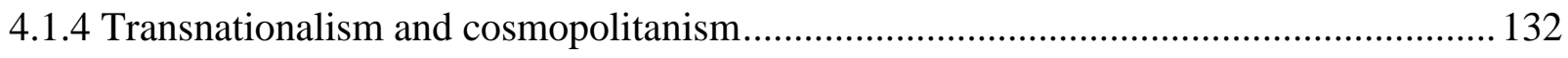

4.1.5 Synthesis: Transnationalism as an approach to study routes ...................................... 133

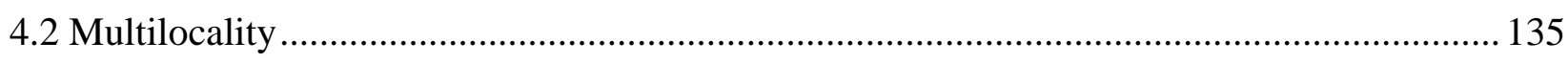

4.2.1 What does 'multilocality’ mean and imply? ............................................................. 136

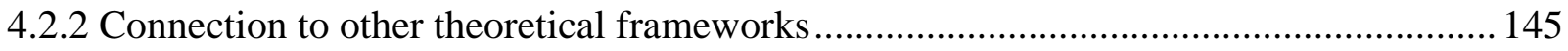

4.2.3 Empirical evidence in multilocality research............................................................. 146

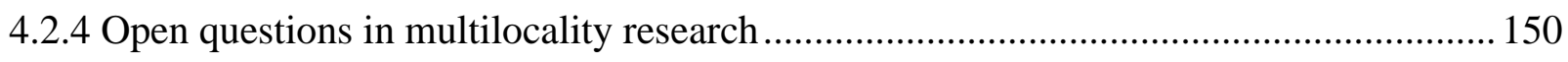

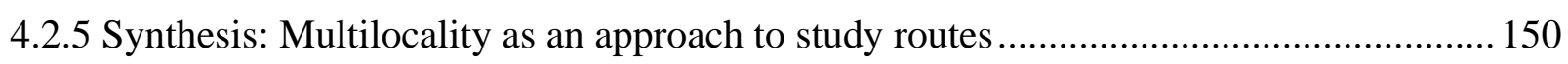




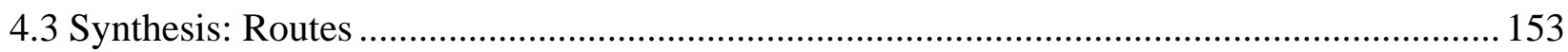

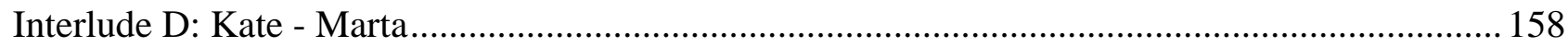

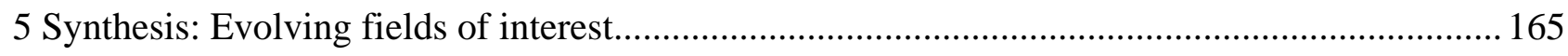

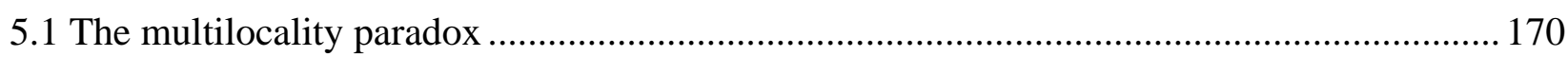

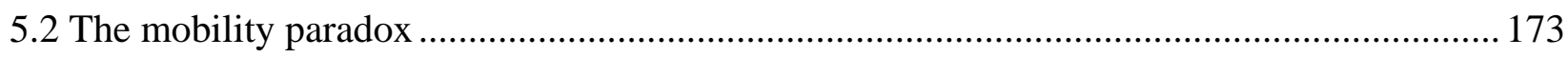

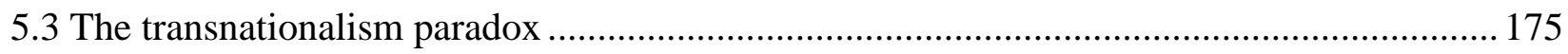

5.4 The character of 'place' in mobile creative knowledge lifeworlds................................... 176

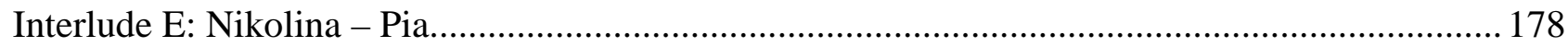

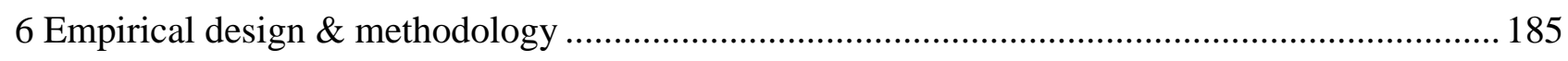

6.1 The phenomenological perspective: studying the lifeworld of others ................................. 185

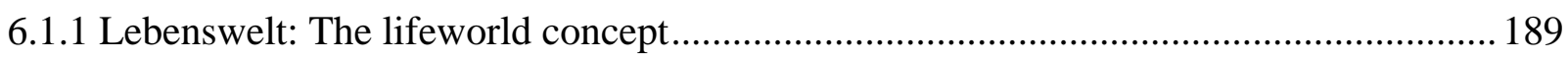

6.1.2 Appräsentation: The cognitive foundation of the lifeworld........................................... 195

6.1.3 Methodological implications of phenomenology ........................................................ 199

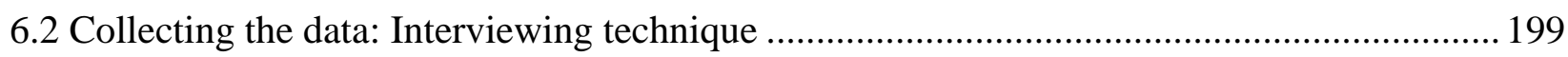

6.2.1 Mixed character qualitative interviews: Biographical narration \& problem-oriented

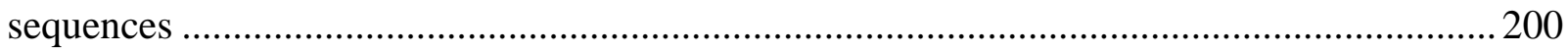

6.2.2 Mental mapping: illustrations of mental cartography .................................................. 202

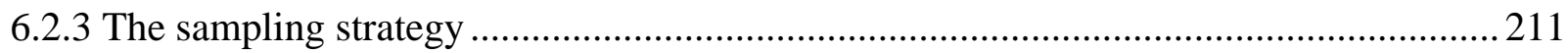

6.2.3 The interview situation: Conversational atmosphere ................................................. 213

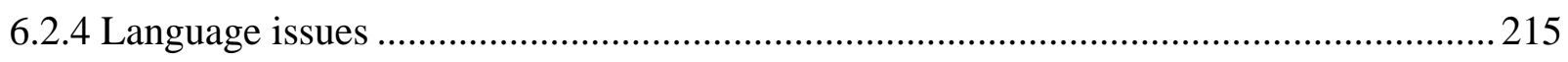

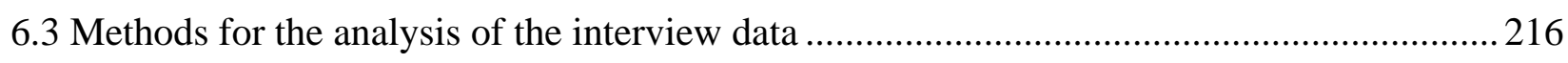

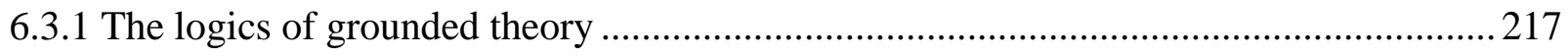

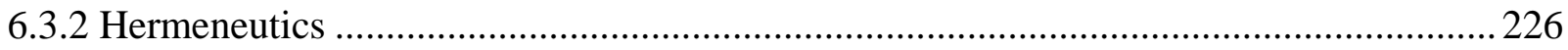

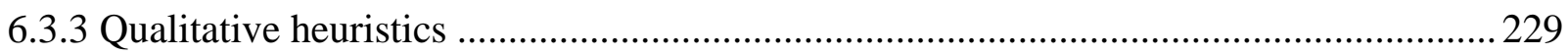

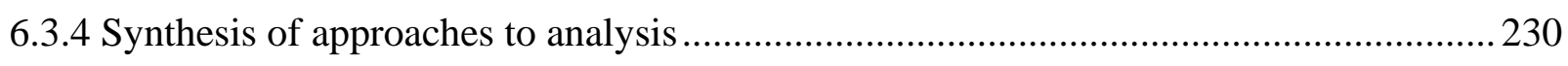

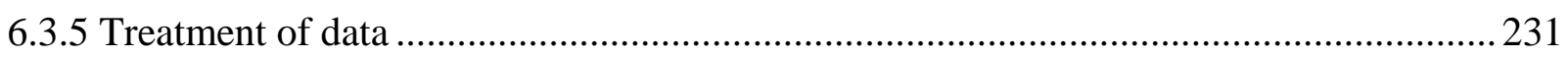

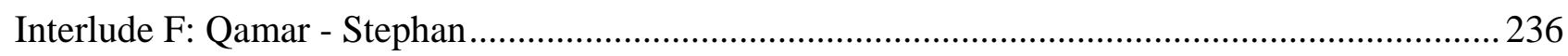

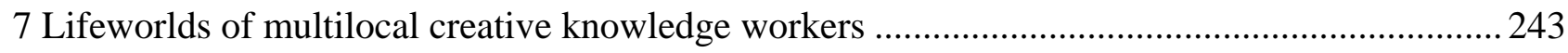

7.1 Places: The geography of my interview partners’ lifeworlds ............................................. 243

7.1.1 Mental maps: Marking one’s own territory .............................................................. 243

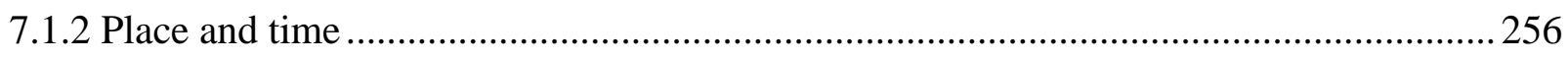

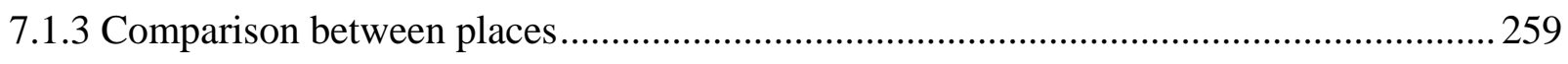

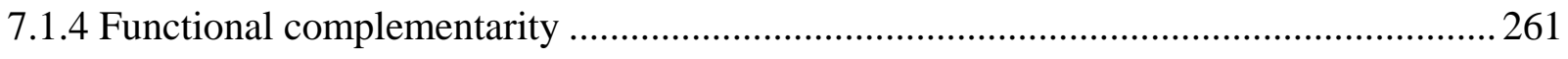

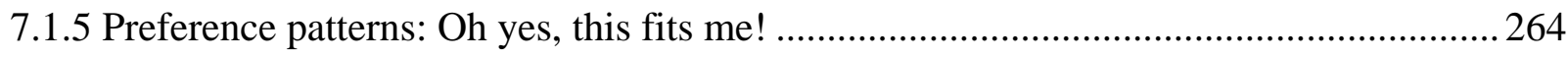


7.1.6 Places patterns between conscious decision taking and outcome of hazard 265

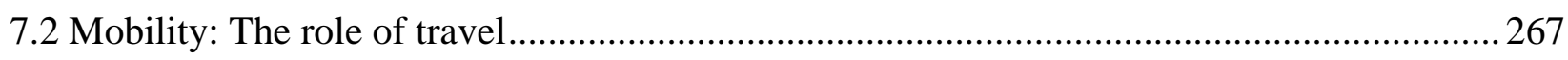

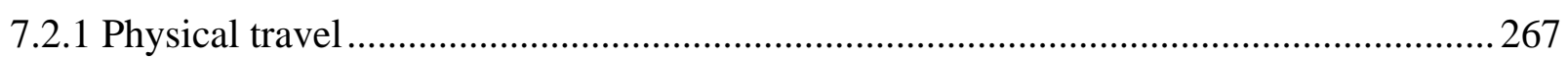

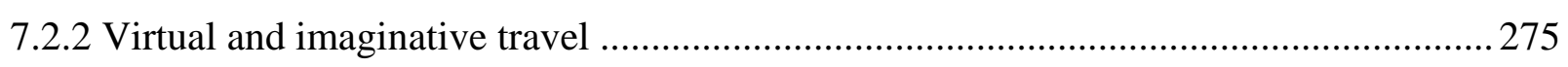

7.2.3 Polyperspectivity as an outcome of travel and the possibility to compare ....................280

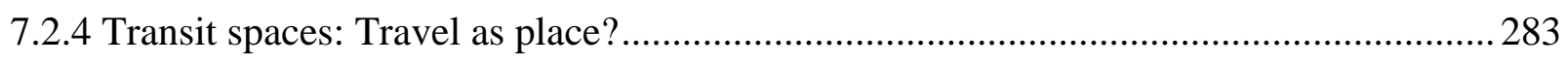

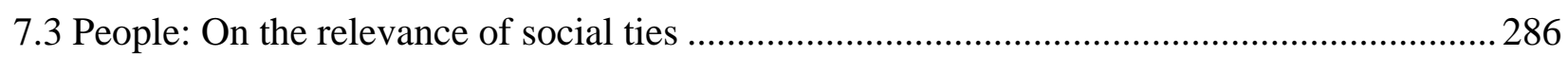

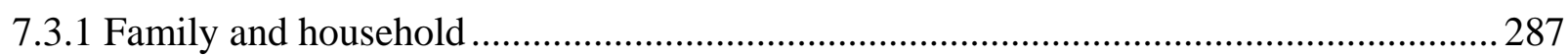

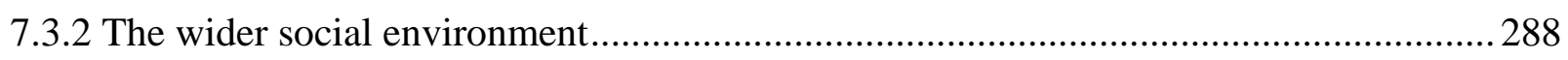

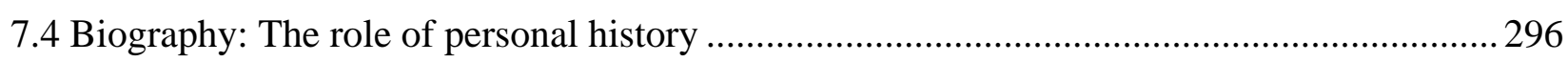

7.5 Profession: The role of multilocality in the creative industries ......................................... 299

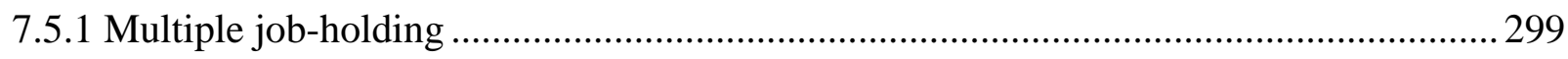

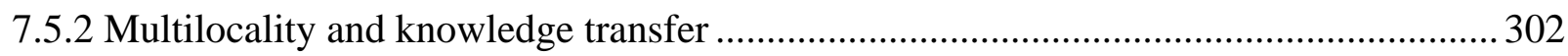

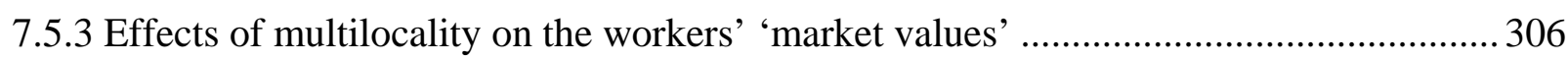

7.5.4 Labour market constraints and the forced nature of multilocality ................................ 310

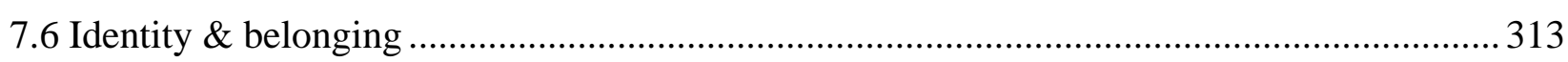

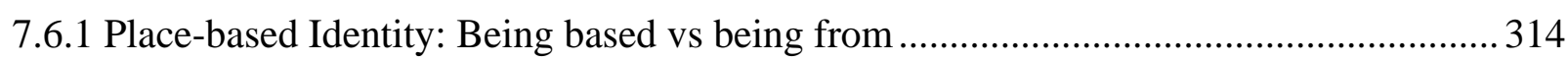

7.6.2 Geographic scales in identification: What about Europe? ........................................... 318

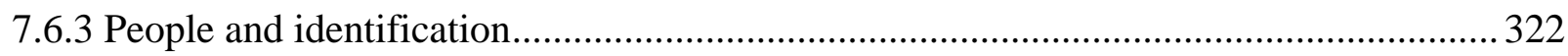

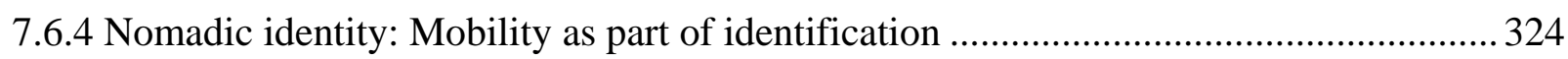

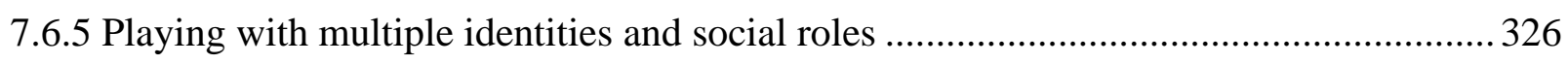

7.7 Resources \& Action: Doing multilocality in creative industries ....................................... 331

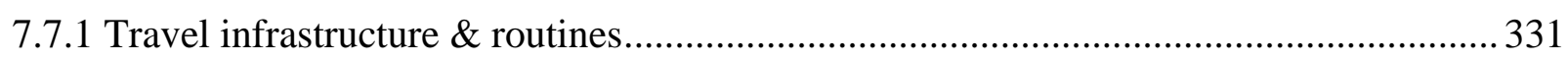

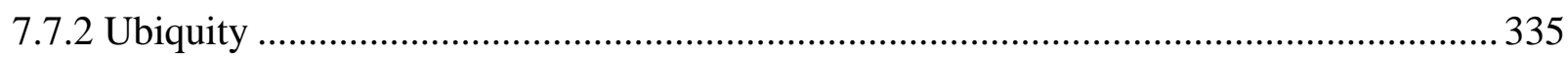

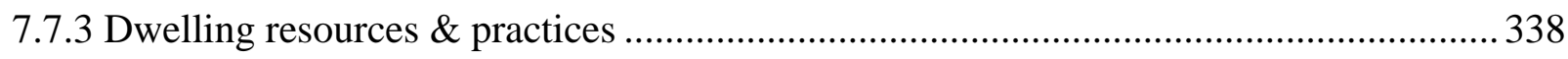

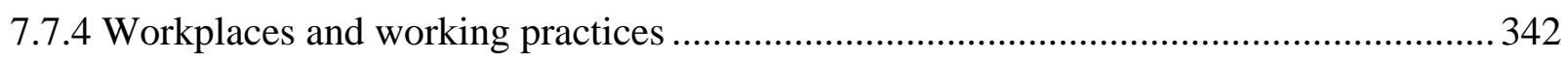

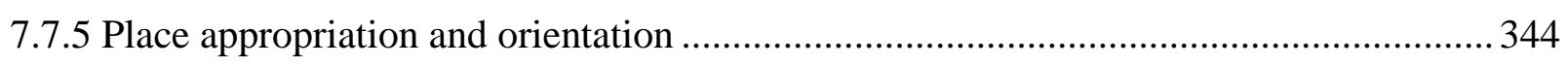

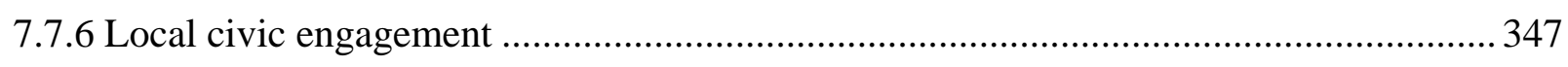

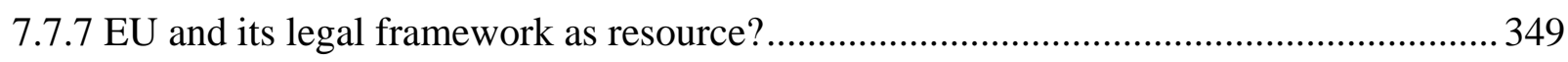

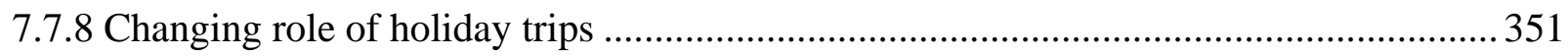

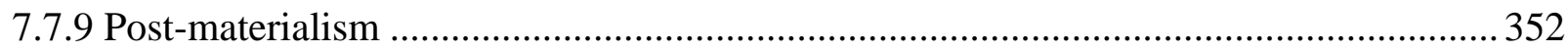

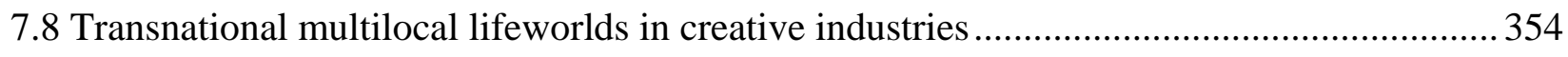

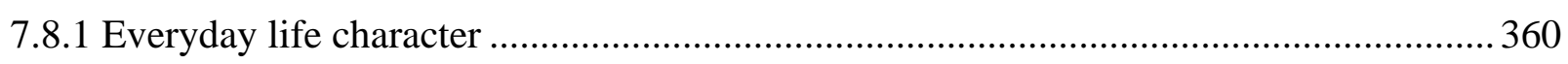


7.8.2 Temporality and future plans 360

Interlude G: Tjark - Valentin 362

8 Creative industries, roots, and routes: Discussion of the findings 369

8.1 Roots and multilocality: Implications for our understanding of belonging in the light of multilocal creative knowledge lifeworlds 369

8.2 Routes: Implications for our understanding of mobility in the light of locally embedded

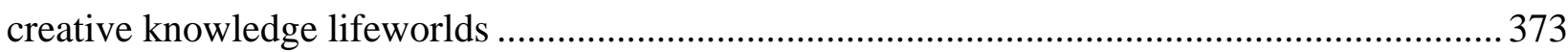

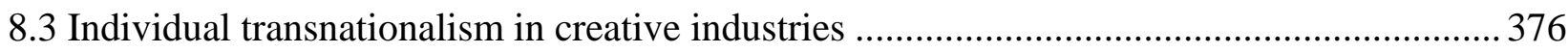

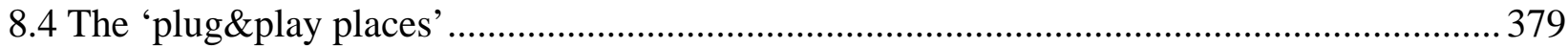

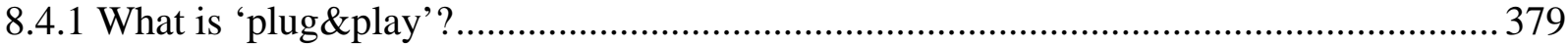

8.4.2 Analogies between the lifeworld and a computer system.......................................... 382

8.4.3 Configuration through place appropriation and orientation techniques ........................ 382

8.4.4 Coordination of the system and mediation between devices ........................................ 384

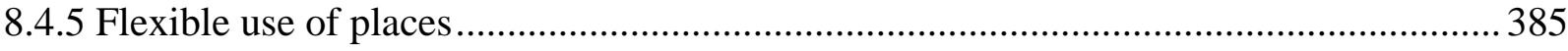

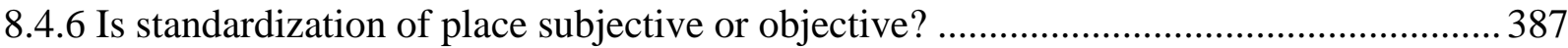

8.4.7 What's the value of knowing about the 'plug\&play' character of places in multilocal

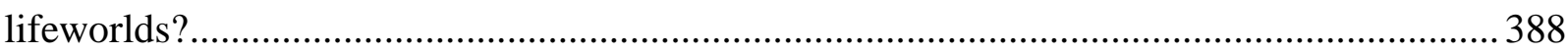

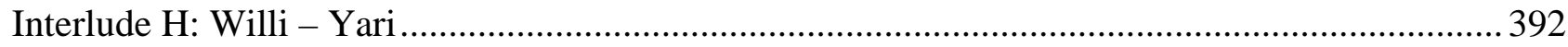

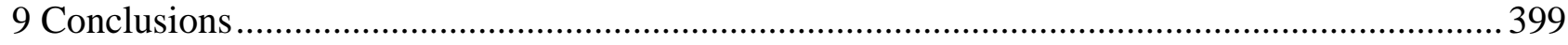

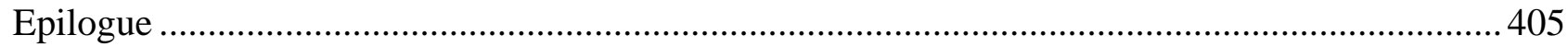

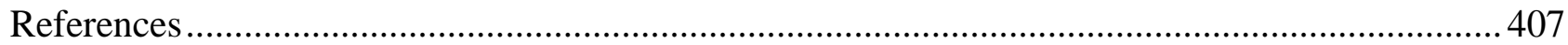

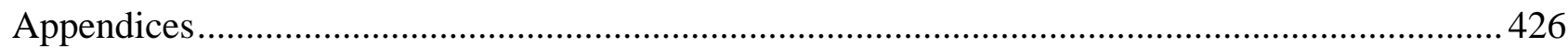

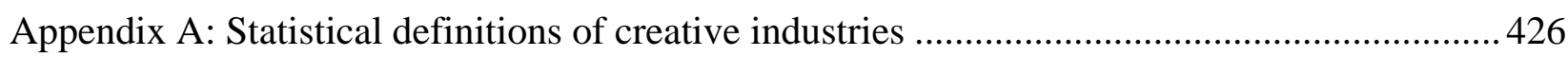

Appendix B: Background information on interview partners, interview situations and mental

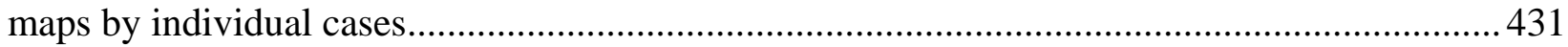

Appendix C: Exemplary interview transcript - Case of Xaverio ............................................. 472 


\section{Acknowledgements}

$\mathrm{A} \mathrm{PhD}$ thesis - though it is written and elaborated by a single scholar for proving their own scientific capabilities - is seldomly feasible without the support of others. The same accounts for my PhD thesis, for which a lot of people have provided the work context I needed.

Thanks to all of you for your support!

To Rosa and Fabian, for standing this time with me, in which I often was not there for you as a husband and father.

To my parents and my grandmother, who helped wherever they could during these years.

To Enzo, Serena, Matteo at the University of Milan-Bicocca, who have trusted in this PhD project and made it possible through the URBEUR scholarship.

To Michela, Francesca Buonocuore, Francesca Menichelli, and all other PhD colleagues; Marianna and Silvia, my former ACRE colleagues; and everyone else in the Bicocca cosmos, who helped me to orientate and accommodate in Milan and the Italian scientific world.

To Sebastian, Joachim, Thilo, Beata and all other colleagues at the Leibniz-Institute for Regional Geography (IfL), who supported me in going to Milan, and who made possible my fieldwork and the elaboration of the thesis through my flexible affiliation to the IfL.

To Gladys and Gianni, for hosting me in Milan; and to all my neighbours in the backyard in Sesto San Giovanni, who took care for me.

To all my interview partners, without whom it would have been very difficult to gather data on my topic.

To Stephanie Voigt, Stephanie Urban, Frank, Claudia, Rosa, who prepared draft versions of interview transcripts.

To Eric, who helped me with the cartography.

To Stephanie Urban, who had to read through all this violation of her mother tongue and who helped me to fine tune my English in this document. Thanks for being so sitting that through!

To Luc, who provided the illustrations at the beginning of chapters 2-9. Luc is a business consultant who is based in Lyon. I met him while spending a year in Grenoble. He now works for a known multinational business consultancy, but his deep passion is painting. His roots in Cameroon, he paints large scale tables which pick up African topics. I asked him to draw me some quick sketches to illustrate my chapters. Thanks for the sketches!

To Elli, alias lagqaffe, who has drawn the wonderful cover illustration. Elli was my roommate during my graduate years, and she has started painting while still finishing her studies in civil engineering. After that she decided that creative industries attract her more than the construction sector, and she now works as a self-employed graphic designer . Thanks for the cover! 


\section{Preface}

I met Aurélien for the first time in 2008, in the context of an FP6 research project which dealt with the development of creative industries in European metropolitan regions. He is a French artist who had recently moved to the city, and it was my task to collect information about his motivation to come to Leipzig, an eastern German city of about 500,000 inhabitants. The project's underlying assumption was that young creative professionals would immigrate from abroad to EU cities such as Leipzig in order to establish their new home base and help the city prosper after decades of difficult economic restructuring. Aurélien was not my first interview partner in this series of interviews with international creative knowledge workers who all helped in their own way to increase the diversity of Leipzig's social fabric. Yet it was a very astonishing interview which confused some of our theoretical assumptions and which made me curious to dig deeper. While a large part of the other interview partners indeed were based in Leipzig, Aurélien's situation was more complex: on the one hand he had taken the immediate decision to buy property when he came to Leipzig for his first visit. He hadn't known the city before, but in an affective moment and with a spirit of artistic experimentalism, he bought an old-brick building in a deprived residential area of Leipzig's deindustrialized and brown-fielded western quarters. I was convinced that something had gone fairly wrong at his former French place of residence, because he was so consequent in investing in a long-term project in Leipzig. When I had my first interview with him, however, it turned out that, in contrast, he had no aspiration to break up with his former French bases. Actually, his big project, of which the property in Leipzig is only a part, is to develop a certain exchange system for French and German artists. Therefore a central issue in his argumentation is to build a bridge between Leipzig and Nice, between Saxony and Brittany, as well as between Germany and France. In this interview, a numerous list of places showed up which he regularly visited, be it for private reasons, for promoting his big project, or for strengthening his artistic career by producing and exposing artwork in both countries. And even more interesting, in the narration the loci that were named revolved in a certain rhythm. All scales - the national in the form of France and Germany, the regional in the form of Saxony and Brittany, as well as the local in the form of Leipzig, Berlin, Paris, and Nice formed a coherent pattern for the localization of his current life. In addition to this pattern of localizing, or basing, one's own life in all of these places and on all of these scales, mobility between these places played an important role in his life.

This pattern - at which I could not look closer within the frame of the FP6 project - caused such a curiosity in me that I decided to make it my own research project. I wanted to find more such life trajectories, and I wanted to combine different individual stories into a more systematic analysis of how mobility and immobility go together in the emergent creative knowledge sectors. Luckily, I also found myself in the position being part of such a creative knowledge work environment. Through the structure of the FP6 project, I got in contact with colleagues at the University of MilanBicocca, who were open-minded and trustful enough to invite me to the URBEUR (Urban and Local European Studies) international graduate school. In combination with my second base at the Leibniz Institute for Regional Geography in Leipzig, I was offered a fruitful work environment in which to realize this project in an international setting. This thesis is the final product of the last years of research in the field of creative industries. However, working internationally includes some difficulties of their own nature. The URBEUR international graduate school at the University of 
Milan-Bicocca makes it possible for their PhD students to write their theses in Italian, German, French and English. I could have easily taken the comfortable way and written in my mother tongue of German, but I decided to write this thesis in English, which I consider to be one of the most important and useful technical tools for the international academic scene. I have come across a variety of German speaking scientists who told me about their hesitation to write in English. I can fully understand their arguments, but I think that writing texts in English is only fair to the international community in which many scholars try to write in English, too, even though it is not their mother tongue. Still, I used quite a lot of German literature to which I refer, and to a smaller extent I also used French, Italian and Spanish references. To improve legibility, I have decided to add my own translations in English in the text, and I have left all quotes in their original language in the footnotes. Therefore the reader who is not familiar with the original language can use my translations to get the point. Please be aware that these are my translations, and have not been checked by the original authors. The same accounts for quotes from my empirical material, where I also translated on my own.

Furthermore, given the latest problems with plagiarism in the scientific field - at least in Germany I decided to put all direct citations from other authors and from my interview partners into italics so that you will easily know what stems from my brain and mouth, and what not. I will also do so with my translations into English, where only the translation originates from my brain, while the original idea of the text itself comes from others. I also put non-English terms into italics for which I could not find the appropriate English vocabulary.

Finally, I would like to point to the 'interludes' between the individual chapters of this thesis. Putting interludes between chapters is not really a common procedure in scientific books. Yet, I was inspired by Adrian Favell's use of grey text boxes which contained extracts of his interview transcripts with his 'Eurostars'. When reading his book, I perceived these text boxes as quite entertaining. So, I also would like to enhance your pleasure in reading this thesis by inserting text boxes in the 'interludes'. There, you will find the summarized life stories of all my interview partners as well as their case specific maps which highlight the individual geographies of their multilocal lifeworlds. If you want to pause and distract from the themes of the chapters, you might take a circuit through these interesting life stories. If you prefer to come quickly to the end, you might skip these interludes without losing the train of thought.

I hope that you enjoy reading it. 


\section{Introduction}

'Multi-locality, a new reality.

A growing number of Europeans enjoy parallel lives - living in Prague and working in Paris or living in Vienna while having a girlfriend in Stockholm. Known as having "multiple habitats," the phenomenon has piqued the interest of sociologists.' Karolina Vránková, for Presseurop.eu/Respekt Magazine, 17 December $2010^{1}$

The quote by Karolina Vránková indicates that a new phenomenon is currently occuring within Europe, which has attracted the attention of social scientists all over the continent. Europeans are increasingly organizing their everyday lives across the permeable borders of different countries. This new form of mobility somehow does not fit into traditional concepts of migration. It is more short-term, less regular and predictable, more individual, and thus rather fuzzy at first glimpse. In order to shed light on this new oscillation between European cities and towns, a new strand of mobility studies has emerged in the German speaking scientific community which focuses on the development of the multilocality concept. Multilocality tries to integrate the contemporaneity of movement and localization. It tries to reconstruct the relation between the mobile individual to physical and social places and space.

In this thesis I will pick up this early work on multilocality, and I will study multilocal lifeworlds in the specific context of the creative industries. The notion of 'creative industries' arrived in Europe through the United Kingdom during the late 1990s. At this moment the deindustrialization in the UK had largely been completed, and the government was looking for new forms of employment for the UK workforce. The UK Labour Party started an initiative to enhance the development of the 'cultural industries' (Labour Party 1997). For political reasons, the early terminology of 'cultural industries' was later transformed into 'creative industries' (Garnham 2005). As such, it was used by the UK Department of Culture, Media and Sport (DCMS) to describe an emerging economic sector, which had not been systematically observed before. Scholars in urban studies and regional policy (Becattini 1991; Blackler 1995; Landry 1996; Helbrecht 1998; Smith 1998) quickly became interested in the topic of cultural and/or creative industries. Creative industries are considered very dynamic economic sectors and in the last two decades they have grown above average within the European Union.

Following the UK Labour Party's 1997 initiative on cultural policy and creative economies, the interest in the creative economic activities has risen in all EU member states. This development has

\footnotetext{
${ }^{1}$ http://www.presseurop.eu/de/content/article/433711-multilokal-total-banal (accessed 31 October 2012).
} 
been supported by EU policy. In 2000, the European Council approved the Lisbon Strategy. This policy document described several developmental goals for the decade until 2010. One of these goals was to make the EU the 'most competitive and dynamic knowledge-based economy in the world, capable of sustainable economic growth with more and better jobs and greater social cohesion' (European Council 2000: 2). The knowledge-based economy should be built on innovative companies which generate new jobs for all Europeans. In particular, the cultural diversity of Europe was mentioned as an asset for achieving this goal and founding a new source of wealth: 'Content industries create added value by exploiting and networking European cultural diversity.' (European Council 2000: 2). The eEurope Action Plans 2002/2005 accompanied the Lisbon Strategy with concrete measures to increase the Europeans' participation in life-long learning programmes as well as their use of the internet.

By the mid-term evaluation of the Lisbon Strategy, critics said that the Lisbon Strategy would most probably fail (cf. Blanke 2004; Social Platform 2004; Tausch 2006; Kok 2004). The final evaluations in 2010 were not much better. In the same year, the EU launched a new agenda for the current decade - the Europe 2020 Strategy - which looks largely like a small update of the Lisbon Strategy from ten years ago. With one out of three priorities named 'Smart Growth' (cf. European Commission 2010a: 5), it again makes the knowledge-based economy a central interest and expresses the importance of investment in research and development as well as education: 'Smart growth means strengthening knowledge and innovation as drivers of our future growth. This requires improving the quality of our education, strengthening our research performance, promoting innovation and knowledge transfer throughout the Union, making full use of information and communication technologies and ensuring that innovative ideas can be turned into new products and services that create growth, quality jobs and help address European and global societal challenges' (European Commission 2010a: 11f.).

Labour mobility has also become an important issue in both the Lisbon and Europe 2020 Strategies. Since the 1957 Treaty of Rome, people have been guaranteed the freedom of movement through all countries in the European Union (Vandenbrande, Coppin et al. 2006). This treaty includes the right to work in any other EU country, and it implemented strict rules against discrimination of EU foreigners in the national law of the member states. Up until today, a main goal of EU policy is to facilitate this cross-border labour mobility as a tool for intercultural exchange as well as against the negative impacts of demographic change and regional disparities within the EU. All EU-15 countries experienced a slight increase of the share of foreign born workforce originating from other EU-27 countries ${ }^{2}$. Analysing European Labour Force Survey Data, Bonin (2008: 17) observed that the share was $12.9 \%$ in 2006, compared to $10.6 \%$ in 1995 . According to Vasileva (2012), 6.6\% of EU inhabitants, or 33.3 million people, were foreigners in 2011. Only one third of foreigners, namely $2.5 \%$ of all EU inhabitants, are EU nationals living in a member state different from their

\footnotetext{
${ }^{2}$ EU-15 refers to the early member states: Germany, Belgium, France, Italy, Luxembourg, the Netherlands, Denmark, Ireland, UK, Greece, Portugal, Spain, Finland, Austria, and Sweden. The EU-27 group contains the EU-15 and the countries which accessed the EU later on: Estonia, Lithuania, Latvia, Malta, Poland, the Slovak Republic, the Czech Republic, Slovenia, Hungary, Cyprus, Bulgaria and Romania.
} 
own nationality. Thus, there are only about 12.8 million EU nationals who might be considered mobile in the sense that they migrated across national borders.

Even though these figures had increased from 2010 to 2011, comparison with other world regions reveals that Europeans are rather reluctant to move to another country or region. As Vandenbrande, Coppin et al. (2006: 71) remark: 'both geographical and job mobility rates remain substantially lower in Europe than in the USA (the usual point of comparison): about 32\% of the US population lives outside of the state in which they were born, while about $21 \%$ of the EU population has lived in a region or country other than their own (Krieger and Macías, 2006); the proportion of foreigners in the total population and in the labour force in the USA surpasses that of Europe (Turmann, 2004); average job tenure in the USA is lower than in any European country (Auer, 2005).' While Europeans generally have a positive opinion on the EU's Freedom of Movement and on going to live and work abroad, they actually do not put this attitude into practice. Figures for movement across borders still score low. Vandenbrande, Coppin et al. (2006: 71) say that 'only 4\% of EU citizens have ever moved to another country in the EU and less than 3\% to another country outside the EU. There is no indication of a mass migration in an enlarged Europe. Asked about their intentions to move in the future, only 3\% of all EU citizens indicate that they might move to another EU country in the next five years.' They conclude that cross-border mobility in the EU will remain low, because Europeans tend to be constrained by 'bound mobility' which is caused by relatively strong family ties, social networks, and - important to bear in mind - cultural contexts.

Particularly this last point is interesting in light of the creative industries debate. Based on the current understanding, creative work is a linguistically and culturally-bound market segment. Thus, at first glimpse it seems to contradict with extending cross-border mobility. Therefore the overall goal of this thesis is to add understanding to which role cross-border mobility might play in Europe's creative industries. What does it actually mean to creative workers, if they live a multilocal life in different countries instead of in a single one? While in the USA people move around within a homogeneous linguistic space and one national regulatory system, Europeans move between largely independent nation-states, with very diverse cultural and linguistic properties. For creative workers this might be both an obstacle as well as an opportunity. On the one hand, the difference between EU countries' cultures and languages could hamper the production of cultural goods and services through creative knowledge migrant workers. In certain creative activities, it might be difficult to translate e.g. 'sense' and 'humour' between different cultural and linguistic systems. It could also become a problem to export such cultural goods and services to other EU countries and their respective linguistic and cultural systems. For example, imagine a German advertising worker who is asked to do a funny TV advertisement in Spain: this poor person will have to learn the Spanish way of joking first. They might be able to learn and adapt to the Spanish context after a while and be able to do a good job in Spain, but then imagine that this German advertising worker comes back to Germany and would like to apply the things they learnt in Spain in a German context: I would suggest that they face the cabeza quadrada problem several times moving between the two countries.

On the other hand, being mobile and learning about a new language and culture could increase the job opportunities of migrant creative workers. Our advertising worker travelling between Germany and Spain might be valuable to companies in both Spain and in Germany, as they know how humour works in both countries. They have a specific ability to translate between cultural contexts, 
which equals an extension of market range for a company which formerly worked only in its own national cultural context. Neither of these possible scenarios has been studied thus far, and therefore it makes transnationally mobile creative knowledge workers an interesting social phenomenon. Different than technical workers, creative knowledge work cannot rely on a universal language such as work in mathematics or computer sciences. Creative knowledge work is linked to linguistic and cultural contexts, and this makes the study of mobility between such contexts an interesting concern.

In light of the two EU policies mentioned above - targeting knowledge-based economy and labour mobility - the aim of this thesis is to take a closer look at what it means to be both a mobile and a creative knowledge worker. Using a set of 25 narratives and problem-oriented interviews with mobile creative knowledge workers, I intend to shed some light on how these people deal with the continuous alternation of cross-border mobility and location in the cultural contexts in their everyday life. I will focus on the following three fields of interest, which will each contain a set of questions:

- The creative knowledge worker as a person on the move. What role does mobility play in the lives of creative knowledge workers? Does high mobility change the modes of identification of creative knowledge workers? Do they develop new hybrid identification patterns, including the mobility itself in different places, jobs and social roles? It is furthermore of interest how creative knowledge workers orientate in physical and social space: do they develop a routine of orientation in social and physical space? Finally, it is of interest what these people do while travelling. How do they perceive and use the transit time and transit spaces between their various places? Finally, how is cross-border mobility linked to creative knowledge work?

- The creative knowledge worker as a locating person. First, the coherence of the specific mix of places in an individual's life has to be studied: why are exactly these places part of their life and how do these places match with each other? Then the practice of locating oneself is an interesting topic: how do these people appropriate new places to make them a part of their own life? Do they develop specific routines to appropriate new places? How do they become members of local communities and cultural contexts abroad? Intuitively, I would assume that mobile creative knowledge workers are combining transnational and local communities. But I would suggest that this combination goes along with an inherent conflict between being in each locus an insider and an outsider at the same time. This also leads to the question of how the relation between scales (local, regional, national, supranational) is dealt with by these people. To which scale do they feel that they belong? Are places equally important, or are there certain hierarchies, and what roles do these spatial contexts play for creative knowledge production?

- The creative worker in relation to places. I suggest that places do not only influence the creative knowledge workers and their lives, but these workers also influence the places in which they live. What does the dialectic of physical absence/presence then mean to the places themselves? Here, I am wondering what type of impact it has on formerly rather stable, immobile and monolocal communities in cities and neighbourhoods if a certain part of a community is not present and available for communal activity on a regular basis. Are these mobile creative knowledge workers still available for the local community? This point 
is as particularly interesting as the creative industries literature suggests: creative knowledge workers have a strong influence on the production of space.

While much research has been done in recent years on the development of creative industries framed in national economies, few insights have been elaborated upon about the small group of creative knowledge workers who live and work abroad, or who commute across national borders. In particular, those creative knowledge workers who have not emigrated - in the sense of a unidirectional and permanent move - but rather circulate in short-term rhythms between two or more countries; they have not been studied, neither in migration research nor in the studies on creative industries. Therefore, this thesis will discuss the above mentioned questions with a particular focus on the intertwined character of creative knowledge work and cross-border mobility. In addition, the literature on creative industries often suggests that creative knowledge workers are a pioneer group which can have a strong impact on places. They are often attributed the capacity to re-define shared images and to initiate renovation processes in the places where they are present. This logic might be reasonable for monolocal and present creative knowledge workers, but what about those who alternate between places? Here again the issue of periodic alternation between absence and presence caused by their multilocality comes into play. So far, there are no empirical studies available which deal with this issue of mobile creative knowledge workers and their impact on places.

To answer these questions, I will first reflect the emergence of the creative industries phenomenon and I will introduce a theoretical figure of the creative knowledge worker based on a discussion of the literature on creative industries (chapter 2). Then (chapter 3) I will examine previous work which tries to understand immobility. In particular, the notion of home and identity seem relevant here. The newer concept of the psycho-social sedentariness will be presented, too. Subsequently, I will focus on two recent perspectives dealing with new forms of mobility. I will discuss the utility of the multilocality concept on the one hand and transnationalism on the other hand. Both are concepts to understand the current mobility patterns in reference to two different geographic scales: the local and the national (chapter 4). Chapter 5 will be a synthesis of the previous and separate chapters. It aims at specifying the central fields of interest for this research project. The empirical part of this thesis follows the logics of grounded theory, as there is very little knowledge about the links between transnational mobility and creative industries. The design of the empirical study must therefore have an exploratory character and will result in conclusive theoretical considerations. I will not test a theoretical model of hypotheses with my data, and for those who would expect that, I must apologize in advance for the disappointment. Rather, I intend to use a phenomenological approach to generate theoretical/conceptual insights for future studies. All of these methodological issues will be presented in chapter 6. Subsequently, I will examine the interviews with creative knowledge workers. I have tip-toed along a line of personal relations, using snowball sampling techniques, in line with paying attention to theoretical sampling. Using this method, I talked to 25 creative knowledge workers who have very distinct backgrounds. I talked with young and old, women and men, parents and those without children, homeowners and individuals who did not even have a registered residence for certain times. I talked with wealthy and less wealthy people. You can get to know these interview partners and their individual stories in the grey text boxes that I have inserted in the interludes between the coming chapters. These text boxes can be read independently of the surrounding text and they provide a dense reconstruction of the persons' 
individual stories. You will still be able to follow the argument of the thesis if you decide not to read them, but you might definitely miss some interesting stories. Using the qualitative heuristics approach, I concentrated my analysis of the interviews on the common, the combining, and the shared issues across the individual stories - chapter 7 will give space to that. In form of a comprehensive overview I will sketch out how the border-crossing multilocal lifeworlds of my interview partners look like. Furthermore, I will have detailed look at these 25 creative knowledge workers mental maps and I will use these to reconstruct the geographies of their lifeworlds. In chapter 8, I will then reflect and discuss my empirical findings against existing theoretical concepts. As a lot of things emerged from my interviews, I decided to select certain issues only. Here, I will also present an empirically derived understanding of 'place' in the context of multilocality, which I call the 'plug\&play places'. Chapter 9 will contain a conclusion and an outlook to future research questions. 


\section{Interlude A: Aurélien - Dirk}

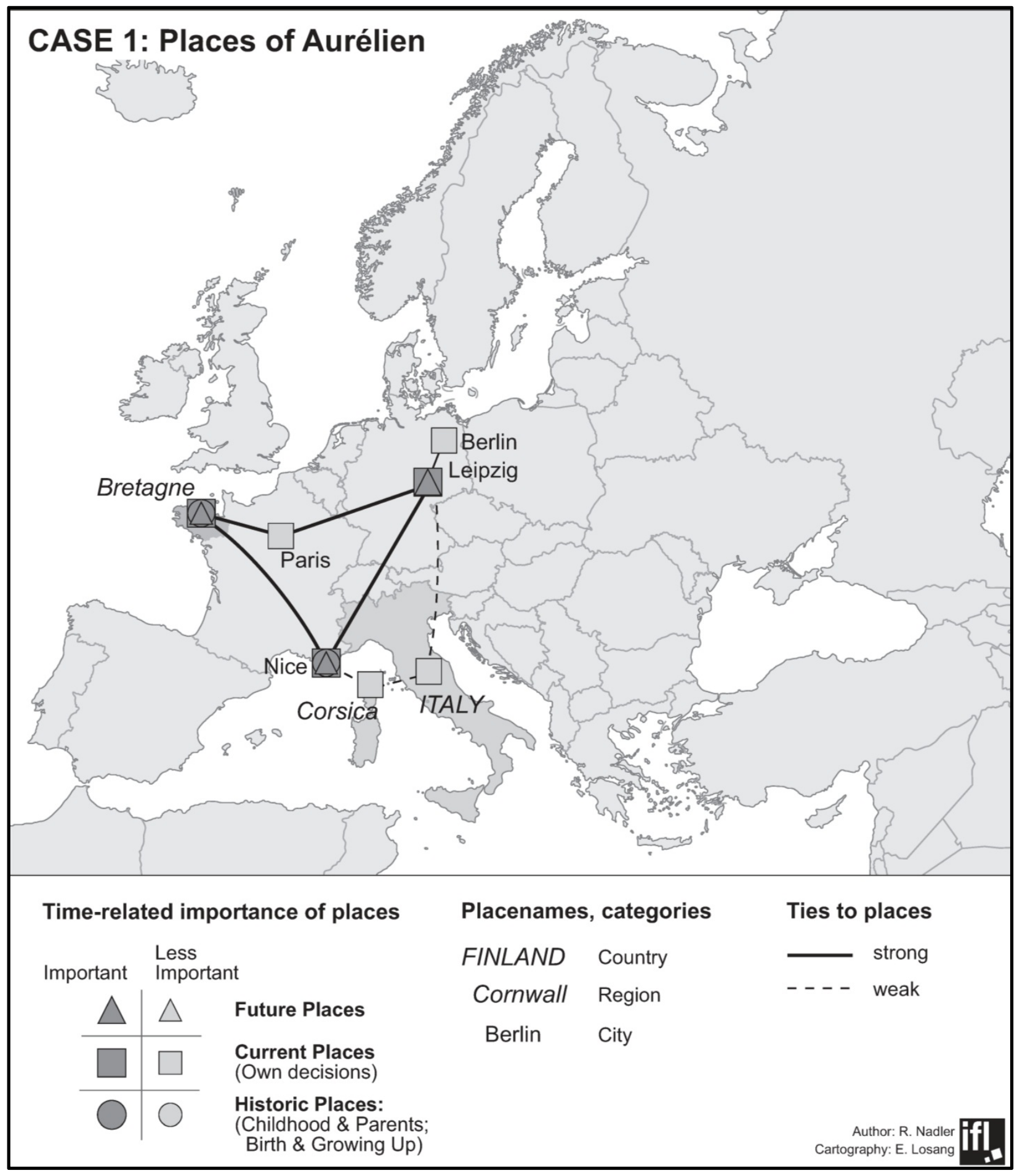




\section{Meet Aurélien}

Aurélien is a French artist in his early 30s. He was born in Nice, but he grew up in Brittany, and only returned to Nice for studying at the École des Beaux Arts. Paris is an important location to him. A lot of friends and his brother are living there, and he often goes there to exhibit art work or to meet some artist colleagues and to work on joint projects. After graduation in Nice he came to Leipzig, by hazard, as he says. He decided to settle more long-term in Leipzig and to start anew after having broken up with his ex-girlfriend. He bought an old apartment block with the money he had made from selling his apartment in Nice. This old building is now his new project in terms of a private person renovating an apartment, but also in terms of an artist renovating other apartments in it to be used for Artist Residency for German, Polish and French artists. He also founded an association which should form as legal framework for fundraising.

He also has a lot of friends in Berlin, who he visits on day trips. At the moment of the interview, Aurélien was dating a German opera singer, who also lived in Leipzig. Aurélien is working as a freelance artist in the field of visual arts and installations. Besides selling his art work in galleries in France, he earns some money with scholarships for organizing cultural and artist exchanges between France and Germany. He also points out that being based in Leipzig now raises his market value in his gallery in Nice, where he sells his artwork. Being from elsewhere makes him exotic - a positive aspect of a transnational multilocal life.

Frequent travel between the France and Germany is important to him and is the basis of his work. Aurélien likes travelling; it is inspiration and discovery for him. On the other hand, he has a strong sense for property, which works as a mental anchor in his nomadic life. Brittany is also always with him when he is in Leipzig or in Nice. Brittany represents the place of his roots, and works as a place of mental resource to him. Thinking of Brittany lets him forget about the social pressure by which he is surrounded when he is in Leipzig, in a foreign language and cultural setting.

Aurélien perceives himself as a French artist who is currently at home in Leipzig. He also refers to his Brittany roots, which gave him his special relationship to property, in the sense of a fisherman, who has his home harbor, but needs to go out to sea for fishing. He uses this metaphor to describe how he perceives his project in Leipzig and his frequent travelling to France and other places.

Aurélien's major professional aim is to establish a European network of artists who support and maintain each other, something like a European social security for artists. His Leipzig-based association addresses this issue by organizing exchange projects for European artists. Artist Residency is the main tool that he uses as support to other transnational artists. He offers to let colleagues to stay in his apartment block. Apart from this European level activity, his association is also part of local artist networks in Leipzig which deal with the development of the city and the specific industrial neighbourhood in which his house is located.

In the future, Aurélien plans to do more projects in Nice; he would like to re-establish his links to the Nice art scene. He also wants to address the official partnership between Saxony and Brittany for interregional artist and educational exchange projects. Finally, in addition to his Nice-Brittany-Leipzig triangle, he has started to build up satellite projects on the Isle of Corsica, where he organized a Residency project with local artists. His personal wish would be to have kids and found a family later on, which could go together with becoming more monolocal again, but he is not excluding staying multilocal for the coming years.

Aurélien's metatopic is the mental aspect that comes along with being multilocal across borders. 


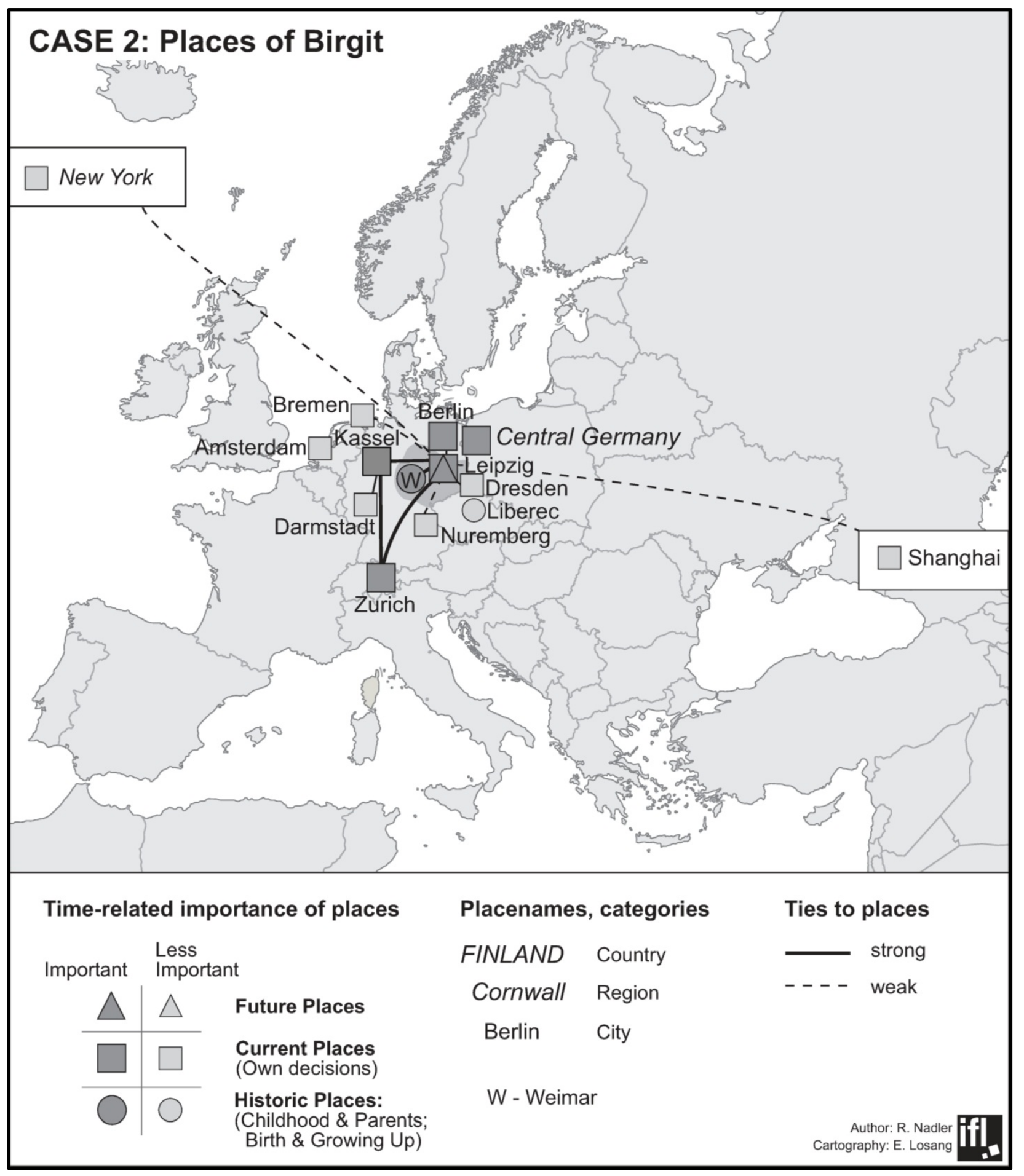




\section{Meet Birgit}

Birgit is a German architect and urban planner in her early 50s. She currently has different jobs: first, she is a part-time professor for urban and regional planning at Kassel University; second, she owns and manages a private planning office in Leipzig; and third, she occasionally works as a freelance consultant for municipalities throughout the world (Zurich, Berlin, New York). Together with her partner, a Leipzig-based photographer, she lives in a loft apartment in one of Leipzig's renovated industrial buildings. The apartment has a nice rooftop terrace with a view of a canal and other renovated factory buildings hosting restaurants and galleries. She considers this apartment her base. Leipzig is the centre of her lifeworld, while Kassel and the university job is rather a satellite project.

She had brought her elderly parents, who were in need of care, from Thuringia's countryside to a retirement home in Leipzig. Therefore, her private life is now concentrated in Leipzig, but she also actively constructs everyday life during travel and in her other locations. She always stays in the same hotel room in Kassel, chatting smoothly with the hotel manager as well as the employees of the university's cafeteria. She always takes the same seat on the high-speed train between Leipzig and Kassel, doing her preparation work on the way out, and reading yellow press on the way back. She buys the same international newspaper wherever she goes. She calls her partner twice a day, to say good morning and good night. Sometimes her partner takes her by car from Leipzig to Kassel, in order to spend more time together. She visits her mother in the rest home weekly. She meets a friend in Berlin at her hairdresser's monthly. Having a positive attitude towards her mobile life, she explains that everyday life character is still a necessity to her in order to feel at ease with being on the move.

Being on the move is something she learnt from her mother, who had to escape with only one bag from the family house in Liberec, Czech Republic, during WWII. Her mother explained to Birgit that the most important thing which one can own is one's brain. Thus, Birgit has a pronounced distance to physical property. She appreciates her own apartment, but she would not seek for more materiality as it becomes a burden in multilocal arrangements. She is in possession of a Bahncard 100, which allows her to use all of the trains in Germany without buying tickets. This Bahncard 100 is a metaphoric thing as well, as she would love to enjoy other services which function in providing access.

Birgit currently has no time to engage in civic projects. She is a volunteer in business associations related to her own professional world, and in her scarce leisure time she prefers to spend time with her loved ones and to remain in tranquility, reading a book or staying at home. She also mentions that all of her recent vacations took place at home in Leipzig due to having a stressful professional life. During her time off she likes to stay in one place without travelling.

As she's getting older, she would like to become more monolocal. She often feels exhausted by the frequent travelling. In order to protect her health, she wants to reduce travelling, and she also wants to become more connected with the Leipzig community, from which she expects a reinforced feeling of belonging. She could imagine starting gardening or writing books. Birgit's main issue is the construction of everyday life and atmosphere in a multilocal life. 


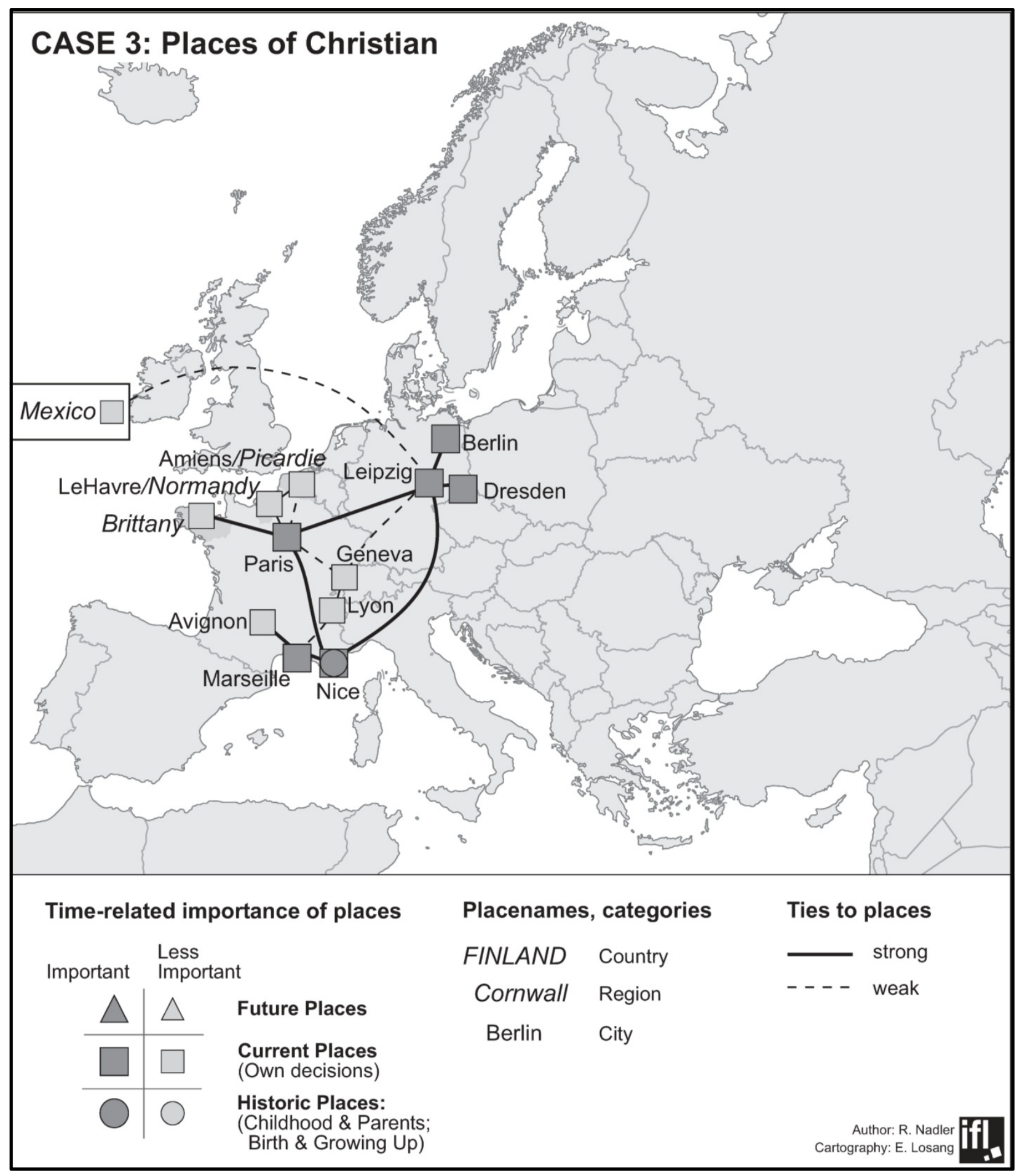




\section{Meet Christian}

Christian is a French artist in his early 30s. He was born in Paris, but his parents frequently moved around in France, so he does not feel that he has his own home that he could consider in terms of representing his roots. He currently lives and works in Leipzig, in Aurélien's apartment block, helping him with the renovation of his building. Christian feels that Leipzig has now become a temporary home base to him, but he regularly has to go to Dresden, too. He lived there before, and is still doing the $2^{\text {nd }}$ year of a post graduate degree (Meisterschule) in Fine Arts at the Academy of Fine Arts Dresden.

Currently he has a scholarship from the Deutsch-Französische Jugendwerk, which allows him to travel and buy material for his artwork, but he cannot live from his artwork - he mainly writes poetry and designs performances - and does all kinds of bread and butter jobs (modeling, construction work, translating, interpreting etc.). However, he enjoys these jobs, because he does not have to produce art according to a buyer's taste. And second, he appreciates getting in contact with very different kinds of people. He does not like having one closed social group (e.g. only artists) to which he belongs. He needs changing social and physical environments as inspiration to his artwork.

Christian is a passionate networker who loves to bring people together from different countries, places and social backgrounds. Mixing different groups means creating mutual social interest within the society. He likes the idea of opening people's minds by confronting them with each other and the unknown. He himself is a person who is always the stranger, he says. He hasn't ever been accepted as local in any of his locations. Thus, he can fully understand the problems of those who do not feel understood in society.

Christian travels frequently between Germany and France. Berlin is an important place to him, as it is an international art market in proximity to Leipzig, where he is currently trying to establish collaboration with more famous artists. In France he mainly collaborates with other artists in Nice and Marseille. He is currently organizing five exhibitions in Germany and France. He has lived in many different places in France, where he still goes to see old friends (Lyon, Picardie, Normandie, and Avignon). He also goes to Geneva, Switzerland on a regular basis as a close friend of his lives there. He only has an apartment in Leipzig; in all other places he mainly stays with friends or in artist residences. Christian also worked in Mexico where he organized an exhibition with local people.

Travelling, to him, means to live a nomadic life. And thus, the main theme for his artistic production is the Cabane, the hut. A hut is a metaphor for installing oneself wherever one arrives, using the material which is present at this place. This also accounts for social relations, as Christian says. Christian would consider himself as a type of nomad as well.

In the future Christian can imagine becoming more monolocal again; he has friends who live very routine lives, with family and property, and even though he doesn't want to live like that at the moment, he does not exclude it for later periods in life. The main issue in Christian's multilocality is the idea of networking and mediating on the gathering of people and ideas from different places. 


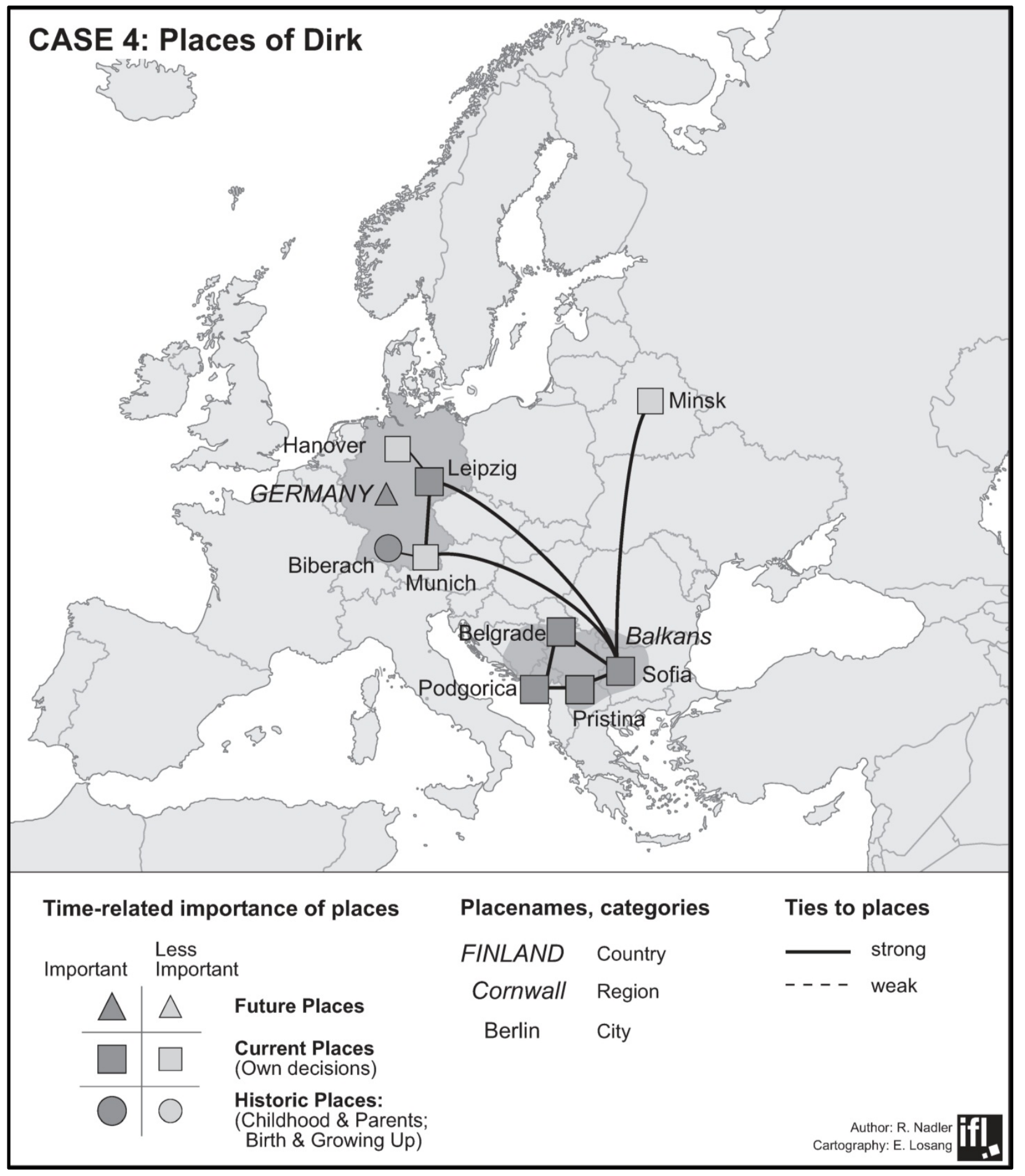




\section{Meet Dirk}

Dirk is a German Slavicist and journalist in his late 30s who grew up in Biberach, a rural town in southern Germany, and then studied in Munich. He is currently working part-time as a researcher for a Leipzig-based research institute, but he lives and does his empirical field work in Bulgaria, as the research project is concerning Bulgaria. He further works as a freelance consultant in the field of adult education policy for the EU in the Balkans (Serbia, Montenegro and Kosovo) and in Belarus. His third field of activity is writing his $\mathrm{PhD}$ thesis, tutored at the University of Leipzig by a professor from Brunswick, about the role of priests for regional development in Eastern Europe. He owns an apartment in Munich, where he is also registered as a resident, but he sublets the apartment to other people, as the factual centre of his everyday life is now Sofia, where he has rented another apartment and where he lives with his Bulgarian girlfriend. He only works when he is in Leipzig and thus he does not need his own apartment. He stays at an apartment of a journalist colleague, who had moved to Berlin without giving up her Leipzig base. In Germany, he also visits his parents in Biberach and friends in Munich.

For work, he frequently travels to the Balkans, where he assists local and regional governments in the field of installing an EU standard adult education system. Here, he cooperates with a Serbian colleague who now operates from Hanover in Germany. He also does this type of work in Minsk, Belarus. Minsk became part of his life when he met a Belarusian teacher who took the same language course with him in Bulgaria. After they became friends, she also became his colleague in his Minsk operations.

He developed an interest for Eastern Europe early on, when floating down the Danube River in 1989, directly before the end of socialism. He passed Hungary, Slovakia, Romania, and Bulgaria, and he liked Bulgaria the most. He then started to live there as an exchange journalist for the German-speaking edition of the national Bulgarian radio channel. By then, he still spent half time in Sofia (the radio job), and half time in Munich (the PhD thesis). Now Sofia has become the centre of his world. This also changed his perception of his southern German home region. He now appreciates it for its natural beauty, the landscape, the clear rivers and lakes. On the other side he now perceives Bulgaria differently. Whereas it was a place of imagination and positive associations during his adulthood, today he knows about the country's major problems such as corruption, ecological problems and the social polarization between rich and poor people. Thus, Dirk's story illustrates the comparison of places and the dynamic character of place perception.

In the future, Dirk would love to become more monolocal and re-settle in Germany with his Bulgarian girlfriend. He wishes to have his own children, and to be closer to his elderly parents again. Dirk's main issue is the way in which multilocality allows him for comparison, and in particular comparison between eastern and western Europe. 


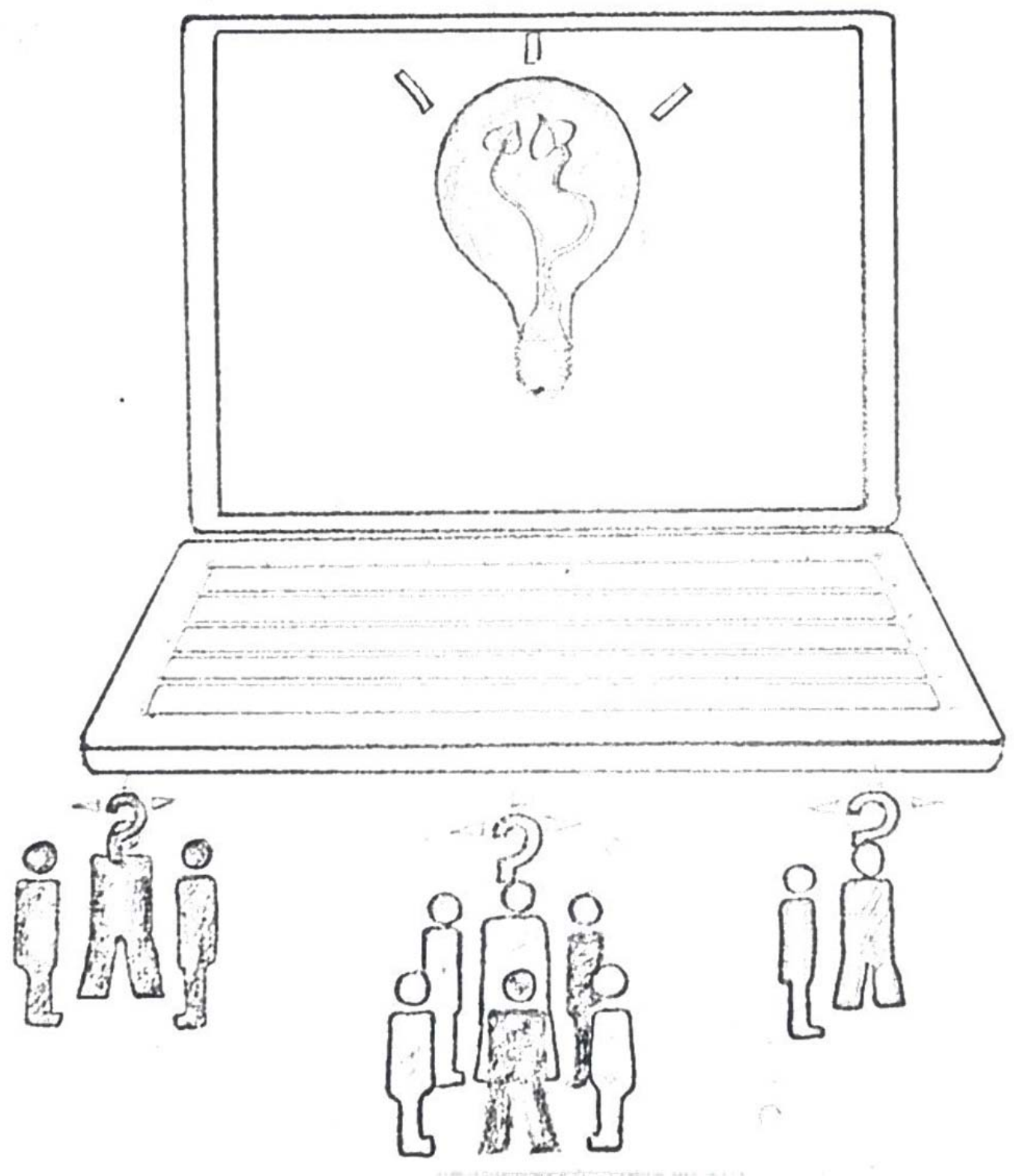




\section{Creative industries}

Scholars of regional policies (see Florida 2002, 2008; Landry 2000; Scott 2006), urban sociology (see Jacobs 1970; Sennett 2006, 2008; Castells 2000a, 2000b; Sassen 2002; Häußermann 1987; Häußermann \& Siebel 2004; Bürkner 2005) and economic geography (see Lange 2007; Musterd \& Deurloo 2006; Musterd et al. 2007) point out that knowledge-intensive services and the so-called 'creative industries' are estimated to be the new urban economy of the knowledge or information society, in other terms also conceptualized as the new accumulation regime structuring our economies (see Brenner 1998; Marston \& Smith 2001). US regional economist Richard Florida comments (2002: xiii): 'Human creativity is the ultimate economic resource. The ability to come up with new ideas and better ways of doing things is ultimately what raises productivity and thus living standards.' Ross also observes (2008: 32): 'Creative industries policy is embraced as the anchor of regional development by governments around the world on the lookout for a catch-up industrial plan. In the business world, creativity is viewed as a wonderstuff for transforming workplaces into powerhouses of value, while intellectual property [...] is increasingly regarded as the 'oil of the $21^{\text {st }}$ century'. These two statements indicate that creative industries are discussed as the next growth machine for matured economies in the post-industrial countries. Throughout the first decade of the new millennium there was a strong presence of the topic in the media, policy debates and academic studies. I will use this chapter to clarify what exactly this sector is about. First, I will contextualize the advent of the creative industries. Then I will present different ways to define these economic activities. Subsequently, I will characterize the creative industries' work life and its impact on the lives of creative knowledge workers. Finally, I will also sketch out my own understanding of a creative knowledge worker, and I will derive some conclusions for the empirical interest of this thesis.

\subsection{The advent of creative industries}

During the 1980s, the UK lived through the Thatcher Era with a neoliberal political agenda. As mentioned in the introduction, the post-industrial UK was looking for a future economic source of income to secure long-term growth. In this process of economic restructuring, the Labour Party (1997) initiated a debate about the role of the 'cultural industries'. When the Labour Party regained the political power, it introduced the term 'creative industries' as used by the UK Department of Culture, Media and Sports (DMCS) (cf. Garnham 2005). Shortly afterwards, the concept of creative industries also became an issue in continental Europe. Additionally, the popularity of the 'creative industries' as a research topic was boosted by the US regional economist Richard Florida (2002) and his popular book 'The Rise of the Creative Class', which found wide reception.

There are various developments in recent history that have helped the emergence of creative industries and the attention they receive today. Many drivers are mentioned in literature - amongst others reduced working time and the increase in leisure time; improved education; growing real income; change in consumption patterns; increase in spending for cultural services and products; etc. (Competitiveness Report 2010: 191). Florida integrates the emergence of creative industries into a sequence of historic innovations, following the former periods of the agricultural era, the 
emergence of mercantilism with trade and specialization, the industrial era, and the organizational age with the extension of bureaucracy (Florida 2002: 45-66). However, I will concentrate on the relation of information and knowledge as well as the role of the internet and modern information and communication technologies (ICT); I think that it provides a systematic understanding of where the discussion around creative industries comes from.

\subsubsection{The information society and the creative industries}

Since the 1960s scientific scholars have been studying the transformation of society into something new called information society. The sociologist Jochen Steinbicker (2001) has provided a comprehensive overview of this topic. In this and the following subchapters, I will mainly refer to his description of the emergence of the information society and the knowledge economy, which he presented in his 2001 book entitled 'Zur Theorie der Informationsgesellschaft: Ein Vergleich der Ansätze von Peter Drucker, Daniel Bell und Manuel Castells.' As the title suggests, he compared three of the main scholars of the information society, Daniel Bell (1976, 1980), Peter Drucker (1969), and Manuel Castells (1997, 2000a, 2000b).

Steinbicker understands Drucker's approach as orientated on a management perspective as common in economics. Drucker aims at providing management professionals with a manual for dealing with the growing importance of information and knowledge for economic production. Steinbicker here refers to Drucker's 1969 book 'The Age of Discontinuities'. In this book, Drucker's central argument is that knowledge is the main resource for the economy in the information society. Steinbicker critically remarks that this macro-level economic approach neglects the micro-level perspective of the individual people. Individual concepts and notions are important for a sociological study of the information society, as Steinbicker says. Therefore he criticizes Drucker's work for remaining incomplete as it is an economic approach, too much focused on knowledge as a resource for the economy.

There are also important elements in Drucker's work that could help to develop a theory of information society. First, there is the idea of an expansion of education within the society which causes a structural change. Then, the boundedness of knowledge in a person's mind is important; this implies the central role of the knowledge worker. The person themself becomes more relevant as they apply knowledge to knowledge - the central activity in the knowledge economy. Furthermore, Drucker concluded that the growing importance of knowledge as a resource, ongoing technological innovation as well as the expanding innovation systems will fundamentally change the education system and scientific research.

Drucker also identified an inner contradiction which is inherent to the knowledge worker and which is typical for the information society. Knowledge workers have the power over the knowledge they have in their brains, thus, they start to feel independent and self-defining, but still they do not exert power over the productivity of this knowledge. This power remains with the employing or commissioning organizations. While knowledge workers consider themselves as autonomous and intelligent, they still depend - similarly to a factory worker in the industrial age - from their company (cf. Steinbicker 2001: 21-48 \& 109-111). 
Steinbicker then turns to Bell's approach (Bell 1976, 1980), which he considers more analytical and a more sociological approach compared to Drucker's. As Steinbicker mentions, Bell's central texts concerning the information society are 'The Coming of Post-Industrial Society. A Venture in Social Forecasting', from 1976, and 'The Social Framework of the Information Society' published in 1980. Two central axioms characterize Bell's thoughts: first, the central position of codified theoretical knowledge, and second, the shift from the industrial production of goods to service sector dominance. Bell added the centrality of the bureaucratization of science to an understanding of information society. This bureaucratization causes the opposition of the professional ethos of scientists against bureaucracy. His notion of a 'third infrastructure', which is represented by the ICT, is also helpful, as Steinbicker remarks. However, Steinbicker remains critical as he sees many of Bell's ideas falsified through actual developments in time. For example, Bell's idea of a changing character of work towards a playful communication between interacting human beings has not come true. A large share of knowledge work is still based on interaction with computers and machines. Also his idea of a communal society, in which social equality has risen again, has not become reality as of today (cf. Steinbicker 2001: 49-77 \& 111-113).

Castells' approach consists of an empirical diagnosis of various developments in current society. He merges his observations into a theoretical draft of the information society which is based on networks. Steinbicker refers to Castells' trilogy, 'The Information Age: Economy, Society and Culture' which was first published from 1996 to 1998 (here editions from 1997, 2000a, 2000b). Better than Bell, Castells achieves an integration of social change mechanisms in his conception of the information society. He explains social change with the interaction of productive factors (such as knowledge in the form of knowledge workers) with the production system. According to Castells, two developments are at the centre of the advent of the information society. First, networks become the dominant form organization. Technological and social networks interact and host the main part of communication and information flows. Thus, a culture of 'real virtuality' will make participation in the technology-based, virtual 'spaces of flows' necessary in order to be part of society. Second, the role of identification and identity once again becomes more important. In particular for the individual, a self-positioning in relation to the predominant networks is important to find a place in society. There is also the emergence of new social movements which oppose themselves to the networks and their oppression. For Castells, this last point represents the new way of social emancipation. In addition, Castells witnesses a solution of traditional class struggle. Knowledge workers increasingly are paid in shares and options of the companies they work for. This means they become co-owners of their own production system. Critically, Steinbicker remarks that Castells' blueprint of an information society cannot claim to be a social theory. It neither provides useful analytical tools nor does it explicitly develop the role of technology. In addition, many concepts of Castells' remain unclear and ambiguous (Steinbicker 2001: 79-108 \& 113-115).

According to Steinbicker, these three authors have delineated helpful contours of current societal developments, but discussions concerning the notion of the information society remain dissatisfying for those who are looking for a new and encompassing social theory. Steinbicker looks for similarities in the three authors' literature on to which a theory of the information society might be grounded. He identifies seven comparable characteristics of the information society which could be found in the three approaches (2001: 109-124). 
First, the information society is based on a new economic system in which knowledge and innovation become the main drivers of economic wealth - as opposed to labour, money, land, or machinery in the agrarian or industrial societies. This includes a change in socio-economic structures. Bell and Drucker suppose that in particular the education sector and scientific research will become the central axes of the information society. Steinbicker, however, is doubtful about that point. He rather thinks that education and research will lose autonomy and become more dependent from the financial capital of private companies (Steinbicker 2001: 115).

Second, the extended availability and capacity of information and communication technology (ICT) is a central asset of the information society. ICT changes the organization of production and consumption. For Castells, ICT plays a major role in the development of the 'space of flows' and a 'real virtuality'. Furthermore, ICT exhibits a strong influence on the new ways of communication, defining the character of so-called 'network companies'. They also cause an informational paradigm in the field of knowledge work. For Bell, ICT is the reason for the emergence of 'intellectual technology'. Drucker argues that ICT helps a restructuring of social and economic organization. All three approaches share the vision of ICT-based economic and social activity as a central aspect of the information society (Steinbicker 2001: 116).

Third, there is a pronounced change in social organization itself. Vertically integrated and hierarchically organized structures have become less important, and social interaction has become organized in more horizontal and flexible ways. Drucker and Castells explain this change independently of the ongoing digitalization and extending use of ICT. For them, organizational change occurs out of the necessity for flexibility, adaptive capacity (life-long learning) and efficiency. As an output of this process of change, Castells mentions, the network organization will be the dominant type of organizations. Drucker speaks of creative and knowledge-based organization. Somewhat opposed, Bell designs his draft around the observation that cultural change precedes organizational change. The changing cultural norms and values put pressure on hierarchical companies and organizations to develop a more social character, and to put less emphasis on economic aspects. Bell stresses the central and axial position of publicly financed scientific research and theoretical knowledge for the economy (Steinbicker 2001: 116f.).

Fourth, in the working world, knowledge becomes the central input. Thus, knowledge workers are strategically the most important group of workers today, and their share on total employment is rising. Again Bell argues differently from Drucker and Castells. For Bell, the central shift in the working world is from secondary sector production (industrial goods and manufacturing) to tertiary production (services). Drucker and Castells rather argue for a changing nature of work itself, irrespective of economic sectors. For Drucker, work is more and more defined by the application and creation of knowledge. This means that formal education is important, less for its content, but rather for teaching people techniques for how to continuously acquire new knowledge. This capability for life-long learning is the central resource for the knowledge workers. Drucker argues that the change in the working world is caused by the expansion of the positive evaluation of formal education in post-industrial societies. Thus, it is first of all a cultural change, concerning norms and values related to a higher evaluation of education. This shift in values and norms is in line with the argumentation of Bell, who is convinced that the information society comes along with a rising importance of post-material values, such as liberalism, focus on education, health, quality of life, culture, etc. Just as Drucker, Castells stresses the role of 'adaptive capacity' in informational work 
environments. However, Castells does not speak of wider shifts in value systems, but only the notion of the culture of 'real virtuality' and the arising bottom-up social movements are an indication of cultural change, yet without direct links to the working world. Steinbicker summarizes that the three approaches share the idea that the growing role of knowledge, technological necessities, and communication will alter the formerly hierarchical forms of control and organization in the working world (2001: 117f.).

Fifth, there is a transformation in the factors that define social stratification. Today, the access to knowledge and education is more and more the central element for a person's position in society. While Bell and Castells speak of talent and skills as the central criteria for being in an advantaged position, Drucker argues that independent of talent and skills everyone has the possibility to become a knowledge worker through education. The growing role of the education system is central in all three approaches. This includes that social position will most probably be defined by access to and use of the education system (Steinbicker 2001: 118f.).

Sixth, the state loses power in regulating the social system. The power relation between the economy and the (nation-)state changes in favor of the global economy. Here, the three authors show significant differences in their interpretations, as Steinbicker remarks. Drucker expects the formerly central position of the state to be devaluated through the rise of competing organizations (e.g. NGOs, multinational corporations). The role of the state will be reduced to a coordinating function. For Bell, the political elite will remain central and will develop new modes of control, being internally diversified through particularistic lobby groups. By contrast, Castells thinks that societal organization will be based on networks, which itself will exhibit power within distinct social spheres. A new 'networking logic' will develop which will determine society. Important networks such as the global financial system, global media, networks of political institutions as well as multinational corporations will form 'spaces of flows' around the globe, in which communication and information flow is based on ICT (Steinbicker 2001: 119).

Seventh, new social conflicts will arise. There will be less of an industrial conflict between labour and capital, but emerging tensions are expected in the field of the service industries, given the growing divide between high-skilled knowledge workers and non-skilled/low-skilled service workers (Steinbicker 2001: 9-11). Bell mentions that a conflict between meritocracy and populism might evolve. Bell and Drucker also envision a conflict between knowledge workers, their individualistic ethos, and the ongoing bureaucratization of the knowledge economy, in particular in the field of scientific research.

Steinbicker concludes his comparison of the three approaches with the observation that these still do not suffice to form a coherent theory of information society. According to him, merging the three approaches is not possible, as individual concepts within them even argue in opposite directions. Yet, two main aspects are similar. First, productive factors change. Knowledge becomes the central resource for production. The relationship between science, state and economy will change, and the economy will be differently organized than in industrial production. Second, the working world will change dramatically. Knowledge workers will be confronted with inner conflicts between their selfunderstanding (autonomy and self-actualization) and their role in the economic system (functioning in bureaucratic companies). Even though he criticized Bell's idea of the more humane character of knowledge work, Steinbicker still believes that in the future work will be more playful. This is 
because it will be happening more between people. In industrial society, work was often an interaction between human beings and large machinery. In the information society, it has become more communicative and interactive in human-human relations, even when mediated through ICT. These two aspects might form a frame for a theory of information society, which still needs further empirical elaboration (Steinbicker 2010: 121).

It becomes clear in this brief documentation of the discussion around the notion of the information society that this concept is clearly linked to the central role of information, knowledge, and innovation. Dealing with and developing new knowledge, trading information and processing information are all activities that have become more and more important in the post-industrial society. The main supporters of this change are the knowledge workers.

How is this discussion on the information society linked to the notion of the creative industries? Looking at recent policy documents, creative industries and creative workers are now described as fulfilling a bridging function between art, philosophy, science and business (cf. European Commission 2010c: 5) and to linking the extension of the information society (European Commission 2010c: 2). We see that creative industries and creative knowledge workers are understood as a central part of the information society which puts the first of the seven elements of the information society that Steinbicker outlined into practice: creative knowledge workers assist in linking the innovation and creation systems closer to the economy and private capital. In addition to being a pioneer group, producing innovation and information, they are a central element in the emergence of what is called information society.

Creative knowledge workers could therefore be considered a specific group of knowledge workers which are part of the advantaged group of high-skilled knowledge workers. Looking at the literature that stems from scholars of the information society, on the one hand, and scholars of the creative industries, on the other hand, we find many similarities in the description of the workers. One can also understand the information society as one of the sociological anchor points of the creative industries. The study of creative industries is often done by regional economists and economic geographers who take an economic perspective. They look primarily for the impact of creative industries on regional development, using a focus on economic and financial aspects (e.g. growth rates, employment, productivity and turnover). However, concerning the social character of creative industries, much has already been announced in the work on the information society (e.g. growing role of knowledge, information, communication; the centrality of ICT; network organization; postmaterial values), thus it is very helpful to look at the sociological results stemming from the 'information society sphere' in order to better understand what creative industries are like.

\subsubsection{The knowledge economy and the creative industries}

Apart from the wider societal shifts to an information society, there is also literature which deals with a stronger focus on the changes taking place in the economic system. Steinbicker's 2001 text book is also helpful recapitulating the emergence of the knowledge economy as a scientific topic. He describes that economists began early to deal with the role of data processing and information for late-industrial production. (Machlup 1962) was the first to speak about the 'knowledge industries'. Fritz Machlup's study 'The Production and Distribution of Knowledge in the United States', from 1962, is considered the ground-breaking oeuvre for the development of studies around 
the 'knowledge economy' (cf. Steinbicker 2001). Machlup tried to quantify how much of the US gross domestic product (GDP) was based on knowledge work. His basic distinction was that of 'knowledge producing activities' and 'non-knowledge producing activities'. Machlup identified a $29 \%$ share of GDP related to the knowledge economy for the US in 1958. He also observed an increase from 11\% of knowledge work in 1900 to 32\% in 1959 (referring to activities according to his definition as knowledge producing).

While Machlup also included the domestic and private work (e.g. of parents who teach their children) or on-the-job training, in the 1970s Porat excluded such non-market activities. Based on Machlup's ideas, Porat divided the economy into four sectors: extraction (agriculture, mining, and resources), producing industries, service activities, and information services (cf. Steinbicker 2001: 15-17). Porat and Rubin then introduced the important novelty (Porat \& Rubin 1977) of distinguishing between a first and a second information sector. The first information sector consists of companies which primarily produce and sell, or trade with information. The second information sector contains the organizational entities of large industrial corporations which have to do with the processing of information as a service to other industrial activities within the same organization (e.g. the advertisement department of a car manufacturer). Porat reports a $46 \%$ share of GDP for the US in 1967 being spread to $25 \%$ in the first information sector and to $21 \%$ in the second information sector. Both authors, in their time, predicted an extensive growth in the knowledge economy, which ultimately did not happen as quickly as expected (cf. Steinbicker 2001:16f.).

Also Japanese authors - with the advent of information's mass distribution through radio and TV dealt with the knowledge economy (cf. Morris-Suzuki 1988). In 1963, Japanese author Tadao Umesao equally developed a four sector model of the economy, including an information sector. Umesao was convinced that the mind would become industrialized and future society's main activity would consist of the production of symbolic value. The Japanese government also commissioned several studies in the 1960s which dealt with the advent of the information society. The notion of 'information society' itself dates back to Yujiro, who suggested that the growing wealth in advanced industrial economies would lead to a growing demand in emotional goods orientated on fashion, lifestyle and quality. According to him this was related to a growing share of information in total production costs of goods (e.g. for research and development, design, marketing) (cf. Steinbicker 2001: 17f.), thus the Japanese authors observed the link between societal and economic changes early on.

Given the rise of the knowledge economy as a wealth generator of the information society, we should also consider the relation of the knowledge economy with creative industries. First, empirical data suggests that creative industries are an important part of the wider knowledge economy. Labour Force Survey data indicates that creative industries have the highest proportion of employees with tertiary education (ISCED levels 5 \& 6): ranging up to $57 \%$ as compared to $26 \%$ of the total EU economy (European Commission 2010b: 199). Changing the perspective to occupation-based numbers, the share of tertiary education even increases to $78 \%$ in creative occupations as compared to 24\% in non-creative occupations (European Commission 2010b: 202f.). I would interpret these numbers as a first evidence for the close interrelation between the creative industries and the education sector. Creative workers consume knowledge and information before entering as well as while working in the creative industries. This knowledge in fact is sold by the educational institutions, which represent a central sector as they trade knowledge within the 
knowledge economy. By contrast, the highly-skilled creative workers 'create' and produce new knowledge, which is sold to the educational sector. Both sectors are strongly interwoven and interdependent. Creative industries produce knowledge, symbolic value and emotional goods, which are then distributed to the wider economy and society through the educational sector.

Another point is the high share of labour costs in total value added in the creative industries. Creative business activities' main production asset is human capital, the creative worker themself, who is used to provide a service or good. Thus, staff costs range the highest on the input side. In other words, labour productivity is generally higher than in the total economy. This again indicates the central role of the creative industries as a part of the knowledge economy (cf. European Commission 2010b, 2010c).

UK data also shows that creative businesses are more innovative than other economic sectors. This refers to different aspects of innovation in the fields of products, processes, and wider encompassing innovations. While 32\% of the UK's creative businesses have introduced new products in the last three years, it has only been $21 \%$ for the rest of the economy. In addition, $16 \%$ of creative businesses made their production processes more innovative as compared to $11 \%$ of all other businesses. Distinguishing between the more relevant new-to-market innovations and less relevant new-to-firm innovations, the difference becomes even more pronounced. Additionally, wider innovations were presented by $40 \%$ of creative businesses and $29 \%$ of all other businesses. Another empirical source, the Community Innovation Survey (CIS) suggests that the main drivers of this innovativeness are the software activities in the creative industries. They have a high output in innovative software products and licences, but advertising and architecture are also drivers of innovation through continuously creating new service models. The European Commission, after reporting this data, conludes that creative industries 'engage in more innovation-related activities, undertake more $R \& D$, invest more in training, and spend more on design than firms in other sectors' (European Commission 2010b: 218ff.). Evidence can also be found for the strong linkages between universities/scientific research and creative industries, with creative industries evaluating fundamental research more important than many non-creative businesses. Finally, also customers and clients are considered an important source of knowledge to be used for innovation and for breaking up with traditional hierarchies between experts and non-experts. In particular, software and architecture firms tend to use these two sources of knowledge in an extensive way (European Commission 2010b: 221).

We can see here that creative industries are prototypical for the character that is attributed to the knowledge economy. They are dominated by service sector activities that require high-skilled labour. And they are based on the elaboration of knowledge through creative processes. Furthermore, they often produce symbolic content, which serves as an intermediate input to industrial companies who want to sell physical goods.

\subsubsection{Internet, ICT and the creative industries}

According to Steinbicker (2001), early scholars in the 1950s tended to think of the extension of automation as well as information and communication technologies (ICT) as a deliberation from the social oppression through labour. The future vision entailed a large share of people working in the service sector, in which automation and rationalization did not seem to be possible. On the other 
hand, there was an infinite variety of new and innovative services that might be invented and made into tradable goods. The emergence of a more leisure-based society was also circulating in that time. Steinbicker (2001: 13-15) critically examines the supposed link between ICT and the emergence of the information society. The rapid integration of the notion 'information society' into political and scientific debates was not caused by, but accompanied the rapid extension of ICT availability, he says. Steinbicker mentions that there were already discussions about information society - e.g. in the 1960s with early micro-electronics as well as mass TV and radio access before ICT became available for the widespread public in the late 1970s. On the other hand, the ICT development path could be traced back to the early $19^{\text {th }}$ century, when no one spoke of 'information society'. Therefore, there is a temporal coincidence of ICT's and information society's advent. But there is no one way causal relation between the two. The link between the two is always dependent on social structures and their capacity to include ICT in societal organization, as Steinbicker mentions.

Though we do not live in a world in which we are released from work, as envisioned in the 1950s, I would confirm that the development of ICT was a milestone for the advent of creative industries. While the technological development and the affordability of ICT itself enhanced the efficiency of the creative production process (cf. Sinan et al. 2012), it was only with the emergence of the internet that new business models could be developed. Today a large part of successful and creative businesses are based on internet-mediated access to clients and customers.

We can find some arguments for this in the Competitiveness Report by the European Commission (2010b) as well; in different ways the internet has been important to creative industries. First, the development of the technology itself - including software development - and the extended provision of ICT infrastructures allowed for an easy-to-access virtual market place. Therefore, on the sales side, the internet is an important issue for the distribution of creative goods and services. In the Competitiveness Report, the European Commission mentions: 'ICT and the internet are leading to new forms of distribution, more choices for consumers, and a more efficient production process' (European Commission 2010b: 191). New business models are evolving in the field of ecommerce. For instance, the advertising sector has gone through massive digitalization, with online advertising summing up to at least $20 \%$ of the EU market. The music industry has been equally affected by the expansion of digital markets, with decreasing sales in traditional retail stores, but an increasing and very dynamic online market (European Commission 2010b: 199). These developments are strongly based on the growing accessibility of clients and customers through the internet. More people tend to be online; they stay on the internet longer; and they have better broadband access. In 2009, an IFPI study even found a correlation (at the level of 0.8) between the availability and use of broadband internet connections, on the one hand, and the size of creative industries in different EU regions, on the other hand (International Federation of Phonographic Industries/IFPI 2009; cited according to European Commission 2010b: 199). Thus, the technological possibilities coming along with ICT seem to be exploited in efficient ways through the actors of creative industries. The European Commission writes (2010b: 218): 'Current means of mass (re)production, mass consumption, and commercialization of artistic/creative content have been made possible mostly by technological advances in the fields of information and communication technology (Cunningham et al. 2004). In fact, creative industries are intense users of ICT innovations in particular, as well as other new technologies. For instance, digital 
technologies and compression methods for audio and video signals that allow efficient storage and rapid transmission with little loss of quality have created new, low-cost means of sales distribution.'

While $17 \%$ of non-creative businesses operated their own e-commerce solutions by 2000, it was already $30 \%$ of creative businesses. Creative industries are early adopters of ICT innovations, as W@tch Survey data from 2005 indicates (European Commission 2010b: 219). Also in the Green Paper on creative industries, the European Commission stresses the central role of ICT, providing 'new opportunities for creators to produce and distribute their works to a wider public at a lower cost, independently of physical and geographical constraints.' (European Commission 2010c: 6).

Recently, the UK based Interactive Media in Retail Group (IMRG) published its 'B2C Global eCommerce Overview 2012', in which it estimates that the global volume of e-commerce was US\$ 961 billion in 2011, which equals a growth of 20\% from the year before. The USA represents the world's largest national market, summing up to US\$ 297 billion. China had a last year's growth rate of $130 \%$, and the EU represents the largest market worldwide, accounting for a total volume US\$ 307 billion. Furthermore, the study suggests that 2.2 billion people use the internet worldwide. The number of internet users is rising constantly, and will reach $50 \%$ of the world's population in the coming years ${ }^{3}$. The US Census Bureau published another study, calculating estimates based on annual business surveys. Here, it becomes obvious that business-to-consumer (B2C) e-commerce only accounts for $10 \%$ of all US e-commerce. Thus, the business-to-business e-commerce volumes are still much larger. In the US economy, e-commerce accounts for $16 \%$ of all value of shipments, sales, or revenues ${ }^{4}$. These numbers highlight that there is a growing sales potential in virtual markets for all types of creative businesses.

On the other hand, the internet has also become a large source for market relevant information and knowledge and thus a productive factor. A study done at the University of California Berkley's School of Information Management and Systems in 2003, estimated that each person on this globe produced about 800 Mbytes of new information in that year. The annual growth rate of information of mankind was 30\% between 1999 and 2002. Furthermore, the authors found 750 Gbytes of information circulated through instant messaging each day, equalling 274 Terabytes a year; emailing accounts for even 400,000 Terabytes a year; 92\% of new information is stored digitally ${ }^{5}$. Even though there might be a lot of methodological issues in these figures, they still provide an estimation of the relevance of the internet as a source for information.

Third, the internet allows for a flexible organization of work. With internet-based communication tools (virtual hard drives, chat rooms, online telephoning) the production of creative products and services could more and more be organized without physical co-presence in one place. This does not mean that face-to-face contacts become unimportant, but teams can be managed in a more flexible and independent manner.

\footnotetext{
${ }^{3}$ http://imrg.org/ImrgWebsite/User/Pages/B2C_Global_e-Commerce_Overview_2012.aspx (accessed 31 October 2012) http://www.internetretailer.com/2012/06/14/global-e-commerce-sales-will-top-125-trillion-2013 (accessed 31 October 2012)

${ }^{4}$ http://www.census.gov/econ/estats/2010/2010reportfinal.pdf (accessed 31 October 2012)

${ }^{5}$ http://www2.sims.berkeley.edu/research/projects/how-much-info-2003/execsum.htm\#summary (accessed 31 October 2012)
} 


\subsubsection{Information, knowledge, and creativity}

At this point it might also be helpful to come back to the different semantics of the three words knowledge, information and creativity, which are at the core of the issues I have discussed above. So far, I have used these terms in an unreflected way; I have done so for practical reasons, in order to illustrate how the ideas of information society, knowledge economy and creative industries can be linked. Still, I think we must come back to these three basic notions in order to understand how the focus on creativity and creative production evolved from the remaining two notions.

Abel (2008) provides a detailed discussion of the notion of 'knowledge'. According to him there are different forms of knowledge which can be differentiated from each other. Very generally one could say that knowledge consists of: 'everyday habits, customs, competencies, and practices as well as science, technology, and institutions of the modern civilized world' (Abel 2008: 11). However, this general understanding does not help very much understanding the character of knowledge. Abel then explicates that there is a narrow and a broad way to speak of knowledge. The narrow way refers to knowledge 'obtained by a methodologically well-regulated procedure bound to justification, truth, and verification' (Abel 2008: 12). This includes the criteria that a person is able to communicate about how this knowledge came into existence, and that its generation is intersubjectively verifiable. The broader definition, by contrast, refers to all everyday life knowledge (know-how) which helps us to orientate in the world and to react appropriately within any given situation. Based on this two-fold differentiation, Abel then introduces a four-fold system of forms of knowledge. First, there is everyday knowledge (unreflected basis for action); second, there is theoretical knowledge (e.g. mathematics); third, there is action knowledge (knowing how to do things); and fourth, there is moral or orientational knowledge (guiding social interaction).

In all these four forms of knowledge one can additionally distinguish between the different ways in which these forms of knowledge can be transported intersubjectively. Explicit knowledge is displayable, as Abel says. This means that other people can also obtain that knowledge through indirect ways independent of direct communication with its producer, such as e.g. reading books. By contrast, implicit (or tacit) knowledge is not displayed. It is inherent in a person's mind and links different things we know in a given situation.

Furthermore, knowledge can have verbal character, which means that it is articulated through language. Conversely, the articulation of non-verbal knowledge is not limited to specific linguistic systems; it can be transported through other channels. Abel also explains that there is a difference between propositional and non-propositional knowledge, which is that propositional knowledge can be expressed in a 'that clause' through words (knowing that something is). On the other hand, nonpropositional knowledge cannot be put into such coherent wording. Finally, there is also a distinction between matters of fact, which consist of existing objects and events, and abilities or skills on the other hand. Matters of fact can be observed and perceived by different people. Abilities and skills are bound to a person, and they refer to the know-how about doing things.

For Abel, knowledge is not something atemporal or innate which exists independently of a person's individual context. Knowledge is embedded in specific cultural contexts: 'The thesis is that, for humans as finite and perspecivist beings, contents of knowledge and forms of knowledge cannot exist independent of the forms, practices, and dynamics of the underlying representational, 
interpretational, and sign system' (Abel 2008: 15). This observation includes the dynamic character of knowledge which could change with time and transportation into different interpretational systems. Abel points out the problems which arise by translating knowledge - in particular explicit, scientific knowledge - between linguistic systems. As mankind has not yet found a 'best' way to organize such translation of knowledge between interpretational systems, we have to accept the plurality of ways of how to generate knowledge.

At this point, Abel also speaks about the 'best and creative brains' (2008: 16) that are necessary to elaborate useful translations between interpretational systems. Here, we can see that knowledge itself has a certain relation to creativity. Knowledge itself is not universally applicable, but it needs creativity to make it interculturally efficient in terms of a knowledge society. I will come back later to what relation creativity and knowledge have.

Let us go on with the notion of information. Abel (2008) explains that information is a common buzzword in the frame of societal and technological change. He remarks that there is currently much confusion between the two terms of information and knowledge. It is not clear anymore if we are living in a knowledge or an information society, thus some clarification is necessary. In a fundamental understanding, information is a bridging process between physical cognition and phenomenal processing in the brain. This involves the logical consequence that information is always treated and processed through the lens of existing knowledge. Information has to be interpreted, and interpretation needs former knowledge. In opposition to the often suggested idea of information existing ex ante of knowledge in a person's lifeworld, Abel thinks that information does not exist independently of knowledge. Information only becomes 'information' when it is perceived and processed by a knowing human being (Abel 2008: 17f.). Self-critically, Abel remarks that in certain spheres, information can become existent before knowledge, e.g. in public social opinions as transported in the media. However, I think that the basic model is more helpful to an understanding of the relationship between knowledge and information: information has to be perceived by human beings; it has to be processed using interpretation, and can then become part of new knowledge.

What is then the role of creativity? An interesting overview about the philosophical understanding of the term is provided by sociologist Ulrich Bröckling. Bröckling (2006: 513) formulates: 'Creativity is tied to the human potential to bring into being something new. Its basis is, first, the power of imagination as the capacity to make the absent present; and second, building upon this, fantasy as the capacity to realize the (as yet) inexistent'. According to Heinrich Popitz, Bröckling explains that creativity contains three overlapping elements, which are exploration (discovery, invention, new knowledge), shaping (producing artefacts), and finally the generation of meaning (interpretation, reasoning). We can see that such an understanding already contains links to the notion of knowledge. Creativity is, on the one hand, the production of new knowledge through exploration, but it is also the semantic and symbolic re-processing of existing knowledge into forms with new meaning and content.

Bröckling then describes six fields which can be used to approach a closer definition of creativity. First, creativity can be understood as artistic expression which is something innate to the human being. Second, creativity can be thought of as production, referring to the relation between the human being and what they produce with their own work. This field is linked to an understanding of 
the craftsmen and artisanal handicrafts. In this understanding there is a connotation of selfactualization through work, which nowadays shows up again in the description of creative industries, though not necessarily producing manual goods anymore. Third, if one focuses on the aspect of problem-solving, then creativity is more related to invention and innovation. In their own lifeworld, human beings are continuously confronted with new situations in which routines and instincts do not suffice for an appropriate reaction. Thus, the unknown situation calls for a new and 'creative' way to deal with it. Bröckling mentions that the figure of the inventor is exemplary for this type of creativity. Fourth, creativity can also be related to the idea of revolution. The term creative destruction explains how people extend borders that were formerly thought of as intransgressible. A fifth field of creativity is articulated in the notion of life itself. Related to biological creation and evolution, creativity also contains an element of vitality, survival and adaptation. Finally, and sixth, creativity also refers to the metaphor of play and purposeless activity. Bröckling remarks that creativity is, thus, the interplay of inventing up the new and reshaping the existent.

'Creativity involves the ability to synthesize', says Richard Florida (2002: 31). Other than intelligence - which is the ability to deal with large amounts of data - creativity serves to combine existing materials, things and concepts into a new and useful way. Florida mentions a four-step model which characterizes creativity (2002: 33). During a creative production, the first step consists of 'preparation'; this means the creative worker studies the problem by logical means. In the second step, called 'incubation', the creative worker's conscious and subconscious minds interrelate. Then, through 'illumination', the creative worker realizes a new synthesis. And finally, through 'verification and revision', the new synthesis is tested for its usefulness. 'Creativity cannot be switched on and off at predetermined times', Florida mentions (2002: 14). Thus, in line with the general idea as described by Bröckling, Florida also relates to the fact that creativity is somehow always present in the life of each individual, yet he is convinced that creativity is also manageable and can have a cumulative effect: 'Creativity involves distinct kinds of thinking and habits that must be cultivated both in the individual and in the surrounding society. [...] It reflects norms and values. [...] Furthermore, creativity requires a supportive environment that provides a broad array of social and cultural as well as economic stimuli' (Florida 2002: 22). Here, he suggests that specific contexts can lead to more creativity than other contexts.

I will conclude this brief and still superficial confrontation of the three notions with the following observation: according to Abel, we can understand information as an input to the development of knowledge. The process of making knowledge from information could be described with creativity, as Abel, Bröckling, and Florida have written - each in his own sense. From that conclusion, it becomes obvious that the advent of the creative industries debate can be traced back to earlier scientific work dealing with how information and knowledge have influenced our post-industrial society and economy.

\subsection{Definition of creative industries}

Making creative knowledge workers the object of analysis in this thesis, it is important to have a look at the discourse around the definition of the sector and its workers. What is this whole discussion about? The basic problem with the definition of creative industries is that it is rather 
difficult to estimate how strong creative work penetrates the economy as a whole. As we have heard above, scholars of the knowledge economy have realised that there is an autonomous sector on the one hand, but there is also knowledge work as part of other sectors such as e.g. automotive, chemicals, or food. The same accounts for creative input, which is part of nearly all industrial goods, even of public services, therefore it is not an easy task to define creative industries and make them distinguishable from other sectors. As with many other issues, there is much variety between different scholars and their viewpoints. However, all the publications share the characteristic that they define creative industries both, on the one hand, in a rather 'soft' way through qualitative descriptions; and on the other hand, in a 'hard' way through statistical analysis according to standardized industrial or occupational classifications. I will briefly examine which definitions could be found in the literature before describing my own understanding in the frame of my research project.

\subsubsection{Soft definitions: the qualitative dimension of creative industries}

An important and early contribution was the book titled 'Creative Industries' by Richard E. Caves (2000). Caves dealt with the question of how creative industries differ from other business activities and could thus be distinguished. In particular, he discussed the different organization of production as compared to other economic sectors. Applying economic contract theory, he studied under which circumstances tasks were organized in-house or as out-sourced market transactions. According to his definition, creative industries are those sectors (2000: 1) 'supplying goods and services that we broadly associate with cultural, artistic, or simply entertainment value. They include book and magazine publishing, the visual arts (painting, sculpture), the performing arts (theatre, opera, concerts, dance), sound recordings, cinema and TV films, even fashion and toys and games.' Caves explains that creative industries are to be distinguished from other economic activities (2000: 2): 'What I stress instead is that creative goods and services, the process of their production, and the preferences or tastes of creative artists differ in substantial and systematic (if not universal) ways from their counterparts in the rest of the economy where creativity plays a lesser (if seldom negligible) role'. Why is that? Caves describes seven basic properties of creative industries that make these sectors different from other economic activities.

First, the demand for creative goods and services is rather uncertain. Creative producers often do not react to an existing demand in the market. They develop goods and services which can often only be sold if the producers create the demand for the product along with the product itself. Thus, creative production is associated with a high uncertainty concerning the sales potential. It contains a high economic risk. Also, creative products and services are 'experience goods' (Caves 2000: 3), which means that the evaluation following the experience of creative industry products is rather subjective and less predictable. The problem is not asymmetrical information - as in many buyerseller-relations - but it is 'symmetrical ignorance': the seller does not have a clue about the expected demand of a creative product, and the buyer does not know what 'experience' he is going to buy.

Second, creative producers care about the quality and the reputation of their ouevre. While not all customers might really be able to appreciate the quality of a creative product, there is also the evaluation of other colleagues which is important to many creative producers. Thus, the creative 
producers put more effort into the production than they are actually paid for. This problem is linked to the first property: the problem of 'symmetrical ignorance' also comes into play here. The creative producer does not know in advance if they have translated a great vision and concept into a creative product which is understood as a 'great' one by the consumers as well. Often the vision is not understood as such, and it does not pay off in financial terms (Caves 2000: 3-5).

Third, the diversity of experts with different specializations who collaborate on the production of a creative product is an important aspect of creative industries. When many people with their individual (artistic) tastes have conflicting opinions about the product as a whole, then collaboration becomes a difficult issue. These conflicts are often not solved through formal contracts, but through a 'rank-order mechanism of 'muscle', as Caves writes (2000: 5). Collaboration is also characterized by what Caves calls a 'motley crew property' (2000: 6), which in economic theory is rather known as multiplicative production function. Whereas in a simple production function each individual input is substitutable, in creative industries this is often not the case. When individual inputs are highly specialized and unique, and thus a necessary piece to the output as a whole, then team collaboration is a decisive and constituting element for a product's realization. However, there is a double difficulty in terms of production teams: on the one hand, individualistic artist characters, with their very specific tastes and self-understanding, need to compromise with other, similarly individualistic artists in order to elaborate a complex creative product. On the other hand, if one of these individuals is not satisfied with the compromise and quits the team, they cannot be replaced by another with a similar qualification ('their input was unique'), and the whole product will not be realized.

Fourth, creative products are often unique and less comparable than other products. Given the particularity that they have to be 'experienced', a consumer can only compare two creative products to each other (e.g. two movies or two songs), if they have experienced and consumed them. Thus, ex-ante pricing is very difficult. This horizontal differentiation of creative products, as economists name it, is causing the 'infinite variety property' (Caves 2000: 6). Products are differentiated in a vast variety of unique appearances, but consumers, looking for similarities to compare prices, overlook these differences. Thus, products which have low production costs will probably be compared and considered as similar to very costly productions. The latter will then make lower profits or will remain unprofitable on the market, as consumers are not willing to pay a price which could reach the profit margin targeted by the creative producer. The infinite variety problem is only limited by the 'first-copy costs' (Caves 2000: 364), which means the fixed costs an output will have to produce a first edition to be marketed.

Fifth, there is also vertical differentiation between creative goods and services offered. Caves illustrates this aspect using the example of a movie production in which the producer will achieve a higher profit if they produce the movie with an A-list actor. Even though a B-list actor would offer the same service to the producer, they will not be contracted as the differentially lower rent does not allow the producer to make the film profitable, so creative industries are characterized by an ' $A$ list/B list property', as Caves says (2000: 7).

Sixth, there is the problem of temporal coordination of creative inputs from different creative agents who are necessary to achieve a creative output (e.g. in a movie production: actors, cutters, special effect engineers, make-up designers). If a project is only in its initial stage and only some money 
has been spent, then a delay is no problem, but at the moment when the production comes close to an end and production costs have been sunk, returns on investment should be realized soon, as inflation devaluates money with the passing of time. Thus, at the end of the production it is very important that all inputs are available at the right time. This problem to coordinate inputs in a temporal sequence is called the 'time flies property' by Caves (2000: 8). This temporal sequence of production inputs also comes along with another problem: the creative producers who provide their input at the initial stage of a project often lose control over the following production stages when other input producers provide their parts. As 'time flies' by, market information might change (e.g. information about an expected return), and consequently the project as a whole could be driven in a different direction. Thus, 'option contracts' are a common model to organize market transaction in creative industries. In an option contract the first input provider hands over the right for decisiontaking in the project to the second input provider. The second input provider guarantees the first input provider that they will take a decision about how to proceed with the project within a predefined time frame, so that the first input provider could still hope for a profitable finalization and soon return on investment. Furthermore, the second input provider will also negotiate with the first one on how much the first one will be rewarded for their input. Subsequently, the same transaction occurs between the second and third input provider, and so on.

Finally, there is the question of the durability of creative products. The main mechanism to realize rents is the copyright, and copyrights have a certain temporal duration according to the legal system, a fact which Caves calls the 'ars longa property' (2000: 9). The major issue here is how these rents can be collected if they occur in form of numerous small units, with costs attributed for the exertion of the copyright ownership. Another question concerns the share of this rent which is guaranteed to each input deliverer of a complex creative product. Often the amount of the rent is only an insecure estimation, and thus shares are difficult to calculate.

These seven basic properties also affect the organizational structure of creative industries. Caves remarks (2000: 16): 'In the creative industries these diverse groups of market competitors turn out to reflect the basic properties of creative goods. For example, the inner and individualistic aspect of creative production mixes badly with the orderly, rule-driven routines of the large, bureaucratic firm. Creative industries tend to be organized so that the most delicate dealings with artists concentrate in small firms specialized to this task, while other firms (usually much larger ones) undertake to combine creative work with large teams of humdrum inputs.

These many small creative firms with high degrees of specialization then need gatekeepers who match them with potential clients. These gatekeepers have a strong power for they can decide which individual small firm or artist to promote in a preferred manner. A lot of small producers in creative industries who have not established good relations to powerful gatekeepers or who have trusted in powerless gatekeepers will remain in a precarious situation. Caves writes (2000: 363): 'The many would-be creative workers who suffer rejection [from the right gatekeepers] either toil in dedicated poverty or settle for humdrum work, while those who experience creative success reap adulation and wealth in what tend to be winner-take-all contests.' In other words, entry barriers for individual creative workers are very high as large-scale distribution systems in creative industries are mainly organized in oligopoly structures. In order to get good access to the market, 'payola' is a widespread phenomenon in creative industries. Payola is the practice of paying for market entry in 
informal ways. It represents a type of corrupting gatekeepers to prefer the promotion of their own creative product to those of other creative producers (cf. Caves 2000: 286-296).

Caves also explains that some properties of the creative industries are part of other economic sectors as well. For example, the way in which information flow is organized (Caves 2000: 367): 'In complex creative industries, a great deal of information flows within and among firms - the A list/B list ranking process and the jostling over nascent projects that might or might not prove successful. [...] Academic researchers in the social and natural sciences conceive of the research process essentially the same way as do creative artists - as a way of identifying new problems of substantial importance and devising compelling solutions to them. Scholarly researchers assign high value to an open culture with unfettered exchange of information.' It becomes obvious here that certain characteristics are similar to mere knowledge work (e.g. science or research and development), as understood before the rise of creative industries/creativity as scientific topic.

To summarize, Caves provides a sound effort to characterize creative industries. He developed in detail his seven properties to differentiate creative industries from other economic activities, and he also later described internal differentiation within creative industries, which I have left out here for reasons of text length. Regardless, he remained vague about which activity in detail to include in or exclude from creative industries. He did not specify individual sectors, but only names exemplary activities. On the other hand, he remains rather narrow in his initial definition and observations described in the book: he only focuses on artistic production and understands creativity as a creation process in an artistic sense (cf. Bröckling 2006, as in section 2.1.4).

The definition in Richard Florida's books (2002, 2005) is equally fuzzy, however, because for Florida the notion of 'creative class' is central as he prefers to speak about the actors instead of the industrial sectors. According to him, creative industries are composed of 'people who are paid principally to do creative work for a living. These are scientists, engineers, artists, musicians, designers and knowledge-based professionals [...].' (Florida 2002: xiii). It is obvious that he includes far more activities than Caves, talking about more than artists. However, Florida's notion of class has to be questioned as it varies significantly from that of the traditional sociologists such as Marx. For Florida, a class is determined by a group of people 'who have common interests and tend to think, feel and behave similarly, but these similarities are fundamentally determined by economic function' (2002: 8). Thus, a class - according to Florida - is already a class when people do the same job and develop similar interests. In the Marx notion, a group of people become a class through a collective identity and collective agency. This is certainly not true for creative professionals, as Florida, a self-proclaimed Marxist, notes himself (2002: xxix). What defines Florida's creative class is the job for which it is paid, namely to 'create', in a work environment valuing individuality, difference, autonomy, flexibility and merit. Differing from the creative class, Florida describes the working class and the service class, whose members mainly 'execute' commanded work.

Within the creative class he distinguished between a 'core of creative workers', who create new ideas, technology and content. Surrounding this core, there are the 'creative professionals' who do jobs in which independent judgment and high education are necessary inputs, and who have to apply the innovative knowledge created by the core of creative workers. According to Florida (2002), creative workers prefer self-expression and open-mindedness (for difference) over 
homogeneity and conformity, with the latter having been necessary in much of other manufacturing and low-end service work. Furthermore, challenging tasks, responsible positions and flexible schedules are important job-related aspects for creative professionals. Even though the term 'creative class' leaves much reason for criticism, it has become famous in Europe as well (cf. Marlet \& van Woerkens 2007).

In 1998 the European Commission follows the UK Department of Culture, Media and Sport (DCMS) in its definition that creative industries 'have their origin in individual creativity, skill, and talent and [...] have a potential for wealth and job creation through the generation and exploitation of intellectual property' (DCMS 1998, cited in European Commission 2010b: 191). It is interesting to see here that the notion of intellectual property is mentioned as a way to generate rewards and profit. This reflects the UK DCMS' understanding that creative industries, in order to be an industry, must be profit oriented and part of the private sector. In particular, the European Commission acknowledges the potential of creative work which takes place in other sectors (European Commission 2010b: 198): 'Creative occupations' is a broader concept than 'creative industries'. It embraces the professions that are 'creative' in essence, no matter whether they belong to the so-called 'creative industries'. [...] These 'knowledge workers' produce intangible assets such as ideas, knowledge, and information that increase firms' value added.' Here again the link to the knowledge economy shows up. The penetration of brain work into other economic sectors as well as the importance of knowledge creation is emphasized.

In the Green Paper called 'Unlocking the potential of cultural and creative industries' the EU Commmission says (2010b: 2): 'In this new digital economy, immaterial value increasingly determines material value, as consumers are looking for new and enriching 'experiences'. The ability to create social experiences and networking is now a factor of competitiveness. [...] the rapid roll-out of new technologies and increased globalization has meant a striking shift away from traditional manufacturing towards services and innovation. Factory floors are progressively being replaced by creative communities whose raw material is their ability to imagine, create and innovate'.

There is a broad variety of other - what I would call - qualitative descriptions of the character of creative industries. I will not go further into detail here, because this section does not aim at a refined definition. Yet, I believe that this brief presentation of some ideas on creative industries helps to understand the specificity of this sector as compared to other economic sectors. Let us keep in mind that creative industries often engage in immaterial production: they solve problems through innovation, creation and imagination. Creative outputs can be emotions, experiences, concepts, values, or formal knowledge. They are composed of small and independent production units, which gather in flexible ways in highly unique and specialized teams around fixed-term projects. Therefore, networking is an important issue in order to bring together the needed skills at the right moment, but also to ensure quality through the provision of reputation. Furthermore, the creative industries are an economic sector in which market risk is asymmetrically heavier a burden to be borne by the many small production units, while a few large corporations - Caves' gatekeepers control distribution and access to sales markets. Finally, there are a few individual stars in creative industries who claim a large share of profits, whereas a large army of creative knowledge workers has to divide the small rest. I think we have to keep this in mind when reflecting the situation of creative knowledge workers later in the text. 


\subsubsection{Hard definitions: the quantitative dimension of creative industries}

I am not interested in discussing statistical hard definitions in detail in the frame of this thesis. I have been involved in projects where this was an issue, however I do not think it is necessary in the frame of this $\mathrm{PhD}$ project as the research questions are not about quantification. Despite this, I will try to illustrate the size and quantity of the 'creative industries' phenomenon by referring to statistical numbers that are mentioned in several studies. Whereas I discussed the rather soft and qualitative definitions of the creative industries in the section before, I will present the hard definitions here. With hard definitions I mean the different selections of statistical codes extracted from the standardized economic statistics. Most common databases are the labour statistics which use a nomenclature of occupations (e.g. ISCO - International Standard Classification of Occupations) as well as business statistics with a system of industrial codes (e.g. NACE Nomenclature statistique des activités économiques dans la Communauté européenne). Definitions - the composition of creative industries according to classification codes - vary strongly from author to author. Thus, it has to be mentioned that numbers are not comparable and have to be considered with caution.

Howkins (2001) provides a wider definition of creative industries which is orientated on concrete industrial sub-sectors. According to his definition, 15 sub-sectors have to be considered as parts of the creative economy: Research and development, publishing, software, TV and radio, design, music, film, toys and games, advertising, architecture, performing arts, crafts, video games, fashion, and art. In 1999 these sub-sectors had a global market size of US\$ 2,240 billion, of which the US creative economy held 43\% (Howkins 2001: 116, cited in Florida 2002: 47). It becomes obvious that this definition is more inclusive than Caves' understanding of creative industries. Scientific activities and crafts are considered a part of creative work, too.

Richard Florida also prefers an inclusive way of defining his 'creative class' while declining to define the industries along industrial classifications (Florida 2002: 327-329). If we have a look at Florida's composition of the 'creative class', we can observe a rather open definition again. According to Florida nearly all activities that have to do with 'brain work' are included. Florida distinguishes his 'Super-creative Core' from 'Creative Professionals' (2002: 328). The former is dominated by occupations in the scientific fields and in engineering, but it also includes the artists, designers and entertainers themselves. The latter group contains occupations that rather deal with providing more complex services to other businesses and private clients. Florida tries to list specific occupational groups. According to him, scientists, IT specialists and engineers are part of the creative class as they develop and create new knowledge and information. Furthermore, managers, lawyers, financial professionals, health care personnel and even salespeople have to be included as well. These latter 'creative professionals' are applying existing knowledge in innovative ways, meanwhile improving applied knowledge through small innovations. Allowing for this openness in the definition, Florida calculates that 38.3 million workers in the US constituted the creative class in the early 2000s. They accounted for a 30\% share in the total labour force. Florida is convinced that the phenomenon is a rather young one, and the creative class has grown in particular since 1980 (Florida 2002: 74ff.). However, there is also widespread criticism concerning this openness in his definition, as there is evidence for multicollinearity between human capital (measured as education levels) and the share of creative class in a place. This drives critics to the conclusion that such a 
wide definition as that of Florida, which includes nearly all high-skilled professions, does not produce any benefit for the explanation of regional economic growth (cf. Markusen 2006; as cited in European Commission 2010b: 214).

Whereas in the USA the discussion about a proper definition of creative industries rather focuses on the agents themselves, the creative knowledge workers, or the creative class (cf. Florida 2002), European scholars tend to refer to economic sectors and use NACE and ISCO code combinations. The ESPON Programme 2013 (ESPON 2013 Programme Coordination Unit 2011), recently published a report on Europe's creative workforce. Here a set of 42 different ISCO-88 4D codes has been used to define the creative workforce (cf. Appendix A). As in the definition of Howkins, crafts as well as scientific research and teaching are included in creative industries.

In its 2010 Competitiveness Report, the European Commission (2010b) limits the focus again, excluding scientific sectors. Crafts are still considered part of the creative industries. The UK government follows this manner of narrowing the sector and uses a very selective definition. In this definition the scientific activities as well as research and development are excluded. Non-profit activities are also not counted as creative industries, as the latter should have a clear 'economic' character.

Elsewhere, the European Commission defines the 'cultural' industries in its Green Paper (European Commission 2010c), listing eight sub-sectors: artistic and monumental heritage, archives, libraries, books and press, visual arts, architecture, performing arts, audio and audiovisual media/multimedia. These sectors are also used by Eurostat for statistical analyses. Furthermore, a functional distinction is introduced in this definition. Cultural industries could be differentiated by preservation, creation, production, dissemination, trade/sales and education (European Commission 2010c: 5). The Green Paper also follows a rather broad approach in defining cultural industries as provided by the 2005 UNESCO Convention on the protection and promotion of the diversity of cultural expression. The European Commission (2010c: 5f.) says: "Cultural industries" are those industries producing and distributing goods or services which at the time they are developed are considered to have a specific attribute, use or purpose which embodies or conveys cultural expressions, irrespective of the commercial value they may have. Besides the traditional arts sectors (performing arts, visual arts, cultural heritage - including the public sector), they include film, DVD and video, television and radio, video games, new media, music, books and press.' In addition to these cultural industries, there are 'creative industries' which 'use culture as an input and have a cultural dimension, although their outputs are mainly functional. They include architecture and design, which integrate creative elements into wider processes, as well as subsectors such as graphic design, fashion design or advertising' (cf. European Commission 2010c: 6).

In the logic of the Competitiveness Report 2010, creative industries are divided into three subsectors: information services (publishing, new media, software), business services (architecture, engineering, advertising, design, photography, translation and interpretation), and arts and entertainment. According to the European Commission, the dominant sub-sector, which is the motor of creative industries, is information services. These information services accounted for $62 \%$ of creative industries employment in the EU-26 (Malta excluded) in the year 2008. Business services made up $29 \%$ of creative industries' employment, so the third sector of arts and 
entertainment only sums up to roughly $10 \%$. Therefore, there is a strong internal differentiation in respect to the importance of sub-sectors within the creative industries.

According to the Competitiveness Report 2010 (European Commission 2010b: 191ff.), approximately 6.7 million people worked in the creative industries in the European Union (EU-27) in 2008. Thus, $3.0 \%$ of the total employment is creative knowledge work (compared to $4.0 \%$ in the USA). The higher share in the USA can be explained by the large audiovisual and computer software sector, which exceeds the European one. In terms of external exports from the EU-27, creative industries account for $4.3 \%$, and make up about 3.3\% of the total GDP. In the period from 2000-2007, employment annually increased by 3.5\%, while total employment in the EU-27 only grew by $1.0 \%$. In the US and China growth rates were smaller, with $1.8 \%$ and $1.9 \%$ respectively. The European Commission also highlights that there is internal differentiation concerning the growth rates, with software consulting and supply, audiovisuals and architecture recently growing faster than other sub-sectors of the creative industries. Computer programming and consulting also make up 37\% of EU-27 total employment in creative industries, followed by architecture (15\%). Employment figures here are based on people employed by creative companies (selected according to industrial codes).

However, there are also creative workers in other industrial sectors. These could be found by using labour statistics and the occupational classification system, in which workers are not registered as belonging to a company but to an occupational group. The European Commission reports that more or less $62 \%$ of creative occupation takes place outside the creative industries (2010b: 198). Calculating occupational instead of employment shares, they maintain that in 2008, in a selection of 15 EU countries, for which Labour Force Survey data was available, 7.7\% of people were occupied with creative work. The most important sub-sector concerning occupation is then composed of architects and engineers. Between 2002 and 2008, there was a growth in creative occupation by $3.1 \%$, with the highest growth for artistic and entertainment professionals as well as social scientists and related professionals.

The recent economic and financial crisis which started in 2008 has also affected the creative industries. In particular, sub-sectors with a strong share of business-to-business (B2B) activities experienced a downturn: advertising's turnover decreased by 12.4\% from 2008 to 2009 in the EU27. Publishing turnover also decreased by $6.8 \%$ as advertising budgets were cut during the crisis. In terms of both employment and turnover, the creative industries in the EU-27 experienced their first decline in the past 10 years, with $3.3 \%$ in employment and $9.9 \%$ in turnover (European Commission 2010b: 200f.).

National and regional governments have also discovered the issue of creative industries, and recently economic as well as cultural affairs' departments and ministries have been rushing to 'count' the extent of creative industries in their own jurisdictions. We can again observe that 'creativity' is inherent to the process itself: governments use rather individualistic and poorly comparable definitions. In Germany, for example, after long discussions a national definition was agreed upon, which includes all economic activities in the fields of music production and interpretation, book authoring and publishing, fine arts production and trade, television and radio broadcasting, performing arts, design activities, architecture, journalism, advertising, the software and games industry, and finally the rather catch-all sector of 'miscellaneous cultural activities' (e.g. 
circus artists, museums, libraries) (Söndermann, Backes et al. 2009). Despite this, definitions still are not consistent. Some regional governments prefer wider definitions, e.g. the Saxonian government also includes craftsmen and producers of musical instruments (SMWA/SMWK 2009). One could suggest that there is a strategy behind all of the definitional chaos, but I will ignore this for the moment.

To summarize, the presented definitions and figures highlight two aspects: First, there is a large variety between individual definitions in the field of hard definitions using statistical classification as well. As for the qualitative descriptions, we again find broader and inclusive definitions along with more exclusive approaches. Thus, it is rather difficult to precisely distinguish creative industries from other sectors which are closely related to it. I will leave this as a statement and I am not tempted to develop my own solution to that problem here, but there is a second and even more important observation: independent of the definitional issues several authors have come to the conclusion that creative industries represent a very dynamic segment of the labour market. Even though the sector is still a rather small one, there is some empirical evidence in these figures that it is becoming more important in relation to other segments. Above average employment growth has come along with a relatively smooth performance during the financial crisis, and both of these facts make creative industries an interesting field of research for the coming years. Some critics, with arguments I would definitely support, point to the neo-liberal and oppressive character of these sectors or even try to diminish the 'creative industries' phenomenon to a mere product of discursive construction by certain interest groups ${ }^{6}$. Finally, I would say that we still do not know enough about it in order to renounce making it a topic; we still do not know if these sectors will turn out to become a stable, long-term growth machine for post-industrial economies or not, and if so, we would need to study in more detail what their presence means not only in economic but also in social terms.

\subsubsection{Public sector activities}

There is an ongoing debate about the role of public activities in creative industries' markets. A large share of creative institutions, e.g. opera houses, orchestras, theatres etc. are financed to a large degree with public money. The same accounts for museums. The question is then, do these activities reflect market participation, or is it part of the state's agency? There are different opinions on this issue.

For example, both the UK government and UNESCO use definitions in which a distinction between economic and cultural activities has been introduced. I mentioned above that the European Commission (2010b) pointed to UNESCO's position that cultural goods and services are those that are provided 'irrespective of the commercial value they may have' (UNESCO, cited in European Commission 2010b: 193). The UK DCMS also changed the notion of cultural industries, as used earlier by the Labour Party, to the term creative industries. The DCMS underlined that cultural services would exclude a broad range of knowledge services that were formerly termed 'new economy', and which made up a large share in economic power of the creative industries. Based on

\footnotetext{
${ }^{6}$ I cannot prove this observation with a precise source, but this is what I have experienced in the last five years of working on this topic.
} 
ideas by Meusburger, Welker et al. (2008), the European Commission writes (2010b: 193): 'While creative industries link creative content to job and wealth creation, cultural industries are not first and foremost defined by their business value.' The UK DCMS even goes further and finally excluded non-profit activities from its definition (European Commission 2010b: 194).

Another approach consists of the 'three sector model' which evolved in Switzerland in the frame of the first national creative industries report. Here, the distinction is made between merely public and private sectors. Additionally, a third sector is introduced which is at an intersection between both. For the Swiss policy makers and scholars of creative industries, the creative industries are mainly the private sector which is profit oriented. The other two sectors, public and intermediary, mainly work in non-profit fields. They are strongly linked to the private one, as e.g. individual actors work in all the three sectors. From the perspective of the creative professionals these three sectors only work as a compound system. Yet, for analytical ambitions the sectors should be divided, and only the economically oriented one should be examined in detail (Weckerle, Gerig et al. 2008: 28).

\subsubsection{Insertion of creative industries into the wider market}

After having described the content with which the creative industries deal and the extent to which they are in place today, I would like to mention some points which are relevant in understanding how the creative industries relate to other sectors. We have already heard a bit on internal structures in creative industries in the '7 properties' by Caves (section 2.2.1), however, there are some interesting additional observations concerning the insertion of creative industries into the wider market.

\section{Intersectoral business relations}

Creative work is not only limited to creative industries. Florida (2002) remarks that there is an ongoing penetration of creative work into other industrial sectors. What he calls the 'rise of the creative class' or the emergence of an 'Age of Creativity' is also visible in the change of tasks and qualification levels in the economy as a whole. Equally the European Commission mentions that 'creative workers can also be found in other skill-intensive manufacturing or business services activities' (2010b: 191). A large share of creative occupation could be found in sectors other than creative industries as defined by selected NACE codes.

Another fact is the close interrelation of creative industries with other industries through value chains. From UK data, it is known that business-to-business relations of the creative industries with other sectors account for $60 \%$ of their total output. In particular, intermediate services and products are provided by the advertising, architecture, software and fashion sub-sectors. Other sub-sectors such as radio and TV, the film industry as well as the arts sector are mainly orientated to private consumption and business-to-consumer relations. The Competitiveness Report reveals that $80 \%$ of turnover in software and 93\% of turnover in advertising stem from business-to-business transactions (European Commission 2010b: 216).

A Danish study recently examined the client structure of creative business-to-business transactions. Using creative industries' shares on intermediate inputs to a sector, it was found that creative industries themselves were the main consumer (with $37 \%$ of all inputs consumed from other creative businesses), followed by the real estate sector (22\%, mainly buying from publishing and 
software companies), the adult and other education sector (21\%, mainly consuming audiovisual services), and tobacco and beverages (17\%, mainly buying advertising services) (European Commission 2010b: 217/246).

Also the knowledge spillovers from creative industries to other sectors are an important aspect. The European Commission remarks (2010b: 218): 'Creative industry firms tend to make use of a large network of weak, heterogeneous relationships that ensure easy access to and fast absorption of new knowledge - an observation which fits well with the evolutionary/systemic view of innovation.' Thus, non-creative companies having business relations with creative companies can profit from this character. Creative industries are attributed external innovation effects that make companies outside the creative sectors more innovative when they have strong links to creative businesses. Based on UK data, Bakhshi, McVittie et al. found that non-creative businesses which double their spending on creative products and services from $3 \%$ to $6 \%$ are $25 \%$ more likely to innovate their products (cf. Bakhshi, McVittie et al. 2008, cited in European Commission 2010b: 222).

In the European Commission's Green Paper, we also find a hint to the reinforced importance of existing links between creative industries and external sectors (European Commission 2010c: 7): 'The global digital shift reinforces the position of important stakeholders such as telecom companies or consumer electronics manufacturers, and coincides with the emergence of new strong global players such as search engines and web based social platforms which - while making significant contributions to the development of the market place - can potentially act as new gatekeepers.'

Based on a paper by Chartrand (1984), the authors of the Competitiveness Report list four different types of impact that the creative industries can have in economic and social spheres (European Commission 2010b: 192). The primary effects could be measured directly in terms of employment, value added, or exports which are rooted in creative industries themselves. The secondary effects could only indirectly be observed. For example, economic spillovers to other industrial sectors stem from creative industries' capacity to provide important intermediate goods and services for other businesses, which help these other businesses to grow. In particular in the light of a growing importance of branding and invidualising consumer goods, creative industries help traditional manufacturers to add value to their products. Tertiary effects evolve from spillovers of innovation (e.g. process innovation, new ideas, creative input) to other sectors. These effects are not quantifiable but can have a major impact on how non-creative businesses produce and sell. Often creative businesses are contracted for a certain task by large traditional companies, and even after the paid service is delivered, the commissioning company can profit from knowledge spillover and innovation acquired during the collaboration with the creative supplier. Quaternary effects are even harder to observe as they are both indirect and non-quantifiable. These effects include the general augmentation of the quality-of-life in a certain place through innovative ways of organizing the economy and the society. In particular, the provision of cultural services and the innovating impact on traditional value and norm sets can be mentioned here. Also the assistance in defining symbolic meanings represents such quaternary effects, e.g. through the development of place-based or groupbased identity concepts, lifestyles, images, etc. 


\section{Internal market structure}

As mentioned above, company size distribution in creative industries shows a specific pattern, which is relevant for how the market itself works. According to the European Commission's Competitiveness Report (2010b), about 95\% of all creative businesses have only up to 9 employees, compared to $80 \%$ in traditional manufacturing sectors. Self-employed people make up 58\% of the total creative industries businesses, with advertising at $67 \%$ level having the highest share of all creative sub-sectors. Translated into employment statistics, this means that $35 \%$ of creative workers are employed in micro firms with less than 10 employees, and $13 \%$ of creative workers being selfemployed. In the European Commission's Green Paper the situation in creative industries is described as follows (European Commission 2010c: 7): 'the vast majority of the CCI's [cultural and creative industries] consist of a myriad of micro-, small and medium sized enterprises, as well as freelancers, co-existing with vertically or horizontally integrated companies. [...] They are typically the risk takers and early adopters and play decisive roles when it comes to scouting for new talents, developing new trends and designing new aesthetics.'

Another interesting fact is that creative industries have strong internal supply-chain linkages between different sub-sectors. The European Commission observed that in particular the advertising and publishing sectors are intertwined. The media sector and relevant content providers (independent writers, journalists, and photographers) also have strong business connections. Thus, creative industries are a self-referential sector to a certain extent, in which only certain sub-sectors link to external markets. This is also reflected in the relatively high share of internal business-tobusiness consumption. In 2004 in the UK, 19\% of intermediate purchases of creative businesses were bought from other creative businesses, accounting for $8 \%$ of total gross output of the creative industries (European Commission 2010b: 216). Interestingly, the large and important creative subsector of software consultancy and supply shows very few internal linkages to other creative subsectors (European Commission 2010b: 202f.). It could therefore be questioned to what degree these activities should be included in creative industries or not. It is at least obvious that the software sector has a specific and unique role within the creative industries: it is the largest activity and the least connected to other creative sub-sectors.

Furthermore, the European Commission points to some patterns in creative industries' markets that could be described as market failure, which would legitimate policy intervention (European Commission 2010b: 223f.). First, comparably to Caves (2000), the European Commission identified the 'speculative character' of revenues in uncertain markets as a major threat to the large group of micro firms and self-employed people; the low predictability makes decision making very risky. Second, creative businesses' revenues are mainly based on first-mover advantages in the markets. But as creative goods and services consist to a large degree of concepts, ideas, knowledge and information, these could easily be stolen or copied before being put onto the market. Thus, a central problem is to find investors without 'whistle-blowing' one's own idea. Therefore, traditional market-based finance models still do not sufficiently work, and public financial assistance as well as a better coordination of venture capital provision is necessary, as the European Commission concludes (2010b: 223). 


\section{Interrelation with society}

Creative goods and services are also subject to strong externalities - both in production and consumption. Value is created mainly through interaction with society. A creative good increases in value when it is applied by a large share of people, and when it gains symbolic value in the society. This implies that market price mechanisms are dysfunctional, and rewards collection is a complex issue. Here, the EU calls for a more pronounced discussion about the regulation of intellectual property rights. There is a large negotiable continuum of practices for the management of intellectual property rights (IPR) between the two poles of Creative Commons licenses and open access policies, on the one hand, and rigid copyright protection, on the other hand (see European Commission 2010b: 224). Similarly, the European Commission argues in its Green Paper (2010c: 6): 'Economic value is being displaced towards the end of the chain, which in certain sectors affects the effective reward for creation. Moreover, parts of the content industries, particularly the recorded content industries, have been severely hit by piracy and losses in sales of physical supports (e.g. CD, DVD), which have damaged their development, shrunk their revenue basis and consequently limited their potential to generate jobs and investment. This is why the industry also needs to develop new and innovative business models.' This could be read as a call for a more rigid regulation of access to creative content, too.

The product itself is no longer the only issue for a creative company. In creative industries the emotional and symbolic loading of products is also an important activity field. With ongoing individualization and diversification of lifestyles, group identities, and individual behaviour, target group specific marketing strategies and product images have become at least as important as the product itself.

New forms of value-adding are also being developed in which the product is no longer sold, but rather leased or rented for a certain time. This business model responds to more mobile and flexible life styles. It led the US sociologist Jeremy Rifkin to speak of the advent of the 'Age of Access', in which material property becomes less important but the access to a variety of products and services is the new driver of growth and prosperity (Rifkin 2000). According to Rifkin, nowadays prosperous businesses often are not paid anymore for delivering a product or providing a service, but rather they earn money through selling access to knowledge, ideas, innovation, or even to emotions. Thus, indeed a growing share of economic activity is no longer considered with trading material goods. There is a certain dematerialization of economic activity, and the main source for remuneration becomes intellectual property (cf. also the European Commission 2010c).

Furthermore, the temporal aspect changes: product life cycles and market trends are becoming shorter. This means that research and development of new and innovative products is more important than ever, and has to be more rapid in order to keep pace with the market. Knowledge has become a crucial resource for the development of new products and services. Thus, analytical tasks are a growing field of activity. Theoretical anchor points like the knowledge society or the information age (Castells 2000b) are helpful to embed the development of creative industries theoretically. But creative industries go beyond these two concepts for they do not only focus on technological innovation and the development of abstract process-related knowledge. They are also concerned with the development of emotions, values, norms and symbolic meaning. Creative knowledge work goes beyond mere economic interests and touches also broader societal issues, as 
could be witnessed with the debates around growing social entrepreneurship, social innovation or the growth of ecological and fair-trade economies.

To sum up, creative industries represent a growing economic sector in post-industrial countries. They are heavily interrelated with traditional economies. One might even argue that the globalization and ICT-induced outsourcing processes that only made creative industries visible as its own business sector, and helped to boost its emergence ${ }^{7}$. Formerly, creative knowledge work was often only a small part of large industrial conglomerates (and still remains so to a certain degree), and it was rather difficult to estimate the amount of it through existing statistics. Today, the outsourced activities have led to the creation of new companies and jobs, which are visible as independent players on the market. These creative activities heavily influence the wider economy as well as society. The internal organization in small units bears risks (e.g. unpredictability of revenue, low market regulation, poor availability of venture capital) and opportunities (e.g. freedom from stasis and bureaucracy, flexibility allowing for creativity). So far, I have outlined how the creative industries as economic sector are characterized. Section 2.3 will put the emphasis on the character of creative knowledge work and the typical description of the creative knowledge worker which can be found in the literature.

\subsection{The creative knowledge workers and their work environment ${ }^{8}$}

Creative industries provide an income to a growing share of Europeans (Wedemeier 2010). Furthermore, creative industries are considered an interface between the formerly opposed systems of capitalist business-making and the idealistic world of free and independent artists and their oeuvre. These two opposing perspectives are increasingly merging, and the creative industries represent the arena where this rapprochement is being played out. On the one hand, it is no longer about mere artistic freedom and independence from capitalist necessities amongst artists, but also about how to capitalize on one's own work (Friebe \& Lobo 2006). On the other hand, the postindustrial economy has to accept that standardized mass consumer goods have to be added by customized and individualized products and small-scale series, which become more important to generate value-added (Friebe \& Ramge 2008; Sennett 2008).

Here, a new type of actor is to assist this merging of the worlds of arts and the economy. The socalled 'culturepreneur' is dominating both of these fields (Lange 2005, 2007). They know how to create new and innovative services and products, but they are also aware of that they have generated a 'product' and not a pure artistic oeuvre. This includes the reflection about commercialization. Characteristically, these culturepreneurs are successful in market niches, which they actively developed with the emergence of the internet and new modes of e-commerce. They are also capitalizing on highly flexible and specialized fields which result from the ongoing outsourcing process in the industrial sectors (Sennett 2006). Yet, one question remains: how is it to work in

\footnotetext{
${ }^{7}$ This argument cannot be discussed in more detail here. However, interested readers can enter into the debate around this idea by reading Sennett (2006).

${ }^{8}$ Main parts of this section were published earlier in a working paper that I have written in the frame of the PhD research at the University of Milan-Bicocca, and which I presented in a slightly revised version during the 8th European Urban \& Regional Studies Conference 15th to 17th Sept 2010 in Vienna/Austria. The conference paper can be accessed here: http://www.dur.ac.uk/resources/geography/conferences/eursc/17-09-10/RobertNadler.pdf (accessed 31 October 2012).
} 
these new fields? To answer this question, we should reflect the main characteristics of creative knowledge work in the light of the character of the industries as mentioned above.

\subsubsection{Immateriality - brain work}

Creative and knowledge intensive work is first of all 'brain work'. It could be defined as 'labour that produces an immaterial good, such as a service, a cultural product, knowledge, or communication' (Hardt \& Negri 2000: 290, cited in Hesmondhalgh \& Baker 2008: 98). It is clear that not all creative knowledge workers share the same degree of 'brain work' compared to material production and more standardized production steps, but the creative knowledge work consist much more of reflection, innovation, and invention than work in other more traditional sectors. So, the remuneration of creative work faces the problems of how to fix prices. Whereas in material production piece rates are relatively easily calculable and negotiable, prices for immaterial production and intellectual work are more difficult to define. At first glimpse, this might seem a trivial observation, but it defines the economic power relations of the entire creative and knowledge markets. We should remember Drucker's ideas about the new struggle of the knowledge worker: they have the power of knowledge, but not of the knowledge's productivity. The economic value of intellectual output is difficult to estimate, as one performance could not easily be compared to another. Remuneration schemes thus stay poorly transparent. Christopherson (2008) documented how e.g. in the cable TV production sector the power relations and price fixing are strongly defined by the TV channel owners. This heavily affected income opportunities of freelance suppliers in TV production.

\subsubsection{Symbolic contents and their distribution}

Gill \& Pratt (2008: 9) recall that - for distinguishing creative work from other work - there is the 'importance of symbolic production'. This means that in creative industries the production of not only basic knowledge, but also interpretations of this knowledge are delivered. Producing creative output frequently means producing lifestyles, identities and meanings. Lange \& Mieg (2008: 233) conceptualize creative industries as 'regionally differentiated markets of meanings in which the production and exchange of culturally relevant, symbolic values or goods is inscribed'. Also, Hesmondhalgh \& Baker (2008: 101) agree 'that the terms 'cultural' and 'creative', as applied to industry and work in recent policy discourse, have been centred primarily on symbolic, expressive and informational creativity'. This means that a large share of creative industries work - as related to the immateriality aspect - is composed of not only thinking up new things, but also giving them a meaning.

This production of meaning or symbolic value could easily be copied or claimed and redefined by others. Creative knowledge products and services, contrary to traditional mass production, add in value with their distribution. Once an idea, a concept or a lifestyle is accepted and used by a broad mass, it becomes dominant. Its 'creator' can expect large revenues, but the way to large scale distribution is difficult to achieve for an individual creator within the creative industries. Gatekeepers (e.g. a publishing company, a music label, a film producer) might refuse to support distribution. Second, there might be competing ideas, concepts and lifestyles which might interfere and interrupt the ascent of another one. There is thus a dilemma of how to efficiently distribute 
while keeping control over the symbolic value and its commercialization. A good example is the music industry in which the digitalization caused massive problems in market organization. The copyright question is linked to this - with positions reaching from 'no copyrights' to stronger regulation (see also description in section 2.2). Another example for how relevant market dominance is in this 'winner-takes-all' competition is to be found in the academic debate about creative industries itself: Richard Florida placed his books and presentations at the intersection of academia and popular science, reaching a huge audience. Even though there are many studies which are elaborated more carefully in academia, it is Richard Florida who has become an iconic figure of creative industries research. He is booked worldwide for presentation in the fields of business development, urban planning, scientific research, etc. His idea of the '3Ts' - technology, tolerance, and talent - became repeated throughout the world. Having a publishing house with powerful marketing machinery selling his books made him a winner figure, while other creative industries scholars who developed probably more robust and reasoned ideas about the creative industries did not succeed in the same way.

Thus one major aspect of creative industries is how a creative knowledge worker gets his symbolic content listened to. The best idea in the world does not help the creative knowledge worker if no one becomes aware of it, or if no one relates its origin to exactly this creative knowledge worker. Here, we can surely relate to Austrian urbanist Georg Franck (1998), who elaborated a draft of what he calls 'Die Ökonomie der Aufmerksamkeit' (Attention Economics). The basic idea of Franck's approach is that attention is a new and very important currency in the world dominated by information and mass media. Attention is a scarce good which can translate into financial value, but also into other forms of revenues (e.g. fame, recognition). In an information society in which immaterial production such as in creative industries is becoming more important, attention is the central criteria for placing immaterial goods in the market. An idea's value is increased with the number of people who become aware of it and accept it as valuable. Therefore, the idea has to be compelling, affect the emotions of the recipients and be communicated in an efficient way. Only as such can a symbolic value-added be generated. This complex system of distribution is often out of reach of the individual creative knowledge worker (even though new forms of web-based distribution are helping to reduce this problem).

Furthermore, attention is differentiated according to its origin: who gives attention to an idea? This is particularly important in creative industries in which reputational systems are crucial to quality management in the whole sector. Peers are competitors, but they can also provide the reputation and attention which can push forward the 'creations' of an individual creative knowledge worker. For example, you might think of a scientist in humanities who needs other humanists' acknowledgement to raise his scientific value in order to improve their fund-raising capacity for research projects. The same accounts for an architect who needs the attention and reputation within the architecture community in order to improve their market value.

\subsubsection{Project based work and irregular time schedules}

'Creative people always have experienced and even cultivated a blurring of time. Writers, artists, musicians, scientists and inventors often have erratic and irregular schedules, working from home and seemingly playing at work' (Florida 2002: 14). The observation that Florida has pointed out 
above also applies to the periodic rhythm in which these creative knowledge workers are sometimes very busy and work long hours, while then having periods without work again. This irregularity is caused by the organization of work in temporally limited projects. Working in creative industries equals a continuous sequence of output-oriented projects which dominate the creative worker's lifetime. Sennett (2006) interprets this development as a new temporal pattern of life, characterized by a dominance of periodicity.

This includes that in creative industries the formerly neat borders between private life and professional life become blurred (von Streit 2010). With the flexible work schedules, creative knowledge workers have the freedom to work whenever they want, not being bound to the 9-5 office hours which are typical in bureaucratic hierarchies in industrial companies. This other side of the model is that creative knowledge workers also have to work when the project has to meet strict deadlines. In this way working at home, in the evening hours or at the weekend becomes a latent imperative in creative industries, causing trouble in family life and private spheres. In addition, there is much insecurity about the future situation as projects mainly provide employment only for a limited time. Thus, the work-life balance in creative industries is a bi-fold story: of deliberation from traditionally rigid work organization on the one hand, and of the emergence of new constraints on the other hand (cf. Brown, Nadler et al. 2010).

\subsubsection{Emotional involvement and affective work}

Hesmondhalgh \& Baker (2008) point to another problem of creative industries work: the emotional character of work. On the basis of interviews with production workers of a talents TV show in the UK, they found that the common argument of affective labour that the creative autonomy pays off for the loss of security could not be supported. Whereas it might be true that there is more creative freedom, this freedom translated into a 'pressure to be creative' among the workers in this production team. In addition, this special segment of TV shows produces emotions (among spectators, among candidates, among families of candidates). In this sense 'emotional labour' becomes 'over-emotionalized'. In addition to their own creative emotions, the freelance workers have to handle the emotions of all these other groups being involved in the production. This leads to negative emotionality among the workers: stress, exhaustion, depression, and conflicts with the candidates, the candidates' families, and the spectators.

Furthermore, there is a strong competition integrated into the production system. Production workers compete to find the best candidates to go ahead with the show. This leads to negative emotions between the workers in the team. Therefore, Hesmondhalgh \& Baker (2008: 115) conclude: 'Our view is that autonomist concepts of 'immaterial labour', 'affective labor' and 'precarity' are at best evocative metaphors rather than theoretical-political constructs with any analytical force.' They opt for a more careful definition and more adequate theorization of the 'affective labour' concept in specific cultural environments in order to understand precarious situations better. Also Gill \& Pratt (2008: 17) point out that there is an important 'role played by affect in generating consent (or even passion) for working lives that, without this emotional and symbolic sheen, might appear arduous, tiring and exploitative'. So, the emotional involvement into creative knowledge work is connected to professional identities in a positive and negative way. The positive effect of this emotional identification is that the workers gain an intrinsic motivation from 
their work, which could not be paid off with more money. This, on the other hand, is simultaneously the negative effect of emotional identification with the creative knowledge work. It delivers a good argument for keeping pay and labor market regulation on a low level. Thus, the emotionality of creative knowledge work makes the workers be exposed to exploitation by commissioning clients and employers.

\subsubsection{High skills vs low pay}

Betzelt (2006) presented a study on the work life of the 'flexible Wissensarbeiter' (flexible knowledge worker). She found these workers to represent a proper type of income earners (Erwerbstyp). Her flexible knowledge workers were characterized by contingent work, low income, and a disappearance of gender bias in the sector. Yet, her workers showed high professional skill levels and were highly educated, and they developed individual strategies to deal with the precarity, in absence of public regulation and social protection. A subjectively coherent understanding of the proper profession (subjektiv-kohärentes Berufsverständnis) is very important in managing risk individually. The individual workers construct sense to what they do; the internalized market risks and the commodification of creative knowledge work could only be faced in collective risk management systems by having such a professional understanding.

Risk management in knowledge sectors, Betzelt found, is organized in ways very different from traditional Fordist unions, because creative knowledge workers are rather individualized. Collective action rather tends to work temporarily and networked. The support systems offer services in the field of business development, marketing, and vocational training for the individual workers. A high demand seems to exist in business knowledge, which is insufficiently taught in humanities from which many knowledge workers start into the labour market. Yet, this collective organization is still rather inefficient and underdeveloped, having no real power to protect against market risks.

Furthermore, Betzelt (2006: 67) points out that precarity is not equally distributed in the knowledge sector: 'Based on intrinsic motivation and a reflexive professional understanding, highly skilled freelancers develop active-reflexive strategies to face the risky conditions, which lead to different, but not necessarily precarious, modes of social inclusion.' Creative knowledge sectors, according to her, are characterized by profiting at both the producers' and the consumers' side from the low income levels of the workers. Thus, she doubts that the current financial organization of the sectors is sustainable for its workforce. From a German perspective, she argues for better social protection in the sector (especially health insurance, unemployment, and pensions) as she has observed that the individual protection potential is too weak.

Also, the social protection schemes could work as a motivating momentum to enter into freelancing/self-employment, which in general must not be precarious when protected. She considers low regulation in creative knowledge sectors as a general problem throughout all countries (to be thought of as a convergence of working conditions), she also sees specific national potentials for installing social protection (divergence of working conditions). First attempts are visible in German social protection to open traditional institutions for the new flexible workers. Finally, she argues for a reform of national income statistics: Better registration of freelance income, better combination of income and household structure patterns. The actual situation of 
freelance creative knowledge workers can only be monitored and social security schemes can be fine-tuned to their needs in this manner.

Similarly, Manske (2007) studied the life of freelancing IT workers in Germany, titling the study 'Precarization on a high level'. She also conceptualizes creative knowledge industries as radicalized markets in which risk is delegated onto the individual freelance worker. Among the web designers she interviewed, she also found them to feel left alone with the insecurities by the government and large enterprises. In a distinction between material and institutional-legal dimensions of insecurity, Manske (2007: 216) mentions the double character of their exclusion: 'The income situation of web designers is insofar precarious as they obviously do not fit neither into the standards of traditional freelance professions [in Germany: lawyers, medical practitioners, architects, etc. with high income and private protection] nor the dependent labor force [lower income but public social protection].' In line with Betzelt (2006), she stresses the subjective professional understanding which results from this mismatch of material and institutional-legal resources on the one hand, and the market realities of the web designers on the other hand. Manske also points out that individual strategies are developed to face the insecurities, which she calls 'eigenverantwortliche Teilhabeprojekte' (self-dependent participation projects). These strategies are composed of self-dependence as well as pronounced future optimism, which leads to a conviction that 'things could be changed'. She concludes that precarity is conceived as self-determined among the web designers of her sample. They strongly stressed autonomy and creative freedom. Manske (2007) also mentions that this understanding of one's own precarious situation - 'aktive Gestaltung prekärer Lage' (active figuration of precarious situation) - is only possible because of the high cultural capital of the web designers.

This therefore brings us to a new dilemma in creative knowledge sectors: the workforce is highly skilled in terms of professional education, often highly talented and motivated, yet the pay for the delivered work is frequently below average. A personal investment into skills and education will not necessarily be rewarded anymore by an above average income.

\subsubsection{Missing regulation and liberal labour markets}

Connected to what we have seen above, Lange \& Mieg (2008) consider creative industries as emerging markets which have to be understood as being in the process of constitution. There are not yet efficient traditional regulation schemes as in established industries (trade unions, industrial organizations, wage agreements, etc.). They even argue that the adaptation of established regulative institutions might even be hindering for receiving attention as an economic force. The emerging markets are only perceived as something 'new' when differences in the production organization can be expressed. Thus, structural insecurity is inherent in the creative industries. There is no traditional scheme for fixing prices, for organizing the market relations, or for rather general evaluations and legitimization of someone/something inside of this market. Lange \& Mieg (2008) consequently identify creative industries as being incompatible with traditional modes of thinking regulation. They suggest conceptualizing creative industries through the lenses of a 'market-as-network' approach, in which the market is regulated in totally new ways of networking reputation, meanings, values, prices, and thus security, within a liberalized and deregulated environment, so regulation and self-organization might be less sophisticated and individual market participants are thus more 
exposed to risk while having less power to negotiate sufficient income to cope with this risk. They have to rely even more on personal networks as a back-up against insecurities.

This fact of missing labour market regulation also means relief from Fordist control mechanisms (rigid structures, hierarchy, financial incentive systems, and bureaucracy). There is a new type of 'soft control' (Florida 2002: 13) which delivers creative workers from these traditional control mechanisms as known from manufacturing or low-end services. In addition to hard financial aspects of work, creative workers are motivated through intrinsic motivation (more in detail see below section 2.3.8). This means they appreciate the freedom of self-management. They can structure their own schedules and are responsible only for the delivery of an output, but it is up to them how they structure their work. Through peer recognition and reputation they also use their work for the creation of their own professional identity, which is not related so much to a company or employer but to the wider field of peer colleagues (cf. Florida 2002).

On the other hand this freedom also means that creative workers often have to bear more of the market risks. As far as the demand in the market exceeds the supply, everything is good for creative workers. But if creative markets have a surplus of labour, the risk of participating in this market is rather high for individual creative workers. The option would then be to avoid market participation through exit strategies, or to diversify risk through multiple job-holding within creative industries or across different labour market sectors.

\subsubsection{Multiple job holding}

Multiple job holding is a common practice in creative industries. On the one hand this is driven by mere economic necessity. I mentioned earlier that creative industries are characterized by the 'winner-takes-all' mechanisms (cf. Caves 2000). There are very few 'stars' who earn a lot, but on the other hand there is a large majority of creative workers who 'starve' in the literary sense. The latter often have to take different jobs in different professional fields to earn their living.

This multiple job-holding also has a functional character, as Florida (2002: 13) mentions: 'Today, the people in my interviews identify themselves through a tangle of connections to myriad creative activities. One person may be simultaneously a writer, researcher, consultant, cyclist, rock climber, electronic/world music/acid jazz lover, amateur gourmet cook, wine enthusiast or micro-brewer. The people in my interviews report that they have little trouble integrating such multiple interests and personae. This kind of synthesis is integral to establishing a unique creative identity.' Thus, having multiple jobs becomes normality and is essential for people to mark their individual identity. The individual curriculum vitae is under current construction. It has to express individuality and distinction from others through a unique combination of professional experience and backgrounds. Here, the multiple job holding - often driven by economic necessity - becomes redefined as a positive property of the individual career. This observation points out a central difference to traditional industrial careers in which a clear linearity without interruptions was considered as indicator for success.

European Labour Force Survey data suggests that around 6\% of people in creative occupations hold several jobs as compared to $4 \%$ in non-creative occupations. In particular, the writers and creative or performing artists (10\%), artistic, entertainment \& sports associate professionals and fashion 
professionals (9\%), social scientists (8\%) and health professionals (8\%) are affected by this phenomenon (European Commission 2010b: 203).

\subsubsection{The role of intrinsic motivation}

In psychology there is the difference between intrinsic and extrinsic motivation in relation to the work in which one is engaged. Intrinsic motivation is nurtured by self-expression, freedom over one's work organization, self-esteem, self-realization, and the pursuit of one's own goals rather than that of others. By contrast, extrinsic motivation is related to financial rewards, the amount of paid vacation, job security etc. Florida (2002) remarks that intrinsic motivation is often more important than extrinsic motivation in creative industries. He identifies three main values that characterize the creative class. First, creative workers are strongly orientated towards distinguishing themselves from the mainstream. They avoid conforming to traditional or modern values and they resist to group-oriented norms. Ironically, this leads to the fact that individuality and non-conformity becomes the new mainstream. Second, creative workers are oriented towards meritocracy. This means they have a strong work mentality. They set goals for themselves which they seriously try to achieve. Thus self-esteem and achievement are central values in this group. Also the reputation in the professional peer group is a big issue, making creative workers very attentive to the feedback they get from their colleagues. This goes along with a diminished importance of material and financial status. It is not so important what you have, but it counts what you know and what you do. Work has to make sense and be in line with the individual value system. Third, creative workers are driven towards environments that signal diversity and openness. Given their individuality and nonconformity they need places where they can feel accepted in their otherness. Also, difference is an important input factor for work, stimulating exchange of different values, convictions and ideas (Florida 2002: 77-80). Florida also cites studies by political scientist Ronald Inglehart and sociologist Paul H. Ray, who have both looked at the changing value systems and norms in the post-industrial societies. In particular, the group of people who are neither traditional nor modern form an emerging segment of the societies. They tend to be orientated towards progressive values. They engage in societal activities that benefit the wider community. They also understand themselves as not orientated in material values, making Inglehart speak of a 'postmaterialist' group. Florida remarks that this shift in norms and values is based on material security. Our post-industrial society could allow post-materialism as industrial times have brought us material security. This means that current orientation to 'quality of life values' could be read as a post-scarcity effect or a reversal of the protestant ethic (cf. Florida 2002: 81f.).

All of these aspects make the creative workers vulnerable to market exploitation. They often underestimate that material security is not granted in the post-industrial society, but they still have to shelter their own material security by doing business; they have to make money with their creativity. As mentioned earlier, creativity is difficult to price, and creative workers themselves often have less interest in developing a price mechanism than their market counterparts, the capitalist businesses, who should buy their products and services. Making money somehow contradicts the post-material and meritocratic values of the creative class. This consequently means that creative workers are vulnerable to power asymmetries in the markets. 


\subsubsection{Gender aspects and compatibility of family and work life}

Henninger \& Gottschall (2007) found that, among cohabiting couples with children, traditional gender arrangements (male bread-winner) are a prevailing pattern of the IT sector, whereas reversed gender arrangements (female bread-winner) were mixing with traditional models in other creative industries. Nonetheless, they also found that women still suffer a feeling of being pressured to be responsible for the household and childcare in both gender arrangements, making a combination of job and household more difficult for them. New forms of household arrangement therefore do not necessarily result in an equal participation of women in labour markets, although reformed labour regulation might foster this.

Studying the satisfaction with job-related aspects among nearly 2,800 creative knowledge workers in the European Union, I also found that male workers are more satisfied with their work in creative industries than female workers (Nadler 2010). However, creative knowledge workers in my study were more satisfied with their work environment when they had children. Thus, I suggest that there is mixed evidence about the social outcome of creative industries work. Against my initial expectation that creative industries might have an experimental character for defining the genderbased roles in a new way, I found remaining dissatisfaction for women. On the other hand, the flexible work schedule, against my expectations, led to higher satisfaction for parents compared to those creative knowledge workers without children.

In contrast, Florida (2002) argues that creative production is not completely compatible with family life and tight social relations. Using the example of historic geniuses, he describes they often had a large group of acquaintances and friends, but they were neither married nor had they children. As psychiatrist Anthony Storr (1988: 103, cited in Florida 2002: 34) said: 'If intense periods of concentration over long periods are required to attain fundamental insights, the family man is at a disadvantage. [...] If Newton had been subject to the demands of a wife for companionship or interrupted by the patter of tiny feet, it would certainly have been less easy for him.'

We can therefore observe that there is no straightforward development of a more family-friendly creative knowledge labour market in which gender equality can be realized. Furthermore, studies are necessary to derive a deeper understanding of the social implications that the proliferation of flexible creative knowledge work in less regulated liberal labour markets will bring along.

\subsubsection{Synthesis: Creative knowledge work in dilemma}

The character of creative knowledge production leads to different dilemmas for the workforce. On the one hand, the products and services are more immaterial, need more symbolic content, yet rely on widespread distribution and attention. They are thus exposed to the threat of plagiarism. The efficient protection of the creative value adding and its commercial exploitation become very difficult.

On the other hand, creative work is linked to being part of the creative knowledge worker's life. Termed 'bohemian' by Florida (2002), these sectors' work provides its workforce with a nonmonetary gain in identification opportunities - with a certain lifestyle or artistic and social avantgarde. Thus, the workforce is more exposed to exploitation risks by market forces (commissioning clients, supervisors in the company, etc.) as other sectors' workforce. In addition, this exploitation 
is often neglected or not even perceived by the creative knowledge workers, who often emphasize the positive features of their work life. A good example for this different perception of creative knowledge work is provided by the opponent interpretation by creative knowledge workers Friebe \& Lobo (2006) and sociologist Bröckling (2007). While the former argue in their book 'Wir nennen es Arbeit' (We call it work) that creative knowledge work is a deliberation from industrial rigidity, the latter calls his book 'Das unternehmerische Selbst' (The entrepreneurial self) in which he argues that creative knowledge work brings along the constraint of continuous work without interruption at the costs of private life. Bröckling calls creative knowledge work a type of self-exploitation.

Furthermore, the creative industries are emerging markets. The character of being 'new' relates to a lack of labour market regulation and inclusive social protection. For now, it could be concluded with the words of Hesmondhalgh \& Baker (2008: 103): 'Cultural workers might be thought of as the pampered children of the bourgeoisie, and they may be relatively privileged compared with other kinds of worker, but the human costs of their working conditions are real enough.' Here, the authors refer to the fact that creative knowledge workers often celebrate themselves and their liberal work environments, yet they seem to underestimate the economic, social and psychological longterm consequences of such a life.

Theoretically, they should be very dissatisfied with the security and monetary aspects of work, but actually they show a high satisfaction with the aspect of emotional and affective work in a liberal environment. I call this the 'trade-off hypothesis': within the satisfaction of creative knowledge workers there is a trade-off between 'hard factors' (pay, job security, contract forms, etc.) and 'soft factors' (self-actualization, freedom, stimulating aspect of work, challenging atmosphere, symbolic production, identification, etc.). The decreasing satisfaction with 'hard factors' might be compensated by a rising satisfaction with the 'soft factors'.

Brown, Nadler et al. (2010), trying to test this 'trade-off hypothesis' using the cases of Birmingham (UK), Leipzig (Germany) and Poznan (Poland), have found hard factor settings of creative work to be disadvantageous compared to the general labour markets: creative professionals had to work above average hours; they frequently had below average income though being highly-educated; they were more strongly affected by temporary employment. These negative hard factor settings also translated into feelings of job insecurity and precariousness. Nonetheless, these feelings are indeed compensated by positive perceptions of the creative work: influence over work, sense of achievement and intellectual challenges were considered main motivators for ongoing professional engagement in the creative sector. Brown, Nadler et al.'s findings (2010) suggest a partial verification of the 'trade-off hypothesis', but it is still unclear to what degree this affects the social sustainability of creative industries' employment.

So far, it is unclear if creative knowledge sectors really develop towards an avant-garde labour market in a social sense as well and not only from a discursive and hedonistic perspective. There seem to be several question marks about the social sustainability of creative knowledge work. Suspicion remains that the over-estimation of intrinsic job aspects leads to an exploitability of individualized creative knowledge workers. Furthermore, more research is needed to clarify if there is any effect of casualised and individualised creative knowledge work on general life orientations and sociality patterns of creative knowledge workers (e.g. stability of partnerships, family founding behaviour, solidarity with other social groups, etc.). 
There is also reason for positive conclusions: work environments in creative industries allow for new forms of self-organization. As several scholars have pointed out, local networks and milieus represent flexible means of social security, however, the modes of how security is built up is not comparable to traditional industrial sectors. While in the latter it was through formal regulation (e.g. collective labour agreements, unions etc.), in the former it is through internal quality management, reputation and trust that security against market risks is established.

\subsection{The creative knowledge worker between arts and science: my own definition}

As we have witnessed so far, defining the creative industries as well as the creative workers is not an easy task. There are different findings about single aspects, and there is much discussion about the best way of creating definitions. So far no generally convincing solutions have come up. Most studies and research projects which deal with creative industries therefore pragmatically use their own definitions. This means I will do so for my research as well.

You might have remarked that I have used the notion of 'creative knowledge workers' so far. I was attracted to simply use the term 'creative class' as used by Richard Florida, but he has been criticized a great deal for that term. I also agree with some of the criticism, but I quite liked the openness and inclusiveness of Florida's definition. It opposes to the exclusiveness of some European definitions (cf. UK DCMS 1998, European Commission 2010a, 2010b). I also liked the idea of basing definitions on actual occupation instead of industrial classifications. Occupation much more indicates if a person is working in a creative job no matter for what their employer is registered. However, what I dislike about the notion of 'class' are its implicit sociological suggestions. Even though Florida tried to distance his understanding of 'class' from common sociological understandings in the tradition of Marx, he still assumes a strong homogeneity of a social group which according to my conviction is very heterogeneous in many aspects. After years of research in creative industries, I do not think that there is something such as a class consciousness amongst the main actors of creative industries. This might change in the future if political and scientific talk about it continues and a broad public and the creative knowledge workers themselves internalize a positive connotation of the term 'creative class', however, I would point out that this group of people is internally very heterogeneous and also pursues the goal of individualization and distinction. Being a good artist or a good scientist means being different from others, standing out from the masses. Instead of a whole class, I prefer to speak of individual 'creative knowledge workers', who are first of all each a case in itself.

Why do I speak of 'creative knowledge workers' instead of only 'creative workers'? I put the two components 'creative' \& 'knowledge' into my term. I understand the adjective 'creative', and creativity as a related noun, in the sense of Bröckling's first field (2006; cf. above 2.1.4) as artistic expression. I use the word 'knowledge' as related to the idea of a more explicit and abstract scientific form of knowledge which can be distinguished from everyday practical or implicit knowledge as well. Thus, knowledge is something that is produced by only a specific group of professionals. Not limiting both these terms here would cause a 'catch-all' problem. The limitations at this point have an epistemological function, I would like to be able to identify my study group from which to sample later on. It does not mean that I think this is the only way to define these two terms; I am aware of and I support other definitions in other contexts. 
The introduction of the two components 'creative' and 'knowledge' has two consequences. First, I merge the two into one term. Thus, I suggest belonging and similarity. I include a large variety of occupations that at first glimpse might differ substantially (e.g. the scientist from the painter), but I think that both related professional fields still share certain characteristics of work life. To me, there is a continuum of a slightly changing nature between a mere knowledge worker and a mere creative artist. So let's take both, a scientist and an artist, as illustrative theoretical figures which represent the extreme of an axis on which most of the empirical reality might be found. Most creative knowledge workers will be positioned somewhere in-between, working in multiple jobs, doing projects at the intersection of arts and science. They share certain features and can be distinguished from other professional groups in other economic sectors. I hope I made clear how work life in creative industries is currently described in the literature (see above section 2.2 \& 2.3), but I will repeat it briefly in the light of the 'artist' and the 'scientist':

- The market is organized in small and independent units, often only freelancing selfemployed individuals who flexibly gather into problem-oriented teams, bringing in their specific specialized skills. Artists often work as freelance workers with a strong focus on individualistic perspective, but they also join into groups to realize larger art works. Scientists are still employed by institutions, but the work within institutions becomes more and more segmented into smaller units, which are problem-oriented as well. The individual scientist can also pursue their very individualistic research projects, just like an artist.

- The work is temporally structured into small, short-term projects which succeed each other. Sometimes with and at other times without interruptions and breaks in employment. Scientists as well as artists are contracted based on project runtimes. When a given project ends and there is no immediate follow-up project, a scientist equally as an artist will face periods without stable income, being dependent on access to social security. The composition of project teams also follows this periodic logic. The teams' composition is continuously subject to change with the succession of projects. This calls for specific social skills such as the capacity to adapt to new colleagues' characters and overlook interpersonal differences. The common goal is often the joint project elaboration and not becoming best friends. Yet, having no stable colleagues anymore forces workers to develop more superficial and quick-to-build social relations that are both functional in economic terms, but also provide a 'friendly and fun' social environment.

- The workforce is highly-educated and has a high affinity to life-long learning. Both artists at arts colleges as well as scientists study and undergo additional professional training during their post-graduate time. Whereas there might be a lot of affective and emotional aspects to work in these fields, there is also a lot of explicit knowledge that has to be incorporated in order to be successful in the labour market. Knowledge gathered through higher education is an important productive factor in these fields of 'brain work'.

- The revenues and profits are redistributed in asymmetrical ways, with a small group of stars earning a lot, and a large majority of workers with low income levels. Thus, for most of the workers it means that the modern logic of return-on-investment in the field of education does not work anymore. This observation accounts for both an artist who has not become Andy Warhol or Damien Hirst as well as for a scientist who has not become famous outside his specific field of academia. In academia there are also examples of individual scientists 
who become 'world stars' and make money with presentations, expertise, or from copyrights on publications (remember e.g. Richard Florida and the Creative Class Group).

- The work is mainly immaterial and non-manual, namely brain work. A lot of work is related to producing ideas, concepts, strategies, knowledge, problem solutions, emotions or symbolic meaning. This includes the idea that production sites can be organized in a more flexible way; oftentimes a computer and internet access is sufficient for production. Thus, flexible work hours and home office models have become part of scientific work, and thus approach the flexible and free working hours of artists. A certain spatial flexibility accompanies both science and arts. If it is using work steps where a computer and the internet are the main resources, the work can be done independent of place.

- Within the markets, access to distribution channels is controlled by single powerful gatekeepers. Thus, profit margins are not only in the hands of individual workers. This equally accounts for an artist who needs a high-rank gallery to make good money with their artwork and is similar in science in which the value of a scientist is measured according to his publications in international journals and his position in citation indices which are based on these publications. The problem then is that there are individual journals which are read more than others, and their editorial boards have power in terms of accepting people for publication or not. Finally, being published and cited increases the market value of a scientist, e.g. in terms of having a higher probability to receive funding for their research.

- Work has a character of self-organisation in the frame of minimal institutional structures. The worker is self-dependent and autonomous: they have to construct and build their own career. There are no clear career paths provided as an orientation structure by the employer or the market. Whereas for artists there have never been such pre-defined career paths, they are also becoming less structured in science. Tenure is becoming less important. Ways of accessing chairs for professorship have been multiplied (e.g. through the introduction of the so-called 'junior professor' positions in Germany). Work contracts have also become more flexible and short-term, forcing scientists into the logic of project rhythms.

- Individual workers continuously compete with each other for resources. In both sciences as well as in the world of arts, scholarships and grants play an important role for individual career advancement. In addition, competition becomes evident in the search for funding of larger projects through foundations or private companies. Finally, the more market-oriented arts and sciences will tend to compete as any other economic sectors' market participant has to do with competitors. Yet, the specific character of artistic and scientific worlds - with small specialist units that are flexibly put together in short-term projects - causes a continuous alternation between competition and collaboration. Individual actors might have competed for resources yesterday and work together on a team today, however, they are unsure what their social relation will be tomorrow. There is less stability and clarity of social relations and roles.

- Workers are highly motivated by intrinsic aspects: self-actualization, personal curiosity as well as satisfaction drawn from work come along with an intersection of professional interest with private interest ('work as hobby'). Thus, these people dedicate a lot of time to work, as work often has a connotation of 'passion'. Working in these sectors is often far 
from being a bread \& butter job, only to earn an income which then finances other spheres of a mere private lifeworld or hobby in which completely different interests exist.

- Pricing often does not follow traditional market models. E.g. the art work is not sold as a mass product with small profit margins and economies of scale. During production it is often unclear what price the final output will achieve as artwork has to be sensually experienced, causing an emotion in a potential buyer. Artwork is sold in unique pieces or small series and then draws value from attention and from discursive elements (e.g. how an expert or a broader public speaks about it / reputational systems). The same discursive elements also come into play when scientists publish their scientific outputs. It is very difficult to project the return-on-investment that financing a scientific project might generate in the future in terms of public attention.

Given these shared aspects of the character of creative knowledge work, I believe that we can think of scientists, artists and of all people in-between as one group, yet within the limits that we focus on this list of similarities as mentioned above.

On the other hand, using 'creative' and 'knowledge', I also suggest difference. I refuse neither the one nor the other. Both words have a more or less equal semantic power, however, I believe that there are underlying differences in being occupied as a scientist, who to me is a prototype of a knowledge worker, producing scientific abstract and explicit forms of knowledge, and a painter, who is prototypical for a liberal and autonomous atom.

Then, there is also a methodological problem that I see: my research project is an exploratory one which will study in detail a small number of individual cases. Thus, I do not study a large group through quantitative data generation (e.g. a quantitative standardized survey) or secondary analysis of existing statistical databases. I conduct qualitative interviewing. I think that for statistical analysis and for quantifying creative industries it is essential to be selective and define precisely. Otherwise, data sets become too complex and would not be manageable anymore, but doing a qualitative exploration of a very specific field - namely transnationally multilocal workers in this sector - I don't think that it is helpful to exclude in an exhaustive way ex ante. Qualitative studies carry the problem of field access and of identifying relevant cases. It becomes difficult to find interview partners in complex and sophisticated qualitative studies with too many and rigid selection criteria. In exploratory studies, following the grounded theory principles, the problem arises from the question of finding relevant cases. Thus, definition should not be too rigid.

To sum up, by creative knowledge workers I will mean those people who are included in the broader definitions of creative industries, including scientific occupations, non-profit cultural activities, craftsmanship and also business services. Finally, in the specific field of creative industries, we have heard earlier that multiple job holding is a common practice. Thus limiting definition too narrowly would probably be dysfunctional as it would not allow capturing the professional hybridity of workers in the creative industries.

\subsection{Creative industries and their relation to places}

In the literature on creative industries, the information society or the knowledge economy a repeated argument is that these new forms of economic activity cluster mainly in selected urban 
agglomerations. At first glimpse, this observation seems counter-intuitive, as ICT and the nonmaterial character of brain work should allow for locating everywhere and independent of place, thus equally including rural and remote areas, yet there is a logic behind this which is mainly derived from the fact that creation and knowledge transfer are linked to face-to-face interaction and communication between people. Therefore, urban environments provide the more advantageous locations compared to sparsely populated areas. Peter Meusburger writes in the introduction to his book series 'Knowledge and Space' (2008: 1): 'In the age of telecommunication, spatial disparities of knowledge have not become irrelevant. Quite the contrary, their significance has increased.'

Creative knowledge workers, managers, and freelancers are supposed to preferably work and live in urban milieus, where they find a critical mass of other people for cooperation, inspiration, and networking in spatial proximity (see Lange 2005). In addition, they find a large number of consumers for their symbolic content in the big cities. Lately, creative knowledge workers have even been discussed as drivers of a process of re-urbanization or the renaissance of the core cities after a long period of suburbanization and urban sprawl (see Wiest \& Zischner 2006; Wiest 2008; Siedentop 2008; Spellerberg \& Wilbert 2008; Gornig \& Mundelius 2012; Kujath 2012). However, not all urban agglomerations attract the creative industries in the same way. 'Places have replaced companies as the key organizing units in our economy' says Florida (2002: 30), and here he means that places such as urban agglomerations have certain location specific assets which either work in an attractive way or not. The main questions are then: how are creative industries linked to place and which features of places are important?

\subsubsection{Urban diversity, open-mindedness and tolerance}

Florida's books (2002, 2008) always rotate around the same line of argumentation: creative industries are crucial for economic growth nowadays. Thus, for a place - let's say a city or a region - it is of utmost importance to attract and retain a certain stock of the 'creative class'. In Florida's argumentation a city is more attractive if it can provide a tolerant and open-minded atmosphere as creative knowledge workers look for inspiration based on difference on the one hand, and a place where they feel accepted with their individuality and non-conformity on the other hand. Florida (2002) methodologically translates this tolerance into a gay index, a bohemian index, a melting pot index, and a racial integration index. The first measures the relative concentration of gay people and the second the relative concentration of people working as artists in a given place. The third allows measuring the relative concentration of foreign-born people. The fourth is a measure for the degree of integration or separation of different racial groups who live in a place. Correlating these indices to data about economic growth, he found that the more concentrated both groups (foreigners and gays) are, the more productive a place is. Florida was often criticized for talking about a causal relation between these variables, yet he does not say that there is such one (Florida 2002: xvii). His statement, however, includes an important finding: tolerant places are more likely to grow than intolerant places. He remarks (2002: xx) that it is about '[...] habitats open to new people and ideas, where people network easily and offbeat ideas are not stifled but are turned into new projects, companies and growth. Regions and nations that have such ecosystems are likely to do the best job of tapping the diverse creative talents of the most people, and thus gain competitive advantage.' Not astonishingly, Florida then explains that the creative class is more attracted by 
large urban agglomerations where diversity can be found - diversity in terms of the people living there, in terms of the ideas and lifestyles being accepted, or in terms of heterogeneous stimulus. These places should necessarily allow creative knowledge workers to express themselves, to experiment and to develop their own identities.

Testing this diversity hypothesis, the European Commission (2010b) found that there is no link between diversity in the EU - as measured in the share of foreign-born people - and the concentration of creative industries. In the EU, creative industries' concentration is strongly related to the population size of a place. 'It is obvious that size matters', says the European Commission (2010b: 213), yet the human capital, measured as the share of people holding a tertiary education degree, is correlated with creative industries.

D’Ovidio adds that in particular, large cities offer a larger opportunity for encounter and inspiration with other creative knowledge workers (2010: 133): 'High density implies close proximity and consequently a high potential for face-to-face interaction and building relationships of exchange, support and trust. [...] It is the diversity of creative workers, when these are highly packed into limited space, that facilitates creativity.'

Therefore, we can observe that creative industries are not equally spread across space. In fact, they tend to concentrate on very specific urban agglomerations in which the potential for exchange is high, and where open-mindedness allows for experimentation and creativity. This is not to say that creative industries cannot be found in rural areas (cf. Ermann 2011).

\subsubsection{Third places, networking and information flow}

Creative industries, as many other economic sectors, tend to cluster in urban agglomerations for their diversity and the input and stimulus that comes along with that diversity. However, there is also another reason why urban agglomerations attract the creative knowledge workers and their businesses. Creative knowledge workers profit from dense social interaction with other creative people and with clients. Business-relevant communication and interaction in creative industries not only happens during the core working hours, but also during what was formerly called leisure time. Evening events such as vernissages or parties are important sites for knowledge exchange, information flow and project planning (Currid 2007; D'Ovidio 2010).

A large part of this creative interaction takes place in a world in-between the professional and the private spheres, also between public places/space and private space. Places with this character between private, the first places, and professional/public, the second places, are discussed as 'third places’ (Oldenbourg 1999; Soja 1996). These third places allow for a reflection about the subjective relation of the creative knowledge worker towards the broader public, and this reflection leads to creation and innovation, therefore, the availability of 'third places' is crucial for creative industries' flourishing.

In the $20^{\text {th }}$ century there was already a growing interest in the 'third places' concept. The starting point was the observation that there was a traditional dichotomy of 'first places' and second places. First places are those places which relate to the private life (e.g. one's own home, a second home in a vacation destination, the grandparents' house etc.). 'Second places' are those ones which are linked to the professional life (e.g. the workplace, the office, professional conferences, locations of 
clients and customers). Oldenbourg (1999) introduced the concept of third places in between the two traditional socio-spatial environments of people. To describe what a third place is Oldenbourg introduced the following criteria which allow for distinction between the private and professional places (1999: 41):

- First, third places have a neutral character, which means that people can come and go without directly being forced to do so. Frequenting these places includes a certain voluntariness.

- Second, third places are defined by social equality. People meet independently of their social status. Social status does not matter within the third place, and access to the third place is not bound to social status.

- Third, these places are characterized by a pre-dominance of communication and conversation in a playful, relaxed and less serious atmosphere. They allow for liberty from professional and private life roles.

- Fourth, third places - next to being accessible for everyone - are neutral in the sense that they provide the feeling of being welcome and accommodated to different social groups. They allow for diversity.

- Fifth, the third places are characterized by a group of people who visit these places on a regular basis. Mainly these people define the atmosphere, styles, and habits, yet these people are not exclusive towards newcomers: they rather invite new people and introduce them to the social fabric of the specific third place.

- Sixth, third places are designed simply but comfortably. They provide a cosy feeling and do not exclude individual groups through specific interior design or modes of conduct. Thus, the built environment is also inviting and inclusive.

- Seventh, the mood of people and the way that interaction takes place has a cheerful character. It is about hanging around with people and making jokes. People respect each other, and hostility and conflict are not part of the environment.

- Eighth, third places can enter into the visitors' emotional awareness. A third place becomes a type of 'home away from home' that has become part of one's own lifeworld.

Whereas Oldenbourg (1999), and later also Putnam (2000) used the idea of the third place in the frame of a declining sense of community in US American cities, there are also a variety of scholars who re-discovered the concept for the explanation of the interrelation between creative industries and their locating cities. More or less obviously, these observations refer to the third place characteristics. Kunzmann (2009: 43) e.g. underlines how important public space and public places are for the emerging creative industries in our cities: 'Even if a large share of cultural life occurs in private space, for attractive cities it is the public spaces that remain the most important places of cultural production and consumption. [They have] an important social function in Europe's future cities, as they are the sites where the oft-cited face-to-face contacts take place. There, people find a stage; they see and can be seen without having to pay entrance fees. For creative business these 
public spaces are indispensable. They provide the urban decoration, which they need for development and attracting attention.'9

The European Commission has also concluded that creative industries tend to locate in urban agglomerations. Calculating location quotients for the year 2008, a significant difference could be observed between urban and rural areas. In some EU metropolitan areas, location quotients, based on occupational data, even rise up to 2.0 or higher, whereas thinly populated areas average at around 0.5 to 0.6 (European Commission 2010b: 209-211). This concentration process is explained by the benefits resulting from agglomeration economies and clustering, e.g. the specialized local labour markets and the available local human capital and tacit knowledge; the knowledge spillovers between creative industries' sub-sectors; the availability of specific collective resources and infrastructures; access to place-based project developments and networks; participation in collective learning; or even supportive local government (policies). Given the mentioned supply-chain linkages between creative industries' sub-sectors, businesses locate close to other potential business partners. For instance, new media companies often settle where traditional media and/or software companies are already located. Equally, advertising companies locate close to newspaper headquarters as well as radio and TV stations.

Furthermore, long-term observations show that the spatial distribution remains relatively stable, suggesting a certain path dependency. The project-based and flexible nature of creative industries also makes it more efficient to be in the hot-spots as it keeps transaction costs for new projects affordable. Face-to-face contacts here play a crucial role for the building of trust and support networks, as well as for the information flow between business stakeholders. Uncertainty in creative industries is so high that direct and unmediated access to the market is crucial for the survival of a business (cf. European Commission 2010b, 2010c; Lange 2007; Lange \& Bürkner 2010). The availability of third places is thus a central issue for creative industries. It is in these places that informal networks unfold their power in organizing the creative knowledge market. In these places creative knowledge workers meet during lunch and coffee breaks or during events and soirées. Business-relevant information can be spread by meeting up 'accidentally', and new project ideas can be developed. Moreover, trust is established in regular face-to-face interaction in third places, defining the membership in local scenes and networks (Lange \& Ehrlich 2008).

\subsubsection{The creative city}

Creative knowledge workers are not only influenced by a city; it is also suggested in the literature that creative knowledge workers and their businesses heavily influence the cities themselves. Accordingly, there has been a broad hype of policies aiming at supporting 'Creative Cities' development (Scott 2006; Landry 2001). A large number of city councils and mayors would like to make their city a 'creative city' to which the creative people should be attracted, and which then

\footnotetext{
${ }^{9}$ Translated by the author from the German text: 'Auch wenn ein Großteil kulturellen Lebens in privaten Räumen stattfindet, sind für attraktive Städte doch öffentliche Räume die wichtigsten Orte kultureller Produktion und Konsumption. [Sie haben] eine wichtige soziale Funktion in den europäischen Städten der Zukunft, da dort die oft beschworenen Face-to-Face-Kontake stattfinden. Dort finden Menschen eine Bühne, wo sie sehen und gesehen werden, ohne Eintritt zahlen zu müssen. Für Unternehmen der Kreativ- und Kulturwirtschaft sind solche öffentlichen Räume unverzichtbar. Sie geben ihnen den dekorativen städtischen Rahmen, den sie brauchen, um sich zu entfalten und um Aufmerksamkeit zu finden.'
} 
should benefit from their presence. How does this work? Currid (2007) e.g. mentions that creative knowledge workers positively affect the 'urban vitality'. They engage in the cultural milieu of a city, offering cultural amenities and organizing a vivid cultural life that consequently also attracts those who work in other sectors, and who want to consume culture as a 'quality-of-life' feature. In addition, tourists might also be attracted by a vivid cultural life.

In particular, in the frame of brownfield redevelopment (the functional redefinition of formerly industrial production sites such as factories and warehouses), creative knowledge workers are considered a pioneer group which often enters into decaying neighbourhoods and uses the vacant buildings for their own purposes. Artists, architects and urban planners as well as craftsmen welcome the often spacious structure and experiment with the attribution of new function and meanings to these structures. Inhabitants from other more established and wealthy neighbourhoods or from outside these cities then become aware of the cultural diversity that is being provided by the creative knowledge workers who redefine decayed neighbourhoods. These people first come to the creative sites and begin to consume the cultural offer, and later on they start to invest in these neighbourhoods. They buy property and increase real estate prices. As a consequence the rental prices increase, many creative knowledge workers cannot afford to live and work there anymore; the so-called gentrification process leads to displacement of the former pioneer groups. The neighbourhoods become wealthier and the cultural scene changes from production-based to consumption-based (cf. Schäfer 2010).

The consequence of this observation is that policies are tailored to make cities creative. As Currid resumes (2007: 455): 'Following this line of thinking, planners and economic developers now seek to provide the amenities, consumption opportunities, and quality of life and place that attract highquality human capital.' For politicians, urban planners and business developers in particular, the emerging debate about creative industries and the related creative city concept arrived at the perfect moment. After the crisis of the New Economy, a new post-industrial economic sector was needed onto which development strategies could be based. Policymakers thus rapidly adopted creative city policies and tried to develop their local creative industries. Such a discoursive focus on creative cities results in a rhetoric that had been criticized early on, because it neglects the associated social costs for the wider urban community. Critics blame this approach for its potential to worsen the situation of the poor and powerless people in the urban social fabric (see Hansen, Andersen et al. 2001). If creative knowledge workers induce gentrification processes, then a concentration of scarce public resources for these actors is aggravating the problems of segregation and polarization in the cities (Ponzini \& Rossi 2010; Evans 2009). Furthermore, Florida mentioned that a creative city cannot be planned from scratch (Florida 2002: xxiii): 'Growing a creative ecosystem is an organic process. [...] The development of such environments cannot be planned from above. The key thing to understand is that the solution lies in the hands of each region - in the knowledge, intelligence and creative capabilities of its people.'

Thus, we can see how creative industries have reached broad public awareness that has caused massive political attention. This political action is often not grounded in professional analysis of the local opportunities and threats that come along with the political focus on creative city development, but this political response mechanism can be observed worldwide as creative industries are suspected to have positive impacts on general regional growth, particularly in metropolitan areas (ESPON 2013 Programme Coordination Unit 2011). 


\subsubsection{Language, culture and national frameworks}

A central element of creative knowledge work is language and culture. In order to produce symbolic values and content, creative knowledge workers need language as a medium to get their message to other people and to produce cultural goods. However, this problem was paid less attention in the study of creative industries. Most case studies on creative industries focus on individual locales and on specific cities or countries. In doing so, they neglect the role of migration and mobility between countries and places for the creative industries and for the cities.

Only few scholars have dealt with the nexus of mobility and creative industries. One such study was the famous book 'Eurostars and Eurocities' by Adrian Favell (2008), in which he studied the life trajectories of young European creative knowledge workers. He calls these young and mobile workers 'Eurostars' as they move freely between different national and regional cultural contexts without feeling uncomfortable. Despite this, he observes that this free movement takes place in a very complex European environment (Favell 2008: 26): 'Even the most casual traveller around Europe quickly understands that the continent today remains a Babel of national distinctions, local customs, and regional peculiarities, which demand of any European an extra-ordinarily high level of nationally specific know-how and local knowledge to feel comfortable living and working outside their country of origin.'

In an earlier work Favell (2003: 422, cited in Pethe, Hafner et al. 2010: 164) commented on Europe's places as locales of a free labour market in a similar way: 'Eurocities remain distinctive, variable environments at the international level, and one has to compete with all the in-built advantages of the local bourgeoisie. Only in rare cases is there a critical mass of foreign residents, such that the structure of the city itself is changed.'

Equally, Pethe, Hafner et al. (2010) stress the importance of language as well as local and national contexts for the attraction of creative knowledge workers within the European Union. Factually there might be a free labour market by law, but there are still large obstacles when it comes to everyday practice of cross-border mobility in Europe. 'The remaining differences between nation states, the incompatibility of pension schemes and social security systems and other hard factors and bureaucratic obstacles as well as soft factors still affect international labour migration.' (Pethe, Hafner et al. 2010: 164). Studying the examples of Amsterdam, Barcelona, Dublin and Munich, they show that it is the degree of cosmopolitan 'look \& feel' of a city in particular which determines how accessible they are for international creative knowledge workers. Dublin e.g. has attracted a lot of international immigration with an English-speaking and open-minded atmosphere, while Munich could become less international as English is less spoken in everyday life and national immigration regulation and tax schemes are disadvantageous. Thus, Pethe, Hafner et al. (2010) emphasize the importance of cultural difference (language proficiency in particular) for the international mobility of creative knowledge workers. The influence of cultural difference is lessened by the internationality of a national/local economy. Particularly, international investment and international headquarters are attracting migrant workers from other countries and therefore induce cultural diversity and otherness in specific locales.

Pethe, Hafner et al. (2010: 187) conclude that the EU labour market is not yet characterized by 'free movement': 'Although the EU as a supranational organization aims to reduce the national 
regulations and wants to level out the differences in the international migration and economic policy, the differences are still more pronounced when compared with the USA which consists of a unified national market.'

Furthermore, Pethe, Hafner et al. explain that it is not only the national and local economic and political context which defines the attractiveness of migration. In creative industries there is a sector-specific importance of the local professional networks that inhibits international mobility: 'If they move to other regions, they often lose those pivotal contacts. This limits their mobility.' (Pethe, Hafner et al. 2010: 188) Finally, there are also problems with the recognition of foreign educational degrees in European countries. In creative industries, which are characterized by the high educational level of its workforce, this is also a disadvantageous aspect for international mobility.

\subsubsection{The role of geographic proximity: Creative industries and local networks}

As we have seen above local context seems to matter in a pronounced way in creative industries, making mobility an adventurous undertaking, but why is local context so important to creative industries? An interesting question which is discussed in this field is the role of geographic proximity in product development. Ganesan, Malter et al. (2005) e.g. studied the product development process in the field of the US optics industry. While optics is not really the core of creative industries, the article still provides a helpful discussion of the theoretical positions concerning the role of geographic proximity. While many of the marketing scholars in business administration understand new product development as an isolated process within one firm, other disciplines such as economics and economic geography have focused on the involvement of different actors in networks and clusters; different forms of knowledge are exchanged and information flows take place within these local clusters. On the one hand, explicit knowledge circulates through formal agreements. On the other hand, there are inter-personal and more informal ways of tacit knowledge exchange between individuals from different firms. A basic condition of both forms of market-relevant information is geographic proximity, which allows for face-to-face contact. This face-to-face conversation then aids the development of inter-personal and inter-firm trust and reciprocity as well as enduring strong relational ties. Furthermore, the concept of agglomeration economies argues that geographic collocation allows firms in the same as well as in differing sectors to profit from externalities such as access to skilled labour and distribution channels, knowledge spillovers as well as market institutions (research institutes, business associations, interest groups).

However, cluster studies reveal few insights about the way that geographic proximity translates into new products. Ganesan, Malter et al. (2005) question the dominant understanding of a causal relation between geographic proximity, strong relational ties and tacit knowledge flows. Using their empirical study in the optics industry, they suggest that strong relational ties might also emerge at a geographic distance, namely through ICT mediation. In particular, they found that email communication was very important for building up relational ties as well as for product development, thus geographic proximity is not the only condition which is necessary for product development. Furthermore, they elucidate that beside informal tacit knowledge, it is particularly 'technical knowledge' which is relevant to product development. 
Ganesan, Malter et al. (2005) distinguish between two forms of knowledge: non-codified and codified knowledge. They suggest that non-codified knowledge is best transferred by face-to-face contact as this mode of communication allows for non-verbal communication and synchronous feedback mechanisms, enriching the understanding and reception of this tacit knowledge. Codified knowledge, in contrast, might also be transferred through ICT mediated ways, therefore it is not so dependent on geographic proximity. Second, there are two different contents of business relevant knowledge: process and product related knowledge. Ganesan, Malter et al. (2005) think of product knowledge as a less complex form of process knowledge. Product knowledge is suggested to circulate more easily through ICT mediated communication, while process knowledge transfer is more likely to need close relational ties and face-to-face contact. In general, they suggest that closer ties are more valuable for any knowledge transfer than loser ties. This means that relational thickness is more important than geographic proximity.

There seem to be hints to the fact that creativity, as a first stage of product development, is largely dependent on tacit, non-codified knowledge, and thus on face-to-face contacts. The second stage of product development, namely prototyping and market introduction, is based more on process knowledge, which itself is more efficiently transferred by face-to-face contacts.

As an empirical result, Ganesan, Malter et al. (2005: 55) formulate 'that (1) geographic proximity is related to face-to-face communication but is unrelated to relational ties, (2) relational ties moderate several linkages in the path between geographic proximity and new product development, (3) face-to-face communication is less effective than electronic communication as a means of knowledge acquisition, and (4) knowledge content has a greater effect on new product development than knowledge form.' Consequently, they argue for a relativised understanding of the role of geographic proximity. Whereas geographic proximity is beneficial to face-to-face communication, it is not straightforwardly related to the strength of relational ties. Given that relational ties are more important for knowledge acquisition, the common theoretical assumption that geographic proximity is generally relevant is not shared by the authors. In other words, if geographic proximity matters, it matters because relational ties in geographic proximity are strong.

Ganesan, Malter et al. (2005) support the idea that in geographic proximity tacit knowledge circulates more easily as it is transferred by face-to-face communication, but they identify more formal types of knowledge as business-relevant, and this knowledge might equally or even better be transferred by email and other ICT mediated communication, independent of geographic proximity: 'Our findings suggest that e-mail and other means of electronic communication are more critical to new product development than is frequent face-to-face contact with external knowledge provider' (Ganesan, Malter et al. 2005: 56). Other studies seem to support this argument. While face-to-face communication might allow for transportation of non-verbal and atmospheric information, it can also be inefficient in terms of providing too much unnecessary information. E-mail and ICT mediated communication is more concentrated on a specific topic, and thus can be more efficient in certain contexts: 'The socially laden nature of face-to-face meetings can actually be counterproductive. In contrast, e-mail appears to help focus communication on the business at hand, thus resulting in more effective transfer of knowledge that is useful for new product development' (Ganesan, Malter et al. 2005: 56). 
They conclude: 'There is no magic that stems automatically from being located near other firms or research institutions in the same industry. Instead, a firm must first develop strong relationships with key knowledge providers to gain access to knowledge, regardless of whether these organizations are near or far.' (Ganesan, Malter et al. 2005: 56)

However, we have to consider that Ganesan, Malter et al. (2005) looked at the optics sector, which could be counted as creative industries if one followed a wider definition including all $R \& D$ activities, but this assumption could also be contested by supporters of a more narrow definition of creative industries.

D’Ovidio focused on more obvious creative industry sectors: fashion, art, entertainment and design. According to her, tacit knowledge transfer plays a crucial role in creative industries, and therefore face-to-face communication is very important, also making geographic proximity a large issue (d'Ovidio 2010: 114): 'There is empirical evidence that face-to-face interaction remains a crucial means of communication for their highly skilled workers, despite the development of new communication technologies and the low cost of their use. The reliance on direct interaction, and the requirement of physical proximity that this entails, are part of the explanation of the clustering of these industries in cities and in specific quarters of cities.'

As in other industrial sectors, face-to-face communication is an important issue not only for the transport of tacit knowledge, but also for the establishment of inter-personal and interorganizational trust. Why is face-to-face communication so important in creative industries? D’Ovidio describes several functional aspects which are specific here. First, creative industries deal with the production of cultural and symbolic goods and services, yet cultural and symbolic contents are perceived very subjectively. Therefore, finding at least a minimum of inter-subjective agreement during product development is important for the success of new products, and such inter-subjective understanding can only be generated by face-to-face discussion in various social contexts. Furthermore, d'Ovidio calls to mind what we have outlined above (section 2.2.1 \& 2.3.3): creative production takes place in small and flexible teams, bringing together specialists from very divergent backgrounds. In order to find a common and inter-disciplinary language for a specific project, faceto-face communication is also relevant: it allows for intensive debate about concepts and their shared understandings.

Furthermore, d'Ovidio describes that local-scale face-to-face contact and the trust built up and being reproduced from it is relevant for the establishment of a local creative community. This local creative community develops its own social dynamic over time, including codes and value systems. New members might be accepted within this community through a socialization process, namely the internalization of these local codes and values. The new members have to share the local culture, habits, and judgements. Acquiring this local cultural capital allows individuals to belong to the local professional community. Additionally, these local creative communities monitor themselves in order to maintain the quality of their products and outputs. Therefore, reputation systems are established, which observe each individual member of the community and their impact on the community itself. If an individual creative knowledge worker increases the personal 'reputational capital', they have easier access to relevant information and are invited to project collaboration more often: 'As in the cultural industries there are no formal credentials that can guarantee the 
creative qualities of a person, an individual's reputation, which derives from being recognised as belonging to the creative community, is of paramount importance' (d'Ovidio 2010: 115).

Finally, local context and geographic proximity are relevant for the unplanned and spontaneous occurrence of situations that help creative inspiration. In particular, the interaction between different networks of the creative industries (e.g. the fashion industry and photography) is necessary to creative processes so that they might lead to successful products.

Through her empirical studies in the fashion industry of Milan and London, d'Ovidio could find evidence that there is another functional aspect to geographic location, though not necessarily proximity between actors. Linking one's own business with a specific location might have symbolic value. Places themselves have identities or images in other places. If these place-specific images are similar or complementary to the businesses' symbolic values, it might be helpful to pronounce geographic origin. In d'Ovidio's study, Milan-based companies use the glamorous image of the built environment in the city centre to sell their prêt-à-porter fashion, while London-based fashion companies operate more in the field of street culture, for which London is also more renowned than Milan. Therefore, London's businesses are also located in the centre of the metropolitan area, but they are distributed around several quarters within it. D’Ovidio says: 'It can be argued that through their location fashion houses establish a link between the aesthetic quality of the built environment and the sophistication and originality of their production' (2010: 121).

Talking to fashion designers in these two cities, d'Ovidio found that geographic proximity allows for maintaining networks and thus strong relational ties. Other than in the study by Ganesan, Malter et al. (2005), fashion designers are somehow more dependent on geographic co-presence to stay in the business, in particular if it is in main centres of the industry: 'The work of fashion designers seems to be all about being engaged in making and maintaining relationships, which requires direct and frequent interaction and therefore co-location of actors. The main demand of their job is to be in the city, constantly reachable and permanently available to meeting people who are part of the fashion system or are engaged in the creative field' (d'Ovidio 2010: 125). D'Ovidio thus considers the fashion industry relationships as a 'contact sport' which is defined by three different scales. First, the individual project team is mainly rooted in the local context. These project teams - here for designing a new fashion collection - meet up in design laboratories often located in fashionable urban neighbourhoods. Then these neighbourhoods and the wider city represent an important scale, where market-relevant knowledge is exchanged between workers from the same field, but different companies and project teams. Third, there is a scale of local interaction which is actually not only linked to the particular profession of the worker, but to the industrial sector as a whole. Here, industrial events such as fashion weeks and trade fairs, fashion label parties and presentations of new collections are important events for meeting with other relevant people, socializing and networking. 'Participation in these events is a must because by being present designers affirm their belonging to the system. It is also during these events that they gain visibility and recognition by the media.' (d'Ovidio 2010: 127) However, it is not only about individual events. Here, the so-called 'third places' become important. D'Ovidio found that her fashion designers have very precise mind maps of where their industry is located within their cities. This includes bars and cafés where fashion industry people hang out and sudden meetings can take place. All of these three levels of local face-to-face interaction are crucial for reproducing and reinforcing one's own position in the market. 
Intersections with other creative industries' sectors are also organized on the local level through interaction in public space and third places within the creative neighbourhoods. D'Ovidio found the London-based fashion designers to heavily relate to artists and seek geographic proximity by locating in the same neighbourhoods. This allows for strong interaction, and this strong interaction with other sectors' creative knowledge workers is crucial for inspiration. D’Ovidio emphasizes that fashion designers e.g. do not talk about the creation process, e.g. discussing a draft model of a fashion piece, with each other; they use e.g. architects, painters, photographers to collect feedback and ideas.

D’Ovidio concludes (2010: 132): 'As they need to be connected in order to function successfully in their profession, time and energy are constantly invested in networking, in seeing each other and being seen in the 'right' places and events.' These right places have the following character (2010: 133): 'Particularly for the quarters established in the last two or three decades, high accessibility, high quality of the built environment, a certain social mix and alternative, bohemian atmosphere are the main characteristics of these places.'

Interestingly, d'Ovidio also found hints to the problems that are associated with the local disembeddedness of creative workers and firms. The traditional Milan-based fashion labels such as Prada or Trussardi are orientating to global markets and hire new and innovative fashion school graduates preferably in England and France instead of employing those who graduate from the city itself. This induces a problem of segregation of the local fashion labour market, with young Italian fashion designers facing difficulties in entering the industry. The economically successful labels do not engage in the local anymore, as their outreach has become global. This also means that the local innovation and creativity system lacks support from the large fashion houses. Such local problems translate into a negative global reception of the Milan fashion scene and the fashion weeks, which have become apparently less interesting, affecting the marketplace in total. 'Monopolized by the 'big names' and lacking new names and faces, the shows are of little interest from the point of view of creativity and originality' (d'Ovidio 2010: 134).

We can therefore see that geographic proximity has an important influence in terms of keeping contact with the particular creative sub-sectors' players. As creative knowledge work takes place in a rapidly changing network structure, it is crucial for the individual worker to be present in a local context (cf. Storper \& Venables 2004). While ICT and the internet allow for global connection, work-at-a-distance and the more focused exchange of explicit and formal market knowledge, geographic proximity ensures face-to-face communication, which translates into trust, reputation and knowledgeability about market relevant developments.

\subsection{Creative industries and mobility between places}

Based on US American experience, Florida suggests that mobility is important among creative knowledge workers: 'Creative Class people are mobile and tend to move around to different parts of the country; they may not be 'natives' of the place they live even if they are American-born. When they are sizing up a new company and community, acceptance of diversity and of gays in particular is a sign that reads 'nonstandard people welcome here" (Florida 2002: 79). Here, Florida underlines his main argument of a 'free-floating' creative class. Being active in different places seems to be a special character of the 'creatives'. The specificity stems from their way of 
moving through space and thereby constructing and symbolically defining their own spaces (see Florida 2002; Lange 2007), which could closely be related to the notion of glocalization (see Brenner 1998). The volatility (in the sense of temporary organization and short product life cycles) of creative industries leads to specific practices of community, such as 'glocal' networking (bridging the local and the global scale) and multilocal acting as one answer to the need for flexibility and constant innovation (see Sennett 2006).

On the one hand, the workers in these creative industries need a certain local rootedness in order to be able to produce their creative services and products from within the networked security of a place and local community. The integration into local networks (being based on spatial proximity) also offers access to tacit knowledge (see Lange 2005; d'Ovidio 2010). This tacit local knowledge is one of the essential economic resources of the creative industries. On the other hand, these managers and workers are highly dependent on the integration into more abstract networks on a global scale (see Florida 2002, 2008). These global networks are facilitated by ICT and modern high-speed transportation infrastructure. Being globally integrated offers another economic resource for the production of creative services and consumer goods. It provides the 'creative class' with information about upcoming trends, new ideas and applications elsewhere on the globe so that the risk of a local 'lock-in' can be reduced (see Grabher 1993). Kunzmann also identifies the need for internationalization in creative industries (2009: 39): 'Creative industries have strong local roots. Yet, similarly as other economic sectors, in times of nearly borderless internationalization actors in the cultural industries are forced to establish durable international networks. Even though they might be heavily dependent on specific locales, they cannot only rely on local and regional markets. This has several implications for individual mobility as well as [...] for strategies of external information and marketing., 10

Yet, mobility seems to be less of an issue among creative knowledge workers in Europe than in the USA. Musterd \& Gritsai (2010), having studied 13 European cities, point out that Florida's assumption of a highly mobile creative class which is driven to culturally vivid and open-minded cities cannot be supported in the European context. First, this is due to cultural constraints between countries. People have to learn languages, native people are less open-minded towards foreigners, and national and local contexts work in very specific and distinct ways. Furthermore, in the European context institutional constraints are very important as an explanation for the lower degree of mobility. Nationally and regionally different education and health systems, pension schemes, labour market legislation as well as bureaucratic issues around business start-ups all inhibit mobility (see also section 2.5.4; Pethe, Hafner et al. 2010). Musterd \& Gritsai resume (2010: 38): 'Our evidence also established that key actors [in creative industries] are much less mobile than some of the literature, notably Florida (2006), would like us to believe. [...] Florida's model may work for single young professionals or bohemians looking for inspiration or a quick career and ready to take off at any moment if they see a better chance. But it does not seem to apply to families with children in a school age, when a perspective of another language and a novel cultural environment may become a serious obstacle. It may also not work for middle-aged couples, where one of the partners is

\footnotetext{
${ }^{10}$ Translated by the author from the German text: 'Kreativwirtschaft hat starke lokale Wurzeln. Doch in Zeiten fast grenzenloser Internationalisierung sind die Akteure der Kulturwirtschaft gezwungen, ebenso wie andere Industriezweige auch, sich dauerhaft international zu vernetzen. Bei aller Ortsgebundenheit können sie nicht nur auf lokale und regionale Märkte vertrauen. Dies hat vielfältige Implikationen für individuelle Mobilität sowie [...] für nach außen orientierte Informations- und Marketingstrategien.'
} 
professionally connected with a certain European area or where the adjustment to other pension rules would also be seriously considered.'

Also Martin-Brelot, Grossetti et al. (2010) critically add that mobility in the creative industries in Europe is strongly dependent on personal trajectories. This includes that people tend to move to places where they are already related to other people. Thus, it is not only the place and its soft factors that work in attracting them, but also the personal relations that a mobile creative knowledge worker has. However, for some creative knowledge workers, being globally integrated is an imperative to stay in the market, it is not only based on personal ties. These workers have to travel a lot between different workplaces, offices, meetings and events. Be it for individual career advancement or the international expansion of their businesses, these people are mobile across borders and engage in different national and local contexts (Pethe, Hafner et al. 2010).

Another aspect of the creative industries is the export activity across borders. According to the export base theory as already developed in the 1950s (Andrews 1953), regional and urban development is much dependent on how good a city or region succeeds in exporting its own goods and bringing in money from elsewhere. Businesses must try to export goods and services if their home markets are characterized by a high satisfaction level of consumer demand. Projecting this idea to creative industries, certain natural problems show up. Creative businesses, with their strong dependency on cultural stimulus and the cultural context, are still focused on their national markets. In particular the language is a central tool to produce and communicate ideas, concepts and information. Furthermore, humour, social values and norms are important frames and inputs to creative production, so going international is not as easy as for other sectors. In addition, creative industries produce more services than goods, and services in general are less tradable (European Commission 2010b: 204). Thus, more research is needed on the topic of 'internationality of creative markets', as the European Commission (2010b: 204) remarks: 'While a number of studies, policy documents in particular, point to the growing importance of trade in creative industry products, and the sound export performance of the creative industries, this issue has until now almost never been studied in a thorough way.' Looking at export figures, creative industries accounted for 3.3\% of total exports in the world in 2005. Yet, this share is slightly decreasing, as other sectors' exports grow more rapidly than the volume traded by creative industries. Given the role of linguistic barriers, it is not astonishing that two thirds of global creative industries' exports are part of design activities which work mostly in a visual and non-verbal way. In the second position, with 13\%, publishing services and goods were exported in 2005.

There is also a geographic imbalance on the global scale. The European Commission's Competitiveness Report reveals that China, the US and the EU-27 account for 66\% of global creative industries' exports (inner-regional exports not included) - with one third by China, a quarter by the EU-27, and the rest by the US (European Commission 2010b). These three world regions also deliver different goods and services to the world market. The EU mainly supplies products in the fields of design, publishing and visual arts to other parts of the world. New media products are also playing an increasing role. Mainly music and audiovisual products were traded across borders within the EU. China's creative exports are also dominated by design products and services as well as arts and crafts products, and it has a rapidly growing share in new media exports. Here, the European Commission mentions that 'these findings are quite intuitive and emphasise the role of common language and cultural norms in creative industries' trade. For instance, prevailing 
cultural and linguistic differences between China and the Western hemisphere make it almost impossible for the Chinese publishing and music industries to compete in world trade' (2010b: 205). In contrast, the US creative industries are competitive exporters of publishing, visual arts as well as new media products and services, yet US creative businesses underperform in exports in the design fields. Even though the exports are only growing slowly in creative industries, the growth is linked to a changing structure between these three world regions. Between 2000 and 2005, the EU27 gained the most in new media, publishing and trading in antiques. It has lost comparative advantage in the fields of video games, interior design and arts and crafts. For 11 EU countries, data was also available in the form of trade balances, showing that these countries imported more creative industries goods and services than they exported. However, between 2000 and 2005 the balance improved due to a more rapid growth in exports. Looking at the character of goods and services traded in creative industries, an important share of royalties and licence fees can be observed: it accounts for $24 \%$ of both imports and exports in the 11 selected EU countries. This again points to the central role of intellectual property and copyright in creative industries even on an international scale (cf. European Commission 2010b).

Looking at Eurostat Business Survey data, it becomes obvious that the internal EU market is less important for creative industries. Only about $13 \%$ of all creative businesses engage in cross-border activities. The main barriers causing this relatively low share are difficulties in the identification of clients in other countries, the lack of international standards for services, as well as language and cultural barriers (see European Commission 2010b: 208).

This review of the literature dealing with the internationality of creative industries suggests that one can observe an increasing integration into global markets. This is more driven by international trade than by international labour mobility. The low degree of international labour mobility is caused by several barriers. First, production is rooted in national and local cultural contexts and linguistic systems. Additionally, the institutional settings differ between countries within the supposedly free EU labour market. This translates into difficulties with the transferability of social security rights as well as the recognition of foreign educational certificates. Given the creative industries' reliance on high-skilled workers, the non-transferability of certificates between countries is a large problem. Furthermore, remembering the often high risk that creative knowledge workers have to bear in their poorly regulated labour market, the non-transferability of social security between countries represents another obstacle to international mobility.

\subsection{Synthesis: Creative industries}

Creative and knowledge-intensive industries have emerged as a new field of economic activity since the beginning of de-industrialization in the 1970s. The emergence of these economic activities is also linked to the individualization process in the society, the general restructuring of the economy as a whole (e.g. outsourcing processes) and the acceleration of product life cycles, as well as the global integration of markets and the related cultural complexities (Lange 2007). This economic sector responds to the need for a more rapid development of new and individualized products and services in more flexible ways. Additionally these creative products and services have to be sold in more emotional ways, not only selling the product but a complete lifestyle. Therefore a higher input of knowledge and creativity is necessary (Musterd, Bontje et al. 2007). 
Creative knowledge work is characterized by a high share of non-manual tasks and brain work. It consists of thinking about new solutions for current problems, thus it entails much conceptual and reflexive work. It is less repetitive and can only difficultly be standardized or automatized. As problem solving and innovation is a rather complex task that often need highly specialized experts for the solution of a single piece of the whole problem, creative knowledge work is organized in continuously changing project ecologies with a changing composition of experts according to the relevant problem to be solved (Lange 2007).

This means that the market is structured in a large share of very small and specialized economic production units (freelancers, micro and small companies) and a small share of large multinational companies controlling distribution (Henninger \& Gottschall 2007; Hesmondhalgh \& Baker 2008; Gill \& Pratt 2008). This structure of creative industries leads to power asymmetries (with a few large multinational companies having power over price mechanisms) which then have consequences for the individual workers.

Thus, creative knowledge workers participate in a very insecure labour market sector, which provides mostly short-term and project-based employment. This insecurity is evaluated as precarity by some scholars (Neilson \& Rossiter 2008). Despite having a comparably high educational attainment, creative knowledge workers face employment and income instability, a comparatively high work load and less leisure time (Brown, Nadler et al. 2010). On the other hand, this flexible market organization is also understood as reflecting the individual freedom of creative knowledge workers (Friebe \& Lobo 2006). They are often satisfied with their work-life balance and indicate being happy with their employment situation because they can actuate themselves, define work tasks and content autonomously and feel free to accept/renounce jobs according to personal interest. This image of the creative knowledge worker can be understood as a traditional trade-off between security and freedom (Brown, Nadler et al. 2010). Creative knowledge workers might be more selfreliant and bear more individual autonomy than other sectors' workers concerning their lives and their work, which might lead to a higher identification with their professional life and a voluntary and playful blurring between leisure and work, between the private and the public (accentuation of the positive side of creative knowledge work - 'freedom', e.g. Friebe \& Lobo 2006). However, they also face a stronger exposure to the risk of self-exploitation, working a lot for a small income due to being too idealistic about their work (accentuation of the negative side of creative knowledge work - 'insecurity’, e.g. Bröckling 2007).

Studying the lifeworlds and spatial attachments of creative knowledge workers is particularly interesting for several reasons. First, due to their stronger insecurity of employment and income, we can assume that they are partly forced into multilocal lifestyles in a stronger way than others. Being mobile and finding work in different places is a chance for income increase and risk diversification. Second, different places are also an input to the production of knowledge and ideas. Combining different local creative networks might mulitply the access to cultural knowledge and resources. Being confronted with the unknown or even the foreign could provide a stimulus for transfer and creativity. Thus, moving around might help to improve the individual expertise and labour value. Third, creative knowledge work deals with symbolic values, therefore it has a strong relation to national cultural backgrounds and language in particular. It is thus of utmost interest to find out how transnationalism can be used as an asset for production in creative knowledge industries. So far we 
have learned that transnationalism could also be a challenge, given the strong rootedness of creative knowledge work in national cultural contexts.

To conclude, I think that the few mobile creative knowledge workers are an interesting group to study in detail. So far, no sufficient empirical results exist on the issue of multilocality and crossborder mobility in creative industries. The field is yet to be explored. In particular, as individualized and autonomous creative knowledge workers are self-dependent, I suppose they must also organize their mobility to a large degree on an individual base. In doing so, they differ from the often studied groups of highly-skilled transnational expatriates who operate in the frame of multinational corporations (Pethe 2007; Favell 2008). Pethe, Hafner et al. (2010: 186) consider expats as those type of migrants 'who have little choice over the country they are seconded to.' Their employing companies 'allocate their employees due to their needs' (Pethe, Hafner et al. 2010: 164). For these expatriates, it is their company which organises their mobility. The company says to where and for how long they have to emigrate. The company organises a relocation service, the school/nursery for the children in the foreign destination, the socialisation for the accompanying partners, etc. However, creative knowledge workers have to construct their careers and employment paths autonomously; in most cases a large transnational corporation is not at hand (except for the few large gatekeeper companies in the distribution activities). They must organize the relocation on their own and are thus forced to deal with all the everyday life problems in the unknown and foreign places.

Mobile creative knowledge workers will then supposedly also differ from the managing elites as described e.g. by Nowicka (2005). Nowicka studied the lifeworlds of high-level employees of the United Nations Organizations. Similar to multinational corporations' expatriates, these administrative elites are also pampered by their organization. Having quasi diplomatic immunity, they can move freely between countries in the world without a necessity to confront national bureaucracy and immigration law. They take part in the UN's own social security schemes, therefore facing no problems of transferability between countries, and they are provided with all of the relocation services provided for private companies' expatriates. Thus, their contact and interaction with national and local culture remains relatively superficial. These people rather circulate within their own cosmopolitanism (Nowicka 2005). In contrast, creative knowledge workers, I would suggest, are forced and volunteer at the same time for contact with local specificity. They might seek contact to the local/national everyday life as this represents a creative stimulus and inspiration. Based on the literature, I would assume that creative knowledge workers, if they are mobile, have a professional curiosity in foreign culture. Foreign culture in combination with their own cultural background might lead to completely new and hybrid creative products.

Given the currently strong focus on expatriate elites in migration studies, Pethe (2007: 228f.) resumes: 'The diversity of different groups of migrants and the variety of processes, which influence the migration are only partly mapped out and not systematically compared. A new research agenda should take this into account. For example, little is known about highly skilled professionals who searched for new positions abroad independently.' I will undertake an attempt to study such highly skilled and self-dependent professionals in the form of mobile creative knowledge workers in the following chapters. 


\section{Interlude B: Emil - Gunnar}

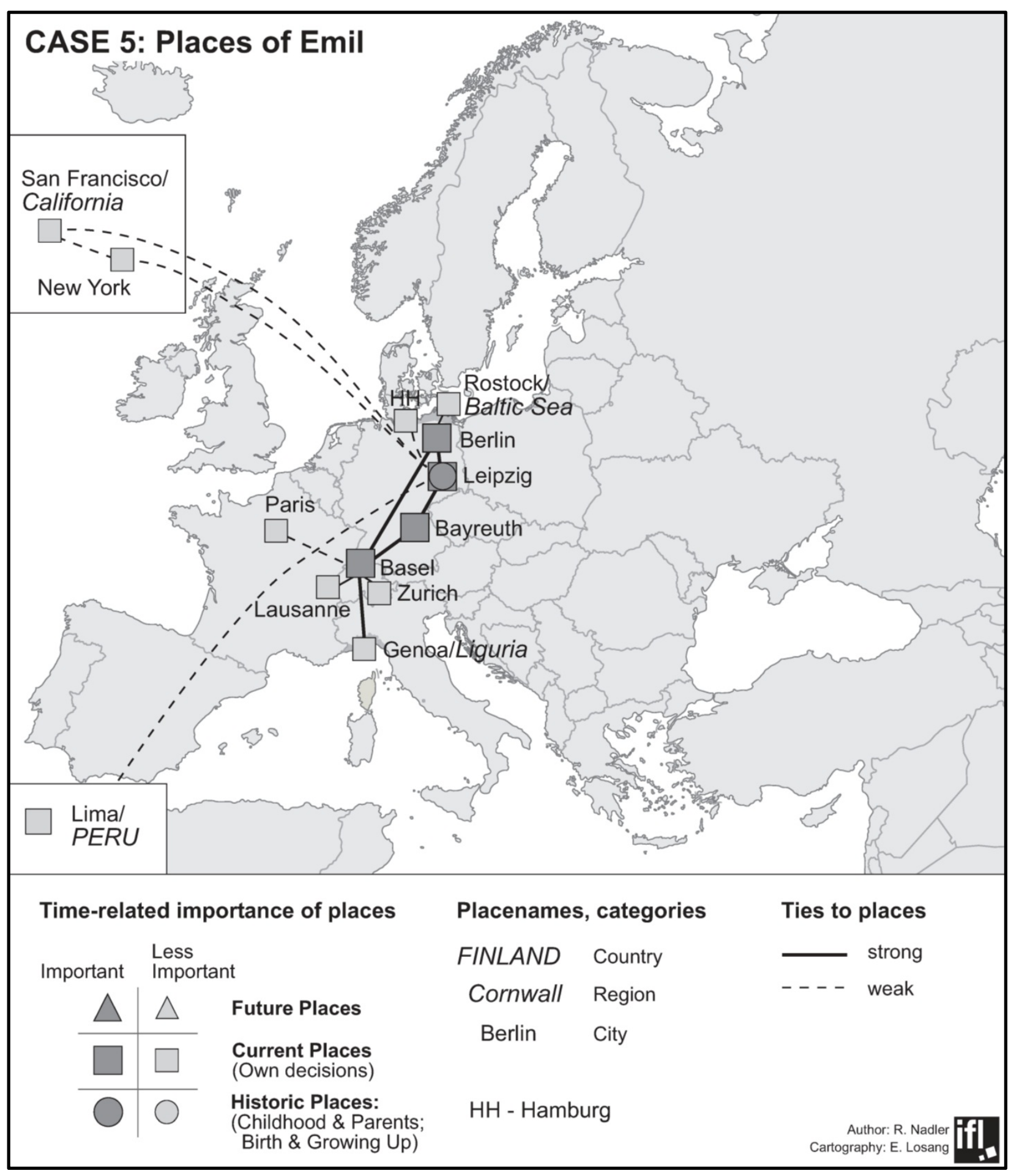




\section{Meet Emil}

Emil is a German political scientist in his 40s who grew up in Leipzig, and which he stills considers his home base, where he has his apartment and where he is enrolled in the universities' $\mathrm{PhD}$ students list. He is currently receiving a $\mathrm{PhD}$ scholarship from the University of Bayreuth. Thus, he also has an apartment in Bayreuth, as he spends about half of the week there. His PhD thesis deals with copyrights, and it is linked to the Law School in Bayreuth and to the cultural historians at the University of Leipzig.

Furthermore, Emil is also living in Basel, where he had formerly lived, studied and worked monolocally for more than 2 years. Now Basel is more important to him because of his Italian boyfriend who works there. They share a large apartment to which they regularly invite friends and colleagues for dinner parties.

Some places are still important to him because he lived there before or because they are related with close people. Rostock and the German Baltic Sea were his home for three years, and he always goes back there for holiday. He also lived in San Francisco, USA, to where he regularly goes to see old friends. For 20 years he and his boyfriend have gone for the Easter Break to Genoa at the Ligurian coast. And finally, he also met a close friend from Lima, Peru with whom he regularly e-mails and skypes, and who helps him pay attention to the news from Peru.

In Basel, he used to work for a wealthy person who donated money to an arts project in her own villa, but Emil stopped working there. His civic engagement is now limited to Leipzig, where he is part of a cultural association which deals with local cultural policy. He organizes events there such as exhibitions, classical music concerts as well as public debates.

Emil loves travelling due to the fact that it allows him to escape from places. He does not feel restricted to one place only. He also considers himself as having an unsteady and mobile character, and a person who needs to travel, but sometimes he would love to have more time in one place, to be able to stay, as he has a lot of close friends, which he wants to spend time with in all of his places.

Emil does not have a precise idea for the future. On the one hand, he sometimes thinks his multilocality is exhausting, the travelling itself, the fact that mono-local people do not call him anymore because he is absent most of the time. But on the other hand he likes to stay multilocal with the combination of Leipzig and its eastern German character and proximity to Berlin and his family there; and Basel, with his completely different and fancier everyday life, including dinner parties, trips to Zurich and Paris, etc. He would not want to have to choose between one of the two cities.

Emil's main issue in the interview was the personal enrichment that can be drawn from multilocality in terms of combining the different locations' offers. 


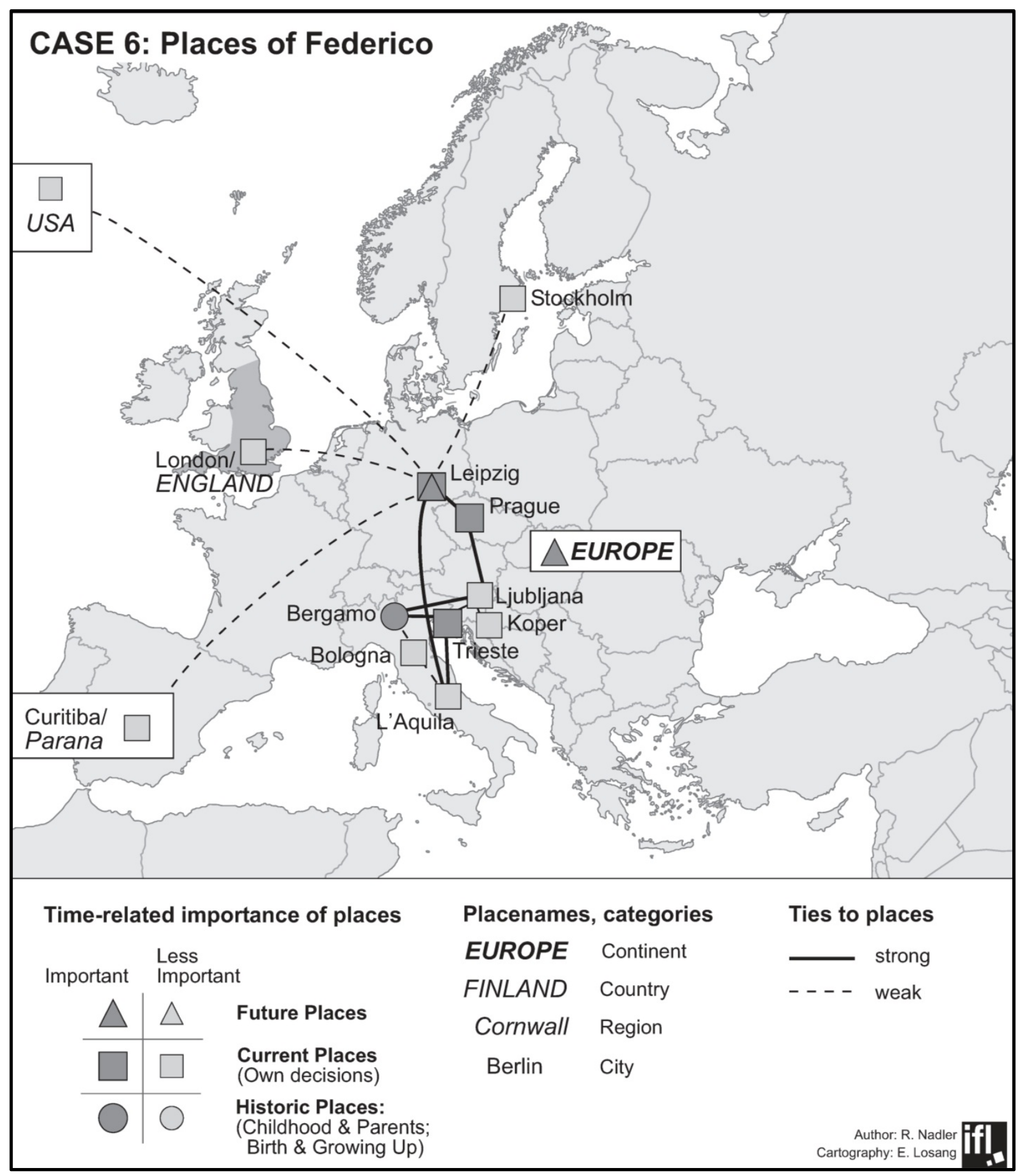




\section{Meet Federico}

Federico is an Italian cultural scientist in his 30s who grew up in Bergamo, but which he would avoid calling his 'hometown'. When he studied in Bologna, he came to Leipzig for an Erasmus exchange year and immediately decided to stay for a longer time. He then finished his curriculum being enrolled in the universities in both Leipzig and Bologna, travelling frequently between the two cities. Later, he did a $\mathrm{PhD}$ at the university in L'Aquila, where he also spent a year for teaching and research, while he continued to live in Leipzig. His main research interest is on transnationalism in Czech and Slovenian literature. Thus, he frequently goes to Prague, Trieste, Koper and Ljubljana, where he has a large professional network and where he works in the libraries and institutes which are cooperating with his institute in Leipzig and where he has a position as a postdoctoral researcher.

Federico likes the frequent travelling because he is quite aware of the different advantages that each of these cities has. He loves Kafka and ancient Prague, but he also loves modern-day Prague's vibrant theatre scene. He enjoys the melting of Italian and Slovenian culture in Trieste. His family also has roots in Trieste, to where his Italian grandmother had to escape during the Tito era's occupation. Thus, Federico had contact with Trieste early on through narrations from his grandmother.

Federico is also a quite reflective person. For example, he prefers taking trains to flying for ecological reasons. And if he needs to fly, he tries to squeeze the best out of this ecological and financial effort. Once going to a conference in Rio de Janeiro he decided to stay for month more, to live and work in Curitiba; he wanted to get in touch with everyday Brazilian life and learn Portuguese.

Today Federico only shares an apartment with an arts student in Leipzig, but he has a surprising travel philosophy: he expects colleagues and friends to host him, because he dislikes staying in hotels. Hotels are anonymous places where one cannot feel and experience the places. He prefers to stay with people who have an everyday life in the places he has to go. This is also related to his passion for suburbs, which for him are the locus of real everyday life, opposed to the touristic city centres in Europe.

Federico engages in cultural events in Trieste that deal with the sometimes difficult situation between Italians and Slovenians. He loves to bring these people together, because through his travel he has experienced that cultural differences exist, but they are not relevant for living together.

For the future, Federico could imagine living a monolocal life in Leipzig, but he believes that the labour market in humanities constrains this idea. He only works on fixed-term contracts, and is currently looking all over Europe for a unlimited position. He sometimes feels that this precarious labour situation is a threat to his future planning.

Fedrico's main story is that of the perception of cultural differences through a transnational multilocal life, which results in a personal tolerance towards others. 


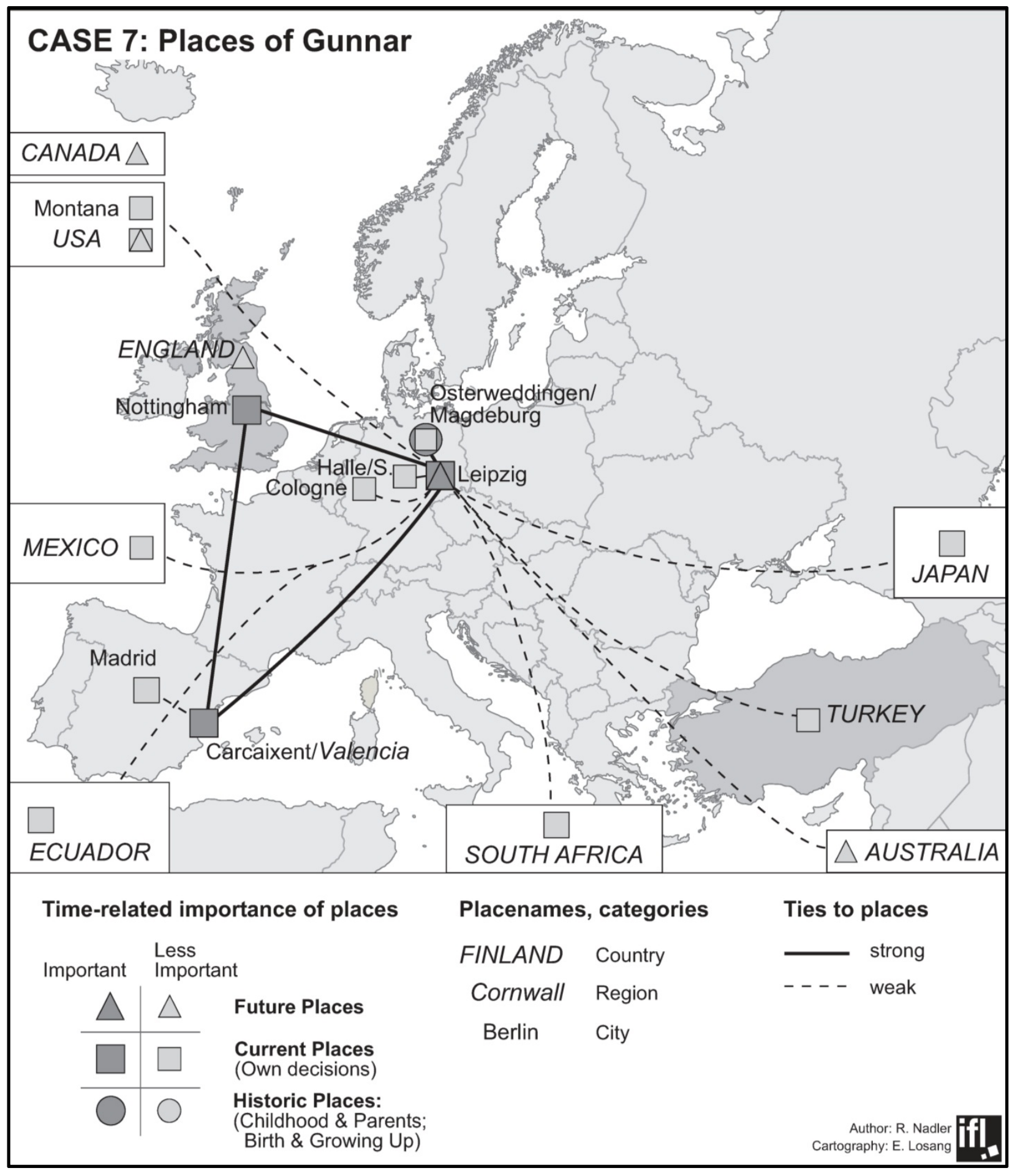




\section{Meet Gunnar}

Gunnar is a German cultural scientist in his late 20s who grew up in Magdeburg and later on went to Halle to study American Studies. During his undergraduate course he decided to do an exchange year at Montana State University in the USA, where he then met his Spanish girlfriend, who was also on exchange during that year and to whom he is now engaged. After finishing his exchange year, he says, his multilocal life really started. He returned to Germany, but transferred to Leipzig University while his girlfriend went to Ecuador to volunteer in a UN programme, so he went to see her there. After one semester she went back to Valencia in Spain to finish her studies and they frequently travelled between Leipzig and Valencia. In that time his girlfriend also volunteered in Ecuador, and he went there to see her. She then decided to enroll in a Master course at Leipzig University as well, and they then spent her first year together in Germany. However, as her Master was an international one, she went to South Africa in the second year. Gunnar then travelled to South Africa to visit her. And for her final semester, she went back to Spain, and they re-established their travel routine between Spain and Germany. So, the first time, Gunnar's multilocality mainly concentrated on keeping the relationship with his girlfriend alive.

When both graduated, they found an apartment in Madrid and worked there for a year. Meanwhile Gunnar was asked by his professor at Leipzig University to come back and work for him, so he decided to go back to Leipzig and start his $\mathrm{PhD}$ while tutoring students and lecturing. His girlfriend then left Madrid and went back to Valencia. So again, they alternated between Leipzig and Valencia. After that year his girlfriend went to Nottingham, England to start her own PhD project. By then, Gunnar had already decided that he was tired of a relationship which for years had consisted of short-term visits and long breaks in between, mainly keeping in touch through daily phone calls. He convinced his girlfriend to move to Valencia in the coming months. Both will continue working on their $\mathrm{PhD}$ projects, and he will stay contracted at Leipzig University, where he will continue to lecture.

Gunnar is also employed by the Leipzig University Hospital as a stem cell courier, which means that he flies around Europe monthly and around the world for two or three days. This has taught him to develop routines in dealing with travelling. He knows to take only the most necessary; he knows how the airports are structured, etc. He thinks that this makes a difference to people who do not travel as much, as e.g. his parents.

Gunnar's multilocal world is mainly a story of how to organize an intimate relationship from a distance. Several times he refers to the psychological stress that comes from not being in the same place. For example, Gunnar described that he rented only a furnished room in Leipzig, as he was living there with the clear intention to leave again, even though he stayed in this room for several years. He also explains that focusing on his relationship from a distance has caused him to not actually live in the places where he was; he more or less lived in a mental projection to the place where she was.

Gunnar was and is engaged in a cultural association dealing with neighbourhood developments, and in the local branch of the Green Party in Germany, but as travelling is exhausting him, he does not feel like actively working there. He prefers to remain a paying member only.

For the future, Gunnar wants to live in one place with his girlfriend and have a family with her although he is quite aware of the difficulties that are included when both partners are working in scientific environments in which mobility is a pre-requisite for building a career. 


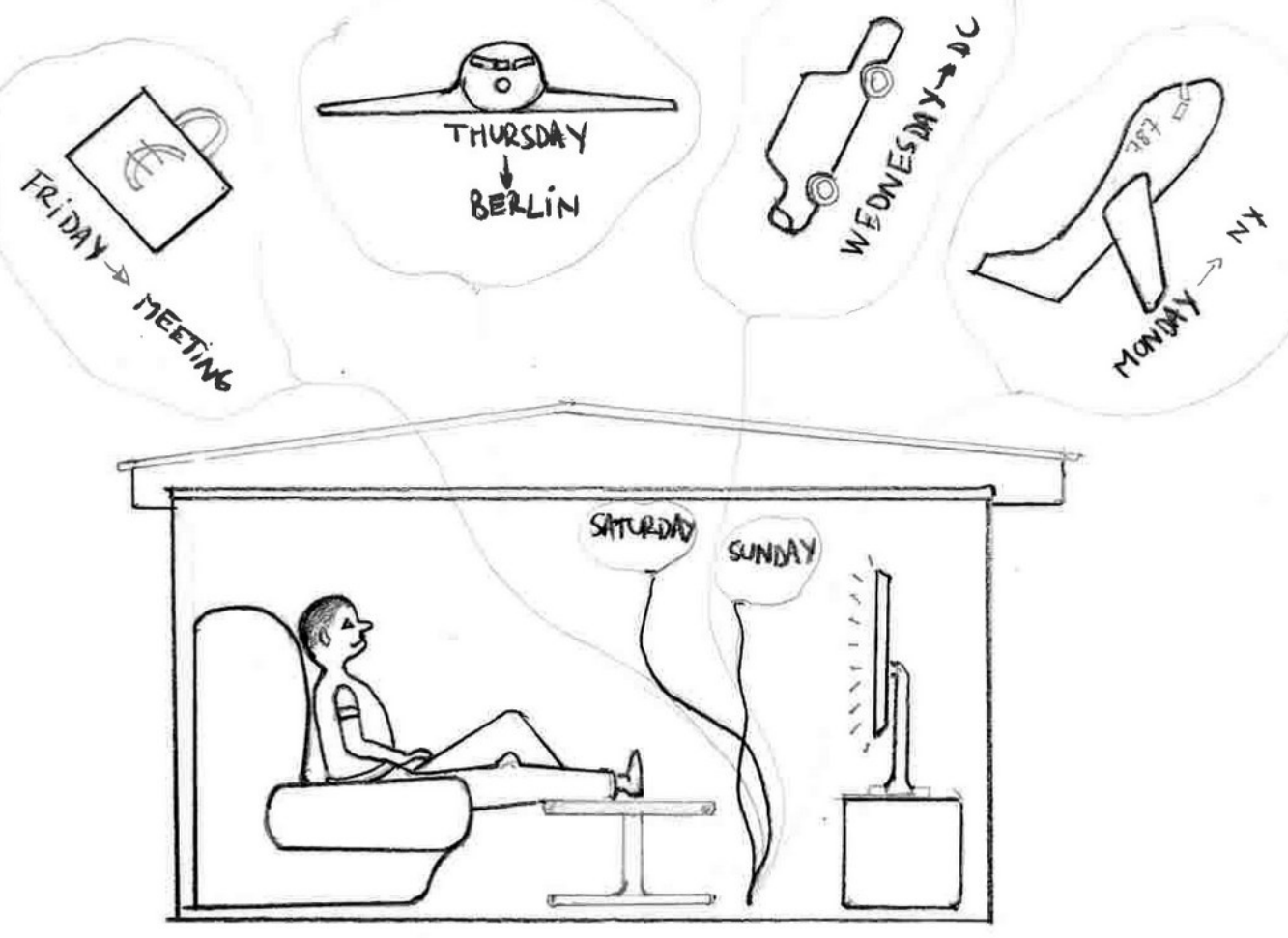




\section{Roots: Understanding belonging}

I must admit that I took the idea of labelling this and the following chapter 'Roots' and 'Routes' from Johannes von Moltke, who did so as well in his book 'No Place Like Home' (2005). He used it to describe from where the idea of the Heimatfilm, a German movie genre, originated (his 'roots') and which development it took (his 'routes'). I rather think of 'roots' and 'routes' in the geographic sense of considering how people incorporate both places and mobility into their lifeworlds, and this idea is actually not mine either: I found it later on in Duyvendak's book 'The Politics of Home' (2011), in which he also distinguishes between 'roots' and 'routes' to describe the two main reference dimensions of home-making practices of 'chronically mobile' people. I think that this pair of words describes quite well the changing of place relations which people develop in a mobile world. I will start with the 'roots': this chapter will deal with the role of places in the everyday life of people. Whereas we have heard in the section before how particularly creative knowledge workers use 'place(s)' in their work life, there is also a wider understanding of how we relate or belong to places. I will try to illustrate this by using the concepts of (1) home/Heimat, of (2) placebased identity, of (3) dwelling/Wohnen, and finally of (4) psycho-social sedentariness. Furthermore, I will reflect the changing nature of place relations by presenting two current approaches by Duyvendak and Dienel, which both look for an understanding of place relations in a mobile world.

\subsection{The concept of Heimat}

One remark in advance: the German word Heimat is a very difficult one when it comes to translating it into other languages, as the word itself is deeply rooted in German history. Applegate (1990), a US American historian, studied how Germans autonomously grounded their Heimat concepts in their own cities and regions (the local scale), while the government sought to artificially construct Heimat on the national scale. She remarks (1990: 4): 'Heimat carries a burden of reference and implication that is not adequately conveyed by the translation homeland or hometown.' Other authors also agree on this difficulty of translation between German and English. For example, von Moltke (2005) faced the same problem, to which he has offered a pragmatic solution: he translates Heimat to 'home' in English, using 'homeland' when it comes precisely to the national scale of Heimat, and 'hometown' when he refers to the local scale of Heimat. I will also be pragmatic here, and use both terms Heimat and 'home' more or less equivalently, even though I am aware of the difficulties of doing so.

The notion of Heimat or home is a central concept in the migration literature. Migration research that deals in particular with return migration after periods abroad as well as the research that deals with expelled 'diasporas', uses this concept to explore how people are 'rooted' and how they refer to certain localities. Scheffel (2012), in his study on returning migrants, provided an interesting review of how the concept of Heimat has developed up to today. According to him, the German word Heimat showed up in the vocabulary in the $17^{\text {th }}$ century, when it referred to one's own house or farm. It described the belonging of a person to a place. With time, the geographic connotation extended to wider areas and was up-scaled. Heimat rapidly concluded the whole village and the local community that existed there. When industrialisation caused a massive migration from rural 
places to the growing cities, many people became even more aware of Heimat. Narrations and discourse from that time tell that those who lived in the industrialised cities suffered from homesickness, which focused on the village and the countryside from where they came. The early urban industrial society was characterized by a Heimat, which was distant and lost to some extent. Heimat became a romanticized image of the countryside (Scheffel 2012). Applegate also notes (1990: 9): 'The Heimat of numerous novels and poems about the countryside and village life was an idyll of local communities, close family harmony, and a domesticated, friendly nature [which] stood in opposition to the city.'

In the following period of nation building, throughout the $19^{\text {th }}$ and $20^{\text {th }}$ century, the notion of Heimat was an instrument of national governments. Heimat was transferred to the national scale. In the 1820s Heimat was up-scaled by politicians from the local level of the community to the level of the state and national society. Applegate describes (1990: 8): 'The [...] administrators were substituting a new principle of state citizenship for the old practice of community control over who belonged and who did not. That so familiar and innocuous a term as Heimatrecht should have embodied this principle reveals an important moment, not just in the confrontation between bureaucrats and hometownsmen, but in the discussion within the progressive bureaucracy on the role of localities in a greater state.' The homeland became the central issue. This moment included a duality between the local and national scale, both of which should be part of the Heimat. The suggestion of that time was 'a basic affinity between the new, abstract political units and one's home, thus endowing an entity like Germany with the emotional accessibility of a world known to one's own five senses. [...] Heimat was both the beloved local places and the beloved nation' (Applegate 1990: 10/11). Applegate analysed in her book how different scales were incorporated by the national scale. She mentions that through media and political discourse the nation as scale of Heimat had 'settled into people's minds', and how the nation then embraced 'their smaller worlds [regional and local Heimat]; Germanness could encompass their diversity' (1990: 13). This observation shows how 'being rooted' in space must not only relate to a place, a local scale. The higher scales often articulate themselves in the local one. I think this quote of Applegate mirrors also the rhetoric of the European Union and its official motto 'United in Diversity', which is only the next step in the up-scaling process. Yet, the nation-state/national society always remained an abstract construct which was difficult to be felt with the own senses. Poets of different periods in German history used the local scale as something what could be grasped and experienced, and what was then constructed as the immediate notch of a nation and national community.

As a result of the World Wars and the fascist regimes in Europe, scientist rejected the use of the notion Heimat throughout the 1960s and 1970s, and many still do so today. However, with rising mobility and global and European integration, Heimat has once again become a central concept in migration studies (cf. Scheffel 2012). For many migrants, the feeling of attachment to a specific place has become an issue again. Von Moltke (2005: 7) remarks: '[...] more recently it has come to serve as a trope for identity politics in an increasingly multicultural society.' This new notion of Heimat implies that people in general, and migrants in particular, construct Heimat actively and self-responsibly. Heimat can no longer be controlled by national governments, because people decide on their own where they feel attached to in a global world.

The problem with the concept of Heimat is that it has existed for many decades, but there is no clear conceptualisation of the word. It is used differently in different periods (cf. Applegate 1990). Anton 
Kaes has concluded that the only possible way of bringing together all of these different connotations is to speak of 'everything that is not distant and foreign' (Kaes; cited in von Moltke 2005: 8). And von Moltke adds (2005:8): 'The longevity of Heimat as a keyword has much to do with this semantic flexibility. One of the defining characteristics of the word may be its adaptability to different contexts. On the other hand, such flexibility poses serious problems for any attempt to pin down its meaning.'

The Italian philosopher Giuseppe Duso (2004: $21^{11}$ ) tries to define it as follows: 'The notion of Heimat refers to a physical, cultural and mental dimension, which gives us the feeling that we are at home in the world around us. We recognise our identity, perceive it as an acknowledged one, and we feel rooted in the world and in a set of relationships. This rootedness makes sure that we are part of an ethic context, which appears as a shared one, and which at the same time is able to found individual action in its very subjectivity.'

Scheffel tries to pin it down even more precisely by explaining that Heimat is based on the imagination, values and norms which a person internalises throughout the socialisation process. Six dimensions are important here (2012: 78):

- First, the spatial dimension describes the physical extension, the actual territory that is part of the individual Heimat.

- Second, the cultural dimension includes values, language, symbols and habits that are related to the place and which are shared with others who also stem from that place. The cultural dimension might be used to distinguish one's own Heimat from other places with a different culture.

- Third, Heimat has a temporal dimension. Only with the passing of time and with experiences that are related to a certain place can this place also become a Heimat. A person has to live in the place in order to experience it, and with experience, a feeling of attachment and belonging evolves. A certain history can be told about that place of Heimat.

- The fourth dimension is the social one. This means that not only the atomic and individual life in a place constitutes Heimat, but also the fact a person shares their feelings with others in that place. Communication is a central issue in defining Heimat. People exchange their experiences and thus identify what Heimat means and what it is. Heimat is an intersubjective concept. This includes the observation that Heimat often refers to a social space in which there is a minimum density of social relations and interaction, and in which there is a mutual understanding for each other which does not need intercultural translation.

- Fifth, there is also a psychological dimension to Heimat. Based on the temporal and social dimensions, people develop an emotional affiliation to a certain place or to a region which they consider Heimat. The emotions are mostly positive, e.g. people feel at ease with places, or they feel they can be themselves without having to play artificial roles. This includes a strong relation to place-based identification patterns - that will be explained in section 3.2.

\footnotetext{
${ }^{11}$ Translated by the author from the German text: 'Der Ausdruck Heimat verweist auf eine physische, kulturelle und geistige Dimension, die uns das Gefühl gibt, daß wir in der uns umgebenden Welt zu Hause sind. Wir erkennen unsere Identität, erfahren sie als eine anerkannte und fühlen uns in der Welt und in einer Reihe von Beziehungen verwurzelt. Diese Verwurzelung sorgt dafür, daß wir Teil eines ethischen Zusammenhangs sind, der als gemeinsamer erscheint und zugleich imstande ist, die individuellen Handlungen in ihrer Subjektivität zu fundieren.
} 
The emotional aspect also becomes evident when people are far from their Heimat and they start to feel homesick.

- Finally, there is an economic and legal dimension that makes up Heimat. The place itself must allow a person a free life in terms of labour market access in order to guarantee the economic survival.

We can see that the concept of Heimat is composed of various intertwining dimensions. By acknowledging these dimensions we can study how people develop an attachment to a place and how they develop roots.

\subsubsection{There's no place like home}

The phrase 'There's no place like home', which comes from the movie The Wizard of Oz, was used by Johannes von Moltke (2005) as an inspiration for the title of his book on Heimat representations in German cinema. But where is this place called home in geographical terms? The problem with Heimat is that it is often unclear to which geographical place, space or scale it might refer. Von Moltke examines this problem with reference to Applegate and Confino. Applegate, he says, breaks down Heimat as a mediator between the local and the national scale, while Confino describes the local as being a metaphor for the national. Principally, it can be said that Heimat describes a relation between people and space. Common suggestions here are e.g. 'the place where one spent their childhood' or 'a place one has elected as a second Heimat' during the course of one's life. Von Moltke (2005: 10) also cites a dictionary from the late 1950s in which Heimat was defined as the territory in which the 'totality of life' is located, and which can be distinguished against other foreign places and areas. This dictionary's definition also includes a connotation of physical abilities of the human being for it says that 'the world of Heimat is necessarily small, for only then it can be experienced completely and be open to that complete familiarity in which humans can take comfort in being at home.' This notion of the experience of the 'totality of life' also shows up in other words as the 'personally inhabited space'. Von Moltke underlines that Heimat is a part of wider space which can be experienced in an individual and immediate way. He resumes (2005: 11): '[...] We think of it as place, as limited terrain that affords its inhabitants respite and protection from incursions originating in the more intangible and abstract spaces beyond its boundaries.'

Yet, von Moltke also makes global mobility an issue in his discussion on the concept of Heimat. Contrary to Scheffel, who uses the rising mobility as an argument to say that Heimat is becoming more important to 're-root' the people, von Moltke implies in a post-modern notion of Heimat that there is an interplay between physically present and distant elements on the local level. This idea was originally developed by Anthony Giddens, who said: 'Place becomes increasingly phantasmagoric: that is to say, locales are thoroughly penetrated by and shaped in terms of social influences quite distant from them' (Giddens; cited in von Moltke 2005: 12/13). Von Moltke thus argues that Heimat should not be equated to one confined place. Heimat must be understood as a geographic relation between different scales e.g. the local and the global, or between distant places. If one stuck to the static and pre-modern version of Heimat as one confined place, one would only reproduce the 'invented tradition', this nostalgic hindsight that was supposed to help people orientate in modern times, as von Moltke says (2005: 14). Yet, there were and are many more connotations to Heimat. There were the legal aspects related to modern national citizenship; there 
were the connotations related to modern urban environments and technological progress and therefore there was always a linking function between modernity and tradition.

The standpoint of Pasonati (2004), who hosted a scientific conference about regional identities in the Austrian and Italian Alps, is also equal. Pasonati generally observes that within Europe, national scale references of Heimat lose ground to the regional scale. At the same time, the European scale is not yet able to work as a Heimat for the people. The whole process of changing geographical references includes a de-territorialisation and disorientation. People are looking for new geographical anchor points. Pasonati concludes (2004: 14 ${ }^{12}$ ): 'Nowadays Heimat can no longer mean the wish to make one's own idea of happiness real in a cosy place in the country and in rural privacy. Rather it is about looking for the new meanings of the word within the context of the natural and general longing for security or any type of anchor place.'

Given all of the current dynamics in global and European mobility, Duyvendank thinks of home as a 'multi-faceted and multi-scalar' phenomenon (2011: 4). Within this multi-scalarity, different scales have different meanings that are inter-related and sum up to a coherent pattern for the individual person. To Duyvendank, in particular the nation, the community on a local scale, and finally the house and household are the main scales of reference for feeling at home. Duyvendank also thinks that the national scale is somehow losing importance, and the local community might become more important than it has been so far.

Gustafson (2001: 680) has also found out that place attachment is related to very different scales: 'Place attachment may be directed toward residence, neighbourhood, city, region, or country (and indeed toward several places of different spatial scale).' Thus, we can see that it is not an easy issue to relate the notion of Heimat to a specific geographical reference. Different scales are intertwined, and also same scale references might exist parallel to each other within a person's idea of Heimat e.g. two or more cities, or two or more regions.

\subsubsection{Home is where your heart is}

This famous phrase makes it clear that there is also an emotional aspect to home or Heimat. For example, it shows up as a metaphor in Kearns \& Smith's (1994) quote on homelessness: 'Someone might be adequately housed but spatially removed from their acutely felt sense of home (i.e. some other place of greater felt meaning or value)' (Kearns \& Smith 1994; cited in Cloke, Milbourne et al. 2002: 13). Cloke, Milbourne et al.'s (2002) subject is homelessness as well. In order to deal with it, they start from the notion of home and then link home and homelessness as mutually influencing aspects. For them, the 'metaphorical homelessness' as described by Kearns \& Smith (1994), includes that the idea of having a home is far more complex than only possessing of access rights to a physical infrastructure. While they remain on the scale of the house or the apartment, I think we can find parallelity to the discussion of Heimat and what Scheffler said about the fifth dimension of

\footnotetext{
12 Translated by the author from the German text: 'Heimat kann heute nicht mehr bedeuten, an einem lauschigen Plätzchen der Provinz und in ländlicher Zurückgezogenheit seinen Traum vom Glück verwirklichen zu wollen. Vielmehr geht es darum festzustellen, welche neuen Bedeutungen das Wort innerhalb der natürlichen und allgemeinen Sehnsucht nach Geborgenheit oder einem irgendwie gearteten Ankerplatz annimmt.
} 
it: Heimat or home has a clear emotional dimension. A person must be able to feel it, to feel at home.

This emotional dimension also shows up in the book called 'The Politics of Home' by the Dutch sociologist Jan Willem Duyvendank (2011). Duyvendak observed that both in western Europe and the United States 'home' has become more and more connotated with an emotion of nostalgia. In western Europe people feel confused by the changes within their immediate neighbourhoods which come along with global migration flows. Formerly homogeneous communities have become diversified. As a nostalgic reaction, nationalists use the nation as a metaphor for home, which still tries to tell the story of a homogeneous national society. On the other side of the Atlantic Ocean, the nation as a homogenous society has never been a similarly strong issue. Here home resulted from a specific feeling for one's own house and family. However, this type of homey feeling is also becoming threatened by global societal changes. The participation of women in the labour market redefines the role of men and women in relation to the family. Family life is eroding, and US Americans feel 'more-at-home-at-work' (Duyvendak 2011: 4). Thus, the emotional feeling and nostalgia focuses on a past harmonious family life, which is subject to change in post-modern labour markets.

Finally, I will come back to one issue: Heimat is something dynamic. Scheffel (2012) points out that people can develop a feeling of Heimat to very different places in life. This means that we should not understand Heimat or home as a life-long stable item in a person's lifeworld.

On the other hand, place attachment can also remain stable in the course of a lifetime: 'It may involve not only the present home place but also, as the case studies above clearly bring out, earlier places signifying childhood and origin in addition to other meaningful places'. (Gustafson 2001: 681) There seem to be places that are more meaningful in a person's life than other places, and these more meaningful places have a higher chance of remaining part of the personal Heimat. Yet, Heimat is subject to change over time, depending on a person's emotional attachment. To conclude, the basic question that results from the Heimat concept is: where does a person currently feel that they belong to?

\subsection{Identity and place ${ }^{13}$}

So far, we have seen that Heimat/home is a difficult concept to grasp. Identity and processes of identification are complex social phenomena, too. They are difficult to operationalise. In sociology 'identity' and 'identification' are mostly understood as describing the relationship between an individual and an object of reference. The latter is often understood as a social group. However, in human geography forms of 'place-based' identification or finally place identity are particularly relevant topics. Let’s have a look at both disciplinary approaches.

\footnotetext{
13 I have presented parts of this section in a conference paper titled 'Identity between places and peers? Insights from a study about multilocal creative knowledge workers in the European Union' during the Annual RC21 Conference 2011 'The struggle to belong. Dealing with diversity in 21st century urban settings', Amsterdam (The Netherlands), July 7-9 2011 (http://www.rc21.org/conferences/amsterdam2011/prog-19.php; accessed 31 October 2012)
} 


\subsubsection{Identity in sociology and psychology}

Identity and identification form a complex issue that takes a prominent role in psychology and sociology. After several decades of research about it, the terms could be found in various scientific dictionaries, which reflect a common sense of definitions. Jenkins for example says (2008: 5):

'As a very basic starting point, identity is the human capacity - rooted in language - to know 'who's who' (and hence 'what's what'). This involves knowing who we are, knowing who others are, them knowing who we are, us knowing who they think we are, and so on: a multi-dimensional classification or mapping of the human world and our places in it, as individuals and as members of collectivities. It is a process - identification - not a 'thing'.'

Similarly, Bhushan (2003: 127) remarks:

'Identification refers to a social-psychological process which involves the assimilation and internalization of the values, standards, expectations, or social roles of another person or persons (for example, one's parents) into one's own behaviour and self-conception. [It thus includes] having a sense of 'oneness' with him, and being able to put one's self imaginatively into his position'.

Hence, identity is the outcome of a process of identification. This process of identification can be understood as the placing of the self in the closer and the wider world. Here, place is not necessarily to be understood as a physical one. Rather it refers to a social place, to a personal understanding of one's own position in society. As identification is a process, its outcome, identity, is not something static which endures once constructed. Rather it changes continuously. Thus, from an analytical point of view, it is more interesting to study processes of identification instead of 'identity' which could not be more than a temporary snapshot. Although an interesting 'starting point', understanding identification is not that simple and we should have a more detailed look at how it actually works.

Weichhart (1990) provides an interesting overview about the origin of identity research. I will repeat his chronology here, as presented in chapter two of his book 'Raumbezogene Identität' (1990: 14-24). Weichhart mentions that the study of social identity dates back to the work of the psychologist Mead (1934), who studied the development of the 'social self'. Mead was a representative of Symbolic Interactionism, who thought of the social self to be a result of processes of social interaction. The most important element of such interaction is verbal communication, through which the individual receives feedback about their appearance as seen by others. This feedback then becomes internalized into the reflection about and the construction of one's own personal identity.

Weichhart (1990) then remits to Stone (1962). Stone developed Mead's ideas about the production of identity by distinguishing between 'appearance' and 'discourse'. While Mead assumed that individuals take roles within an interaction, Stone criticized that role-taking is not explained by Mead. Stone says it is especially the moment of 'appearance' - before verbal communication which helps individuals to understand the other and their role. Only then can 'discourse' emerge namely the communication about specific content between interacting individuals. Stone distinguishes between two processes which are related to that 'communicative situation' and which lead to the development of identity. First, there is the 'identification of' the interaction partner in the moment at which the interaction starts. This process is based on the 'appearance' of the other. Once 
having identified the other, a second process of 'identification with' the interaction partner begins, in which the conversation or 'discourse' about an issue might lead to a feeling of sameness. Therefore, this approach for explaining identification consists of a distinction between the cognitive perception of the interaction's environment and the actual content of the social interaction (cf. Weichhart 1990: 15f.). Later, Stone (2005: 143) summarised: '[...] identity is established as a consequence of two processes, apposition and opposition, a bringing together and setting apart. To situate the person as a social object is to bring him together with other objects so situated, and, at the same time to set him apart from still other objects. [...] To have an identity is to join with some and depart from others, to enter and leave social relations at once.'

According to Weichhart (1990), Graumann (1983) adds a third process of 'being identified' to Stone's two perspectives of 'identification of' and 'identification with'. He assumes that people are not only actively identifying others and deciding about identifying with them, but they are also identified by others as having a certain characteristic. Being identified means to be ascribed social attributes. The pattern of social attributes with which a person becomes ascribed will be internalised during the process of socialisation. Graumann also broadens Stone's 'identification of'. According to Graumann, it should be labelled 'identifying the environment' which actually includes psychological processes of experiencing sameness or difference with other objects. For him, this identification consists of categorising the environment and thus being able to orientate within it. Finally, Stone's 'identification with' is modified by Graumann into a 'identifying with one's environment': people identify with persons and things in their environment which are as they themselves would be. They work as 'models' or 'reference groups' which shape the appearance of the respective individual (cf. Weichhart 1990: 16ff.).

Finally, there is also a distinction between 'personal identity' and 'social identity'. Concerning personal identity, Doise (1998: 23) remarks: 'Personal identity can be considered to be a social representation, an organizing principle of individual positioning in a field of symbolic relationships between individuals and groups. [...] Not all social relationships in which we take part are equally present in our minds. Depending on situations, various relationships become salient and give rise to different identity dynamics.'

By contrast, Mühler \& Opp (2006) describe identification, according to Tajfel's Theory of Social Identity, as a process in which an individual actively tries to be as similar as possible to a social group which it personally evaluates in a positive way. As a consequence, being recognized as part of that reference group by others leads to a positive social identity and individual satisfaction. Social actors thus continuously also engage in the amelioration of the social group's perception in the whole society in order to maintain their positive social identity. However, there is a difference between self-ascription and external ascription by others.

We can see that identity as discussed in sociology and psychology is a complex interplay between individual perception of one's own position in society and the external perception of this position by others. The individual reflects on what others think of them. This represents a continuous evaluation of reference groups. Furthermore, Graumann has already pointed to the role of physical objects and things as well as the physical environment for processes of identification. 


\subsubsection{Place-based identity}

Weichhart (1990), as a human geographer, is interested in the cognition of space. He tried to combine geographic with sociological and psychological theory in order to understand what role 'place-based identity' might play for an individual as well as for the society as a whole. Weichhart (1990) suggests that identification processes could also be based on 'reference places' instead of reference groups/persons. As long as the relevant place has a certain symbolic function and could be defined with a certain character, an individual might also be identified by a relation to such a place (e.g. place of birth, place of residence). Not only the individual level might be subject to placebased identity, but also collective identities could emerge which help to characterize a group of people from a certain territory. Here, the traditional 'we-they' divide comes into play: Once a certain group of people internalizes an ascribed identity, it starts to use it to distance itself from others, and for this distinction this group might also start to express claims to a certain territory which they understand as their own.

Since the 1970s, 'place' has increasingly showed up as one referential dimension in studies about identification. Here, place is integrated into the process of identification in terms of an individual's or group's cognitive representation about that place (Weichhart 1990, p. 20). This cognitive representation helps people to distinguish 'this' place from 'other' places. In this sense places are thought of as objects of the everyday lifeworld, which become part of the spatial cognition as well as the communication between individuals. They help people to orientate both in a physical (denotative) as well as a psychological (connotative) way. In this sense, places could be used to identify a spatial environment according to Graumann's 'identifying the environment' or Stone's 'identification of'.

Yet, also Stone’s ‘identifying with’ or Graumann’s ‘identifying with one’s environment' and 'being identified' open a second perspective on the role of place in processes of identification (Weichhart 1990). Here, place is not functionary to spatial orientation, but it works as creating a place-based individual or collective identity which allows its users to group others into being 'from here' and 'from there'. Individuals use certain places as part of the social self. On the other hand, they are also ascribed place-related parts of their identity by people from outside this place. Weichhart concludes (1990: 98f.): 'Space-related identity must thus be interpreted as 1. the subjectively perceived identity of specific places and 2. their collectively perceived identity [...] 3. those spatial segments of environment that an individual includes in his/her self-concept [and 4.] the identity of a group that considers a certain segment of space as a partial element of the ideological representation of the 'we-concept' or as defining criterion for the determination of identities of other groups ('theyconcept')'.

Place-based identity fulfils several social functions. First, it helps individuals to maintain their 'egoidentity'. Individuals use spatial reference in order to find security, to stimulate activity, to interact socially and set symbols, as well as to individuate themselves. Second, spatial identity is used to stabilize social systems by integrating different space-related sub-systems into the super-ordinate level. It thus provides reference and orientation and facilitates social order, communication as well as expectation. Third, it leads to group cohesion and solidarity and thus fosters social integration (Weichhart 1990: 99f.). 
Weichhart describes collective place-based identities as 'symbolic communities', which have a low emotional involvement of the participants, and in which the spatial reference is understood as delimitable. Group binding is a result of symbolic binding and group loyalty. It is less an outcome of jointly inhabiting a relevant territory. Thus, there is not necessarily real interaction between the group members. Here, also Anderson's ‘imagined communities' concept could help an understanding of place-based identity (Anderson 1983). The imagination of the character of a community from a certain territory could cause a feeling of attachment and belonging. An individual must not necessarily have met personally the community's members in order to identify with that community. To identify with this community it is sufficient to know what type of image this community has and if oneself would like to be identified as a member of that group as well (cf. also Phillips 2002).

Finally, there is the question for the most relevant spatial scale of reference, which Weichhart supposes to be the local and the neighbourhood. He assumes (Weichhart 1990: 100): '[...] that the cognitive as well as the self- and 'we/they'-related aspects of place-related identity are equally orientated on a continuum of scale ranging from personal space to national and supranational entities and that the aspects are focussed on the various scales depending on the specific context of action and situation. Seen from the perspective of the individual, however, the local level turns out to be the principal dimension of reference. The experience gained on this level of space-related identity may be transferred to other objects of reference on different scales by means of generalisations based on similarities'.

Most people thus oscillate between different geographic scales when it comes to defining their spatial identities. Yet, the cognitive departure for this oscillation starts mostly at the local level which can be experienced in everyday life. The oscillation is organized as a process of transference of local cognitive models to the more abstract supra-local levels. The level of reference is dependent on the specific situation in which an individual is asked for their identity. The immediateness of the local level becomes the most important factor for the predominance of the local level in individual place-based identification (Weichhart 1990: 78). Weichhart argues that the local level is a sphere which can be experienced directly through the corporeal senses (seeing with the eyes, touching with the hands, smelling, hearing, etc.). One can walk through a neighbourhood, a city, but walking through a region might become a sporty enterprise. Finally, walking through a country or a continent really needs courage and fitness. Here, Lynch's concept of 'legibility' might also play a prominent role, too (Lynch 1960). Lynch described that places have different degrees of legibility; there is a varying simplicity in orientation. This idea also brings us back to the physiological senses, giving the local scale a prominent role. However, when it comes to supra-local identification scales, external influences also affect the individual perception (e.g. nation building, place branding, etc.). These external images and identities might be internalised by individuals (Mühler \& Opp 2006).

Mühler \& Opp (2006) operationalise 'regional identification' as a process in which a territorial unit becomes an 'object of attitude' (Einstellungsobjekt) for an individual (2006: 18). A person actually does not identify with a geographic place itself but with a group of people that are characterized as living in or originating from a place/region. In the perception of an individual this people's origin gives them a certain characteristic to which this individual might feel emotionally or affectively related. The individual might evaluate these characteristics as positive. Based on this positive evaluation, they might then develop a sentiment of belonging to this group of people. Place-based 
identification is thus a type of identification in which a social group is the origin of the feeling of attachment. Yet, this feeling is focused on the mediating object of the 'place' or 'region'. It is about identification with a social group whose unifying moment is a 'place' (Mühler \& Opp 2006: $22^{14}$ ): 'Often [we] speak of a 'regional' or 'supra-regional identification', and here we mean 'identification with a group, which amongst others can be characterized by the belonging to a specific region'.'

To conclude, place-based identification is composed of two major reference categories: objects and persons. Physical objects (e.g. buildings, landscapes, etc.) work as directly influencing positive or negative associations with place. However, people who are identified by others as stemming from a place - or that self-identify with a place - base these place-based parts of their identity also on the social components of a place (cf. the concept of 'socio-scapes' as described by Albrow 1997). A place becomes characterized by the people who inhabit it. Being from there is then only a simplification of the actual connotation being like the other people from this place. Thus, the social composition of a place translates into its image and identification character.

\subsubsection{Place-based identity in the European Union: some empirical insights}

In the European Union, European integration is supposed to have a strong effect on processes of identification among EU inhabitants. Mühler \& Opp 2006 criticize that this wide-spread hypothesis is not empirically grounded. They used a panel survey with about 1,000 Saxonians to test some common hypotheses and to study the dynamics of their identification between 2000 and 2003. Basically, they assume that different geographic scales might be combined in processes of individual place-based identification, and that their hierarchies might vary in time. They also look for the inter-dependencies between identification and engagement with a region.

Mühler \& Opp use two main indicators to measure place-based identification: 'feeling part of a certain place' and 'being proud of originating from a certain place'. Three hypotheses guide their analysis (2006: 45-49): (1) a higher quality of life increases regional identification; (2) primary socialization (being born and growing up) in a region increases regional identification; and (3) conflict situations, stressing the population of a region, increase regional identification. Furthermore, they suggest that regional identification causes: (1) region-oriented behaviour (regional consumption, representation of the region); (2) engagement in regional politics; (3) regional orientation - personal interest in regional developments and societal life, low willingness to move; and (4) regional centrism - degradation of other regions and people from other regions, especially when other geographic scales are missing in the individual identity. These four supposed effects of regional identification are combined in the notion 'regional focussing' (regionale Fokussierung) (Mühler \& Opp 2006: 49). Mühler \& Opp’s sample was drafted in the Saxonian City of Leipzig and in the rural district of the Ore Mountains (Erzgebirge) in southern Saxony.

The findings of Mühler \& Opp (2006) suggest that identities were stable between 2000 and 2003 (about $50 \%$ of respondents did not change their identity patterns). Only the identification with Europe rose by $8 \%$. Regional identification with Saxony also grew. Furthermore, their results show

\footnotetext{
${ }^{14}$ Translated by the author from the German original: '[Wir] sprechen oft von 'regionaler bzw. 'überregionaler Identifikation' und meinen 'Identifikation mit Gruppen, die u.a. durch Zugehörigkeit zu einer Region zu charakterisieren sind'.'
} 
the smaller the geographic scale, the stronger the identification. The immediate local environment was the most important geographic scale for place-based identity. This supports Weichhart's assumptions concerning the local scale importance in place-based identity (cf. section 2.2.2). In addition, Mühler \& Opp found that there is a significant difference between the urban and rural sample in terms of identification with the local level: in the rural sample this scale is far more important than in the urban sample. There were no differences between the rural and the urban population concerning the European identification. However, between 2000 and 2003 identification with Europe was more stable among the urban people (Mühler \& Opp 2006: 136f.).

Testing for their initial hypotheses, Mühler \& Opp came to interesting results. The socialisation hypothesis: Mühler \& Opp (2006: 230) have found that the place of birth and where a person was raised have no dominant impact on place-based identification. Rather positive associations (such as e.g. the evaluation of regional dialects) during primary socialisation are an important base of regional identification. The duration for which a person has lived in one place is also not a facilitating factor for identification. Rather regional identification is a life-long learning process. As Opp \& Mühler (2006: 231) put it, regional identification is not something given with birth and childhood, and it could be unlearnt. On the other hand, a new regional identity could be learnt later in life, mainly based on positive experiences in a region and, interactively, with people from a region. The latter two are considered cumulative causation.

The quality of life hypothesis: this hypothesis could not completely be verified by Mühler \& Opp. Respondents in their study did not report linkages between quality of life and increasing regional identification.

The conflict hypothesis: Mühler \& Opp could verify the conflict hypothesis at least partially. When it comes to place-based identification, the regional (Saxonian) identification was largely influenced by a perceived conflict with or discrimination by western Germans. The perceived discrimination increased regional identification on a sub-national level.

Basically, Mühler \& Opp (2006) identified two distinct sub-groups of people according to their regional identification. First, there are people who feel that they are part of a regional group which is subject to discrimination by other regions' groups. They base their identification on the shared threat from 'outside', pronouncing a rather conservative and exclusionary group character. The second sub-group is composed of people who have moved to a region later in life and have 'learned' the regional identification mainly based on the satisfaction with the quality of life in their new environment. This sub-group is rather open-minded and inclusionary. Furthermore, the first sub-group expresses more negative perceptions of EU institutions and policy; the second sub-group is more positive about EU issues.

Mühler \& Opp (2006) also analysed linkages between the different scales of identification. To repeat, general discourse suggests that European integration has a positive impact on the development of a European identity among Europe's inhabitants. The reciprocal causality could also be assumed: European integration is legitimized and can take place successfully only if there is a common European identity. Both of these assumptions would have the potential to diminish the relevance of the national and sub-national scale for place-based identification. Mühler \& Opp (2006) criticise both these hypotheses as empirically not robust, and they refer to the Theory of Cognitive Balance (kognitive Gleichgewichtstheorie) according to Heider (1958), which stresses a 
proportional link between the different cognitive elements which are inclusionary along a hierarchy. Mühler \& Opp translate this theory into the scales of identification (region being a part of the nation, nation being part of the EU, etc.). Their empirical results show that a stronger national identification is linked to a strong European as well as regional identification. This means that the rise of European identity does not automatically substitute national identification; it accompanies and complements national and regional identification.

Further, Mühler \& Opp suggest that European identification is not only linked to regional and national identification, but also to the positive or negative evaluation of EU institutions and policy. They found individual policy decisions (e.g. Euro as joint currency; Schengen; Eastern European accessions) to be relatively unimportant, whereas EU institutions played a more prominent role in determining EU identification.

Finally, Mühler \& Opp (2006: 231) studied the relationship between the Heimatgefühl (personal feelings about home) and regional identification. Their findings suggest that Heimatgefühl is something given by birth and the place where one spent the first years of childhood. This feeling about a place of the 'personal roots' does not change in time and is not subject of any processes of cumulative causation. However, it is not automatically translatable into a feeling of regional identification, which is influenced by other biographical developments later in life.

Thus, we can see that home and regional identification are different things. We should be aware of that and not mix them when speaking about the ways in which people are rooted in place and space. Yet, both concepts - home/Heimat (section 3.1) and place-based identification (section 3.2) - allow for interesting insights into the origin of the emotional side of belonging to a place, or belonging within a space. Let's now have a look at a concept which more strongly focuses on a social practice, namely that of dwelling in a place.

\subsection{Dwelling and living in a place ${ }^{15}$}

The German term wohnen, for which I will use the English term 'to dwell', is listed in several sociological dictionaries. Herlyn (2003) e.g. describes dwelling as one functional element of cities. According to Herlyn the basis for being able to dwell is an apartment or a house, which is characterised by the fact that a person or a group of persons exert their exclusive right of disposal over this physical space. Inhabiting an apartment or house, which we can also term 'dwelling', is organised in a specific logic that allows for a strict differentiation between private life and the various public spheres of life (work, hobbies, associations, neighbourhood). The locational decision of where to dwell has consequences on many other aspects of one's lifeworld. Neighbourhoods have different images, which can translate into neighbourhood effects, and they are differently equipped with infrastructures that define opportunities for living one's own life. Furthermore, the neighbourhood in which a person dwells also marks how much a person lives in it. Herlyn remarks

\footnotetext{
${ }^{15}$ This section was part of a conference presentation titeled 'Multilokalität und die Bedeutung von Orten: Die Beispielgruppen Großeltern in Nachtrennungsfamilien und kreative Wissensarbeiter/-innen.' which I held in collaboration with my colleague Giulia Montanari during the Dortmunder Konferenz 2012 'Mobilitäten und Immobilitäten’ in Dortmund, 10 February 2012 (cf. Nadler \& Montanari 2012).
} 
(2003: $\left.377^{16}\right)$ : 'Generally speaking, the social relations within a neighbourhood can be considered an hinge through which the distanced contacts within the smaller group are linked. Although the neighbourhood in the modern city has lost many functions for adult inhabitants, and against the social distance which is characteristic for city life, there are still various relationships that are rooted in the neighbourhood. Children and elderly people rely on neighbourhood relationships which remain important functional elements in their everyday life. Yet, one can observe that inhabitants of large cities live in a way which is more oriented towards family than towards the neighbourhood.'

Häußermann \& Siebel (1996) understand dwelling as one partial aspect of a person's 'conduct of life’ (Lebensvollzug). According to Elias' idea of the life style, Häußermann \& Siebel speak of a 'dwelling style', which is characterized by four distinct functional elements (1996: 15): first, dwelling refers to all activities that are not part of the work life. Thus, dwelling refers to private life. Second, the household which dwells is considered a social unit, which is composed of the family. Thus, the dwelling itself is the private place of the family. Third, a dwelling has a sociopsychological dimension. The dwelling is the place where a person is alone with themself in absolute privacy and intimacy. Activities that are subject to a sense of shame take place here. Fourth, dwelling is regulated on a societal level through norms and institutions. Apartments and houses are traded on a market. Finally, Häußermann \& Siebel also acknowledge that dwelling understood as both an act and as the house/apartment - has a symbolic value. How and where a person dwells signals social status and can result in social distinction (1996: 44).

Also Stracke-Mertes (2003: 156) understands dwelling as one of the human's basic needs. This need to dwell can be satisfied by one's own apartment/house which allows for complete selfdetermination concerning social proximity or distance. It represents a retreat from social control. The dwelling is the space/place where a person controls their own territory. Apart from the exceptions, no other person can interfere in this territory. Within the limits of social norms and rules, a person can do whatever they want within their 'own four walls'. This territory is protected from the outside world and is therefore strongly related to the personal identity. Within the dwelling a person is relieved from social roles and can become themself again.

Goffman (1959: 106ff.) thus understands the dwelling as a 'back region' which allows people to relax from the social roles that they have to play in the public spheres. Social norms are only valid in a limited way. In action space theory, the dwelling is considered 'the most important pocket of local order', around which a person organizes their everyday life (Ellegård \& Vilhelmson 2004: 284ff.; Kramer 2012: 90).

In the light of increasing global mobility, dwelling as a social practice is changing quickly. One outcome of this change is that people and families dwell in different places at the same time. This means that the above-mentioned characteristics of dwelling become threatened by new empirical models. Weiske, Petzold et al. (2009) deal with that issue in their study on multilocal households.

\footnotetext{
${ }^{16}$ Translated by the author from the German text: 'Allgemein sind die gesellschaftlichen Beziehungen im Stadtteil als Scharnier zu verstehen, durch das sich die distanzierten Kontakte in der kleinen Gruppe verbinden. Wenn auch in der modernen Stadt die Nachbarschaft für die Erwachsenen weitgehend entfunktionalisiert ist, gibt es doch noch trotz aller stadttypischen sozialen Distanz zahlreiche nachbarschaftliche Kontakte, die v.a. für Kinder und alte Menschen wichtige Funktionen im alltäglichen Leben übernehmen können. Trotzdem gilt: Der Großstädter wohnt weniger nachbarschaftlichorientiert als vielmehr familienorientiert.'
} 
Thus, they add to the discussion that dwelling cannot be understood anymore as one household living in one apartment. Dwelling can still mean to be a household, but this household refers to several apartments that are used in everyday life.

This idea of Weiske, Petzold et al. (2009) leads to difficulties in understanding the social practice of dwelling. According to the more traditional views (cf. above Häußermann \& Siebel 1996) dwelling was a social practice which helped the individual person to draw a neat line between the public/professional and the private spheres of everyday life in one place. Now people are starting to have two such dwelling places? What does this mean? Scholars who deal with the study of such multilocal dwelling acknowledge that then dwelling cannot be thought of as such concentration of social functions in one specific locale. The listed social functions (distinction private/public sphere, social back region, intimacy, etc.) are distributed to different locales, yet administrative systems are not prepared for this. For example, people are still registered mainly in one place, having one specific address, paying taxes in one specific place. Therefore, Hilti (2009) suggests understanding 'dwelling' not according to an all-encompassing concentration of life spheres around one place. She prefers to focus on the dwelling's main function: It provides a place where to 'stay overnight'. If someone stays overnight, then he dwells.

Stock (2009) observes that 'dwelling' is more difficult to define in a mobile or multilocal world. According to Stock, dwelling can be described as the social practice of 'dealing with space' (sich mit seiner räumlichen Umgebung auseinandersetzen; Stock 2009: 108). This practice happens all the time and everywhere. Dwelling thus is independent of one's own apartment and of registered residency. It becomes also independent of 'familiar' and of 'foreign' places (Eigen- bzw. Fremdorte). The same as Hilti, Stock's reflection reveals that dwelling today has to be thought of differently than some decades ago. It is no longer about defining the centre of the private sphere of life in exactly one place from which a person starts every day into the public outside world. However, I believe that both approaches also highlight the difficulties of re-defining dwelling in the mobile world.

Schier (2009) tries to shift the attention from a place to the social group of the family. She suggests looking at family interaction in everyday life instead of studying apartments and neighbourhoods. This includes the idea that people 'dwell' within their family compound. Then multilocal dwelling becomes analytically approachable again, and the multiplicity of places does not disturb the operationalisation for empirical studies.

The different approaches to define 'dwelling' show that this social practice, which is heavily related to place, is changing quickly. It stills remains a central issue in order to understand how people are rooted in space. Even though it is changing its character, dwelling remains the private basis for the public social life. It remains somehow the 'back region', as Goffman (1959) called it. The clear borderline between private and public place which was formerly marked by the apartment door seems to have blurred. With more places becoming part of 'dwelling' as social practices, it can be assumed that semi-public dwellings are emerging. So far, first attempts of conceptualisation of dwelling in a mobile world have remained unsatisfying. On the one hand, making the definition of dwelling less precise runs the risk of making it a catch-all notion, yet sticking too much on the joint physical location of all functional elements of dwelling in one single place is not the only reality 
anymore. A new way of understanding dwelling is necessary to understand how people relate to place, and how they accentuate belonging by dwelling somewhere.

\subsection{Jung’s concept of psycho-social sedentariness}

Recently Matthias Jung presented another approach to study the rootedness of multilocal people ${ }^{17}$. Jung (2012) developed a model which he calls 'psycho-social sedentariness' (psycho-soziale Seßhaftigkeit). In an everyday understanding of 'sedentariness' the central property is the monolocality which is represented by settledness in one specific geographic place.

Jung, however, mentions that sedentariness also includes other aspects which are not necessarily bound to geographic location. His model is based on the observation that there are three main aspects of sedentariness. First, people are corporeally sedentary. A person has to acknowledge that they are positioned in their body and will experience their own lifeworld through this incorporation. If a person does not accept their own body, they cannot become sedentary in any other way. Second, a person is psychologically sedentary. One's own life plan and own practice has to be part of an individual relation to oneself. This means a person must develop a personal identity and a consciousness about themself. Here it is important that a person is capable of anticipating their own future and to make plans and develop strategies for their own life. And third, a person is socially sedentary in which they are part of different forms of community. This includes different levels from the family to the wider society.

For the individual sedentariness, a fundamental issue is that all of these three aspects are defined by congruence between a person's individual perception and the objective situation in which they find themselves in a given moment in life. All of these three aspects are not necessarily related to physical space. Jung uses the example of scientists or artists to illustrate how sedentariness can have a rather abstract and non-physical character $\left(2012: 2^{18}\right)$ :

'People who exhibit professions which are oriented to self-actualization and in which they are not only bearers of partial social roles, but in which they are involved with their personality as a whole, as a total individual, are more emphatic. Contrary to people who understand their job merely as a means of earning money, people who work in artistic and scientific professions as well as religious virtuosoship are typically sedentary in an abstract way, namely in a thing. This type of sedentariness must not necessarily be bound to a specific locality as long as a minimum level of favourable conditions allow for dedication to this thing.'

\footnotetext{
${ }^{17}$ The ideas presented in this section are based on an unpublished manuscript by Matthias Jung, which he presented during the bilingual workshop 'Ici et là: Ressources et vulnérabilités de la vie multilocale', which took place 17-19 May 2012 in Basel, Switzerland. I also had the possibility to discuss this concept with Matthias Jung after his presentation. A first publication on this concept is currently being prepared.

${ }^{18}$ Translated by the author from the German text: 'Personen, die in Verwirklichung ihres Lebensentwurfes Tätigkeiten ausüben, in die man nicht nur als Träger partialer Rollen, sondern als ganze Person involviert ist, als Individuum in seiner Totalität, wie beispielsweise der in einem emphatischen Sinne, d.h. im Unterschied zu bloßem Erwerb verstandene Beruf, das künstlerische und das wissenschaftliche Handeln oder auch das religiöse Virtuosentum, sind typischerweise abstrakt sesshaft in einer Sache, diese Sesshaftigkeit muss aber nicht mit Notwendigkeit an eine bestimmte Lokalität gebunden sein, solange nur günstige Bedingungen dafür gewährleistet sind, sich dieser Sache widmen zu können.'
} 
This includes the methodological difficulty that one cannot directly record this abstract psychosocial sedentariness. It has to be reconstructed, using case contrasting, from biographical narrations, as Jung indicates.

Leaving aside the corporeal sedentariness, Jung then examines how the two forms of psychological and social sedentariness were constituted in different moments in history. He starts his reflection with the pre-modern and traditionalist cultures. Here, a person was basically pre-defined by birth. A person was born into a position in the social system of the place where they lived, and this social position was regulated by norms. It included a given set of rights and obligations. A person did not have much flexibility in defining their own position if they wanted to be accepted by the community. Other than a social role that one could leave without being sanctioned, a negotiation about the social position often led to the expulsion from the community. People were not allowed to develop individual life plans, but life was planned for them, as Jung remarks. The only freedom that a person had was to build a secret opinion about this social position. Yet, the way they acted had to be in line with the ascribed position. In this pre-modern culture, psycho-social and geographic sedentariness were congruent.

Second, Jung examines the sedentariness in nomadic communities, which are built around a culture of mobility. Jung observes that European scholars often have difficulty in considering nomadic communities as sedentary ones. Nomadic life is associated with immediateness, asocial behaviour and a lack of plans for the future. Jung, however, mentions that nomadic people are psycho-socially sedentary even though they are not settled in a stable place. Nomads incorporate the cognitive modes, the behaviour, the rites, the symbols and the communication styles of a mobile community. They are sedentary in mobility. Jung says that this is the differentiating criteria to people who move a lot, such as in US American society. Those who move a lot remain psycho-socially sedentary in immobility but physically they are mobile. The nomads are both psycho-socially and physically sedentary within mobility. Empirical evidence for the difference between physical and psychosocial sedentariness is given by the problems that occur if nomads are forced to settle somewhere by national governments; then they remain psycho-socially mobile, while they are physically constrained to immobility.

Third, Jung looks at the sedentariness of those people that today are termed 'non-sedentary'. Their situation is rather precarious in terms of psycho-social sedentariness. They perceive and understand their current life situation as a temporary passage, which is a transition to a more stable and normal life. Thus, they have not developed a psycho-social sedentariness in their current life. This goes along with a difficulty in developing realistic plans for the future that will lead to a better match between individual perception and objective situation.

Jung's studying long-term unemployed people and their psychological situation found that in particular one's own parents and the family of origin as well as the current social contacts are of importance in order to develop one's own psycho-social sedentariness. One's own family provides the basis for developing an independent life which is then reflected in the social contacts. Examining his cases, Jung finds that people often have only a few anchor points in life which work to establish psycho-social sedentariness. In particular, if these anchor points are highly unique and not substitutable, and if people do not exert power over their existence, they might end up in 
psycho-socially precarious situations. Specific psycho-social sedentariness patterns can thus cause situations that are in differing ways advantageous or not for a future independent life.

What Jung offers with this approach is a way of understanding the rootedness of people, which is not directly geographical, drawing back on physical space. Even though it is only an initial draft, Jung describes a way of how to understand a more abstract type of belonging 'somewhere' and of belonging particularly in one's own life.

\subsection{Mobility and its effects on roots}

In the literature, there are different positions on what increasing global mobility might do to all these forms of roots that we have discussed so far. Duyvendank (2011) - in his interesting book 'The Politics of Home' - investigates the ways in which increasing global mobility affects the local feelings of belonging and home. Duyvendak provides a comprehensive review about the current discussion, which relates mobility to the fact of being rooted to place. Many scholars tend to think of people becoming placeless and uprooted. For example, Morley \& Robins (1995: 121, cited in Duyvendak 2011: 7) think that mobility 'is provoking a new experience of orientation and disorientation, new senses of placed and placeless identity'. Rapport \& Dawson (1998: 5f., cited in Duyvendank 2011: 7) mention that 'Movement has become fundamental to modern identity, and an experience of non-place (beyond "territory" and "society") an essential component of everyday life' . Both statements indicate that mobility alters the way in which people root in space or to places. But how in particular does this happen?

Table 1: Strategies to feel at home in a mobile world; Source: Duyvendak (2011: 12 \& 15); Own redesign: Merge of Duyvendank's tables 1.1 and 1.2

\begin{tabular}{|c|c|c|}
\hline & Mobility seen negatively & Mobility seen positively \\
\hline $\begin{array}{l}\text { Universalist position: } \\
\text { generic places }\end{array}$ & $\begin{array}{l}\text { Threat: no attachment to } \\
\text { particular places possible; } \\
\text { People lost in space: no } \\
\text { strategies for home-making } \\
\text { available }\end{array}$ & $\begin{array}{l}\text { Opportunity: no attachment } \\
\text { to particular places } \\
\text { necessary; } \\
\text { Chronically mobile people: } \\
\text { the hotel chain strategy for } \\
\text { home-making; } \\
\text { Looking for generic places }\end{array}$ \\
\hline $\begin{array}{l}\text { Particularist position: } \\
\text { particular places }\end{array}$ & $\begin{array}{l}\text { Threat: strong attachment to } \\
\text { particular places necessary; } \\
\text { Defensive localists: my } \\
\text { house is my home strategy; } \\
\text { Defending their safe haven } \\
\text { against invasion }\end{array}$ & $\begin{array}{l}\text { Opportunity: strong } \\
\text { attachment to particular } \\
\text { places possible; } \\
\text { Elective belongers: the } \\
\text { mobile home strategy; } \\
\text { Avoidance of generic places }\end{array}$ \\
\hline
\end{tabular}

Duyvendank reports two main ideas. On the one hand, there are those scholars who argue for a declining relevance of place for the personal feeling of belonging and attachment. On the other 
hand, there are arguments for a growing relevance of place in a mobile world. Both positions then include differing visions of how places will look in the future. Finally, they also differ in their way they think of mobility itself, as something positive opposed to a more negative view.

Table 1 shows the different positions that Duyvendank has identified in the literature. Generally, the 'universalists' think that places lose their particularity under the influence of global mobility. The particular meanings of individual places become relativised. People increasingly have social relations with people in other places, and thus their everyday practices become partly disembedded from single places. Famous scholars such as Castells or Heidegger observed that mobile people become more and more 'homeless' and 'detached' from places and end up in 'spaces of flows' (Duyvendak 2011: 8f.). Notions such as the 'homeless mind' by Berger (1973) or the 'general condition of homelessness' by Said (1979) point out that mobility used to be perceived as a threat to traditional feelings of belonging in a stable home place. Others such as Braidotti (1994) praised the 'postmodern nomadism'. Despite the different evaluation of mobility, universalists agree on the fact that places might lose their particularity (cf. Duyvendak 2011). The upper left field in the matrix describes the universalists' position, which considers rising mobility as a threat to people's feeling of attachment and belonging. People will become 'lost in space' and they do not possess strategies for efficient home-making in a mobile world. The upper right field in the matrix represents a more positive evaluation of increasing mobility. The suggestion here is that a group of chronically mobile people consider mobility as an opportunity, and they look for generic places with a standardized design all over the world, which allow for a feeling of home independent of geographic location. Duyvendak calls this pattern the 'hotel chain strategy' of rooting in space. These chronically mobile people will not need particular places anymore. They feel at ease in standardized places.

On the other hand, mobility might also re-root people to their places in new forms, such as e.g. 'glocal' arrangements. Duyvendak calls this the 'particularist' position. Again we can find two different evaluations of rising mobility. Those particularists who stand for a negative evaluation of mobility suggest that a strong attachment to particular places is necessary for people to feel at home. Therefore, mobility is considered a threat. This assumption is based on the fact that there are a large number of people who actually do not move themselves, but see their beloved home place being changed by incoming mobile people (e.g. international immigrants). They oppose global mobility defensive and localist strategies (cf. table 1 lower field left). By contrast, those particularists who think of mobility as a positive thing, stress that the rising mobility allows for more flexible home-making practices. Mobile people have the chance to choose the one which they like the most from different particular places. An alternative strategy would also be to become mobile and take one's own belongings on the road (Duyvendank's 'mobile home strategy'; table 1 lower field on the right). These people mainly avoid generic places and look for and switch between authentic places.

Another important contribution concerning the link between mobility and place stems from an international group of mobility scholars who have developed the concept of 'spatial partnerships' (Raumpartnerschaften) (Dienel, Meier-Dallach et al. 2004). Dienel (2004, 2009) describes that the starting point for their work was the observation that people are becoming more mobile and more multilocal in particular. This means that people increasingly feel attached to several places at the same time, including feelings of being at home and rooting one's own identity in several places. A second observation was that there are certain multilocal systems. Specific places are connected 
more than others because a large group of people circulate between exactly these two places. Third, the authors observed a significant difference in the character of places that are connected with each other. The authors speak of 'contrasting places' (Kontrasträume). The main pattern on which they focus is the link between large cities and more or less close rural areas with nice and attractive landscapes. As a result of significant amounts of commuters, these places become mutually orientated towards each other (aufeinander bezogen), and they have a certain complementary character which is evaluated positively by the multilocal people. These places thus enter into a 'spatial partnership' (Raumpartnerschaft). Berlin and the Baltic Island of Usedom, Hamburg and the North Sea Island of Sylt and Zurich and the Engadin Mountains are a few examples. The authors suggest that such multilocality based connections between places signify a 'new proximity' (neue Nähe) between formerly distant and unrelated places.

Dienel (2004) explicates that the condition for Raumpartnerschaften is a minimum of repetition of movements between places, which leads to a different perception of places, different from e.g. tourists who are 'first-time-arrivals' to a place. People who found Raumpartnerschaften through their circulation use places in the frame of their everyday life. They do not arrive for a first time to new places. Thus, they become interested in the places to a stronger and less superficial extent than tourists normally do. The link that individual multilocal people create between places is called informal Raumpartnerschaft (cf. Dienel 2004: 21, 29ff.). Against this type of Raumpartnerschaft there is also a more collective type of institutional Raumpartnerschaft, which emerges when political and social actors have become aware of these relations between two places. These types of institutional Raumpartnerschaften are based on the individual informal ones, thus on the concrete socio-spatial practice of a significant mass of individuals. They could be imagined as a type of twin city partnerships, but between contrasting places such as villages and cities.

Raumpartnerschaften allow for social innovation as individual multilocal people seem to behave distinctly in their different places. Thus, in one place they would do what they would or could not do in the other place. They experiment with different modes of behaviour which can be transferred between places. Dienel also offers a possible explanation for such place-differentiated behaviour (2004: $\left.24^{19}\right)$ : 'Historio-sociological and anthropological interpretations today mainly agree on the fact that the search for complementary experiences, outside of routinised everyday work life, is the main driver for modern leisure travel: the spatio-complementary travel and soujourn behaviour is an expression for the longing for complementary possibilities to behave and experience.'

Dienel thinks of multilocality as an escape from the perceptional constraints related to the life in the industrialised cities. Multilocality is not an escape from one place to another because of material deprivation in the original place. Rather it should be understood as the search for a different experience of time-space, which cannot be offered by contemporary city life. Thus, people look for contrasting and complementary experience.

\footnotetext{
19 Translated by the author from the German text: ,Historisch-soziologische und anthropologische Deutungen stimmen heute weitgehend darin überein, dass hinter dem Verhalten des modernen Freizeitreisens die Suche der Reisenden nach komplementären Lebenserfahrungen außerhalb ihrer Alltags- und Arbeitsroutine verborgen ist: Das räumlich-komplementäre Bewegungs- und Aufenthaltsverhalten ist Ausdruck für den Wunsch nach komplementären Verhaltens- und Erlebnismöglichkeiten.'
} 
Related to the contrasting character of places, people also act in a contrasting/complementary way in different places. Dienel defines (2004: $26^{20}$ ): 'The notion of 'contrasting place behaviour' on the one hand represents the need for the experience of spatial contrast (e.g. between the place of residence and the place of vacation), on the other hand it expresses the wish to act out one's own scope of experience in social terms, namely to live within one life different social roles, characters and lifestyles.'

Thus, mobile people want to combine distinct, spatially rooted 'sub-lifeworlds' within their total lifeworld. The individual person perceives her/his own informal Raumpartnerschaft first of all through a mental and cultural perspective. While most mobility studies rather focus on transport, the exchange of goods and materials or even information, the study of Raumpartnerschaften calls for a focus on space perception and individual biography. Even when the involved places might be the same for two different people, their meaning will vary strongly, and this variation is not only based on standard socio-economic variables such as gender, class, age, income or education. It is strongly influenced by personal history, socialisation and cultural background. In addition, the immediate spatial environment in which people act in their places are of upmost importance for their perception. Thus, a focus on the local scale is important. Dienel lists several variables that might be helpful for studying informal Raumpartnerschaften (2004: 27): place of origin; place of childhood/growing up; mobility of parents during own childhood; own places after childhood; living places; reflection about home; origin of the partner; children and their spatial relevance; individual interests in professional and leisure sphere. Thus, contrast originates from the very individual perception of it, rooted in individual biography, yet at the same time it has also an intersubjective as well as an objective character.

In particular, travel itself between places is an important aspect in understanding how people perceive informal Raumpartnerschaften. Dienel describes travel as follows (2004: $27^{21}$ ): 'Travelling to the contrasting place is a switch into another lifestyle and accordingly into the other character. [...] The physical movement towards the contrasting place equals stripping off the character of the recently left other place. Travel can be a means to an end, for the shift into the contrasting place, but it can also be an end in itself.'

Once an individual traveller has a specific interest in the trip itself, travelling becomes an end in itself, allowing for specific emotions and psychic states. If travelling takes place on regular routes and combines the same places over longer periods, then people engage in what Dienel calls 'raumpartnerschaftliches Verhalten' - a specific type of behaviour in the context of individual spatial partnerships, in which travel plays an important role (2004: 27).

Dienel comes back to a definition of Raumpartnerschaft when he examines the notion of place/space through the distinction of two factors influencing its character. First, there are Gestalt factors that define how people perceive these places or spaces. These are mainly defined through

\footnotetext{
${ }^{20}$ Translated by the author from the German text: 'Der Begriff "Kontrastraumverhalten” steht zum einen für das Bedürfnis, räumliche Kontraste (z.B. zwischen Wohn- und Urlaubsort) zu erfahren, zum anderen für den Wunsch, einen räumlichen Erfahrungsspielraum auszuleben, mehrere Rollen, Existenzen und Lebensstile innerhalb eines Lebens zu verwirklichen.'

${ }^{21}$ Translated by the author from the German text: 'Der Weg in den Kontrastraum ist der Wechsel hinüber in den anderen Lebensstil bzw. die andere Existenz. [...] Die Bewegung zum Kontrastraum ist das Abstreifen der zurück bleibenden Existenz. Sie kann Mittel zum Zweck, zum Wechsel in den Kontrastraum sein, sie kann aber auch Selbstzweck sein.'
} 
landscape and elements in landscapes. Landscapes can both be natural (hills, lakes) or artificial (e.g. architecture, built environment in the city). They define the Gestalt of a place/space. On the other hand there are Flux factors that are related to travel/movement/mobility. These factors are specific lines on which one can move, e.g. roads, train lines, subways in cities, rivers or even airports. They are related to transit and transit spaces. Kontrasträume show normally different patterns of equilibrium between Gestalt and Flux factors. Furthermore, Dienel says a spatial partnership (Raumpartnerschaft) refers to (2004: 2922) 'the interdependent relatedness of action and the mutual influence to repeated joint acting with a long-term character and which includes a certain spectrum of common aims, patterns of behaviour and projects.'

This means that Dienel understands Raumpartnerschaft as the relations that exist between actors in different places, not between the places themselves. These relations between actors are specific and binding (verbindlich), they constrain other alternative ways of acting. As Raumpartnerschaften could also be informal, in the form of lived practice by individuals, they consist not only of actors who are related to each other but also of identities and parts of the lifeworld. Here Dienel uses the notion of multiple place-based identity (multiple Ortsidentität). He says (2004: 30f. ${ }^{23}$ ): 'The individual person not only has one plac, with which they identify so strongly that this place could be considered belonging to them, and the person belonging to this one place respectively. These individuals have two or even more places to which they feel they belong. [...] These places become partners by means of the individual who identifies with different places.'

In both forms, the informal and the institutional Raumpartnerschaft, the partnership relation can have varying degrees of intensity. For informal Raumpartnerschaften, Dienel mentions that a possible empirical differentiation might be based on the frequency and duration of visits to a place, on the character of dwelling in these places, on the financial resources used for living in each place, or on the geographic distance between places. Yet, also places that are seldom visited or even not at all (anymore) might be of relevance if they have a strong emotional impact on individual persons. These places might e.g. be those which are referred to as Heimat or where a person lived in the past. Dienel speaks of 'reference places' (Referenzgebiete) here. Finally, differences might also occur according to the way in 'how' people look for contrasts and complementarity between places. So far, only few insights exist about the categories that make up contrast/complementarity (cf. Dienel 2004, 2009).

\subsection{Synthesis: Roots}

In this chapter I have asked how we can understand the ways in which people relate to places and how they develop a belonging. First, I have presented the German notion of Heimat, which I translated - conscious of all difficulties - to the English term 'home'. Heimat and home are terms

\footnotetext{
${ }^{22}$ Translated by the author from the German text: 'Wechselseitige Bezogenheit des Handelns und auf gegenseitigen Einfluß, auf wiederholtes gemeinsames Tun, das auf längere Sicht angelegt ist und ein bestimmtes Spektrum an gemeinsamen Zielsetzungen, Handlungsmustern und Projekten umfasst.'

${ }^{23}$ Translated by the author from the German text: 'Das Individuum hat dann nicht nur einen Ort, mit dem es sich so sehr identifiziert, dass dieser Ort als zu ihm gehörig angesehen wird bzw. umgekehrt das Individuum zu diesem Ort, sondern es hat zwei oder noch mehr Räume, mit denen es sich eins fühlt. [...] Über den Einzelnen, der sich mit den betroffenen unterschiedlichen Räumen identifiziert, werden diese Orte zu Partnern.'
} 
which are often used as everyday vocabulary by most of the people when they are asked for their geographic belonging. We have seen in section 3.1 that Heimat contains more than the reference to a small cut-out of the geographic world. Further important aspects are the social relations, the personal history and emotions. The literature on Heimat also suggests that not all aspects will fall together in one specific place anymore when mobility is becoming an issue.

I have also presented the origin of the term place-based identity. This concept also uses places and people simultaneously to explain the foundations of geographic roots. The images of places are main references for place-based identities. Exactly these images of places are then to be understood as composed of both physical as well as social elements. Once a person creates roots through identification with a place, they have perceived the place itself positively as well as the people living in it. As the perception of a place is an important issue here, the local scale was said to be the most important for the creation of place-based identity, even though other scales always interfere in place-based identity. However, larger scales (such as regions, nation-states or the European Union) are more abstract and cannot be perceived with one's own senses (walking, seeing, smelling, touching, hearing, etc.), therefore they become less relevant for identification and a feeling of belonging can more easily be felt on a local scale (e.g. a city, a neighbourhood, a village and its inhabitants).

This observation has brought us to the notion of wohnen/dwelling, which is understood in sociology as a social practice of drawing a neat line between the private and the public spheres of one's own life. In particular, the apartment, seen as the materialisation of one's own personality, represents an important physical aspect of a person's belonging in geographic terms. Yet, mobile people today seem to face difficulties in expressing where they are actually dwelling at the moment. This difficulty is observed by sociologists who are trying to revise modern conceptualisations of 'dwelling'. As in the notion of Heimat/home the several properties of 'dwelling' as a practice as well as 'the dwelling' as a place do not necessarily merge in the same geographic location anymore. Dwelling often happens across different places. However, social scientists still cannot answer sufficiently how this takes place.

Finally, I have presented a recent and still unpublished approach to understand rootedness through the notion of 'sedentariness'. Sedentariness is used in everyday vocabulary as a synonym for rootedness. The social scientist Jung, however, breaks it down to physical and psycho-social components. For Jung, the sedentariness can be described as the congruence between the selfperception and the objective situation of a person concerning three aspects: the corporeal sedentariness within one's own body, the psychological sedentariness within one's own personality, and the social sedentariness within different scales of community ranging from the family to the wider society. If individual perception and objective situation are not congruent, a person might lack a feeling of belonging. Furthermore, we have witnessed in Jung's ideas that sedentariness additionally consists of physical (geographic) sedentariness, which Jung equates with immobility. If mobility rises, the traditional superposition of physical and psycho-social elements of sedentariness becomes questionable. On the other hand, Jung refers to nomadic societies in order to underline that physical sedentariness might also be equalled with mobility. Nomadic tribes can indeed be considered sedentary, namely sedentary in mobility, in a culture of mobility. The question is now which patterns of sedentariness will arise in a post-modern society with increasing mobility. Will we also become rooted in a culture of mobility, and what does this imply? 
The 2x2 matrix of Duyvendak, which I presented in section 3.5, suggests one possible answer to this question. Here, the changing nature of places (division between generic and particular places) and the evaluation of mobility (positive versus negative) are the distinctive variables for the creation of 'belonging' in a mobile world. Duyvendak's approach describes the diversification of people's home-making strategies. Some will appreciate the opportunities to find new types of 'homes' (such as mobile homes, or generic and substitutable places) that come along with mobility; others will emphasize the threats. The latter will increasingly have problems feeling at ease with their changing environments (threatened by invading migrants) or they will feel 'lost in space' when their particular places become generic ones. As a second example, I introduced Dienel et al.'s idea of the spatial partnerships. Dienel's suggestion is that mobility is not randomly affecting all geographic areas and all people. There will be some people who circulate in regular forms between selected places. The regularity of this mobility will then lead to individual and institutional links between these places, which he calls spatial partnerships (Raumpartnerschaft). These spatial partnerships will have effects on individual feelings of belonging; they provide a context for multiple and hybrid forms of place-based identity, including different places.

To conclude, rootedness in place shows up in all of these concepts as a mixture of physical and social environment. Both the physical place and the social interaction/contacts a person has within this place form the decisive fundament of belonging and rootedness in space. Whereas in traditional and also modern societies the physical and the social elements of belonging were mostly located in the same place, this superposition increasingly seems to have vanished in recent times. This process is paralleled by an increasing diversification of forms of belonging. Formerly collective identities become increasingly individualized, and in everyday life, traditional terms such as Heimat/home and identity become loaded with various individual connotations, thus it is becoming more difficult to study them. Scholars are trying to adapt the common concepts of belonging and rootedness to the new empirical reality, yet these adaptations are only at an initial stage and empirical tests have remained explorative and insufficient so far. 


\section{Interlude C: Henriqua - Juliana}

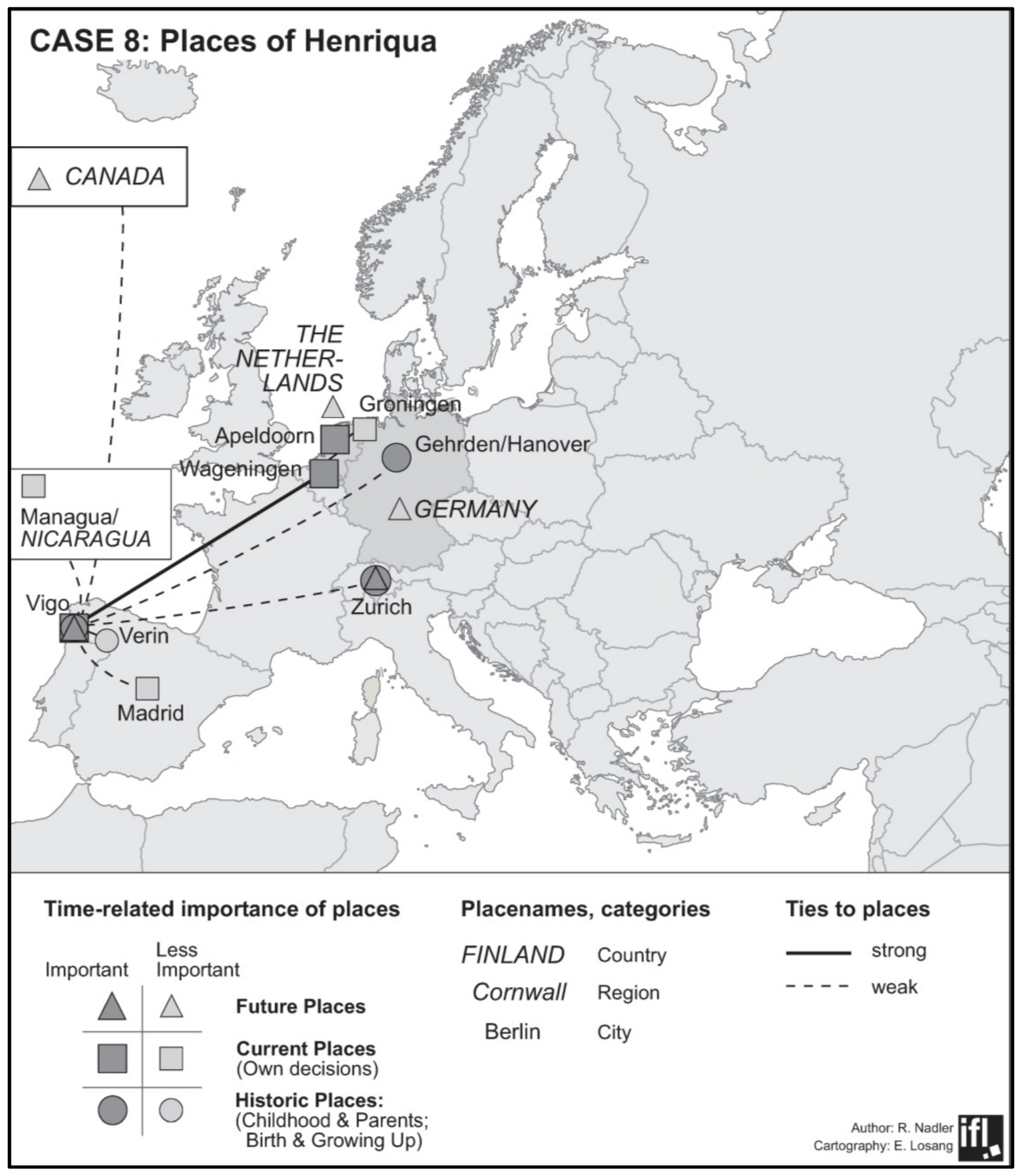




\section{Meet Henriqua}

Henriqua is a Spanish postdoctoral researcher in the field of regional development in her late 30s. She was born in Hanover, Germany to immigrant parents who returned with her to Galicia when Henriqua was four years old. She then grew up in the small town of Vigo, which is located on the border to Portugal. When she was younger, her cousin lived with her family and was like a brother to her. During summer vacation each year, both went to Zurich, where her aunt, his mother, lived at that time. This made her feel comfortable with travelling and discovering new places. In her 20s she even went to live there for nearly two years. After that she went to live in Madrid for three years before she returned to Vigo. Upon returning to Spain she worked as a German language teacher before she joined the University of Vigo for European research projects. For her $\mathrm{PhD}$ years she enrolled at the University of Wageningen in the Netherlands, where she started to work in a postdoctoral position which is actually related to field work in Galicia. She therefore now frequently travels between Vigo and Wageningen.

The first day she arrived in Wageningen she also met her Dutch husband. Both have bought an apartment in Wageningen by now and they have a small daughter. The three moved to Vigo when she started with her postdoctoral field work for her Dutch employer. They rented their apartment in Wageningen to other people, and were able to move into an apartment of her Zurich-based aunt in Galicia, which the aunt herself does not use. For Henriqua this was a move back to her home and to her roots and bases. But for her husband it was a completely new situation: he had to learn Spanish, and had difficulties finding a job. This made her family ties even stronger. For Henriqua, her family is the most important issue, more important than a job or career. As it is a binational partnership, her parents-in-law are in Apeldoorn in the Netherlands, and she often combines her business trips to Wageningen with family visits in Apeldoorn.

Henriqua does not pronounce a specific relation to her professional multilocality and mobility. She thinks that it is exhausting, and that travelling has lost its exotic character: it has become a necessary routine. This includes the fact that she does not feel like travelling on holiday anymore. On the other hand, she also likes it a bit for the fact of learning new languages and seeing new cultures.

Henriqua also mentions that she would miss travelling in a more mono-local life, because she is someone who feels bored being part of only one local context. That being said, she is not part of any associations, clubs or civic engagement groups. She thinks that these groups are too stable, and always circulate around the same topics and have a dominant pattern of thinking, which she dislikes, so she rather focuses on her family and some hobbies in her leisure time.

For the future, Henriqua says that she would love to be more monolocal and based in Vigo so that she can keep her family - daughter, parents, and grandparents - together. In her childhood years in Germany, she didn't have that contact to her grandparents, and she doesn't want such geographic separation for her daughter and her own parents. This monolocality however should be related to regular business trips in order to escape from the local context. However, she is also aware of the difficult labour market in Galicia, and has applied for a job in Canada, but she could also imagine moving to Germany, Zurich or the Netherlands if necessary.

Henriqua's story is mainly about the episodic character of multilocality - place relations changing with time, but also about how to keep a family together in a multilocal life. 


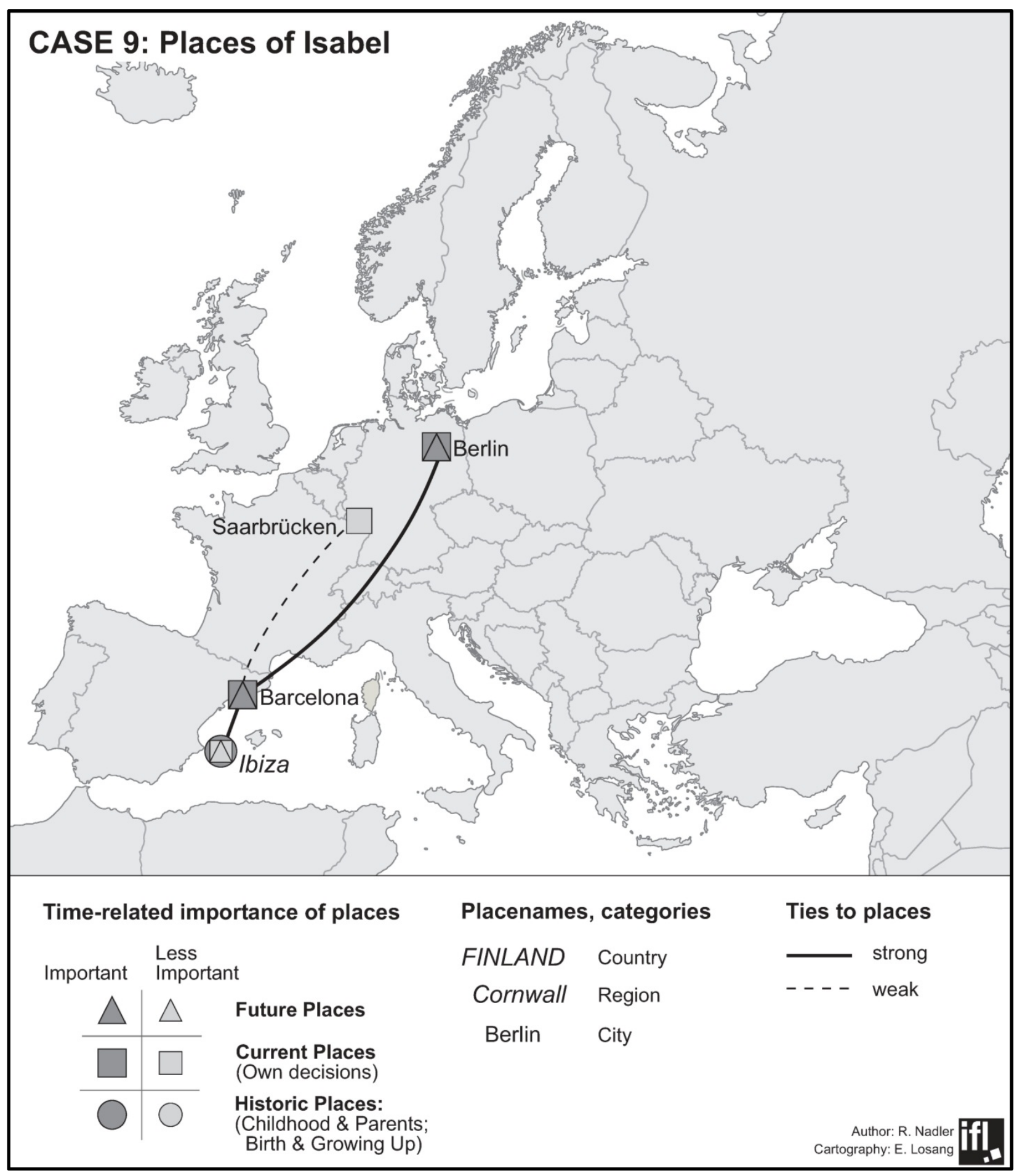




\section{Meet Isabel}

Isabel is a Spanish interpreter and translator in her 40s. She grew up on the Isle of Ibiza and later studied at the university in Barcelona. During that time she also came to Saarbrücken/Germany for an exchange year, and where she then stayed for nearly twelve years. She liked Germany, but she also didn't want to leave Spain for good, so she became specialized in German and English as her working languages. This allowed her to develop a transnational multi-locality including Germany and Spain. She says that she feels very happy with this situation, because both places, Berlin and Barcelona allow her to be very different people. In Barcelona she is a very outgoing person (partying a lot, enjoying life, the seaside, the easy-going mentality of the people), on the one hand, and helps her divorced brother to raise his children, on the other hand. In Berlin, by contrast, she is a more reflective person, focusing on work and on serious issues in life such as environmental protection and politics. Finally, she often visits her parents in Ibiza during weekend trips from Barcelona. While at her parents, she is mostly their daughter and lets them take care of her. Isabel also pays attention to the fact that her different worlds do not become mixed, which would disturb her. Thus, e.g. she does not like to invite friends from one place to join her in the other place.

Isabel mentions having problems with her social relations as she perceives herself as a very unsteady person who has to travel a lot to be happy. She also does not like making plans, she loves spontaneity. This does not work with most of her more mono-local friends who live routine lives. She often disappoints her friends by leaving spontaneously to her other places. This includes problems of having stable love attachments.

Isabel wanted to engage in the places in which she feels at home. She thinks that she is a very happy person, and she wants to give something back to other people who need help, so she went to several NGOs, but none of them wanted to work with her, as she can not tell definitely when she has time to work for them. She cannot bind herself to regular engagements, as her life has such an irregular rhythm. She says she seldom spends more than three days in one place, not knowing where she has to go for interpreting jobs afterwards. Isabel is working on a very professional level. She interpreted for the Pope, the Spanish Prime Minister and other high-ranking politicians. This includes a mode of conduct which makes her available on a short-term basis to her clients. She has both her apartments equipped with clothes that have a fits-to-all character, so that she can flexibly pack things for two or three days of business trips. She does not use office infrastructures anymore, and works on a freelance basis.

For the future, Isabel wants to continue with her mobile life. However, she recently bought an apartment in Barcelona which works as an anchor point in her mobile world. She has deposited all her important belongings there, such as photo albums, official documents, favourite clothes and her shoe collection. Yet, being so mobile and working in an international field, she is not very interested in local issues. She thinks it's boring and often narrow-minded. This means that she also has only a small circle of friends who live a similar life and who really understand her and share her mentality.

Isabel's story is mainly about the advantageous outcomes of multilocality, specifically to be able to live several different lives in different social and physical contexts. 


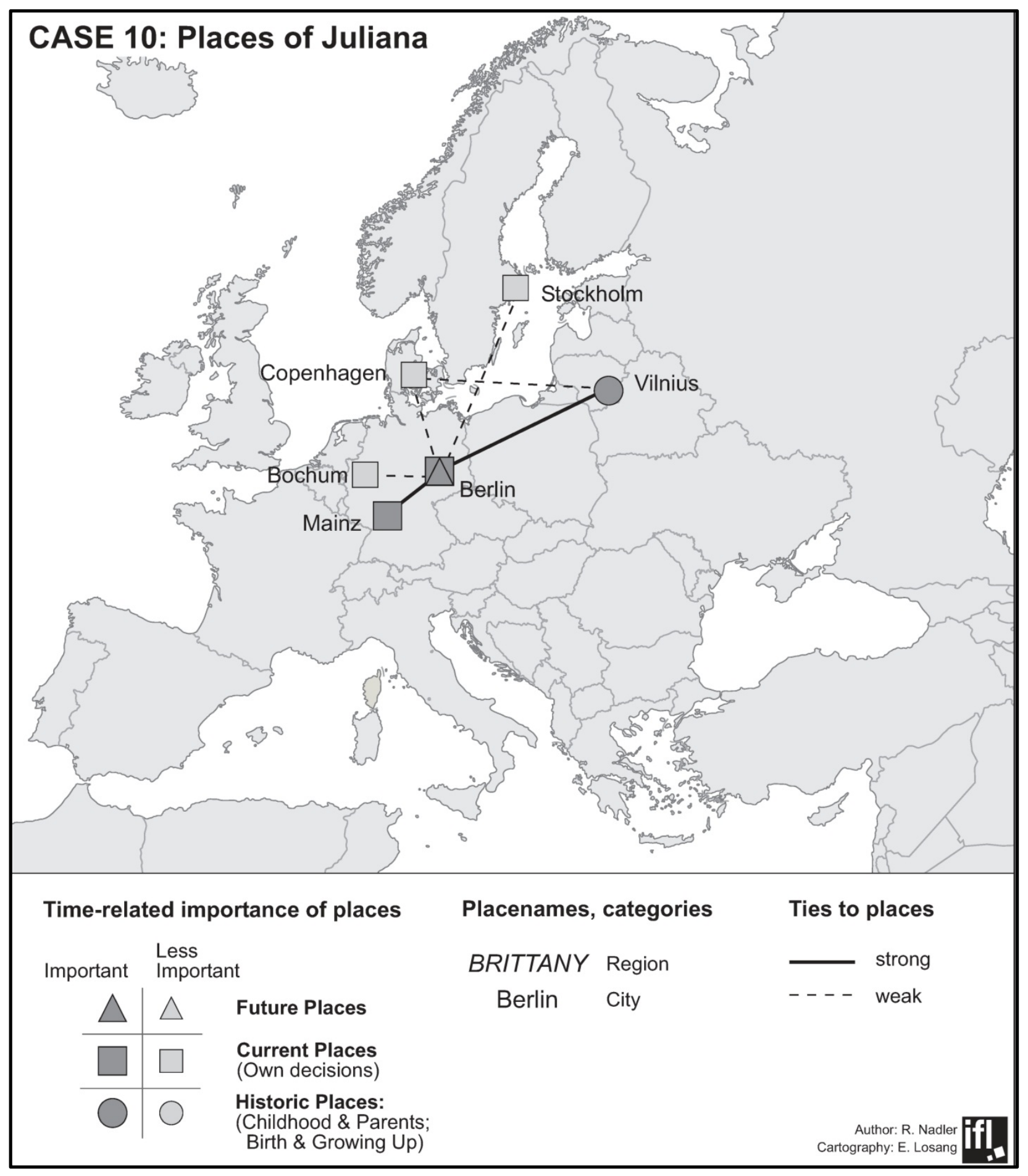




\section{Meet Juliana}

Juliana is a Lithuanian junior professor in the field of Ethnology. She is in her 30s and has lived in Germany for about fifteen years now. She actually came to Germany for her husband, who is German, and whom she met during her exchange year in Copenhagen, Denmark, at the time of her undergraduate courses in Lithuania. After the exchange year, she decided to move to Berlin and finish her studies in Germany. She even continued on to do her PhD at Humboldt University in Berlin. As she already had her first daughter by then, and as her husband was working in Bochum in the Ruhr area, she decided to do her fieldwork in Lithuania, where her parents could take care of her daughter while she worked. She then frequently travelled between Berlin and Vilnius.

Nowadays she and her family still live in Berlin, where they have now two small children, and where her mother-in-law is living. She now works as a junior professor at the University in Mainz, while her husband has a postdoctoral research position in Stockholm, Sweden. She says that this spatial pattern is very difficult for the organization of a family everyday life. They can only stand that situation due to the fact that both have flexible working times, and as her mother-in-law and some nannies help them with the kids in Berlin. It even comes to situations in which both parents are out of town for work while the children have to remain with others. Juliana doesn't actually like this multilocal pattern and would prefer to be more stable. She considers it as a forced type of mobility, which lets her feel guilty that she cannot always be with her children. Managing her family and her career involves tight time management, e.g. she says that train rides between Mainz and Berlin are weekly moments for concentrated reflection, reading and writing, while Berlin itself is more dedicated to family, and Mainz to university administration and teaching.

Juliana also describes that moving to Germany was not easy. She had a lot of problems with bureaucracy as Lithuania was not yet part of the EU. Her high school degree wasn't accepted, she had to take different subjects in university than in Lithuania, and she had to apply for visa, proving her financial independence. After getting married things became much easier, she says. Yet, she still wants to make the EU as a labour market an issue of her work; she wants to study - based on her own experience - how different rights are granted to mobile workers from different nationalities, and how the national and the European are actually interwoven in individuals' lives.

Juliana mentions that she cannot currently find the time for civic engagement as she is very busy with work. She and her family are part of the Christian community in Berlin, but they do not actively engage in it. Furthermore, she had been part of a Lithuanian community supported by the Lithuanian embassy in Berlin. While she found this very helpful in the beginning of her Berlin life, she became bored by it quickly and stopped going there. By contrast, Juliana says she is engaged politically because her scientific work always contains political elements.

In the future, Juliana wants to live in one place and be monolocal with her husband and children. She and her family would like to stay in Berlin. Therefore, they would also accept that one partner commutes to another city. Moving the family would be the last option, done only if it doesn't work any other way in Berlin.

Juliana's multilocality is mainly a story of forced mobility, which has resulted from her double ambitions as a mother and a scientist. She actually doesn't like it, and is looking for strategies to travel less. 


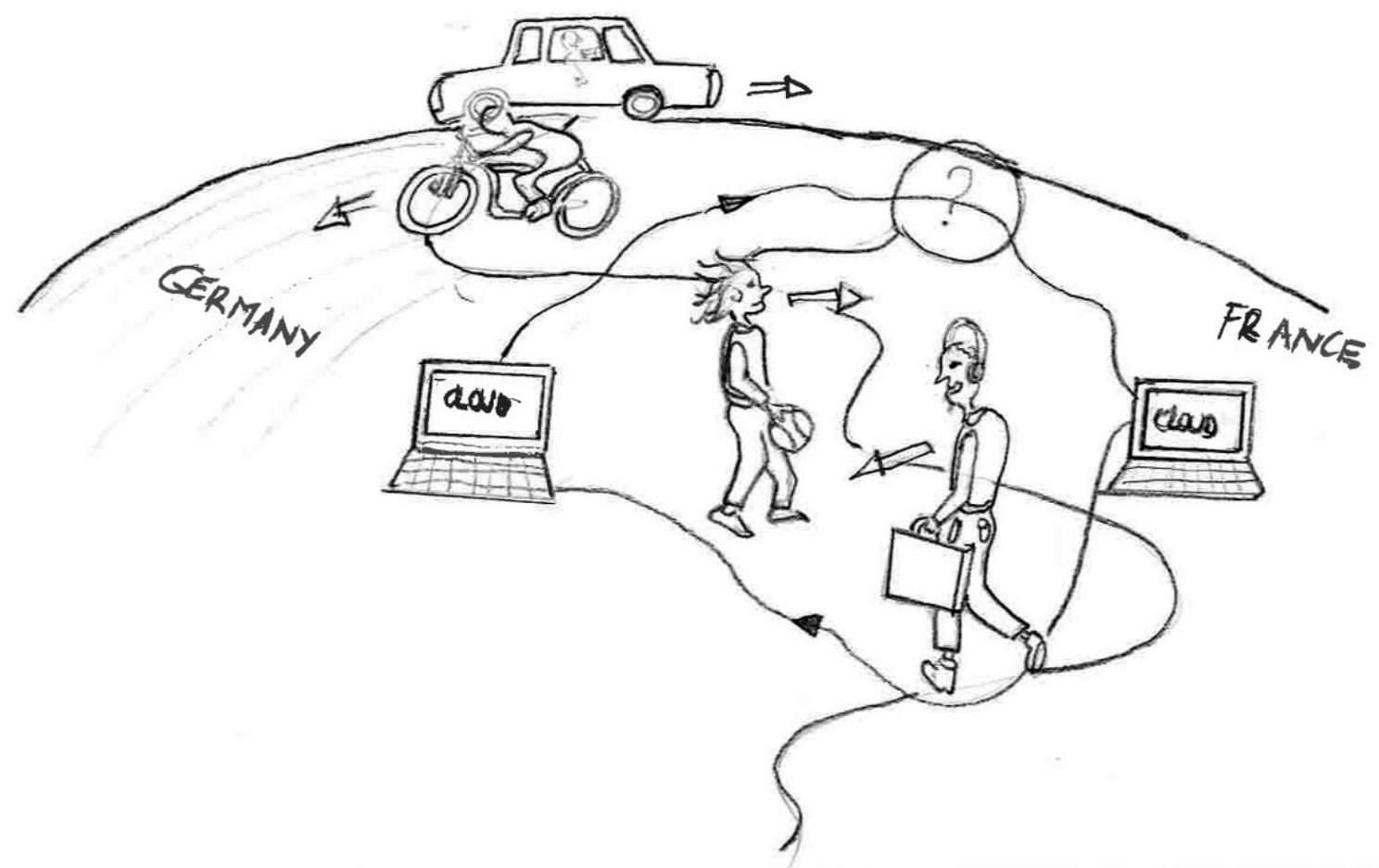




\section{Routes: Understanding mobility}

'I have come to think of this additional geographic dimension of socioeconomic class in terms of two groups that I refer to simply as the mobile and the rooted. The mobile possess the means, resources, and inclination to seek out and move to locations where they can leverage their talents. They are not necessarily born mobile, nor are they inevitably rich. What the mobile understand is that pursuit of economic opportunity often requires them to move. [...] A far greater number constitute the rooted - people who are tied to place.'

Richard Florida (2008: 79-80)

As Richard Florida's statement highlights, there is a group of people who live a more mobile life than the rest of the population. We thus have to think about what mobility means in contemporary society. This chapter will be dedicated to the study of mobility as it is discussed in current sociological and geographical analyses. I will focus on two promising notions which I deem relevant to my study. First, the idea of an upcoming transnationalism is helpful as it lets us understand how lifeworlds of people do not fit into and do not relate to only a single nation-state. The social space in which people live today is increasingly going beyond national boundaries and transgressing the former restrictions that come along with these boundaries. Second, the concept of multilocality is taking a similar perspective, yet on a smaller geographic scale: namely the local instead of the national. In order to understand how people who circulate between different places on a regular basis relate to exactly this circulation and to the involved places, multilocality research has emerged in the German speaking world of social sciences (mainly in Germany, Switzerland and Austria).

\subsection{Transnationalism}

Since the early 1990s, the notion of transnationalism has become part of social scientific studies which deal with the lifeworlds of people living 'in-between' two or more states (Brickell \& Datta 2011a). Migration scholars in particular have adopted the concept in order to understand the new forms of circular migration that have occurred with the increasing integration of global (labour) markets. Principally, transnationalism is about the various linkages that mobile individuals develop to different nation-states and national societies. The pioneers of current transnationalism research, Glick Schiller, Basch et al. (1992: 1/2) defined:

'[...] transnationalism as the processes by which immigrants build social fields that link together their country of origin and their country of settlement. Immigrants who build such social fields are designated 'transmigrants'. Transmigrants develop and maintain multiple relations - familial, economic, social, organizational, religious, and political - that span borders. Transmigrants take 
actions, make decisions, and feel concerns, and develop identities within social networks that connect them to two or more societies simultaneously'.

Glick-Schiller et al., who studied migrant groups from the Philippines and several Caribic Islands in New York, found that the transnationalism concept is subject to six premises. First, the existent sociological categories of nation, ethnic group, culture and society no longer suffice to grasp with circular forms of migration between nation-states, and they help little to study transnationalism. Second, changing migration patterns are strongly interwoven with the extension of global capitalism and the worldwide integration of markets. The spatial re-organization of production as well as the increased dynamic of relocations has made migrant workers a more vulnerable group. Their response could be seen in the transnationalisation strategy, which equals risk distribution. Third, transnationalism must be understood as grounded in the daily lives, activities and social relations of migrants. Fourth, these transnational migrants are forced to confront, draw upon, and rework different national, ethnic and racial identity constructs. Fifth, sociological concepts have to be re-conceptualized: mainly class, culture, society, nation, ethnicity and race. Here, Glick Schiller, Basch et al. (1992) criticise cultural studies approaches (e.g. Hannerz's (1990) idea of an opposition between cultural homogenisation and cultural creolisation) for underestimating the migrants' capacity to mediate between existing cultures. Sixth, while transnational migrants are constrained by national hegemonic contexts, they also actively reshape these contexts through their activities.

Yet, transnational situations do not only occur in migrational contexts; they can also exist in the everyday life of physically immobile persons. The migration scholar Ludger Pries thus prefers to differentiate between three different social scales instead of between physical mobility (migration) vs. immobility (no migration). According to Pries (2008), transnationalism is the conscious and opportune connecting of two or more national backgrounds (referring to nation-states, national legislation, national culture, national language etc.) by private individuals (micro level), by economic corporations and non-governmental organizations (meso level), and by state actors (macro level). Other than in more traditional approaches of internationalism/internationalisation, the interaction between national governments only plays a minor role in transnationalism. Actors at the micro and meso level who 'transcend' the nation-states boundaries represent the main focus.

Pries also wants to avoid 'transnational' being used as a label for everything that crosses borders, so he only applies the term to phenomena of social change that cannot be studied in the frame of 'methodological nationalism' (cf. discussion as brought up by Beck \& Grande $2010^{24}$ ). Pries says (2010: $10^{25}$ ): 'Transnationalism in the narrow sense looks for the social phenomena and social relations which are spanning different local units in different national societies, and which are

\footnotetext{
${ }^{24}$ Beck \& Grande criticise social and political theory and methodology for referring only to national frames. They say (2010: 412): 'Until recently, social theory has taken the nation-state as its implicit unit of analysis; the terms 'society' and 'culture' implicitly referred to what have been perceived as discrete, self-contained and relatively homogenous entities bounded by national borders, institutions and legal frameworks. Accordingly, theory has operated with an unquestioned assumption of a neat correspondence between nation, territory, society and culture.' As a consequence they argue for a 'methodological cosmopolitanism. This view is also shared by some transnationalism scholars such as Wimmer \& Glick Schiller (2002).

${ }^{25}$ Translated by the author from the German text: 'Der Transnationalismus im hier verstandenen engeren Sinne dagegen betrachtet vor allem solche Sozialphänomene und sozialen Beziehungen, die sich über mehrere lokale Einheiten in unterschiedlichen Nationalgesellschaften hinaus erstrecken, die relativ dauerhaft sind und vergleichsweise dichte Interaktionen beinhalten.'
} 
relatively stable and include comparably dense interaction.' Pries later adds (2010: 1326): 'The terms transnational and transnationalisation are understood here as cross-border phenomena, which - still rooted locally and in different national societies - constitute relatively enduring and tight social relations, social networks or social spaces.'

He also speaks of an equal distribution of activities to the involved places. Frequency and density of social relations are the central criteria in making transnationalism a new phenomenon, and for counteracting criticism from the part of historians who might argue that transnationalism has always existed and is nothing new. For Pries, the early mobility of people was characterised by a rather monolocal territorialisation of the lifeworld, which is no longer the case in contemporary forms of transnationalism.

According to Pries, transnationalism - other than many globalisation concepts - does not expect a declining relevance of national borders and nation-states. Nation-states and all aspects of the lifeworld that are based on national regulation maintain their power in defining a person's life. There is no straightforward deterritorialisation.

Pries (2009) rather argues that different locales are combined within one lifeworld across remaining national borders. Transnationalism studies focus on new forms of socialisation (soziale Vergemeinschaftung). Consequently one could understand globalisation as the sum of all transnational ties in the world. Pries thinks of transnationalism as one ideal type of the internationalisation of 'socialisation' (which he calls Vergesellschaftung). He distinguishes transnationalisation from six other ideal types (Pries 2009: 592-595). Four out of these ideal types of the 'internationalisation of socialisation' might still be studied according to the assumptions of 'methodological nationalism' (mainly the assumed superposition of geographic and social space on the national scale): 1) internationalisation as the process of intensifying relationships between nation-states; 2) re-nationalisation as the answer to internationalisation and globalisation by closing one's own borders and re-concentrating on one's own nation-states' internal socio-cultural life; 3) supra-nationalisation, which is the opposite answer to re-nationalisation, through upgrading sovereignty to a geographic scale above the nation-state but below the global scale; 4) globalisation as the process through which internationalised relationships between nation-states increasingly take place globally and not only within limited world regions. The super-position of social and geographic space must not necessarily be questioned in order to study these processes. According to Pries (2009) the only slight criticism stems from those scholars who argue for a diminishing relevance of geographic/physical space for socialisation (the 'virtualisation argument' as e.g. can be found in Urry’s ‘Mobilities' (2000) and Castells’ 'Spaces of flows’ (2000b)).

On the other hand, there are also processes of internationalisation of socialisation which call attention to geographic space, but which cannot be studied using a methodological nationalist perspective (cf. Pries 2009: 594f.): 5) glocalisation, which is a circumscription for the dialectic between the global and the local scale, including processes of new bordering and reterritorialisation; 6) the spread of diaspora situations, which refer to the fact that one social space can be spread to very different geographic spaces across national borders and with orientation to a

\footnotetext{
${ }^{26}$ Translated by the author from the German text: 'Mit den Begriffen transnational und Transnationalisierung werden hier grenzüberschreitende Phänomene verstanden, die - lokal verankert in verschiedenen Nationalgesellschaften - relativ dauerhafte und dichte soziale Beziehungen, soziale Netzwerke oder Sozialräume konstituieren.'
} 
shared homeland; 7) transnationalisation, which represents the emergence of new 'trans- and plurilocal configurations' above and beyond nation-states. Thus, it becomes clear that there is a set of different processes happening at the same time, which might be confounded with transnationalisation. However, as Pries has presented, they have a different character and need differentiated epistemological approaches. Transnationalism consists of the social practice of combining different national contexts and its opportunities. Therefore it should be studied with distance to methodological nationalism (cf. Glick Schiller, Basch et al. 1997; Pries 1997).

Interestingly, Pries also argues that transnationalism can only exist as long as the nation as an anchor point or 'reference' is available (2010: $12^{27}$ ): 'It is only possible to speak of transnationality or transnationalisation if and as long as nations, nation-states and national societies still exist - the transnational as something exceeding the nations can only exist as long as there are nations as reference points for the denotative.'

Transnationalism as a field for studying current phenomena of cross-border mobility thus also takes into consideration that mobility (here cross-border migration) and place (here the nation as materialising in the local) are strongly interrelated.

Furthermore, Schmiz refers to Itzigsohn, Dore Cabral et al. (1999, cited in Schmiz 2011: 21), who introduced a differentiation of transnational migration according to the migrants' degree of involvement into transnational social spaces. Studying Dominican transnational migrants, they found that there is a continuum between 'narrow' and 'broad' transnationalism. Itzigsohn, Dore Cabral et al. used three dimensions to measure the involvement: frequency of trips between countries; institutionalisation of a person (meaning the formality of membership in organizations); involvement of a person (meaning the frequency of participation in collective agency). If transnational migrants score high in these dimensions, they are part of a narrow transnationalism. By contrast, if they travel seldom, or are not institutionalised or personally involved, they might be considered representatives of broad transnationalism. Additionally, Itzigsohn, Dore Cabral et al. (1999: 324) presented four dimensions of transnationality: 1) economic; 2) political; 3) civil societal; and 4) cultural. One could then study the narrowness, or respectively the broadness, of transnational ties according to these dimensions.

\subsubsection{Transnational social spaces \& transnational social fields}

Pries started to study Mexican family networks that included a part of the family members living (at least temporarily) in the United States. Even though his research starts from the observation of migrational movements, Pries derived some more general conclusions about transnationalism. Pries (2008) observes that the former unity of 'geographic space' and 'social space' - as found in the modern nation-state with a belonging national society - is being disrupted more and more. The nation-state is still an important frame of reference for social life in post-modern societies, but social interaction also takes place by 'transcending' its national boundaries. The social interaction on the level of individual lifeworlds is increasingly taking place across borders, and thus in a more

\footnotetext{
${ }^{27}$ Translated by the author from the German text: '[Es] kann von Transnationalität oder Transnationalisierung nur solange und dann die Rede sein, wenn (noch) Nationen, Nationalstaaten und Nationalgesellschaften bestehen - Transnationales als Nationen überschreitendes bzw. übergreifendes kann nur solange existieren, wie es Nationen als Bezugspunkte des zu Bezeichnenden gibt.'
} 
or less 'transnational' way. This means that we have to think in a new way of social space and its relation to geographic space (cf. Pries 2010).

To approach the term 'space', Pries (1997: 17) draws back on Simmel’s (Simmel 1903) 'Soziologie des Raumes' (sociology of space). Simmel described three main properties of social space. First, there are different types of social formations. Some social formations are geographically exclusive. This means that one social formation does not accept another similar social formation in the same territory (e.g. where one nation-state is, there is no other). On the other hand, there are social formations which can co-exist in geographic space (e.g. different religious groups in the same city). Second, Simmel thinks of social space as something which has a border, delimited to other social spaces. Built up for distinction, this border consequently leads to geographic borders. This idea includes the logic that a border is not a geographic fact with a social impact, but vice versa (cf. Pries 1997: 17). Third, Simmel observed that geographic space allows social space to materialise (locate) and concentrate (density of social interaction). Pries further outlines that in sociology such a social space concept persisted for a long time, including the super-position of geographic space (national territory) and social space (national society and culture). This super-position was only questioned upon the arrival of Luhmann's Systemtheorie, as Luhmann thought of societies as consisting of communication. Consequently society had to be looked at on a global scale, not a national one, because communication does not stop on national borders (cf. Pries 1997: 17).

Pries therefore suggests conceptualising geographic space not as a container (e.g. like a national territory), but as a relational space in which objects are located and related to each other. Social space should then be thought of as characterised by lifestyles and the different forms of cultural, social, economic and symbolic capital (as derived from Bourdieu). In sum, the individual lives in their own 'action space', which has a geographic and a social dimension. Here, Pries draws back on the work of Hägerstrand (1970) and Giddens (1985). In particular, Pries makes use of Giddens' concepts of 'locales' and 'regions'. According to Giddens 'locales' are the specific socio-spatial settings in which everyday social interaction takes place, and 'regions' define a specific 'zoning of time-space' in which an individual navigates in everyday life (Giddens 1985: 271f., cited in Pries 1997: 28). Pries applies Giddens' ideas to the specific context of transnationalism and speaks of 'transnational social space'. Transnational social spaces represent the emancipation of social space from geographic space. Pries says (1997: $34^{28}$ ): 'We suggested, to understand transnational social spaces as new spaces of social relations (Elias 1986: 'soziale Verflechtungsräume'), which are diffuse in terms of geographic space as well as multilocal, and which constitute at the same time a not only temporary social space. This social space is an important reference structure for social positions and positioning and it defines everyday life practices, (professional) biographic projects and identities of the people. Furthermore, it indicates the existence of social relationship beyond the limits of national societies (Pries et al. 1995; Pries 1996).' Transnational social spaces thus call for a concrete social interaction between people, and this means that people must be physically mobile

\footnotetext{
${ }^{28}$ Translated by the author from the German original: 'Wir haben vorgeschlagen, Transnationale Soziale Räume als neue „soziale Verflechtunsgräume“ (Elias 1986) zu verstehen, die geographisch-räumlich diffus bzw. multi-lokal sind und gleichzeitig einen nicht nur transitorischen sozialen Raum konstituieren, der sowohl eine wichtige Referenzstruktur sozialer Positionen und Positionierungen ist, als auch die alltagsweltliche Lebenspraxis, (erwerbs-) biographischen Projekte und Identitäten der Menschen bestimmt und zugleich über den Sozialzusammenhang von Nationalgesellschaften hinausweist (Pries et al. 1995 und Pries 1996).'
} 
across borders. Yet, these spaces have an imaginative and virtual dimension, Pries adds. Pries even calls them 'imaginative or virtual places’ (1997: 24).

Schmiz (2011: 22f.) adds that transnational social spaces are always linked to different geographic spaces as they include different locales in different nation-states, however, a transnational social space is defined by an equal distribution of activities and importance across all involved places. This means that social interaction and ties are dense and stable in time. Furthermore, it is not possible to define a dominant geographic centre within a transnational social space. Schmiz also explains that transnational social spaces are 'imagined' spaces that come into awareness only after concrete transnational social interaction. Thus, the extension of transnational social spaces has to be imagined as individual and subjective.

Bommes (2002) appreciates the emergence of the concept of 'transnational social space'. Transnationalism is basically the disintegration of society from the nation-state, but if one disintegrates society from the nation-state, one has to reintegrate it somewhere else. Bommes thinks of 'transnational social spaces' as exactly such an approach of reintegration of the sociological studies of migration, and he lists six critical points which follow his conviction that the concept of transnational social space is still insufficient (Bommes 2002: 94-98). First, transnationalism rejects the national as defining spatial scale. It then draws back on a spatial category - 'space', and uses it to re-draw new borders that also rely on territoriality. Second, the use of 'space' as a category is misplaced as it is one of the main irreducible dimensions of sociology (next to time or society), and as such 'transnational social spaces' are delimited in spatial terms. Third, the concept does not suffice to replace the 'national society container' as it represents a container in itself. In terms of structural theory it does not help much to better understand society. Fourth, it is unclear to what degree transnationalism will remain its own paradigm or only a temporary experience of criticism in nationalism. Fifth, nation-states still structure opportunities through setting legal frames, therefore, transnational social spaces cannot become an independent space as they are dependent on the existence of the nation-state. Sixth, the central role of the 'network' as a structural pattern of transnational social spaces is problematic. Networks are seldom operationalised in transnationalism studies. Transnationalism scholars often use this term without explaining which structure they are analyzing and referring to, and how these structures come into being. To sum up, Bommes mentions that transnationalism and transnational social space are concepts that have developed without reference to social theory. Whereas he positively evaluates Vertovec's (2001) idea of a 'transnational social formation', he would prefer to include the 'theory of functional differentiation' into transnationalism. According to this theory, 'space' would not be a given container in which social structure can develop. Rather, 'space' could be an outcome of migration phenomena. The relevance of such a spatial category for the development of social structure in the frame of transnationalism is still empirically unproven.

Instead of transnational social space, Glick Schiller, Basch et al. (1992: 1f.) described the existence of the so-called 'transnational social field': 'We have defined transnationalism as the processes by which immigrants build social fields that link together their country of origin and their country of settlement. Immigrants who build such social fields are designated 'transmigrants.' Transmigrants develop and maintain multiple relations - familial, economic, social, organizational, religious, and political that span borders. Transmigrants take actions, make decisions, and feel concerns, and develop identities within social networks that connect them to two or more societies 
simultaneously.' While the concept of a transnational social field is still less developed here, it was later on elaborated more precisely by Roudometof. Roudometof (2005) suggests that there are three different forms of transnational socialization: (1) 'transnational social spaces', which are interaction spaces based on the cross-border mobility and migration of people (as described, among others, by Pries); (2) 'transnational social fields', which refer to the rather formal relationships between institutions and organisations, but which do not necessarily include physical mobility across borders by the involved individuals; and (3) 'transnational communities' formed by migrants' social networks. A 'transnational social field' is the sum, or totality of the conditions for individual transnational acting. Such conditions could be legal regulations (in particular concerning migration as well as work and residency permission), economic and socio-cultural relations, or finally the individual involvement in transnational situations (e.g. bi-national partnerships, multilingualism, double citizenship). Given this definition, even non-migrants can potentially become part of a transnational social field (cf. Schmiz 2011: 15). Physical mobility is no pre-condition for membership in and the ability to shape transnational social fields. In contrast, transnational social spaces have a more exclusive character. Membership in transnational social space calls for concrete, often face-to-face interaction with people in 'other' national contexts. Physically immobile people thus cannot become part of the transnational social spaces, but of course they can become involved in transnational social fields (cf. Roudometof 2005). Using this definition, Roudometof changes a bit the idea that Glick-Schiller et al. had about transnational social fields; it allows for a better distinction between the 'spaces' and the 'fields'.

Also, Mau, Mewes et al. (2008) differentiate between a specific transnationalism which is based on migration and mobility on the one hand and a more general transnationalism of the local and immobile people on the other hand. The latter is sometimes to be understood as a consequence of the former, e.g. when migrants re-shape the character of formerly autochthonous neighbourhoods in the large cities. Here, Bürkner (2005) critically remarks that the idea of 'transnational social spaces' have something mystical because their fundaments have a largely cultural character and they widely neglect all economic reasons for transnationalism. Transnational structures might also develop out of economic necessity, and are not only a cultural phenomenon.

Historians might argue that transnationalism has always existed since nations were constructed, yet the new qualities and quantities of transnational experience suggest changes in the reflection about the social self and the belonging in wider parts of the (still rather) nationally organized societies. It is often supposed that the transnational experience of nearly all parts of the national societies leads to the emergence of more hybrid or multiple identities on the individual level (Walker 2011). Therefore, in particular the individual experience of transnationalism is an interesting field for sociological research. To be specific, transnational migration research might offer new objects of analysis: individual lived experience, social networks, and the perception of mobility in-between places.

\subsubsection{Transnational migration}

Studies dealing with transnational migration developed as its own field within the wider topic of transnationalism Faist (2000). Especially the academic fields of sociology, cultural studies, anthropology, and geography have developed a growing interest in transnational approaches to 
study new forms of migration, which no longer fit into the unidirectional and permanent pattern of $20^{\text {th }}$ century labour migration (Pries 1997). Schmiz (2011: $14^{29}$ ) comments: 'Since the 1980s, a large novelty in international migration research has been the focal shift from studying the migrant actors and their uni-directional or bi-directional changes in geographic location towards an understanding of migration as a continuous state of a person. The approach of transnational migration applies this understanding and emphasizes the repetitive and cyclical migration of people.' These new forms of migration are supposed to feed into the re-composition of the relation between geographic and social space. Transnational migration and its specific character of a back and forth mobility between different geographic spaces (such as nation-states, regions or cities) causes a break in the mutual exclusiveness of social and geographic space. Pries describes that the current geographic spaces host several independent social spaces (to be witnessed in multicultural society, in global cities, etc.). Today different social spaces are layered on top of each other within the same territory. Additionally, social spaces may extend in geographic terms to different poles. They become geographically multi-polar and thus intervene with formerly foreign territory. Even though they are not the only group, transnational migrants are suspected to be the main actors enhancing these processes.

Translated to the lifeworld of transnational migrants, Pries observes a transnationalisation of the social world, caused by the 'increasingly border-crossing references in the life of the people' (Pries 2008: $7^{30}$ ). The sedentary life seems to lose influence; the importance of one individual local reference frame, and thus one local community, as dominant among earlier generations, gives space to a more extended reference frame including several local communities in different countries. This plurality of life locales leads to problems for sociological studies as it evades from traditional and place-bound concepts, as Kalwa $\left(2010^{31}\right)$ observed in her studies on Polish transnational migrants in Germany: 'The main property of circular migration is a specific simultaneity of two ways of existing for the migrants, who function 'here and there', and who experience different forms of discrimination as well as of (cultural or social) ascent. The migrants incorporate different (yet often similar) social roles in both of their places. These social roles become fundamental in the construction of the migrant's identity. The life in continuous circulation causes an existence in a difficult-to-grasp cultural space between two worlds. This space is a symbolic area in the frontierland, which Zygmunt Bauman considers as space of potential success of those who autonomously define the frontiers within it. By definition, such frontiers are fluent and provisional (Bauman

\footnotetext{
${ }^{29}$ Translated by the author from the German text: 'Eine große Neuerung in der internationalen Migrationsforschung stellt die seit den 1980er Jahren erfolgte Abkehr von der Betrachtung der AkteurInnen und den von ihnen vollzogenen uni- bzw. bidirektionalen Ortswechseln hin zum Verständnis von Migration als dauerhaftem Zustand dar. Der Ansatz der transnationalen Migration greift dieses Verständnis auf und stellt die widerkehrende, zyklische Wanderung von Personen in den Vordergrund.'

${ }^{30}$ Translated by the author from the German text: 'zunehmend grenzüberschreitenden Lebensbezüge der Menschen.'

${ }^{31}$ Translated by the author from the German text: 'Das kennzeichnende Merkmal der Pendelmigrationen ist eine spezifische Gleichzeitigkeit zweier Existenzweisen der Migrantinnen, die „hier und dort“ funktionierend sowohl verschiedene Formen der Diskriminierung als auch des (kulturellen oder sozialen) Aufstiegs erfahren und in beiden Räumen unterschiedliche (wenn auch scheinbar ähnliche) soziale Rollen erfüllen, die sich im Konstruktionsprozess ihrer Identität als Migrantinnen widerspiegeln. Das Leben im Pendel ist die Existenz in einem schwer zu fassenden Kulturraum zwischen zwei Welten. Er ist eine symbolische Zone zwischen den Grenzen (frontier-land), die Zygmunt Bauman als Raum des potenziellen Erfolgs derjenigen bezeichnet, die darin selber die Grenzen bestimmen, welche in diesem Raum per Definition fließend und provisorisch sind (Bauman 2003). In der Konsequenz hat die Erfahrung der Pendelmigrantinnen in sich etwas vom liminalen Schwebezustand des „weder noch“ (between and betwixt), was eine komplementäre Darstellung, das Begreifen der Gesamtheit der Erfahrung erschwert, die sich der Macht präziser Kategorien und unbeweglicher Perspektiven entzieht.’
} 
2003). As a consequence the experience of these circular migrants can be characterized as something betwixt and between, just like a liminal state of uncertainty. This property of circular migration makes it difficult to comprehend such an experience in its totality and to describe it in complementary ways. It simply evades the power of precise categories and of immobile perspectives.' This quote highlights the necessity to overcome the methodological nationalism in social sciences, at least for the study of such border-spanning mobilities as described in transnational migration. The traditional concepts and categories of migration research are no longer sufficient.

Based on a literature review in the field of transnational migration, Schmiz (2011: 17f.) describes the life of transnational migrants in their own transnational social spaces and the wider transnational social fields. In particular, for processes of social inclusion and assimilation - two standard issues of traditional migration research - transnational migrants have several alternative strategies. First, they can reject inclusion and assimilation in the destination country and alternatively focus on their transnational social space. Second, they can still try to assimilate in traditional terms in the destination country. Third, they can opt for multiple and simultaneous forms of inclusion in different social spaces, namely the destination country as well as the transnational social spaces. Empirically, all these forms have been observed so far.

\section{Transnational migration, language, and culture: Transculturality}

Linguists also dedicate attention to transnational migration. In the recently published Handbook of Multilingualism and Multilingual Communication, Monica Heller (2007) asks, if we are currently witnessing the globalisation-based advent of wider multilingualism. With the growing importance of symbolic goods languages becomes more important for economic production. At the same time, these products circulate in extending ways across the globe. Heller thus is convinced that multilingualism becomes more important for everyone: 'It seems likely that fewer people are protected from these processes; more and more, we all have to deal with the multiplicity of material and symbolic resources, language(s) among them, as part of our daily lives' (Heller 2007: 540). As a consequence, in her linguistic studies she observed tendencies to both reinforce monolingualism through a standardisation of language as well as counter-strategies of maintaining linguistic diversity. Heller is convinced that more studies at the intersection between linguistics and transnationalism are necessary in order to understand how people's mobility leads to the distribution of languages and multilingualism. She concludes (2007: 548): 'This is a historical moment of discursive shift, one in which the political economic conditions of the globalized new economy are intimately tied to new ways of using language, and new ways of understanding what language is, and how it is connected to social identity and social relations. Transnational multilingualism is a first sign, and a key site for discovering what those changes are all about.'

As Schmiz (2011) points out, language is considered an important tool in becoming included in any type of social space. With transnational migration, it is suggested that bilingualism and multilingualism will increase and lead to different forms of language-based social positions. Different situations are possible here. Transnational migrants can remain excluded in one of the national contexts of their transnational social space, as they master one language better than the other. Second, they might be equally proficient in the relevant languages in their respective countries. And finally, they might also have difficulties with all languages, not having mother 
tongue level in any of 'their' languages. This would most probably be the case for secondgeneration migrants or people who have migrant parents.

The interest is not, however, limited to languages and linguistic systems. In the frame of transnationalism, cultural studies deal with the relatedness of migrants to nationally defined cultural systems. On the one hand, it becomes obvious that transnational migration puts the traditional concept of 'multiculturalism' into question, which relies on rather traditional forms of permanent immigration and the existence of stable and homogeneous ethnic groups in national societies. Prato (2009: 16f.) remarks that transnational migration calls for a reconsideration of how integration into cultural contexts takes place today: 'Contemporary policies of multiculturalism cannot therefore [meaning the extending transnationalism] ignore the significance of the links established at the intersection between the local, the national and the global.' Here Prato acknowledges that different geographic scales have an interrelated influence on cultural practices through transnational migration. While Prato still thinks that the concept of multiculturalism can be adapted to transnational migrants' realities, other scholars criticise the term for its implicit assumptions that 'cultures' are distinguishable (Stock 2010; Conti 2010). Multiculturalism describes the co-existence of different cultures which have a certain geographic origin; they are linked to specific territories. As such, a multicultural society in a specific country is composed of a native culture, which 'tolerates' immigrated cultures on its side. These immigrated cultures are supposed to be 'authentic' and unchanged, and to reflect the cultural situation of the country of origin (cf. Stock 2010).

The situation which is generated by transnational migration is better understood as a transcultural instead of a multicultural one, because transnational migrants 'transgress' the boundaries of national cultures and re-compose them to new forms. Hühn (2010) studied German pensioners who circulate between Spain and Germany. As a theoretical lense she used Welsch's definition of 'transculturality' (Welsch 2000), which she translated into the following aspects: first, the crossing of borders and thus the transgression of national culture is essential; second, the emergence and creation of some new cultural phenomenon must be observable; third, a transcultural phenomenon must be describable independent of national culture or as overarching national cultures (this fact includes the parallel existence of traditional national culture and new hybrid transculture); fourth, transcultural phenomena are those that can be related back to national culture. Hühn (2010) could empirically find all these four aspects in the cultural practices of her transnational German pensioners. Thus, she thinks of transnational migrants as drivers of transculturalism.

Equally, Conti (2010) adds that the formerly rigid and static conceptualization of culture has become obsolete, as cultural studies meanwhile have shown that culture is mainly supported and continuously changed by individual persons. Contemporary culture does not exist independent of the people. Based on the work of Clifford (1994), Conti describes that the individual person is culturally dynamic: an individual continuously incorporates new forms of cultural belonging through participating in ever new 'collectives'. These collectives can have two characteristics, as Conti argues according to an idea from Hansen (2009). On the one hand, they can be defined by destiny (gender, generation, place of birth, social position of the parents). On the other hand, collectives can be defined by personal interest (hobby, place of residence, attitudes, profession). Each individual is thus part of different collectives, and as such 'multi-collective' (Hansen 2009). As Conti (2010: 180f.) recalls, Hansen thinks of culture as rooted in such collectives. According to Hansen, collective cultures emerge out of the basic necessity to reduce the environment's 
complexity and heterogeneity. In different geographic scales, people build different institutional frameworks which facilitate everyday interaction through standardization and homogenisation. Standards, symbols and norms then guide interaction, and they form a collective culture. For Hansen, the most important scale here is the local one as geographic proximity is supposed to enhance frequency of interaction, and thus the value of setting up collective culture. Translated into the life circumstances of transnational migrants, this means that they are part of different collectives in different places and countries, and as such they enact knowledge about different cultural systems. They know about the differences and they know how to navigate in this difference. As a consequence, they become both the bearer and the designer of these systems (cf. Conti 2010).

Based on this theoretical reflection, Conti concludes that people transgress all types of cultural systems, as they incorporate membership in different collectives, because the mixture of collectives is individual for each person. Thus, what might seem coherent on the individual level of the person is not congruent with a collective system anymore: 'Any interaction can thus be considered as 'transcultural' (Conti 2010: 182 ${ }^{32}$ ). Consequently, transculturality is the rejection of the idea that cultural systems can be distinguished from each other, as they are interwoven through the individual persons' mixture of membership in collectives. Finally, Conti uses Balducci's (1990) metaphor of the 'global man' (essere umano planetario) to explain the difference between intercultural and transcultural settings. Balducci mentioned that all individuals on this planet are interdependent and exchange with each other in more or less direct and conscious ways. Therefore they should employ an inclusive state of mind. Conti (2010) uses Balducci's idea to define the transcultural setting in which two individuals acknowledge each other as individuals, and they try to include each other by creating a completely new culture of communication. By contrast, in intercultural settings two individuals perceive each other as representatives of 'other' homogenous and collective cultures, and they try to approach the culture of the other.

Transnational migration is thus conceptualised as a powerful driver of changing cultural patterns, leading to more hybrid, transgressive and individualized forms of culture. An interesting empirical observation in this context was provided by Toplak (2011), who studied a group of transnational migrants, who are also professionally engaged with culture: Toplak looked at Slovenian artists who have migrated to Argentina. As a main result, she found that the transnational life experience translates into the artistic work (e.g. paintings) of the case study group. In particular, those Slovenian artists who frequently travelled between Slovenia and Argentina were drivers in the creation of a new mixed style of artistic production.

\section{Transnational migration and identity}

Whereas the spread of transcultural phenomena may be one aspect in defining belonging in a new way, there are several other aspects that add to 'culture' in defining belonging and in particular identity (cf. Samers 2010: 276f.). Migrants’ identity is also influenced by class, gender, race, generation etc. While there is no space to go into detail here, I would like to highlight that transnational migrants are supposed to develop multiple and hybrid identities (cf. Schmiz 2011; Pries 2008). Glick Schiller, Basch et al. (1992) already observed the phenomenon of a multiplicity in transnational migrants' identity. Glick Schiller, Basch et al. found that in particular social groups

\footnotetext{
${ }^{32}$ Translated by the author from the German text: 'Damit kann jede Interaktion als ,transkulturell' gesehen werden.'
} 
such as hometown associations are important anchors and reference frames for transnational identification. They (1992: 11) resume: 'Within their complex web of social relations, transmigrants draw upon and create fluid and multiple identities grounded both in their society of origin and in the host societies. While some migrants identify more with one society than the other, the majority seem to maintain several identities that link them simultaneously to more than one nation.' This quote highlights again that one could imagine transnational migrants as developing several simultaneous and different or also new hybrid forms of identity, which extend national identities. Equally, Kaiser (2011) understands transnational migrants as having different social roles in different places. This multiplicity brings along a redefinition of the main question concerning identity (Kaiser 2011: 54 ${ }^{33}$ ): 'The great problem which occurs here is no longer the durability and the modern question for 'Who am I?', but the post-modern question for 'Do I incorporate all social roles which might become relevant to my existence?' Multiple appropriation of space, polycentric place relations and transnationalism are phenomena, which must thought of as linked to changing patterns of identity construction by actors in the light of contextual changes caused by globalization and pluralisation.'

The notion of Heimat/home seems to persist as an anchor point of identity, and it will remain related to existing spatial scales such as the nation, the region and the city. Against the suggested argument of an increasing supra-nationalisation of identity, Schmiz (2011: 19 ${ }^{34}$ ) says: 'This [opponent] position is based on the argument that traditional national and regional models of identity and belonging will neither dissolve in a global world society nor in a post-modern and immaterial 'space of flows', and it will not be replaced by supranational constructs such as the EU. Rather, the notion of home, which is strongly linked to a national identity and to an imagined community (cf. Anderson 1996), will keep its important role for transmigrants. Relationships and networks between people on the local, regional, national, global and transnational scale will not replace each other. Rather, the forms of living together will increasingly be differentiated (cf. Pries 2005: 21).'

Transnational migrants are thus suspected to develop new hybrid forms of identification while referring to existing categories. Here, Goeke (2004) criticises that transnationalism studies are often poorly linked to social theory. In particular, the question of how transnational migrants develop feelings of belonging to various places at the same time cannot be studied without using social theory. This includes the fact that identification patterns are difficult to assess. Goeke argues for a more precise use of the 'hybridity' concept as an analytical lense instead of an observable variable. He says (2004: 196): 'Cultural hybridity moves the axis of differentiation from an external division

\footnotetext{
${ }^{33}$ Translated by the author from the German text: 'Das große Problem, das sich hier ergibt, ist nicht mehr die Dauerhaftigkeit und die modern Frage nach 'Wer bin ich?', sondern die postmoderne Frage: ,Habe ich alle Rollen im Repertoire, die von mir gefordert werden könnten?‘. Multiple Raumaneignung, Ortspolygamie und Transnationalismus sind somit Phänomene, die in Zusammenhang mit Veränderungen der Identitätskonstruktionen der Akteure unter den sich wandelnden Bedingungen der Globalisierung und Pluralisierung stehen können.'

${ }^{34}$ Translated by the author from the German text: 'Dieser Ansicht liegt das Argument zugrunde, dass sich traditionelle nationale und regionale Modelle von Identität und Zugehörigkeit weder in einer globalen Weltgesellschaft noch in einem postmodernen immateriellen ,Raum der Ströme' auflösen, noch durch supranationale Gebilde wie die EU verdrängt werden. Vielmehr spielt die Heimat, die eng mit einer nationalen Identität zusammenhängt und auf eine vorgestellte Gemeinschaft rekurriert (vgl. Anderson 1996), weiterhin eine wichtige Rolle für TransmigrantInnen. Verflechtungsbeziehungen und Netzwerke von Menschen auf lokaler, regionaler, nationaler, globaler und transnationaler Ebene werden sich nicht gegenseitig verdrängen oder ersetzen. Vielmehr wird es zu einer weiteren Ausdifferenzierung der Formen des Zusammenslebens von Menschen kommen (vgl. Pries 2005: 21).'
} 
between self and other to an internal plurality of differences. This is why hybridity should be understood as an analytical tool but not as a mode of self-description, especially since it is often a nameless and hidden category.' In transnational social spaces, identification is not only limited to the 'self' and 'other' description in relation to national frameworks. Transnational migrants have the possibility of defining and being defined as someone in between the national 'self' and 'other'. Goeke uses the illustrative image of the transnational migrant as a 'translator' (2004: 195): 'From an outside perspective, they might be labelled as 'hybrids'. They are continuously involved in processes of translation between the supposedly 'pure' elements.' This translation is mainly a reflection on the personal 'impurity'. Studying Balkan migrants in Germany, Goeke found that migrants also take into account aspects of 'places' and 'professions' when they reflect on their personal transnational identity. While places seemed to lose their purity and specificity and thus are less opportune for identity patterns, jobs and professional environments were increasingly included in identity by these migrants. Goeke reports that transnational migrants actively compare two or more national reference frames which are available for identification, yet they do not show loyalty to the existing collective identities. Rather they develop strongly individualised means of identification which provides them a certain freedom from national identitary constraints and is subjectively perceived as more reliable. The main anchor point here becomes often one's own biography (cf. Goeke 2004).

A specific biographical approach to transnational migrant identification is provided by Ursula Apitzsch (2003) as well as Lutz (2004). Apitzsch understands transnational social spaces as invisible structures of political, cultural and legal intersections, which provide guidance to transnational migrants' biographies, and which are the context of life experience and perception. Apitzsch thus argues that it must be the empirical project of transnationalism research to uncover these invisible structures within migrants' biographies. 'Biography as place of transnational and transcultural space is the intersection of collective constitution and individual construction' (Apitzsch 2003: 72, cited in Lutz 2004: 211). Lutz acknowledges that Apitzsch's approach is helpful in addressing latent cognitive patterns in migrants' transnational life experience, however, Lutz prefers to think of 'biographies' as 'articulations' rather than 'places'. According to Lutz, place is an atemporal notion that does not include the historic dimension of biographies, which only become what they are over the course of time. Understanding 'transnational biographies' as 'articulations' allows respect for the dynamic character of a biography. If one thinks of articulation as the process of linking the subjective 'self' to surrounding discourse, then one must acknowledge that during the course of a lifetime, a biography is a continuous sequence of disarticulation and rearticulation of certain discursive elements (Lutz 2004: 212f.). Here, Lutz refers to the concept of articulation as introduced by Stuart Hall in the 1990s in his articles on 'cultural identity' (e.g. Hall 1996). Lutz explains that a transnational migrants' self-positioning (including elements of identity) is mirrored in biographic narration. Furthermore, this biographic narration is subject to change according to a continuously reflected discursive environment in which the migrant is embedded. Thus, in order to study transnational identities it is essential to assess the logics behind biographic articulation and narration.

Scherke (2011) also points out that transnational migrants often identify with different scales at the same time. Based on a study amongst migrant children in Germany by Dannenbeck (2002), she recalls that these migrants often answered the question 'Where are you from?' with spatial 
categories that are not reflecting nationality. Rather regional and local references were provided. Furthermore, other non-spatial references were used by these migrants. Gender and religion showed up as adding to spatial categories.

Another approach to studying hybridity in transnational identity is provided by Mecheril (2011). Mecheril says that affiliation (Zugehörigkeit) is the key to an understanding of hybrid identity. Zugehörigkeit/affiliation is generally composed of three components: Mitgliedschaft (membership), Wirksamkeit (effectiveness), and Verbundenheit (attachment). The first component, membership, is generated by the self-reference to and acceptance of others to be part of a certain group whose members have a specific idea of what this group is about. A person will be considered a member of a certain context (e.g. a social group) if they feel that they themselves belong to it, and if others tell them that they belong there. Then there is the component of 'effectiveness', which describes how effective the affiliation can become in terms of permitting and prohibiting well defined activities and habitus to its members. Third, Verbundenheit/attachment describes the emotional belonging of a person to a context that they are part of. This includes moral convictions and obligations, the cognitive and practical familiarity with the context (Vertrautheit), and finally material aspects as well.

Whereas traditional patterns of affiliation could be labelled 'legitimate' as they represent situations in which all three components are congruent, transnational migration often leads to 'illegitimate' situations of hybrid belonging. Mecheril basically defines 'hybridity' as the transgression and rejection of binary distinctions. It refers to a state of mixture in which the supposedly irreconcilable is reconciled with something new (cf. Mecheril 2011: 48). Here, transnational migrants represent a type of social actors who develop hybrid forms of identity which are composed of multiple forms of affiliation to several national contexts, and in thus they transgress national identity and reject the binary distinction between being part of the nation and being a foreigner. Rather they might identify as both or even as something completely new, being in between and evading traditional national contexts of belonging.

\section{Transnational belonging and civic participation}

Samers (2010: 279-297) discusses in detail how transnational forms of belonging interrelate with citizenship and civic participation. Screening the existing literature on migration studies, Samers (2010: 280) explains that transnational belonging could be described as bi-focal, creolized and representing a double engagement 'in which migrants constantly think of the 'here' and 'there', often in contradictory ways'. This means that in extreme cases they might feel to belong neither here nor there, or on the other hand to belong equally here and there. In both cases there is a continuous reflection about one's own position. Migration scholars are arguing about how to understand this form of belonging. While some think of it as an antipode to traditional assimilation of migrants in the host country, other argue for a possible parallelity of both processes - the incorporation into transnational environments and into the host country's society. Thus, the national scale and the involved nation-states play an important role in defining the frame for belonging, e.g. through providing access to (double) rights to vote, to access social security schemes, to work, to reside etc.

The point of belonging is that it is mainly perceived on a local scale, in the urban environment, where everyday life takes place. Here, Samers draws back on Vertovec's discussion of the notions 
of 'trans-localism', 'trans-locality', 'trans-regionalism', or the transnational village (cf. also Vertovec 2001, where the specific importance of the sub-national scale in transnational migration is outlined. Also, Smith's (2001) concept of 'transnational urbanism' stresses the relevance of the urban scale, as most travel of transnational migrants takes place between specific cities in different countries, and not necessarily the country as a whole. Samers remarks (2010: 286): 'We should expect migrants to have diverse transnational and other attachments (trans-local, trans-regional, etc.) within and across multiple territories. These attachments are likely to produce different forms of political participation.'

Thus, looking for the ways in which transnational migrants engage in the different territories to which they feel they belong also calls for a differentiated approach that includes the interference between geographic scales. Samers cites the work of Koopmans (2004) who pointed out that local civic participation in particular is heavily dependent on nationally granted opportunity structures. While participation has been made easier through changes in citizenship and naturalisation legislation, many countries still exclude migrants from central aspects of political life such as elections. According to Samers, migration literature hints at different scales of migrants' political engagement. On the national scale, transnational migrants' engagement is often defined by a double orientation to the political context of the host and the home country. Here, transnational migrants often engage in the transfer of political culture and political tools. Furthermore, their engagement might aim at changing the policy of one of 'their' countries towards the respective other countries. On the local scale, hometown associations are often cited as an example of transnational migrants' engagement for a specific locale in the home country. In the host country, municipal 'consultative committees' have been installed in recent years across European cities, in which migrants are asked for advice and for their needs when it comes to the provision of local public goods and services. Samers (2010: 296) concludes: 'Migrants negotiate these multi-scalar realities in the context of 'super-diversity', sometimes by rejecting the norms and practices of the country of immigration, sometimes by adopting them, and sometimes by constructing, navigating, or negotiating complex transnational (or diasporic) identities, which involve attachments to real or imagined 'homelands'. [...] Dual nationality, jus domicile, and multiple belonging do not overall seem to dissuade political participation in the country of immigration. Quite the contrary, it may stimulate political involvement as countries of origin and destination become tied by large overseas communities and intertwined cultural, political, social, and economic networks.' Finally, he underlines that national, regional and local political opportunity structures mainly define such migrant political participation. While many of these issues are still problematic in terms of migration between the EU and so-called 'third countries', migration within the EU could already be considered a showcase of 'postnationalization’ (Samers 2010).

\section{The specificity of the European Union}

Studying retirement migration using the case of transnational German pensioners in Spain, Kaiser (2011) also points out that the European Union provides a specific framework for the development of transnational migration. In particular, the possibility for free movement of people has been extended over the last decades. The right to choose freely where to live and work was first granted to workers in the 1960s; then it was extended to self-employed people and entrepreneurs. Nonemployed people such as pensioners and students could also move to any other country in the European Union in the 1990s, and in the 2000s the last barrier was abolished. People who intended 
to stay for more than three months in a foreign country did not have to ask for a residency permit anymore; they could now simply register as a resident of a place abroad. Furthermore, other institutional contexts were also standardized in the European Union. The most important novelty was the introduction of the Euro as a common currency, but smaller changes also occurred in the fields of banking and the access to bank accounts, income tax law, access to insurance and social security systems, or the possibility to buy property in a foreign country (cf. Kaiser 2011: 104f.). Difficulties might still remain for transnational migrants, if e.g. social security schemes are not transferable to other countries or compatibility is not established between two nation-states' systems (Kaiser 2011: 54f.).

\section{The transnational family}

Körber (2011) studied how families change in the frame of transnational migration. She observed that social scientists (such as John Urry and Richard Sennett) perceive that the increasing labourmarket driven needs for mobility in global capitalism lead to an erosion of kinship and family ties. According to this position, 'family' comes into existence by spending time together in one place. This includes face-to-face interaction and relative immobility. If people become more mobile, they will increasingly be forced to break up with such family structures, as the argument goes. Körber disagrees with such a point of view, as it reflects an essentialist concept of family which is rooted in 'methodological nationalism' (2011: 93). It suggests that family can only exist in geographically sedentary lifestyles.

In studying several transnational migrants' family ties, Körber and her research team found that family today works also as a type of 'imagined community' in virtual ways, which add to physical co-presence. Families today are becoming more and more multilocal. In addition to their function as an economic unit, they also become mythological reference frames. They inherit nostalgia and kinship, which can be described as 'proximity at a distance'. Therefore, ICT development was a necessary condition as it allows for interaction and the maintenance of relations in virtual ways. Family today is nothing stable anymore, which continues to exist. At a distance, family has to be reproduced every day. Here, Körber (2011) refers to Schier \& Jurczyk's concept of 'family making' (Familie als Herstellungsleistung) (Schier 2007). Family has to be thought of as a sequence of physical absence and presence, in which collective agreements no longer define the ways in which family comes into existence, but conflicting interests of the individual family members are negotiated against each other. Transnational migration thus allows for extending the range of opportunities for families and does not necessarily destroy them. At the same time, it also brings along new duties and expectations to the individual family members (as e.g. expected submission of remittances).

Empirical studies in the field of transnational families have resulted in well-known concepts such as the 'global care chain', the 'transnational parenting' or 'long-distance mothering', which all mainly stem from a focus on female labour migration in the field of domestic and care work. Here, transnational migration mainly refers to the migration systems that exist between the global south and the global north, or between the European East and West. These migration systems are characterized by young women, often mothers, who emigrate to more wealthy countries in order to take positions in private households, where both partners are working full-time. Thus, they have to 
leave their own children and parents back home, and care for children and elderly people in a foreign country.

\subsubsection{Transnational entrepreneurship}

A growing interest in transnationalism can also be observed among economic geographers and regional economists who have found 'transnational entrepreneurship' to be an extending phenomenon (Drori, Honig et al. 2009; Henn 2012). Drori, Honig et al. (2009: 1001) suggest that transnational entrepreneurs could be defined as follows: 'We [...] define TEs [transnational entrepreneurs] as social actors who enact networks, ideas, information, and practices for the purpose of seeking business opportunities or maintaining businesses within dual social fields, which in turn force them to engage in varied strategies of action to promote their entrepreneurial activities.' It is remarkable how clearly this definition encompasses non-material aspects: ideas, information and practices. This observation points to the fact that transnational entrepreneurship is a type of business-making which successfully exploits the resources that two or more distinct and territorially limited markets might provide. The competitive advantage of transnational entrepreneurs lies in the access to market relevant knowledge and information in two or more countries. Drori, Honig et al. mention (2009: 1002): 'By virtue of their unique geographical affiliations, they may be in a unique position to exploit opportunities either unobserved, or unavailable, to other entrepreneurs located in a single geographical location.'

Drori, Honig et al. (2009) also point out that transnational entrepreneurship has to be distinguished from international entrepreneurship. Other than many international businesses, transnational entrepreneurs are rather working in small scale business units that operate just like local businesses in a way that they must face local and national institutional constraints. Yet, other than local businesses they can compare institutional settings and try to shape them to their own advantage.

Schmiz (2011) remarks that transnational entrepreneurship must be differentiated from immigrant or ethnic businesses which mainly operate in destination countries of migration. Transnational entrepreneurs use two or more countries and their cultural contexts in their business models, yet this does not mean that both contexts are equally important parts of the business itself. Empirical studies have shown that highly-skilled transnational entrepreneurs often focus on markets in the host country, while low-skilled migrants rather tend to focus on home country markets (cf. Schmiz 2011: 24). Drori, Honig et al. (2009) add that ethnic entrepreneurship is distinctive in the sense that ethnic entrepreneurs heavily rely on local resources in the context of the host country. Furthermore, their business environment is characterized by co-ethnicity. On the one hand, most of their clients stem from their own ethnic group, or customers and business partners are ethnic fellows. Finally, ethnic entrepreneurship can be considered a counter strategy against the failings in traditional forms of integration. If immigrants do not feel able to become part of the native majority of society, engaging in ethnic businesses is an alternative for economic and social integration.

In contrast, transnational entrepreneurs supersede the constraints which arise from ethnic entrepreneurship and its limits to one's own ethnic group. They engage independently in two or more countries, and while not necessarily rejecting the ethnic component, they also make use of other resources. As such, Drori, Honig et al. (2009) speak of a bi-national and non-assimilating group of migrants who engage in transnational entrepreneurship. This means that for transnational 
entrepreneurship the personal experience of migration is one of the key resources for business making, therefore transnational entrepreneurs also differ from international entrepreneurs, who do not necessarily have a migrant background. Furthermore, whereas international businesses often try to create standardization and harmonization, transnational entrepreneurs understand cultural and institutional difference between countries as their resource.

Equally, Faist (2000) observed that there is a shift from ethnic businesses to transnational entrepreneurship among Turkish migrants in Germany. Using the empirical example of Turkish people in Germany, Faist conceptualises entrepreneurship of migrants as a three-step model. Immediately after arrival in the host country, these migrants mainly submitted remittances back to Turkey, which were used for both consumption and investment in small start-ups in Turkey. In a second step, Turkish migrants invested their accumulated financial capital in Germany, where they founded start-ups that could largely be grouped into the category of ethnic businesses - mainly in the field of private consumption (restaurants, grocery shops, craftsmanship, travel agencies). In the third step, Turkish people now increasingly invest in businesses in which both the host and the home country are part of the business environment. Typical sectors here are textile production and distribution, which requires larger sums of start-up capital, or import-export businesses. In this transnational economic space, transnational migrants could exploit insider advantages that result from languages skills, cultural knowledge and pluri-local business networks (which are often supported by family members and friends in both contexts).

Equally, Drori, Honig et al. (2009) explain that transnational entrepreneurs are characterised by the capacity to recognize their insertion in two distinct cultural contexts as their advantage. They make use of their symbolic and cultural capital, different knowledge and skills. Thereby, one can observe that transnational entrepreneurs are tending to apply cosmopolitan instead of ethnic cognitive models. Their social context is the transnational one, not any context bound to one specific group, yet they are able to realise local cultural specificity and respect it.

A particularly interesting aspect in transnational entrepreneurship consists in the interrelatedness of culture and business. According to Drori, Honig et al. (2009), culture has to be seen as something which is not static and given; rather the individual can influence, shape and change it. Transnational entrepreneurs are considered a group of business makers who have a strong capacity to analyse and exploit culture and cultural difference, while at the same time being able to re-shape culture according to their needs. In reference to Putz (2003), Drori, Honig et al. (2009: 1009) remark: 'TEs adopt diverse schemas of meaning and strategizing within varied symbolic orders "leading action in social practice, namely as a 'repertoire' which offers various options of how to act and to which agents can have reflexive access" (Putz, 2003, p. 557). Such an approach views TEs as agents who have to redraw the boundaries of their cultures in order to follow action paths and routines embedded in both practice and diverse symbolic orders.' The same accounts for market and social institutions in the host and home country of migration, which are both influencing transnational entrepreneurs' opportunities and being modified by transnational entrepreneurs. Here, Drori, Honig et al. (2009) also highlight the role of social capital and the embeddedness in local networks in multiple ways. This type of double social embeddedness provides security and access to resources, and as such, it can be considered a guarantor for market power. 
Another interesting input to the discussion about the role of transnational entrepreneurs stems from economic geographer Sebastian Henn (2012), who studied Indian entrepreneurs in the diamond sector of Antwerp. Referring to cluster theory, Henn mentions that the study of cluster dynamics mostly focussed on internal and local structures. Only recently, new approaches include the external linkages of localized industrial clusters. However, these external linkages are still poorly studied and conceptualised. One attempt was made by Bathelt, Malmberg et al. (2004) who think of the local cluster as a frame for knowledge circulation among local cluster members. They call this internal knowledge flow 'local buzz'. Additionally to the local buzz, there are so-called 'global pipelines' which link different clusters and lead external knowledge exchange.

Henn tries to shed light on this aspect by using the transnational entrepreneurs in the diamond sector as an example for how these 'global pipelines' of local clusters might work. In his literature review, Henn emphasizes the role of family links in enabling and supporting transnational entrepreneurship. Furthermore, he cites other authors who stress the role of both local and international business relations in emerging knowledge-intensive economies such as IT. For cluster development, the ability of transnational entrepreneurs to 'translate' between different contexts is mentioned as advantageous (cf. Henn 2012: 499). Henn summarises (2012: 504): 'As they are more or less simultaneously engaging at two or more different locations, TE are able to draw on a worldwide resource base. Research on the interface between TE and regional clusters so far has come to the conclusion that TE contribute to the evolution of global production networks, to a transfer of knowledge into existing clusters and to the forming of new clusters in their home regions. Put generally, TE have been found to be beneficial for the development of clusters both in their home and in their host country.'

The example of Indian diamond traders shows that the exploitation of double knowledge about Indian consumer and European producer markets caused the success and accession of Indian traders into the world market. Furthermore, they traded in a cosmopolitan way, not differentiating their business partners by ethnic groups. At the same time, they kept their knowledge within their own ethnic group for two reasons: the traditional Belgian and British diamond traders underestimated their new colleagues from India, and the Indian community was very reclusive. As such they became successful transnational entrepreneurs who were neither fully integrated in Antwerp, Belgium nor in Palanpur, India. They operated in their own transnational social space. Consequently, the Belgian diamond sector was confronted with increasing competition and lost dramatically in market power and size. This example shows that transnational entrepreneurship can have a strong influence on the local context, both in positive and negative ways.

Still, the main problem for transnational entrepreneurs is the relatively high cross-border moveability of production factors such as financial capital or intermediary goods, while the movement of people - such as the entrepreneurs themselves - is often limited by the legal regulation of migration. Therefore, empirical studies found that double citizenship is a good indicator for transnational entrepreneurship (cf. Schmiz 2011).

Drori, Honig et al. (2009: 1014) conclude: 'The phenomenon of TE implies a distinct opportunity structure which enables those immigrants who found and maintain businesses to benefit from "two worlds" as a crucial factor for survival, a way of "breaking out," and/or a method for providing competitive advantage.' Furthermore they mention that research must take into account the specific 
character of transnational entrepreneurship: namely, that transnational entrepreneurs are social actors who reflexively operate in different social contexts, and who have to handle multilevel organizational insertion.

\subsubsection{Transnationalism and cosmopolitanism}

A current question in studies of transnationalism is to what degree this growing transnational life experience affects the individual cosmopolitan attitudes of the people (Mau, Mewes et al. 2008). The concept of 'cosmopolitanism' dates back to the work of classic sociologists such as Kant, Simmel and Schütz, who had developed the character of a 'cosmopolitan stranger' who gets in contact with a locally rooted social community. This description led to the idea that there is an opposition of the cosmopolitan and the local, as Ossewarde (2007: 372) describes: 'The cosmopolitan is flexible and mobile enough to move through localities and jobs while the local is rooted and fixed in neighbourhoods and workplaces'.

Cosmopolitanism is conceptualized as a state of mind, an attitude, which allows a person to detach from a local context and to critically distance themself from it. The cosmopolitan person knows different local contexts and is able to compare them. The locally rooted only knows their own and is thus unable to take the perspective of people from other local contexts. The cosmopolitan person lives in a more abstract world, relying on what Merton calls 'knowledge about' - a technical knowledge, which is applicable in different locales. On the contrary, the locally rooted uses their 'knowledge of acquaintance' which only works in their own local context (cf. Ossewarde 2007).

This idea of cosmopolitanism goes along with a normative understanding about cosmopolitan attitudes. Kant's vision of the 'world citizen' and a 'global citizenship' is the base for normative conceptualisations of cosmopolitanism (cf. Beck 2002). The cosmopolitan person ideal typically accepts that all people are part of one and the same humanity. Although every person might be socialized in a specific local culture, the cosmopolitan acknowledges this difference of origin while rejecting any judgments or prejudices. The culturally other is considered a human being with equal rights who deserves to be respected. As a consequence, this normative approach to cosmopolitanism argues for a world citizenship and questions the legitimacy of the nation-state.

In the current literature on cosmopolitanism, the global-local opposition is quoted. The general argument is that the ongoing 'transnationalisation' of the national societies leads to a split into a more open-minded and tolerant cosmopolitan group, on the one hand, and a closed and ethnocentrist group of locals, on the other hand. It is suggested that the winners of globalisation, mostly brain workers employed in multinational corporations and international political bodies, form the cosmopolitan group, which is detached from the local context and problems (Duclos 1999). This group partially retracts from local solidarity and engagement (Andreotti, Le Galès et al. 2010). In contrast, the losers of globalisation, mostly blue collar workers in industrial companies, form the local narrow minded part of the societies (Mau, Mewes et al. 2008), which fear the dissolution of the stable frame of national reference.

Yet, other positions are based on the conception of 'glocalisation' (Roudometof 2005), which stresses the simultaneity of becoming more global and cosmopolitan as well as remaining rooted locally. Here, cosmopolitan attitudes co-exist with a national and local orientation within the 
reference system of a person. Depending on the thematic field, an individual might take a more cosmopolitan and inclusive position, or a more exclusive and local standpoint. These positions only form two extremes, and the empirical reality is supposed to be situated on a continuum in between.

Pries (2008) is rather critical towards the cosmopolitan standpoint. He thinks of his transnationalism studies as something separate. According to Pries, cosmopolitanism is too closely linked to the normative idea of a world society, something that Rumford (2008) called the 'one-worldism'. In this position it is assumed that there will be one world society to which everyone feels a belonging and in which every person is related in his consciousness with all other people in the world. For Pries, transnationalism rather deals with a non-holistic or universalist perspective. Transnational lifeworlds are only connecting selected places, and not the world as a whole.

\subsubsection{Synthesis: Transnationalism as an approach to study routes}

In section 4.1, I offered an overview about some - definitely not all - aspects that evolve around the topic of transnationalism. Transnationalism - as a scientific field of interest and conceptual framework for empirical study - provides some interesting ideas on how to grasp cross-border mobility between countries in the frame of global capitalism. With the political, economic, and technological innovations that have occurred in the last decades, social organization has also changed worldwide, leading to increasing interaction across national borders. The concept of transnationalism allows approaching new forms of circularity and exchange between actors from different national contexts. Pries $(1997,2008)$ showed how transnationalism adds to other forms of internationalised socialisation. Some of them might still be studied using methodological nationalism, which is basically understood as the superposition of geographic (national territory and political sovereignty) and social space (national society and institutions). Others - such as transnational socialisation - must be conceptualised as driven by and driver of disintegration of geographic and social space.

I focused here on the micro level, on the individual transnational migrant and their characterisation. Obviously there are other levels such as the meso and the macro level (cf. Faist 2000). However, I deem this individual level as the most relevant to my study of mobile creative knowledge workers. It has been shown that transnational migrants are understood as a new type of migrant who circulates between two or more countries. This form of migration includes that transnational migrants elude traditional approaches of integration and assimilation in a host country. Rather, they proactively pick from both countries what they need and what they like. It is impossible to lock them into 'national containers'; they create and operate in their own transnational social spaces, which are characterised as a situation 'in-between' and with the maintenance of social ties in different countries. These ties are more stable and include more frequent interaction than was the case for traditional forms of unidirectional migration.

Transnational migration is supposed to support the development of transcultural phenomena and multilingual settings: it changes the way in which people identify. Increasingly, multiple and hybrid identities show up and confound the traditional idea that people are rooted in one national culture and society. This includes that 'society' itself becomes less rooted in one bordered nation-state. Furthermore, transnational migration literature points out that new questions will arise around the issues of citizenship and civic engagement. So far, national legal and regulation regimes are still 
exclusive when it comes to transnational migrants with a different citizenship. However such transnational migrants are increasingly part of all nation-states in the world, so equal participation in the public social life must somehow be negotiated for them. They often feel that they belong to countries of which they are not officially acknowledged as citizens with equal rights (e.g. voting, political activism). I have also mentioned that the European Union represents a specific setting for transnational migration in this respect as the Citizenship of the European Union, introduced in the Maastricht Treaty of 1992, grants additional transnational rights to national citizenship to European citizens. Last but not least, also on the very individual, micro-sociological level of interpersonal relations - the social unit of the family was presented as a showcase - changes are evident. Families increasingly work according to the idea 'proximity at a distance', meaning that not all family members will continue to live in the same place. Families are also becoming transnational and as such must be actively constructed across borders by its members.

I have also referred to the literature on the economic impact of transnational migration. Here, the figure of the transnational entrepreneur was presented. Clearly, not all transnational migrants also become transnational entrepreneurs, but if they do so, they seem to be distinct from international, ethnic or emigrant entrepreneurs. Transnational entrepreneurs 'translate' between different nationally influenced business environments and exploit their knowledge about these differences. The given insider advantage that they have provides an opportunity for business models. Depending on the degree to which they share their knowledge with their local business communities in their countries, these transnational knowledge stocks might become global pipelines of knowledge transfer, and as such helpful to regional economic development.

Finally, I briefly hinted at the ongoing debate about the role of transnational migration for the diffusion of cosmopolitan attitudes. On the one hand, scholars argue that increasing cross-border mobility and personal experience of migration will enhance cosmopolitanism, as migrants' experience of being a foreigner causes cultural flexibility. On the other hand, more sceptical positions argue against such automatism. Here, the argument is that transnational migration only involves selected places and not the world as a whole.

Even though transnationalism allows for a better understanding of contemporary cross-border mobility, there are still open questions and criticism. Schmiz (2011) thinks that transnationalism has not still provided sufficient definitions of the 'transnational migrant'. Is it unclear according to which criteria a migrant can be labelled 'transnational' or not? How should this be measured? Should one use the frequency of trips, of other exchange activities? Which exchange activities are relevant? Are only economic activities relevant, or cultural and identitary ones as well? Schmiz therefore calls for the elaboration of a definitory catalogue, including criteria which are found in empirical research.

In addition, much empirical work focuses on one national group within one transnational social space without internal differentiation, and there is a strong focus on remittances and ethnic groups: both approaches include the problem that analytic units (nation and ethnicity) are considered as naturally given. Schmiz argues that the analytic units should better be defined from case to case using relevant criteria (Schmiz 2011: 35). Schmiz also criticizes the idea of an opposition between assimilation and transnationalism; she would prefer to study this opposition empirically, and assumes that there might be more of a co-existence between assimilation and transnational 
belonging. Additionally, transnationalism studies so far have brought no results in terms of the longevity of the phenomenon. She suggests conducting more long-term inter-generational studies to see how transnational settings evolve over time.

Kaiser (2011) adds that the role of family ties has still not been studied sufficiently. So far, transnational migrants are often looked at as individual actors, yet much of their decision taking happens in the frame of discussions in the family. Are family ties inhibiting or facilitating transnational migration?

Bommes (2002) critically examines transnationalism - through the use of the transnational social space as a main concept - is still referring mainly to spatial notions, which suggest geographic 'container spaces'. However, empirical studies have not been able to prove their real existence, thus the concept remains metaphoric. One answer could be Apitzsch's concept of transnational social space as rooted in personal biography (cf. Lutz 2004: 210ff.).

After reading through a large bulk of transnationalism literature, I had a bi-fold impression. I found it positive that in all these texts the issue of geographic scale was mentioned. I was astonished how clearly transnationalism acknowledges the importance of the study of the everyday life of transnational actors - not only migrants - and the specific role of the local level within transnational life experience. Yet, the negative impression I had is that there is poor information on the concrete role of the local scale within the national scale. The importance of the local scale is mentioned often, but then it is not further specified. Furthermore, there is only little information on the individual perception, feelings, emotional and socio-psychological aspects that accompany the transnational migrant. Also, the point of a possible interrelation between these cognitive aspects and the concrete action of transnational migrants has not been profoundly developed.

Hence, I think that the concept of transnationalism is already quite promising for the study of current cross-border mobility, but it remains deeply rooted in the 'nation' and the national scale as reference categories, even though criticising the methodological nationalism (in that I share the some of Bommes' critical points (2002). On the other hand I must admit that so far I cannot answer the overall question of what transnationality would be without the nation: trans ity? And what would it be if it were more consequent in the focus to the local scale: probably translocality? I will therefore use section 4.2 to switch to another current debate which provides such a consequent study of the mobile everyday life on the local scale - multilocality.

\subsection{Multilocality ${ }^{35}$}

In one of the 2009 issues of the journal Informationen zur Raumentwicklung (Information on Spatial Development, Issue 1/2.2009), published by the German Bundesamt für Bauwesen und Raumordnung (The Federal Office for Building and Regional Planning), 'multilocality' is introduced as an analytical concept which can be located between the notions of '(circular) spatial mobility' and 'migration'. Several authors from Vienna (Weichhart), Bonn (Sturm, Meyer), Dortmund (Reuschke), Chemnitz (Huchler, Weiske), Munich (Schier, Zierold), Frankfurt/Oder (Petzold), Basel (Duchêne-Lacroix, Winkler), Zurich (Rolshoven), Sitten (Stock), and Berlin

\footnotetext{
${ }^{35}$ I have also published parts of this section in the Italian journal Sociologia urbana e rurale (cf. Nadler 2011b).
} 
(Dienel) discuss 'multilocality' theoretically and then provide first empirical evidence. It should be mentioned that the assembled authors are also founding partners of the German speaking 'Netzwerk Multilokalität' (multilocality network).

While transnationalism and cosmopolitanism deal with geographically distant and culturally different reference systems, multilocality is explicitly focused on a smaller geographic scale - the local one. Here, the local scale is supposed to be the most important for the experience of the everyday life and the cognition of space (Weichhart 1990, 2009). Thus, multilocality scholars analyse the combination of different locations in an individual's everyday life. Rolshoven defines (2006: $181^{36}$, cited in Weichhart 2009: 1): 'Multilocality means Vita activa in multiple places: the entire active everyday life takes place in diverse locations which are visited for more or less long periods and which are used in more or less functionally divided patterns'.

Based on Hägerstrand's 'action space model' (1970), which defines the territory used for the daily activities as reachable by an individual from the location of their own dwelling, multilocality looks at ways of spatially organising everyday life alternatively to traditional migration and circular mobility (e.g. commuting). Migration - in its traditional understanding - means the spatial relocation of one's own home, and accordingly, of the related action space from place A to B. In contrast, commuting mobility is understood as the maintenance of the home in a stable location A and the flexible design of the daily action space around that location. Now multilocality is defined as something in-between these two socio-spatial options. The multilocal individual combines two homes in the different locations of $\mathrm{A}$ and $\mathrm{B}$, and seeks to profit from the amenities of both of these locations' daily action spaces (Weichhart 2009).

The central question is then, how do people experience this everyday alternation between two distant places? Suggestions range from a positive evaluation of 'free movement' to the negative feeling of 'being forced' into this specific way of life (Rolshoven \& Winkler 2009). On the one hand, circulating between two homes could have a deliberating effect on the multilocal person. It might give rise to multiple identities and social roles, help to raise personal profits, and be stimulating. On the other hand, some multilocals lead such a life because they 'need' to do so in economic terms. They often experience multilocality as an external constraint to their monolocal identity and orientation. Social disintegration and re-integration of the multilocal individual into local contexts are thus central topics in multilocality research (cf. Duchêne-Lacroix 2009). The relationship between an individual's feeling of belonging and the actually frequented territories also represent a field of interest, which is studied by looking at the individual capacity to produce spatial relations by residing/Wohnen (cf. Stock 2009).

\subsubsection{What does 'multilocality' mean and imply?}

For the theoretical considerations, four papers within this issue of the journal are of major importance. First, there is Weichhart (2009), who introduces the topic in a very general way and provides an overview about the mutual positioning of 'multilocality' to 'migration' and 'daily

\footnotetext{
${ }^{36}$ Translated by the author from the German text: 'Multilokalität bedeutet Vita activa an mehreren Orten: Der tätige Lebensalltag in seiner Gesamtheit verteilt sich auf verschiedene Orte, die in mehr oder weniger großen Zeiträumen aufgesucht und mit einer mehr oder weniger großen Funktionsteilung genutzt werden.'
} 
circulation'. Then there are Rolshoven \& Winkler (2009), who rather confront the multilocality concept in relation to the cultural and social science understanding. Third, Duchêne-Lacroix (2009) asks for the cognitive and emotional impacts of multilocality, especially when it comes to personal relationships between people. Finally, Stock (2009) explains his notion of Polytopizität as enriching the housing focused multilocality research.

Figure 1: Multilocality as seen by Pavel Reisenauer/Respekt Magazine. Source: Pavel Reisenauer 2010, http://www.presseurop.eu/de/content/article/433711multilokal-total-banal (accessed 31 October 2012)

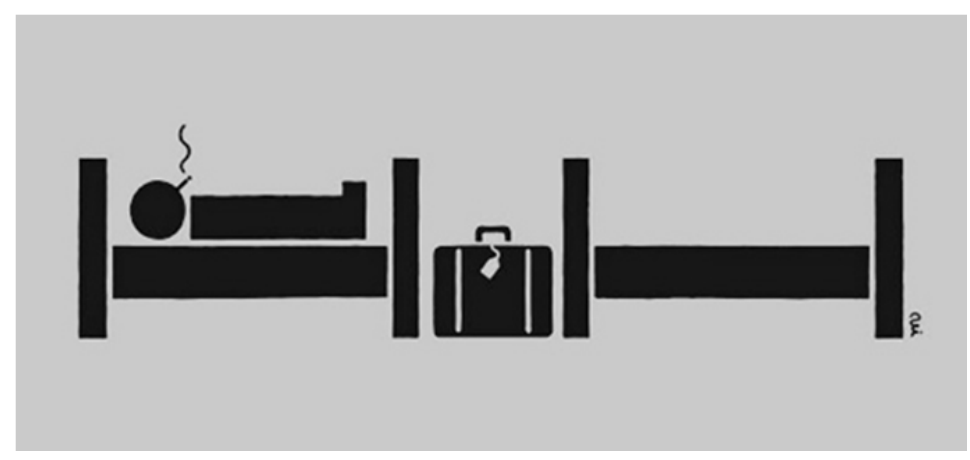

\section{Multilocality in-between migration and daily commuting}

Furthermore, every person links the needs to specific activities in the daily routines. There are places which are made for sleeping and resting, there are places which are made for working, and there are places which are made for pleasure, enjoyment, and leisure. However, these places are not necessarily located in the same geographic area, so human activity is always composed of travel between different locations in order to best satisfy the everyday needs. Here, Weichhart understands multilocal acting as a strategy to increase the chances of the satisfaction of all everyday needs of a person. Within a tolerable limit of transaction costs (such as travel, temporal and monetary efforts, etc.) people will opt for a combination of different places. Here, one could see a first difference towards traditional circular spatial mobility understandings. Everyday needs and life are not considered constraining the daily action space. Rather everyday life is conceptualized as an element which influences the combination and integration of several locations. It is more about giving a feeling of home to the act of travelling than limiting travelling within the daily geographic outreach from one single home base. Home and everyday life overcome the mono-centric locational orientation. As Weichhart (2009: $2^{37}$ ) indicates, historically, people have always been somehow multilocal: 'Thus, it would be an appropriate strategy for a person to locate in a spatial position in

\footnotetext{
${ }^{37}$ Translated by the author from the German text: 'Für einen Menschen wäre es deshalb eine angemessene Strategie, eine räumliche Position einzunehmen, an der er oder sie eine möglichst günstige Kombination an Nutzungsofferten vorfindet, welche die subjektiven Nutzungsansprüche optimal komplementieren. Solche Orte sind natürlich nicht eben häufig und einer bestimmten Person nicht jederzeit verfügbar. Eine alternative Strategie ist es deshalb, zwischen verschiedenen Orten zu changieren und deren einander ergänzende Standortofferten wechselweise in Anspruch zu nehmen. Eine solche Strategie erscheint vor allem vor dem Hintergrund spärlicher Ressourcen sinnvoll. 'To be on the move' war für Jäger- und Sammler-Gesellschaften sowie für jede Form des Nomadismus die optimale Überlebensstrategie.’
} 
which he or she finds the most preferably advantageous combination of utility offers which optimally complement the subjective utility demands. These places, of course, are not numerous and not always available for a certain person. Hence it is an alternative strategy to change between different places and to use their place-specific offers, which complement one another, in an alternating way. Such a strategy appears especially reasonable in environments characterized by scarce resources. 'To be on the move' was the optimal survival strategy for hunter-gatherer societies as well as for every kind of nomadism.'

Similar definitions are also wrapped into different terms. Steinbrink e.g. uses the term 'translocality', which he defines as (2009: 90f. ${ }^{38}$, cited in Hühn, Lerp et al. 2010: 17): 'a social and economic unit's system to secure one's own life, whereby its members are not all located in the same place ('translocal household'). It includes the coordinated use of the material and immaterial resources which are provided at the different locations, as well as the balancing of strategic agency of all members in these different locations with the aim of securing long-term existence and satisfying the (individual and collective) needs'.

Here we can see that there is a utility maximising orientation as explanatory factor for multilocal action. However, such multilocality is limited by human needs for rest and sleep. Fundamentally, as Weichhart (2009) explains, is the fact that the human being, in between two days' time of activity, needs a place to stay and rest/sleep - which is the home. This home has the character that it is often related to property or long-term usage rights (owning/renting a house/an apartment), and therefore could be considered a counterpart to mobility and movement ${ }^{39}$. Rolshoven \& Winkler (2009: $101^{40}$ ) equally state that in cultural studies and sociology, 'dwelling' and 'mobility' are not consequently combined as they are thought to be at opposite ends: 'In western societies the home is the symbol as well as the expression of being settled. However, when you look at it closer, it does not stand for the actually realised practice of staying but rather for the wish to stay: for the wish to locate oneself and to 'belong.'

Therefore, the logic of maximising the utility of a location (of the home/residence) for an individual's activities follows an 'everyday life oriented logic' (alltagsweltliche Logik), which is guided by an individual's/household's subjective perception of the opportunity to maximise utility. Weichhart's (2009) principal argument is: an individual's/a household's location predefines his/her/its daily action space ('reachable from home in daily rhythm circulation', Weichhart 2009: $5^{41}$ ). Thus, it affects the utility of this location for the individual's socio-economic development (Lebens- und Verwirklichungschancen). This indicates as well that there might be links between the

\footnotetext{
38 Translated by the author from the German text: 'Ein System der Lebensabsicherung einer sozialen und ökonomischen Einheit, deren Mitglieder sich nicht alle an demselben Ort aufhalten (,translokaler Haushalt'). Es umfasst die koordinierte Nutzung der an den verschiedenen Orten zur Verfügung stehenden materiellen und immateriellen Ressourcen sowie die Abstimmung des strategischen Handelns der an den verschiedenen Orten lebenden Mitglieder mit dem Ziel der (individuellen und kollektiven) Bedürfnisbefriedigung und der langfristigen Existenzsicherung.'

${ }^{39}$ In the German language, real estate is called 'Immobilie'. Here, we could see the semantic opposition of home/house/dwelling and mobility.

${ }^{40}$ Translated by the author from the German text: 'In den westlichen Gesellschaften ist das Haus sowohl Symbol (Inbegriff) als auch Emblem (Ausdruck) der Sesshaftigkeit. Genau gesehen steht es jedoch nicht für die realisierte eigentliche Praxis der Sesshaftigkeit, sondern vielmehr für den Bleibewunsch: für den Wunsch, sich zu verorten und ,dazu zu gehören'.'

${ }^{41}$ Translated by the author from the German text: 'von der Wohnung aus durch tagesrythmische Zirkulation erreichbar', reachable from home in daily rhythm circulation'
} 
(multi-)locational decision and social mobility. However - and that is the news about multilocality - this everyday life does not only start from one place in the morning and returns to the same place at night. Rather it 'takes place at different places'.

According to Weichhart, the industrial age represented the moment when people could achieve the highest utility at a single location. The design of the modern city, after a large wave of urbanisation, with its public transport systems, the rising availability of individual car use, the industrial production facilities, the modern housing blocks, and the decentralized concepts of service provision led to maximised utility in relatively small action spaces. However, since global economic integration, governmental supranationalisation, the affordability of long-distance travel (e.g. low-cost airlines, high-speed trains) and the development of ICT developed dynamically in the second half of the $20^{\text {th }}$ century, the action spaces of people became more extended geographically. Nonetheless, this extension is different from the medieval, pre-industrial forms of 'nomadism'. The post-industrial characteristic of action spaces' extensions is that the geographic range became much larger than it was before industrialisation. Due to transport and communication technology today, very geographically distant places can be combined in the everyday Lebenswelt (lifeworld) in order to exploit the utility potential of both places. Rolshoven \& Winkler (2009) call this the emergence of 'mobile living practices', which can be evidenced statistically by increasing residential relocation, labour mobility, leisure mobility and finally a rising amount of secondary household maintenance in nearly all western countries. This form of combining two distant places for everyday life, of which the geographic distance could not be surmounted in a daily commuting rhythm, leads to multilocality.

Figure 2: Terminological conventions of commuting and migration research; Source: Weichhart 2009: 6; translated by the author in accordance with Peter Weichhart

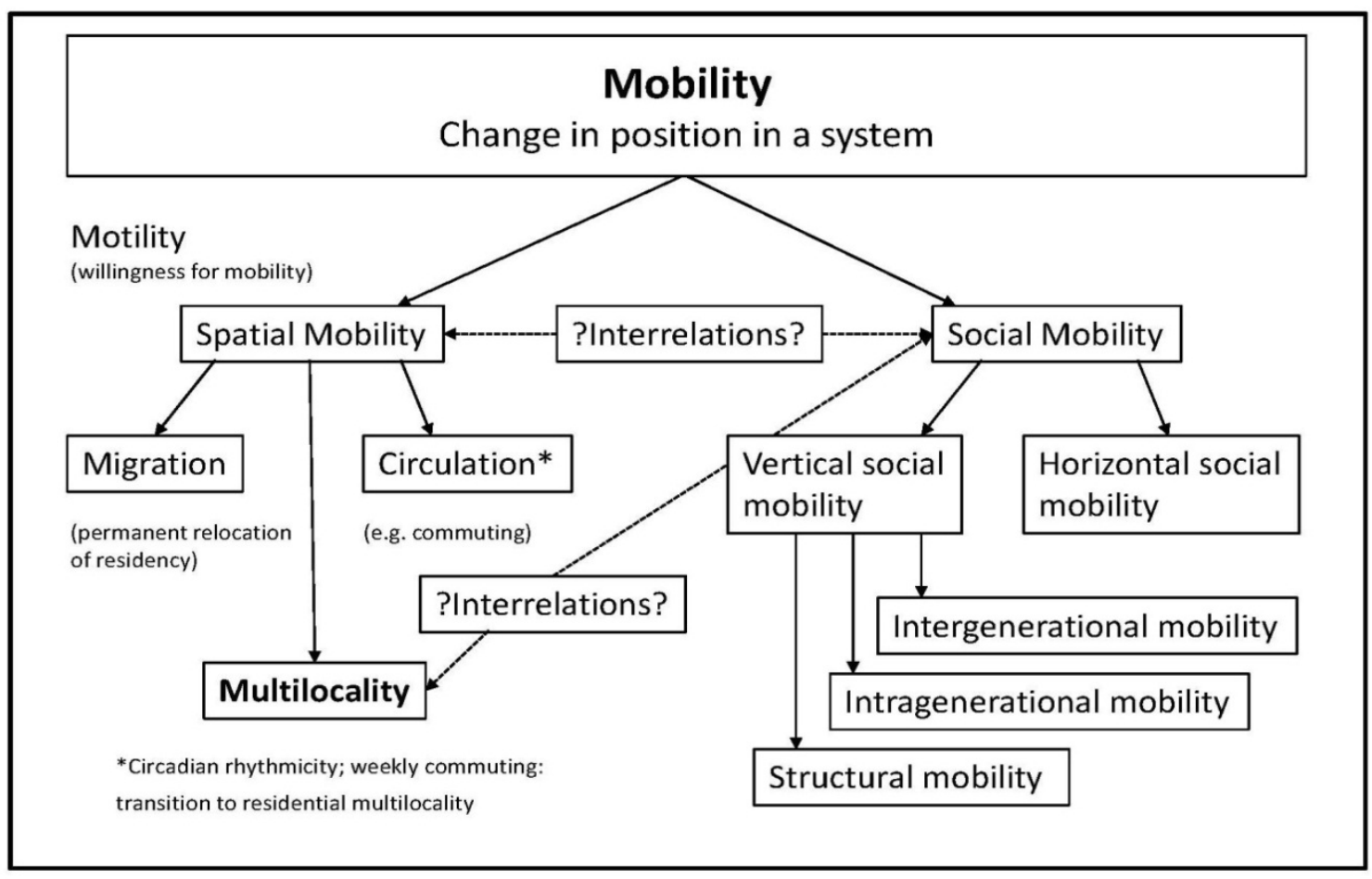


Figure 2 illustrates how Weichhart conceptually situates 'multilocality' as part of spatial mobility between the circulation type of mobility and the notion of migration. Weichhart (2009: $6^{42}$ ) says: 'Spatial mobility is used to describe the various movements between the locations of human activity. Thereby, today it is mostly distinguished between migration and circulation. Migration means a permanent relocation of the residence; circulation all the daily rhythm, out-of-home activities which start from the home and lead back home at the end of the day (work, shopping, leisure activities etc.). Daily commuting would be an example of such circulation. However, a commuter who only comes back home at the weekend because they are forced to maintain a second home at their work's location already represents a case of multilocality.'

In figure 2, it becomes obvious that Weichhart (2009) understands multilocality as positioned between the other two notions of spatial mobility - which are migration and circulation. Circulation here is mostly linked to what is explained as spatial mobility in relation to transport geography (cf. Gather, Kagermeier et al. 2008), in which a daily travel routine takes place (e.g. commuting).

Weichhart also stresses the differentiation between forms of spatial mobility according to the main criteria of 'time' (e.g. everyday movement vs. unique move with permanent intention) and 'residency' (daily action space from fixed unique home vs. one-time relocation of the unique home). Again, multilocality is situated 'in between', because it describes a spatial strategy of individuals and households, in which the home is no longer composed of one single location. Multilocality means living in several homes and cognitively living one's everyday life at geographically distant places at the same time.

Second, the concept of multilocality does not only challenge the 'single home' paradigm of the traditional spatial mobility approaches, it also questions the temporal dialectic between daily circulation and the long-term or even permanent move between locations. Multilocal individuals do not necessarily have only these two temporal dimensions in mind when they move around between their different homes. Their stay could take only a single day, or several months, or even years. The important criterion in distinction to a leisure or business tourist in the definition of a multilocal person is that the multilocal one is characterised by the 'everyday life' taking place at different locations. So, 'everyday’ infrastructure and services within the multilocal life are fundamental.

Weichhart (2009) notes that multilocality is considered by other scholars to be a particular sub-type of migration, somehow a status between migration and settlement, yet this understanding does not really reflect, as he says, the 'lifeworld-existential' (lebensweltlich-existenziellen) and 'cognitiveemotional' (kognitiv-emotionalen) sensual configurations of multilocality. Therefore, he prefers to understand 'multilocality' as being an independent social practice equal (gleichberechtigt) migration and circulation.

He then links multilocal living to the action space concepts (cf. Hägerstrand 1970). Opposed to migrants or commuters, the multilocal individual achieves the combination of two or more complete

\footnotetext{
42 Translated by the author from the German text: 'Räumliche Mobilität wird auf verschiedene Bewegungsvorgänge zwischen Standorten menschlicher Handlungen bezogen. Dabei unterscheidet man heute meist zwischen Migration und Zirkulation. Migration bezeichnet eine permanente Wohnsitzverlagerung, Zirkulation alle tagesrythmisch ablaufenden außerhäuslichen Aktivitäten, die von der Wohnung ausgehen und wieder zu ihr zurückführen (Arbeiten, Einkaufen, Freizeithandlungen etc.). Tagespendeln wäre also ein Beispiel für Zirkulation. Handelt es sich jedoch um einen Wochenendpendler, der am Arbeitsstandort mit Notwendigkeit eine eigene Unterkunft benötigt, dann liegt bereits ein Fall von Multilokalität vor.'
} 
action spaces. They can live an everyday life at two or more different places by operating from two or more homes. The commuter however can only partly live an everyday life in the different locations (in the one location where they work, in the other where they have their leisure and private life). Migrants, in contrast, only live in one place, but use their knowledge from former places in their everyday life at the new location. Weichhart (2009: $8^{43}$ ) stresses the importance of two homes as a basis for the possibility of everyday life in multiple localities: '[Multilocality], however, is based on the availability of a second home in addition to the original or primary one, which can be used as an anchor point for everyday life at the second location.' Therefore, there are different locations which all serve as connectively rooting everyday life.

\section{Multilocality from a phenomenological perspective: multilocal lifeworlds}

Rolshoven \& Winkler (2009: 101f. ${ }^{44}$ ), however, do not speak of the connecting but rather the 'disconnecting' function of different locations. They prefer to stress the mobile character of multilocality instead of the rooting aspects. According to them, the multilocal practice of 'dislocating' equals a disconnecting of everyday life spheres: 'From the perspective of the moving person, mobility is a practice of dislocating, which disconnects different everyday life spheres from each other. The life takes place at different places - at home, while travelling, at the work or study place, at other places, where meetings take place, where everyday obligations are fulfilled or leisure time is spent - but still it remains one and the same life.'

It still seems unclear to what degree multilocal living could rather be characterised as the 'connecting' or 'disconnecting' of places and activities with or from each other. It is ate least combining different locations within the everyday life sphere, and most probably it depends on the perspective one would take.

Rolshoven \& Winkler (2009) approach multilocality from a cultural science-phenomenological perspective as a relational, actor-centred process, and thus the result of strategies and practices of everyday life and individual auto-positioning (Selbstverortung) (2009: 99 ${ }^{45}$ ): 'We could thus understand multilocality as a way of life according to Raymond Williams and his concept of culture as 'the whole way of life'.'

Dwelling is also a central term in Rolshoven \& Winkler's conceptualization of multilocality. They criticize social science for applying a too static understanding of dwelling. For them, dwelling is rather a lifeworld (lebensweltliche) category, which leads to thinking of it as an activity. This activity is fundamental in order to understand multilocal ways of life. Early indicators of specialised infrastructures for multilocals might be seen in the development of 'temporary housing' services offered at more and more places, offering attractive and flexible homes for medium-term stayers with furnished apartments and domestic services.

\footnotetext{
${ }^{43}$ Translated by the author from the German text: '[Multilokalität] wiederum setzt voraus, dass neben der ursprünglich bestehenden Wohnung eine zweite Behausung verfügbar ist, die als Ankerpunkt des Alltagslebens an einem zweiten Ort genutzt werden kann.'

${ }^{44}$ Translated by the author from the German text: 'Aus der Perspektive des sich Fortbewegenden ist Mobilität jedoch eine Praxis der Delokalisierung, die unterschiedliche Alltagssphären voneinander entkoppelt. Das Leben spielt sich an mehreren Orten ab - daheim, im Unterwegssein, am Arbeits- oder Ausbildungsort, an anderen Orten, an denen Begegnungen stattfinden, Alltagsbesorgungen unternommen oder Freizeiten verbracht werden - und es bleibt doch ein und dasselbe Leben.'

${ }^{45}$ Translated by the author from the German text: 'Wir dürfen daher Multilokalität als Lebensweise im Sinne des von Raymond Williams formulierten Kulturbegriffs als the 'whole way of life' verstehen.'
} 
However, for Rolshoven \& Winkler (2009), multilocality always has to be thought of as making possible as well as forcing people into mobility. According to the 'network capital' notion of Larsen, Urry et al. (2006), they say (2009: 103f. ${ }^{46}$ ): 'Having to move could be an escape in all its various forms, just as having to stay could be called forced locating. [It is about] mobile surrogateplaces, a kind of flying carpet for translocal people on which one often takes a seat voluntarily, but more and more often also involuntarily. [...] A growing flexibility of life spheres has resulted due to the structure of the modern lifeworld, which forces as well as makes possible both the distribution of actual living to different places.'

Such a multilocal life, with its communicative and cognitive links between different locations, could have a significant impact on those living it. One the one hand, it might foster the individual action potential and the stabilisation of identity. On the other hand, it might have negative effects on the social networks of the person; it might cause psychological tensions and a perceived cut from a person's localised roots.

\section{Multilocality and personal ties}

Duchêne-Lacroix (2009) adds to Rolshoven \& Winkler's observations on emotional consequences and personal relations. He derives his understanding of multilocality from the cognitive aspects and imagines current societies as mobile societies in which a person's individual places are becoming more and more fragmented and multiplied. He calls this process the Archipelisierung (archipelagoisation) of life. Caused by globalisation, European integration, economic liberalisation and transnational networking, life is taking place more and more on different 'islands', as well as across national borders. Thus, social life and society no longer coincides with national political territories. This conceptualisation of border traversing shows similarities to the basic ideas of transnationalism (Pries 2008), the post-national 'sociologies' of Urry (2000) or the refusal of 'methodological nationalism' (Beck 2007). Duchêne-Lacroix describes the constituting character of these islands as follows (2009: $88^{47}$ ): 'The belonging of the islands to the great whole consists certainly of observable habits (e.g. the frequency and duration of sojourn at, or the travel to and between certain places, or the number of visits) or of facts (such as property, locations of work and residency, etc.), but also of subjective elements (the feeling of belonging, intensity of the commitment, etc.).'

He questions if the particular empirical perspective for multilocality research has to focus on 'subjective' indicators, such as affective belonging, or rather on objective indicators, such as durations of sojourn. According to him, the subjective aspects are important to catch the cognitive and emotional dimensions of multilocal living. He conceptualises multilocality as a 'temporal parallelism of presence and absence' at the involved places. The physical presence on one of the

\footnotetext{
${ }^{46}$ Translated by the author from the German text: 'Sichbewegenmüssen kann Flucht in ihren vielfältigen Formen bedeuten, ebenso wie Sesshaftgemachtwerden Zwangsansiedlung heißen kann. [Es geht um] bewegliche Surrogat-Orte, um eine Art fliegende Teppiche für Translokale, auf die man sich häufig freiwillig, aber immer öfter unfreiwillig setzt. [...] Aus den Strukturen der modernen Lebenswelt resultiert eine wachsende Flexibilisierung der Lebensbereiche, die die Verteilung des Lebensvollzugs auf mehrere Orte zugleich erzwingt und ermöglicht.'

${ }^{47}$ Translated by the author from the German text: 'Die Zugehörigkeit der Inseln zum Ganzen entspricht gewiss beobachtbaren Gewohnheiten (z.B. Häufigkeit und Dauer der Aufenthalte oder Reisen in und zwischen den Orten oder die Anzahl der Besuche) oder Fakten (Eigentum, Lokalisierung der Arbeitsorte, der Wohnorte etc.), aber auch subjektive Elementen (Zugehörigkeitsgefühl, Intensität des Engagements etc.).'
} 
'islands' always includes the risk of disintegration at all other places (or islands). Thus, he constructed four dimensions of integration which become effective at the place of presence and which compensate for the perception of disintegration at the other places.

First, there is a 'functional' dimension, which is mostly characterised by normatively ascribed roles of family members. Each individual has to fulfil functional expectations at the place of presence (e.g. child care, household maintenance, construction of family everyday life, etc.). Second, the 'social' dimension of integration is linked to expectations of loyalty towards a social group at the place of presence. This means that a person - especially in their professional life - is evaluated by other social group members such as colleagues according to their presence and commitment to the shared group/organization/place. Multilocal persons react to this localized social pressure by developing individual adaptation strategies (e.g. strategic presence mobility - strategische Anwesenheitsmobilität) in order to keep in touch with social contacts and to have access to local informal knowledge. This leads to the third 'cultural-cognitive' dimension, which according to Duchêne-Lacroix (2009) is characterized by the continuous updating of specific information about the life in one place. This dimension is about the informational asymmetry and differing socioprofessional relevance of the multiple places within a multilocal life. Each locus disposes of a specific set of informational value for the multilocal person. Information at one place might be professionally irrelevant, but it is very relevant for personal reasons. Other places, however, are more relevant on a global scale and thus information is consumed rather for professional reasons. Some places are connected to ICT-supported, continuous informational flows; other place's information is only updated sequentially and through more traditional channels (e.g. newspapers or phone calls). The crucial point is, in order to be included in the different places, one has to know what is going on there. Fourth, there is an 'identitary' dimension to integration. Identity construction, for multilocal people as well, is based on long-term habits, appropriation and perpetuation of international and local roots. This is what Duchêne-Lacroix (2009: 95 ${ }^{48}$ ) calls the 'stable point in a modern nomadic career'. Duchêne-Lacroix (2009) concludes that these four dimensions are more important for the organisation of everyday multilocal life than geographic distance. He says (2009: 95f. ${ }^{49}$ ): 'In spite of increasing multilocality, traditional expectations about presence remain at the internationally distributed places. Caused by their sporadic absence, multilocal people are challenged to align a locationally dispersed life with the normality and implicitness of life at each of the involved places. This often does not work out. [...] Only if the presence achieves a certain intensity [not duration of stay], it becomes possible for multilocal people to gain a foothold in a milieu, to appropriate a place, to tighten a position, etc.' Thus, multilocal living is also linked to the act of continuously switching between different and localised roles in order to cope with the expectations of the locally rooted others.

\footnotetext{
${ }^{48}$ Translated by the author from the German text: 'stabilen Punkt in einer modernen nomadischen Karriere'

${ }^{49}$ Translated by the author from the German text: 'Trotz zunehmender Multilokalität bleiben traditionelle Anwesenheitserwartungen an den international verstreuten Orten erhalten. Aufgrund ihrer sporadischen Abwesenheit sind Multilokale gefordert, ein örtlich verstreutes Leben mit der Normalität und Selbstverständlichkeit des Lebens an jedem der beteiligten Orte abzugleichen. Dies gelingt oft genug nicht. [...] Erst wenn ihre Präsenz eine gewisse Intensität [nicht Aufenthaltsdauer] erreicht, ist es für Multilokale möglich, in einem Milieu Fuß zu fassen, sich einen Ort anzueignen, eine Position zu festigen etc.'
} 


\section{Multilocality as Polytopizität}

Stock (2009) develops an even more refined approach to multilocality. For him the traditional, conceptual opposition of dwelling/house/residence (as a place of being rooted and feeling familiar) and the mobility/mobile life is not providing a sufficient understanding of multilocality in all its commitment. According to him, current sociological thinking - though it might refer to postmodern, transnational, cosmopolitan perspectives ('liquid modernity', 'spaces of flows', 'mobile cultures') - still applies this traditional and dialectic opposition. Therefore, scholars today overestimate the 'uprooting' or 'disembedding' effect of the post-modern necessity to be mobile. He, as well as Rolshoven \& Winkler, distinguishes between multilocality research which is focused on the more narrow 'dwelling' aspect (maintenance of multiple residences) and multilocality as a broader concept, which also includes everyday life practices and cognition.

His term Polytopizität refers to a new conceptualisation of dwelling (Wohnen), which could be termed as 'making geographies'. According to Heidegger's idea of 'being on the earth', Stock includes every spatial aspect of everyday life into the term dwelling/Wohnen. In other words it is about the 'capacity to produce relations to space' (2009: 108) ${ }^{50}$. As Stock believes, Heidegger was still much of a monotopical scholar. In Heidegger's analysis about rural places with small action spaces, multilocality did not exist. Consequently, Stock, (2009) also bases his approach on action theory and the analysis of the lifeworld according to Werlen (1997). According to Werlen's approach, the social construction of space is not only limited to the question of how space is produced in the Lefebvrian sense, but it is also asked for the social practices of individuals to relate themselves and their everyday lives to different places. Wohnen (living/housing) is then to be understood as a social practice not 'in space' but 'with space'.

Stock (2009) develops three analytic dimensions for studying Polytopizität. First, wohnen is always confronted with solving spatial problems - this problem solving process might be called Wohnpraktiken (dwelling practices). Second, 'spatial capital' (cf. also Lévy 2003) is always involved, which is the capacity to surmount geographic as well as cultural and cognitive distance. Here, Stock (2009) uses the notion of Wohnregime (dwelling regime) for the description of this analytic focus. Third, wohnen is also related to giving sense and symbolic value by practicing a certain life style locally. This is what Stock calls 'dwelling style' (Wohnstil). He summarizes that, due to the ongoing functional differentiation of societies, the linkage between 'practice' and 'place' becomes more and more multiple according to the specific qualities of places for certain activities.

Stock (2009) distinguishes then monotopical (monotopische) from polytopical (polytopische) Wohnstilen (dwelling styles). Whereas familiar places are geographically close to the location of the dwelling for the monotopical style, the polytopical style is more complex, with familiar places (Eigenorte) within geographic distance, while at the same time unknown, or foreign places (Fremdorte) as also in proximity. Polytopizität, thus, is characterized by a differentiation of geographic and identitary/symbolic proximity, or as Stock (2009: $114^{51}$ ) puts it: 'From a spatial perspective, mobile individuals should be considered 'geographically plural individuals'.'

\footnotetext{
${ }^{50}$ Translated by the author from the German text: 'Kompetenz der Herstellung räumlicher Bezüge'

51 Translated by the author from the German text: 'Mobile Individuen sind aus räumlicher Sicht als 'geographisch plurale Individuen' zu bezeichnen.’
} 
Stock's 'geographically plural individual' - in distinction from monotopical people - disposes of particular capacities: (1) the capacity to construct place-based identities in multilocal ways; (2) the capacity to transform unknown places into familiar situations by appropriation techniques; (3) the capacity to manage absence/presence; (4) the capacity to touch down in place or to settle only temporarily and periodically; (5) the capacity to manage a multilocal spatio-temporal orientation; (6) the capacity to interpret one place differently according to situational demands; (7) the capacity to relieve from local situations and to become independent from monolocal reliance.

This conceptualisation of polytopical lifestyles and geographically plural individuals enriches the residentially focused multilocality approaches by not limiting definitions to residential questions. Wohnen - in the sense of a registered residence - might geographically and administratively take place at only one location, but it still can happen 'multilocally' from practical as well as cognitive, emotional, symbolic and cultural perspectives of the lifeworld.

By referring to these four theoretical papers, I have demonstrated that multilocality is a concept which is primarily defined according to the objective criteria of (1) maintaining different residences (or dwellings) and switching between them with the possibility to (2) perform and organize the individual everyday life in each of these locations. However, multilocality is more than this objective definition. In addition to its central elements, 'multiple residency' and 'everyday life', it could also be considered a certain (3) lifestyle and thus a momentum of cultural distinction. Furthermore, (4) there are very subjective and cognitive aspects to multilocality, including integrative dimensions for compensating the physical absence/presence duality as caused by multilocality. Finally, (5) multilocal living can be understood from a broadened approach to dwelling/housing/residing (Wohnen), in which multilocality is not necessarily defined by multiple residency but rather by multilocal relationships between space and everyday practice.

\subsubsection{Connection to other theoretical frameworks}

However, the concept of multilocality is not detached from other sociological frameworks. Weichhart (2009) briefly indicates how the concept of multilocality might be connected with broader theoretical frameworks. The overall research interest on multilocality has to be seen as oriented on action theory (Weichhart 2009: $12^{52}$ ): 'Multilocation is a process which - without any doubt - is based on the decision-making competencies of the individuals, and which is related to their capacity to become effective as a 'source of contingency' and to induce consequences of action in the social and physical world. [...] Social-geographic approaches of action theory seem to be especially appropriate from the perspective of the locational relatedness of every action.'

Then, Weichhart (2009) also mentions the 'time geography' based on Hägerstrand (1970) as a possible theoretical reference for multilocality research. Especially the notion of 'constraints' as developed by Hägerstrand might be helpful in order to understand the limiting effects of a dwelling's location on the multilocal individual's 'action space' and location benefits as well as the

\footnotetext{
52 Translated by the author from the German text: 'Multilokation ist ein Prozess, der zweifellos in die Entscheidungskompetenz der betroffenen Akteure fällt und auf deren Fähigkeit beruht, als ,Quelle von Kontingenz' wirksam zu werden und Handlungsfolgen in der sozialen und materiellen Welt zu produzieren. [...] Vor dem Hintergrund der Ortsbezogenheit des Handelns bieten sich besonders sozialgeographische Varianten der Handlungstheorie an.'
} 
multilocal strategies to surmount these limitations (Weichhart speaks of Reichweitensysteme range systems; 2009: 12).

There have also been some recent innovations in the field of migration research which might be applicable to multilocality. The current debates about transnational migration, circular migration, social networking, and finally the new migration economy with its notion of 'subjective place utility' could be used here.

Last but not least, Weichhart mentions the vast theoretical work which has been done to explain 'social change'. Here, one might refer to the concept of the 'liquid modernity' according to Bauman (2000), as well as scholars of individualisation Beck (1992) and flexibilisation Sennett (2008) might be of interest. Furthermore, there is the cultural capital theory according to Bourdieu, which could assist a better understanding of the symbolic value of multilocal lifestyles and the specific residential locations. Furthermore, Bourdieu's notion of 'capital' might be useful. Here, Weichhart points to Dirksmeier (2007), who has researched the 'residential capital' of certain housing locations.

\subsubsection{Empirical evidence in multilocality research}

This section will provide an overview about what kind of empirical research has been done so far in the German speaking countries, especially by the Netzwerk Multilokalität. Apart from understanding multilocality as a new perspective on empirical phenomena, the central question is what kind of evidence exists for empirically observable and new multilocal phenomena?

Sturm \& Meyer (2009) both work for the Bundesamt für Bauwesen und Raumordnung (Federal Office for Building and Regional Planning). This governmental institution maintains the largest database on housing and residential location of the people living in the German territory. The data is continuously updated from the residential registry and the micro census. Furthermore, there are certain large German city regions which take part in the registry of residents on the level of city quarters. According to Sturm and Meyer, this is the best dataset - among the few existing ones which could be used to estimate quantities and characteristics of the multilocal people in Germany.

Technically, the calculation is a simple difference between registered people with a 'main residence' (Hauptwohnsitz) and the fewer with a registration based on a 'secondary residence' (Nebenwohnsitz). However, this is only an approximation for the real number of multilocal arrangements as there are several measurement errors.

From analysing the registry data, Sturm \& Meyer (2009) found that there are certain social groups in Germany who are over-represented in the secondary residence data: minors with divorced parents in different locations, students and professional trainees, professionals in certain economic sectors (such as media, politics, etc.) and elderly people with secondary residences in an attractive countryside. These groups mostly locate in inner-city quarters. The locations of the secondary residences tend to trickle into the outskirts of the large cities.only in cities which have already introduced the secondary residence tax.

Furthermore, the municipalities are confronted with different problems when planning their development. Multilocal persons are difficult to register, and it is still unclear to what degree they could be involved into the local community life. Sturm \& Meyer (2009) suppose that the locally 
rooted opt much more for participation in civic engagement than multilocal people do. In times in which municipalities apply governance models which rely on civic participation, this might become a future problem. Rolshoven \& Winkler (2009), however, critically remark that such statistical data analysis could never cope with the complexity of multilocal everyday life arrangements, as they are only a brief static image of the real world. Furthermore, they think that in order to apply statistical methods, a technical definition of multilocality is needed. Such a universal definition, they say, does not exist so far. They refuse to apply quantitative approaches to multilocality research and suggest (2009: $104^{53}$ ): 'An ethnographic exploratory approach is probably generally necessary for this topic.'

Dienel (2009) presents a case study on multilocal arrangements between central urban cores and peripheral rural regions, using the example of Berlin and the Baltic Sea island of Usedom. He develops his notion of Raumpartnerschaft (spatial partnership) which might assist a planner's way of dealing with temporary inhabitants in declining rural areas. He describes the spatial partnership as consisting of two opponent places which have a contrasting character. The urban cores (e.g. Berlin) are mostly the places with stable routines; the peripheral rural secondary homes are places for testing new behaviour (e.g. Isle of Usedom). Thus, multilocal living and secondary residencies might be a chance for declining rural areas for social renovation and regeneration. Spatial partnerships might thus have the complementary character of integrating central growing cities and distant rural peripheries. The involved planning authorities should actively assist such a spatial partnerships by identifying partner cities/regions and then providing appropriate infrastructural and service offers for the multilocal people Dienel (2004).

Schier (2009) looks at the family level. She considers family an everyday construction process - a 'family making' - and asks how this construction process is challenged by multilocal lifestyles. Due to the professional necessity for mobility and the increasing rates of divorces, families today live more and more as social networks in multiple households which are often also located in different places.

Based on data from a study on family organisation, she explains that there are two basic types of multilocal families. First, there are those which are characterised by mobile professionals who have to travel a lot. Here, the 'being together' or 'far away' could not be planned and is very irregular (e.g. employees in the film industry). Second, there are families who are characterized by people who are working during the week in another city and come back home at the weekend. This type is more regular and routinised, and family members could better anticipate the periods of physical copresence. Both of these forms are to be understood as professionally induced multilocality. Schier (2009) then says there is also family induced multilocality (e.g. by divorce, by generational division of the household, etc.).

Schier (2009) concludes from her observations that families today are increasingly stressed by multilocal arrangements when it comes to living life stages together, taking care of each other, or keeping physically and emotionally in touch. Today, the difficulty is not only to find time for family, but also to find a common place to meet and to be with each other. The everyday

\footnotetext{
${ }^{53}$ Translated by the author from the German text: 'Möglicherweise braucht es für dieses Thema grundsätzlich einen ethnografischen exploratorischen Einstieg.'
} 
organisation and constructing of a family life is becoming more and more difficult. Distant family life is characterised by an active process of 'family making'. Schier cites different innovative types of 'family making' (e.g. mobile families travelling together, planned forms of visiting each other, 'virtual family'), which lead to the question of flexible and innovative designs of support infrastructure (child care, flexible schooling, municipal media points). She also points out that multilocal living is characterised by spontaneity. Family life planning is becoming more and more corrupted by unpredictable timetables for work, and thus for presence/absence.

Huchler, Dietrich et al. (2009) looked at the case of flight personnel. According to them, flight attendants are the prototype of a multilocal person whose job already contains the dissolution of temporal and spatial barriers (Entgrenzung) up to crossing time zones. They asked how these aviation employees manage their everyday life between flying around the world and being home and caring for the family. They found that there are specific infrastructures provided by the airline companies in order to support the mobile employees. In addition, the flight personnel also rely on support from local, private acquaintances and family members.

They point to the fact that multilocal living has significant disadvantages for the individual sphere. The maintenance of personal relations to loved others becomes difficult, and the professional necessities collide with demands from the family. Thus, multilocal living needs support systems which compensate the disadvantages. These systems already exist in the aviation business, but they have to be extended to other professional groups which have only been confronted with multilocal living recently. Nonetheless, Huchler, Dietrich et al. (2009) are rather pessimistic about the extension of appropriate compensation and support systems. According to them, the growth of lowcost carrier segments is more a sign of a decrease of support for multilocal flight attendants and pilots than a representation of a stable example for how to organise support for multilocal employees.

In her study, Reuschke (2009) combined mobile lifestyles and housing demand. She found that the flexibilisation of work environments and the pluralisation of job holding lead to an ever increasing importance of job-related commuting over a long distance. Reuschke questions the common understanding of a strict distinction between a person's 'primary private home' and the 'secondary professional residence'. She found that multilocal arrangements rather follow an alternating pattern. People do equally use different locations, not necessarily preferring one over the other. Therefore, demand for the quality of the secondary home does not much differ from those of the primary home. The location choices are similarly complex for each of these several localities.

However, Reuschke (2009) states that there is also a gender gap. Men apply the traditional type of multilocal arrangements more with a suburban or even rural primary residence and a secondary home in large urban areas. In contrast, women have 'modernised' multilocal behaviour by rather combining one urban pole with another urban pole. She concludes (2009: $41^{54}$ ): 'For lifestyle and mobility research based on the settlement-specific patterns of multilocal types of living, more detailed questions have resulted which ask about the everyday practice and mobility of

\footnotetext{
${ }^{54}$ Translated by the author from the German text: 'Aus den siedlungstrukturellen Mustern multilokaler Lebensformen ergeben sich damit für die Lebensstil- und Mobilitätsforschung vertiefende Fragestellungen nach der Alltagspraxis und Alltagsmobilität von ,komplementären rurbanen Lebensstilen ' einerseits und multipolar-urbanen Lebensstilen andererseits.'
} 
'complementary rurban lifestyles' on the one hand and multi-polar urban lifestyles on the other hand.'

Equally, Rolshoven \& Winkler (2009) point to a certain connection between multilocality and gender issues. They expect the extension of multilocal lifestyles to provide emancipatory potential for women who - by switching between and combining different places - might overcome monolocal cultural restrictions on their individual development.

Weiske, Petzold et al. (2009) try to develop a certain typology of multilocal actors. They construct their typology based on a set of semi-structured interviews along two dimensions in a matrix. First, there is the physical or spatial movement (in how many places do people actually live?); second, there is a rather cognitive aspects of social mobility (in how many or at which places do people actually construct the 'sense' of their (multilocal) lives?). They found that there are types which could be mainly differentiated by their degree of 'mental openness' towards multilocal arrangements. As Rolshoven \& Winkler (2009) also mention, multilocality could be perceived as 'freedom' or as a 'constraint'. There is always an individual perception of one's own multilocality. The types described by Weiske, Petzold et al. (2009) underline this idea empirically. The fact that they found their types of multilocal households mostly being only temporarily multilocal is particularly interesting. Especially the biographic moment - at what moment in life multilocality is lived - seems to be decisive for the mental openness towards and individual understanding of multilocality.

Hilti (2009) refers mostly to the residential aspects of multilocality. Her understanding of multilocality is slightly different from the one offered by Weiske, Petzold et al. (2009), in that she does not so much focus on the perception but rather on the differentiation between 'multilocality' and 'multilocal housing'. She defines (2009: $78^{55}$ ): 'Multilocal housing is a specific type of the multiple locating of people. [Multilocal housing] implies the existence and the use of more than one residence, or more generally: of more than one dwelling. [...] In contrast, multilocality as a wider term refers to the broader understanding of an everyday life multilocality, which has some overlapping with concepts such as daily-rhythm circulation, but which is also related to a more philosophical understanding, rejecting formal constraints. Multilocality, then, is [...] the active everyday life distributed to different places.'

She uses this distinction to better be able to reconstruct the everyday lifeworld of her case studies between Liechtenstein, Switzerland and Austria. According to her, the trespassing of administrative boundaries stresses people due to resulting bureaucratic issues. European administrative systems have not yet adapted to multilocal living. Thus, she says, multilocal housing in its official understanding (as two or more residences) no longer coincides with multilocality as a whole (with all the existential problems in peoples' lifeworlds). This disintegration of lifeworld and bureaucratic definitions causes trouble, because in having to deal with two or more bureaucratic systems, multilocal individuals face several definitional inconsistencies. Practically, people who live

\footnotetext{
${ }^{55}$ Translated by the author from the German text: 'Multilokales Wohnen ist eine spezifische Form der mehrfachen Verortung von Menschen. [Multilokales Wohnen] impliziert das Vorhandensein und die Nutzung von mehr als einem Wohnsitz, oder allgemeiner: mehr als einer Behausung. [...] Demgegenüber umfasst der Überbegriff Multilokalität ein weiteres Verständnis einer (alltäglichen) Mehrörtigkeit, welche zum einen Berührungspunkte mit Konzepten wie der tagesrythmischen Zirkulation aufweist und zum anderen einer eher philosophischen Auffassung folgt, die auf formale Einschränkungen weitgehend verzichtet. Multilokalität ist dann [...] der auf mehrere Orte verteilte tätige Lebensalltag.'
} 
multilocally also use multilocal housing, but they cannot sufficiently be registered as multilocal residents as registration legislation is too old-fashioned to handle this growing phenomenon.

\subsubsection{Open questions in multilocality research}

Weichhart (2009) points out that there are still a lot of open questions in the field of multilocality research. First, no one has ever quantified multilocality, which is partly caused by unfinished conceptualisation as well as weak data availability. However, it might be interesting to know how strong the phenomenon has become, and how multilocal groups are characterised concerning other socio-economic indicators. Second, he suggests that the multilocal actors are not a homogenous group. Rather one should investigate on the different existing 'types' of multilocal individuals and their specific multilocal arrangements. Third, from a sociological perspective, multilocality could be interesting when it comes to asking how individuals switch between 'social positions', 'roles', and group participation when they change location. Does multilocality facilitate multiple social identity construction? Fourth, there is the aspect of how families or households deal with multilocal arrangements from the logistic point of view. How do they trade-off multilocal transaction costs against the benefits of multilocality?

Another focal point of further research might be on the rather cognitive aspects of multilocality. When people switch between different places, what kind of character do these places have according to the individual perception? Are places then characterised primarily according to their function? Or are there other aspects which are important for their characterisation?

Then there is the question for the impact of multilocal living onto the places themselves: how does a large group of multilocal actors in a city change its internal structure and functional systems? Here, one has to also study the impacts on the composition of local communities and the availability of multilocal people for local civic engagement. Furthermore, it might be interesting to have a look at processes of exclusion and gentrification on the level of city quarters induced by multilocal lifestyles.

Finally, the transition process between the two or more locations might be of interest. Multilocal people travel a lot, which means high temporal and financial costs. How do they perceive these passages of travel? What do people do in the times when they switch between places? These questions could somehow be linked to the research by Watts \& Urry (2008), who are already working with 'mobile methods' in order to study how people use 'travelling time'.

\subsubsection{Synthesis: Multilocality as an approach to study routes}

The multilocality concept as presented above offers a useful extension of perspectives on spatial mobility research. It systematically closes a definitional gap between the long-term character of migration and daily rhythm circulation. In times of a differentiation of mobility types, the multilocal patterns are gaining importance.

However, the first attempts to define multilocality and distinguish it from other terms of mobility studies do not always lead to a satisfying result. The approaches presented in this literature review still lack a clear operationalisation of certain aspects of multilocal lifeworlds. 
First, most of the scholars try to use a residentially founded approach to define multilocality (see e.g. Weichhart 2009; Sturm \& Mayer 2009). I believe that the orientation to define multilocality by the criterion of two or more households ignores the fact that in everyday practice multilocal arrangements might not always be necessarily characterised by multiple residency, but more generally by the everyday life taking place in different locations. Some of the scholars correctly imagine multilocality as a 'vita activa at many places'. Yet, in the end they limit themselves when it comes to operationalising the vast whole of such a 'vita activa' by only using the indicator of residential practices; this only reflects a small detail of a person's everyday life.

The most convincing answers to this problem are provided by Rolshoven \& Winkler (2009) or by Stock (2009): they try to integrate the complexity of everyday life into the research on mobility with their approaches. They do not fear touching on 'difficult-to-understand' topics such as cognition, emotion, sense-making, or symbolic contents in relation to multilocal living. I suggest it is very necessary to keep a definition of multilocality open to cases which are rather 'mentally multilocal' (in reference to Stock's image of a 'geographically plural individual') than actually being physically or even only residentially multilocal.

This leads to another problem of the presented approaches: they are still too much focused on physical travel in space. Clearly, a person who never surmounts geographic distance could less easy become a multilocally minded one. The ancient philosopher St. Augustine of Hippo already observed: 'The world is a book, and those who do not travel read only one page.' However, an exclusive characterisation of multilocals as constantly travelling and switching between fixed places does not support the intention to systematically analyse the various manifestations of multilocality especially not the virtual forms multilocality. Although at the moment being monolocal in the sense of physical immobility, I think a person might have had a very mobile life in the past. In present time, this person could still be very mobile mentally when e.g. keeping social networks alive, or combining different places cognitively within their everyday life.

Here one could refer to Larsen, Urry et al. (2006) and Urry (2000) who use the notions of 'imaginative' travel and virtual travel. Imaginative travel is the 'thought' or 'wished' movement to socially and/or geographically distant places, which is also an indicator of the capacity or openmindedness to switch between and integrate locations into one's everyday life. According to Urry, imaginative travel is enhanced through mass media and the development of ICT. Today people can easily become informed about distant and foreign contexts without even travelling there physically. As Urry (2000: 69) puts it, imaginative travel is that 'by which distant events, personalities and happenings are mundanely brought into the living room and transform everyday life. As a consequence we imagine ourselves sharing events, experiences and personalities with many others, with whom we constitute certain kinds of community'. Urry thinks of the resulting community as a global one, and thus contrasts Anderson's 'imagined community', which is mainly rooted in the nation. Imaginative travel could then also feed into 'motility' - the general willingness and mental capacity to travel (cf. Gather, Kagermeier et al. 2008; Kaufmann, Bergman et al. 2004).

Virtual travel, however, is part of what Larsen, Urry et al. (2006) call the 'network capital' of an individual. Virtual travel sometimes substitutes physical travel; sometimes it also causes physical travel. However, it always enriches the network capital of a person in a mobile society, in which this type of capital becomes decisive for social inclusion. A definition of multilocality should be 
open to these type of 'non-physical' aspects, which are also important for the construction and practicing of multilocal arrangements.

Then, there is the temporal dimension of the presented multilocality approaches. Most of the scholars still stick too much to a periodical understanding of time in relation to multilocality. The length of sojourn or the frequency of absence/presence is still thought as a rather stable cycle linked to a place. This, however, excludes several groups of mobile people from being multilocal according to such a definition. What happens with irregularly travelling business people? People who do always travel, but do thereby locate in a more random and less predictable patterns, according to business necessity - are they multilocal or not? They do not necessarily return in periodic rhythms to the same locations, however, they are cognitively not monolocal. Or what about private travel, a person visiting a friend, and during this sojourn being hosted in the friend's house? The same might account for family visits. When do people become a multilocal in these cases? The length of the stay or the frequency of the visits is not really a good criterion for distinguishing multilocality. Duchêne-Lacroix (2009) is quite right in pointing out that it is not about this periodic temporal understanding, but about the 'intensity' of the sojourn at a place. Here again, emotional and cognitive aspects might help facilitate a better understanding of multilocality than objective criteria.

A rather secondary critical point concerning the current concept of multilocality might be the strong focus on job and family as dominant reasons for multilocal behaviour, and the image that places are functionally divided - in the sense that one place is related to family and the other to the professional sphere. In the end there might be a lot of other motivators for multilocal arrangements. Multilocality could also be thought of as being multi-functional, and not only a combination of mono-functional places. People do not always travel for only one reason; different functions are often combined within one trip. This is an awareness that spatial mobility research in the field of circulation/daily mobility/commuting has already achieved (cf. Gather, Kagermeier et al. 2008), and multilocality should also take this into consideration. When social complexity should be addressed in the study of multilocality, then simple causal models using monofunctional distinctions might not serve the ends.

A different topic concerns the construction of identity within the lifeworlds of multilocal people. Some scholars such as Stock (2009) or Rolshoven \& Winkler (2009) have pointed that out, but there is more to it than the individual construction of self-perception, or of using multilocal living as a creator of a self-ascribed image. What about the notion of 'home' and belonging in the residentially focused multilocality conceptualisation? What role does home play? How are terms such as house, residence and home linked to each other? From a rather practical perspective, this means that multilocal people's conceptualisation of home has to be looked at. Does home in multilocal arrangements turn into something placeless and more related to social categories such as family, friends, networks, or even milieu?

This leads to a last lacking point in current multilocality definitions. So far, not much has been said about how to integrate the cognitive aspect of spatial appropriation and orientation in space. Only Stock (2009) has remarked that there is a dichotomy between unknown, foreign places (Fremdorte) and familiar places (Eigenorte). But how should one imagine the current work of adaptation that multilocal people have to perform when switching between places? Particularly nothing has been 
said about orientation as a part of adaptation. Multilocality - when it is not only understood as a repetition of the same trips - is confronted with the constant demand for orientation in new environments. How does this interfere with what Stock (2009) calls the 'capacities' to be multilocal? When people constantly have to leave familiar situations and adapt to new ones, do they develop behavioural patterns or routines for how to deal with this highly stressful demand? And how do such multilocal routines lead to a change of our cities' physical structure and services - in the sense of Duyvendak's 'generic places'?

The multilocality definitions are not complete, yet, they surely were not meant to be so. Multilocality is not yet a sufficiently defined concept with which to work. A common sense of what to integrate or not has not yet been achieved. Rather, the quoted approaches represent the 'status quo' of multilocality research, which is still in its founding process. The Netzwerk Multilokalität has presented interesting theoretical approaches and empirical evidence which leads to the conclusion that this is a promising future field of empirical research. There are a lot of interesting ways of thinking of 'multilocality'. The variety of perspectives leaves open a vast area for field work.

To summarize, I believe that the complexity of multilocality as a social phenomenon makes qualitative approaches seem more appropriate for multilocality studies. In addition, the data availability from census and citizen registry is dissatisfactory. Furthermore, a rigid technical operationalisation of multilocality - as necessary for quantitative analysis - does not match the informational ampleness which the phenomenon provides for social science.

The multilocality concept thus provides an interesting (re-)new(-ed) field under the umbrella of both social and spatial mobility research. Empirical designs for multilocality research still have to be tested. Exploratory qualitative studies might serve to found a more substantial understanding of multilocality, and careful efforts of quantification might be helpful. In contrast, it seems still too early for large comparative and complex quantitative studies.

\subsection{Synthesis: Routes}

As I have shown in this chapter, 'transnationalism' and 'multilocality' provide two research topics which have intensively dealt with how we could understand the increasing mobility between places. The interesting aspect in both of these approaches consists in the attempt to combine spatial mobility ('routes') and belonging ('roots'; cf. chapter 3). Obviously, there are some linkages between transnationalism and multilocality research. First, conceptual frameworks of both approaches refer to the social theory of modernisation, and in particular to the most recent approaches under the key words of 'second modernity' (Beck \& Grande 2010) or 'liquid modernity' (Bauman 2000). Both research programmes make use of the idea of an increasingly differentiating social reality, in which individualisation and global integration cause ever more complex patterns in social reality. This is often cited in the linguistic metaphor that social reality in the 'first modernity' could be grasped with in 'either-or' (entweder oder) dialectics, whereas in the 'second modernity' it increasingly became 'both-and' (sowohl als auch) continuum (Weichhart 2010). Transnationalism as well as multilocality frame their main questions within this theoretical reference and also try to overcome its limitations. 
Second, both concepts refer to perspectives which focus on the connections between the social and physical space of an individual. I have presented Ludger Pries $(2008,2009)$ as a reference for the idea that social and physical space lose their formerly given superposition, yet there are a variety of other scholars (Faist 2000; Glick Schiller 2005; Steinbrink 2009; Dienel 2004, 2009) who independent of their background in transnationalism or multilocality research - have formulated and argued their conceptual approaches in a similar way: within one physical space more and more social spaces co-exist and transcend each other, while at the same time individual social spaces increasingly exist across administrative borders.

Third, both fields agree on the observation that the everyday life of individual people and/or households is an interesting field of empirical study as it allows the understanding of the complexity of 'transgression' phenomena from the viewpoint of the actors themselves. I do not wish to negate the relevance of the meso and macro levels. Faist $(2000)$ as well as Dienel $(2004,2009)$ have convincingly shown how individual border-crossing phenomena are reflected in processes of institutionalisation at higher levels, and how these meso and macro level structures influence the micro-level of the individual actor. However, I read both bodies of literature as an invitation to study the lifeworld of mobile persons. The interesting question is how mobile people relate to the 'both-and' phenomena which seem to characterise their lives.

Fourth, both approaches not only deal with the combination of mobility and belonging, but they also focus on the parallel existence of different countries and places within the lifeworld of the people. A lot of research questions circulate around ideas of multiple belonging to different territories and they ask about the impact that it has on place-based identification. In particular, the design and character of places that co-exist in a person's life are still relatively unknown. Some scholars have already explored this field of research and first insights have appeared. Here again, we are approaching the issues we could find in section 3.5 about Duyvendak's types of belonging in particular to generic places. Besides the idea of the 'generic places', the idea of the 'non-places' is mentioned in transnationalism studies as a possible type of place relatedness (cf. Kaiser 2011: 53). 'Non-places' as a concept date back to Marc Augé (1995: 77f.) who says: 'If a place can be defined as relational, historical and concerned with identity, then a space which cannot be defined as relational, or historical, or concerned with identity will be a non-place.' In Augé's logic, 'places' always include a historic dimension into their present existence. To Augé, the prototype of such a 'non-place' is the transit space that mobile people pass while travelling, e.g. airports, train stations, highways, airplanes, trains, or ships. Augé's main argument is that through the time we spend in travelling, we increasingly pass through space instead of being in places, thus we perceive the time of transit as a rush through different landscapes which are chained like in a 'theme park', but we do not have contact with them. The time of travel is mostly spent in 'non-places' which are characterised by uniformity and the absence of organic social life.

Fifth, both approaches acknowledge the relevance of the local scale. While multilocality directly points to it, the word transnationalism rather suggests a focus on the national scale. However, looking at the results of transnationalism research, I found that the locale is always thought of as the scale which is best perceivable or liveable for individual people; it can be experienced with the physical senses (cf. section 3.2). Thus, transnationalism research reflects the national scale quite often through the lens of local everyday life experiences. The national, in contrast, is an abstract appearance. While it is already difficult to experience the local - let's say a city - in all its 
complexity and range, this is even more difficult for higher scales such as the national. Having said this, one also has to mention that multilocality research pays little attention to the national scale.

However, the general suggestion of both these strands of the literature on mobile lives is that people today are less settled and rooted in stable, long-term, monolocal, mononational patterns than in earlier times. People are suspected to develop more dynamic and short-term patterns of rootedness and belonging. This includes the necessity to combine the local and the national (and sometimes even supra-national or global) scale in one's everyday life. Still, there is a majority of people who are immobile in the sense that they do not physically move across national borders, or that they do not live multilocally across administrative boundaries of municipalities. These people are orientated to geographical stability and long-term belonging, focusing on anchor point and orientating on their 'one' main place, yet their monolocal lifeworlds will be affected by global influences as well. These people might become part of a more imaginative and virtual forms of mobility, even if they do not travel physically.

A still small but growing group of people interprets the arrival of the global/transnational in the local as an opportunity in life. These people rather opt for physical mobility. This mobility includes a broad variety of patterns that might be situated between the extreme poles of unidirectional longterm migration across borders, on the one hand, and the daily commuting rhythms between two places, on the other hand. Multilocality and transnational migration represent two mobility patterns somewhere in-between these two poles. From a historian's perspective, they might have existed for long time, but they have a new character and relevance in our societies.

This phenomenon is studied through the perspectives of transnationalism and multilocality, which both share the interest in understanding the interplay between mobility and belonging. One could illustrate that transnationalism looks at the life of people from 'above' - from the national scale; and multilocality takes a look from 'below' - from the local scale. I imagine that most realities of people's lives actually happen in a field somewhere in between, thus a combination of both perspectives might provide a more detailed understanding of current lifeworlds in the globalising societies.

What we have not dealt with is the precise examination of terminology. The word stem clearly indicates a reference to a geographic scale, yet there are a variety of prefixes (such as pluri-, multi-, trans-, or inter-) and suffixes (-ism, -ity, -isation) which are put in front or behind the word stem. In both multilocality and transnationalism research, terminologies are not used based on a shared understand and common definitions. For example, this is reflected in the open question: who is a transnational person, or equivalently who is a multilocal? There is no neat line to draw between inclusion and exclusion of cases in an empirical study. Additionally, there is the large problem of translating terms into other languages. I do not wish to go into detail here, as I do not deem it helpful at this point to elaborate a clear distinction between terminologies, but I rather agree with Oßenbrügge (2004: $17^{56}$, cited in Hühn, Lerp et al. 2010: 22) who says that for the study of transphenomena it is more important to operationalise 'what should be considered the entity for social integration'.

\footnotetext{
${ }^{56}$ Translated by the author from the German text: 'was als Instanz sozialer Integration betrachtet werden soll'.
} 
For the interested reader, a very helpful book is 'Transkulturalität, Transnationalität, Transstaatlichkeit, Translokalität' by Hühn, Lerp et al. (2010). In this book Hühn, Lerp et al. (2010) explain that from a linguistic perspective it could be formulated: the prefix 'multi-' indicates 'many'; the prefix 'pluri-' means 'several'; the prefix 'inter-' refers to 'between'; and the prefix 'trans-' means 'beyond' or 'moreover'. The suffix '-ism' hints at an ideology and normative circumscription; the suffix '-ity' describes a social fact or situation; and finally the suffix '-isation' draws on a process. While this makes sense from a theoretical perspective, the practical use of terms in the literature is often less reflected. In his brilliant reflection on the use of terms in the German speaking academia, Petzold (2010) mentions that what he understands to be 'multilocality' is mainly labelled as translocality in the English speaking scientific community, as e.g. visible in the collection of papers on 'Translocal Geographies' edited by Brickell \& Datta (2011b). Equally, Steinbrink (2009) uses the term 'Translokalität' in German, but Petzold would differentiate the terms 'multilocality' and 'translocality'. He suggests understanding 'translocality' as a particular form of 'multilocality' in which a person develops emotional and social attachment to various places of the own lifeworld. As an antagonism, he would understand 'interlocality' as another form of 'multilocality' in which a person has one central place for attachment, and other places of the own lifeworld do not have a similar binding function. However, this definition of the prefixes contradicts Pries' (2009) argumentation about 'transnationalisation' and 'internationalisation'. To Pries the 'trans' component is part of the 'inter' term, while the 'inter' term is also part of itself. Hühn, Lerp et al. (2010) cite the example of Welsch's use of the term 'multiculturalism', which they would rather call 'interculturalism'. Furthermore Hühn, Lerp et al. (2010) as well as Weichhart (2010) also provide very helpful theoretical reflections on the denotative and connotative meanings of 'trans-'terms.

An interesting question remains: how does the arrival of globalism, or transnationalism affect the attitudes of people towards people and culture from distant places in the local sphere of everyday life? The literature on cosmopolitanism remains divided about this issue. While there are authors that argue for a growing cosmopolitanisation of the world society, there are others who suggest a retreat into national and regional cultural spaces.

Furthermore, there is still more research necessary in order to understand how the local and national scale are linked in mobile lives. This includes the question for the development of supra-national scales that might become relevant in people’s lives. Pries' (2009) definition of supra-nationalisation allows first insights, but the European Union is theoretically the perfect empirical field for studying the supra-nationalisation of everyday life.

Finally, the issue of the corporeality of the human being has also showed up in transnationalism and multilocality research. It is discussed in relation to the problem that people cannot be equally physically present in all their places and countries at any one given moment. In these two research agendas it is always about empirical situations in which absence and presence co-exist, however, most studies only point to this issue and do not elaborate on it in more detail, therefore there is a need to better look at how this contemporaneity of 'being here and not there' is included in individuals' lifeworlds.

I will conclude this chapter with the words of psychologist Dean Keith Simonton (1999: 207; as cited in Florida 2002: 33) who said that, 'creativity is favoured by an intellect that has been 
enriched with diverse experiences and perspectives', and I hope that the study of transnational multilocality becomes a fruitful endeavour for better understanding the role of place and mobility in creative knowledge workers’ lifeworlds. 


\section{Interlude D: Kate - Marta}

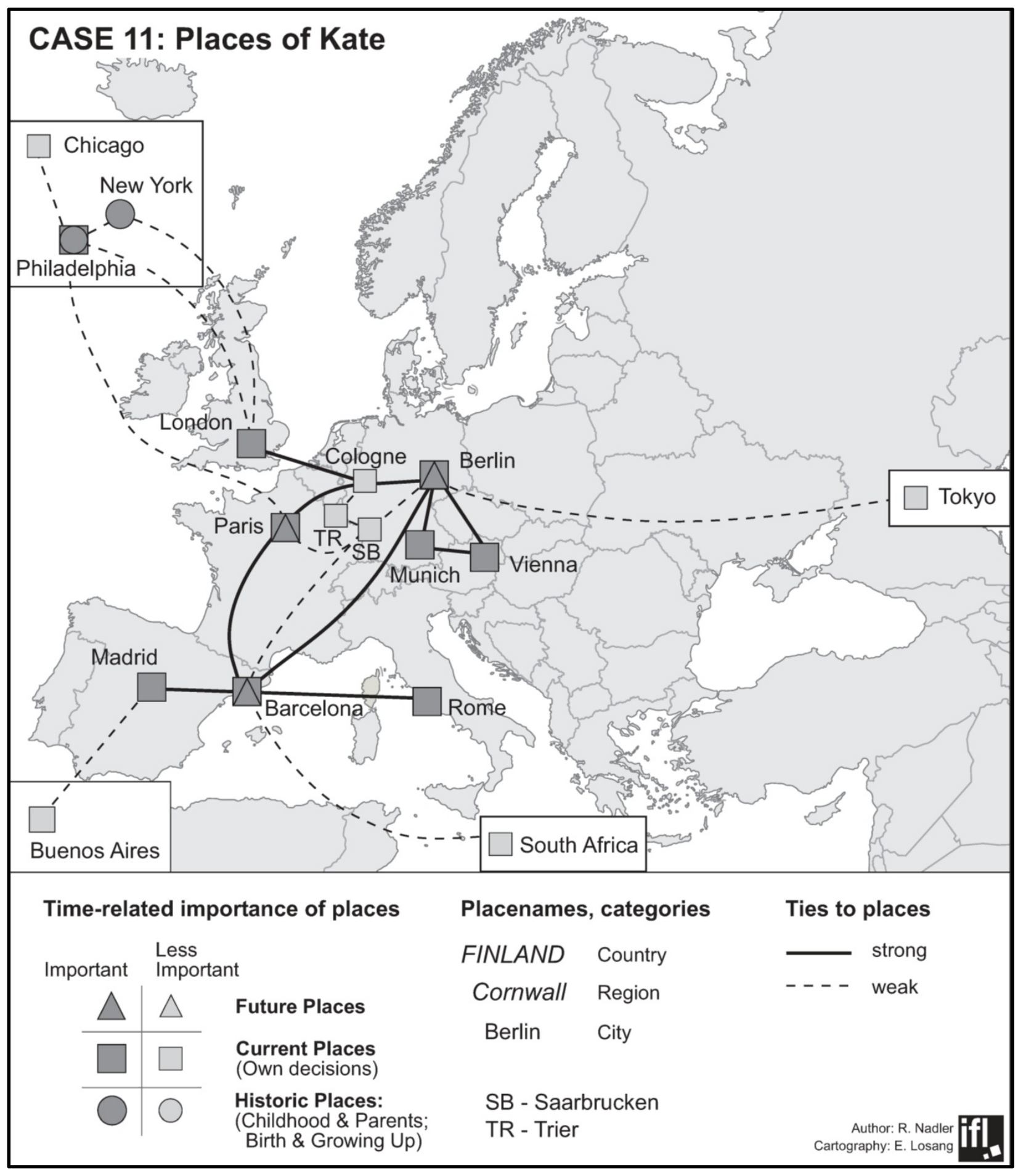




\section{Meet Kate}

Kate is a 60-year old US American artistic director who supervises the film dubbing of major Hollywood productions into European languages. Kate was born in New York, but was brought up in Philadelphia. When she finished high school at the age of 17 , she wanted to escape the narrowness of her family life, and she decided to study Linguistics in Chicago. Two years later she left the US to continue university in Paris. Since then she has never really left Europe. She met her German exhusband in France, whom she married in her younger years. After graduation in Paris, both decided to move to Saarbrücken in Germany; she did her $\mathrm{PhD}$ in linguistics there. During her $\mathrm{PhD}$ time she started teaching at the university, which she continued to do for many years. The topic of her PhD thesis was errors in jokes and humour which occurred during dubbing US American movies to European languages. Twenty years ago, large Hollywood studios became aware of her talent and they started hiring her for the supervision of the dubbing process in Europe, mainly in France and Germany.

Kate says she has always loved travelling and during her time as undergraduate she often backpacked through Europe. She was therefore very happy about the temporary relief from her mono-local family life - house, university job and husband. The parallel expert job that she started doing for the film companies took her to Paris and Berlin, where major dubbing studios are located. She gradually became so much more attracted to this second job that she quit her university position in Saarbrücken ten years ago and started extending her range in the other job. She added Spanish dubbing, including frequent travel to Barcelona and Madrid. She has recently also added Italian dubbing, which means trips to Rome. And meanwhile also Vienna and Munich showed up on her mind map, as the dubbing industry is growing there, too.

Travelling has reached a point in her life that she only stays in one place for three or four days before moving to the next one. This stressed her partnership, so that she got divorced from her husband, who was more mono-local and couldn't stand her absence anymore. Kate feels much better now; she uses the metaphor of a bird that needs the freedom to fly around. She kept a small apartment in Saarbrücken for a while, but she rarely spent time there; it was more or less a large wardrobe. Thus, at some point she decided to leave this apartment, and she lived without having an apartment and her own address for several years. She emphasizes that not having her own - as most other people have - made her feel free, it made her feel proud. Yet, her life is characterized by routines: she always stays in the same rooms of the same hotels in the cities she visits regularly. In each hotel she leaves two suitcases with clothes and toiletries. Her clothes are always cleaned by the hotels and put into her rooms before she arrives. Two years ago, Kate rented an apartment in Barcelona, which is on a roof floor. It works as her 'magic carpet' up in the sky. She emphasizes that she did not rent it to become more rooted again, but she acted in the heat of the moment, seeing the apartment and wanting it; four months ago she bought it.

Yet, Kate still needs some anchor points in life to stand the mobility and hectic life on the move. She spends Christmas and New Year's Eve in Philadelphia at her brother's every year where she sees her nephews. She also takes some weeks off each year during summer to go to Trier to an Artist Summer School, where she dedicates herself to painting in a meditative environment.

Having passed the age of 60, Kate does not want to stop travelling. She can imagine working less, but she never wants to become a settled and retired person looking back on her younger years. She loves her life as it is. 


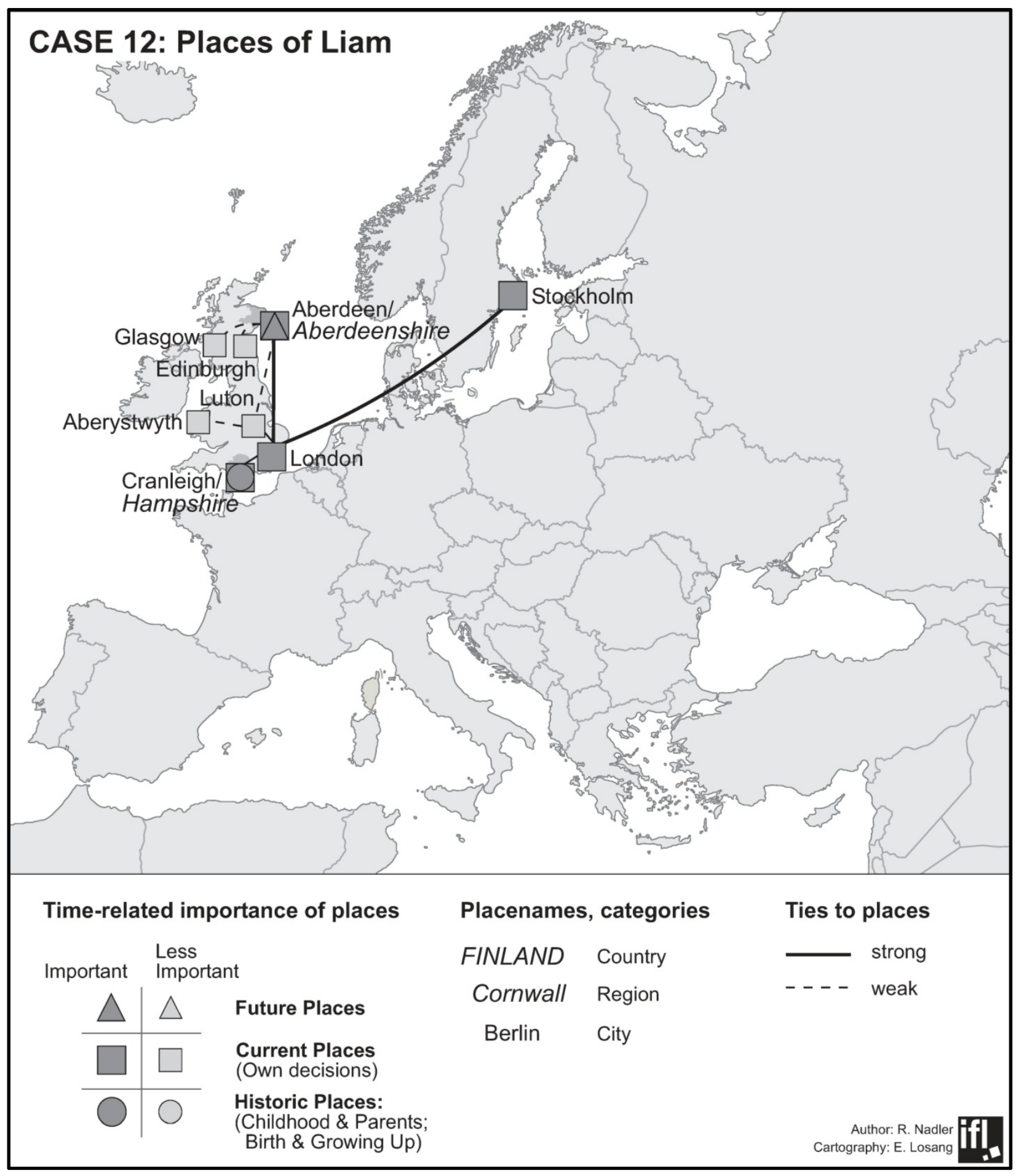




\section{Meet Liam}

Liam is an Englishman in his 60s who has a senior research position in the field of Regional Development. He was born and grew up in Cranleigh, in the southern English county of Hampshire. After school he started a teacher training course, which he quit after a while. He then went to Aberystwyth in Wales. The small and quiet university town at the seaside was the perfect location to reorientate in life, he says. During his years in Aberystwyth he also met his Scottish wife. After graduation, he found a job in Luton, in the North of London. He worked there as a teacher in a school in which ethnic gangs were active. He characterizes Luton as a counterpart to the peaceful life at the Welsh seaside in Aberystwyth. Luton was busy, crowded and a violent atmosphere was part of his everyday life. When his first son was born, he did not want to raise him in that kind of environment, so he started looking for a new job elsewhere.

He found a job in Aberdeen in Scotland, where his wife was from, so both were happy to go there. They sold their Victorian town house in Luton, and moved into a detached house in Aberdeenshire, outside of the city and far from any neighbours. To Liam, Aberdeenshire represented the move back to the peaceful countryside, in which fresh air, less ethnic and class consciousness, and small and nice schooling became features of his and his family's life again. Liam was reinforced in his decision to leave urban Luton when he heard that one of his former students was killed on the street in racist gang violence.

Liam lived a rather monolocal life for many years, having two more sons in Aberdeen and working for only two different employers in the city. His third son was diagnosed with learning difficulties and mental health problems, so Liam and his wife had to take care of him in a more extensive way, and were not very mobile for a long period.

At some point in his career, he thought more internationality could be good for his CV and he started working in European projects and got to know many people from all over Europe. During this time he collaborated with his current employer, a multinational research institution in the Scandinavian countries based in Stockholm. One day the director of this institution told Liam that they were looking for an experienced senior researcher, and Liam applied in order to have another reference on his CV.

As Liam did not fulfil the criteria of knowing a Scandinavian language at the moment of being hired, the director of this institution decided to buy Liam's workforce from the University of Aberdeen. Thus, Liam is legally employed in Aberdeen, but factually he works for the institution in Sweden. This arrangement includes a time schedule which foresees that Liam spends one week a month in Stockholm at the office, and three weeks a month he travels elsewhere for the project or can work at his home office.

For six years, Liam has been circulating between Aberdeen and Stockholm, always passing through London's Heathrow Airport. The Stockholm institution pays for his travel and provides a small apartment for him in Stockholm's city centre. Liam uses the London stops as a possibility to regularly visit his elderly mother and sisters, who live in the South of London, in Hampshire.

Apart from the amenity of family visits, he doesn't actually like this multilocality. In two years, when his contract finishes, he will look for work in Aberdeen again, and he wants to stay there, building up a small farm with his son; his other two sons still live in Scotland, in Glasgow and Edinburgh. So Liam's story is mainly one of multilocality as a temporary stage in life. 


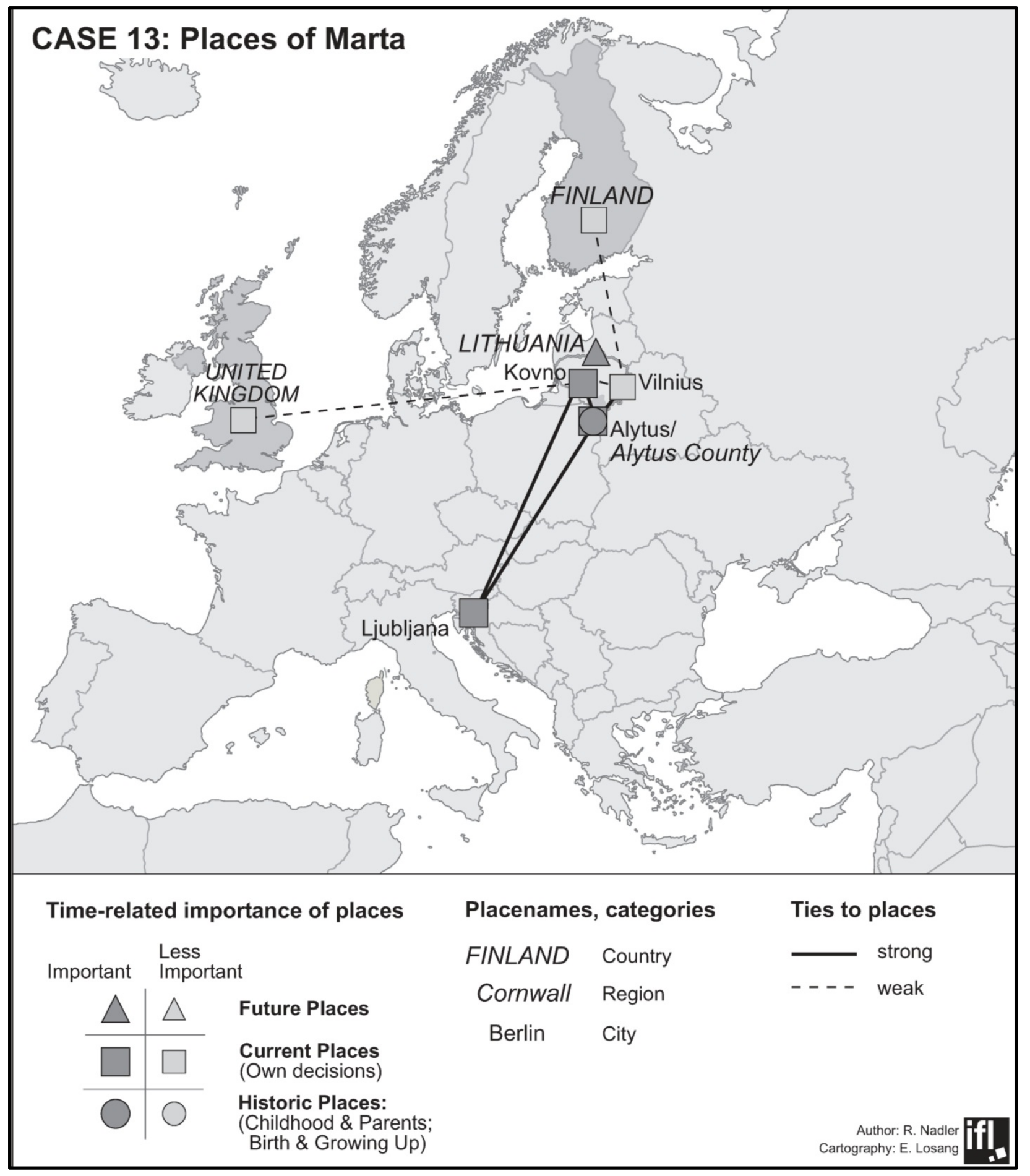




\section{Meet Marta}

Marta is a Lithuanian researcher in her late 30s. She currently lives in Ljubljana, Slovenia, where she has founded a private research office in the field of Regional Development. Her latest project deals with developments in Lithuania, therefore she frequently travels between Lithuania and Slovenia.

Marta was born and grew up in a small rural town in Lithuania. After her high school graduation she went to university in the city of Kaunas. During her semester breaks, Marta also went to the UK, where she learnt English and worked to finance some travelling there. When she finished her degree, she found her first job in marketing for a Vilnius-based company. As she did not like this private sector work, she went back to academic research in the field of rural development. When Lithuania joined the EU in 2004, she became very involved in European projects. When she decided to do a $\mathrm{PhD}$ she gravitated towards a Finnish research institute which was quite advanced in rural studies. So she went there to start her PhD and that's where she met her Slovenian boyfriend, but since the Finnish institute did not allow her to continue with her current research project at the Lithuanian research institute where she kept an affiliation, she decided to quit the $\mathrm{PhD}$ in Finland.

Continuing work in Lithuania, she was permitted project funding for a new European project, and she decided to take the money with her and live in Slovenia with her boyfriend. She founded a company with him which could work as a research institute in European partnerships. Through this research institute which is based in Ljubljana, she is currently working on a case study which focuses on her Lithuanian home region. Thus, she can be close to her boyfriend, she has a decent job and she can frequently travel to Lithuania, which she still considers her homeland, and from where she never detached.

Even though Marta has now spent several years in Slovenia, she reflects on why she still doesn't speak the language. Her work in the European projects is communicated in English, most Slovenians speak English, and the Slovenian language is difficult for her to learn. Thus, most of her life in Slovenia is in English instead of Slovenian.

Marta does not have a special empathy for her own mobility. She tries to reduce travel time itself by buying quick connecting flights between Slovenia and Lithuania. She always has to change planes in Frankfurt or Brussels, and she does not want to waste time in airports. Marta also describes that she only travels with hand luggage, only taking her laptop and some work papers, which she reads through during the travel time. When she arrives in Lithuania, she often stays at her sister's place in Kaunas, and since her sister is the same size as she is, she always uses her clothes when she's there.

Marta was part of a folkloristic association earlier, but she doesn't have the time anymore. In Slovenia, she thought about joining the Lithuanian community which is related to the Lithuanian embassy, but the embassy did not respond to her, so she did not go on. If she had more time, Marta could imagine participating in some local policy projects, provided that people in the group were nice and the project made sense. She is critical, as she thinks that in such local initiatives lobby groups compete, and there is a negative emotional atmosphere.

For the future Marta plans to leave Slovenia again, as she has neither a concrete job opportunity nor does she feel at home there. Her relationship with her Slovenian boyfriend is no reason to make her stay, she says. Even if the relationship worked fine and she had children, she would prefer to raise them in Lithuania. Her current boyfriend, however, wants to raise his kids in Slovenia, so there is still some communication necessary. 


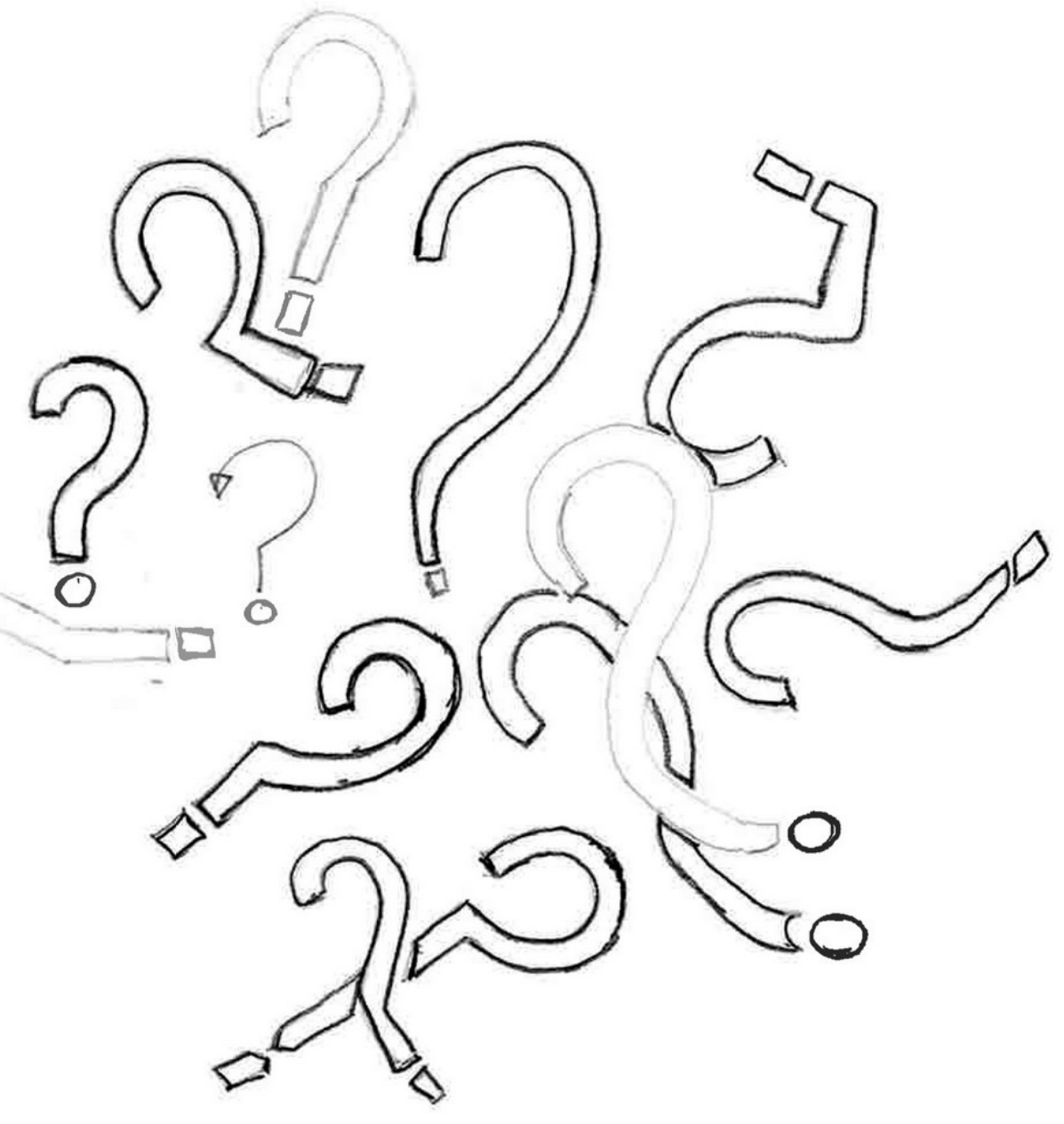




\section{Synthesis: Evolving fields of interest}

This chapter is a synthesis of the previous chapters. In particular, it is dedicated to elaborate the connections between the three thematic dimensions of (1) creative industries, of (2) place attachment and belonging (roots), and of (3) mobility studies and multiple place attachment (routes). The aim of this chapter is to derive the central research questions for this research project.

I began this project with an empirical observation during the ACRE research project ${ }^{57}$ : there are transnational migrants who work in creative industries and who frequently circulate between specific places, or locales, in different countries, and who somehow make use of local social networks. Therefore, I drew on the literature on creative industries to understand the specificity of the economic sector (chapter 2). Then, I referred to concepts such as home/Heimat, place-based identity, dwelling and sedentariness in order to understand how people are rooted in space and in places (chapter 3). And finally, I studied literature on mobile lifestyles such as transnational migration and multilocality (chapter 4). Now I will use the insights from these chapters in order to sketch out three 'theoretical figures', or ideal types in the sense of Weber (cf. Werner 2009), to illustrate some paradoxical constellations between the different discussions on creative industries, 'roots' and 'routes'. Based on these paradoxical situations, I will sketch out the relevant research questions which form different evolving fields of interest for this thesis.

\section{Merlin}

The first theoretical figure is that of 'Merlin'. Merlin is a textbook example of a creative knowledge worker. On the one hand he holds a part-time position as a researcher in communication and media science in the local university; on the other hand he works as a freelance graphic designer for an advertisement agency. The reason for pursuing these two duties is that Merlin's position at the university was not offered to him as full-time employment, as there are not enough financial resources to pay for a full-time position. Whereas Merlin would perhaps prefer to work full-time at the university, he appreciates his current situation of having two jobs; therefore multiple jobholding is evaluated both positively and negatively. Merlin has always loved drawing and painting, and he later on he taught himself to work with graphic design software. During his graduate studies he always designed flyers for student parties or covers for music releases from music bands and DJs of whom he was a friend. At the moment he likes working in the advertisement agency once in a while and without tightly regulated duties. He can earn some additional money there while pursuing an activity that he enjoys. Furthermore, he acknowledges that his two jobs nurture each other: what he studies from a theoretical perspective and what he learns from research at the university helps him to improve the semantics and symbolism of his graphic designs for the field of advertisement. In addition to this, he learns a lot of practical things and observes empirically while working in the advertisement agency. This gives him an advantage for scientific research, as he is already part of

\footnotetext{
${ }^{57}$ More information on the ACRE project and its results are available under: http://acre.socsci.uva.nl/index.html (accessed 31 October 2012)
} 
the empirical field. However, he feels stressed by this life between the worlds of publically financed science and private market advertisement. Both sectors work completely differently. In science things are regulated in rigid ways, he has to respect the hierarchy and bureaucracy at the university. People live in a theoretical bubble, often far from the 'real world'. On the other hand, he sometimes feels dissatisfied with the speed of work in the advertisement agency, where he has no time to reflect on what he is doing. Even though clients expect individualised products and services, his boss has introduced some standard templates for these services in order to raise the productivity of this brainwork. Whereas things are sold as tailored to the specific needs of the company, they are actually also partly what all other clients receive. The continuous switching between these two worlds thus also brings Merlin a lot of emotional and mental stress. In addition, the pay in both jobs is still below average. Merlin holds a Master's degree from a university, yet he has the feeling that the time and money he invested in studying does not really pay at the end of the month. Furthermore, his two jobs lead to bureaucratic problems. His health insurance is related to his employment at the university, but the health insurance company only accepts a limited range of hours being worked in other jobs. Merlin does not have a regular number of hours that he works in the advertisement agency, so he frequently has to go and explain to the health insurance officers that he cannot guarantee to not exceed that limit, but he cannot predict when and for how long such an excess might occur. Similarly, he pays benefits to the unemployment insurance from the salary he receives in the university. Whenever his temporary contracts as a researcher finish, he has to register for unemployment benefits and explain his professional identity so the labour office can look for new employment for him. However, the officers there do not understand that he can do several things: research, communications, and advertisement. In their logic he has to have one professional identity. These things both stress and amuse Merlin, as they show him how much he falls out of standard cognitive models and bureaucratic logics. Given all these difficulties, he always reports being happy in what he is doing. He can pursue his different interests (theoretical and practical) in a given professional field (communications). He can keep himself updated, his intellectual inputs are very welcomed and praised by his colleagues and bosses, and the people around him are friendly, interesting, and stimulate him to begin new projects. Furthermore, in his years in this city he has constructed a large social network of acquaintances and friends. His sister works in the city, too, and her networks are also available to him. Through these networks, he continuously receives informal and up-to-date information about new developments, projects and actors in the local market. This gives him the opportunity to think up new project ideas and set up new project teams and collaborations. He can also switch between his two institutional affiliations when it comes to organisationally embedding a new project. If neither the university nor the advertisement agency are the right place to pursue an interesting project, he can also do it as a freelancer in other flexibly designed organisations. The informal information flow in the city's networks is important in order to participate in the market and being a recognised player in the field. Merlin would not have the opportunity to get jobs as easily in other cities where he does not know anyone. In order to remain integrated and recognised, Merlin combines the useful with the pleasant - he attends several social events during evenings and weekends in which the local creative avantgarde is meeting. During these parties, work life and private amusement are inseparably intertwined. This also has disadvantages, as Merlin remarks. People seem to circulate mentally around themselves in the city, so once in a while he goes on small trips to other cities where he visits friends and relatives. These trips are used to open his cognitive horizon again from the local 
lock-in situation in his city. He lets himself be inspired from what he sees elsewhere and it helps him to refresh his work back home. Even though Merlin loves his life as it is, he sometimes thinks of how it would be if he had a family: he's in his 30's, still young, and at the moment everything is going well with his girlfriend, but in certain moments he wonders if he would enjoy having a more stable family life with children, something more stuffy and less bohemian than now, and then he concludes: 'Well, let me think about that later, there’s still some time'.

\section{Hannah}

The second theoretical figure is that of 'Hannah'. Hannah is the textbook example of a person deeply rooted in her local environment. Hannah was born in One City, and she grew up in one and the same neighbourhood where her parents are still living today. She recently moved to another neighbourhood where she and her husband could afford to buy an apartment which is large enough for them and their two small children. She would have preferred to buy one in her childhood neighbourhood and closer to her parents, but prices were too high. Hannah loves her life in One City, and she feels that this place is her home and where she can be herself. It sometimes makes her nervous to have to travel when her boss sends her around. In other cities she has the impression that people are working completely differently, and she feels uncomfortable among them. When she arrives in different places she already longs for the moment when she is back in 'her' city, in One City, and close to her family. In such situations, she realises even more how strongly she is related to her city and the people there. She understands their humour and can laugh with them; she has a lot of friends and acquaintances who are very supportive in everyday life. She meets people coincidentally on the streets and chats about the local gossip or the annoying political decisions of the City Council of One City. Needing to go on business trips once in a while, Hannah also compares the physical environment and the built infrastructure in her city to that of other cities; she definitely thinks that her city is more beautiful than any other she has visited, so when she is elsewhere she feels homesick. Actually, she only likes to travel if it is for the family vacation, when she can go with her husband and her children. In these moments it does not matter where they go, but that they go together. Yet, Hannah prefers to stay in the country when travelling with the children, as she does not trust health care abroad, and she is also not very fluent in foreign languages. If something happened with the children, she would not feel prepared to deal with foreign institutions (e.g. going to see a medical doctor in a hospital). Her best friend, who she has known since kindergarten, had problems with her small daughter while travelling abroad last year and she told her it was a 'nightmare'. Therefore, Hannah does not mind spending vacation time in the country. As a consequence, Hannah would always identify as being from her city and having the nationality of her country, in which things run 'better' than in other countries. In the future, she definitely wants to stay where she is. She could not imagine living elsewhere. However, Hannah is aware of the changing conditions in which her local lifeworld is embedded. The European Union is affecting her everyday life more and more. She does not really know what to think of that. On the one hand, she is a simple person and knows too little about actual coherences in that matter, so she would not consider herself competent enough to express a clear opinion, but her gut instincts make her feel opposed to these influences from 'above' and elsewhere. She also observes that the social composition of her neighbourhood is changing. Looking only at her own apartment building, she observes that the nice neighbours - those who had always helped her with carrying the buggy 
upstairs when her children were still smaller - moved out three months ago. Mr Miller had applied for a better job in another city. A month ago new neighbours moved into the Millers' apartment. She's met them in the stairway when they moved in, but since they have not spoken much. These people only go to work when Hannah has already brought the children to the kindergarten and has already worked for hours in the office, and these new neighbours only come home late from work, bringing all their daily shopping. Hannah thinks: 'Where do they actually shop? The poor cashiers who have to work so late!' Hannah has observed that the new neighbours have a licence plate from a foreign country, and she is not able to pronounce their family name which is written on the door bell. Hannah wonders about the changes around her, and she is actually very curious about it. She latently feels that the continuity of the existence of 'her' city is becoming threatened by those coming in from elsewhere and re-defining it. This includes the impression that she has to somehow defend the stability of her lifeworld. On the other hand, she knows too little about where this all comes from and where it will lead. For the moment she continues observing and trying to form an opinion.

\section{Daniel}

The third theoretical figure is that of 'Daniel'. Daniel is the textbook example of a multilocal and transnational person. The same as Hannah, Daniel was born in One City and he started attending school there. Four years before his graduation, his father took a new position in Second City, so the family moved there and Daniel had to leave all his friends and make new ones in Second City. As a teenager this is not always easy, but Daniel managed quite well and learned to adapt his personality to this different context so that he was accepted by the other teenagers in Second City. Actually, after a while he appreciated the fact that the family had moved and that he was able to compare the two cities. His old friends in One City loved to hear his stories from Second City, and his new friends in Second City were interested in stories from One City. Sometimes Daniel also took his friends from one place to show them around in the other place. As Daniel had experienced how it feels to be the 'new' guy from elsewhere, he quickly became friends with Thomas who came from Second Country with his parents. While Daniel's classmates in Second City were interested in One City, they were not interested in Second Country stories from Thomas. Daniel was; and Thomas' stories about Second Country made Daniel even more curious to go there. Consequently, he went to Third City in Second Country for an exchange year, where he learnt how it feels to be a foreigner, to express oneself in a foreign language and to live in a more or less different culture. Daniel learnt to compare perspectives and opinions, and he learnt that his own national background does not represent the only lens for looking at the world. After graduation, he was even more curious to learn more about foreign countries. He started studying in Fourth City in Third Country, and he was very happy that his parents supported his decision. This allowed him to follow his personal interest in discovering the new and unknown while being able to break out from the known. In Fourth City he met Anna, who comes from Fifth City in Fourth Country. They became a couple and still live in Third Country. Daniel has taken a job in the local branch of a globally operating company. The company frequently sends him around Europe to provide services to different clients. When Daniel's boss realised his cultural skills and foreign language proficiency, he offered Daniel to prepare market entry and set up the first branch of the company in One Country. Daniel liked the idea of re-enforcing links to his country of origin. Whereas his home has become Fourth City, 
where he shares an apartment with Anna, where they found an international circle of friends, and where he appreciates the cosmopolitan atmosphere, he still feels rooted in One Country, too. Upon accepting the job, he had to spend much time in One Country, and his boss decided to locate the first branch to Sixth City, where Daniel rented a furnished apartment which could be used quickly but also left again without much effort. From that time on, Daniel circulated between One Country and Third Country, between Sixth City and Fourth City, between his work life and his private life. Additionally, he used some weekends in One Country to go and see his parents in Second City, or old friends in One City, and on other weekends he met up with Anna in Fifth City in Fourth Country to see her parents. This multilocal arrangement allowed Daniel to combine the amenities of different places and countries within his own lifeworld. Anna also started to travel to One Country more often and her life became more mobile. Anna and Daniel also developed new techniques to keep in touch while physically separated; they used Skype calls to update each other about the daily news. Sometimes they sent each other small gift boxes when they found something which reminded them of each other in the place where they were in a given moment. hey also became frequent travellers, and developed routines in dealing with airports and train stations, and they became more relaxed in problematic situations with spontaneous delays and changes in travel plans. They somehow felt at home in all their places and also in their travel between these places. This also translates into identitary problems. When Daniel meets other people and has to identify, he sometimes feels problems in expressing himself according to the schemes of expectations other people have. Others most often expect a person to be from one place in one country. But Daniel feels to be from several places in several countries, which are all part of his personality and his lifeworld, and which in combination make up his identity. He accepts with humour how others expect him to answer, and according to the situation he identifies with one out of several different identities. Sometimes he mirrors their expectations; in other situations he rather prefers to confuse the others. Indeed there are more serious problems in such a lifestyle. Being located in different places means that he is often present in the one place while he wants or needs to do things in the other place. Sometimes he wants to embrace Anna in Fourth City after she had trouble again with her boss, but he is in Sixth City. Sometimes he is in Fourth City, but he would need a certain book that he forgot in Sixth City. In other moments, he finds himself in the mood to go and have a beer with his best friend in Second City, while being annoyed by Anna's father Fifth City who is trying to convince him to take a more monolocal job again in Fourth City in order to have a 'real' everyday life with Anna, which could be a basis for founding a family. In all these moments when he briefly feels dislocated in the immediate situation, he remembers all the advantages of such a lifestyle. He has learned so many perspectives and contexts that he has become very relaxed about the anxieties about a decreasing stability of lifeworlds which monolocals often share. He knows that one can individually shape one's own life in a set of alternative directions; there is not just one way that things should be done. Sometimes, when Daniel goes to see old friends in One City, he also meets Hannah on the streets. They know each other from his early school years, but both Daniel and Hannah feel relatively uncomfortable in these situations when they meet coincidentally. Both being polite people, they stop to say hello and chat a while. However, while chatting they have very few things to share. Whereas Hannah is only well-informed about things in One City, Daniel could only chat about things concerned with living in-between Third Country and One Country, or comparing life in different cities. There is something of a mental barrier between their lifeworlds and the topics they could speak about. So, they both decide to keep it short and go on with their lives: 'Have a 
good time, bye!' Daniel then also thinks about how his life would be if he lived like Hannah, and he thinks: 'No, that wouldn't suit me! My life could probably be a bit more focused in geographic terms, but not only in one place.’

The three theoretical figures describe the sometimes conflicting contexts in which people today are constructing their own lifeworlds. In particular, the increasing mobility to which people adapt in very individual ways leads to a great variety of lifestyles, or as Braidotti (2011: 3) says: 'At the start of the third millennium, a diffuse sort of nomadism has become a relevant condition for a great deal of world's denizens.' The increasing individuality of lifeworlds also causes tensions with organisational structures and institutions in which individual actors are embedded and by which they are influenced - their lives simply do not fit bureaucratic and scientific expectations anymore. Weichhart (2010: $66^{58}$ ) states: 'In the second modernity it has become even more difficult than before to describe and understand the world, that what we call the 'reality'. With reference to Beck, Bonß et al. (2004), Weichhart mentions that scientific description and understanding today studying phenomena in the second modernity - increasingly has to 'differentiate in inclusive terms', while it 'differentiated in exclusive ways' during the first modernity.

I understand this last idea as an invitation to look for the junctures of supposedly exclusive phenomena. In relation to the theoretical figures of Merlin, Hannah and Daniel, who I described above, the task is now to imagine a possible 'inclusive differentiation' of their lifeworlds, a combination of all the three theoretical figures within one theoretical figure. Here, I came across several paradoxical elements which reveal interesting research questions.

\subsection{The multilocality paradox}

The three strands of literature - as illustrated in the three theoretical figures - express different understandings about the individual's place relatedness and belonging. Creative knowledge workers such as Merlin identify mainly through their professional identity. This is composed of different professional roles which all feed into this identity, however, they are relying on the local networks and as such they are also identifying with one specific place in which they are strongly embedded in professional terms. These places often provide economic resources. A place can be known elsewhere for a very specific segment of creative knowledge work. For example, Hollywood and recently Bollywood have become centres of the global film industry, Berlin is known for its electronic club music, Milan and Paris for their fashion design. Therefore, being based and identifying with a very specific locale might make sense to creative knowledge workers. They can use place affiliation as a symbolic input for self-branding, for expressing their market value. However, these place identities are not stable in time. Places can lose their reputation in the market. For example, the book industry which was famous in Leipzig, lost its importance after World War II, and Frankfurt developed as the main location of publishing houses and all institutions in this sector. Depending on their specialisation, it can make sense for a creative knowledge workers to stress their place attachment and belonging at a given moment, and to make it part of their personal

\footnotetext{
${ }^{58}$ Translated by the author from the German text: 'Es ist in der Zweiten Moderne noch schwieriger geworden als früher, die Welt, das, was wir die ,Wirklichkeit' nennen, zu beschreiben und zu verstehen.'
} 
identity. On the other hand, creative knowledge workers are also suspected to shape their environments more actively than other groups. They sustain the development of 'creative cities', with all its consequences (gentrification, displacement and segregation, brownfield redevelopment, etc.). The rooted people such as Hannah mainly identify with one individual place and with its related higher scales such as a region or a country. Furthermore, this type of place-based identity is stable in time, and it is relatively easy to treat it in empirical study, as things seem clear. Hannah is the ideal type of place-based identity and rootedness. It becomes more difficult if one looks at the more mobile case of the multilocal transnational people such as Daniel. Obviously, they are not only related to one place, but to several places.

Here, the paradox consists of the fact that place-based identity and rootedness is theoretically described as something which is based on personal experience and spending time in one place. We have learnt that in identification processes symbolic content - be it related to places, objects, or persons - is important as a reference for achieving a coherent self-conception about 'who I am', 'who else is like me', 'who is not like us', and 'how we are different from each other'. If it comes to place-based identification, references might be the place itself, with its certain ascribed characteristic and symbolic value (the narrow sense of placed-based identification). In a wider sense, place-based identification could also relate to a group of people who inhabit a certain territory (direct interaction or only an imagined community). This is already a complex process when it comes to dealing with one place. Now, transnationalism and multilocality research teach us that people live their everyday lives in different nations and different cities simultaneously. Here it becomes difficult: whereas multilocality and transnationalism research provide first insights into how we could understand place-based identity as part of multilocal arrangements, literature on creative industries does actually not speak about multilocal arrangements. Knowing that places and place attachment are important resources in creative knowledge work, it sounds rather contradictory to be mobile and to be a creative knowledge worker.

As a consequence of this paradoxical situation, I think the following intriguing questions have to be studied when it comes to mobile creative knowledge works:

- On a very descriptive level, which places actually constitute the action spaces of multilocal creative knowledge workers?

- How does place-based identification work in the context of multilocal creative knowledge work?

- How much of identification of creative knowledge multilocals is actually based on place?

- Are there any other categories of identification emerging among creative knowledge multilocals (social groups, profession, or mobility instead of rootedness)?

- What impacts do their multilocalities have on their potential to actively shape their cities?

- What relation exists between different places in the perception of multilocal creative knowledge workers?

- Are places equally important, or can we observe an unequal distribution of meaning and importance between places within one multilocal lifeworld?

- Are creative knowledge multilocals still ‘citizens' of a particular place, who engage in local civic engagement, neighbourhood initiatives and governance activities? 
The literature allows some theoretical expectations here: if people travel a lot and their everyday lives are distributed to different places in different nations, then getting involved locally as well as nationally might become more difficult. The interplay of absence and presence leads to a continuous interruption of identification processes - which themselves could be imagined as a fractioned development path. The face-to-face interaction with people in the involved locales/nations is continuously interrupted, and it is difficult to deepen or stabilise relationships. Upon arrival in a new place, the inner logic of social life in one place could not be learnt as profoundly as in monolocal situation with long experience and detailed insights. Thus, the social element of place-based identity is less accessible. When arriving in new places, the multilocal creative knowledge workers do not know the people in these places. The multilocals do not know how the locals are, and if they want to identify with them - in the sense of an imagined community. Consequently, it could be suggested that place-based forms of identification are difficult to establish in multilocal settings. Creative knowledge multilocals might opt for other reference categories for their individual identity - such as professional peers, loved ones or family members - which are comprehensible/less complex even while being geographically absent or distant.

Further, it might also be that the combination of different localities/places in their lives leads to a development of more hybrid relations to places. In particular, creative knowledge workers might make use of different and hybrid place attachment in terms of career building or input and stimulus for their work. Florida, for example, suggests that, in line with non-conformity, a valued orientation among this group, creative workers might exhibit a 'mixing of multiple creative identities' (2002: 78).

Furthermore, this multiplicity of identity might also translate into difficulties in feeling a sense of belonging to one place. Petzold (2010) has pointed out the phenomenon that some multilocals who he studied actually liked being multilocal, and they indeed developed a multiple attachment to various places. Petzold terms this observation as translocality. In contrast, he also found a group of people who actually have to integrate several places into their everyday life, yet they perceive their multilocality as enforcement by external constraints (e.g. labour markets). Consequently, the latter do not equally feel to belong to 'all' their places. They rather have one central place in their lifeworld. Petzold calls this phenomenon 'interlocality'. He then puts the translocality versus interlocality continuum on the horizontal axis of his 'crosshairs' of multilocality research (cf. 2010: 251). Weiske, Petzold et al. (2009) studied multilocal households and the ways they give sense to a multilocal life. They also found a type of households which they call 'dispatch' (Verschickung). This type perceives multilocality as a displacement of individual household members. The household members who are actually travelling do not develop wider relations to all their places; rather, they are very selective. They remain cognitively monolocal, while physically travelling. Given these scientific results, I would assume that if a person does not feel that they belong to a place, the respective feeling of solidarity/empathy for other people at this certain place might be low. I suggest that creative knowledge multilocals might also develop such selective forms of belonging and rather use places for their individual purposes rather than completely living in them as 'caring' and responsible citizens. This means that their availability for civic engagement is rather small, and their involvement in local issues and local development is less probable. 


\subsection{The mobility paradox}

The figure of Hannah - the locally rooted - definitely shows a stable lifeworld which is affected by the increasing mobility only in mediate ways. Other people who are more mobile and arrive at her place are causing changes the in nature of her place, yet Hannah only moves around if necessary for her job, or rarely for private holidays. In both her private and professional life, travelling is a subordinated element. Merlin - the creative knowledge worker - is described as a person who is embedded in a place but who needs to escape from it once in a while to find inspiration elsewhere. To Merlin, travel has a more positive connotation than for Hannah. Additionally, his travel often includes private and professional interests in one and the same trip. For Merlin as well, travelling and mobility is not a central element in his lifeworld. He is rather travelling in mental terms when it comes to creation and thinking up new things. Finally, Daniel - the transnational multilocal - is someone whose life is heavily influenced by physical mobility. It is a very central element, and he is somehow rooted in mobility.

The paradoxical constellation is that mobility is still considered an antagonism to belonging and rootedness. Traditional perspectives would argue here for exclusiveness, as Petzold (2010: 240f. ${ }^{59}$ ) points out when arguing for the novelty of the multilocality perspective: 'Dwelling and mobility are generally thought of as contradictory phenomena and they are related to disparate activities: dwelling, understood as sedentariness, is considered a pre-condition for the development of enduring social relations, shared cultural knowledge and last but not least the formation of local identification. By contrast, mobility means movement, flux and disembedding from the mentioned social, economic and cultural local structures.' Here, dwelling is one constituting element of being rooted in a place, which only serves as an example. Other constituting elements such as place-based identity, the feeling of belonging in terms of Heimat/home and psycho-social sedentariness might be thought of in similar ways as opposed to continuous mobility. If we now look at the creative knowledge worker who is actually making use of their local embeddedness for professional ends, the mobility induced disembeddedness should even equal a professional disadvantage. So, the main questions in this respect are:

- What does mobility mean in the lives of mobile creative knowledge workers?

- Is mobility eroding the stability of lifeworlds and professional situations of mobile creative knowledge workers?

- How do mobility and belonging interrelate in terms of personal meaning and professional symbolic value in the context of creative knowledge work?

- Can mobility become a constituting element of identity and belonging for creative knowledge workers?

- Can mobility even become a professional advantage?

- How can the absence/presence duality be understood?

\footnotetext{
${ }^{59}$ Translated by the author from the German text: 'Wohnen und Mobilität stehen jedoch gemeinhin in einem Widerspruch zueinander und werden mit disparaten Handlungen verbunden: Wohnen als Seßhaftigkeit gilt als Voraussetzung für die Entwicklung stabiler sozialer Beziehungen, kulturell geteilten Wissens und nicht zuletzt für die Ausbildung einer lokalen Identifikation. Mobilität meint demgegenüber Bewegung, Fluss und Entbettung aus den genannten sozialen, ökonomischen und kulturellen lokalen Strukturen.'
} 
- How do mobile creative knowledge workers appropriate and adapt to new places?

- How do they integrate new places into their lifeworld?

- Have they developed routines in spatial orientation and appropriation?

Again, we could formulate some assumptions which result from the literature. First, we can think of mobility as being both beneficial and unfavourable to workers in creative industries. On the one hand, it might be that living in between two places can multiply the resources that are available to the individual creative knowledge workers. Access to several local networks might be valuable in terms of having more options to participate in interesting projects, having more stimuli for creation and innovation, as well as having better access to clients and customers. On the other hand, the duality of absence and presence comes along with mobility. In particular, the aspect of absence might be harmful to the integration of a multilocal creative knowledge worker into his various local networks. Local and more immobile people might feel irritated or even disenchanted by such a circular behaviour of mobile creative knowledge workers. As a consequence, the multilocal creative knowledge workers might be refused complete membership in local networks during moments of presence. Here, Dirksmeier (2012) provides the first valuable insights. He has studied how a Bavarian village was affected by a high share of multilocal people from larger cities. These multilocals bought property in the village and they mainly use that property as second homes during weekend trips or vacation, however, most of the year these houses and apartments are not inhabited by the multilocals. The temporary absence becomes visible in the public street life of the village (e.g. through closed window shutters, untended gardens). This phenomenon results in a negative village atmosphere, and the native immobile inhabitants feel disturbed by their multilocal compatriots. Dirksmeier therefore asks to include the impacts of the absence/presence duality on places, on physical and social environments, into the study of multilocality. He says (2012: 68f. ${ }^{60}$ ): 'The interdisciplinary research on multilocality is currently concentrating on the multilocal individuals and disregarding the perspectives of the respective continuously present inhabitants of the frequented spaces. Research results that declare multilocality as a genuinely positive aspect can only be achieved in this manner. [...] Particularly human geography would pose and answer further interesting, and in future more relevant questions for research if it applied a theory of multilocality which keeps in mind phenomena of absence and its impacts. ' Thus, we can see that mobile people seem to have an influence on the atmosphere and vitality of the local communities in the places that form part of their everyday lives. Finally, we should probably question a perspective which understands society as consisting of a group of 'mobile' people on the one hand and a group of 'immobile' people on the other hand. With his concept of Polytopizität, Stock (2009) presented a perspective in which embeddedness (Verankerung) and disembeddedness (Entankerung) have to be thought of as existing parallel in mobile lifeworlds. Similarly, Bonß, Kesselring et al. (2004) suggest that there is no opposition between the 'mobiles' and the 'immobiles'. In the second modernity, a more differentiated pattern has emerged in which new mixed types occur, e.g. the

\footnotetext{
${ }^{60}$ Translated by the author from the German text: 'Die bisherige interdisziplinäre Multilokalitätsforschung konzentriert sich momentan auf die multilokalen Individuen und vernachlässigt den Blickwinkel der jeweils dauerhaft anwesenden Bewohner der frequentierten Räume. Nur so können Forschungsergebnisse erzielt werden, die Multilokalität als genuines Positivum deklarieren. [...] Gerade die Humangeographie würde mit einer solchen resultierenden Theorie der Multilokalität, die Abwesenheitsphänomene und deren Auswirkungen mitdenkt, weitere interessante und in Zukunft akut werdende Forschungsfragen aufwerfen und beantworten.'
} 
'mobile immobiles' or the 'immobile mobiles'. Also Petzold acknowledges that such new combinations of mobility and sedentariness could be observed as empirical phenomena. With his 'crosshairs' of multilocality (Petzold 2010: 251), he presents a theoretical and terminological framework in which the possible theoretical perspectives could be found on the vertical axis - a continuum - between the extreme poles of 'focussing on sedentariness' and 'focussing on mobility'. According to him, individual lifeworlds will always consist of both mobility and immobility, but it depends on the researcher's perspective how this combination is looked at.

\subsection{The transnationalism paradox}

Hannah is a person who is deeply rooted in her city. Thus, her main reference frame for everyday life is the local, yet during her rare trips abroad she becomes very aware of her nationality and what this means in terms of her everyday life. Thus, she knows that the national frame embraces her local place. The national influences her local life, but Hannah does not always consciously perceive herself in the national context. For Merlin, the picture is a bit different. As a creative knowledge worker, Merlin is also rooted in the local, however, his professional field brings along a strong consciousness about the national scale. In particular, the cultural aspect of the nation, and less so the region, is also important to him. Working as a creative knowledge worker, culture is one of his main resources on which to draw. He needs to be proficient in the language, he must understand puns and equivocations, and he has to have a sense of humour and be able to joke. In sum, he needs to understand the symbolic and semantic system of the culture in which he operates. Thus, he deals with the spatially located culture in his place. In contrast, Daniel compares different such cultural environments to each other. By doing so, we can suspect Daniel of creating his own 'transculture' which is not placed in a specific locality, but which is rather bound to his person and his social networks which transcend geographic barriers.

The paradoxical situation here is that a creative knowledge worker who relies on their competence in playing with a locally or nationally embedded culture cognitively does not fit with transnational mobility. While one can still imagine that multilocality which occurs within one nation and one national culture might cause fewer problems, I think it is rather unclear how creative knowledge workers cope with different cultures in transnational settings. This again reveals some interesting questions:

- How are the national and local scales actually interrelated in creative knowledge workers’ transnationality?

- Do reference frames become multi-scalar?

- Do creative knowledge workers also relate to supra-national scale (e.g. Europe, European Union)?

- Do transnational creative knowledge workers develop transcultural products and services, or do they rather stick to the international exchange of national ones?

Again I would present some preliminary theoretical assumptions. Given that people's reference frames consist of different scales since nation-states have superseded the city states, it is nothing 
new that place-based identification and belonging is somehow multi-scalar. However, due to European integration and globalisation, new scales have emerged (e.g. EU) or have become less abstract/can be better experienced (the global through all forms of available physical, imaginative and virtual travel). The importance of individual scales for societal organisation has also changed ('the revival of the region as scale'). If subnational and supra-national scales are added to the catalogue of possible scales for place-based identification, one could suggest that creative knowledge transnationals might integrate them more easily into their reference frames because their frequent travelling allows them to experience e.g. the supra-national scale more directly in their everyday life. Andreotti, Le Galès et al. (2010) assume the hypothesis that transnationally mobile people withdraw to a certain degree from local engagement as they at least partially 'exit' the local scale - in the Hirschman sense - to profit from a transnational scale. Andreotti et al. studied managers in the larger European cities of Milan, Paris, Madrid and Lyon, and they found that there are different types of transnationally mobile managers. First, there are managers whose physical mobility is rather low, and who base their transnationalism largely on virtual practices. These managers often stick to local and national contexts. These rooted managers - even though working in transnational environments - strictly relate to the local community, having large circles of friends and family members in their neighbourhood or city and using local public infrastructures and services. Andreotti et al. conclude that this finding contradicts the hypothesis of the 'deterritorialised mobile and cosmopolitan elite' which stands opposed to the normal local people (Andreotti, Le Galès et al. 2010: 13ff.). On the other hand, there are managers whose physical mobility is high and who also engage in virtual transnationalism. These managers indeed develop partial exit strategies from the local and national contexts. These exit strategies concern the interaction with local neighbours and participation in local associations and initiatives. Even though they might profit from local offers, they do not actively engage for the benefit of the local community (Andreotti, Le Galès et al. 2010: 15f.). Furthermore, we could also argue that the national scale will lose its dominant power for identification once other categories emerge. In particular, the immediate local level - the quarter of a city (often illustrated as a city centre location with old-industrial urban fabric) is of symbolic importance for creative knowledge multilocals. Thus, I suggest that the local level is very important for them, whereas the national might exist in a subordinated position if the local is combined with the supra-national through transnational life experience. A characteristic of creative knowledge workers is their emphasis on tolerance and openmindedness - close to a Kantian cosmopolite. We could thus also suggest that the global scale might be an important scale for creative knowledge transnationals.

\subsection{The character of 'place’ in mobile creative knowledge lifeworlds}

So far, I have unreflectedly used the term 'place', and this has a reason. Looking at the abovementioned phenomena from a geographer's perspective, it would be intriguing to look at 'place' by using existing theoretical perspectives. However, I would like to keep my perspective unlimited from such theoretical considerations. Following the logics of ground theory (cf. details follow in the subsequent chapter) I will look at my study group's own - and thus an empirical - understanding of 'place'. Place, which I so far think of as a central term to vaguely circumscribe the local scale, seems of central relevance in the literature on creative industries, 'roots' as well as 'routes'. Without defining it precisely, all these strands of literature mention it as an important category of 
the individual lifeworld of people. Therefore, I would like to leave it open for my interview partners to define, how 'place' can actually be characterised such as in the context of creative knowledge transnationalism and multilocality? Answering this question is also one main aim of this thesis.

As a final remark, I would like to stress that this research project is an exploratory one, and I understand these sets of questions around 'creative knowledge multilocality', 'creative knowledge mobility', 'creative knowledge transnationalism' and 'places in creative knowledge lifeworlds', which I outlined in this chapter, as fields of interest that will guide the empirical fieldwork and the analysis. However, these fields of interest should not be understood as a rigid catalogue of research questions that should all be answered in the frame of this research project. They do not reflect a straightforward operationalisation process as used in quantitative research designs. My aim is to answer as many as possible, but I do not expect to find an answer for everything - as outlined above - which interests me. 


\section{Interlude E: Nikolina - Pia}

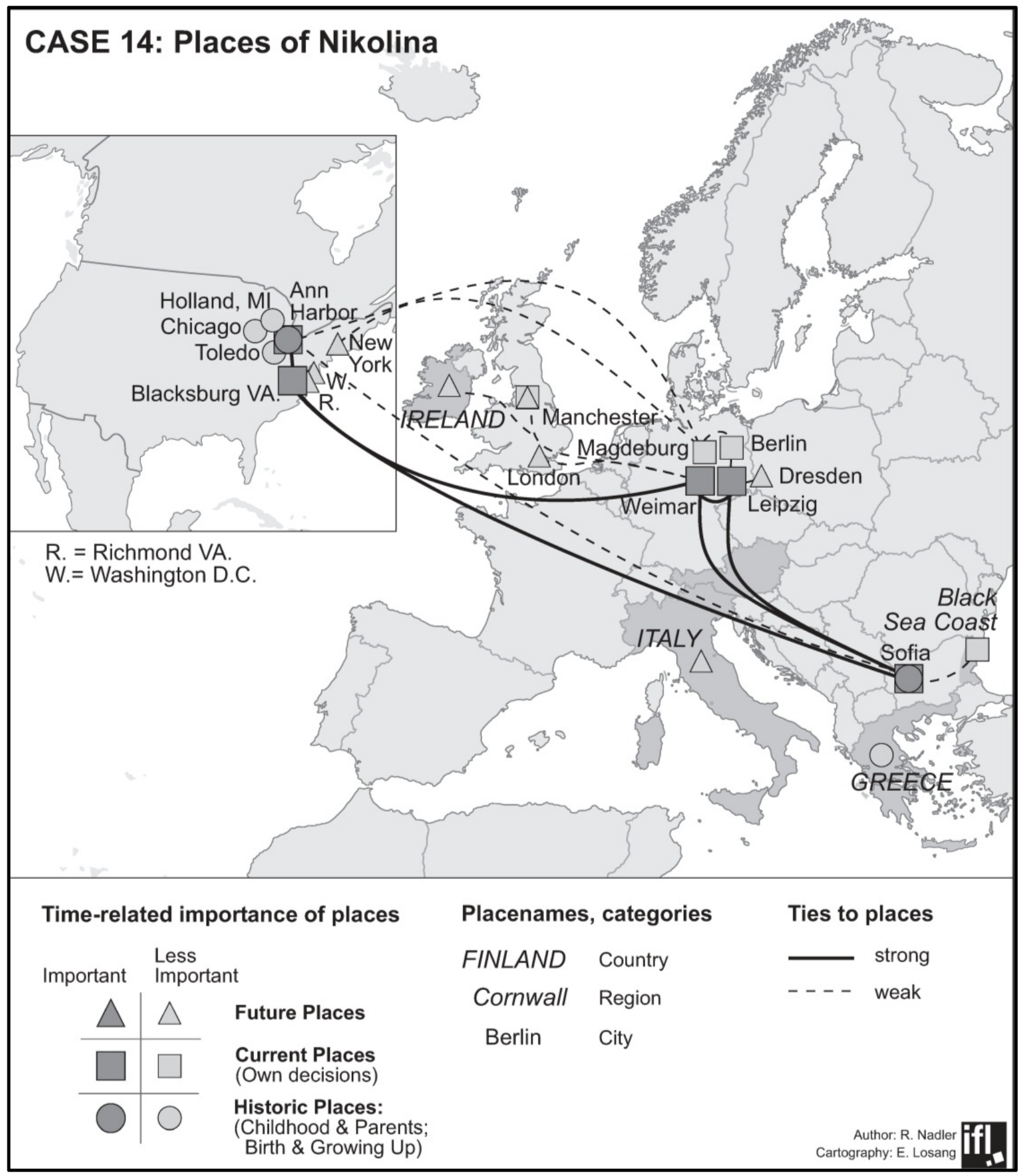




\section{Meet Nikolina}

Nikolina is Bulgarian-American Master's student in her 20s. She studies at the Bauhaus-University in Weimar, and she works as a student research assistant at a research institute in Leipzig, where she is involved in projects dealing with environmental issues in Bulgaria. Nikolina was born in Sofia, Bulgaria, but she moved with her mother to Toledo, Ohio, when her parents separated when she was six years old. She and her mother moved to Ann Arbor, Michigan later on, a small university town which she loved for its international students and the progressive atmosphere.

Throughout her high school years she kept her ties to Bulgaria, and went to Sofia for a month each year during summer vacation. She visited her father, her two Bulgarian grandmas and her cousins and family friends there. After high school finished, she went to Europe to refresh her Bulgarian language skills. She first arrived in Magdeburg, where she spent some months in an exchange family. As she didn't get along with them, she moved to Bulgarian friends from childhood who lived in Berlin, and after a few months she went on to Sofia. She describes her stay in Bulgaria as a time of liberalization from cultural constraints in the US. She partied, made new friends and enjoyed life, spending a lot of time on the Black Sea beaches.

Nikolina went back to the US, when her parents had moved to Blacksburg, Virginia. Her mother had started to teach at the university, and Nikolina joined her parents to study International Studies and Environmental Policy. During summer breaks she always went to Sofia and Ann Arbor. During that time she stayed at her grandmother's in Bulgaria; and at different friends' places in Ann Arbor. She went to Sofia again during the last semester of the 4-year programme. Formally, it was considered an exchange semester, but she used it to improve her scientific language skills in Bulgarian. After having handled her graduation bureaucracy in the US without going back there, she applied to universities in Amsterdam, Bologna, Freiburg and Weimar. In Weimar she arrived for her Master's programme, and from Weimar she came to the research institute in Leipzig, where she is currently working as a student assistant.

Nikolina has a special relation to travelling: she loves it for accessing new languages, cultures and ideas. Due to her personal history, she also has problems identifying her home. She mentions that home is manifold to her, including Sofia, Ann Arbor and Blacksburg, but she would distinguish home from the concept of being based in one place. Mobile people, including her, have bases instead of homes, places which have a temporary and more utilitarian character in life and where there is less emotional attachment. Nikolina also uses the concept of humbling in order to illustrate how social orientation in new places works: you subordinate your own culture to that of the new place. The incoming traveler or foreigner does not impose his own perspectives on the local people, in order not to seem arrogant. The social connection to a new place can only work like that. Her personal trick to get attached is making jokes with people and laughing.

Nikolina was engaged in environmental protection in the US, fighting a coal mine project in the mountains near Blacksburg. She feels that she is only temporarily based in Germany, so she doesn't get involved. She would love to do something for environmental protection in Bulgaria, but as she is based in Weimar, she cannot manage that.

In the future, Nikolina would like to move to another country, probably Italy, the UK or the Netherlands. She also thinks about moving to New York, but she has no concrete plans. Nikolina's story is mainly that of adapting attentively to foreign local communities, without imposing her viewpoints and opinions. 


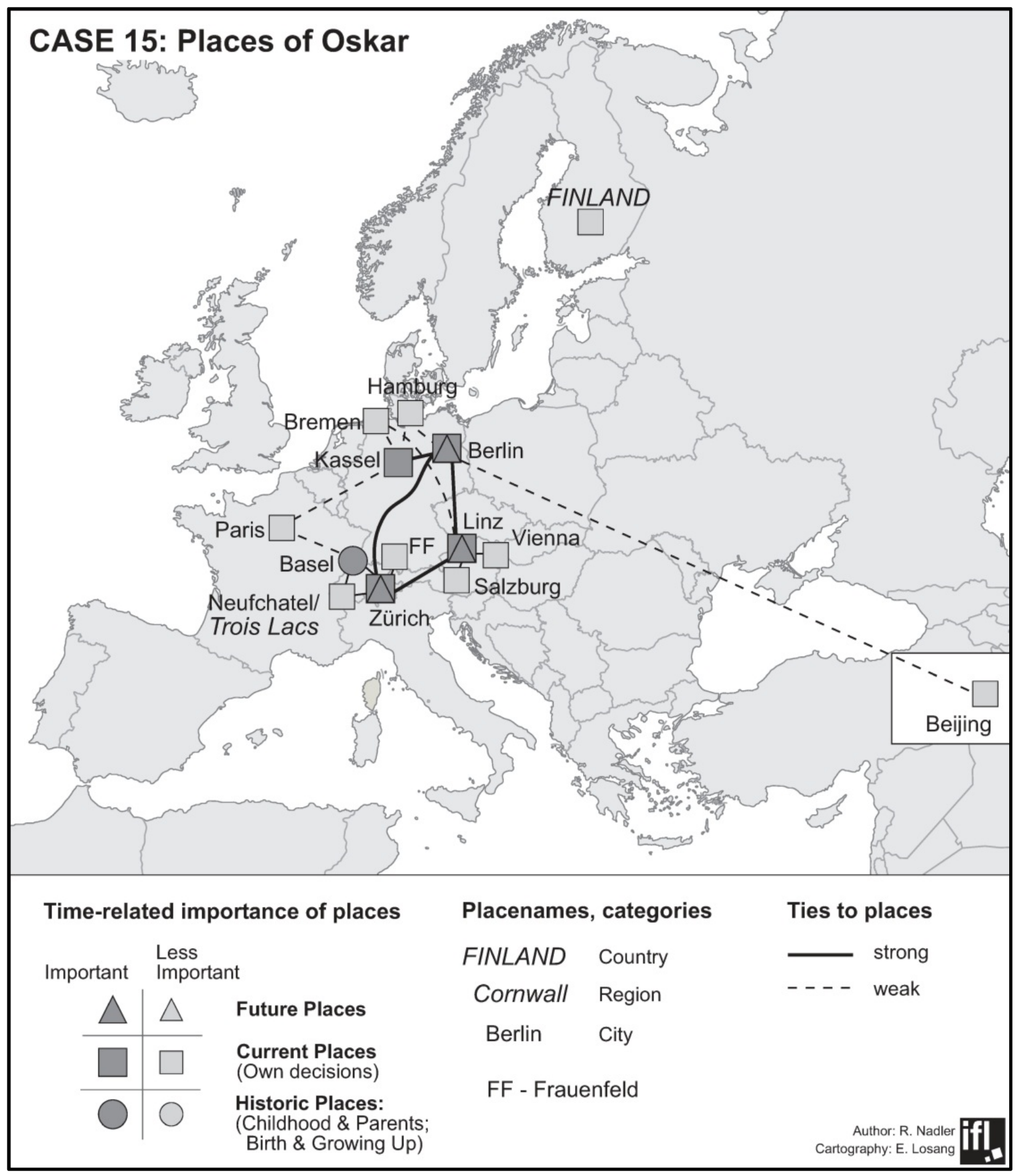




\section{Meet Oskar}

Oskar is a Swiss cultural entrepreneur in his 50s. He studied arts and ethnology and worked as a teacher in arts before becoming the director of a design museum in Zurich. He did this job for about 15 years, organizing cultural events and publications. But after a while he wanted to do something different than his stable job. Luckily, at that time the national Swiss outdoor event in the cultural field had to be planned and Oskar took the position of the Artist Director. The job was very stressful and a lot of work for about four years, so Oskar decided to become self-employed afterwards. His first jobs took him to Bremen and Linz. In Linz he stayed for some years to curate a cultural capital. And he liked Linz, so he bought a former tennis club house at the Danube River. Then his jobs in Berlin started, currently three different projects, and he started jobs in Hamburg and Paris, as well as smaller ones in Switzerland. Today his self-employment has turned into a firm based in Zurich with five employees.

Oskar describes himself as a person in motion. He always circulates between Linz, Berlin and Zurich, lately passing through Kassel, where his new girlfriend from Linz is working. He runs three apartments with very individual qualities in these cities, and it is important to him to have his own places with his own things. He has business suites in each apartment, sports shoes and his toiletries. This is important to him as he distinguishes the places from other places where also regularly goes for work - e.g. Hamburg and Paris - but where he stays in hotels.

Oskar also has difficulties saying where he comes from or where his home is. He says all the three places, Linz, Berlin, and Zurich, are his home - they merge as individual pieces into a coherent pattern of feeling at home. When it comes to identities, Oskar often changes his answers according to a given situation. This includes an opportunistic mode of doing certain things to avoid bureaucratic problems in the countries: e.g. even though it is more expensive, he only uses his Swiss mobile phone, in order to signal German financial authorities that he lives in Switzerland, while in Linz he considers himself to be from Berlin and Zurich, in order to emphasize his international experience.

Oskar does not participate in any civic engagement activities. First, he understands his work as closely related to local and regional development. E.g. he met in Berlin with Chancellor Angela Merkel or President Wulff to discuss the role of Humboldt Forum for Berlin as German capital, or he met highranking Austrian politicians during his activities for cultural capital in Linz. Thus, all of his work has to do with political issues and civil society. Second, Oskar has developed a certain realism towards politics and politicians: if he had more leisure time, he would prefer to play basketball instead of joining a local initiative.

For the future, Oskar says he will not make plans. He is very dependent on short-term developments in the market; he only plans three months ahead. In general, he likes his life spread around the three cities of Zurich, Berlin and Linz: he wants to remain three-fold at home. However, in any given circumstance he might change to a more dyadic pattern if his economic situation requires it.

Oskar's story is mainly that of a multi-local life in which places have a pronounced equal status. Oskar terms this a 'polyperspective' on life, which lets him see local situations with other eyes. 


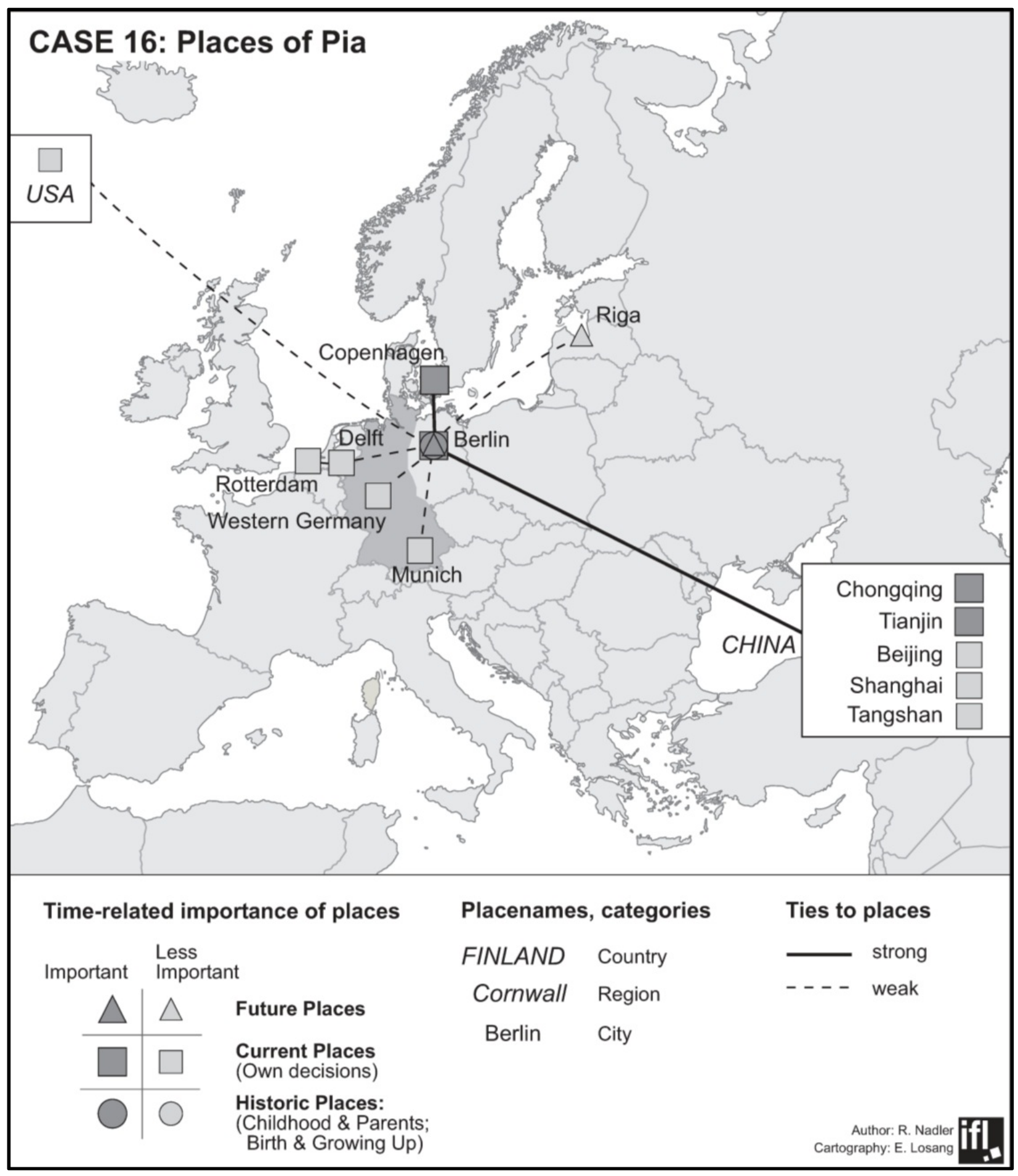




\section{Meet Pia}

Pia is a German architect in her 30s. She runs her own architecture firm in Copenhagen and works as a teacher at a university in Berlin. Pia was born and raised in Berlin, which she still considers as her home and the central place in her world. Pia studied architecture in Berlin, and during this time she went for an exchange year to the University of Delft in the Netherlands, adding on an internship in Rotterdam, which is one of the main locations in the European architecture scene. She started assisting a professor at the university back in Berlin while writing her thesis, and also began participating as a freelance architect in international competitions. Half a year after graduation Pia and her current office partner won second prize in an international competition and seized the moment to found the Copenhagenbased architecture firm. Pia then went to live in Copenhagen, where working as a young architect is easier than in Germany. During that time she started commuting between Berlin and Copenhagen, retaining links to the Berlin university. She also started to become more international by taking jobs in China.

Due to her strong emotional attachment to Berlin, she decided to move back to Berlin once the Copenhagen firm was launched successfully. She used her current part-time position in the university to re-enter the German labour market and social security, and is preparing the launch of a Berlin branch of her Copenhagen firm, in which she will play the leading role. Her firm now has 25 employees, and is mainly managed by her partner. Pia considers herself to be a free-floating atom which looks for new architecture competitions and initiates the firm's participation in these. She sees herself as the more creative head of the firm. The firm is very successful in Scandinavia and China and tries to use these references to enter the German market. Pia appreciates the weekly alternation between Copenhagen, Scandinavia and Berlin, Germany. She profits from the possibilities of young architects on the Scandinavian market, and this experience helps her in the more difficult German domestic market.

Furthermore, Pia frequently goes to the Netherlands to realize projects with her former employer, but also to visit the renowned Dutch Institute for Architecture (DIA). The DIA allows her to study marketrelevant trends in architecture at an early stage. Furthermore, she looks for skilled architects to hire for her firm in Copenhagen. She says that having worked in a Rotterdam architecture firm provides the most valuable skills to architects in Europe. With her firm's focus on industrial harbour conversions, she was recently invited to work in Riga, Latvia, and she is trying to extend business in the Baltic countries in general. Similarly, her current project in China deals with the harbour development in Tianjin. To Pia, her multilocality is both a professional necessity, in terms of escaping the low-pay German architecture market and learning new skills in other countries, as well as the realization of her expectations that working as an architect is related to travelling the world and visiting new and exciting places, therefore Pia is very satisfied with her life and when comparing herself with other architects, she believes that she is in an advantageous position. She combines her home feeling in Berlin, having friends and family there, with the work and professional spheres she can freely live in Copenhagen. Pia mainly identifies herself as a Berlin girl. She was raised in the city, and she feels that others associate positive things with the city: it's a cultural hot-spot and an electronic music scene. She also adds that she is linked to Copenhagen, as the city is widely known for its ecological progressiveness and openminded people. Formerly, Pia was part of a choir in Berlin, but she is currently unable to do any voluntary activities, as she is irregularly in her places, and does not have time. In the future she wants to continuously travel less and be able to stay for in Berlin longer times. Her general aim is to hold a fulltime professorship before she turns 40 . She would include new places in her pattern if it would help her career. Her general story is about straight career planning in combination with a strong attachment to her hometown of Berlin. 


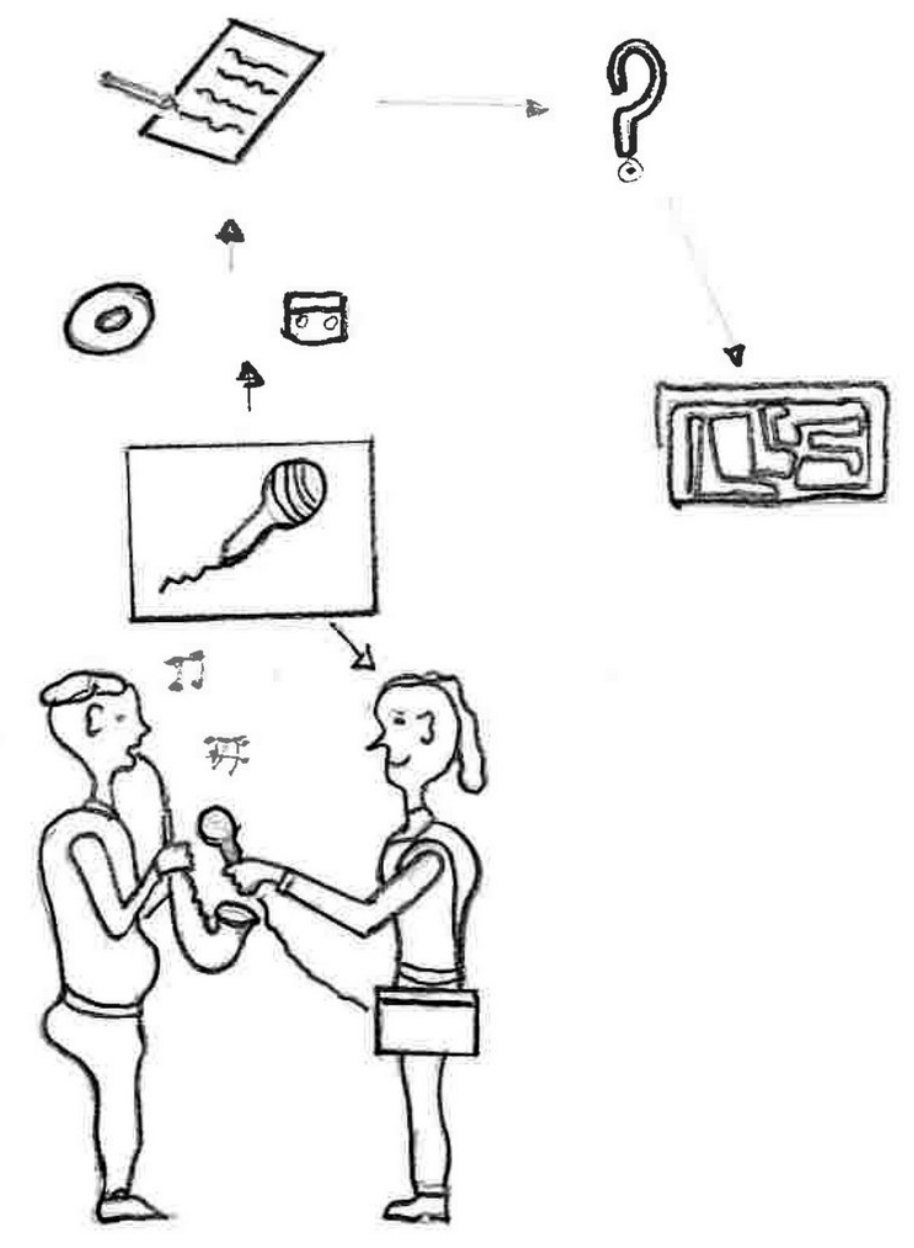




\section{Empirical design \& methodology}

Given the lack of systematic research on the specific group of 'mobile creative knowledge workers,' a qualitative approach seeking for exploration was chosen for this study. Applying such a methodology necessitates considering the character of the gathered data. I chose to take a very close and detailed look into a small number of cases in order to understand the complexity of the relation between mobility and belonging in creative knowledge workers' lives. Furthermore, I am interested in finding out about a possible importance of individual mobility in creative industries - thus another aspect which makes it even more complex. I will start this chapter with an introduction to the phenomenological perspective in empirical research. In the context of this perspective, the notion of 'lifeworld' - which I have used a lot without explanation - is introduced. It should help us to understand the overall frame for what I am studying in my individual cases (section 6.1). I will then speak about the method of how I gathered my data. Here, you will find information on my sampling strategy, on the character of the interviews I have done, on the mental mapping method that I used, and also a reflection on the interview situations and atmospheres (section 6.2). I will dedicate a third part of this chapter to the mode of analysis, describing in what I root my technique: namely, grounded theory, objective hermeneutics and qualitative heuristics (section 6.3).

\subsection{The phenomenological perspective: studying the lifeworld of others}

Phenomenology is a philosophical approach to science which became widely noticed through the work of Edmund Husserl in the $19^{\text {th }}$ century. Basically, it is the study of conscious action. A phenomenological perspective applied to human beings and social interaction understands the human being as existing in a state of 'consciousness', in which all action takes place with 'intention' and has 'significance'. Intention is based on the reflection about the difference between how things should be, and how they actually are. The human being embeds a large share of action within conscious reflection, and thus relates intentions to this action. Intentionality, however, is dependent on mutual understanding. Two interacting people have to have a shared understanding of this intention. Consequently, reference frames are an important issue in the study of social interaction if seen through a phenomenological lens. Basically the phenomenological position says: it's all about interpretation. People interpret what others signal them through their action, and the sociologist's role is to interpret the interpretation of others. This brings us to the notion of the 'lifeworld', which could be thought of as a person's individual reference frame based on previous experiences and personal knowledge stock (I will come back to this in section 6.1.1).

The phenomenological perspective in sociological research is based on early work by Edmund Husserl and Alfred Schütz, and it was re-discovered by Luckmann (Hitzler \& Eberle 2008; Grathoff 1995; Eberle 2000). Husserl (1969 [1936]) observed a crisis of European science which he found to be based on the fact that scientists did not take the real and existing lifeworld of most people into consideration. Scientists were often imprisoned in their theories and abstractions that were distanced from the social realities. Husserl wanted to illustrate the pre-existence of 'lifeworlds', independent of their potential scientific observations. For Husserl, the lifeworld is the original sphere. It is the pre-existing foundation of everyday practice and reflection. It existed before 
scientific observation. Lifeworlds are always individual. Each person lives in its own lifeworld in which, through scientific study, evidence could be found for more formal social structures (cf. Hitzler \& Eberle 2008: 109/110).

Alfred Schütz (in co-authorship with Luckmann; see Schütz \& Luckmann 2003) later adopted these early reflections of Husserl. Schütz observed that social sciences, in opposition to natural sciences, are dealing with recapitulating social 'sense making' and 'significance'. According to Schütz, we can only find larger societal structures of sense making by looking through the lifeworld of the individual.. Sociological research is thus the reflection about the similarities between lifeworlds and their sense making arguments. In this epistemological perspective the 'lifeworld' is both a reference frame for social sciences as well as their empirical object of study. On the basis of his own lifeworld, the sociologist should try to understand the lifeworlds of others in a given thematic field. The aim is to identify universal structures of subjective constitution (Hitzler \& Eberle 2008: 111).

Schütz looked for a secure way through which sociology could understand (Verstehende Soziologie). He refers to Weber's understanding of sociology as a science which seeks to understand subjective sense making related to individual action and practice. How people give sense to their individual action must be analysed. As Hitzler \& Eberle resume (2008: 112 ${ }^{61}$ ), 'Explaining social phenomena based on individual action necessarily calls for the study of the subjective sense that this action has for the acting individual.' In Schütz' explanations, sense making works through conscious reflection (Bewusstseinsakte). A person experiences different situations in life, and they give these experiences a certain sense related to other experiences. A superposed system of more abstract senses develops through a process of grouping single experiences in a person's mind. Thus, a single experience receives a certain sense in reflection on former experiences.

The interpretation of the sense of individual experiences might change with time and with the accumulation of new experiences as well as a change in the personal sense system. Schütz's main interest is to understand how people understand each other even though they cannot look into the other's mind. He thinks that mutual understanding evolves from signification. Sense is transported through signs that have to be interpreted by the recipient on the basis of their personal experience and knowledge, and in the frame of a situative relevance system (situatives Relevanzsystem). The recipient thus can only partially understand what the sender of a sign means, as the sender's experience and situative relevance system is different from that of the recipient. The closer and the more contemporary both sender and recipient are in space and time, the better they can understand each other, as relevance systems and experiences might be more similar.

As a consequence, social sciences have to acknowledge that sense in the social world is prestructured. It is a structure of 'primary order', while the scientific abstraction and theoretisation is a structure of 'secondary order', based on this primary order. Schütz defines two methodological aspects that become relevant. First, subjective interpretation is the central tool in developing sociological models, which are based on observed action and the exploration of sense in this action. Second, the sociologists' concepts and models have to be adequate. This means they have to reflect

\footnotetext{
${ }^{61}$ Translated by the author from the German text: 'Soziale Phänomene aus den Handlungen der beteiligten Individuen zu erklären, muss daher heißen, auf den subjektiven Sinn zu rekurrieren, den diese Handlungen für die Handelnden selbst haben.'
} 
the sense systems and the understandings of the studied individuals and their lifeworlds. They should be understood by the studied individuals as they use common language, sense, and concept. This should avoid sociologists from constructing theories and concepts which do not exist in the real world (cf. Schütz \& Luckmann 2003).

Reflecting on Schütz's work, Hitzler \& Eberle (2008: 114) conclude that experience is the central issue in the phenomenological perspective. Phenomenological analysis of the 'lifeworld', for example, means looking for shared and more general sense making and interpretation in society, which could be characterised as the 'objective'. Furthermore, how these objective senses are internalised by the individual and how individuals change these objective senses has to be studied. For the scientific practice, this implies a necessity to systematically reconstruct multiple experiences. Consequently, there is no objective facticity (objective Faktizität), but only subjective constitutions of consciousness and sense making. Hitzler \& Eberle indicate that between individual lifeworlds - including their relevance systems and stocks of experiences - there are several 'intersubjective' overlaps which facilitate orientation in everyday life. Shared interpretations are a necessary tool for living together. This means that social practice is first of all a communicative action which includes a large share of interpretative work. Interacting subjects have to define the degree of overlapping between their individual lifeworlds. Hitzler \& Eberle (2008) also point to the fact that the lifeworld is divided into so called 'small lifeworlds', which Marx termed 'specific worlds' (Sonderwelten). These fragments of the lifeworld enable relating single experiences to a shared, inter-subjective stock of knowledge (e.g. the professional world, the family world). Only a particular relevance system is applicable in each 'small lifeworld', and thus complexity is reduced. Hitzler and Eberle illustrate this with the growing differentiation of human knowledge stocks into small expert knowledge systems, shared and understood by selected members, but which are not understood by non-members. In particular, in societies characterised by individualization and differentiation, the logic of studying individual lifeworlds becomes a necessity. Kurt (2002) remarks that social phenomenology deals with the consciousness about the consciousness of others. Kurt refers to Husserl's understanding of consciousness as the experience of the position of the 'Me' (Ich) in relation to another relevant 'object'. For Husserl, the Ich and the other object form opposite poles in the mind. Through controlled reflection and reconstruction -which is the sociologist's job the constitution of society and its internal relations should be made possible. Social phenomenology could be brought to the point in the following phrase (Kurt 2002: $253^{62}$ ): 'The core of this argumentation is the idea that the human being is a relation which relates in understanding ways to itself and to others through setting and interpreting symbols.'

This goes along with a growing relevance of hermeneutics and explorative methodological designs. Hitzler \& Eberle (2008: $118^{63}$ ) comment: '[Opposed to the phenomenological insight that different

\footnotetext{
${ }^{62}$ Translated by the author from the German text: 'Den Kern der Argumentation bildet der Gedanke, dass der Mensch ein Verhältnis ist, das sich Zeichen setzend und Zeichen verstehend zu sich und zu anderen verhält.'

${ }^{63}$ Translated by the author from the German text: '[Gegenüber der phänomenologische Erkenntnis, dass unterschiedliche Wissensarten relevant sein können] setzt das Testen von Hypothesen im Rahmen des deduktiv-nomologischen Erklärungsmodells sozusagen implizit - voraus, dass Menschen unter gleichen Bedingungen gleich handeln. In Gesellschaften mit vorwiegend traditionaler Orientierung ist dies zwar oft der Fall, in modernen Gesellschaften jedoch lediglich im Bereich von Routinehandlungen. Je mehr moderne Gesellschaften durch Enttraditionalisierung, Optionensteigerung und Individualisierung geprägt sind (Gross 1994, 1999) und je öfter die Akteure ihre Situationen um- oder neu interpretieren, desto kontingenter wird ihr
} 
types of knowledge might be relevant], the testing of hypotheses in the frame of deductivenomological approaches to explanation - somehow implicitly - requires that people act equally under equal conditions. This is often the case in societies with mainly traditional orientation, but in modern societies this only happens in the sphere of routinised action. The more modern societies are characterized by detraditionalisation, the increasing options [for acting] and individualisation (Gross 1994, 1999), and the more often individual actors re-interpret their own situation, the more their knowledge and action becomes contingent, the more also the predictive power of 'if-then' assertions fades, and the more urgent explorative and interpretative research designs become (cf. also Hitzler 1997, 1999b).'

Equally, Kurt (2002) mentions that objective positions in science have only limited applicability. They have their value in studying objects that are independent of subjectivity; they could be applied to measurable 'quasi-natural' laws (Gesetzmäßigkeiten), and often they are embedded in inductive approaches. Yet, phenomenology is a completely different perspective; it is not about a description of the obvious and visible facts, but it deals with the question how these facts become obvious. The central focus in social sciences is on 'phenomena' such as experience, perception, sense making and consciousness. It is first of all a reflexive process into which the scientist also has to bring in his personal experiences and lifeworld. Kurt says (2002: 10 ${ }^{64}$ ): 'The starting point of social phenomenology is not social realities but symbolic materials which are interpreted in respect to the social sense that can be expressed through them. The aim of understanding in social phenomenology is the development and application of ideal types, which can explain inter-personal relationships.'

Given the fact that creative knowledge workers use symbolic content as a central resource for their work, I think it becomes useful to take a phenomenological perspective for the analysis of the interviews. Remembering the words of Kurt (2002) that human beings are relational and they send and receive signs and symbols, the creative knowledge worker per se could be understood as an obvious object to study. Creative knowledge workers continuously send signs and produce symbols; they also interpret signs sent to them by others in reflection about their own role in society and their own perception in the peer group.

Furthermore, phenomenology might also be helpful in studying the perception and consciousness of space. In that sense, the humanist geographer Anne Buttimer (1976: 280) already referred to the value of a phenomenological understanding of individual space perception in the 1970s: 'Traditional phenomenologists have recognized that man, the cognizing being, is anchored in a physical and social world, and that this 'world' situation influences the meanings and intentionality of his consciousness.' I believe that social phenomenology is a useful reference in this research project in order to provide a framework for understanding the positioning of the self in the world.

\footnotetext{
Wissen und Handeln, desto mehr verkümmert die Prognosefähigkeit der Wenn-dann-Aussagen und umso dringlicher werden explorativ-interpretative Forschungsdesigns (vgl. dazu auch Hitzler 1997, 1999b).'

${ }^{64}$ Translated by the author from the German text: 'Ausgangspunkt der Sozialphänomenologie sind dabei keine sozialen Realien, sondern zeichenhafte Materialien, die im Hinblick auf den sozialen Sinn, der in ihnen zum Ausdruck gekommen sein könnte, interpretiert werden. Das Ziel der verstehenden Sozialphänomenologie ist die Bildung und Anwendung von Idealtypen, die zwischenmenschliche Beziehungsformen erklären können.'
} 


\subsubsection{Lebenswelt: The lifeworld concept}

A central term of social phenomenology is 'lifeworld'. The concept of lifeworld was mainly developed by Alfred Schütz, who was inspired by Husserl's idea of the 'Welt der natürlichen Einstellung' (world of natural attitude) as well as Scheler's 'natürliche Weltanschaung' (natural worldview) (cf. Esser 1991: 12). Esser (1991) identifies three main characteristics of Schütz’s lifeworld/Lebenswelt. First, people do not question the knowledge which is relevant for living in the lifeworld. Second, this lifeworld knowledge has a certain character itself. Third, there are systems of relevance which make certain knowledge a part of a lifeworld or not.

Concerning the first point, Schütz (1944) formulated the idea that each adult person automatically has a certain knowledge stock available. This knowledge stock consists of knowledge gathered through former experience in a given context, and which was transformed into a type of manual for future behaviour in the same context and in similar experiences. A functional element, here, is the assumption of constancy (Konstanz); in the lifeworld, the constancy of the lifeworld structures, the constancy of the relevance of one's own knowledge for a life in exactly this lifeworld, and the constancy of our own capacities to influence our lifeworld are all unquestioned by a person. Only situations of strong ruptures in life, e.g. as described in the figure of the 'stranger' being unfamiliar with a new place, allow questions to arise (cf. Schütz 1944). If the usual practices and knowledge for problem-solving no longer work in a different context (a new situation, an unknown place), then we think about the knowledge that we apply in action routines.

What's the specificity of the lifeworld knowledge? Esser (1991) explains that the lifeworld knowledge is structured in a system of plans and zones with different ranges (Reichweiten). Furthermore, this knowledge is ordered through a process of typification. We construct typical situations or problems and ways to act successfully and satisfyingly, which also means solving problems in an appropriate way, then these types are continuously tested by everyday life experience. If we are confronted with a new problem for which we cannot apply our lifeworld knowledge, we develop new lifeworld knowledge to be used when a similar problem shows up again in the future. Here it is sufficient to only develop a basic knowledge that suffices to solve the current problem, but not to solve the problem in the absolutely best way. This pragmatism is essential in the lifeworld, as there is less time and space for analysis and reflection about details. If we thought about everything in detail, problem solutions would become too complex and we would not be able to react to problematic situations anymore. In many situations, we act in a way in which it is less than $100 \%$ probable that we are doing is the right thing. This is simply because we cannot afford to search for complete information for solving a problem the best way.

This nature of the lifeworld knowledge includes the fact that people are normally 'experts' meaning they have an understanding of 'why' things work the way they work - in only a limited number of thematic fields. In the majority of fields people are laymen (Laien); they only know 'how' things work, yet no longer 'why'. The more distant a thematic field is from the person's lifeworld, the less they know 'how' things work, until only rudimentary knowledge of 'what' things work remains. On the fringes of lifeworld knowledge, we then only find 'beliefs', 'blind trust', mere 'acceptance’ and sometimes even complete ‘ignorance’ (Esser 1991: 15). 
Lifeworld knowledge is also integrated in a double sense. First, individual knowledge fragments are integrated within the person's individual knowledge stock, allowing for appropriate acting in the own lifeworld. This integration is important so that a person can apply everyday life theories (Alltagstheorien), which are causal assumptions between means (Mittel) and ends (Zweck). On an individual level, a person disposes of a set of thematic knowledge fragments which are all related to each other through a super-ordinated unity, and which as a whole make sense for the person and give sense to their life and actions.

Second, a person's thematic knowledge is integrated into inter-subjective knowledge systems (Esser imagines them as institutions) which allow people to forecast other people's behaviour. Lifeworld knowledge has thus a fundamentally inter-subjective character. This inter-subjectivity of knowledge is defined by what Schütz calls the 'reciprocity of perspectives'; people can take others' standpoints. Additionally, it is also defined by the pre-existence of some parts of the knowledge. Typifications and sense relations are normatively pre-existing the individual person in a society. They will be internalised by the person during the socialisation process. Finally, inter-subjectivity is also defined by the institutionalization of knowledge. Two not directly interacting people can share institutionalised knowledge which still makes their acting appropriate. Esser (1991) formulates the example of a person sending a letter through the institution of the postal service and the postman who delivers the letter. Both people have not communicated directly, but the letter reaches the destination.

Finally, 'relevance' is important in the organisation of knowledge. Depending on individual motives and interests, not all people share the same knowledge, as only certain thematic fields are relevant for the one, but not for the other. According to Schütz, there are two types of relevance: motivational relevance (Motivationsrelevanz) and thematic relevance (thematische Relevanz). The first refers to the fact that people select relevant aspects within a given situation based on personal interests and super-ordinated motives that are important for their own life. Esser mentions that in particular language and communication are important for defining this motivational relevance (1991: 18f. ${ }^{65}$ ): 'In this we can see the paramount importance of communication and language for the (discharged) execution of everyday life's actions; not in the transfer of immediate contentual 'information', but in its continuous and ascertaining reference to the momentous structure of relevance each time, as well as the respective and sufficiently routine action which promises success.'

Thematic relevance occurs when the routinised acting, which itself is based on motivational relevance, no longer works in situations that are so new that one's own lifeworld knowledge is not sufficient to master the situation. In such a situation, one's own knowledge becomes questioned and more relevant knowledge is added or put into new relations with other existing knowledge. With the words of Esser (1991: 1966): 'Before [related to motivational relevance] the definition of a given

\footnotetext{
${ }^{65}$ Translated by the author from the German text: 'Hierin liegt die überragende Bedeutung von Kommunikation und Sprache zur (entlasteten) Abwicklung des Alltagshandelns: Nicht in der Vermittlung unmittelbarer inhaltlicher ,Informationen', sondern in ihrem fortwährenden vergewissernden Verweis auf die jeweils bedeutsame Relevanzstruktur und das damit verbundene und hinreichend erfolgversprechenden Routinehandeln.'

${ }^{66}$ Translated by the author from the German text: 'Zuvor war die Definition der Situation ein Teil der Lösung des Problems, jetzt ist sie selbst das Problem.'
} 
situation was part of the solution to a problem, now [related to thematic relevance] it [the definition of a given situation] is itself the problem.' Thematically relevant knowledge is built up in these circumstances until a new and sufficient routine for the definition of the formerly new and unknown situation is developed.

For Esser, the lifeworld is mainly structured by clearly identifiable relevance structures, by routines and typifications of situations, as well as by institutions of ritualised behaviour. They have the function of making the everyday behaviour less difficult and more efficient. They are necessary because of the limited time and resources to continuously question everything and to deal with surrounding world's complexity.

Everyday life and the lifeworld

Schütz \& Luckmann (2003) also thematise the difference between the 'theoretical lifeworld' and the 'everyday lifeworld', which they both understand as 'modes of being'. As a starting point, they formulate their definition of an 'everyday lifeworld' (2003: $29^{67}$ ): 'The specific segment of reality which the awake and ordinary adults in a mindset of sanity and reason simply take for granted should be understood as everyday lifeworld. We consider everything as 'simply given' which we experience as unquestioned, all situations which are unproblematic to us for the time being.' The everyday lifeworld is the frame for the experience of life as such, in which there is no distanced and theoretical reflection necessary, as it would instead be for answering questions in the scientific mode of thinking. Schütz \& Luckmann say (2003: $42^{68}$ ): 'While this general opaqueness of the everyday life stock of experiences appears as a deficiency from the viewpoint of theoretical knowledge, one has to recall that in the natural mindset I am dominated by the pragmatic motive. The stock of experiences serves the solution of practical problems. In the theoretical reflection I can make doubt a methodological principle. In contrast, in the world of everyday life, I am interested in being able to orientate in routinised ways by my behaviour. The interpretations that are sedimented in my knowledge stock have the status of a manual: if things are so-and-so, then I will act so-andso.'

The lifeworld as such is inter-subjective, as it includes other people. There are inter-subjective overlaps between individual lifeworlds, e.g. shared modes of interpretation, or mutual understanding. In particular in the everyday life modus, several things of the lifeworld are taken for granted and are not questioned (Schütz \& Luckmann 2003: 30):

1. The physical existence of other people;

2. The consciousness of other people, which is similar to one's own consciousness;

\footnotetext{
${ }^{67}$ Translated by the author from the German text: 'Unter alltäglicher Lebenswelt soll jener Wirklichkeitsbereich verstanden werden, den der wache und normale Erwachsene in der Einstellung des gesunden Menschenverstandes als schlicht gegeben vorfindet. Mit ,schlicht gegeben' bezeichnen wir alles, was wir als fraglos erleben, jeden Sachverhalt, der uns bis auf weiteres unproblematisch ist.'

${ }^{68}$ Translated by the author from the German text: 'Während diese allgemeine Undurchsichtigkeit des lebensweltlichen Erfahrungsvorrats vom Standpunkt theoretischen Wissens als ein Mangel erscheint, muß daran erinnert werden, daß ich in der natürlichen Einstellung vom pragmatischen Motiv beherrscht werde. Der Erfahrungsvorrat dient mir zur Lösung praktischer Probleme. Im theoretischen Denken kann ich den Zweifel zum methodologischen Prinzip machen. In der Welt des Alltags liegt mir dagegen daran, mich routinemäßig in meinem Handeln orientieren zu können. Die in meinem Wissensvorrat sedimentierten Auslegungen haben den Status von Gebrauchsanweisungen: Wenn die Dinge so und so liegen, dann werde ich so und so handeln.'
} 
3. The environment and its elements are the same for oneself and the surrounding others, and they have the same meanings (Bedeutungen);

4. The possibility to interact with others;

5. The possibility to communicate with others;

6. The pre-existence of a structured cultural and social world, in addition to the natural world, which works as a shared reference frame for myself and others;

7. One's own situations are only to a limited extent caused and produced by oneself.

In this sense, the lifeworld influences our behaviour through its enframing function, but we also structure and influence our lifeworld through the way we act in everyday life. Living in the modus of the everyday lifeworld means that we think and reflect in pragmatic ways. We base our interpretations of individual situations on an internalised knowledge stock which is our reference frame and which is based on our former experiences, as well as the experiences of others provided to us through communication. 'Every lifeworld interpretation is an interpretation in the frame of something formerly interpreted, within a general and rather familiar reality' (Schütz \& Luckmann 2003: $33 / 34^{69}$ ), and people trust in the continuity of their knowledge stocks' validity (Gültigkeit). This implies the basic assumption that the world is constant and does not change, so that knowledge remains functional. In the everyday lifeworld, a person continuously relates new experiences and situations to previously experienced situations. Thereby, situations can principally be understood as problems that have to be solved. Former situations led a person to develop proper solutions. In everyday life, we are continuously confronted with new situations, and we first try to solve their problems with solutions recorded in our knowledge stock. We group solutions in typologies, and we use them to pragmatically and rapidly solve new problems, without having to reflect on it. Sometimes we do not even experience this consciously. That is the essential character of the everyday lifeworld. Things are self-evident (selbstverständlich).

Yet, what happens if our problem solutions do not suffice in solving new problems? Then individual knowledge stocks are reflected consciously in the background of the current situation/problem, and they become adapted. This process can include a modification of existing typologies until similar experiences can be lived without questioning and reflection again. On the other hand, we can also face the problem that our typologies are in themselves incomplete to master our life. Then not only a type has to be adapted, but new types have to be created. This normally occurs if we are confronted with completely new situations in unknown environments. 'Familiarity is only familiarity in relation to typical things, while the atypical aspects of the individual horizon remain undefined, because a typification concerning them proved superfluous - within the respective past interpretational situation' (Schütz \& Luckmann 2003: $40^{70}$ ). This means there is certain rationality in the way people create their knowledge; problem solutions are only temporary and not valid forever (for the time being/bis auf weiteres). Third, there is also the basic problem that the knowledge stock, in form of a catalogue of problem solution strategies, is not integrated

\footnotetext{
${ }^{69}$ Translated by the author from the German text: 'Jedes lebensweltliche Auslegen ist ein Auslegen innerhalb eines Rahmens von bereits Ausgelegtem, innerhalb einer grundsätzlich und dem Typus nach vertrauten Wirklichkeit'

70 Translated by the author from the German text: 'Vertrautheit ist lediglich Vertrautheit mit Bezug auf Typisches, während die atypischen Horizontaspekte unbestimmt bleiben, weil sich mit Bezug auf sie eine Typisierung als überflüssig erwies - in der jeweiligen vergangenen Auslegungssituation.'
} 
logically into a meta-order. In everyday situations, a person can experience that one reference frame (Bezugsschema) is not sufficient for mastering the current situation, and they start to use other reference frames in order to find a solution. This includes that reference frames are reflected in their relation to each other, and a process of ordering the knowledge stock takes place.

Schütz \& Luckmann later characterise the style of experience (Erlebnisstil) that is connected to the everyday lifeworld (2003: 69/70). For them, the everyday lifeworld is particularly this part of reality in which mutual understanding is possible. It is the sphere of the lifeworld in which the self's own action (Handeln) causes changes. These changes then are perceived by the self, and they reveal the mutual influence between the self and the others. One's own way of acting is directly evaluated by others, and a feedback is inter-subjectively produced and experienced. This particular style of experience consists of:

1. Attention to life: in a cognitive mode of being completely awake and attentive to what happens around the self;

2. Epoché der natürlichen Einstellung: there is no doubt about the existence of the outer world and its objects; there is limited reflection about a possible other character of the world;

3. The dominant form of acting is corporeal and intentional (sinnvoll);

4. Others are experienced as consciously thinking fellow human beings (Mitmenschen) who share an inter-subjective world in which communication and interaction is possible;

5. Self-experience is bi-fold: The socially determined 'self' exists in form of social roles; and the free 'I' acts.

The time frame which is relevant in everyday life is defined by the junction of world-time and 'inner time' as a shared structure of time within an inter-subjective world.

Also Grathoff (1995) deals with the specificity of everyday life (Alltag). In particular, he tries to explain the relation of everyday life to the wider lifeworld. Grathoff remarks that many studies use both terms as mutual substitutes. Yet, he sees the necessity for distinctive definitions. Lifeworld is defined by Grathoff as follows (1995: 93 ${ }^{71}$ ): 'For a first approximation 'everyday life' should be described as the concrete and vivid encompassing plenitude of the empirical experience of actors', who - mutually orientating towards each other, relating to absent others, and heading forward to the future - look for orientation in, and define situations according to the historic and biographic inventory of an always pre-existing society.' Everyday life thus refers to a continuous sequence of stories, situations and action that happen in a pre-structured social world. Other than everyday life, the lifeworld is first of all a phenomenological concept, which builds the frame for everyday life. Lifeworld refers to the sensual-conscious corporeality (sinnhaft-bewusste Leiblichkeit), while 'everyday life' consists of the actual living enframed by this lifeworld.

In everyday life, there are certain social facts (Sachverhalte) that could be studied directly, but in the phenomenological lifeworld concept they are linked to other terms, as Schütz described. Grathoff (1995) explains that social relations are translated into inter-subjectivity; social consciousness becomes intentionality; social sense could only be observed through reduction; and

\footnotetext{
${ }^{71}$ Translated by the author from the German text: 'In erster Näherung soll mit 'Alltag' die konkrete und lebendige, umfängliche Fülle der Erlebniserfahrung von Handelnden bezeichnet werden, die, aneinander sich orientierend, auf abwesend Andere sich beziehend und auf Zukünftiges zugehend, im historischen und biographischen Bestand einer stets vorgegebenen Gesellschaft ihre Orientierung suchen und ihre Situation definieren.'
} 
communication could be reflected in the typology of the lifeworld. Grathoff makes it more explicit (1995: 106-112). Social relations in everyday life are often equated to relationships between persons. By contrast, inter-subjectivity in the lifeworld concept refers to the relation between preexistent 'objectivities' of an interaction as well as its subjective experience and conscious communication about it. Grathoff also thinks that phenomenology and the lifeworld concept only allow for a study of action (Handlung), but not of acting (Handeln). This is due to the fact that Schütz's phenomenological position conceptualises everyday life's consciousness (Bewusstsein) as intentionality (Intentionalität) in the lifeworld. Intentionality is characterised by the conscious copresence of the 'intending' and the 'intended'. This means there is more to interaction than mere conscious acting. Third, Grathoff argues about the difference between social sense in the lifeworld and in the everyday life. According to Schütz, there are different and finite sense areas. Lifeworld forms the overall frame for all these finite sense areas. The lifeworld is somehow a holistic horizon. Yet, everyday life constitutes its own finite sense area (Sinnbereich) and its own cognitive style within the lifeworld. Other sense areas would e.g. be the game, the scientific reflection, the fantasy, the dream, which all have their own cognitive styles. Grathoff remarks (1995: 109' ${ }^{72}$ ' While the lifeworld represents the encompassing horizon of sense for all finite sense areas (Schütz has never distanced from this conception by Husserl), the distinguished sense area of everyday life is limited.'

Finally, Grathoff also explains how scientific and everyday life typologies (Typiken) go together. As for the second, he remarks (1995: 110f. ${ }^{73}$ ): 'We are born into a world (as Schütz says), which is pre-structured by typologies mediated through conversational language. Already in the earliest stages of language acquisition we perceive dogs as typical dogs, mothers and friends as typical ones. It is not the specific and unique opposite, but it is the type rooted in former experience which guide the way we act in our everyday life.' In the everyday life sphere, typologies thus are transported and learned through language, and they also evolve. In scientific reflection, ideal types based on a Weberian conception, are the counterpart of typologies. For everyday life it is now important to understand communication as a mode to transcend one's own subjective everyday life and let others participate in the experience of their own everyday life. Communication in everyday life is thus a mode to immediately experience what the other person has experienced. Doing so, communication helps to generate shared types within a typology. At each moment, the personal environment is interference between one's own and other people's everyday life.

Here, two different styles of transporting a message through communication become relevant. First, the everyday life sphere only knows 'types of experiences', and each experience told within a communication receives sense from another person through linking it to a pre-existing type. Second, in the lifeworld phenomenology, the question is what holistic system stands behind the typologies of the everyday life?

\footnotetext{
${ }^{72}$ Translated by the author from the German text: 'Während die Lebenswelt der umfassende Sinnhorizont aller finiten Sinnbereiche ist (Schütz hat sich nie von dieser Husserlschen Konzeption distanziert), ist der ausgezeichnete Sinnbereich des Alltags begrenzt.'

73 Translated by the author from the German text: 'Wir werden in eine Welt hineingeboren (so Schütz), die von der umgangssprachlich vermittelten Typik stets vorgeprägt ist. Schon in den frühesten Phasen unseres Spracherwerbs nehmen wir etwa Hunde als typische Hunde, Mütter und Freunde als typisch solche wahr. Nicht das konkret-einzigartige Gegenüber, sondern der in Vorerfahrungen gründende Typ bestimmt meist unser Alltagshandeln.'
} 
I think this introductory review of the social phenomenology's central terms 'lifeworld' and 'everyday life' helps to frame the exploratory research on a micro-sociological level. In particular, the descriptions of how knowledge stocks are organized and how they are used in pragmatic ways in everyday life while they are reflected more deeply in non-everyday life modes of being might prove helpful for approaching some of the questions that I have developed in chapter 5. For example, I would assume that it can help to understand how multilocal creative knowledge workers can orientate and appropriate new places in their multilocal lifeworlds. The questions that deal with the role of the transnational momentum in their lives might also be better reflected through the lens of the phenomenological perspective.

\subsubsection{Appräsentation: The cognitive foundation of the lifeworld}

Another central term in social phenomenology is the technique of Appräsentation/appresentation. Schütz \& Luckmann speak of appresentation as a mode of crossing the borders of time and space (2003: 634-641). On space and time experience in the everyday lifeworld they say (2003: $71^{74}$ ): 'The wide awake human being is, in their natural mindset, mainly interested in that specific sector of their everyday lifeworld, which is located in their range and is spatially and temporally ordered around the centre of themself as a person. The place, where I am, my present 'here', is the starting point for my orientation in space; it is the zero point of my coordinate system, within which the orientational dimensions, the distances and perspectives of the objects in my surrounding field are defined. In relation to my body, I group the elements of my environment in categories such as 'to the right', 'to the left', 'above', 'below', 'in front of', 'close', 'far' etc.'

This aspect of space-time, which is immediately (unmittelbar) experienced is called the present range (aktuelle Reichweite). This part of the world depends largely on my physiological perceptions, and it is mainly structured in close and distant elements. The experience of this world is very much related to the present moment. It is also subject to change, once the person moves physically in space. When a person moves, the formerly distant becomes closer, while the formerly close becomes distant. The present range continuously changes.

Yet, while being in one present range, a person thinks of other former and past ranges, and as such the past ones transcend the current one. If there is the possibility that the person can go back to the former one, and things and elements in it could be expected as being order as before, then it is a 'recoverable range' (Wiederherstellbare Reichweite). Schütz \& Luckmann formulate (2003: 72f. ${ }^{75}$ ): 'Thus, I assume - ceteris paribus - the one sector, which was formerly in my present range, as constant, or as constantly changing. Further I know that I only have to move like this and that, I have to do this and that, in order to bring this sector back into my present range.' This means that

\footnotetext{
${ }^{74}$ Translated by the author from the German text: 'Der hellwache Mensch ist in der natürlichen Einstellung vor allem an jenem Sektor seiner alltäglichen Lebenswelt interessiert, der in seiner Reichweite liegt und der sich räumlich und zeitlich um ihn als Mittelpunkt anordnet. Der Ort, an dem ich mich befinde, mein aktuelles ,Hier', ist der Ausgangspunkt für meine Orientierung im Raum, er ist der Nullpunkt des Koordinatensystems, innerhalb dessen die Orientierungsdimensionen, die Distanzen und Perspektiven der Gegenstände in dem mich umgebenden Feld bestimmt werden. Relativ zu meinem Leib gruppiere ich die Elemente meiner Umgebung unter die Kategorien rechts, links, oben, unten, vorn, hinten, nah, fern usw.'

${ }^{75}$ Translated by the author from the German text: 'Ich setze also - ceteris paribus - den Sektor, der vormals in meiner aktuellen Reichweite war, als konstant, oder konstant veränderlich, an. Ferner weiss ich, daß ich nur diese und jene Bewegungen ausführen, diese und jene Schritte tun muß, um diesen Sektor wieder in meine aktuelle Reichweite zu bringen.'
} 
there is a broad variety of spatial sectors which were formerly experienced by the individual, and which in sum define the total world in the 'personal recoverable range'. Obviously, this total world, or personal recoverable range, continuously transcends my present range.

If we translate this past orientation into a future orientation, we could speak of an 'attainable range' (Erlangbare Reichweite). This spatial range is defined by all possible and probable present ranges in the future which I can imagine as realistic on the basis of former experiences. All these future ranges depend on subjective probabilities (Wahrscheinlichkeitsstufen) and on technical and physical 'possibilities'. The first ones are based on individual future plans and hierarchies between different plans, while the latter are defined by the technologies that a society offers for spatial mobility (e.g. trains, air connections). The conscious reflection about these probabilities and possibilities are based on past experiences and the resulting estimation for future options. The security that a person has in guessing 'attainable ranges' is dependent on how far away these options are in social, temporal and spatial terms. The less distant they are, the more probable the attainability.

Finally, there is also a social aspect in relation to physical space. While in the everyday lifeworld modus of thinking it could be imagined that a person can change the physical position with an opponent other in order to enter into the opponent's present range. Yet, the perception of this 'present range' is dependent on the individual physiological capacity (ears, eyes, etc.) and might thus differ. One person cannot experience the identical present range of another person even if they exchange their position in physical space. On the other hand, as two persons' 'present ranges' can overlap in large parts, one can speak of a 'shared environment'. Here, biography comes into play: While for one person the present range could be based on former experiences of the same (in the sense of a 'recovered range'), this range could be a new experience for someone else (in the sense of a recently 'attained range'). In terms of future options, some ranges could be imagined as probably attainable by one person, but not by the other. Furthermore, not only a dual intersubjectivity in the given moment could be the case. Such space-time experiences are also shared with third persons and the society as a whole as a system of spatial structuration. It defines the distribution of intimity or anonymity, of the foreign (das Fremde) and the familiar (das Vertraute), social distance and proximity, and this differentiation influences how we experience our lifeworld in spatial terms to a strong degree.

\section{Appresentation, time, and the phenomenological understanding of perception}

Generally, people tend to speak of perception in the form of realising something or someone. Mainly this refers to the corporeal nature of the human being: We see with our eyes, we smell with the nose, we touch with our hands, we hear with our ears. Yet, a mere physiological perception is limited to the immediate spatio-temporal setting, as Schütz \& Luckmann indicate (2003: 591 ${ }^{76}$ ): 'Everyone continuously stretches their own spatial and temporal limits of experience, but they exceed these limits - seldom appalled - as a matter of course.' Therefore, in phenomenology 'perception' is rather orientated to what happens in the minds of people while they perceive. For phenomenologists, perceiving something or someone means a state of reflection, in which the immediate happening is put into relation with former experiences. Thus, the one immediate aspect

\footnotetext{
${ }^{76}$ Translated by the author from the German text: 'Jedermann stößt immer wieder an die räumlichen und zeitlichen Schranken seiner Erfahrung, überschreitet sie aber in Erinnerung und Handlungsentwurf mit der größten - nur selten erschütterten Selbstverständlichkeit.'
} 
that is perceived in a given moment is more than what we perceive physiologically, as former knowledge is added to the immediate physiological perception and thus changes it. Husserl, for example, says that human beings tend to see things that they physically cannot see. The same accounts for all other physiological senses; there has to be more to perception than a mere physiological character. So, there is always a parallelity of physiological perception and mental reflection. Husserl calls this mechanism appresentation (Appräsentation) (cf. Kurt 2002: 51-55). Present things merge with the 'non-present' things in the perception of a person. Kurt illustrates this with a person looking at a building. This person will see the front façade as they are physiologically unable to look through the walls. Nonetheless, the backside or the inner structure of this building is also integrated in the perception as an 'imagination' which is based on former experience. Husserl conceptualises appresentation as a process of 'passive synthesis', in which the consciousness independently of the Me (Ich) and spontaneously - connects the immediate perception of a given moment with former syntheses of past experiences. Kurt explains this with another example: we recognise melodies by only hearing individual tones, or we imagine rain, seeing grey clouds. This is always appresentation.

For appresentation, it is also important that former knowledge stocks exist which are already abstracted in form of typologies. When the person sees the façade of a house, they will come to appresentation if they have already seen exactly this house's interior and back side, or if they have already seen similar façades on other houses, which they have mentally grouped into a type of houses. Therefore, appresentation always needs additional information which stems from the memory and the stock of experiences, and adds to the actual physiological perception.

Appresentation is not only relevant for the study of perception; time is also an important aspect. As appresentation is a process of connecting the past with present situations and experiences, they always have a temporal relation. Time is a fundament of the constitution of everything, says Husserl (cf. Kurt 2002: 55). Relating two experiences to each other, time also separates and distinguishes different experiences in an uninterrupted temporal sequel. Here, two things happen parallel: we always finish one experience, and a new experience starts. And the same moment, we always relate each of these new experiences to a former one. Thus, we continuously relate experiences. According to Husserl, the past, the present, and the future are thereby always related to each other. Husserl uses specific terms for these temporal dimensions. The past is called 'retention', the present is called 'impression', and the future is called 'protention'. Current impressions are always framed by retentional and protential aspects. We always order a situation to what happened right before and to what we expect to happen right after (cf. Kurt 2002: 57). As an illustration, Husserl used a music concert, in which the audience is always aware of the currently playing tone, putting it into a sequence with the former and the following tone. A complete melody could only be understood as a melody, as a coherent unit like this.

Husserl also distinguishes between a present consciousness and the memory. Experiences, which are followed by new experiences are sliding into the 'retentional field' of the consciousness. They are then still relatively close to the present experience, and they are placed in the 'present consciousness' (Gegenwartsbewusstsein). The more experiences follow afterwards, the more this first experience slides away from the present one. From a certain moment it leaves the 'present consciousness', and is no longer part of the present imagination. It then becomes part of memory and can only be made conscious through active remembering (Erinnern). While retention is part of 
our immediate experience and perception, the memory is not. Memorising, or remembering, is the act of re-experiencing some past experience as detached from the present moment. Similarly, future experiences are divided into protention and expectation. Protention, like retention, is part of the immediate experience and perception. Actively made conscious expectations are farther away in the future (e.g. plans, hypotheses).

While these temporal modes of perception rather refer to the passively perceiving Ich, there are also active perceptions. An important notion here is 'apperception' (Apperzeption) which refers to the fact that a person never only perceives the one experience that is perceived physiologically in the moment. The person will also put this perception into a 'perceptional field', as the example of the spider on the wall illustrates. A person might see something dark on a white wall. The direct perception is that of a dark point on the wall, and only through appresentation the person will be able to interpret if it is a blotch or a spider. Then, through apperception the wall and the room itself are also consciously perceived. Husserl says (Husserl 1973: 493, cited in Kurt 2002: 63 ${ }^{77}$ ): 'As such the world is timelessly conscious, continuously apperceived; while I am only focused on this and that as a part of this world, I am still aware of what else is there in the background or behind the immediate.'

Equally, Schütz \& Luckmann (2003) discuss a person's means to cross the borders of time and space. They think of such means as helping to make everything co-present (mitvergegenwärtigen), which could not be perceived and experienced directly and at the moment. Such means can consist of:

- Anzeichen (indications), which refer to something other than themselves, to something hidden or absent; information about things which are out of the temporal and spatial range of a person; help to access the consciousness of others;

- Merkzeichen (bookmarks), which form bridges to the future; they consist of the active and structured making of memories for later moments and situations;

- Zeichen (signs), which are a mixture of Anzeichen and Merkzeichen; the main means of inter-subjective communication, e.g. language; important to cross borders to other people;

- Symbole (symbols), which are a means to transfer the sense of something, of an object, between sense structure of everyday life and other sense structures.

All these means are based on former appresentation, and they are the basis for future appresentation. Schütz \& Luckmann (2003) mention that appresentation is actually the key for the existence of a lifeworld. If people were not able 'to appresent', they would only experience situations which could not be related to each other, and it is exactly these relations which define the lifeworld. Appresentation, they say, is an active process of the consciousness in which links and concepts - based on associational processes - of former information are put in relation to present experiences. A person can only understand the significance of a present situation through appresentation.

Transferring the idea of appresentation to places, we can study how creative knowledge workers deal with their multilocality. The parallelity of different places must somehow leave traces in the

\footnotetext{
${ }^{77}$ Translated by the author from the German text: 'So ist aber Welt immerfort bewusst, immerzu apperzipiert; während ich davon nur auf dies und jenes einzelne gerichtet bin, habe ich doch das Mitdaseiende im Hintergrund wahrgenommen.'
} 
consciousness and sense making of these multilocals. I suggest that in particular, for the duality of absence and presence, the idea of appresentation could provide a useful perspective.

\subsubsection{Methodological implications of phenomenology}

In terms of empirical methodology, the phenomenological perspective calls for a concentration on sense making and sense structures in peoples' lives and in society. Grathoff (1995: 120) argues that a Cartesian understanding of objectivity and measurability of the social facts, as well as an apodictic understanding of evidence is no longer appropriate. Science has to be thought of as part of the lifeworld and somehow related to the everyday life sphere or modus. Scientific field work is not free of values - as suggested by nihilistic positions. The phenomenologist reflects themself in the light of their field. This reflection also allows for a closer relation between science and everyday life, which is necessary for a scientific understanding of the empirical world.

Kurt defines phenomenological fieldwork as follows (2002: $\left.149^{78}\right)$ : 'This preoccupation aims at a scientific understanding of everyday life ways of understanding the social reality. The leitmotif of such an understanding is the reconstruction of this construction, which orders and structures the interrelatedness of human beings. [...] Both ways of understanding intend to interpret the social sense of the self-evident aspects of the lifeworld.'

Yet, while everyday life construction of sense comes along with personal interest, preferences and opinion, scientific reconstruction should be free of these. This position somehow opposes Grathoff's (1995) neglecting of the possibility of value free science. Furthermore, everyday construction happens in the frame of necessity to (re)act, while scientific reconstruction is free of such pressure. Third, typologies in everyday life are practical and necessary to live, to master one's own life (Lebensmittel), whereas in scientific reconstruction typologies serve knowledge acquisition (Erkenntnisgewinn) and have a formal character that helps the formation of theories. In everyday life things are easily and subconsciously understood; in science, questions are raised and consciously answered to exactly understand these everyday life ways of understanding.

As far as I have studied this literature, the philosophy of social phenomenology calls for heuristic approaches that seek 'sense making' practices through the analytical technique of reconstruction - it is about understanding phenomena in the everyday life modus of others (the empirical sample) from the perspective of the theoretical modus of the researcher. Given that there are overlaps between the researcher's and the studied person's lifeworld and the different modes of being, some theoretical reflection which can help to generate heuristic models in this study of these overlaps is necessary.

\subsection{Collecting the data: Interviewing technique}

In this section I will explain how I gathered my empirical data, which consists of 25 in-depth qualitative interviews and related mental maps for each studied case.

\footnotetext{
78 Translated by the author from the German text: 'Diese Beschäftigung zielt auf ein wissenschaftliches Verständnis des alltagsweltlichen Verständnisses von sozialer Wirklichkeit. Das Leitmotiv dieses Verstehens ist die Rekonstruktion der Konstruktion, die das Aufeinander-bezogen-Sein von Menschen ordnen und strukturieren. [...] Beiden Verstehensweisen geht es darum, lebensweltlich Gegebenes auf sozialen Sinn hin auszulegen.'
} 
6.2.1 Mixed character qualitative interviews: Biographical narration \& problem-oriented sequences

For this research project, I decided to use a qualitative approach, as sufficient data on transnationally multilocal creative knowledge workers does not exist so far. The idea of the project is to explore the thematic fields as outlined in chapter 5. Thus, a qualitative design is more appropriate than a quantitative study. There are many pointers in the methodological literature that argue in this direction, too (e.g. Hitzler \& Honer 1997: 10f.; Wessel 1996: 132ff.). Qualitative interviewing is the preferable methodological choice for exploration of new thematic fields, for the generation of empirically grounded theory, and for the development of a catalogue of scientific terms that originate not from science but from the empirical field itself. In this sense, Scherke (2011: 84 ${ }^{79}$ ) remarks, '[...] it could be observed that in more recent migration studies there has been an increasing concentration on qualitative methods. These methods seem more appropriate for examining those processes of 'in-betweenness', of cultural transgression and of the dissolution of borders, which come along with processes of transnationalisation. Narrative biographical interviews, participant observation and ethnographic studies allow for a detailed reconstruction of actors' practices in everyday life [...] [and they are] more suitable to break traditional patterns of thinking.'

In the theoretical literature on methods in qualitative interviewing, a variety of prototypical interview situations and formats are described. Among these, we can find the biographical narration, on the one hand, and the problem-oriented interview, on the other hand (cf. Hopf 1995). The biographical narration is a form of interviewing in which the interviewer provides very little information on the research topic. The interviewee is confronted with a certain moment in their life from which they should start a narration about their own biographic history (cf. Bichi 2002). All narration is recorded no matter of what topic the interviewee focused on. This type of interviewing aims at registering the individual life's topics of an interviewee. A central question is: what events/situations/contexts were important in their life? The interviewer only interferes with rather little guidance of the narration, e.g. asking for more details to a certain issue that comes up during the interview. The interview technique remains a very open one, depending a lot on the biographical aspects and narrations that an interviewee is willing and able to provide.

In the subsequent analysis, the researcher tries to reconstruct how things have evolved in the interviewee's life in relation to the research topic. The advantage of this approach is its openness to all kinds of information. These interviews can generate very detailed and rich narrations that allow for a reconstruction of very complex patterns. It is also very useful in studies that deal with individual reasoning of people, or in studies that focus on lifeworlds. The disadvantage is that it can also generate too much information which is not relevant for the study. The problem is also that interviewees, when speaking about their life, start to become very detailed and often drift away

\footnotetext{
79 Translated by the author from the German text: '[...] ist in jüngeren Studien zum Thema Migration eine zunehmende Konzentration auf qualitative Methoden feststellbar. Diese scheinen besser geeignet als quantitative Methoden, um jene Phänomene des „Dazwischen“, der kulturellen Übergänge und Entgrenzungen zu erfassen, die mit Prozessen der Transnationalisierung einhergehen. Lebensgeschichtliche Interviews, teilnehmende Beobachtung und ethnografische Studien erlauben die detaillierte Nachzeichnung des Alltagspraxis von Akteuren [...] [und sind] besser geeignet, überkommene Denkschemata zu durchbrechen.'
} 
from the researcher's interest. Work then becomes difficult when the researcher has to identify and eliminate the unnecessary information during the analysis.

The problem-oriented interview is an approach with a stronger guidance through the interviewer. The research topic is divided into problems, or sub-topics that might be relevant to the study. Then an interview guideline is developed which should help the interviewer to stay focused and to structure and guide the narration of the interviewee. Other than in standardised quantitative interviews, the qualitative interview does not necessarily call for precisely formulated questions to be asked. Rather, the interviewer uses the guideline with key words to remember all problems to be covered during the interview. Yet, it is still dependent on the interview situation, when, how and how detailed the single problems are talked about. The advantage of problem-oriented interviewing is that it allows for an economic way of gathering information for a pre-defined topic. It limits the 'informational noise' that comes along with more open interviewing techniques. It is also very suitable in situations where comparison between different cases of interview partners should be structured in advance. The disadvantage of such problem-oriented interviewing is that the interviewer basically pre-defines the incoming information by making certain problems an issue during the interview, while not mentioning others. Interviewers must also listen very attentively to what people say in order to not interrupt narration too early and limiting information, as well as to not ask for certain problems again when the interviewee already spoke about them in the frame of another problem earlier.

I understand such descriptions in the literature as prototypical interview situations, and did not pressure myself to stick rigidly to methodological theory during my field work. I think it is more relevant to react to individual interview partners' ability to speak on their own. Some interview partners are more able to provide rich biographical narrations than others, who would need more guidance throughout the interview. I therefore decided to design my interviewing techniques as a mix of both mentioned prototypical techniques. This means I started the interviews with a narrative stimulus as done in biographical narrations. My stimulus consisted in asking my interview partners for 'their places'. While the precise wording was dependent on the situation, the question was more or less: could you please describe to me what you would consider your places? The question partially astonished my interview partners and they were not able to deal with it right away. This was good for me, as I immediately had their attention and I could start a first discussion about the notion of 'my place'. This discussion revealed the first information on what makes peoples' places 'their places'.

If people were not able to answer this rather abstract question, I then made it more precise so that they might orientate on their own biographical history; they might start with where they were born, where they grew up, where they live today, and why. Then my interview partners regularly started a longer narration, while at the same time sketching these places as a mental map on a sheet of paper, as I had asked them to do.

When the narrations about their places had finished, I started to orientate the interviews towards my fields of interest (cf. chapter 5). From there on, interviews became a bit more problem-oriented, but I remained attentive so as not to interfere in narrations provided by my interview partners. I also paid attention to guiding the interview and moderating the change to a different field of interest without looking at my key notes. In other empirical studies using qualitative interviewing, I had 
found that having very large questionnaire guidelines printed in bold letters on A4 pages distracted the attention of the interviewee. Interviewees are curios about the questions and often try to have a look at such guidelines if they are on the table during the interview. Understandably, they want to know what they will be asked, or how long the interview will continue. I perceived this as a disturbance to the interview situation.

Figure 3: Key word paper used during interviews

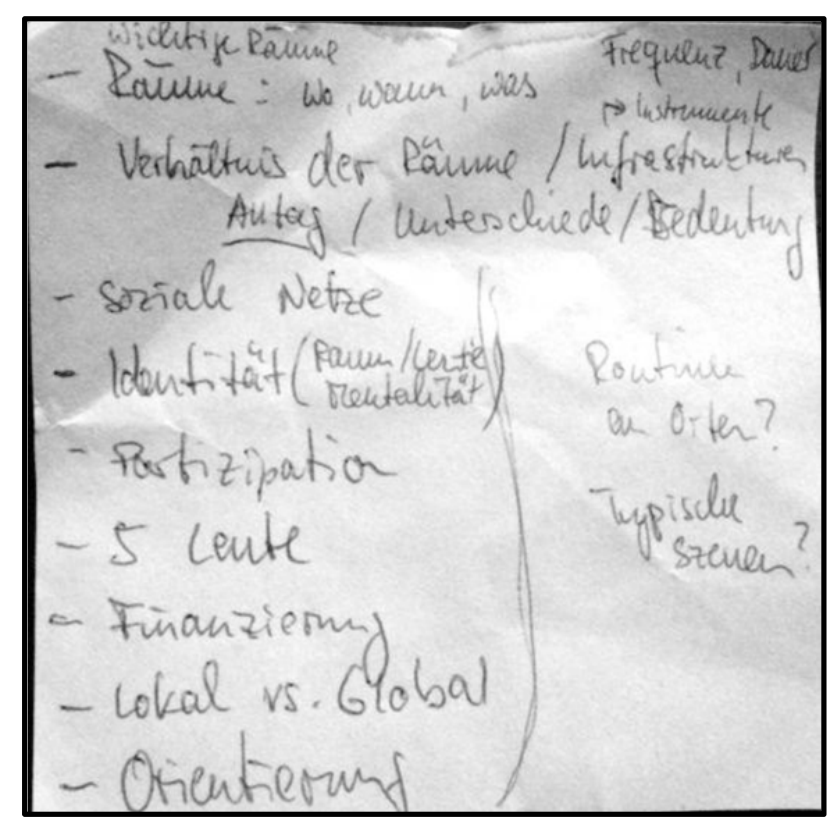

Therefore, I decided to note key words on a very small sheet of paper for this study (see figure 3 above), and I left this sheet of paper covered throughout my interviews. I only took it out when the interview really halted, or I had forgotten what else to ask. However, as far as I could, I tried to not look at it during the interviews. This proceeding has helped my interviews to retain a conversational character in which the mental map of the interview partner was the only item on the table which guided their attention and therefore the narration.

\subsubsection{Mental mapping: illustrations of mental cartography}

The idea of mental mapping derives from research done in the 1960s on the perception of space. Early work was done by Kevin Lynch (1960), who studied how people perceived and orientated in urban environments. His main interest was to base urban planning on individual perception of the built environment, and thus to increase the quality of life. Lynch explained that spatial or environmental perception focused on a distinct set of five attributes (1960: 47ff.):

- Paths, which represent the most important attribute. Paths can also be termed ways; they have a linear character and link other spatial attributes with a point character.

- Edges, which also have a linear character, but their specificity is that of dividing two areas from each other. They signify breaks and interruptions in a territory, and at the same 
moment the edge itself works as a joint between the divided areas. The more different the character of the divided areas, the stronger is the importance of the edge.

- Districts, which have an areal character. They have an internally homogeneous character. A person can enter and exit them, and can identify them as a distinct district compared to other districts upon entering it. This often includes that the district contains characteristic physical elements, e.g. buildings or city squares.

- Nodes, which have a point character in mental maps. They are attributed with encounter and intersection, e.g. city squares or big cross roads. Given this character, they force people to take directional decisions, which basically lead to the fact that people are more conscious and attentive at these locations. It is a spatial localisation of consciousness. As a result, mental map descriptions of nodes can become very precise and detailed. Nodes are also often entrance points to districts.

- Landmarks, which have a point character, but on a smaller scale than nodes. Landmarks interrupt the homogenous continuity of the spatial environment, e.g. large buildings, progressive architecture, historic monuments, etc. Through this character, they help people to pin down their mental maps.

Lynch also found that while drawing their mental maps on paper, people were following a certain logic of progression. The people he interviewed mainly started drawing a central starting point, a location, which was used during their everyday life movements. Then they drew the main directions into their maps, namely paths and nodes. Afterwards, they added the edges as a basis for then putting the important districts. And finally, they made maps more detailed by adding more precise information such as landmarks (cf. Lynch 1960: 113ff.).

In reference to Lynch's work, Downs \& Stea wrote the ground-breaking book 'Maps in Minds: Reflections on Cognitive Mapping', which was published in 1977 (I will refer to the German translation, which was published in 1982). In this book they explain that there is a difference between cognitive mapping as a process, an activity, and the mental map as a result, a tool for everyday life. Concerning cognitive mapping, they formulate (1982: 23) that it is a capacity to collect, order, save, memorise and process information on the spatial environment. Moreover, they think of this capacity as being dependent on age (or maturity) as well as the utility of mental maps and the stock of knowledge a person has. The output, the mental map itself, then serves as a tool to support spatial decisions, such as where we need to go, and how we can get there. Basically, mental mapping is about organising and processing one's own spatial knowledge (Raumwissen) in order to orientate and take individually perfect location decisions. Cognitive mapping is a flexible and everchanging process. Downs \& Stea (1982: 44f.) explain that through cognitive mapping, a person develops a set of varying perspectives which help to master different situations. It is a process of continuous learning by which a person is able to understand the complexity of relations between a place, its people, possible activities and efficient pathways through this complex. Rather than merely knowing something about a place, it allows for knowing a place like the back of one's hand.

Downs \& Stea (1982: 24) then define a mental map as a structured representation of the spatial environment, which is based on a snap-shot of a given moment, and thus subject to change. Furthermore, it is always a very individual imagination of how the surrounding world looks. Other people might have different mental maps, even though they are looking at the world from the same 
position in space. If we reflect what Downs \& Stea offer with these definitions of cognitive mapping and mental maps, we can see the conceptual proximity to the elements of social phenomenology. In the field of mental map research, the everyday lifeworld is also a key modus of experiencing the world, and again, life is split into individual situations - here perhaps rather spatial situations - which are reflected in a cognitive process including all former situations. Finally, the experiences are processed into typologies and structured knowledge in the mind, which form the preliminary mental map.

In addition to this, there is a strong link between personal identity and mental maps. Downs \& Stea (1982: 49) point to the fact that personal identity is strongly influenced by our knowledge about spatial environments. They term this phenomenon the 'sense of place' (Ortssinn). Only having a detailed mental map on a place, we can evaluate it in the light of our own values and convictions and we can decide to include it in or exclude it from our personal identity. If we do not know something about a certain territory (a city, a neighbourhood, a region), we are less probable to identify with it.

Downs \& Stea (1982) also outline why mental maps are so important in everyday life. Everyone is confronted with a general spatial problem. This spatial problem consists of the following elements of information: the 'where' of things and people, the 'what' at a certain location and the 'when' of things happening/being located in space. In order to answer these questions and solve our personal spatial problem, we need to know. According to Downs \& Stea, knowledge is characterised as experiential knowledge (Erfahrungswissen), as learning by doing. They reject the structuralist hyposthesis of a pre-defined capacity to acquire knowledge, imagined as a stencil which allows for knowledge infiltration or not. They also reject the pedagogy conceptualisation of knowledge as something which is uploaded into the brain (Auswendiglernen). To them, cognition, as composed of knowledge and comprehension of this knowledge, is a result of the reflection about spatial experience. During interaction with others and with the spatial environment, a person learns and incorporates this learning into their knowledge stock. The latest knowledge is thereby included into a set of typologies. These generalised typologies help to master subsequent situations and evaluate them as specific experiences in relation to the past. Downs \& Stea reflect this on the example of a 'street'. When coming to a new street, a person already knows something about it, as they developed a type 'street' during their lifetime which also might be differentiated into several subtypes. Thus, this person must not learn anew what a street actually is, but they can immediately look for other more relevant information: where can I do what in exactly this street? How is this street different from the other streets?

Furthermore, Downs \& Stea (1982) elaborate on the 'where' question and the related cognitive knowledge stocks. The ,where' has to be distinguished into the two elements 'identity' and 'location'. Downs \& Stea think that identity is not so important here because when it e.g. stems from names, it might not be unique (e.g. cities with the same name in different federal states, or geographic names such as four corners), whereas location is always unique. 'Location' is more important; it indicates where something is, and how I can reach it from where I am now. It is composed of situation and direction! A location could be described as a situation within a coordinate system, or it could be described by how to reach it from a certain other location (direction); both forms of circumscribing location are central contents of mental maps. 
Related to location, 'distance' is important (Downs \& Stea 1982: 73). It is basically the perception of distance, and as such it is relative, and a cognitive element. The interesting questions for mental map research are: What factors influence our estimation of distance? Which distance measurement units are used in thinking about cognitive distance? Furthermore 'direction' and 'directions' are distinguished by Downs \& Stea (1982: 79ff.). The difference is that 'direction' is something like North, East, West, or South, whereas 'directions' is the description of where to find a location. This first one indicates a location within a coordinate system, and the second one refers to a path to a location, e.g. from my own current location. Using both 'direction' and 'directions', people actually develop the capacity to orientate in space. Orientation is to be thought of as the capacity to cognitively relate individual locations to each other, so that it is functional in one's own lifeworld.

It is important to mention that mental maps have a dynamic shape; they change with time. On the one hand, a perceiving person gets more used to the surrounding environment by passing it more often. This includes that more details are perceived and added to the mental map. On the other hand, the built environment itself is subject to change, through e.g. urban planning (cf. Scholz 2011). A mental map is thus a dynamic representation of spatial knowledge which is based on cognition and perception. Automatised and intuitively spatial information enters through physiological perception into our mind, and it is processed into knowledge through cognition there (cf. Downs \& Stea 1982).

Ploch (1995: $24^{80}$ ) also defines mental maps as the interviewees' 'own perspective on the world, their drawn worldview'. Mental maps are often striking; they contain typologies and also textual explanations. She also points out that one should not forget that mental maps only represent a 'snapshot' of the given moment. The interviewee draws what appears relevant to him, in another moment this might be something completely different. As such, Ploch argues, mental maps do not allow concluding on an 'overall picture' (Gesamtbild).

I came across this idea of mental maps early on when I wrote my diploma thesis, but it did not fit in there, so I kept this latent idea to use mental mapping as a method later on, and I found it matched perfectly with the topic of this project. The only thing that would change for me is the scale when compared to Lynch's work. Lynch applied mental mapping to urban environments, and thus to the local scale. In the literature, also other examples are given of how to use mental mapping as a technique for capturing people's perception and cognition of space. Scholz (2011) studied the individual ideas that people had about the Euroregion of Saar-Lor-Lux, shifting the application to a regional scale. This largest cross-border labour market in the European Union was her empirical field for conducting interviews with several thousands of Saar-Lor-Lux inhabitants, including highschool students, who should describe their region. What Scholz then did in the analysis was to put different maps the one over the other, just as different layers. Then she looked at differences and similarities in the maps. Scholz found that indeed people were using the spatial attributes as described by Lynch. They put in the national borders (edges); they drew the countries or parts of the Euroregion on all sides of the borders (districts); they put major highways and train connections (paths); and some even sketched landmarks into their maps. In addition to mental map research on a local level, Scholz found that people referred to very different geographical scales when speaking about 'their region'. Checking for words related to space, Scholz discovered that local, regional,

\footnotetext{
${ }^{80}$ Translated by the author from the German text: 'ihre Sicht der Welt, ihr gezeichnetes Weltbild.'
} 
national and specifically the Euroregion's names were continuously mixed in individual maps. Then she also found that people used detailed descriptions including clichés based on collective identity of inhabitants. Inhabitants from French parts of the Euroregion attributed specific characteristics to the German parts of the Euroregion, using available stereotypes about Germans, for example. Thus, mental maps do not only consist of the descriptions of mere spatial attributes, but they also contain descriptions stemming from characteristics related to the people living there. This confusion of place and people was also observed in other fields of research such as studies on place-based identity (cf. Weichhart 1990; Weichhart, Weiske et al. 2006; Mühler \& Opp 2006). Therefore, mental maps carry more information than mere topological content.

Methodologically, mental maps are interesting as they lead to a distinct atmosphere between interviewer and interviewee (Ploch 1995). While in qualitative interviews the interviewer, consciously or sub-consciously, interferes and guides the dialogue, they become a marginal figure during the moment when an interviewee is drawing their mental map. The opponent for the interviewee is not the interviewer, but the sheet of paper and the pencil. The interviewer has less possibility to influence what shows up on the paper. The interviewer can only influence the mental map's content through the wording of their invitation in the initial invitation to draw.

What did these results tell me for my research project? Looking at the difference between Lynch's and Scholz' scales, I thought it would be more interesting to scale it up. Scholz could only have found the scale issue in such a way, and I wanted to do mental mapping without even defining scale, which I would assume of as an approach even more open to scale issues. Lynch and Scholz had a clear spatial focus in their research, a specific city or a region. Me, I do not have such a clearly formulated spatial area that I want to study. I wanted to keep my research rather open, so I did not tell my interview partners to which spatial scale they should refer in drawing their maps. What I was interested in was the individual 'action space' (cf. Hägerstrand 1970) as a pattern of different places. Therefore, I asked people for 'their places', and to draw me a map of these places.

There is another issue in mental map methods: how much cartographic assistance is provided by the researcher to the interview partner? There are several approaches to this. Some studies used prestructured maps in which orientational elements are given, e.g. highways, train lines, administrative boundaries, and capitals for regional scale level and above, or city squares, streets and neighbourhood boundaries for studies on the local level. This allows the interviewees, in particular those who are afraid of drawing incorrectly, to have a minimum of orientation for their own elements that they would put in, but it also pre-structures the way in which an interviewee draws. In addition, it predefines the scale of the map, which makes scale variations between cases of a study nearly impossible. This approach is quite good for studies in which there is a certain focus on a given spatial area. It allows, in particular, the recording of information about the variety of size relations between individual perceptions, e.g. studying how large and where people locate a city square within a pre-structured district or street grid.

Another approach consists of providing nothing - just a blank sheet of paper. This approach has the advantage that it allows for flexible data collection. The interviewee is not forced into a scale and into a pre-defined section of the environment. They can decide freely what scale and section to put into the map. This makes analysis more difficult, but the collected material can be richer in individual case-specific information. Scholz also used this approach, not providing orientational 
elements to her interview partners, yet Scholz made a clear spatial reference in forms of verbally mentioning that the study is about the Euroregion Saar-Lor-Lux. I also decided for this option, and I did so for another reason as well. In Downs \& Stea's book on mental mapping, they pointed out that mental maps do not only consist of geometric information (Downs \& Stea 1982), mental maps also consist of verbal information translated into little narrations as well as of images which can be translated into little drawings. We also find hints of this in Scholz's (2011) study, because she mentions in her analysis that people used stereotypes and judgements of other people to describe their vision of the Euroregion. I also became convinced that gathering such data necessarily called for a blank sheet. If you put streets and borders onto a map as orientation, it is already predefined that this mental map should have the character of a geometric cartography in a given territory. Yet, leaving a sheet of paper blank as it is, mental maps can take different characters, e.g. more symbolic elements. In her study on mental maps of Frankfurt-based university students, Ploch (1995) observed that her respondents associated Frankfurt with 'traffic, cars and noise'. However, they only drew this in symbolic or textual forms, while not drawing streets and cars. Ploch interpreted this observation as an indicator for the fact that the students reproduce 'collective images' instead of 'personal experience'. She must have been a bit disappointed as she formerly criticised human geography for studying exactly such collective images instead of personal living spaces. She says (1995: 38 ${ }^{81}$ ): 'In spite of all the traffic chaos mentioned, the 'Frankfurt' of the mental maps neither knows streets nor cars. The question arises if the typical Frankfurt is less the 'personal one' than the 'one of the others'.'

I would rather say that this is an interpretational misunderstanding by Ploch. It might be that Frankfurt-based students did indeed draw 'their' Frankfurt, but they did it in a way (namely symbolic) which was not expected by Ploch (namely cartographic). Even coming back to the approach of Downs \& Stea (1982), we can see that mental maps are very much thought of by most scholars as a topographic map. Little other content and forms are imagined. I am not sure if this is sufficient. Some people's mental maps are probably not maps as we researchers expect them to be.

Furthermore, mental maps are not only functional in terms providing generic information, but they can also be helpful in structuring interviews. Ploch (1995) studied 34 mental maps of university students which they had to draw concerning the 'Frankfurt Region' right in the beginning of connected interviews. Later in these interviews they had to name characteristics which circumscribe the Frankfurt Region. Ploch found that those who had actually drawn the city and not the region also circumscribed the city and not the region in their interview narration. She concludes (1995: $36^{82}$ ): 'This could be interpreted as an indicator that drawing 'personal' maps in the beginning of an interview defines the spatial horizon of all future statements during that interview.'

Having said this, I integrated mental mapping not only for the maps itself. The general purpose of my interviews was to learn about how multilocal people relate to their different places, and I

\footnotetext{
${ }^{81}$ Translated by the author from the German text: 'Trotz Verkehrschaos kennt das Frankfurt der Mental Maps weder Straßen noch Autos. Und es stellt sich die Frage, ob das typische Frankfurt nicht das „eigene“, sondern das der „anderen“ ist.”

${ }^{82}$ Translated by the author from the German text: 'Das könnte als Hinweis gewertet werden, daß die “eigene” Zeichnung am Anfang einer Befragung, den räumlichen Horizont für den weiteren Verlauf des Interviews vorgibt.'
} 
thought that if my interview partners would start speaking about their complex spatial pattern, it would be helpful to have a scheme for the narration which they would draw themselves, and to which we could both come back during the interview. Furthermore, during her own empirical field work, Ploch was surprised about the way respondents liked drawing maps (1995: 28 ${ }^{83}$ ): 'We observed that many interviewees had fun drawing and inventing symbols, and that they seemed surprised about their own creativity. I would estimate this playful and creative reflection on their personal living space as another quality of the method.' Therefore, there is also an entertaining connotation of mental map drawing.

\section{Analysis of mental maps}

Ploch observed in the 1990s that mental maps are increasingly being used as a research method in ethnology and anthropology, but the achieved results are often moderate compared to the results from other methods. Ploch thinks that this is due to the limited analysis techniques which exist so far. She lists several limitations that she observed in 'mental map projects' (1995: 23f.):

1. Mental maps often only serve an illustrative function;

2. In comparative settings, they are only used as an exemplary material which circumscribes a phenomenon as a whole;

3. There is no discussion about analysis techniques (at least in cultural anthropology).

Ploch observes (1995: $24^{84}$ ) that scientists might also be confounded by the type of information that mental maps generate: 'The aesthetic moment of the drawing itself is impressive, and perhaps scientific researchers are particularly affected, as their 'world' is dominated by spoken and written language.' Ploch appeals to the scientist's courage to dismantle the mental map and analyse its individual components. These components might then be compared across cases, always keeping in mind their insertion in the whole picture of the mental map, in the map's totality.

For a systematic analysis of mental maps, there is one big barrier: the respondent's individual skills to draw. These skills are often thought of as being dependent on educational attainment, and as such somehow related to socio-economic positions. Furthermore, Downs \& Stea (1982: 23) already pointed to the fact that these drawing skills are influenced by age, utility and knowledge about topics. We already heard that one can understand 'mental maps' as an activity, and less so as an object that we dispose of. It is an active process of abstracting the empirical world, in which the degree of complexity depends on the actual experience of a given area in the everyday life. Here Ploch observes, while some argue that more educated people have a higher skill to abstract and draw a map, others argue that it is in particular this better educated peoples' skill to abstract the real world into textual formats which makes it more difficult for them to draw in non-textual ways (somehow equalling the scientists' hesitation to work with mental maps as described above).

\footnotetext{
${ }^{83}$ Translated by the author from the German text: 'Wir beobachteten, daß viele Befragte Spaß am Zeichnen, am Ausdenken von Symbolen entwickelten und zum Teil selbst überrascht schienen von ihrer Kreativität. Diese spielerische und kreative Auseinandersetzung mit dem eignen Lebensraum möchte ich als weitere Qualität der Methode werten.'

${ }^{84}$ Translated by the author from the German text: 'Das ästhetische Moment der Zeichnungen besticht; und davor sind vielleicht gerade Wissenschaftler, deren "Welt” von gesprochenem Wort und Schrift dominiert ist, nicht gefeit.'
} 
According to Downs \& Stea (1982), the first level of abstraction consists in a person's perception of the environment. Ploch (1995) says, this is rather a passive process of 'making one-self comfortable in a spatial surrounding'. She argues that there is also a more active process of 'adapting' to this surrounding, and adaptation calls for active involvement (Auseinandersetzung) with the spatial environment. A mental map is only developed as a second level of abstraction after that active process of adaptation. Ploch explains (1995: 25f. ${ }^{85}$ ): 'I understand these signs [as drawn in mental maps] as an interviewee's effort of symbolisation. Through drawing, they appropriate their own environment - in abstract ways - and give significance to it. They order the personal world for the interviewer. With this consideration, I will also emphasize that this activity [of drawing] could be understood as an active process which already takes place during the first level of abstraction. By giving significance to their space, people (re-)design and change it; they actively appropriate it. And this is documented in the drawings.' As such, we can understand mental mapping as both the process of abstracted appropriation of space and mental maps as a drawn output mirroring exactly this process. For systematic analysis of these mental maps, researchers should now ask themselves (Ploch 1995: 26):

1. How relevant was my own scientific expectation for the interviewee while drawing their mental map?

2. How relevant, in terms of 'to-be-drawn', are the self-evident things for the interviewee?

3. How far can I derive conclusions on the interviewee's values, attitudes, and behaviour?

Ploch (1995) then discusses the different perspectives on mental maps in human geography and in cultural anthropology. Human geographers mainly applied mental map research for capturing collective 'images' of places and territories. Ploch refers e.g. to Kevin Lynch's study 'The Image of the City'. Ploch criticizes that this approach largely generates mental maps which are reproductions of 'learnt images', which often do not stem from individual experience and knowledge, but from the wider public images in the mass media. By contrast, cultural anthropologists do not understand human beings as shaped and informed by outside 'images', but as active creators of such images (and culture in more general ways). As such, the research question is different. It asks for people's techniques of image creation, and thus space appropriation. It understands the mental map as an image of the individual's own lifeworld and its relevance systems. Which places and territories are used by the interviewees to satisfy their needs (for security, identity, self-actualization etc.)?

For the systematic analysis of their own empirical project, Ploch and her colleagues started with a typology according to the dimension of spatial relations (Raumbezüge). They differentiated between wider maps (Landkarten; representing the wider spatial environment and larger action space), local maps (Ortskarten; one's own place of residence as focus), and symbolic maps (symbolräumliche Karten; rather abstract and artistic, containing symbols). Here they found that those who had recently moved to the research territory were more likely to draw local maps than those who were natives and drew wider maps instead (1995: 29).

\footnotetext{
${ }^{85}$ Translated by the author from the German text: 'Ich verstehe dieses Zeichnen als Symbolisierungsleistung des Befragten. Mit dem Zeichnen eignet er sich seine Umwelt - abstrakt - an und verleiht ihr auf einem Blatt Papier Bedeutung, er ordnet dem Forscher seine Welt. Zudem möchte ich damit zum Ausdruck bringen, daß diese Aktion auf einen aktiven Prozeß schließen läßt, der sich bereits auf dem ersten Abstraktionsniveau vollzieht. Indem Menschen ihrem Raum Bedeutung verleihen, ihn (mit-)gestalten oder verändern, eignen sie sich ihn auch aktiv an. Dies wird ebenso in den Zeichnungen dokumentiert.'
} 
Further, she found that their respondents' mental maps are not representing the totality of the lifeworld. Rather, they are focused on the familiar territory of the everyday life, while the lifeworld seems to be a wider sphere encompassing a larger territory. Ploch calls this result the 'zoom effect' (1995: 29). Additionally, this familiar territory is not completely familiar, but rather the 'everyday life ways' within it are familiar, the ways through this territory.

Concerning orientation in space (räumliche Orientierung), she refers to Greverus' 'kulturökologisches Raumorientierungsmodell', which understands the living space as space of satisfaction (Satisfaktionsraum). Only if a space satisfies a person's needs, will the person identify with it and include it into their spatial orientation and mental maps. Here four dimensions are relevant: an instrumental one, a political-strategic one, a socio-cultural one, and a symbolic one. Basically, Ploch found that the socio-cultural dimension is the most relevant as mirrored in the mental maps. The instrumental one follows (with a dominance of consumption elements, e.g. shopping places over production elements, e.g. the workplace, places from the professional sphere). The symbolic one comes third (e.g. using natural landscape elements, which Ploch interprets as a sign of distinction against urban space). The political-strategic dimension is rather irrelevant.

Generally, Ploch argues that mental maps can be analysed as follows (1995: $31^{86}$ ): 'There is a first layer of the individual character of these images 'of the world in our minds'. Over this layer, there is a second layer of the 'specific', which, looked at as a whole, represents the 'general', and from which we can also derive the 'particular' with the help of the Raumorientierungsmodell [as presented by Greverus].'

Ploch says that there are two different content dimensions which can be found in mental maps. First, respondents draw 'their places', which Ploch understands as an individual level of significance. Through a comparison of these contents between cases, the researcher could discover similar significance patterns. Yet, as she says, these similarities are not necessarily based on jointly formulated and shared sense. Second, respondents also draw 'ideas of places', which rather represent an interviewee's guess about a collective image. Contents of this latter dimension are drawn more seldom, and they have to be uncovered through more specific questioning (Ploch 1995: 39f.)

Furthermore, Ploch interprets that the few 'ideas of city' that she could see in her mental map studies, as opposed to the many descriptions of 'their cities', is an indicator for the decreasing individual estimation that places might be shaped by the individuals themselves. Ploch writes (1995: 40 ${ }^{87}$ ): 'This competence of actively appropriating the environment - and changing and reinterpreting spaces - becomes stunted equivalently to the extension of politically and bureaucratically formulated general authority of professional planning.' In particular, this last point is interesting in the light of creative industries' literature. There, creative knowledge workers are suspected to be very active agents in the field of space appropriation and re-definition.

\footnotetext{
${ }^{86}$ Translated by the author from the German text: 'Über den individuellen Charakter dieser Bilder "von der Welt in unseren Köpfen" zieht sich eine Folie des Spezifischen, das zusammengenommen das Allgemeine abbildet, aus dem wir mit Hilfe des Raumorientierungsmodells - auch - das Besondere ermitteln können.'

${ }^{87}$ Translated by the author from the German text: 'Diese Kompetenz der aktiven Umweltaneignung - zu der auch Veränderungen und Umdeutungen von Räumen zählen - verkümmert im gleichen Maße wie die politisch und bürokratisch formulierte Allzuständigkeit von Planung steigt.'
} 
Therefore, one could suggest that their maps should contain more 'ideas of space' than 'their space' elements.

\subsubsection{The sampling strategy}

My sampling strategy orientated by my own definition of the target group (cf. chapter 2). The first criterion was: I looked for people that worked in creative industries - according to my encompassing definition. Second, I chose people as interview partners who lived multilocally. And third, the multilocality should take place across national borders, thus having a transnational character. In the description of qualitative heuristics (cf. section 6.3 below), contrasting cases are mentioned as a tool to increase variation between individual cases. I tried to vary my sample according to the following variables: family situation, age, professional field, place patterns (EastWest; North-South; EU vs. intercontinental), and nationality.

Obviously, such a catalogue of criteria is already complex and describes an ideal way of sampling. The problem is that there is no data available from registries or other sources about multilocal people, as it is simply not possible to register it (cf. section 4.2). Also transnationality is not registered, so I had to rely on other ways of approaching interesting interview partners. I started with two interview partners who I already knew, and who fitted into my sample. Then I decided to use the snowball sampling strategy: I asked my interview partners, if they could suggest further interesting interview partners. Such a strategy obviously suffers the danger of producing a very biased sample, so I tried to not stick too much to snowball sampling in the social networks of my two initial interview partners. Instead, I also approached my own social networks, my colleagues and my friends and asked them for interesting interview partners. On the other hand, in exploratory research the bias can be relativised by respecting variation between cases. This qualitative design does not aim at completely unbiased representativity.

Also Currid (2007), who studied creative knowledge people and their social interaction in the case of New York, used such a mix of sampling methods, because otherwise it is difficult to find interview partners. Currid describes her experience with qualitative interview sampling in the field of creative industries as follows (2007: 457): 'I found interview subjects through a variety of methods, including cold-calling, snowball sampling, and intentionally selecting particular subjects. I found, as is true throughout the cultural economy, that credibility and social networks influence access [to interview partners]. Therefore, while I obtained some interviews simply by looking up phone numbers or email addresses and contacting my subjects without introduction, in many cases I obtained contact information for possible interviewees from other cultural producers. While this approach is not unbiased, people to whom I was referred by others were more likely to grant interviews than those I contacted without a reference.'

\section{Composition of the sample}

Through a mix of different channels, I could realise a sample which varies in several ways according to the above mentioned variables (see table 2; for more detailed information see appendix $\mathrm{B}$ with case description as well as the individual life stories as to be found in the inserted grey boxes in the text). 
Table 2: Overview of the interview sample

\begin{tabular}{|c|c|c|c|c|c|c|c|}
\hline No. & Name & Gender & Age & $\begin{array}{l}\text { Nation- } \\
\text { ality }\end{array}$ & Partnership & $\begin{array}{l}\text { Chil- } \\
\text { dren }\end{array}$ & Profession \\
\hline 01 & Aurèlien & Male & $30 \mathrm{~s}$ & French & Yes (German) & No & $\begin{array}{l}\text { Artist \& director of cultural } \\
\text { association }\end{array}$ \\
\hline 02 & Birgit & Female & $50 \mathrm{~s}$ & German & Yes (German) & No & 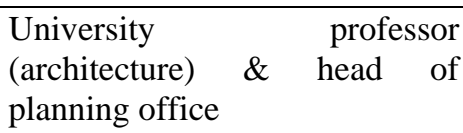 \\
\hline 03 & Christian & Male & $30 \mathrm{~s}$ & French & No (Ex German) & No & $\begin{array}{l}\text { Artist, model, translator \& } \\
\text { construction worker }\end{array}$ \\
\hline 04 & Dirk & Male & $30 \mathrm{~s}$ & German & Yes (Bulgarian) & No & $\begin{array}{l}\text { Slavicist, journalist } \\
\text { consultant in adult education }\end{array}$ \\
\hline 05 & Emil & Male & $40 \mathrm{~s}$ & German & Yes (Italian) & No & $\begin{array}{llll}\begin{array}{l}\text { Political } \\
\text { activist }\end{array} & \text { scientist } \& \text { cultural } \\
\end{array}$ \\
\hline 06 & Federico & Male & $30 \mathrm{~s}$ & Italian & No & No & Cultural scientist \& activist \\
\hline 07 & Gunnar & Male & $20 \mathrm{~s}$ & German & Yes (Spanish) & No & $\begin{array}{l}\text { Cultural scientist \& stem cell } \\
\text { courier }\end{array}$ \\
\hline 08 & Henriqua & Female & $30 \mathrm{~s}$ & Spanish & Yes (Dutch) & 1 & $\begin{array}{l}\text { Scientist in field of regional } \\
\text { development }\end{array}$ \\
\hline 09 & Isabel & Female & $40 \mathrm{~s}$ & Spanish & $\begin{array}{l}\text { No (Ex was } \\
\text { German) }\end{array}$ & No & $\begin{array}{llll}\text { Interpreter } & \text { for } & \text { high } & \text { rank } \\
\text { politicians } & & & \end{array}$ \\
\hline 10 & Juliana & Female & $30 \mathrm{~s}$ & Lithuanian & Yes (German) & 2 & Ethnologist \\
\hline 11 & Kate & Female & $60 \mathrm{~s}$ & $\begin{array}{l}\text { US } \\
\text { American }\end{array}$ & $\begin{array}{l}\text { Yes (Ex was } \\
\text { German) }\end{array}$ & No & $\begin{array}{l}\text { Linguist \& Artistic Director for } \\
\text { Movie Productions }\end{array}$ \\
\hline 12 & Liam & Male & $60 \mathrm{~s}$ & English & Yes (Scottish) & 3 & $\begin{array}{l}\text { Scientist in the field of regional } \\
\text { development }\end{array}$ \\
\hline 13 & Marta & Female & $30 \mathrm{~s}$ & Lithuanian & Yes (Slovenian) & No & Freelance researcher \\
\hline 14 & Nikolina & Female & $20 \mathrm{~s}$ & $\begin{array}{l}\text { US } \\
\text { American- } \\
\text { Bulgarian }\end{array}$ & - & No & Geographer (student assistant) \\
\hline 15 & Oskar & Male & $50 \mathrm{~s}$ & Swiss & Yes (Austrian) & 1 & $\begin{array}{l}\text { Head of firm organizing } \\
\text { cultural events / moderation }\end{array}$ \\
\hline 16 & Pia & Female & $30 \mathrm{~s}$ & German & - & No & $\begin{array}{lll}\text { University } & \text { teacher } & \text { in } \\
\text { architecture \& head } & \text { of } \\
\text { architecture firm } & \end{array}$ \\
\hline 17 & Qamar & Male & $40 \mathrm{~s}$ & $\begin{array}{l}\text { Chechen- } \\
\text { German }\end{array}$ & $\begin{array}{l}\text { No (Ex were } \\
\text { Dagestanian and } \\
\text { Russian- } \\
\text { German) }\end{array}$ & 2 & Artist \& Landlord \\
\hline 18 & Ronja & Female & $30 \mathrm{~s}$ & Danish & Yes (German) & 1 & Journalist \& urbanist \\
\hline 19 & Stephan & Male & $30 \mathrm{~s}$ & German & Yes (Danish) & 1 & $\begin{array}{llll}\begin{array}{l}\text { Architect } \\
\text { university }\end{array} & \& & \text { teacher } & \text { at }\end{array}$ \\
\hline 20 & Tjark & Male & $30 \mathrm{~s}$ & Danish & Yes (Austrian) & No & $\begin{array}{l}\text { Architect, Interior designer \& } \\
\text { teacher at university }\end{array}$ \\
\hline 21 & Ugo & Male & $30 \mathrm{~s}$ & Chilean & Yes (Chilean) & 1 & Scientist in field of urbanism \\
\hline
\end{tabular}




\begin{tabular}{|l|l|l|l|l|l|l|l|}
\hline 22 & Valentin & Male & 30 s & German & Yes (German) & No & $\begin{array}{l}\text { Actor, Juggler, Film maker \& } \\
\text { Freelance pedagogue }\end{array}$ \\
\hline 23 & Willi & Male & 30 s & German & $\begin{array}{l}\text { Yes } \\
\text { Argentinian- } \\
\text { Belgian) }\end{array}$ & 2 & $\begin{array}{l}\text { Scientist in Architecture \& } \\
\text { Urban Planning }\end{array}$ \\
\hline 24 & Xaverio & Male & 20 s & Italian & No (Ex German) & No & $\begin{array}{l}\text { Freelance photographer \& } \\
\text { sociologist }\end{array}$ \\
\hline 25 & Yari & Male & 30 s & Mexican & - & No & Hip hop artist \\
\hline
\end{tabular}

As shown in table 2, I have an age gradient, ranging from a young student towards interview partners in their 60s approaching retirement. I interviewed parents and people without children; I interviewed singles and people in stable partnerships; I interviewed people who own apartments or houses, other people who rented, and even one indicating to have lived through moments in life without renting nor owning other dwellings, not even having keys to somewhere. I interviewed people closer to traditional scientific occupations, and others being freelance artists. Yet, most of my interview partners have various jobs and indeed are situated in-between different professions. I interviewed people who considered themselves multilocals before the interviews, and others who did not; I interviewed people from different nationalities, with diverging place patterns. I interviewed people who (have) lived in bi-national partnerships, while others had not experienced bi-national partnerships. The big challenge is now to analyse this variety of cases with a focus on finding similarity (as demanded in the approach of qualitative heuristics).

\subsubsection{The interview situation: Conversational atmosphere}

In general, I decided to keep the interview situation as natural as possible. Vonderach (1997) has pointed to the importance of natural interview atmospheres in order to generate rich narrations. Given the fact that it was problematic to fix interview dates with my busy and mobile interview partners, I still did not want to get stressed by the often small time frames that we had. In addition, I had never met most interview partners before. Thus, the interview itself was also the situation of getting to know each other. A more relaxed interview situation, I hoped, would facilitate the trust that my interview partners would have in me, and this again, I thought, would improve the narrations. The situation that I wanted to create should be close to a private conversation, and not an artificial interview, in which one asks and the other answers. Such a procedure also allows for a better quality of interview data and it is one part of quality management in qualitative research designs (cf. Mayer 2008).

There were two main aspects that should help me to generate such a conversational atmosphere. First, as far as I could, I tried to combine my interviews with something to drink or to eat, and I invited my interview partners in order to compensate for their time. As my interview partners were very busy, but normal people who needed to eat, I thought it might be easier to convince them to an interview, if the time was to be used multi-purpose: namely, for doing the interview and for eating. Serving something to eat and to drink also allowed for a character of meeting with some acquaintance for a joint lunch, coffee break or dinner, instead of going to a scientific interview. 
Second, I tried to avoid any hierarchical situation in the interviews. Even though I had not met all of my interview partners beforehand, I still tried to address them using the German ' $D u$ ', the French and Spanish 'Tu', instead of the formal 'Sie', 'Usted' or 'Vous'. Obviously this problem does not arise in English language interviews. Being informal, however, needs a bit of sensitivity. Some people might feel offended. In two cases, I obviously was not sure and preferred to stay 'formal' in order to not destroy the situation by being 'impolite'. Hierarchical distance in interviews might also result from the fact that the interviewees feel confronted with a scientist and feel that they know less and drift into a pattern of shy behaviour. The scientific interviewer is then psychologically in a higher position, and he might not get true information as interviewees might respond as they think the interviewer expects them to. This is often the case in biographical interviews, in which the interviewer enters into the core of privacy, and in which interviewees look to legitimise their own life. Yet, the phenomenon might also occur the opposite way around. In particular in expert interviews (e.g. with politicians, managers etc.), the interviewees take the stronger position as they are the one who knows that they know more about a certain issue and that the interviewer has come exactly for that. Thus, information here might also not be the truth, as experts are often people trained in external communications and public relations.

The point is that both hierarchical problems cause an artificial character of the interview. On the one hand, the interviewer gives the interviewee the feeling of being studied as a private person, and the interviewee might fall into a defensive position. On the other hand, the interviewee knows for sure that she/he is in the offensive position and can try to make use of it. I think the key to avoiding hierarchical situation is to build up trust. The interviewee must be able to trust in the fact that the information that they provide during the interview is not disadvantageous to them afterwards. An interviewer can only avoid having either the problem of individual legitimisation or prepared PR talk in this manner.

One way to build up trust is to signal the interview partner that both interviewee and interviewer belong to a same social group, that they share something, some experience or knowledge. Here, also Charmaz (2008) remarks that an interviewer's actual involvement not only helps the interview situation, but also the analysis at a later stage. If a researcher is involved in the empirical field, Charmaz argues, they can better reconstruct the social reality for deriving theoretical outputs. Obviously, this involvement in the empirical field is not always a given, and an interviewer should be honest and never lie about such things by pretending. Yet, in my case it was obviously the case that I also lived through periods in which I was multilocal, and I let my interview partners know about it. This quickly helped for the generation of a conversational atmosphere in which my interview partners did not try to legitimise their life, as they had the feeling of speaking to some 'fellow multilocal' and started to reveal information that otherwise probably would not have been reported automatically. The interview with Willi serves as an example for how this might work (see transcript Willi lines 848ff.). In the interview the conversation took a new dynamic when I started to speak of my own personal situation, and the interviewee started asking me. He later on also revealed new information again, even though for me the interview was already finished. So, I think it was a helpful decision to avoid any hierarchy and to create a conversational atmosphere in which interviewer and interviewee are more or less equal partners.

I also had direct feedback to the interviews by my interview partners directly to me, or even to other people, who mediated that we met. My interview partners expressed that they found the interview 
situation very relaxing and they started to pause in their busy everyday life. The interviews, and in particular drawing the mental maps - which some had never done before - made them reflect on their own situations. Based on these reflections they could think in a funny way about their lives and how their lives should be in the near future. I can confirm what Ploch (1995) mentioned: the interviews in combination with mental map drawing were perceived by the interview partners as funny, inspiring and a moment of reflection outside the immediate everyday life. Actually, they liked it as a brief time-out.

\subsubsection{Language issues}

I also have to point out that my interviews were conducted in different languages. I met people from different national backgrounds, and not all of them could speak English or German properly. So, I offered to do interviews in the language they preferred, provided that I knew a bit of that language, too. Obviously, such a proceeding leads to a lot of language issues. These linguistic difficulties might lead to problems in maintaining a sensitive use of concepts and wording during the interview. Scherke (2011: $88^{88}$ ) also comments on that: 'Another problem for qualitative social research in relation to migration phenomena consists of the language in which an interview is conducted. The life practice of migrants is often characterised by multilingualism. Depending on the context, migrants spontaneously switch between languages or they develop hybrids. Yet, sufficiently qualified interviewers who are able to deal with this phenomenon are often lacking. [...] Qualitative interviewing calls for an intensive attention towards the opponent, in other words interviews that are conducted in a foreign language cause either inhibition for the interviewee or problems for the interviewer, because both cannot be sufficiently spontaneous in their responses to the other.' While Scherke describes this problem, she does not offer a solution to it. She only points out that essentialist and traditional concepts (e.g. ethnicity, nationality) are necessary in some contexts of sociological research in order to understand the resulting problems in multicultural empirical fields. On the one hand, these concepts and adscriptions (Zuschreibungen) make sense to analyse discrimination, which is often based on exactly these traditional concepts. On the other hand, the concepts are part of the lifeworld of migrants, and as such have real consequences in their lives.

A major problem which not even linguists might solve is the translation of individual terms and the related semantic content or concepts between languages. For example, I faced this problem with my central German terms zu Hause and Heimat, which even I could not even translate into English. While some scientists might argue that wording in questions is also a very sensitive issue when it comes to multilingual studies, I would argue that this problem was only of a secondary nature in this project. I formerly had worked in European quantitative studies in which questionnaires were

\footnotetext{
${ }^{88}$ Translated by the author from the German text: 'Ein anderes Problem für die qualitative Sozialforschung im Zusammenhang mit Migrationsphänomenen stellt die Frage nach der Sprache dar, in der ein Interview geführt wird. Die Lebenspraxis von Migrantinnen und Migranten weist häufig eine ausgeprägte Mehrsprachigkeit auf. Je nach Kontext kommt es zum spontanen Wechsel zwischen Sprachen bzw. werden auch Mischformen entwickelt. Vielfach fehlt es jedoch an entsprechenden qualifizierten Interviewern, die in der Lage sind, diesem Phänomen Rechnung zu tragen. [...] Qualitative Interviews erfordern jedoch ein intensives Eingehen auf das Gegenüber, d.h. in einer fremden Sprache geführte Interviews erzeugen entweder bei dem bzw. der Interviewten Hemmschwellen oder beim Interviewenden Probleme dahingehend, dass nicht spontan genug auf die Äußerungen des Gegenübers eingegangen werden kann.'
} 
set up in different languages - and yes, wording is a big issue there. However, I think that qualitative studies have an advantage here, as we are in a situation of conversation. If the interviewee does not understand the question, he can ask for a clarification. If the interviewer thinks that the interviewee has not understood the question, he can ask a question differently. Thus, I dealt in a pragmatic way with the multilingual setting: I simply tried without hesitation.

Coming back to the example of the zu Hause/Heimat problem, in other languages I tried to take another way by circumscribing the topic with other words, which I knew from the foreign language, paying much attention to what my interview partners would answer. In some cases I also tried to discuss the language issue directly with my interview partner if I knew they spoke German as well. For example, you could look at the interview with Kate (lines 227ff.). As a dubbing expert, I though, she was the right person to discuss the translation of Heimat/zu Hause into English language. The interview was done in German, but as she is an English native speaker and a linguist, I tried to figure out the translation aspects with her. Similarly, you can look at the interview with Christian (lines 245ff.), in which I used the German words in a French interview. Christian was not even astonished about it and answered without hesitation. This in fact supports Scherke's observation on the spontaneous switching between languages amongst migrants.

I would conclude that in multilingual settings, such as this study, language can become an issue. Misunderstandings and the impossibility to translate certain concepts word by word into other languages have to be taken into account during the analysis. Nonetheless, I think that in a qualitative study language issues can be controlled much better during the interview than in quantitative settings.

Finally, given the specificity of my study group, being transnational migrants, the language issue is relative also from another point of view. My interview partners all crossed national borders and they are multilocal in a transnational context. This means they have to deal with language issues within their everyday life, and they have developed a certain expertise and relaxed position towards it. They are used to conceptual clarifications between languages from their everyday life, so it was no problem during the interviews to also reformulate questions as well as answers in order to provide an understanding on both sides of the interviewer as well as the interviewee.

\subsection{Methods for the analysis of the interview data}

This section is dedicated to the introduction of some main ideas about how to analyse qualitative data which originates from interviewing. I will first describe the emergence of the grounded theory approach that was, at the moment of its first appearance on the scientific rim, a very progressive and controversial idea. It was rejected by a lot of scientists, yet it offers a philosophy of understanding qualitative research as something that is nurtured by the empirical data. It is very helpful to understand how the generation and first treatment of data could work. Then, I will briefly speak about the idea of hermeneutics. Hermeneutics provide an approach for the detailed and reconstructive analysis of qualitative data. And there are many scientists who use hermeneutics for analysis. I will focus here on the specific approach of Vonderach (1997) which he calls Geschichtenhermeneutik, in which a special attention is paid to the single stories that make up a person's life. Finally, I will also introduce the approach of qualitative heuristics, which Kleining \& Witt (2000) developed. 


\subsubsection{The logics of grounded theory ${ }^{89}$}

The grounded theory approach for the analysis of empirical data was first introduced by Glaser \& Strauss (1967) and their book 'The discovery of grounded theory: Strategies for qualitative research'. This book was written with the intention of overcoming the cleavage between scientific theory and empirical reality. In an interview for the German Journal für Psychologie, Strauss stated that they wanted to introduce a new research practice which derives empirically grounded theory from the data and does not - as was/is common - uses the data in order to verify or falsify preconceived theories (cf. Legewie \& Schervier-Legewie 1995). In reference to Glaser \& Strauss, Kelle (2005) describes that the 1960s' world of Humanities was dominated by a few 'theoretical capitalists' and a large mass of 'proletariat testers' (PhD students and young researchers). The latter were commissioned by the former to empirically test their 'grand theories'. Kelle comments (2005: paragraph 2): 'One of the main purposes of Glaser's and Strauss' 'Discovery book' was to challenge the hypothetico-deductive approach which demands the development of precise and clear cut theories or hypotheses before the data collection takes place'. The grounded theory paradigm is not a scientific theory; rather it is a methodological frame for the analysis of the data. The grounded theory perspective describes a specific relationship between empirical data and scientific theory, in which empirical data lets formal, substantive theory 'emerge'.

This concept was criticised from the beginning as it contradicts other approaches to data analysis which prefer the use of preconceived theory for generating and analysing data. Since the initial presentation in 1967, the concept was thus constantly reworked by Glaser and Strauss, and it finally led the two authors to break up and to develop it in two different directions. Whereas Strauss \& Corbin (1990a, 1990b) paid attention to the 'anti-grounded-theory' criticism and continued to enhance the practical dimensions of grounded theory, Glaser $(1978,1992,1998)$ is more closed towards critiques and focused on a more empiristic discussion which sticks to the original version of the grounded theory concept (Legewie 2005). However, the conceptions of grounded theory of both Strauss and Glaser have been misinterpreted and misused for different types of qualitative data analysis.

\section{Grounded theory according to Glaser}

In order to clarify what is grounded theory and what is not, Glaser (2004) addressed the qualitative social researchers with the article 'Remodeling Grounded Theory'. He writes (2004: paragraph 6): 'I hope to relieve GT [grounded theory] of the excessive scientism brought on it by those worried about accuracy and what is 'real' data when creating a scientific product. I hope to give explanatory strength to those PhD dissertation level students to stand their GT grounds when struggling in the face of the misapplied QDA [qualitative data analysis] critique by their seniors and supervisors.'

After citing common misinterpretations of grounded theory, he introduces a short guideline for how to apply the grounded theory method. According to him, grounded theory development starts stringently without any theoretical pre-considerations. While other methods of qualitative data

\footnotetext{
${ }^{89}$ Main parts of this section were published earlier in a working paper that I have written in the frame of the PhD research at the University of Milan-Bicocca. The paper can be found on the university’s website:

http://www.sociologiadip.unimib.it/dipartimento/ricerca/scheda.php?idUser=246 (accessed 31 October 2012).
} 
analysis start with literature review and the construction of a theoretical framework in order to design an empirical data collection process, Glaser is convinced that 'all is data' (2004: paragraph 12). The researcher looking through the grounded theory lens has to be open-minded for every kind of data sources (interviews, field notes, statistical tables, scientific literature, observations, secondary data, etc.) and to what this data tells him. The view of the story that the data tells has to be open and should not be limited by preconceived theoretical considerations. Scientific literature in this sense is one among many data sources whereas it is not the precondition to start research. The objective of this method is ' a set of carefully grounded concepts organized around a core category and integrated into hypotheses’ (Glaser 2004: paragraph 41). Which steps are necessary to achieve this goal?

\section{Theoretical sensitivity}

How does the researcher get there? In order to achieve this goal, the researcher has to enter into a cyclic process of constant reflection about the relation between the empirical data and the emerging theory (circumscribed as 'systematic circularity' as in Flick 2006b: 37; 'iterative process' as in Charmaz 2008: 156; 'zig-zag process' as in Creswell 2007: 64; 'tacking back and forth' as in Clarke 2007: 424). Therefore, theoretical sensitivity is a fundamental necessity in the Glaserian grounded theory conception. This means that the researcher has to trust his own preconscious processing of the data without yet knowing where it will lead him. He has to be open to confusion in the beginning of the research. Furthermore, he must be able to use first upcoming theoretical insights and hypotheses in order to design the subsequent empirical stages. Even the research problem is unknown before and will be discovered in the process of reflection about the data. Glaser's suggestion is to start right away using the first data source available. The initial step is to find any general concern showing up in the first data. This 'running the data open' is assisted by questions such as: what is actually happening in the data? What is this data a study of? What is the main concern being faced by the participants? (cf. Glaser 2004: paragraph 48).

\section{Open coding \& theoretical sampling}

At this moment of research, open coding of upcoming main concerns/interests has to be applied. Thereby, grounded theory coding differs from other qualitative data analysis methods as it asks for 'action' and not for 'topics', aiming at explanation rather than description (Charmaz 2008). The first codes then indicate a direction which should be pursued. It leads to the development of first categories for analysis and first hypotheses, and with the aid of these hypotheses the theoretical sampling of further data sources can be conduted. The process of theoretical sampling does not aim at a representative sample: 'Cases are chosen as it becomes clear that they can contribute to theory generation' (Harding 2006: 132). With these cycles of data collection, the subsequent data analysis, the category and hypotheses development, and the theoretical sampling of the research become more focused. The narrower the point of interest becomes, the more the coding switches from an open character to a selective type of coding the data (Glaser 2004). In this subsequent stage of 'focused coding' some initial open codes are selected which have a high 'analytic momentum' (Charmaz 2008) or 'theoretical capability' (Clarke 2007). They are put into categories for further theoretical development. Theoretical sampling, here, supports the teasing out of hidden properties of the first categories, which derived from open coding. Further data sources are chosen in order to clarify uncertainty in categories' properties. Finally, a core variable (which should be central and 
should account for a large share of variation in the data) will emerge and be used as the anchor point for the construction of a grounded theory.

\section{Constant comparative method \& analytic induction}

According to Glaser (2004), two basic features make this process a systematic research methodology. First, the constant comparison between the collected, empirical data and the developing hypotheses/theoretical framework assists to remain 'systematic' during theory generation. In an ongoing reflection these two spheres are mirrored against each other. The hypotheses/categories have to be relevant (as indicated by the data), they must fit every used data, they must work with each other in the final grounded theory and they have to be modifiable (in case other data reveals contradictions).

Here, Glaser suggests a three-step comparison. When starting with the research, one only works with incidents in the data (indicators), which should be compared to each other. When uniformity is found among incidents, first concepts should be evolved by the researcher. In the second step of comparison, these concepts are compared (tested) by further incidents. When concepts have proven to fit, they will finally be compared to other evolved concepts. The main interest in comparison, thus, is to find contrasting evidence in the empirical data, and to modify hypotheses in a manner that they fit a broad social reality resulting from the empirical data. This process of 'contrasting' the hypotheses with further data and concepts is called 'analytic induction'. The analytic induction's 'decisive instrument is to analyze the exception or the case that is deviant to the hypothesis' (Flick 2006a: 5). As long as the hypothesis can be destabilised by contrasting evidence, it has to be modified $^{90}$.

Flick (2006b) also points out that the constant comparative method helps to identify 'ideal types'. These types should be derived using the instrument of 'minimal comparison' (of cases within one type) and the 'maximal comparison' (of cases in different types). In the end each empirical case should fit one of the constructed ideal types.

Once a certain uniformity between concepts, categories, types, and hypotheses is achieved a more abstract and formal theory could be constructed which integrates all the single parts. This moment is called 'theoretical saturation'. It means that further data collection and data analysis do not generate new - in the sense of contrasting - insights. Work with the empirical material is then finished, and only the sorting of the ideas follows. Charmaz (2008) alludes that the term 'theoretical saturation' should not be misunderstood as the moment when themes come up repeatedly in new data; theoretical saturation is reached when the properties of categories are clear, and hypotheses have sufficient explanatory quality. In addition, she points out that many grounded theory studies claim theoretical saturation without documenting it.

\section{Memo writing}

This leads to the second systematic necessity of grounded theory methodology: the documentation of the theory generation process. Therefore, Glaser \& Strauss (1967) introduced the procedure of

\footnotetext{
${ }^{90}$ The concept of analytic induction has been criticised by some authors. Yet, Flick (2006a: 5) affirms its usefulness for grounded theory: 'Analytic induction has been criticized as it does not [...] provide a means for establishing causal laws and universals. There are question marks against the generalization of case studies and external validity in general. Nevertheless, analytic induction has its own importance as a procedure for assessing and developing analyses by the use of negative cases.'
} 
'memoing'. Glaser later defines (2004: paragraph 61): 'Memos are theoretical notes about the data and the conceptual connections between categories. The writing of theoretical memos is the core stage in the process of generating theory'.

These memos document the researcher's hypotheses about the connections between categories and their properties. In addition, they show the attempts of how to integrate them into a generated, consistent theory. Memos are the framework for exploring, checking, and developing ideas as well as documenting the research process and progress (Charmaz 2008). The memos are most useful when they are titled, referenced to their source data, compared to other memos, and their generation process is documented as well as their consequences for the study's progress.

In a final process - when theoretical saturation is reached and uniformity among categories exists the sorting of all the researcher's memos generates the conceptual outline for the articulation of the theory grounded in the empirical data. This process of organising the memos also has to follow a certain system. The researcher could start with any memo and then should refer it to the core variable/category. Subsequently all other memos are sorted around this focus and put in relation to each other so that every memo fits into the resulting theoretical frame. Grounded theory methodology, here, assumes that the social reality and each piece of it are completely integrated. The grounded theory researcher thus has to uncover the integration of each of his memos into his own grounded theory. The resulting theory should explain 'as much variation as possible' with 'the greatest scope possible’ using 'the fewest possible concepts' (Glaser 2004: paragraph 74).

During this process of reflection and documentation, Glaser (2004) states that the researcher will finalise their study by writing up a substantive, conceptual theory with general implications. This systematic process of theory generation prevents them from simply itemising a list of descriptive findings. The resulting grounded theory has to fulfil the following criteria of scientific quality:

1. Conceptual representativeness (which means explaining function in other contexts/for other phenomena as well);

2. Comprehensibility (for both researcher and interviewees/empirical sources of social reality).

The resulting, profound, and inductive insights of the grounded theory method represent a 'rhetoric' of the social psychological world (Glaser 2004).

Critiques on the grounded theory approach of the Glaserian school

After years of being used, misused, interpreted, and misinterpreted, the grounded theory approach of Glaser and Strauss has been exposed to a large strand of critical literature. Among them, Thomas \& James (2006) give a comprehensive overview about major concerns. The first critique refers to the use of the term 'theory'. The argument is based on the quality criteria that a scientific theory is scientific when it is predictive. The problem about the grounded theory approach is that it accepts the dynamic and changing character of the social reality. Thus, the grounded theory - already from its self-understanding - is only limited to a certain case, and it only is relevant for limited time. It could not be universal. And if it is not universal, it could not be a theory, but only a particular narrative or description. A second doubt about grounded theory methodology arises from the claim to be purely inductive. The researcher's property to be totally dispassionate about the field of study is a characteristic neglected by critiques of grounded theory. According to these critiques, a researcher always possesses of some ex ante knowledge and should not deny this, but should make 
use of it. Third, the image of the 'emerging theory' which is to be discovered is criticised because a theory cannot pre-exist 'hidden' in the data. Rather, it is an active process of invent-ing/thinking a theory, which equals the process of interpretation as in other analysing methods.

The second critique about the distance to ex ante knowledge as formulated by Glaser \& Strauss (1967) is also addressed by Kelle (2005). This problem consists of the contradiction between, on the one hand, the claim to start research without theoretical preconceptions (letting theory 'emerge') and, on the other hand, the necessity to integrate pre-existing theoretical knowledge into the first steps of grounded theory building by analytic category development (theoretical sensitivity). In Glaser \&Strauss' primary book, The Discovery of Grounded Theory (1967), the two authors could not solve this problem. Later, both of them followed different approaches of how to overcome this paradox. Kelle writes (2005: paragraph 5): 'Since the 1960s it is one of the most crucial and widely accepted insights of epistemology and cognitive psychology that [...] the construction of any theory, whether empirically grounded or not, cannot start ab ovo, but has to draw on already existing stocks of knowledge.'

Yet, the Glaserian school still sticks to a rather 'fundamentalist' understanding of how to do grounded theory construction. According to Kelle (2005), Glaser - in his 1992 monograph 'Emergence vs. Forcing: Basics of Grounded Theory Analysis' - accused Strauss of 'forcing' data by applying too rigid 'coding paradigms'. Glaser (2004) strictly resists to the use of pre-existing knowledge in grounded theory construction. He tries to overcome the initial paradox (see above: emergence of theory vs. theoretical sensitivity) by clarifying the idea of 'theoretical coding'. Whereas 'substantive coding' is the process of applying open codes to the empirical data - the substance - in order to find emerging categories, 'theoretical coding' represents the researcher's set of tools to conceptualise the relations between the 'substantive codes' in the final product: the integrated grounded theory. Glaser defines a set of 'coding families' which describe the ordering of the social world. These coding families contain 'terms from highly diverse theoretical backgrounds, debates and schools of philosophy or the social sciences' and could be used to create causal models from the substantive codes (Kelle 2005: paragraph 13). In these more than 15 coding families, rather formal epistemological terms like 'causes', 'contexts', 'consequences', 'conditions' are mixed together with sociological concepts like 'norms', 'values', or 'beliefs'. Thus, Kelle criticises (2005: paragraph 14): 'In order to develop theoretical models about empirical phenomena formal or logical concepts (like 'causality') have to be combined with substantial sociological concepts (like 'social roles', 'identity', 'culture'). A major problem with Glaser's list of coding families is that it completely lacks such a differentiation between formal and substantial notions.' Equally, Charmaz critically states (2008: 159): 'What Glaser includes in a given coding family sometimes seems arbitrary and haphazard. The coding families are not necessarily mutually exclusive, and their boundaries are often indistinct.'

Therefore, on the one hand Glaser's approach to conceptualise the role of pre-existing theoretical knowledge in grounded theory procedures does not seem sufficiently clear; for inexperienced researchers this coding scheme is especially of less help. On the other hand, Glaser does not seem willing to integrate pre-existing knowledge into his blueprint of grounded theory methodology. He follows a rigid understanding of induction. 


\section{Grounded theory according to Strauss \& Corbin}

What is the difference of the Straussian approach? The general structure of how to do grounded theory construction equals the Glaserian way. The difference could be found in the way of solving the initial paradox (emergence of theory vs. theoretical sensitivity). Strauss \& Corbin (1990a, 1990b) - instead of coding families - suggested a 'more straightforward and less complicated' way of how to start coding; they use a 'coding paradigm', as Kelle mentions (2005: paragraph 15). This coding paradigm consists of four items (or 'types of categories' as termed by Creswell 2007: 64): 'conditions', 'interaction among actors', 'strategies and tactics', and 'consequences' (Strauss 1987: 27-32). The items should guide the researcher in the process of open coding the data.

Axial coding

Once a first code ascription is applied to the different incidents, the process of 'axial coding' is crucial in the Straussian grounded theory construction. Axial coding means putting each code into relevant relations with other codes, and thus checking for logical axes between the codes. If relevant, these axes between the codes help to find a substantive theoretical model, often presented in a visual model (Creswell 2007: 65). In doing so, six criteria should be looked at in order to construct the relations between the codes: (1) phenomena, at which action and interaction are directed; (2) causal conditions, which lead to the occurrence of these phenomena; (3) attributes of the context; (4) additional intervening conditions, which influence the phenomena; (5) (inter-)action strategies used to handle the phenomena; and (6) consequences of (inter-)action (Kelle 2005: paragraph 17).

\section{Causal-conditional matrix}

The resultant axial model represents the image of (inter-)action strategies found in the data. Creswell (2007) points out that Strauss \& Corbin have even taken the analytic process a step further, integrating the substantive theory into a causal-conditional matrix. This conditional matrix is a coding device consisting of concentric circles ranging from the individual via the group, the organisation to the community, the region, the nation and the global world. It should help to integrate micro-sociological interaction theories into a more universal level. Yet, Creswell (2007: 65) indicates that this step is seldom undertaken by the researchers due to a lack of time and financial resources.

Summing up, Strauss \& Corbin follow a pragmatic and interactionist perspective. This perspective makes their approach very useful for micro-sociological studies focusing on interaction and action. However, for macro-sociological, rather system theory-oriented studies the coding paradigm of Strauss \& Corbin could have less practical value (Kelle 2005). Here, the less defined coding families of Glaser might be more open to a greater variety of theoretical perspectives.

Nonetheless, the Straussian approach seems to be attractive to a larger share of grounded theory users. The advantages compared to Glaser seem to be (1) a more precisely guided coding, which also makes grounded theory application feasible for young inexperienced scholars, and which lets research become focused quicker, (2) a more explicit understanding of pre-existing knowledge, which makes its utilisation more legitimate, and (3) an action theory approach, which makes it applicable for micro-sociological exploratory research (cf. Keller 2005). 
In respect to Glaser, Keller concludes (2005: paragraph 23) that his fundamentalism might only be part of a strategy to defend sovereignty of interpretation: 'The suspicion arises that the parlance of 'emergence' fulfils the function to legitimize a specific style of research: [...] following this rhetoric a researcher who follows the 'right path' of grounded theory cannot go wrong since the concepts have been emerged from the data.' Other authors also seem to vote for a more liberal understanding of grounded theory as in the Straussian way (Clarke 2007; Creswell 2007; Harding 2006). Clarke (2007: 437) equally states: 'Careful readers will recognize my very deep appreciation for and usage of many facets of GT [grounded theory], yet I am in deep disagreement with many of Glaser's recent fundamental(ist) points, and turn instead to Strauss.' Clarke's preference for the Straussian school originates in the larger open-mindedness towards the further development of grounded theory. Glaser strongly defends their jointly elaborated initial version. The new Straussian version is flexible, and Strauss does not reject enhancements.

\section{Enhancements of grounded theory}

After more than four decades, grounded theory has been applied and enhanced by several authors. Kelle, for example, points out that grounded theory construction could gain a clearer understanding, if it would refuse 'naïve empiricism', and if it would prevent the 'inductivist self-misunderstanding' (2005: paragraph 24). He therefore suggests conceptualising grounded theory reasoning as not only inductive but first of all abductive. In reference to the philosopher Peirce, Kelle explains that abduction is a third form of logical inference - next to induction and deduction ${ }^{91}$. Abduction consists of rearranging the facts so that new and unexpected experiences do not seem surprising anymore. In this innovative process, parts of existing knowledge which could each alone not explain the new empirical facts are put together in a new way in order to have a better fitting theoretical framework ${ }^{92}$. That is exactly what is done in grounded theory when constant comparative method and theoretical sampling is applied (see also Charmaz 2008: 167).

Consequently, Kelle (2005) discusses the falsifiability (understood as degree of empirical content) as a criterion for the applicability of theoretical preconceptions in grounded theory processes. According to the Popperian school of deductive hypothesis testing, hypothesis are only sound when they can be tested empirically. Thus, abstract and formal theories are considered difficult to use; they are simply too universal to be tested with a single sample. However, Kelle thinks that especially these abstract and formal theories might be very useful for the grounded theory researcher in order to structure the first analysis. In order to avoid the risk of neglecting better fitting formal theoretical concepts, he advocates the use of different - even opposing - concepts as analytical 'lenses'. In exploratory grounded theory concepts, categories of everyday and common sense knowledge additionally might assist in structuring data. These formal and everyday concepts might help to find more empirical categories for the proper grounded theory ${ }^{93}$. Therefore, the use of

\footnotetext{
${ }^{91}$ Induction means to reason from studied cases about the unobserved cases of a same kind in a finite population, so general statements are derived from empirical evidence of a sample. Opposed to induction, deduction means to combine a general premise with an empirical statement about a case in order to generate a further statement about that case (Hammersley 2006a).

${ }^{92}$ Kelle (2005: paragraph 31) describes: ,In making abductive inferences, researchers depend on previous knowledge that provides them with the necessary categorical framework for the interpretation, description, and explanation of the empirical world under study. [...] The framework which guides empirical investigations should be modified, rebuilt and reshaped on the basis of empirical material.'

${ }^{93}$ Kelle (2005: paragraph 40) concludes: 'Previous theoretical knowledge can be used at any stage of the process of empirically grounded theory construction if the researchers draw on theoretical concepts with limited empirical content. [...] Thereby, the
} 
preconceived knowledge - termed 'theoretical pluralism' by Kelle (2005: paragraph 44) - is legitimised not only by the fact that it facilitates the initial search for categories, but it also helps to develop these categories from the beginning on in very substantive ways in the sense of analytic induction. The pure 'emergence talk' thus becomes a more systematic process.

The corroboration of empirically grounded categories and theory is also considered necessary by Kelle. Consequently - and in opposition to Glaser's epistemological fundamentalism - one has to accept the fallibility of a grounded theory. For Kelle, it is a 'methodological fact that empirical research can never provide a final proof for theoretical propositions but only cumulative and always provisional evidence' (2005: paragraph 46). So, for further grounding the theoretical concepts it is necessary to search for empirical and theoretical evidence and counter-evidence. Applying these three suggestions (abductive inference as logic, acceptance of preconceived knowledge, and corroboration of grounded theory), Kelle argues, grounded theory research could overcome its initial contradictions.

Creswell (2007) broaches the issue of theoretical saturation. The problem in theoretical saturation, he states, consists of knowing when theoretical saturation is actually achieved. The moment of theoretical saturation could be a long time coming or even be invisible to the researcher. He suggests using 'discriminant sampling' in order to speed theoretical saturation. Discriminant sampling consists of gathering data from cases which are sampled according to the similarity to the initial cases studied. With these similar cases one can test if the developed theory holds true.

In addition, Creswell (2007) no longer refers to Glaser when describing current types of grounded theory studies. He differs between the Straussian school and the approach of Charmaz (2006). According to him, Charmaz stands for a constructivist interpretation of grounded theory, which is less structured than the Straussian version. It is characterised by the absence of a coding paradigm.

Charmaz (2005, 2006) refuses the single focus on the interactional frame (from which the Straussian coding paradigm is derived); she advocates an interpretative approach to collecting and analysing the data, which accounts for social complexity. She focuses on the values, views, ideologies, assumptions, and power hierarchies of the interviewees, instead of paying too much attention to the methodological accuracy of the research process. The 'reconstruction of social reality' becomes more central. The methodological complexity of the Straussian school limits the flexible use of grounded theory. The researcher should only use a very open, 'active coding', leading him to a grounded theory, which is always suggestive and incomplete (Charmaz 2005). Thereby, expressing the discovered relations should best be done in 'gerund phrases' - accounting for the process character of the social world (Charmaz 2008; in agreement: Clarke 2007).

Charmaz agrees with the Glaserian standpoint that Straussian methodology is too rigid. She argues for more flexibility in the method and advocates for the 'emergence idea'. Theoretical insights will better emerge liberally from the data than be put into the Straussian causal-conditional matrix after applying the coding paradigm. However, she distances her constructivist idea from Glaser's idea of the distanced position of the researcher (Charmaz 2008: 160): 'The constructivist position views research as an emergent product of particular times, social conditions, and interactional situations.

researcher may start qualitative analysis by using heuristic concepts and may then proceed to the construction of categories and propositions with growing empirical content.' 
Constructivists argue that researchers' perspectives will direct their attention but not determine their research. Unlike the view held by Glaser that researchers can and should remove themselves from the influences of their disciplines and the conditions of their research, constructivists aim to make these influences explicit.'

Thus, Charmaz' understanding of 'emergence’ is different from the Glaserian one. She considers both the empirical findings as well as the research methodology as 'emergent'. Glaser \& Strauss, by contrast, only conceptualised the empirical findings as emergent. Charmaz makes clear that it helps grounded theory methodology to integrate the subjective situation of the research, defined by the opinions, pre-considerations, and choices of the researcher. The researcher is guided by upcoming themes during the process of research, and they have to integrate this state of 'being guided' into the theory construction. Charmaz (2008) disagrees with both Glaser as well as Strauss \& Corbin about the use of codes. For her, codes - no matter if coding families or a coding paradigm - force the data. Another suggestion of Charmaz concerns the field of study: she advises personal affection. A grounded theory study becomes better when the researcher is personally involved in the field of study. They then might have a better capability of reflecting the empirical world and the relations with the theoretical frame.

Clarke (2007) offers another enhancement of the initial grounded theory concept of Glaser \& Strauss. She starts her conceptualisation with a summary of current critiques from within and outside the grounded theory community. The most common critiques refer to the fact that Glaser \& Strauss thought up the method in the 1960s as opposed to the, by that time, dominating positivism, and then they were unable to adopt it to later evolving scientific perspectives (such as neopositivism, constructivism/postmodernism, theoretical functionalism, symbolic interactionism/poststructuralism). According to Clarke, both Glaser and Strauss tried to cope with criticism: Strauss (in collaboration with Corbin) focused on a symbolic interactionism perspective, opening up to the researcher's influence on the data collecting; Glaser turned to a more functionalist perspective, neglecting the researcher's involvement. For Clarke, Strauss' position seems more professional and an honest attempt to 'make the post-modern turn' ${ }^{\text {,4 }}$. Strauss admitted that researchers and research are always situated in specific structural conditions, yet Clarke criticises Strauss' causal-conditional matrix for being too abstract and not giving empirical specification or clear explication (2007: 432).

Subsequently, she introduces her 'situational matrix', which she frames with the question (Clarke 2007: 432): 'How do these conditions appear - make themselves felt as consequential - inside the empirical situation under examination?’ She claims to offer a matrix in which conditions of the situation are conceptualised as being in the situation. She thinks that these conditional elements 'are' the situation and hence need to be specified ${ }^{95}$.

\footnotetext{
${ }^{94}$ Clarke explicates (2007: 431): 'Strauss moved partly, and I would say significantly, around the postmodern turn. Thus it is with Strauss that I have asked, 'How, then, can we meaningfully incorporate analysis of the precise ways in which particular contexts may matter into the processes of doing qualitative research?"

${ }^{95}$ Clarke suggests (2007: 432): 'I offer a situational matrix. Here, conditions of the situation are in the situation. There is no such thing as 'context'. The conditional elements of the situation need to be specified in the analysis of the situation itself, as they are constitutive of it, not merely surrounding it or framing it or contributing to it. They are it.'
} 
For bringing Strauss' causal-conditional matrix 'more fully around the postmodern turn' (2007: 433), Clarke conceptualizes her 'situational analysis' as a multi-site research - integrating human and non-human data sources. The focus is widened also to discursive material and cultural objects, which are considered to be constitutive and consequential for phenomena under study. Situational analysis, consequently, offers three 'cartographic approaches' (Clarke 2007: 434): (1) a situational map - analyzing relations between human, non-human, discursive, and other elements of the research situation, (2) social world/arenas map - interpreting the inquiry's meso-levels like collective actors, arenas of commitment and discourse, non-human elements, and (3) positional maps - analyzing the inquiry’s positioning on axes of difference, concern, and controversy.

To sum up, Clarke proposes to shift the focus from a mere action regard to the analysis of the situation - its elements and their relation - a researcher studies. The analyst is thus able to empirically 'construct' the complexity of a situation (Clarke 2007: 435f.). The analysis of the relation of all constitutive elements in the situation helps to overcome the scientific differentiations (e.g. between macro-/meso-/micro-levels) and simplifications (leading to the underestimation of important issues), to position the researcher in the research, and to address solid and fluid power structures (answering to neo-Marxist critiques against grounded theory).

To conclude, grounded theory - since its initial presentation by Glaser \& Strauss (1967) - has become a popular methodological and analytic approach in qualitative field research. Yet, the grounded theory concept had to face criticism -from both outside as well as inside the user community. The original idea of grounded theory was to develop a research understanding opposed to the dominating quantitative, deductive, and positivist perspectives of the 1960s. With the evolution of scientific understanding, the grounded theory concept was enhanced by different users in different directions, amongst which the constructivist way is one of the most visible at the moment. Two different nuances of constructivist grounded theory have been presented: the interpretative one by Charmaz (2008) and the relational one by Clarke (2007). Grounded theory, refined according to postmodern scientific knowledge, more than ever represents a useful tool for approaching the contemporary, complex social realities in exploratory as well as deepening stages of scientific research. While it is a flexible and 'emergent' method, it is nonetheless systematic - if used according to some guidelines (open-to-theoretical coding sequence, theoretical sampling, memo writing, cyclic reflection about data and categories, theoretical saturation, analytic induction and abductive inference). In addition, it allows for a deeper analytic comprehension than mere descriptive methods of qualitative data analysis.

\subsubsection{Hermeneutics}

Hermeneutics is an important methodological standpoint which helps to understand the logics of qualitative research and interviewing. To put it in simple words, hermeneutics provides a guide to the interpretation of things that others have said, whether in interviews or in written form. The main problem of interpretation is that the interpreter has less information on the context of a written or spoken text. Thus, identifying the right intention and meaning of the producer of a text is a difficult task. While this is less a problem in the frame of everyday communication and interaction, it is a central issue for scientific research. Hitzler \& Honer (1997) stress that in particular social sciences face this problem, because, other than for natural sciences, the data that is gathered is pre- 
interpreted through the interview partners. Thus, the scientific analysis of interviews is an interpretation of 'second order'.

Depending on the research design, the interpretation of 'first order' that occurs during the interaction between researcher and interview partner can vary a lot. Methods that use observation or analysis of secondary data (e.g. of speeches by politicians, photos, videos) have fewer difficulties in separating first and second order interpretation. In methods in which the researcher interacts directly - e.g. in a more or less unnatural context of a scientific interview - the analysis must contain a separation of first and second order interpretations. Hitzler \& Honer say that qualitative interviews then differ from quantitative data generation, because the former simulate a quasi natural context. In qualitative interviews mostly language of the everyday life is used and the researcher can influence how close the interview atmosphere is to the interviewee's everyday life (cf. section 6.2.3).

According to Hitzler \& Honer (1997: $23^{96}$ ), the very basic idea of hermeneutic analysis is 'to methodologically controlled - break through the superficial informational content of a text in order to find the underlying (this means somehow 'latent' or 'hidden') layers of sense and significance, while at the same time making and maintaining reasonable this process of reconstruction.' Other than a mere content analysis (e.g. described in Mayring 1995), hermeneutic analysis tries to discover meaningful relations between individual aspects in an open way. In content analysis, categories are set before looking at the interviews, while in hermeneutics the interviews themselves reveal important categories that are linked to each other. One can only reconstruct sense and meaning in people's lifeworlds in this manner. Therefore, it is important to neglect one's own preexisting knowledge, which might be relevant for and influence the interpretation of data. Hitzler \& Honer (1997: $24^{97}$ ) say it is important 'that the interpreting researcher plays dumb on his own stock of knowledge, that he pretends to not be aware of or to not have this knowledge, with the aim of being able to constitute the phenomenon of inquiry in a way which is rectified from his/her routinised cultural connotations, and as such in a 'new' way.' Hermeneutic approaches use doubt, reflection and the neglect of rapid understanding as main tools to uncover the inherent structures of everyday knowledge. The assumption 'to know' something is fundamentally questioned. This philosophy of understanding the knowledge creation process could be equalled with a 'divestment of epistemological naivety’ (Entkleidung epistemologischer Naivität) (Hitzler \& Honer 1997: 25).

A particularly interesting approach in the field of hermeneutics is the Geschichtenhermeneutik, which was introduced by Vonderach (1997). This approach focuses on the reconstruction of individual biographies. The basic idea is that sense making is oriented on single stories and situations that occur in a person's life. The reconstruction of sense making therefore needs to go through story telling. The sense that a person gives to their life derives from the entirety of their single stories. Vonderach thus thinks that researchers have to look for a reconstruction of the overall life story (Lebensgeschichte) of the interviewees. Based on Wilhelm Schapp's

\footnotetext{
${ }^{96}$ Translated by the author from the German text: ,[...] methodisch kontrolliert durch den oberflächlichen Informationsgehalt des Textes hindurchzustoßen zu tieferliegenden (d.h. eben: in gewisser Weise „latenten“ bzw. „verborgenen“) Sinn- und Bedeutungsschichten und dabei diesen Rekonstruktionsvorgang intersubjektiv nachvollziehbar zu machen bzw. nachvollziehbar zu halten.

${ }^{97}$ Translated by the author from the German text: ‘dass der Interpret sich gegenüber seinen eigenen Wissensbeständen „künstlich“ dumm stellt, dass er also so tut als kenne bzw. hätte er sie nicht, um so das infrage stehende Phänomen von seinen kulturellen Routinekonnotationen „gereinigt“, d.h. quasi „neu“ konstituieren zu können.’
} 
Geschichtenphilosophie (1976), Vonderach explains that these stories have a socialising function; they bring people together and they are the basis for identification. During the course of a life, stories build up on former stories. Each situation is reflected afterwards by an individual and it becomes a story. Former stories guide how a person acts in subsequent situations. During this process of lining up stories during a lifetime, a person develops structures to order these stories. It is the researcher's job to discover these underlying structures and to build a grounded theory from such procedure (Vonderach 1997: 176).

Vonderach suggests that a natural interview atmosphere in particular is important to generate storytelling (1997: 176f. ${ }^{98}$ ): 'Following the logics of interpretative sociology, especially if it considers the concept of stories as important, communicative research methods are particularly appropriate, which allow for an expression of the interviewees' 'meanings' and 'stories', in which a person in question experiences themself and their social world in relation to sense and in orientation towards action. This is best achieved through methods which mainly equal everyday life and its ways of 'natural' communication. Open conversation or 'open interviews' are particularly suitable here [...]'.

The selection of interview partners should follow the logics of theoretic sampling and contrasting cases. During the analysis, the researcher should neglect the 'homology hypothesis', which was developed by Schütz for narrative interviewing. Schütz thought that the stories which interview partners are telling can directly be understood as true. However, Vonderach would understand these stories as an ex post reflection about a past situation. Thus, what a person tells now is not necessarily identical with what happened at the respective moment in the past. The researcher should thus concentrate on the overall life story. The first step of analysis is then to write up a case specific life story based on the single narrations from the interview. In a second step, the current life situation and the individual plans for the near future are evaluated, then all this information is merged into individual case reconstructions. The researcher basically writes an external life story about the life story of the interviewee.

To reconstruct this life story, Vonderach suggests a four-step model. First, a sequential analysis of the interview must be elaborated. The researcher should pay attention to the ways in which interviewees acted in specific situations in their life that are relevant for the research topic. These situations are told in individual sequences during the interview. The researcher must then think about possible alternative ways of acting in that given instant. The resulting difference between the two - the actual acting and the theoretical alternatives - can be used for first analytical formulations about underlying structure. Second, the specific interview situation must be looked at. In particular, it is relevant to reflect on how the interviewee tries to present themself in a given interview context and atmosphere. Therefore, the researcher must interpret meaning and things in experimental ways, comparing different possible interpretations of what an interviewee said. Third, the researcher puts

\footnotetext{
98 Translated by the author from the German text: ,Dem Ansatz einer interpretativen Soziologie, insbesondere wenn sie dem Geschichtenkonzept eine wichtige Bedeutung zukommen lässt, entsprechen dabei vor allem kommunikative Verfahren der Sozialforscher, welche geeignet sind, die „Bedeutungen“ und die „Geschichten“, in denen die betreffenden Menschen sich selber und ihre soziale Welt sinnbezogen und handlungsorientiert erfahren, zum Ausdruck gelangen zu lassen. Dies erreichen am besten Erhebungsmethoden, die den „natürlichen“ Kommunikationsweisen des Alltagslebens möglichst nahe sind. Offene Gespräche bzw. „offene Interviews“ sind dafür besonders gut geeignet, [...]'.
} 
the puzzle together: For each case the individual sequential interpretations are put together into a total life story, which includes information about 'relevance', 'preference', 'orientation' and 'acting' of each interviewee. These life stories should be formulated in a way that they serve the specific research topic. In this first stage, empirically grounded categories should be elaborated. In a fourth step, the researcher then tries to abstract central orientations and concepts that structure the way a person acts within their lifeworld.

Finally, the individual cases are compared to each other. This comparison can focus either on similarity or on differences, or on both. It might help a development of a typology across individual cases. Vonderach resumes (1997: 18499): 'In the course of comparison across cases an impression arises of the similarities, commonalities and differences of life stories. This impression cements to the recognition of typical, case-crossing structural relations or gestalt patterns.' Given these explanations of hermeneutics, the methodological proximity to ground theory becomes obvious.

\subsubsection{Qualitative heuristics}

Qualitative heuristics is a method described by the German sociologist Gerhard Kleining at the University of Hamburg. During the 1990s he developed this specific heuristic method that he understands as a guideline for systematic exploration and discovery in qualitative data. He describes his method as linked to 'introspection', a method widely used in psychology. The roots of this method can be found in early psychoanalysis, phenomenology and grounded theory (cf. Kleining \& Witt 2000). Kleining \& Witt explain that qualitative exploration was repelled for a while from social sciences, when quantitative measurement became a methodological imperative following neo-Kantianism. Kleining \& Witt want to re-establish systemic exploration in the social sciences.

According to Kleining \& Witt, four basic rules have to be followed in order to achieve fruitful discovery from qualitative data. First, the researcher has to approach the empirical field in an openminded and flexible way. This includes that they must react appropriately to empirical observations in the fieldwork, which means practically that they have to adapt the personal pre-conceptions to the empirical realities. Whereas this might be a natural step in research, Kleining \& Witt (2000: paragraph 8) remark: 'Alas, discoveries in many cases contradict general scientific beliefs which are hard to give up and may even cause crises within the process of research itself. The rule suggests a reconsideration of the researcher's scientific position if the data consistently are not in agreement with information taken for granted.'

Second, the topic of research is flexible during the research process. The actual research topic only becomes evident once the research is finished. Given the project-based nature of contemporary social science, this might seem an illusionary thing, but according to Kleining \& Witt, it allows for true exploration. In case a topic becomes irrelevant or part of another topic during the research, the empirical fieldwork should not be interrupted, but the project should be titled with different wording. New knowledge can only come to the surface in this manner. This second criteria includes a researcher's open-mindedness towards discovery by chance.

\footnotetext{
99 Translated by the author from the German text: 'Im Verlauf des Fallvergleichs entsteht ein Eindruck von den Ähnlichkeiten, Gemeinsamkeiten und Unterschieden der Fallgeschichten, der sich zur Erkenntnis typischer fallübergreifender Strukturzusammenhänge bzw. Gestaltsmuster verdichtet.'
} 
Third, the data collection process should involve techniques to maximise the 'structural variation of perspectives' represented in the sample. Variation should not be limited to sampling. Kleining \& Witt argue that variation in methods in general as well as in formulating questions is an important way of gathering rich empirical material. One could also translate that point into quantitative approaches, in which individual variables seem to be more influential than others, and then one could diversify variables in a given empirical design while collecting data. Variation also has a temporal dimension; one can look at certain empirical facts from different temporal perspectives: past, present, or prospective.

Fourth, Kleining \& Witt describe that the main focus during analysis must be the search for similarities instead of differences. They refer to notions such as 'analogies', 'homologies' and 'accordance', and they describe the research process as follows (Kleining \& Witt 2000: paragraph 11): 'The analyst starts grouping those parts of the protocols or observations which are most similar to other parts and continues to group the groups tentatively, suggesting headlines for the groups and then headlines on top of headlines thus progressing from concrete parts to a more and more abstract general whole which nonetheless keeps concrete details. Proceeding in this manner, the overall pattern, showing the structure of the topic, will gradually emerge. All data have to be considered and incorporated, and not only a selection of it as an example ("100\%-rule of accordance"). The analysis is integrated into the process of data collection and mutually dependent on it.'

The overall goal of qualitative heuristics is to construct an atmosphere of dialogue between researcher and empirical data/case, which comes close to an everyday conversation. It is a succession of questions and answers in which questions are not rigidly pre-defined. This procedure, as Kleining \& Witt (2000: paragraph 12) explain, will help to 'adjust the epistemic structure of the researcher to the structure of the phenomenon and brings it in line with itself.' Equivalently to the ground theory's call for theoretical saturation, qualitative heuristics tests the validity of analysis through the 100\%-rule, which means that all data fit into the analysis, and further data does not contest and change that same analysis. However, one has to bear in mind that all social data are only historic moments, and they are subject to change with time.

\subsubsection{Synthesis of approaches to analysis}

The presented analysis techniques of grounded theory, hermeneutics and qualitative heuristics are profoundly argued in epistemological terms. However, the practical usage is poorly explained. After studying this methodological literature, I found myself confronted with more questions than answers. I had expected a manual for analysis, but now I know this does not exist. Or, even if one existed, there would probably be another which argues exactly the opposite way around. Therefore, it remains in the hands of the individual researcher to find their own method of analysis orientated towards these theoretical descriptions of the methods. In order to find one's own way, it is helpful to recall the similarities between these three approaches.

The three discussed approaches to the analysis of qualitative interviews reveal several basic similarities. First, all these approaches distance from simple content analysis. Content analysis suggests that we can look at individual fragments within interviews, and that we can hold the content of these separated fragments as true (Mayring 1995). Furthermore, content analysis uses 
pre-selected and theoretically derived analytic categories and coding systems. By contrast, grounded theory, hermeneutics as well as qualitative heuristics call for a more cautious and doubtful way of analysing the data. Here, the content - which lies at the surface of the spoken word - is not considered the actually interesting data. Rather, the underlying and latent sense structures should be looked at, and then the researcher should use these underlying sense structures to generate conclusions. Second, other than in content analysis, coding schemes and categories are not preselected on the basis of theory, but they are derived during the analysis from the empirical data. The researcher asks the empirical material for these relevant categories. Here, all three approaches point to the central role of 'emergence of analytical dimensions and categories' through a cyclic process of switching between theory and empirical data during the process of analysis.

The end of this process is derived once theoretical saturation is achieved. This means - in the words of Kleining \& Witt's '100\%-rule' - that all cases must fit into the empirically derived theory, or hypotheses, or concepts. None of the empirical cases should derange the theoretical abstraction that was elaborated during the analysis. Furthermore, additional cases should be reasonably assumed to not alter this theoretical abstraction.

The focus on variety during the sampling process and to analytical perspectives is also mentioned in each of these approaches. In order to reach theoretical saturation, a great variety of individual empirical realities should be taken into account, and the technique of finding theoretical abstraction starts from contrasting individual cases and studying similarity and difference.

Finally, the relation of the separable interview fragment to the interview story is also thematised in all approaches. Whereas content analysis also uses individualised interview fragments, the presented approaches here demand the reflection on the interrelatedness of single parts of an interview and the wider picture of the whole interview. The latent and actual sense of an interview passage can only be reconstructed through embedding it into the general life story and its sense structures. Therefore, the basic reconstruction of the life story is also necessary.

I think that an orientation on these similarities helps to analyse the interviews and the mental maps in an appropriate way. Thereby, I understand the methodological theory as a description of an ideal typical proceeding which provides a reference frame. I will now explain my own method of analysis.

\subsubsection{Treatment of data}

The recorded interviews were transcribed according to a given set of transcription rules. I decided to keep transcription rules simple. Reinders (2005) explains that transcription can follow different rules, which have varying degrees of reporting the 'para-verbal data' (such as breaks, interruptions, noise). Here, different ways of formatting the text breaks could be selected. Score notation (Partiturschreibweise) shows where para-verbal data influenced the continuity of the interview by specific text settings. Using standard line breaks makes it easier to read the text, but one has to use symbols to report para-verbal data. In addition, there are different ways of writing down the spoken language, which uses different grammar than written language. One can also use standard grammar such as is used in written language. Then there is literary circumscription (literarische Umschrift), a way of typing which words are written down as understood from the audio file. This means leaving 
out individual letters or syllables, but also merging two words in the manner that the people spoke. This technique is interesting when people have spoken with strong accents or even in dialects, where vocabulary differs from standard language. Third, there is the technique of using eye-dialect, which uses phonetic elements to report how people have pronounced words. And finally, one can also write down transcripts according to the International Phonetic Alphabet. Before the transcription, the researcher has to decide and protocol the decision (cf. Reinders 2005). Here, Hitzler \& Honer (1997) emphasise that transcriptions must be complete, meaning the complete length of an interview, but the researcher should take a pragmatic decision about the degree of detail.

Table 3: Transcription rules

\begin{tabular}{|l|l|}
\hline Symbol & Meaning \\
\hline$\ldots . / / /$ & Interruption and change in who speaks \\
\hline$\ldots$ & $\begin{array}{l}\text { Unfinished sentence or thought, without interruption by other } \\
\text { speaker }\end{array}$ \\
\hline [thinking] & $\begin{array}{l}\text { Additional explanations to spoken text; also: para-verbal } \\
\text { information (noise, gestures, information on longer interruptions) }\end{array}$ \\
\hline$[\mathrm{XY}]$ & Spoken text that was not understandable during transcription \\
\hline$[\mathrm{XY}$ _interpretation] & $\begin{array}{l}\text { Spoken text that was not understandable during transcription, but } \\
\text { where interpretation was tried during transcription }\end{array}$ \\
\hline
\end{tabular}

As I did not aim at a detailed psychological analysis of my interviews, I decided to keep transcription rules simple. I also decided to use standard grammar of written language. I think that if no detailed linguistic study of the interviews is the goal, then all other types of writing transcripts make it far more difficult for other scholars to read the transcripts. Finally, I did my interviews in different languages, and phonetic transcription would have been very costly to do, without necessarily providing more relevant information.

I then used small interview protocols to provide background information on the interviewee and on the interview situation and atmosphere, which for an interested reader might be valuable information to understand the quality of the data gathered. You can find one sample transcript and the interview protocols of my interview partners in the annex.

\section{Analysis procedure}

I have tried to orientate to the four step model provided by Kleining \& Witt (2000) in order to structure my empirical data collection and analysis. Indeed, I entered the field with an open and hardly structured interest. I also tried to stick to the second criteria: I started with a set of doubts which I translated into a first draft of the general project context. Then, the description of my research project indeed changed with time and I adapted it to the empirical realities that I faced. For example, in early stages of my project I conceived of my study group as being 'global nomads', moving around just as detached from places and local contexts, which then turned out to be wrong, as it did actually not show up like this in my early interviews. I then changed my own perspective, 
understanding my study group not as global nomads, but as people alternating between mobility and rootedness. I also applied the third basic criteria of qualitative heuristics: structural variation. Indeed I sampled in a way to increase variety within the sample: I sampled different age groups, I sampled women and men, I sampled homosexuals and heterosexuals, I sampled people with and without children, I sampled in different sub-sectors of creative industries. Then I also varied interview atmospheres, going to my places as opposed to going to my interview partner's places, or even doing interviews in third places. I also changed the order and wording of questions. However, what I always tried to stick to was to create an atmosphere of a natural conversation and reacting flexibly to the mood of talking of my interview partners. Finally, I strongly focused on similarities in my data's variety, while I analysed my interview partners' stories. Analysis followed a three-step model:

1. Reconstructing the life story. In reference to Vonderach's Geschichtenhermeneutik (1997), my analysis started with looking at the individual cases in order to understand the overall life stories of each of my respondents, as well as the interview specific story that was told during the interviews. I here added data that was publicly available on the internet, and which helped to embed and extend the information as provided during the interview itself. You find summarised versions of these case specific stories throughout this entire thesis in the grey textboxes. They aim at providing information on the cases, and they should allow for a contextualisation of the individual information drawn from the interviews.

2. Mapping the geographic range of the everyday lifeworld. In the next step I looked at the interviewees' drawn mental maps and I compared them, case by case, to their narrations during the interviews. Here, I gave specific attention to information that concerned 'space' and 'places'. At this stage of analysis, I wanted to find out more about the individual action space of my interviewees, thus determining the geographic extension of their everyday lifeworld. Furthermore, I used this step to compare the richness of geographic information as provided in the mental maps as compared to the narrated interviews. Finally, I merged geographic information from the interviews and from the mental maps into new case-specific maps which help to analyse, visualise and illustrate the geographic set-up of the transnational multilocality of my interview partners. The mental maps can be found in the appendix. The maps, which I derived from the synthesis of 'mental maps' and 'interviews', accompany the 'life stories' (the grey text boxes) throughout the text in this thesis.

3. Searching for similarity across cases. In a third step, I then compared the individual cases to each other. Based on the idea of grounded theory, I started writing memos - yet not in such an ordered manner as demanded by Glaser \& Strauss (1967) - on my observations, and for coding the interviews' text. Then I reflected the individualised and coded sequences in the light of the entire interview and the life stories of the interview partners (as asked for in hermeneutics; cf. Vonderach 1997) as well as the other cases. At this stage, I also referred the empirical analysis to the theoretical considerations, and I grouped codings into categories according to my 'fields of interest'. Here, obviously the clarity of the interrelatedness between different codings and fields of interest became clearer. In reference to Kleining \& Witt's qualitative heuristics (2000), I focused on similarity and commonality. From this step of analysis, I derived slightly abstracted categories which were grouped into dimensions that are finally important components of my interview partners' lifeworlds. 
4. Theoretical abstraction. In a final step, I merged insights from the three former steps into a synthetical analysis. From this analysis I derived the final theoretical output, which consists of the heuristic concept of 'plug\&play places'. The subsequent chapter will introduce the empirical findings from these individual steps of analysis. 


\section{Interlude F: Qamar - Stephan}

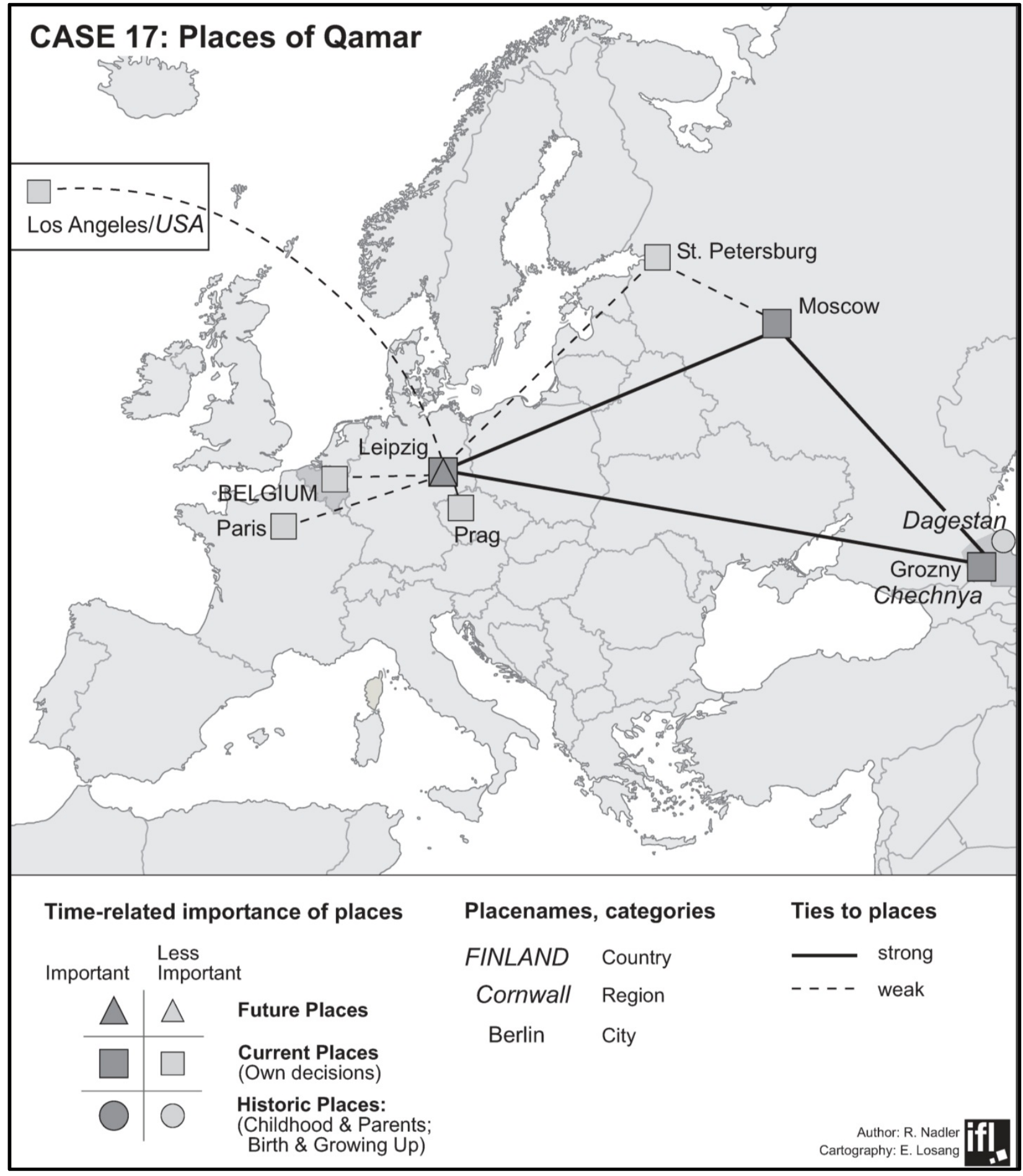




\section{Meet Qamar}

Qamar is a freelance artist in his 40s who is specialised in large scale paintings. He also offers a broad variety of other styles such as silhouettes, drawings, or family portraits. He was born in the Caucasian territory of Dagestan, where he spent his childhood and which he still nostalgically calls his home. His family belonged to the Chechen minority in Dagestan. After school graduation, Qamar went to St. Petersburg (Russia) and studied Fine Arts. In 1989, during the Perestroika period and his years at university, he started travelling westwards. He stayed in Paris, Prague, Leipzig and several other places where he earned money as a street artist. Once he had finished his studies, Qamar moved to Leipzig due to its low living costs and where he had met someone to help him with the visa. He started to study again at Leipzig's Academy of Visual Arts. Qamar mentions that he knew already as a small child that he wanted to become an artist, so he never considered a 'plan B' in life. During his trips around Europe, he often slept in vacant apartment blocks to save money on rent. In 2008, he finally bought an old apartment block in the eastern part of Leipzig. The ground floor is used as an atelier, and above it he rents out some of the apartments. The other renters in his building are also artists: a moviemaker, a tattoo artist, and an artist specialised in sacral arts.

At least once a year Qamar goes to Chechnya for vacation. He goes to meet friends and family, but he also mentions to be far more famous there than he is Germany or the western world. Frequent meetings with high rank Chechen politicians (ministers, the president) made him a public person in Chechnya: he is known as a Chechen artist representing his country in the West. People in Chechnya expect him to have made lots of money and being able to support artists back at home. However, Qamar cannot meet these expectations as he struggles to survive in economic terms in Leipzig. In Germany, his Chechen background gives him the opportunity to be contracted for workshops by associations that deal with the integration of immigrants. Other than that he does not engage in civic associations. He is not interested in politics, and also says that he prefers to be working alone and silently, without broader interest in the broader public of Leipzig or his Chechen home.

Going to Chechnya always leads him through Moscow, where he regularly stops by for some days at artist friends' places, and where he goes to exhibitions to keep up with the Russian arts development. There he meets Russian painters and writers. Qamar also loves travelling to the USA. During the winter months, he leaves his Leipzig apartment for a few weeks. With the money that he saves on heating his Leipzig apartment and atelier, he can rent a room in Los Angeles, and he shares an apartment with other artists and lets himself be inspired by the Californian way of life. Thereby, the Russian community in Los Angeles was quite helpful in providing access to the city and its artist networks. Qamar regularly meets up with and works for people in the show business in Germany, the USA and Chechnya (famous Russian clown Oleg Popov; the Ukrainian-German boxers Klitschko brothers etc.).

Qamar identifies himself as a world citizen. He is convinced that all conflicts in the world stem from nationality and religion. Thus, he rejects such collective concepts in his own identity. But having tried his luck in second-hand car trading, he met people who are completely different from him. During that time he learned that discussing politics and political opinions with people is a waste of energy. He therefore prefers to focus on the person's character and not on their political world view. For his own artist career he is thinking about using a pseudonym which does not allow him to be identified with any nation or religion. In the future, Qamar wants to stay in Leipzig, where he considers his building as something of his own, and where he likes the silent working atmosphere. However, he will always travel to get inspired for his art work. Qamar's story is mainly that of an artist with a bi-fold identity: mainly unknown in Germany and fairly famous in his Chechen environment. 


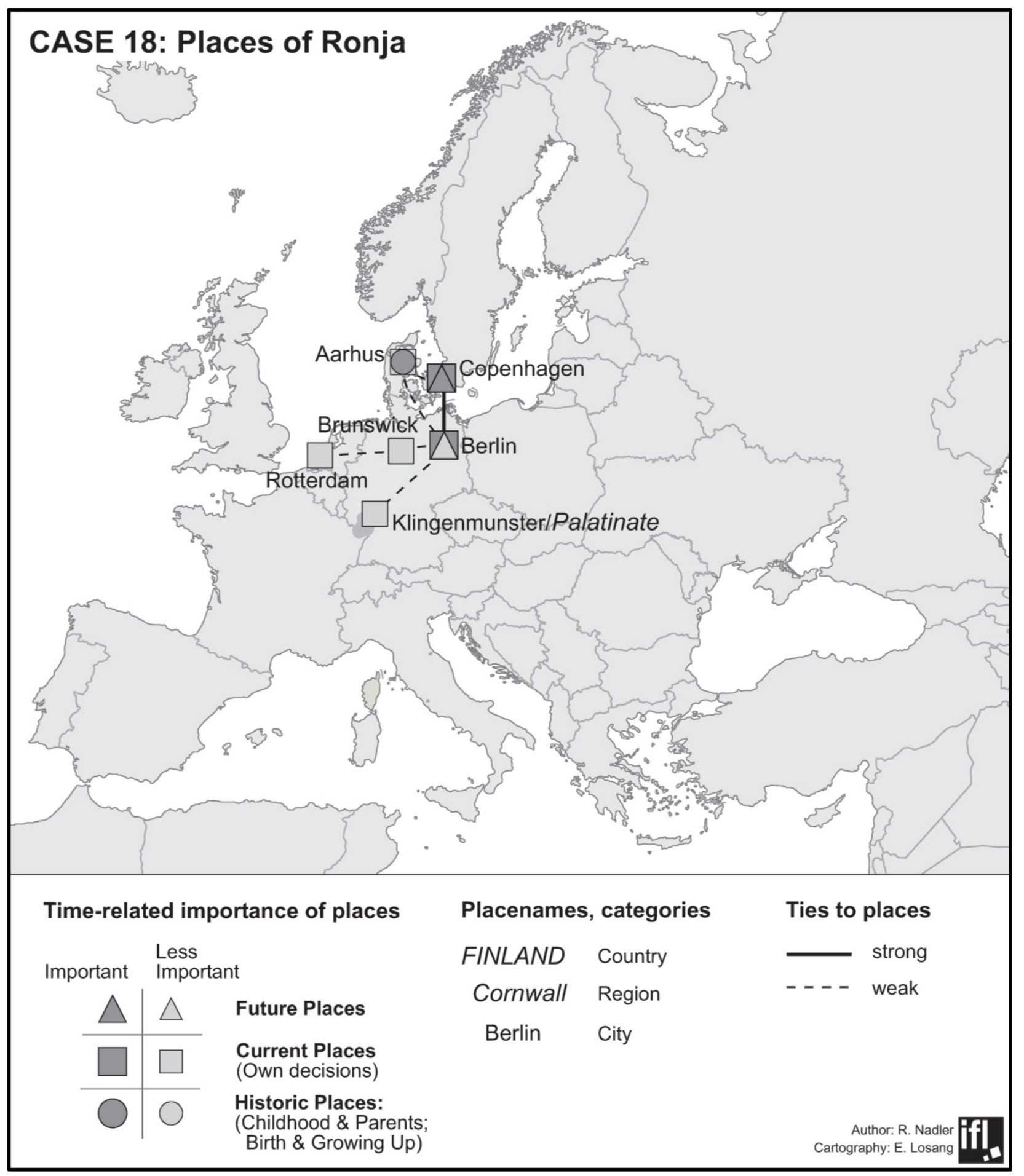




\section{Meet Ronja}

Ronja is a Danish journalist in her 30s. She grew up in Aarhus and later studied literature in Copenhagen. During these years in Copenhagen, she often travelled to Berlin as she liked the city. Then she decided to actually move to Berlin and finish her degree there. She met her current husband Stephan, who is a German architect. At that time Stephan was still studying architecture, and he worked in Rotterdam. This was the reason that Ronja also moved to Rotterdam for 7 months, where she did an internship with some historians specialised in architecture. However, she also took temporary jobs in Copenhagen. During that time she circulated between Rotterdam, Berlin and Copenhagen.

When Ronja finished her degree in literature studies, she moved back to Copenhagen and started to work there. Her husband followed her after graduation, and started to work as an architect in several Copenhagen based firms. Both always preferred Berlin to Copenhagen, and once Ronja was pregnant, they decided to move back to Germany, as she would take care of the baby and Stephan wanted to quit the private architecture offices for a job in the university environment.

Even though Ronja wanted to look for a job in Berlin, she still takes temporary jobs in Copenhagen, e.g. the organization of an Arts and Design Fair; she regularly flies from Berlin to Copenhagen for these jobs. She is convinced that being based in Berlin is advantageous. In Denmark, Berlin has a very international, metropolitan image, and Ronja thinks that the Berlin arts and architecture scene is very inspiring and interesting. Thus, her foreign work experience in Germany and Berlin in particular is helpful for getting jobs in Denmark.

With the arrival of her baby, Aarhus once again became relevant for Ronja, as she went to see her parents, who were now grandparents, more often. To Ronja, also her bi-national partnership is an important issue in her life. She appreciates that she and her small child will have a family life with two national frames of reference. Furthermore, she can also experience the ridiculous bureaucracy in the European Union in this manner. For example, she was not able to give her child a name as German and Danish authorities did not agree on the legal situation. Her Danish health insurance also did not want to pay for the costs of giving birth in Germany, but Ronja accepts these situations with humour and wraps them into entertaining storytelling.

Having recently become a mother, Ronja says that she would appreciate finding good employment in Berlin, where she lives with her husband. However, given the availability of low cost air connections to Copenhagen, she could also imagine commuting between Berlin and Copenhagen once she re-enters the professional world after the childcare months. In the future, she will keep her private life based in Berlin, since she loves the city. Unfortunately, Berlin has a difficult labour market for creative people, as there are so many creative workers arriving from all over Europe. There are more and better paid jobs in Copenhagen, so her choice will be any arrangement between Copenhagen and Berlin, but definitely no smaller towns or rural areas.

Ronja mentions that she follows the professional debates about gentrification in Berlin, but she does not engage actively in any initiative or association. She says that most initiatives support only one oversimplified standpoint concerning a certain topic, but she mostly prefers to look at different points of view in order to understand the complexity of things. In addition, she thinks of her job as a journalist with interest in urban development and architecture is already a type of engagement with local development issues. In the future, she would love to do more, e.g. working in the Quartiersmanagement (publically funded neighbourhood initiatives in German cities). Ronja's story is basically that of a mobile young mother who lives a transnational life and is trying to combine family and career. 


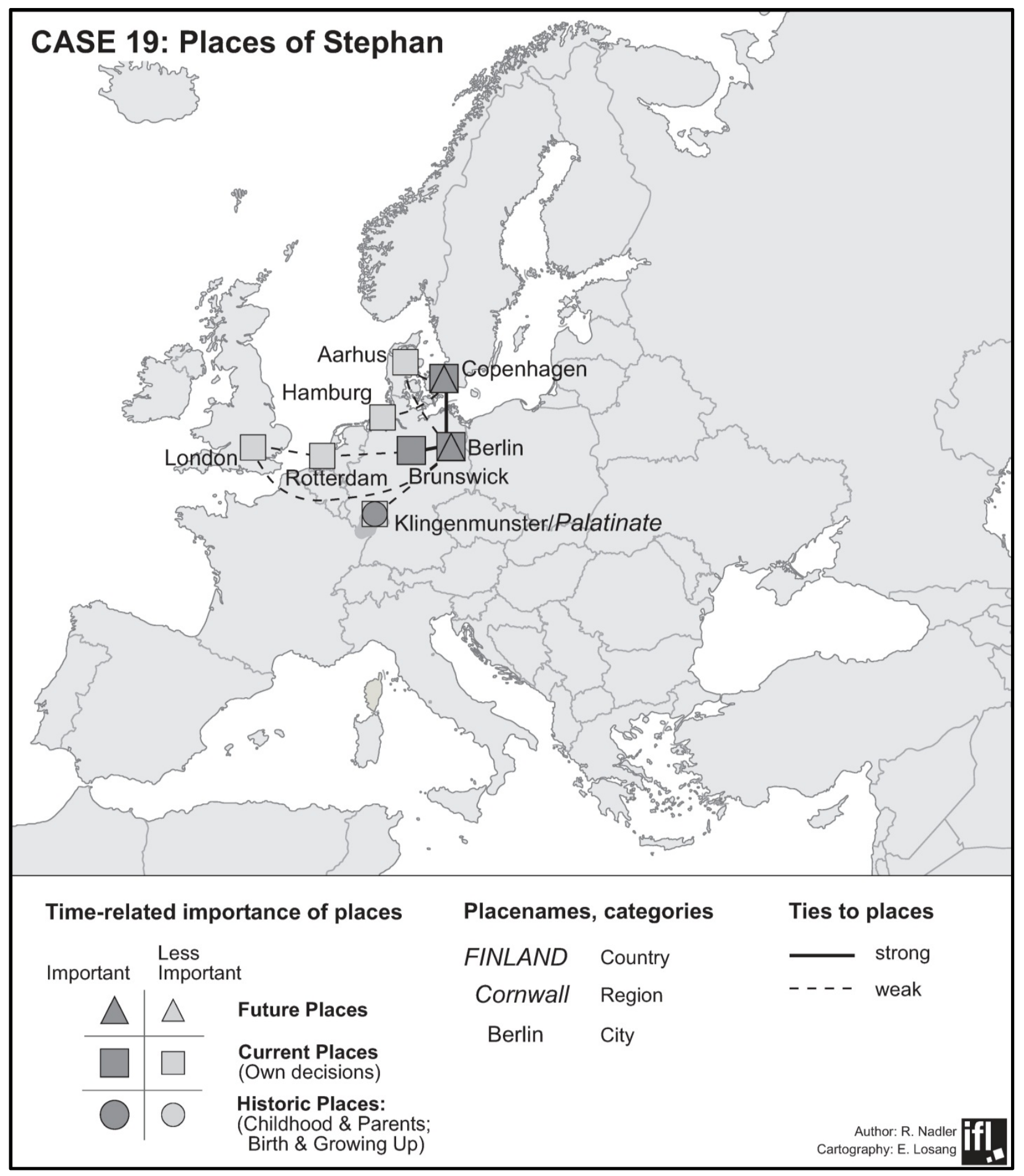




\section{Meet Stephan}

Stephan is a German architect in his 30s who grew up in a village in the Palatinate region, close to the French border. After school graduation he went to Berlin and studied architecture, where he also met Ronja, who has now become his wife. Since he left his village in Palatinate, he has not spent more than three years in one and the same city. From Berlin, he went to London for a few years and worked as an architect and then he came back to Berlin and finished his studies. Later on he went to Rotterdam for three years, and finally he moved to Copenhagen with Ronja and worked in several Danish architecture firms.

After a while, he was tired of working in architecture firms in Denmark and he took a teaching position at the University of Technology in Brunswick. He commuted on a weekly basis between the private apartment in Copenhagen (the weekends with Ronja), his room in a shared apartment (the evenings with friends and family) in Berlin, and his office in Brunswick (his job during day time). When Ronja was pregnant, they left their apartment in Copenhagen and moved into a new apartment in Berlin. Now Stephan mainly commutes from Berlin to Brunswick, and Copenhagen is seldom visited.

Stephan thinks of himself as a European. In his childhood at the French-German border he wondered what national borders are actually good for. He did not understand why some kilometres away people lived in a different country, yet spoke German. Later his own experience of living abroad in Rotterdam, London, or Copenhagen did not cause a feeling of living in a stranger environment than when he moved to Hamburg or Berlin. He therefore thinks that there is something such as a common European culture which is distinctive to other world regions, and he identifies with Europe as a whole, rejecting the idea of a national identity.

Still, when he is abroad he is always identified as a German, but he has experienced that in architecture it is a competitive advantage to emphasise the personal internationality and interest in foreign work experience. Architecture is a global labour market, and therefore a certain interest in other countries and cities is a necessary prerequisite for career advancement. However, with his parenthood he can no longer afford the high level of mobility. Therefore, he is looking for a professional stabilisation in Berlin or Copenhagen, while being sure that Brunswick is only a temporary stage in life.

For Stephan, living mobile life is a personal enrichment in the sense of having the ability to discover and 'live' foreign places in a way which is not possible as a tourist or exchange student. He loves to learn new languages and cultures. To him, the important aspect for really 'living' somewhere abroad is defined by the fact that he does not have a clear end date for that particular sojourn. He also refers to the negative aspects of mobility as actually being a necessity in his business while also being a constraint to his family life.

Stephan does not engage in civic associations or local initiatives in his places. He has a lot of ideas for projects and he also reads a lot about local news, but he does actually not have the time to do anything besides his jobs and his family.

Stephan's story is mainly that of an architect who is trying to escape the flexibility and mobility demands of his internationalised labour market, as he wants to provide his young family with geographic stability. 


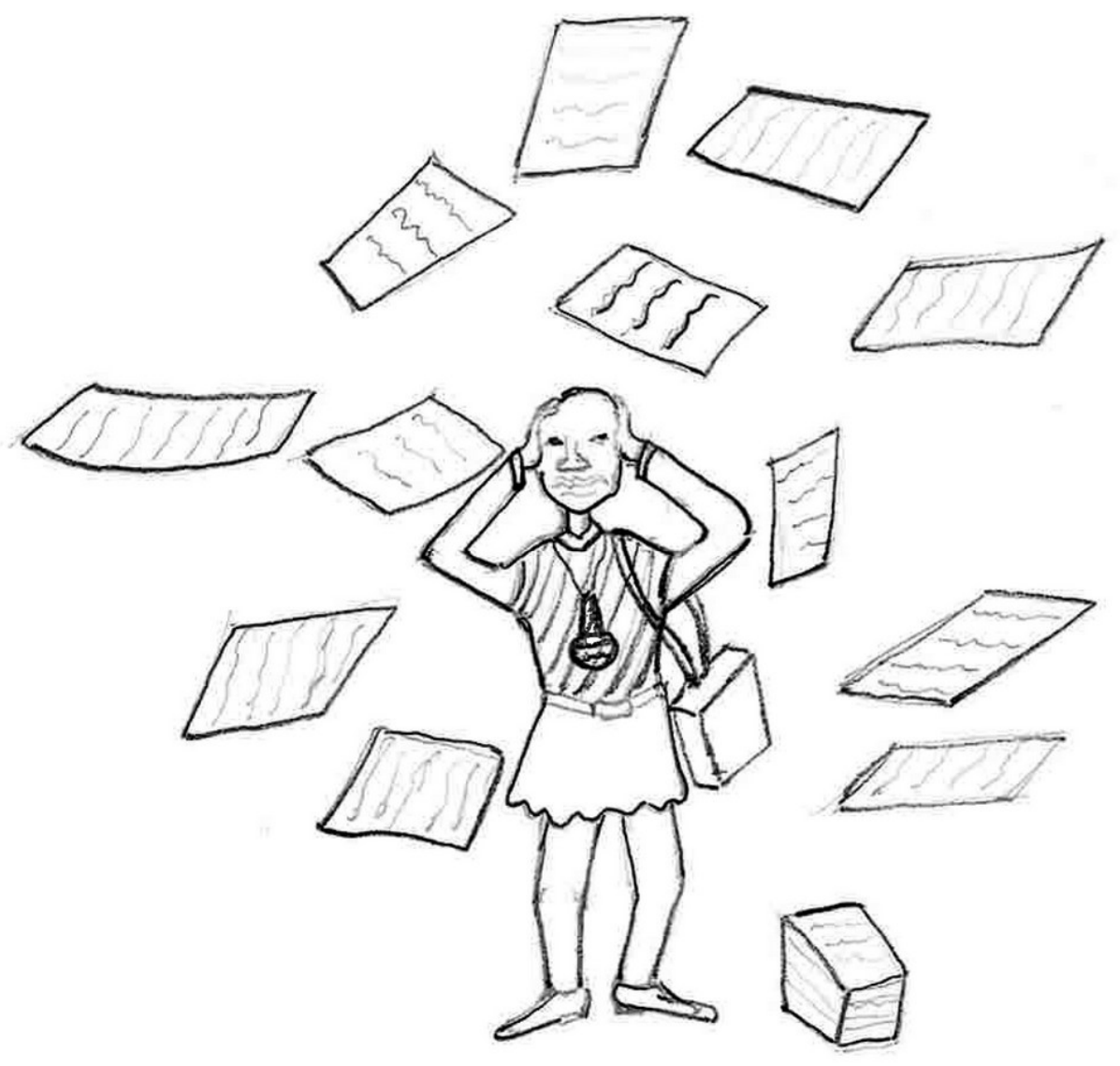




\section{Lifeworlds of multilocal creative knowledge workers}

This chapter is dedicated to the empirical results of the analysis of my 25 interviews and mental maps which I undertook in the period between 2010 and 2012. Following the philosophy of grounded theory, I will describe the categories that have shown up while studying the interviews, and I will follow the logic of qualitative heuristics, searching for similarity within variation, as described in the previous chapter. This entire chapter will adhere very tightly to the original empirical material. I will use the subsequent chapter in order to discuss the relevance of the empirical findings in the light of theoretical considerations as outlined in chapters 1-5. This chapter is structured as follows: first, I will present the descriptive findings from analysing the narrated and drawn mental maps of my interview partners. Then I will elaborate on the patterns of 'place' from the perspective of my interviewees (both section 7.1). The following sections (7.2 through 7.5) will present empirical results which are related to further central dimensions in multilocal lifeworlds. Thereafter, I will highlight the resulting outcomes of the perception of these dimensions, namely identity patterns and the respective feeling of belonging (section 7.6) and the specific action patterns (section 7.7). Finally (7.8), I will introduce a scheme which summarizes the former sections, and which I derived as a theoretical abstraction from my previous analysis. This final section will also be used to discuss the relationship between the individual dimensions as outlined in the sections before. This chapter must be understood as a comprehensive and encompassing perspective on my interview partners' multilocal lifeworlds. Chapter 8 will be used to further synthesize this rich material in the light of the fields of interest for this thesis (cf. chapter 5).

\subsection{Places: The geography of my interview partners’ lifeworlds}

This section is dedicated to the analysis of the geographic constitution of my interview partners' lifeworlds. I looked at their mental maps and the geographic information that stems from the interviews in order to understand which places are actually parts of their lifeworlds and how large their action space is. I also analysed meanings of places which my interview partners have revealed. As I mentioned above, I started my interviews by only roughly explaining to my interview partners what my research project was about, in order to not suggest too much content-wise orientation beforehand. Immediately after starting the interviews, I asked my interview partners to draw me a map with the 'places that are important' in their lives. This playful method and the related narrations which the map stimulated revealed which places, and spaces in-between these places, formed the territory, or action space, in which my interview partners were active.

\subsubsection{Mental maps: Marking one’s own territory}

The action spaces of my interview partners are individual, and as my interview partners all frequent different territories, they are also very distinctive from one another. Knowing about this fact, it is relatively difficult to compare the individual mental maps in order to find a more objective pattern across all the maps. Other mental map studies do this by directly comparing cartographic elements, and I think they can do this as they deal with a selected territorial detail, e.g. a neighbourhood, a 
city, or a region. Scholz (2011) e.g. analysed the hundreds of mental maps about the predetermined region of Saar-Lor-Lux through layering one map over the other and comparing cartographic content and topology of content. In such a way she could derive rather objective conclusions on the perceived geographic range of the Saar-Lor-Lux Region, or it was possible to evaluate the size of the French as compared to the German sectors of the region according to respondent's nationality. However, my own mental maps did not work with a predetermined territory. I left it to my interview partners to decide on what they would draw. I did not ask them to draw me 'Region A' or 'Region B'. Instead I asked them to draw me a map of 'their places'. As such, my mental maps are even more individual in their geographic range, and thus more difficult to compare as they do not orientate towards a shared geographic territorial range. Despite this fact, I tried to find patterns of similarity in the way in which my respondents actually drew their mental maps, and I asked myself what this might say about how places and space are imagined in a multilocal life.

When I analysed my mental maps, my first observation was that not all maps were cartographic maps, as I, as a geographer, had somehow implicitly expected. The mental maps which my interview partners drew had very different styles and represented different forms of content. Therefore, I will first present typical 'contents' and 'styles' of the mental maps of multilocal creative knowledge workers.

\section{Cartographic elements}

Even though not all mental maps took the shape of geographic cartography, the cartographic elements still represented main components. First of all, 'points' and 'small circles' represented places and the concrete localisation of the lifeworld. These place-related symbols could also vary in their illustration in order to provide further content. For example, Christian used small circles (cf. figure 5) for drawing his places, and then he added a variation of putting 'no cross', or a cross 'inside' or 'besides' the circle in order to express different degrees of importance of his places. Furthermore, points, or even small lines were used to signify 'transit places' which somehow marked a break on a longer line connecting two places. Birgit used this symbol (cf. figure 4, within the dashed lined circle) to underline the relevance of the Erfurt train station as a transit place where she has to change trains on her regular trips between Leipzig and Kassel.

\section{Figure 5: Point on line to express transit places in Birgit's mental map}

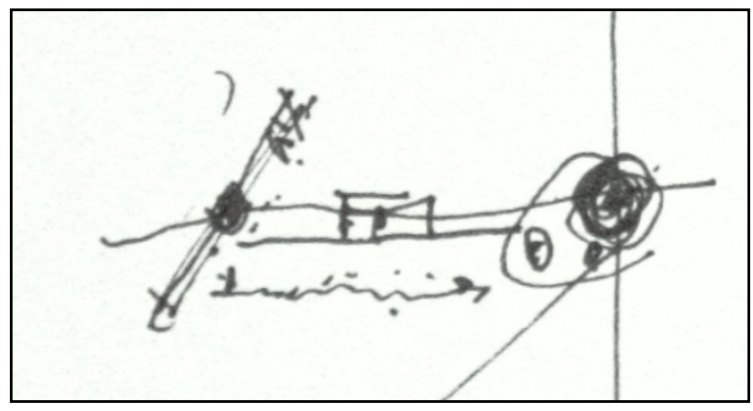

Figure 4: Variation of circle symbols to indicate places in Christian's mental map

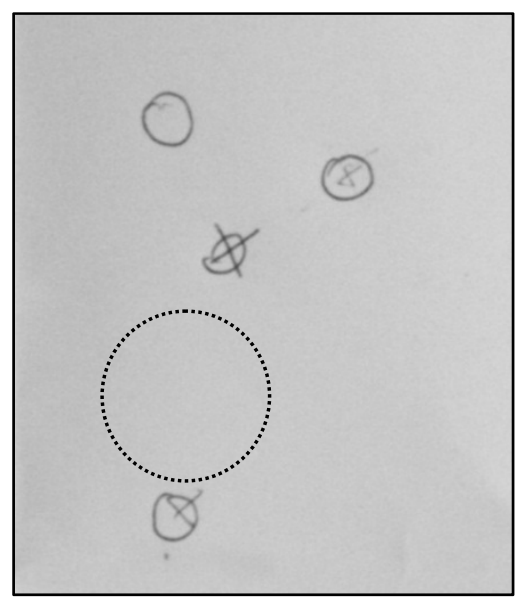


Second, connecting lines represented important cartographic elements which were used to define relationships between places. These lines referred to both actual travel routes on the one hand and abstract imagined connections on the other hand. These lines could also be added to further information through symbols such as arrows. Isabel, in her mental map, used arrow ends on her lines to underline the mutual directedness of her places (cf. figure 6).

Figure 6: Arrow lines in Isabel's mental map

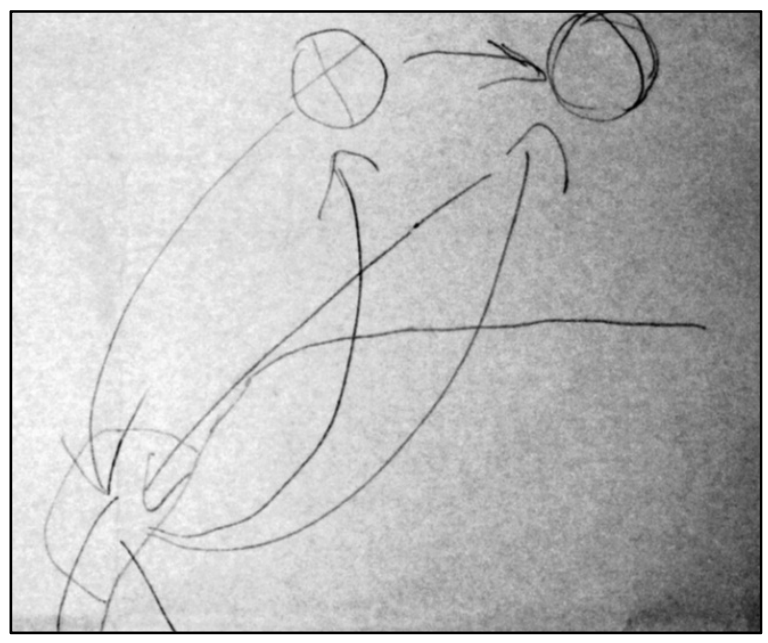

Figure 7: Large circles to explain edges of districts in Dirk's mental map

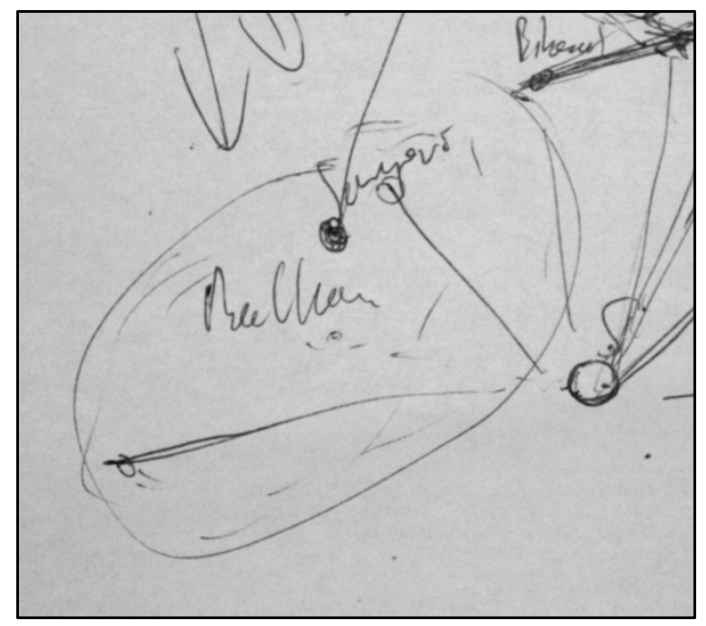

Third, larger circles were drawn in the mental maps by some interview partners in order to distinguish specific sub-territories in the personal lifeworld's geography. Such a bordering was used to describe the integrity of a specific geographic area. These areas are to be regarded as something homogeneous and different from the other areas in their own lifeworld. Dirk used this technique to emphasise the role of the Balkans (cf. figure 7); Marta also used this drawing tool to express the duality of Slovenia and Lithuania in her life; Oskar underlined the difference between western Switzerland's Trois Lacs Region and the wider Zurich area in the East. In Tjark's mental map we can see that he draws a border around Berlin and Copenhagen to express where his life mainly takes place.

Finally, topographic borders were also drawn in the mental maps. In contrast to the large circles, these borders aimed at images which are true to original topography. They mainly had the function of providing orientation while drawing, yet they also represented the geographic reference to one's own action space. Europe as a continent, or smaller segments of it, were most often illustrated (e.g. in the mental maps of Federico, Isabel, Kate, Liam, Nikolina, and Willi). If part of one's own lifeworld, other continents also showed up (South America in the mental map of Ugo, North America in the maps of Kate and Nikolina, or even all continental borders around the Atlantic Ocean as in the map of Yari). Then the globe as a whole was drawn and the Eurasian land mass was put on it (cf. Pia's mental map in figure 9). Topographic borders from individual countries were represented as well. We can find Germany and Denmark e.g. in Ronja's mental map, or the UK in Liam's drawing (cf. figure 8). Therefore, topographic knowledge actually seems to influence the mental maps of my interview partners. Crossing national borders on a regular basis, they also refer 
to formal political maps and the drawn borders and known symbols such as points and small circles for places, lines for connections between these places, and larger circles for areas.

\section{Figure 8: National borders in Liam's} mental map

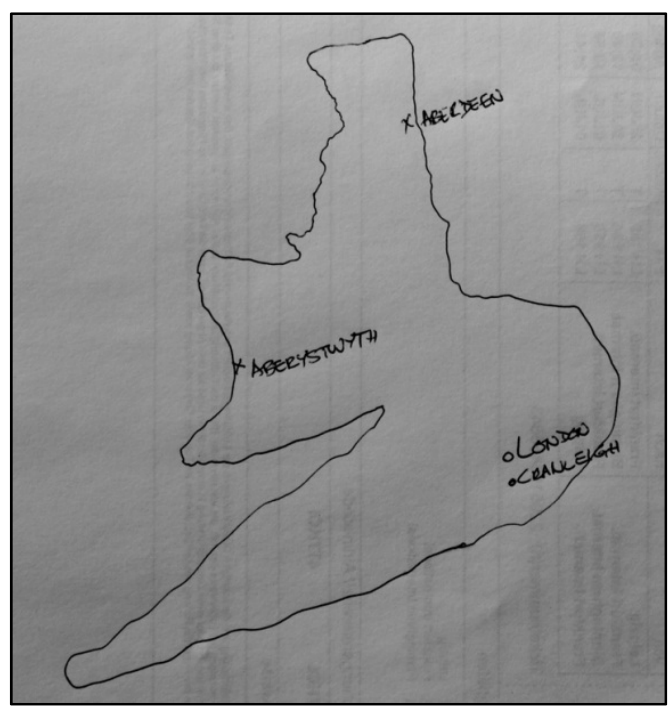

\section{Figure 9: Continental borders in Pia's mental} map

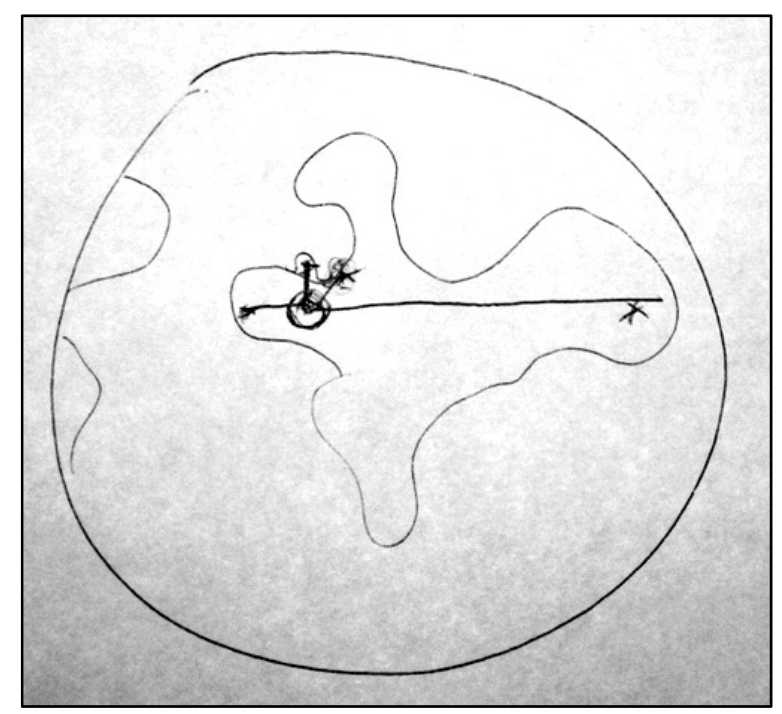

Here, the logics of space perception as described by Lynch (1960) once again become visible. Whereas Lynch studied the local scale of the city and its neighbourhoods, the set of mental maps provided by my interview partners showed that the scale was largely a continental to global one. However, the ways in which the geographic space is categorised is similar. The main difference consists of the fact that people in the Lynch's study, or those described by Downs \& Stea (1973, 1982), could actually perceive the geographic territory that they were asked to draw. The local level can be experienced through immediate physiological perception. The respondents could walk through their neighbourhood and perceive 'paths' (e.g. streets), 'edges' (e.g. the border of a park or an apartment block), 'districts' (e.g. the park as opposed to the shopping mall), 'nodes' (e.g. important intersections where transport routes cross), and 'landmarks' (e.g. skyscrapers, town halls, opera houses of remarkable architecture).

In my study the geographic space in question cannot be perceived immediately by my interview partners; the scale is too large. Thus, the mental maps of their own action space are necessarily ones which come into existence through a process of cognition in which physiological perception, former experiences and memorised images are combined with formal geographic knowledge of higher scales (e.g. political cartography as learned through an atlas). The described cartographic elements in my mental maps are rather to be understood as an image which is composed of information stemming from different sources. Nonetheless, we can also see that my interview partners, similarly to Lynch's local studies, order their action space according to 'paths' which are represented in more abstract ways as the connecting lines between places (e.g. frequent travel routes, or also ICTmediated communication channels), to 'edges' (e.g. the topographic border, or the larger circles around distinguished areas), to 'districts' (e.g. the territories within the shapes formed by the line elements) and to 'nodes' (e.g. the important transit places during their trips such as airports or train stations). Lynch's 'landmarks' could not be found in the mental maps of my interview partners; this 
does not mean that they do not perceive them, but the scale of the presented mental maps is so large that cartography becomes more abstract.

\section{Textual explanations}

In addition to the cartographic elements, textual components were also put into the mental maps by many interview partners. The written text had different content. First, text included geographic names of places and their related abbreviations. We can find examples in the mental maps of Dirk, Kate, or Marta. Furthermore, the text is also used to circumscribe the function of places, as could be seen in Emil's mental map. Xaverio also used text to add information about his activities in his places. Furthermore, distance between places is indicated by text. For example, Henriqua highlighted how close two of her Spanish places, Vigo and Verin, are located by using a textual explanation (cf. figure 11). Finally, Willi used textual elements to add information about the time dimension (figure 10). He explained durations and periodicity through the indication of years of stay as well as of presence times in individual places in percentage values.

Figure 11: Text used to explain periodicity and durations in Willi's mental map

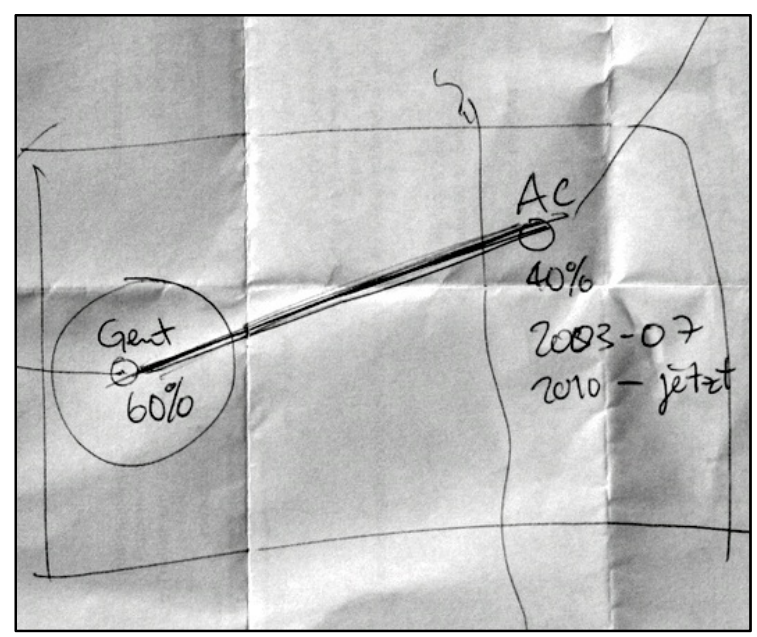

Figure 10: Text used to explain distance in Henriqua's mental map

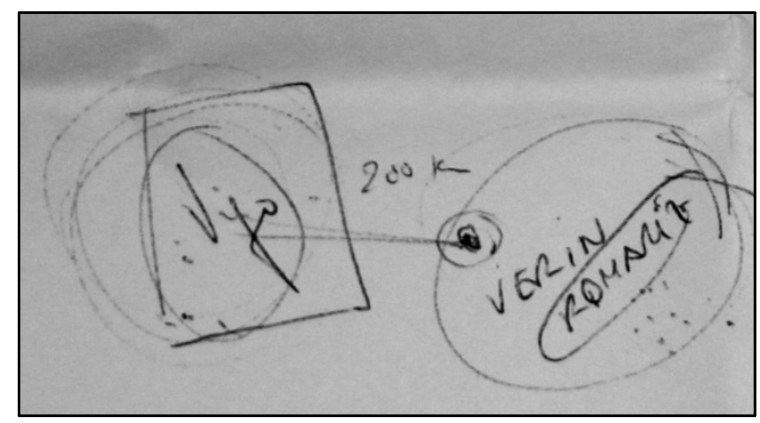

\section{Sketches}

Above I have mentioned that there are maps that actually are not cartographic maps. Having said this, I mean that the elements that were used to draw these maps are not following the logics of ordering basic cartographic elements such as lines, shapes and points (which are also the basic geometries available in geographic information systems) into a topology. Mental map drawings can also consist of little illustrations or sketches which represent important aspects of a person's geography of the lifeworld. As already mentioned, the time dimension is important in mental maps as well. Mental maps not only contain spatial information but also temporal information. For example, Birgit's map shows dashed lines. These dashed lines do not signify connection between places, as there are no points (places) at their ends. Birgit used these lines to express the periodicity of presence in her different places (the same that Willi has done through text). In her map one can see a line next to the point which represents Berlin in the upper part of the map. A horizontal line of 
dots is drawn next to the Berlin point (figure 12). Here, Birgit wants to express that she goes to Berlin many times a year, but only for short periods, mainly for day trips. By contrast, Zurich is represented in the corner to the lower left. Here, the horizontal line indicates fewer trips to Zurich, yet these trips are longer and include more overnights stays (figure 13). Therefore, a distinct type of dashing was used here to express time as related to places.

Figure 13: Periodicity as sketched for frequent short trips in Birgit's mental map

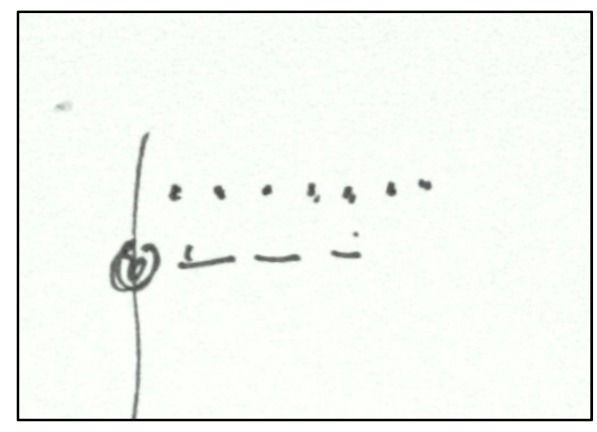

Figure 12: Periodicity as sketched for seldom trips with longer stay in Birgit's mental map

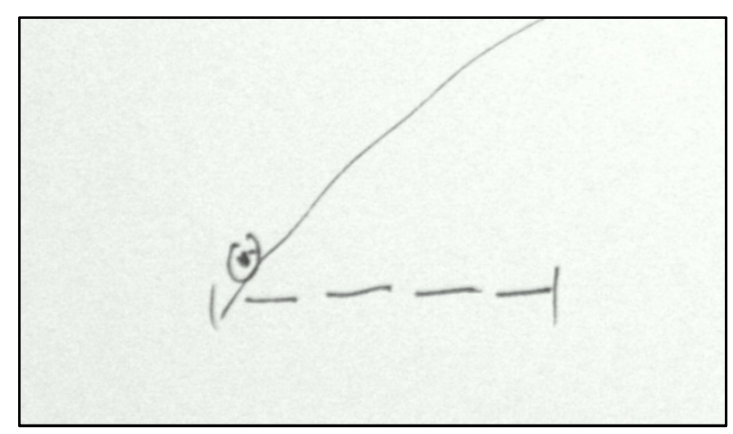

Time is also 'sketched out' in Oskar's mental map (figure 14). In the lower segment of his mental map, we can find a timeline. This timeline starts with his birth, and it contains an indication of the years in which significant changes in relation to his lifeworld's geography have occurred.

Figure 14: Timeline as sketched in Oskar's mental map

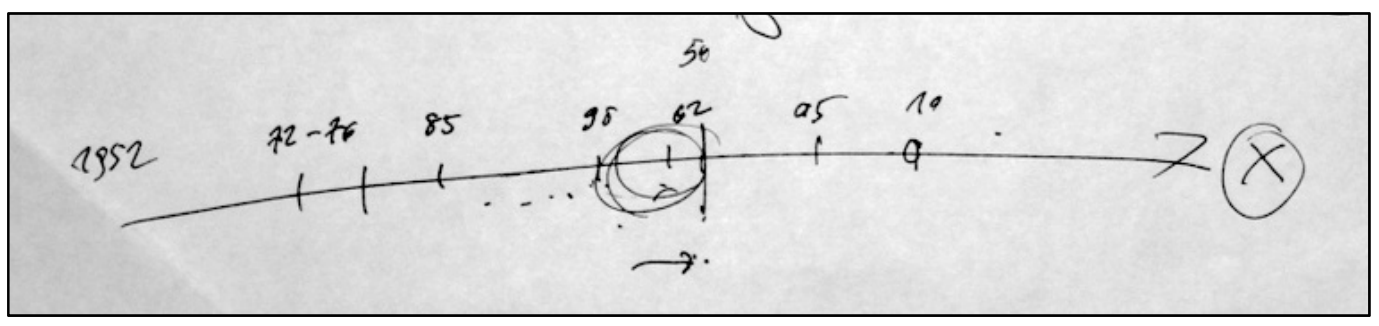

There are also sketched elements referring to other content. For example, both Nikolina as well as Ugo drew little aeroplanes into their mental maps. The transnational multilocality of Ugo takes places between Chile, Italy and Germany, while that of Nikolina includes the USA, Germany and Bulgaria. In particular, the intercontinental trips are then related to long flights and the aeroplanes become a symbol of these passages (cf. figures $15 \& 16$ ). Qamar makes even more consequent use of little sketches to draw his mental map. His mental map only contains one cartographic element, which is the national border of Chechnya. All other places are represented by landmark symbols. We should not relate these landmark symbols with the landmarks in the sense of Lynch. For Lynch, landmarks are everyday life facilitators of orientation within the urban space, but Qamar does not continuously pass the landmark symbols in his everyday life. He does not orientate with their help. For him they are icons which symbolise a place as a whole. 
Figure 16: Airplane as symbol for travel, sketched in Ugo's mental

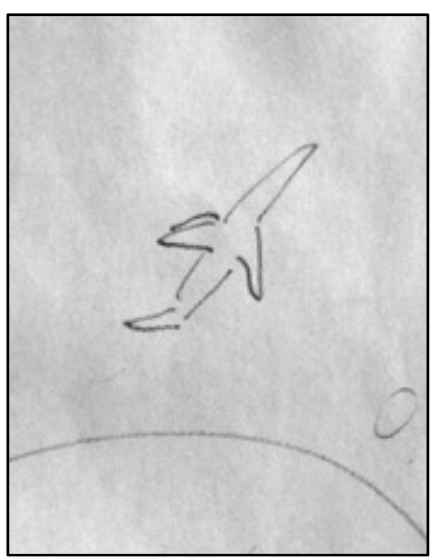

Figure 15: Airplane as symbol for travel, sketched in Nikolina's mental map

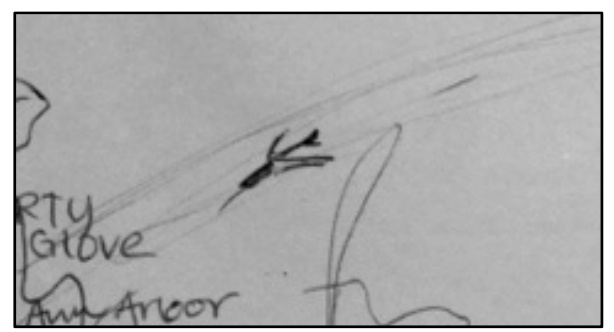

\section{Schemes}

Finally, I also found that mental maps might take the shape of what are known as 'mind maps' in psychology. Gunnar's mental map represents an example for that (cf. figure 17); he actually does not use any cartographic elements. His mental map consists of text and arrows that link the text. As in Qamar's mental map (figure 18), there is no topological information describing the locations in space of individual places towards each other. Gunnar's mental map is rather a scheme of his biography, in which he lists the different stages of his life. In text form, he also provides information about places and times. His mental map is definitely not a cartographic map.

Figure 18: Sketched biography in Gunnar's mental map

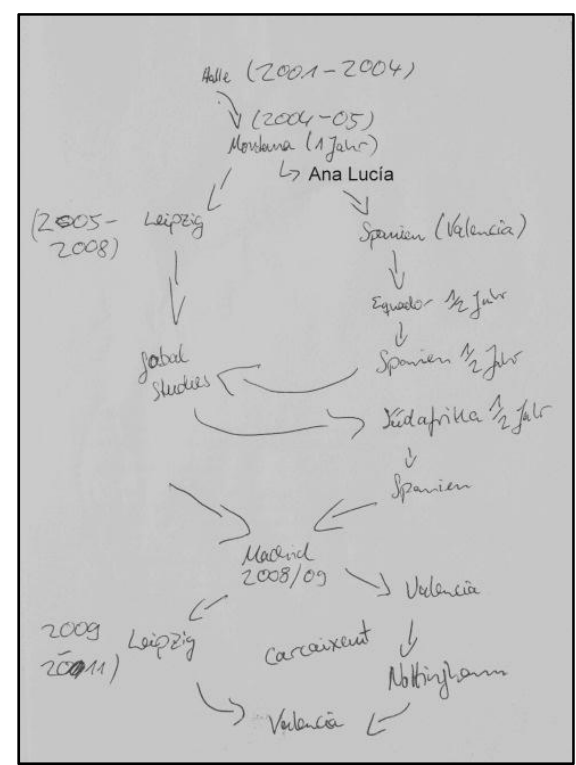

Figure 17: Iconic sketches in Qamar's mental map

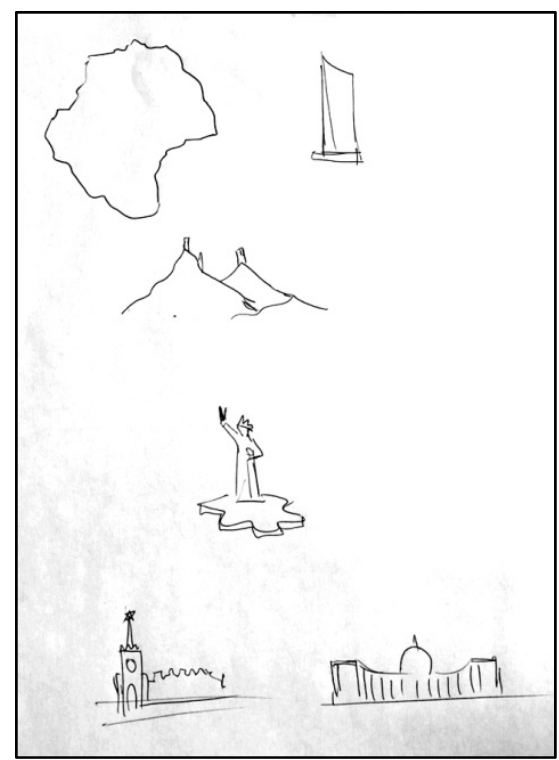




\section{Multiple component maps}

Another observation that I made during the analysis of the mental maps is that some maps actually consist of different individual components. These components are independent units that can be read on their own; they are self-contained and provide separate information. They also refer to the other components of the mental map. Such multiplicity of components can be a reflection on the different geographic scales. For example, Ronja and Tjark’s mental maps both show individualised components which refer to the local scale on the one hand and describe the national scale on the other hand. In Ronja's mental map (figure 20), there are two components on the left side which show Germany and Denmark, and which reveal the location of 'her places' within the countries. On the right side there are two components which show Copenhagen and Berlin, and which provide an explanation of the precise neighbourhoods within the city that are relevant to her. Similarly, Tjark actually used three distinct sheets of paper to draw the three individual components of his mental map. The first one contains a global perspective in which New York is shown as positioned against Rotterdam, Berlin, Copenhagen, and Aarhus. The second component is a zoom in on Berlin in which Tjark highlights the local scale of his action space there. His third component is equivalent to the second, but it provides details for his local scale in Copenhagen.

Furthermore, multiple components can also have an illustrative character. Ugo's mental map shows a small sketch which looks like a mountain. This mountain is a circumscription of Loncopanguo, a small village in the foothills of the Andes, close to Concepcion. Here, his family has a small piece of property where he is used to going when he wants to relax from the stress of everyday life in the bigger cities such as Santiago and Concepcion. Therefore, in addition to the cartographic description, the illustrative sketch is its own carrier of information.

Figure 20: Multiple components in Aurélien's mental map

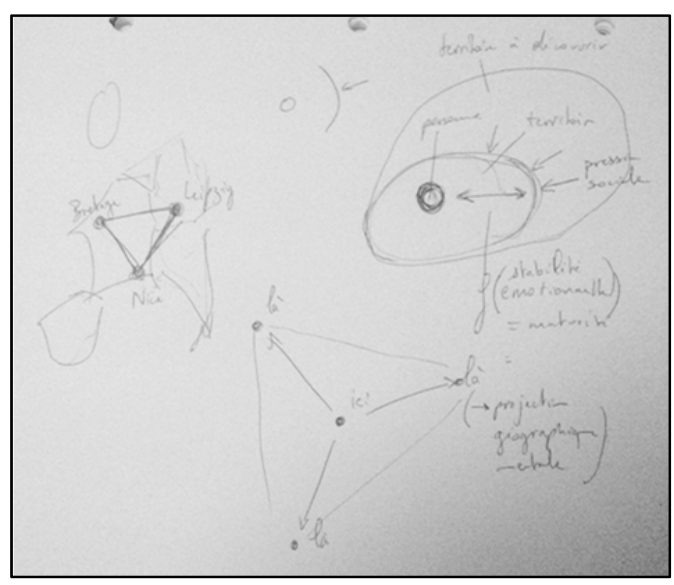

Figure 19: Multiple components in Ronja's mental map

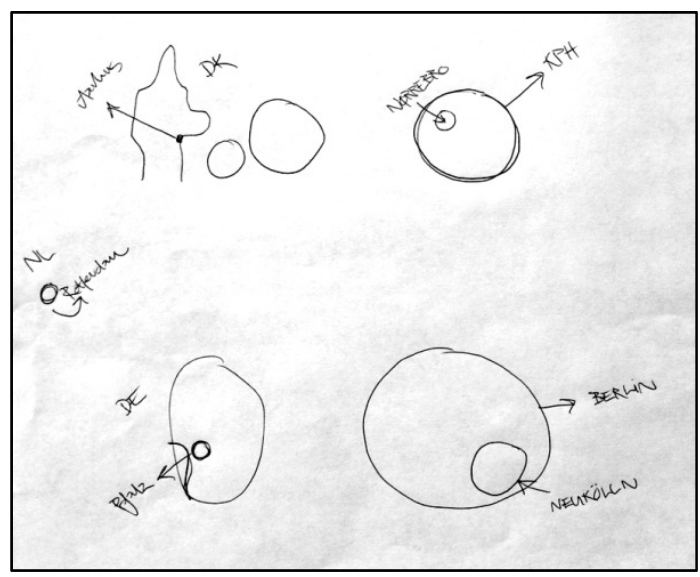

Finally, additional components in mental maps might also refer to schemes which illustrate rather abstract lifeworld concepts. Aurélien's mental map (figure 19) serves as an example here. He first drew a cartographic component of his map which highlights Nice, Leipzig and the region of Brittany in Germany and in France. While later speaking about the relation between these places, he started to refer to the influence of emotional stability on the range of his action space, and in parallel he illustrated that conceptual idea in the upper right corner of his mental map. 
Subsequently, another thought came up in his narration which was linked to the idea of emotional stability. This new thought deals with the concept of 'mental geographic projection', which represents a way of understanding how he relates to his places. He also added this concept to his mental map as its own component.

\section{Summary: Composition of mental maps}

Mental maps not only contain cartographic elements; there is a range of other carriers of information within them, and individual mental maps can also be made up of multiple components. I therefore suggest trying to understand mental maps in a broader sense. We should not think of mental maps as a drawn cartographic map. It is an image, an illustrated representation of how people perceive of the geography of their lifeworld. People use different 'carriers' for their relevant information, and they have different techniques in putting the parts together. Therefore, we should be open to the variety of data and mental map designs which we might face in empirical field work.

My analysis has shown that my interview partners group the single elements of the geography of their lifeworld into different types of drawing. The most important elements in these mental maps are places and their connection lines. Furthermore, distinct areas (or districts, as Lynch would call them) exist in my respondents' mental maps, which are separated by border lines. They structure their action space according to these elements and the mental maps then include information about the topological relations between these elements. In addition to these spatial contents, my interview partners also add temporal information. Therefore, space and time are always thought of as things which go together. The question which now arises is what significance do these individual elements have in the multilocal lifeworld? I will turn to this question again in section 7.1.2. I will draw some methodological conclusions in advance, which I derived from the application of mental mapping during my field work.

\section{Methodological utility}

Apart from the actual data that I collected through the mental maps, the drawing of mental maps is also interesting in terms of research methodology. There are two observations that I made during the field work. First, for me it was helpful to capture my respondents' territory, and to know from the beginning of the interview where the life of my interview partners actually 'takes place' so I was better able to guide the interview narration and pose my questions. While the interview partners drew their mental maps, they were forced to reflect about their action space, and they explained slowly and understandably which their places actually are. This also gave me time for reflection and thinking about the next steps in the interview.

Second, for my interview partners this was a helpful exercise, too. Many of my interview partners do not have much time as they are always busy with travelling between their places. This means they also do not have much time to reflect on their lives, and less so to reflect on the geography of their lifeworlds. Through this task of drawing a mental map, they were spontaneously forced to enter into such a reflection. After the interviews I had very positive feedback about this playful and enjoyable part of the interviews. My interview partners liked drawing these maps, and they also expressed that it was a completely new experience and really helped to make certain things clearer to them. This had already translated during the interviews into a high willingness to reflect on why certain places and other contents showed up in their maps - actually very useful information for 
answering my question how my respondents relate to place and space. Additionally, the drawn mental maps helped my interview partners to structure their narration. They could check for what they had already mentioned, and what was still missing in relation to 'their places' within their maps, and looking back on their maps, new things also came to mind while speaking. Also cognitive psychologist Barbara Tversky (1992: 493) pointed to this phenomenon when she said that 'map drawing provides a better cue to a missing map element than does a description as it is closer to the original stimulus and there are visual/spatial cues pointing to its absence.' In her experiments she tested if people can better recall spatial information using verbal description or visual drawing. She found that people actually combine both, and depending on the type of input verbal or visual - they can better recall information in the same form as the input was provided. Yet, visual ‘depictions' have supportive function to verbal 'descriptions’ (cf. Tversky 1992).

I would like to stress that through the experience with the analysis of the mental maps in a study on multilocal lifeworlds, I do not think that mental map drawings should be used as a solitary method. The complexity of a 'lifeworld's geography' goes beyond the study of the perception of a single neighbourhood or city. It includes different scales, and thus it makes it more difficult for respondents to draw easy-to-understand maps. Many of my interpretations, which I have presented above, were only possible as I could use my qualitative interviews and field notes from directly after the interviews as additional data. I doubt that any external person would come to similar conclusions only by looking at the drawn mental maps. However, the mental maps - as I have mentioned above - were very useful in combination with the interviews. Therefore, I would definitely argue for the use of mental mapping methods, but I would do so with the suggestion to use them in a mix with other data generation methods (possibly interviews, video recordings, field notes, internet research). In the next section I will therefore illustrate how I analysed my mental maps in combination with my interviews and how I translated them into 'case maps,' which are a synthesis of information from both data sources.

\section{Mental map between drawing and narration: The generation of personal case maps}

It is important not to forget about the fact that mental maps are only a snap-shot in a given moment (cf. Ploch 1995). If one asked the same interview partners to draw the mental map on another day, the map might contain different information. This does not mean that the mental map, which is inside the respondents' mind, is not consistent or something random. Rather, the problem lies in making it conscious and processing it ad hoc for expression. Furthermore, not all people have the same skills in expressing a mental map by the means of drawing, therefore I decided to compare the drawn mental maps to the interview narrations. The interviews themselves took much longer than the mental map drawings, and therefore the probability was high that I would receive additional geographic information. Furthermore, I suggested that those who felt less comfortable with drawing might reveal their information by talking about the mental map instead.

I will illustrate the value of this procedure with the example of Marta. During the interview, Marta was appalled by my request for a mental map. In the beginning of the interview I asked her for the mental map. I asked: 'Could you draw me a map of the places that are important for you?' (cf. Marta; line 22). She replied that she did not understand the question, and that she actually only related to her home country Lithuania. I did not insist on the drawing, so I asked my question for 'her places' again, giving some more precision to my question. This time I left out the part with the 
drawing. Immediately after, Marta started two narrative sequences which were rich with geographic information. The narration revealed that she was born in a small town where she also grew up. She explains that she went to Kaunas (Kovno) for her university studies, and then she moved on to Vilnius. She speaks about geographic information, but she hesitated to actually draw it. Later in the interview she reveals information about her stay in England when she was a university student, and her time in Finland, where she worked for a while. She also speaks about her life in Slovenia. So, there is a lot of narration which is actually the clue to her mental map.

By the end of the interview, I asked Marta again for her mental map and she replied: 'I really don't know how it has to look like. I am not understanding the... the idea of the map' (cf. Marta; lines 419-420). Basically, she did not want to draw, but she also did not want to snub me, and she finally drew me a mental map (cf. figure 21). If one now compares the content of what she was willing or able to draw and what she was telling me in the interview, one could argue that drawing is not necessarily always the best way of capturing a mental map. Some interview partners are indeed hesitant or shy to draw even though everyone disposes of a mental map. Therefore, I believe that mental map studies must take into consideration different ways of generating mental map data.

Figure 21: Marta's mental map: an example of sparse data generation through drawing

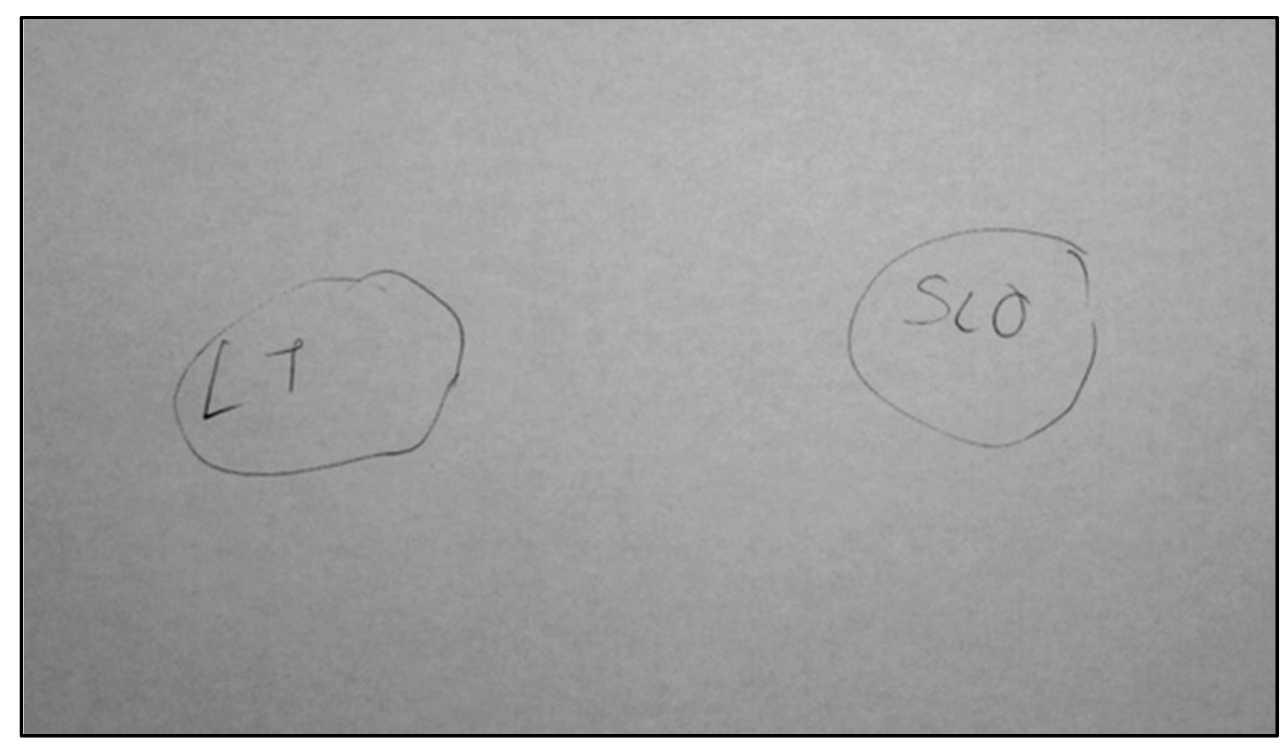

Obviously Marta is an extraordinary example in my sample. However, as a consequence of this observation, I also scanned all other interviews for additional geographic information in the narrations, then I used this additional information along with the one from the drawn mental maps to generate new case (specific) maps. These new maps are my scientific analysis and interpretation of my interview partners' drawings and narrations. Furthermore, I have used a cartography which translated the very individual mental maps into directly comparable ones. The maps that I 
generated $^{100}$ share a common symbol system and one could easily put them on top of each other in order to find further similarities across cases.

Figure 22: Marta - Case map, as result of merged geographic information from mental map drawing and interview narration

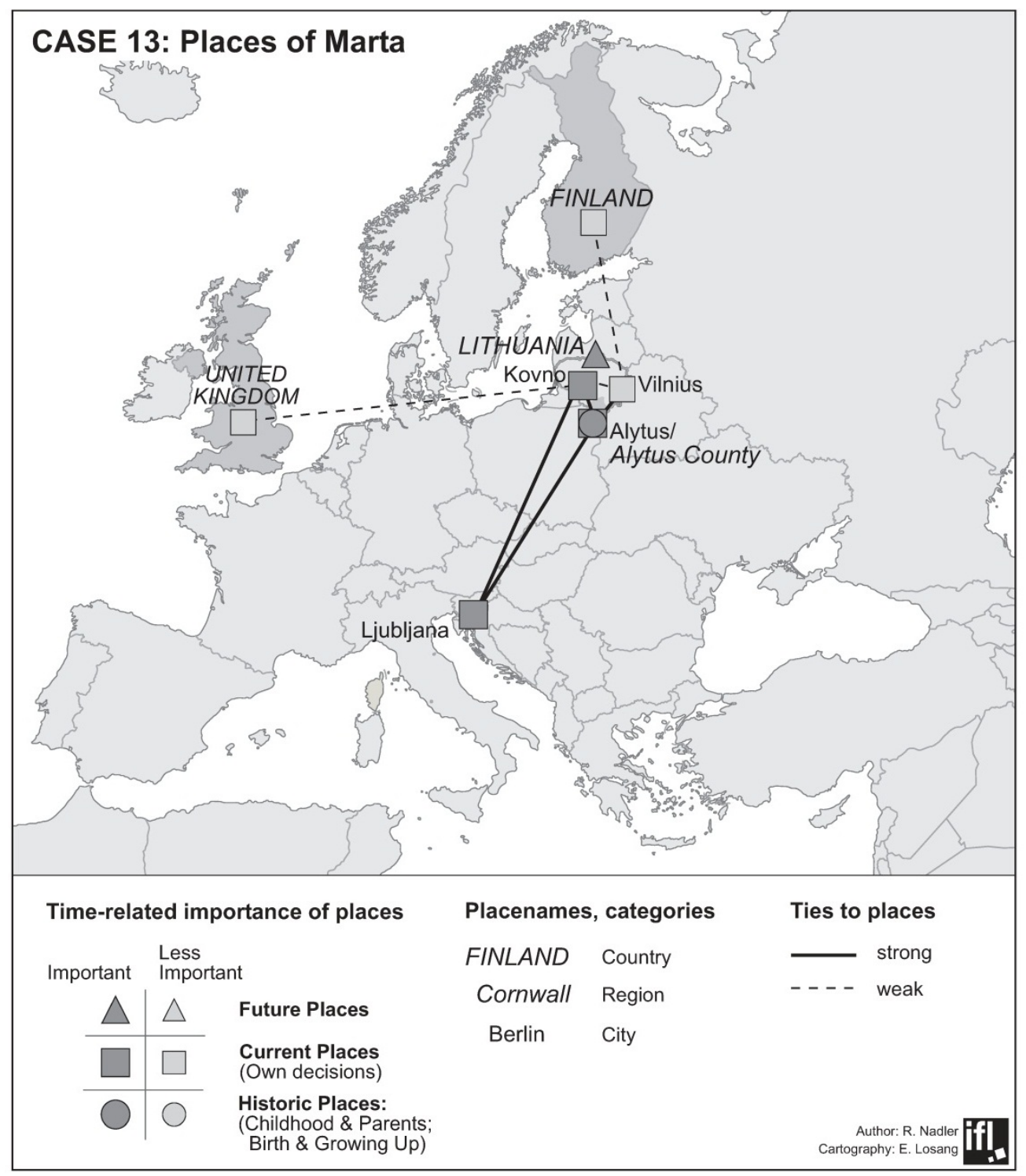

This analysis has shown that the combination of the two sources 'narration' and 'drawing' enriched my own case maps. It completed information on the individual action space (cf. figures $21 \& 22$ for the case of Marta). Therefore, I would like to repeat that mental maps do not exclusively come along in the form of drawings, but also in the form of narrations. Having said this I would support the Dual Coding Theory, which was presented by Allan Paivio (1971, 2006). Paivio suggested that

\footnotetext{
${ }^{100}$ Here I would like to thank Eric Losang at the Leibniz Institute for Regional Geography for his support in the technical realisation of the case maps.
} 
both visual imagery and verbal data are important for learning and knowledge creation in cognitive processes. If people are asked for something, they start to recall these verbal and visual knowledge segments and combine them in their expression of the answer. The same accounts for the mental maps that I have presented here.

\section{Similarities across case maps}

The individual case maps are presented throughout this whole document next to the grey boxes containing the individual life stories. Through the analysis of the interviews (in particular the analytic step of reconstructing the life stories) and mental maps, I have derived the set of symbols for the case maps. There are two important aspects in relation to the maps' legend. First, I grouped my interview partners' places according their importance as well as to their moment of appearance in the individual life stories. Here, I also distinguished between 'important' and 'less important' places. Both categories signify importance, and clearly not 'unimportance,' as I think that the places mentioned by my interview partners in the course of the interviews have a general significance, otherwise they would not have been mentioned. However, a difference in importance was obvious in the narrations about these places. The differentiation according to the moment of appearance is orientated on the concept of appresentation as in the phenomenological lifeworld literature (cf. chapter 6.1). Here, the perception of one's own lifeworld follows the trinity of past, present and future. If you remember, the terminology of lifeworld scholars was 'retention', 'impression' and 'protention'. Analysing my interviews, I also found this cognitive distinction in relation to places, but I changed the categories slightly. The past and present were translated into the 'historic places' and the 'current places' (I will come back to this in section 7.1.2). This terminology follows the logic of the main biographic stages in life which I have found to be part of my interview partners' cognition. My interview partners differentiated between personal history, which refers to the time span between birth and leaving the household of the parents to start their own life. Here, locational decisions that affected which places were part of their lifeworld and which not were taken by others (mainly their parents). Thus, historic places are those which were chosen by others for my interview partners during their childhood and adolescence. Current places then represent places that were actively and self-reliantly chosen by my interview partners once they started to live their own lives. This mainly started with moving to another city for university studies. Finally, there are future places where my interview partners could imagine or already plan to live in the future. They include ideas about how the good life should be as compared to the current one. However, information about future places was not provided by all of my interview partners, or it was less precise in the ideas of other respondents.

A second distinction is then presented in how strong the individual places are currently linked to each other in the geography of my interview partners' lifeworlds. I have derived this information from the narrated temporal information concerning physical and virtual travel (time spent in these places, frequency of trips between the places, periodicity, the communication flow). Furthermore, I looked at signifiers of relationship in the mental maps (e.g. line elements, arrows, etc.).

Even though I do not wish to go into detail, a look at the derived case maps reveals some repeated patterns across the individual cases. First, it becomes clear that for most of my respondents there is a 'core action space' which includes selected places from the overall mix. This core action space often has a tri-polar shape, whereas it could also remain bipolar, or seldom have even more than 
three poles. The corners of these shapes consist of current and important places, which are linked through strong ties. Furthermore, I observed that this core action space is mainly located within Europe, and less so in intercontinental ways. Even within Europe, the places which make up this core action space are located rather close to each other. I think this could be interpreted as evidence that 'geography still matters'. ICT as well as affordable fast travel connections have not automatically led to globalised action spaces on the level of the individual. The geographic distance that has to be bridged through travel is still a decisive factor for managing a multilocal life.

Around this core action space we can find satellite places that are rather located in a peripheral position in the own geography of the lifeworld. They represent less important places and they are tied weakly to the core action space. Here, I also found places on other continents than Europe, but intercontinental connections are not only seldom in terms of occurrence across the individual lifeworlds, they are also less relevant for those interview partners who engage in them. Therefore, my interview partners' action spaces consist mainly of a segment of Europe. Within Europe the action spaces can be distinguished according to a core of two or three places, and a group of less relevant satellite places around this core.

It is also interesting to look at the relations of historic, current and future places. Across all cases the dominant pattern is that my interview partners intend to live in the future in one or more of their current places, and in particular current places in the core action space. Future places are thus often places which are already now a component in their multilocal lifeworld. I interpret this finding as an indicator for a high satisfaction of my interview partners with life in these places. They currently experience how it is to live there, and they can easily develop ideas of a future in these places, too. This also reveals that multilocals should not be equated with global nomads who drift to ever new places. While my interview partners are multilocal and travel a lot, they seem to seek stabilisation in their spatial patterns.

In contrast, the historic places are less often part of the current core action spaces. After having left these places, once the development of their 'own life' started, my interview partners maintain mainly emotional attachments to these places. They could even be important in their current lifeworld, yet they are weakly linked to the core action space. While analysing the character of the historic places of those few cases where the future places fall together with the historic places, I found that there are two types. First, interview partners who stem from large cities (e.g. Pia, Ugo or Yari). Their historic places are part of their current core action space, and they represent possible future places, too. Second, there are interview partners that originate from rather rural areas and small towns. Looking at their life stories, it becomes visible that they maintained connections to these historic places and might opt for them as a future place (e.g. Aurélien, Henriqua or Marta). However, for the other cases where historic places are not part of the current core action spaces and are not equal to future places, a pattern of urbanisation is obvious. They originate from smaller towns and they do not see their future in these towns but rather in the larger cities where they are currently living (e.g. Birgit, Federico, Gunnar, Juliana etc.).

\subsubsection{Place and time}

Next to the mere analysis of the geography of lifeworlds, a main focus of my research is also to understand the meaning of these places in life. Analysing the interviews, my initial observation is 
that places could draw relevance from very different moments in life. As found in the analysis of the mental maps, the interviews also reveal that time in relation to places is a dominant issue. As I have already mentioned, my interview partners cognitively separate places that have historic relevance in their lifeworlds. They are places that have the character of not having been chosen freely by the interview partners themselves. These places are somehow 'given' within their life. This refers mainly to the place of birth, and the places in which my interview partners grew up and spent their childhood. These places became important in life through happenings and decisions that were out of the reach of my interviewees, e.g. when parents frequently moved with their children. Historic places often have the character of rural small towns, and leaving them was often linked to the individual urbanisation, moving to larger cities.

Gunnar e.g. speaks in a pronouncedly disparaging way about the people who have stayed in his 'historic place', a small village close to the eastern German city of Magdeburg where he grew up. He thinks that these people are those he was not interested in earlier in school, or that these are failed people who have not been able to make something out of their lives by leaving the city. This statement includes that he consider the option of going away, being mobile, as a type of winner strategy, while those who stay are the losers:

'Uh it is already an agglomeration [Leipzig], while somehow in Magdeburg there is nearly no one anymore, because the people have moved all over the world. I mean, everyone who has achieved a university degree moves away from Magdeburg. I mean no one wants to stay there. What should one do there? Those guys from my school who still live there are also the ones I did not have contact with before. Apart from two exceptions: two poor folks who have never finished their studies, and still live with their parents. They veg out, these poor ones' (Gunnar; lines 400-407 ${ }^{101}$ ).

This quote highlights that the often rural historic places are not perceived as personal future places. Still they also fulfil a clear function of being an emotional or mental base for one's own personality. My interview partners describe these historic places in which they grew up as places which work as an anchor point in their mobile lives or which work as a place to return to and relax from a stressful and hectic current life. Their childhood seems to be related with peace, silence and a life without sorrow.

For example, Aurélien says that Brittany and Nice are two places that he did not decide to have in life, but that were given to him in a way through his individual biographic background:

'Let's say, these two cities, these places have been given to me. They were given to me by birth. [...] You were born in Nice, but your family stretches out to Brittany, so you permanently travel between the two, for seeing the family, social and familial stability' (Aurélien; lines 91-95 ${ }^{102}$ ).

\footnotetext{
${ }^{101}$ Translated by the author from the German text: 'Äh es ist schon ein Ballungszentrum auch, währenddessen irgendwie Magdeburg da kaum noch jemand ist, weil die Leute natürlich auch in alle Welt verstreut sind. Ich meine, jeder der irgendwie es schafft halbwegs 'nen Universitätsabschluss zu machen, zieht ja weg aus Magdeburg, ne. Also da will ja irgendwie keiner bleiben. Was soll er da auch tun, ne? Die Leute, die jetzt aus meinem Jahrgang noch da sind, sind so Leute, mit denen ich auch vorher nicht so viel Kontakt hatte. Mit zwei Ausnahmen: zwei arme Gestalten, die irgendwo nie ihr Studium geschafft haben, und bei ihren Eltern noch wohnen, und so vor sich hinvegetieren, die Armen. ‘

102 Translated by the author from the French text: 'Disons que ces deux villes, ces deux lieux m'ont étaient donnés. C'est me donné de naissance. [...] Tu naîtras à Nice, mais ta famille étira en Bretagne. Donc tu voyageras en permanence entre les deux, pour voir ta famille, stabilité sociale et familiale.'
} 
These places therefore refer to personal history. On the other hand, there are also places from the past but which have been selected through the conscious decision taking of my interview partners. These are places in which they have lived for some time, and which are thus considered 'their places'. They must not necessarily be part of the core action space today, but they were chosen selfreliantly by my interviewees after the moment they left their parents' house, mainly from the moment they left secondary school. With the graduation from school my interview partners started to take over responsibility for their lives and the spatial decision belong to that responsibility. Independent of their current importance, these places have a link to their present life and their present places, which are the places that currently are most important within the individual multilocal pattern of my interview partners. In the latter, the present places a lot of current everyday life takes place, but links are maintained to the self-chosen places of the past. I subsume all these places under the notion of 'current places', which refers to the fact that these places were all thread like pearls on string by my interview partners and that they influence their current life. However, they are not all parts of the direct present places in which my interview partners actually live. The present places of living are mainly part of the core action spaces, and they are places where my interview partners have dwellings. These past 'current places' are more peripheral in the present action space. In a temporal dimension, they influence life from further away, yet they are part of the current 'individual life’ of my interview partners.

Finally, there are also places that have a more prospective character, and which I therefore will call future places. My interview partners expressed a clear idea of where their future should 'take place', where they want to live later on. Often these future places are part of the present core action space. They are places where my interview partners currently live, and about which my interviewees have developed clear images. These images contain positive evaluations of their current life and thus allow for a positive estimation of these places for their own future. Aside from some exceptions (cf. 7.1.1), these places are larger cities.

These three basic temporal dimensions cannot clearly be distinguished in terms of defining a time range from when on a place switches between the three temporal dimensions. It is not possible to sketch out how much time of one's 'own life' has to pass until a historic place becomes a current place. The future outlook also does not have a precise time frame. It is more a diffuse perspective into what should come after the present situation. Therefore, a simple conclusion is that individual places can be part of different temporal dimensions at the same moment. Place A might be the place of birth, but it remains a place lived at in the present. Or place B might be relevant now, and also shows up as the major place in someone's personal future perspective. I also found that places do not really disappear from the mind maps of my interview partners. Even historic places are regularly visited by my interview partners, and they keep their links to these places alive; the individual case maps illustrate these findings.

The second way in which time is related to places is constituted by how regularly and for how long the current places are visited. The interview partners report a great variation of rhythms in which they come to the places that are currently part of their lives. Some places are visited in weekly alternation, while others might only be visited for a few days every year. The interplay of rhythm of alternation and duration of stay in each place is a very complex issue. It becomes obvious that the 
whole place pattern of the individual lifeworlds is dynamic. Rhythms of alternation as well as durations of stay are changing quickly if external conditions change. In extreme cases, places get a virtual or imaginative character. This means that the interview partners do not visit them for a long time, but they keep their links to these places alive in an imaginative form (e.g. thinking consciously of these places; remembering them) or in virtual forms (e.g. following the news about the places on the internet; speaking to people in these places by e-mail and phone). Places can also return to a more intensively visited rhythm again. As such the individual pattern of multilocality is not stable but very dynamic and subject to change. Places can lose importance, but they might regain it again.

This can be illustrated by Henriqua's description of periodicity. During her adolescence she used to travel regularly to Zurich, as her aunt and some cousins lived there. This gave her the opportunity to practice her German language skills that she was about to lose after her parents had moved from Germany back to Spain. During these years she enjoyed the freedom that the holiday trips to Zurich provided. Far from her parents and in companionship with her cousins, she could go out and do what she wanted. However, place patterns are far from stable in my interview partners lives. Henriqua says:

'Well, to Zurich I stopped going, but I used to travel [there] a lot. Since I was 13 every, almost every summer. Uh... and now my aunt doesn't live there, and I still have some friends. But let's say I don't miss them. Between the Netherlands and Spain... well, I told you we are travelling now because also of job reasons. When the job stops, I think, we will visit the Netherlands for... 3 times a year, or four. It depends on the circumstances' (Henriqua; lines 186-190).

Her pattern shifted from Spain to Switzerland to Spain to the Netherlands later in life. Today she is married to a Dutchman. They have a small child, and she has a work contract in a Dutch university. The family is living in Vigo, Spain, so they frequently have to travel between the two countries. Hennriqua knows that the frequency of trips to the Netherlands will most probably decrease once her work contract finishes. Then trips will only have a private character, to see her in-laws, the grandparents of her daughter. Nonetheless, Zurich and Wageningen are 'her places'; they are part of her lifeworld.

This observation of the interrelation of time and place makes it nearly impossible to define in objective ways if multilocality is evident or not. A simplifying definition is not adequate for studying the broad variety of multilocality. For example, defining that multilocality is a given when a person alternates between places on a weekly basis, spending three days here and four days there, would automatically exclude other important places which are probably less often visited physically. The temporal aspect of multilocality is far more complex. Therefore, I think the pragmatic solution to the definition of multilocality, deciding if a place is part of one's own everyday life or not, remains in the hands of the interview partners themselves.

\subsubsection{Comparison between places}

A second interesting point is that my interview partners also told me regularly about the way they compare their places to each other. The different experiences that they make in their places cause a more or less conscious reflection about these places of which my interviewees become aware in 
different situations in everyday life. This concerns mainly small things, but it can also refer to more basic reflection of a wider and more abstract nature.

Xaverio e.g. works and lives in Berlin, Milan and Rome. His frequent trips to all these places allow him to compare, and he describes it as follows:

'It's funny, because they are... three completely different cities. So, for me it's like if I, in every city I can find something. And I, I'm starting to know what I can find in each of them. So, I know that Milan has a certain type of a rhythm, connection, people, a way of life. That Berlin, it's more quiet, it's more easy to live there, the quality of life is higher, and uh there is more culture. Rome, it's amazing, because it's a place where the culture was born, I mean. But at the same time, it's a little bit more traditional, I mean, in respect to Berlin. But for me, Berlin is... it's the way to, to have new feedback. When I want to, to know something new, and to have new feedback, uh... normally I go to Berlin" (Xaverio; lines 56-64).

This quote highlights how the assets and the character of the different places in one's own lifeworld are perceived. In another section of his interview, Xaverio explains that Milan is the place where he earns his money; he has a lot of jobs as a photographer in the fashion industry. Therefore, what he refers to Milan's rhythm etc. in the quote above basically means that everything in Milan is about staying connected in the business world. In contrast, Berlin is the place where he finds inspiration for his personal artistic photography, which he then likes to exhibit and develop in Rome. Xaverio's description is an example of the continuous comparison in which my interview partners engage.

Dirk equally describes an episode of everyday life: he compares Bulgaria to Germany when it comes to organising the things one needs such as the internet connection for one's own apartment:

'Many things are easier [in Bulgaria]. For example, simply when you are living somewhere and you need internet, then you can order it. In Germany it's a whole rigmarole until you get it and so on, it takes at least two weeks. Back in the days it was four weeks, yeah. And there it was like, you call, then a guy comes over and lays a cable up to your apartment, outside along the façade of the building [laughs]... uh and then it works within two hours. And if you don't pay it, then you don't pay it, they simply turn it off. Once you pay it again, they switch it on again. Uhm much, much more... somehow unintentionally more friendly. More service- uh service-oriented, because it is less obstructed through these unbelievable structures and institutions and all that as in Germany' (Dirk; lines 223-232 ${ }^{103}$ ).

Through this constant comparison, the place-specific characters and assets become visible to my interviewees. This comparison of places leads to two consequential aspects in the lifeworld: first, my interview partners order their places cognitively according to the perceived 'assets'; second, they develop preferences for places.

\footnotetext{
${ }^{103}$ Translated by the author from the German text: 'Viele Sachen sind einfacher. Zum Beispiel Internet, einfach wenn du irgendwo wohnst und du brauchst Internet, dann kannst du dir das bestellen. Das ist in Deutschland ein Riesentheater, bis das dann kommt und so, da vergehen zwei Wochen. Also früher waren es noch vier, ja. Und da war es halt so, da rufst du an, dann kommt einer und legt das Kabel, und zwar einfach außen an der, an der Fassade hoch [laughs]... äh und dann geht das in zwei Stunden. Und wenn du es nicht zahlst, dann zahlst du es halt nicht, und dann ist es abgeschaltet. Und wenn du es wieder zahlst, dann stellen sie es wieder an. Ähm viel, viel... ungeplant viel servicefreundlicher. Service- äh serviceorientierter, weil es nicht so durch unglaubliche Strukturen und Institutionen und so alles verbaut ist wie in Deutschland.'
} 


\subsubsection{Functional complementarity}

A second interesting aspect is the 'functional complementarity' which I observed between places. In the beginning I wondered if places would be substituting each other in the multilocal lifeworlds of my interview partners, in a sense that they would all provide equal facilities for living everyday life. However, during the analysis I rather found that there is often a clear differentiation between private places, being part of leisure time, private life, personal biography, and job-related places. Already when I asked my interview partners to draw me a map of their own places, they were wondering to which places I was referring. They cognitively differentiated between these main categories, and they wanted to know which ones they should draw. Private places are linked to the personal history. These places are less often visited. They might have lost a bit of their relevance in favour of more recent places, but they remain important in the lifeworld of my interview partners. They actually refer to the temporal dimensions of 'historic places' and the 'past current places'. The interviewees regularly return to them, even in long rhythms, or they keep these places in their memory. Then there are private places that are related to the current private life and leisure activities ('current places', where people are actually present in terms of living there now). These are places that my interview partners associated with family and their partners (if they have one). In these private places, my interview partners often own their apartments or they have rented some dwelling. Their personal belongings (e.g. official documents) are deposited in these places. They mainly chose these places voluntarily. These places are part of the future projections of their own life ('future places'). My interview partners feel satisfied at these places; this includes that they try to spend a lot of time there.

These places have a predominant character over the others, which are places that have a professional character. Often my interview partners describe the relation between their places as a hierarchical pattern in which one private place represents the central location of the lifeworld, while several other places are scattered around as 'satellites'. The central location is then described as the basis of current life.

In the professional places, their lives mainly circulate around work. This includes the fact that my interview partners do not make very much effort to construct a sense of belonging to these places. The places often were not chosen voluntarily but rather they showed up in the lives of my interview partners through professional necessity. Thus, there is a connotation of temporariness that comes along with these places. These places are actually frequented as long as my interview partners are contracted for a job there. This means that my interview partners preferably use flexible and temporary forms of dwelling. It could be a hotel which is used for sleeping in these places. It could also be a rented and sparsely equipped apartment (I will come back on the issue of dwelling forms in section 7.7).

This functional complementarity is visible in Juliana's narrations. Juliana frequently commutes between Berlin and Mainz. Berlin is her private family place and the centre of her lifeworld, and Mainz only recently became one of her places. Some months ago, she took a position as a professor at the university in Mainz, but she would have actually preferred a position in Berlin. With a little self-irony she explains: 
'Probably this sounds a bit decadent, but we [she and her husband] always make jokes, or I... I always tell my husband, that I do not clean up, I do not cook, I don't do anything over there [in Mainz]. Because, my family, because here [in Berlin] I am continuously busy with such things because of the kids, so that over there I mainly don't do such things. Until now, I also have not seen very much there, because it was in the winter semester. So I always left work in the evening, and then it is dark and cold. I also do not know very many people there. Meanwhile, yes, in the summer it is more lovely. And yes, then I go to the city centre once in a while, or I eat out with my colleagues. Of course I enjoy that, because I... here I have the time... I have very little time. The time to go out without having to do anything. I don't know, as parents we always have to negotiate about who can do what and when and so on [laughs]. And there, I also like the work, I like teaching, the students. They are good, like everywhere. I think, these young people who are curious and so on' (Juliana; lines 306-318 ${ }^{104}$ ).

This quote reveals that Juliana starts to see the advantages of separating the work and private sphere according to the two places of Berlin and Mainz. Initially, she did not want to go there and leave her family in Berlin. However, she is rediscovering parts of life - like going out without having to organise child care - which she no longer had in Berlin. Therefore, she draws a line between the two places and the related functions which they provide. Mainz is functional in terms of providing a job, professional social contacts and a lifestyle without the responsibility of the children. Berlin is functional in terms of providing a home base for her family, her private social networks, and her private life in general. Both places are functionally complementary.

My interview partners also do not often engage in socially connecting to these professional places. Social contacts are often limited to the immediate professional environment, e.g. colleagues, business partners, students. Thus, the wider local community is not always approached equally by my interview partners in all their places. I also observed that my interview partners often hold multiple jobs, and then these different jobs can be related to different places.

Birgit e.g. has a part-time position as a professor at the university in Kassel, and at the same time she is the owner of a private planning office in Leipzig, which she manages herself. So, to her Leipzig and Kassel are two places which are clearly separated in professional terms and concerning her social networks. She stresses that Leipzig is her 'base station':

'What I find important is the image that I have very clearly decided for Leipzig as a central location [in her life], even though I hold this professorship. This comes with the knowledge that there is this other leg I stand on economically, the 'office', which is somehow an existential issue. But it's also, I would say, a [personal] attachment. This attachment to this place [Leipzig], because it's simply, my family lives here, Thomas [her partner] and our relationship, my mother [...] In any case, I simply

\footnotetext{
${ }^{104}$ Translated by the author from the German text: 'Das klingt vielleicht so ein bisschen dekadent, aber wir lachen immer, oder ich... ich erzähle immer meinem Mann, dass ich dort überhaupt nicht aufräume, nicht koche, nichts mache. Weil, also meine Familie, weil ich hier [in Berlin] so also natürlich ständig mit solchen Sachen wegen den Kindern beschäftigt bin, ähm dass ich dort solche Sachen meistens überhaupt nicht mache. Bisher habe ich da auch nicht sehr viel gesehen, weil das Wintersemester war. Also ich war abends immer dann von der Arbeit, und es ist dann dunkel und kalt. Und ich kenne auch noch nicht so viele Leute dort. Mittlerweile, ja, jetzt im Sommer ist das schöner. Und dann, ja, ich gehe manchmal so in die Stadt, oder gehe halt Essen mit Kollegen, und so. Das mache ich gerne, natürlich, weil ich... hier habe ich die Zeit... also sehr wenig Zeit. Auch so Zeit einfach um frei irgendwo rauszugehen. Ich weiß nicht, wir müssen das ständig als Eltern das aushandeln, wer wann was machen kann, und so. [laughs] Und ich arbeite dort auch gerne, ich unterrichte gerne, finde die Studenten gut. Also die sind alle, ich denke überall, gut. So junge Menschen, die neugierig sind, und so.'
} 
want to live only in this city. In Kassel, I do not have an apartment, nowhere to stay, but I live in a hotel. And I prefer to organise it rather temporarily. I think, this also affects the relationships, if one says decisively, my bed is my bed, and only there. And the other thing is a different situation' (Birgit; lines 47-61 ${ }^{105}$ ).

Birgit later also adds about Kassel:

'I really have few contacts in the city, and I am not so much involved into the urban community where one might have friends or spend leisure time, or one does whatever. This is more or less only the cosmos of the "university"' (Birgit; lines 84-87'06).

In this description of functional complementarity between places, also a hierarchical relation between the individual places can be found. Places mostly have different degrees of importance to my interview partners. I had already mentioned this in section 7.1.1, where I explained that I have translated this finding into the legend of the case maps. According to the importance of a place in their own lifeworld, my interview partners selectively engage in attaching to the places. This includes various forms of relating to people in these places (see section 7.3) or also different dwelling practices (see section 7.7).

Looking at the entire relational pattern of places in each of my studied lifeworlds, I would say that they can best be described as functional complementarity. The single places fulfil certain roles in the lifeworlds; they have a clear function and offer specific resources and opportunities. Only in sum, these functionally complementary places allow the integrity of my interview partners' lifeworlds. If one place wasn't part of the pattern anymore, the lifeworld would change its character. To my interview partners the whole pattern makes sense.

Aurélien illustrates this integrity or unity of the places in his lifeworld with a very illustrative and animal-like circumscription. He says:

'Okay, quote unquote, me, I have urinated on my territory, on my territory in Leipzig, in Brittany, in Nice, you know. That's somehow my thing' (Aurélien; lines 80-81 ${ }^{107}$ ).

This means to him that he has ordered and structured his places into a coherent spatial pattern that represents his territory. Therefore, we should keep in mind that places in my interview partners' lifeworlds are ordered in a logical manner of functional complementarity which can include hierarchical structures, and these hierarchical structures are also reflected in preference patterns, on which I would now like to elaborate.

\footnotetext{
${ }^{105}$ Translated by the author from the German text: 'Was ich in jedem Fall wichtig finden würde, wäre auch das Bild, dass ich mich ziemlich eindeutig für diesen Mittelpunkt Leipzig entschieden habe, obwohl ich diese Professur angenommen habe. Mit dem Wissen, da gibt es noch diesen, dieses andere wirtschaftliche Standbein ,Büro', was irgendwie auch eine existenzielle Frage ist. Aber auch 'ne, ich würde mal sagen 'ne [persönliche] Bindung. Und auch die Bindung an diesen Ort, weil es einfach, weil hier die Familie lebt, also Thomas und die Beziehung, meine Mutter [...] Auf alle Fälle äh möchte ich einfach nur in dieser Stadt [Leipzig] wohnen. Ich habe in, in Kassel keine Wohnung, auch keine Bleibe, sondern wohne da wirklich im Hotel. Und organisiere das dann auch lieber so temporär. Das hat, glaube ich, für die Beziehungen Auswirkungen, wo man sagt, wirklich eindeutig, mein Bett ist mein Bett. Und nur da. Und das andere ist wirklich eine andere Situation.'

${ }^{106}$ Translated by the author from the German text: 'Ich habe auch wirklich wenig Kontakt innerhalb der Stadt. Und bin da auch nicht so in diese Stadtgesellschaft eingebunden, wo man Freunde hat oder Freizeit verbringt. Oder was auch immer tut. Sondern das ist dann wirklich dieser Kosmos ,Uni'.'

${ }^{107}$ Translated by the author from the French text: 'Donc, entre guillemets, moi, j'ai pissé mon territoire, sur mon territoire à Leipzig, en Bretagne, à Nice, tu vois. C'est un peu mon truc.'
} 


\subsubsection{Preference patterns: Oh yes, this fits me!}

The places themselves also have a distinct character. My interview partners describe that they prefer one place to the other for place-specific aspects that are independent of their own lifeworld. Some places have a more positive and attractive character than others. For example, an international atmosphere is mentioned as a positive property of places. Low rent and living costs have been associated with preferred places as well. On the other hand, a negative aspect of place is e.g. when the built environment is rather ugly and not appealing. As mentioned above, my interview partners continuously compare their places. They look for things in that places that suit them, and meanwhile perceive things that disturb them. Thereby, these properties of a place are not so closely linked to specific people in these places. Places are perceived as having a specific character independent of the social networks that are available to my interview partners. The latter are also existent and relevant, but I will come back to this later. Here, it is really about the places themselves.

Tjark provides an interesting description of his comparison between Berlin and Copenhagen and the resultant evaluation:

'Uhm... Berlin is interesting because uh... because of the fact that there is so much space, and it's green. And it is not so $100 \%$ capitalistic. Not yet. And I hope it remains like that. This means in terms of urban planning and so on, for example you can see it in each street, it is less regulated. For example the areas in front of all the cafés, they actually belong to the City Council, where the trees are and so on. But then each café or kindergarten and so on, they have constructed their own little world. This would never happen in a city with more control. It's more like: Berlin is always a bit more bottom-up and less top-down. This means less politics and more autonomous initiatives of the citizens. And that's one of the large assets of Berlin, that uh... that is one of the big reasons why Berlin is a super interesting city. [...] Copenhagen is, it's more regulated. Copenhagen is also wealthy. So, Copenhagen has far more money than Berlin. On the other hand it is also a smaller city, and as such it is easier to control' (Tjark; lines 583-631 ${ }^{108}$ ).

What you can see in this quote is that Tjark translates the perception of the place-specific assets (Berlin as less controlled and more bottom-up; Copenhagen as wealthier and more regulated) into an evaluation and preference for Berlin. Berlin is interesting, whereas Copenhagen is regulated and controlled. In later interview sequences he explains that he perceives Copenhagen as a city in which material status is very important and the general atmosphere of Copenhagen is about successful life stories. In Berlin things are more relaxed. This means that Tjark has developed a preference for Berlin, and this is reflected in the fact that he calls Berlin his favourite future city.

\footnotetext{
${ }^{108}$ Translated by the author from the German text: ‘Ähm... also Berlin ist interessant wegen äh... wegen dass es unglaublich viel Platz hat, und es grün ist. Und dass es nicht so 100\% kapitalistisch ist. Noch nicht. Und das, hoffe ich, es bleibt so. Das heißt mit Stadtentwicklung und so, sieht man zum Beispiel auf jede Straße, es ist nicht so reguliert hier. Das sieht man zum Beispiel, dass vorne jede Café, gibt es einen Bereich, das hört, gehört eigentlich so der Stadt, da wo die Bäume stehen und so weiter. Aber dann hat jedes Café oder Kindergarten und so was eine kleine Welt aufgebaut. Das würde nie passieren in einer Stadt mit mehr Kontrolle. Das ist eher so: Berlin ist immer ein bisschen mehr bottom-up und weniger top-down. Das heißt weniger Politik und mehr so Eigeninitiative von den Bürgern. Und das ist eine riesige Stärke von Berlin, dass äh... das ist einer von den großen Gründe, warum Berlin eine super interessante Stadt ist. [...] Kopenhagen ist, ist reguliert. Kopenhagen ist auch reich. Also, Kopenhagen hat natürlich mehr Geld als Berlin. Aber es ist auch eine kleinere Stadt, so dass es einfacher ist zu kontrollieren.'
} 
Also my other interview partners engage in such comparison. The comparison is important to find out what are places in multilocal pattern which are providing the opportunities and atmospheres that my interview partners need. Through comparison they discover the appropriate places. Henriqua, who alternates mainly between Vigo (ES) and Wageningen (NL), uses the phrase 'Oh yes, this fits me!' to circumscribe how she feels when she comes to Vigo after having been in Wageningen for a while. The character of the places quasi exists ex ante, before my interview partners arrived in the places, but they only become aware of the specific character once they are there by 'using the place'.

\subsubsection{Places patterns between conscious decision taking and outcome of hazard}

My interviews also revealed some interesting findings in relation to the way in which my interview partners actually decide on locating somewhere. As we have just seen, my interview partners develop preferences for places by comparison, and these preferences influence their future location decisions. However, the question is how did they decide for their current places? Obviously a lot of places have actually not been chosen for the place itself. Decisions rather orientated to other aspects of the lifeworld, such as professional issues or social ties. Here, places are only indirectly chosen, namely in the frame of another conscious decision for a job, to move closer to some relevant persons, etc.

However, there are also 'current places' of which the presence in the lifeworlds of my interview partners is considered as an outcome of coincidences. This does not mean that my interview partners did not take a decision to go to these places, but the decision was not consciously following a precise plan. Rather, my interview partners 'drifted' into the places by circumstances that they had not actively provoked.

For instance, Aurélien could not really explain to me why Leipzig had become one his places. Whereas Brittany and Nice can be characterised as 'historic places' which entered his lifeworld while he was still not of age to decide on his own, only Leipzig was added to his multilocal lifeworld at a later stage and is one of his current places. However, Aurélien says:

'Then there is Leipzig. This, it was finally an objective coincidence of life which made me uh... arrive in Leipzig. And for the moment I have decided - so it's actually a type of decision - to say: me, it's those three places there' (Aurélien; lines 98-100 ${ }^{109}$ ).

In addition to Nice and Brittany, Leipzig has become his third place, and the decision to make it his place was a conscious one, but the point is that Aurélien cannot really explain why he came to Leipzig for the first time. This happened somehow by coincidence, as he says. He actually did not know anything about the city beforehand, and he did not have any job offer there. He actually did not exactly have a clear reason to go and live there.

On the same note, we can find the absence of a clear decision taking process in respect to places in other interviewees’ narrations. Dirk describes how Minsk and Hanover appeared on his case map;

\footnotetext{
${ }^{109}$ Translated by the author from the French text: 'Puis qu'il y a Leipzig. Ce, c'était finalement un hasard objectif de la vie, qui m'a fait euh... arriver à Leipzig. Et pour l'instant j'ai décidé - donc c'est un rapport de décision - de dire que: moi, c'est ces trois endroits là.'
} 
neither had been his intention. He intentionally went to Bulgaria. Coincidentally, he met a young woman from Minsk who participated in the same Bulgarian language course, so he began travelling to Minsk to visit her. In another one of his stories about one of his jobs in the Balkans where he works as a trainer and consultant for structural improvement in adult education, Dirk says that his main partner in this job is from the Balkans, but recently moved to Hanover from where he now runs these adult education projects in eastern Europe. Therefore, he has not chosen Hanover voluntarily or intentionally, yet it is now part of his lifeworld, as he needs to go and see this professional partner once in a while. The interesting fact then is that one of his adult education projects brought him to Minsk, where his client, an organisation for adult education, is simultaneously the employer of his private friend, the young woman with whom he shared a language course in Bulgaria. Dirk, to a certain degree, drifted into this place pattern. He remarks:

'If you have to do with these and those persons, and one also likes having to do with them, then this does not mean that one would not get along with other persons. But this circle of people is also a bit of a coincidence. And it is probably something like that with places as well' (Dirk; lines 383$\left.386^{110}\right)$.

Nikolina also cannot really explain why she chose Weimar as one of her places. Weimar is where she is currently doing a Master's degree, but she had applied in several other locations across Europe. Nikolina says:

'I grew some seeds in applications in Italy and in Germany, like in Freiburg I applied, or in Amsterdam I applied. And I got in in most of the places, but uhm... why didn't I... it was just like a blind choice. I really don't know why I went to Weimar. I mean, I could have gone to Amsterdam, but the programme there was like really, really research uhm... -based. And I wanted to do something more practical. And uhm... the program in Freiburg was like... was like really, really political. So, I wasn't sure if I wanted to do that. And then in Italy, like I wanted to live in Italy. I definitely do. But uhm... I heard that like it's easier to be like a foreign student in Germany" (Nikolina; lines 340-347).

Here you can see that the appearance of Weimar on her case map was not an intentional decision for Weimar, but rather an intentional decision against all other places. Weimar then was left on the list of places where she was accepted.

A final example should be enough to describe this point of coincidences of place patterns: Qamar explains why he has actually come to Leipzig, which today is the geographic centre of his lifeworld. Qamar describes:

'This was a, a coincidence. In St. Petersburg, I had earned my living outside on the streets as a painter, there I made my bread. And one met a lot of tourists, with whom one talked, and uh... and, and, and... One had, it turned out that a German lady invited me. Because uh at that time the visa was, for... in order to get a visa, you had to have an invitation. Without invitation it was... there

\footnotetext{
${ }^{110}$ Translated by the author from the German text: 'Wenn man mit den und den Personen zu tun hat, und halt auch mit denen zu tun haben mag, dann heisst das ja nicht, dass man nicht auch mit anderen zu, auch zu Recht kommen könnte. Sondern das ist halt auch ein bißchen zufällig der Kreis dann. Und so ähnlich ist es bei den Orten wahrscheinlich auch.'
} 
were no travel agencies during that time... it was in the beginning of the Perestroika. In '89 I came here for the first time.' (Qamar; lines 69-74 ${ }^{111}$ )

Again, this quote highlights that even Qamar somehow drifted to Leipzig; he did not have a precise plan of making Leipzig one of his places. Looking at these examples, we should bear in mind that the geography of the creative knowledge workers with whom I spoke is not completely an outcome of conscious decision taking. Rather, some places also show up in life and then the interviewees grabbed the chance that was offered to them.

\subsection{Mobility: The role of travel}

In addition to place patterns, mobility is one of the central dimensions in the multilocal lifeworlds of my interview partners. In this section I will elaborate on different aspects that showed up during the analysis of my interviews which are all related to the topic of mobility. This includes physical travel itself, but it is also about the ways in which my interview partners think of imaginative and virtual travel. Furthermore, I found that physical mobility also causes a type of mental mobility or polyperspectivity. Finally, physical travel happens across space, and my interview partners develop specific relations to the transit places and space through which they have to pass when circulating between their places.

\subsubsection{Physical travel}

My interview partners all travel in various regularities between the places that form their multilocal lifeworlds. Physical travel is a central aspect in their lives and it has a certain meaning to them which is more distinct from the meaning that it might be for monolocal people. The main difference consists of the fact that travelling over long distances and across borders to my interview partners has something of an everyday life character. It is nothing special in terms of organising it. My interview partners distinguish the way they travel from that of other peoples' travelling style. Other people often travel in leisure time or they travel for holiday. In such cases, travel organisation might be an extra-ordinary task, and it might make people nervous, however, my interview partners describe that travelling is so much part of their everyday lives that preparation does not make them nervous anymore. They sometimes even do not think about it consciously - it simply happens. Valentin is a specific example here: his physical travel is actually less job-induced and rather a private pleasure to him. Valentin is strongly orientated to outdoor activities and climbing. The jobs as a graphic designer, film producer and pedagogue teaching school children juggling are only a means to an end for travelling. He is not career-oriented, he works to save money for travelling, and as such, he has travelled across Europe, Asia and Australia looking for perfect climbing locations. In particular, he loves travelling alone and letting himself drift around, as he explains about his last trip to Australia:

\footnotetext{
${ }^{111}$ Translated by the author from the German text: 'Das war ein, ein Zufall. In St. Petersburg hatte ich als Straßenmaler draußen so mein Stipendium verdient, da mein Brot verdient. Und da hat man auch viele Touristen getroffen, mit denen auch geredet, und äh... und und und... Da hat man sich, hat sich so ergeben, dass eine deutsche Dame mich eingeladen hatte. Weil äh früher war Visum, für... um das Visum zu bekommen, brauchtest du so eine Einladung. Ohne Einladung gab es so... es gab keine Reisebüros noch in... Es war Anfang der Perestroika. '89 bin ich das erste Mal hier gewesen.'
} 
'I actually did not want to do the typical Australia thing, this Work and Travel, but I wanted to go there simply because it's summer while it's winter here, and I wanted to go climbing. [...] And I think, in comparison to many others who did such an Australia trip immediately after graduation from school and told me about it, I have discovered many places which one will not see if one follows these ordinary touristic Lonely Planet routes. Sure, one sees awesome things while following Lonely Planet, but there are also a lot of beautiful things to see if you leave these routes' (Valentin; lines 63-83 ${ }^{112}$ ).

Valentin's quote shows how one's 'own physical travel' is constructed as distinct and alternative way of travelling. Even if Valentin is a specific case as he does travel more for private pleasure, my other interview partners also show this pattern of understanding their own style of travelling as something distinct from touristic forms of travel.

For my interview partners, this physical travel has positive and negative connotations. They perceive them both in parallel, and there seems to be a type of individually perceived tolerance threshold which defines whether the positive or the negative is more noticed. This tolerance threshold is made up of the frequency of physical travel. In this sense, Liam e.g. says that until a certain limit he enjoys travelling and being mobile, because it takes him to places to which he would not normally have gone:

'Providing it's only once a month and there aren't too many other trips to other places in the month, I enjoy it. Uhm what does it mean to me? I don't know. It's uh... it's a source of frustration sometimes especially when there's too much snow or, or British Airways are on strike. Last year was a bit of an... in November, December last year was a nightmare, yeah. But it's nice, too. I suppose I, I have seen small parts of many countries, European countries, that I wouldn't have ever seen otherwise. But I, I do tend to see the, the street between the hotel and the meeting room. And I, I think it's a bit of a waste, that I don't see much more" (Liam; lines 318-324).

Liam underlines that to a certain degree travelling is fun, and he would appreciate profiting more from visiting all of these new places. Basically, his job does not allow him to spend much leisure time in the new places to which he goes, and the regular trips to Stockholm also do not leave him much time to discover the city. Nonetheless, he would like to spend more time discovering. On the other hand, he clearly says that it depends on the quantity of trips he has to do. Travelling is also associated with stress, time pressure, problems in air and train connections etc. Stephan also points out that travelling's positive aspects are sometimes overrated and he would argue to not forget about the stress that accompanies it:

'In everyday life it is simply annoying. I mean, I also try to convince myself of the fact that it is something great, and clearly it is. But uhm to miss your train connection somewhere at night, and then only arriving the next morning in Copenhagen because one could not fly the same day for whatever reason, this is simply a pain in the arse, and exhausting. Therefore, I think it is something

\footnotetext{
112 Translated by the author from the German text: '[Ich] wollte jetzt nicht dieses typische Australiending da machen, dieses Work and Travel-Teil, sondern ich wollte da eigentlich nur hin, weil's halt Sommer ist im Winter und klettern. [...] Und hab echt, ich hab glaube ich, so verglichen zu vielen, die mir das erzählt haben, die früher nach dem Abi so eine Australienreise gemacht haben, echt super viele Orte entdeckt, die, die man halt nicht so sieht, wenn man diese normalen Touristen-Lonely-Planet-Routen abklappert. Man sieht natürlich geile Sachen, wenn man Lonely Planet folgt, aber es gibt auch Superschönes zu sehen, wenn man vielleicht dann abseits dieser Routen sich bewegt.'
} 
you can only do for a limited time. This is nothing desirable in any form' (Stephan; lines 260$\left.276^{113}\right)$.

This tolerance threshold hints at the fact that there is a certain optimum balance between mobility and staying/immobility. Physical travel must always be thought of as happening between places, and these places are stations of rest, or where life becomes stabilised and anchored. Birgit e.g. describes that her mobile life needs Leipzig as a fixed anchor point. Leipzig is associated with stabilising and continuous elements of her lifeworld: her partner and her mother, her office, and her own apartment and household. Birgit says:

'Well, you need to have that [a stable place as an anchor point] somehow, otherwise it would be more difficult to handle this... this continuously being on the move, and the presence times in other places, at least I think so. [...] I miss my own bed. And I, I do everything so that I can be there as often as possible. Then I also prefer to leave very early in the morning, and I come back home the same day. This I do. So, that, that uh... and this has also to do with, I believe, one needs to have one fixed place. At least for me it's like that. And I can imagine that this is the same for everyone' (Birgit; lines 64-66, 509-513 ${ }^{114}$ ).

The places which represent anchor points in the mobile lifeworld are often associated with loved ones, with family members, one's partner, the children and close friends. This means that another important negative aspect is the distancing effect of physical travel. If one has to leave a place, one often also has to leave the people there. This becomes a problem particularly for my interview partners who have small children or who have to take care of their elderly parents. Henriqua thinks that travelling becomes something so ordinary after a while that she cannot really find any pleasure in it anymore. It's more of a habit and a necessity, and since she has become a mother, it is even more negative in her perception:

'I remember the first European project I was, I loved to travel. And I thought: Oh, now we are going to meet in Italy, and then in Germany, and then in Ireland. Now, when I have to travel, I think: Pooh, I have to travel. And it's not stressing, but it's not that I find it so exciting anymore. But why, also my circumstances are different. I have my baby at home, and I will be three days without seeing her, and I think, well, this is also good for me, because I can rest. But also I miss her. Many things are influencing that. But also you get tired of travelling. Because I have friends who uh... we have been speaking about it... who have to travel a lot because of their job, recently. And now they have discovered, what I always told them, that when you have to travel, uh you have five meetings in half a year or in one year, it's not anymore so exciting. When it has to happen like regular, you know, it's like uh... because also you get tired of it. I mean even when you don't have

\footnotetext{
${ }^{113}$ Translated by the author from the German text: ,Im täglichen Leben ist das auch einfach nur nervend. Ich meine, ich versuche mir das auch immer so einzureden, dass das so ein bisschen was, was Tolles hat. Und das hat es natürlich auch. Aber ähm nachts irgendwo den Anschlusszug zu verpassen, und dann erst am nächsten Morgen in Kopenhagen zu sein, weil man irgendwie an dem Tag gerade nicht fliegen konnte, aus irgendwelchen Gründen auch immer, das ist einfach nur total nervend, und anstrengend. Und von daher ist es einfach was, was man auch nur für eine gewisse Zeit machen kann, glaub ich. Also das ist einfach nichts, was irgendwie, in irgendeiner Form erstrebenswert ist.'

${ }^{114}$ Translated by the author from the German text: 'Also das muss man irgendwie haben, sonst hält man diesen... dieses Unterwegssein, und diese anderen Anwesenheiten an anderen Orten, also jedenfalls nach meinem Gefühl, schwerer aus. [...] Ich vermisse mein eigenes Bett. Und ich, also ich tue alles daran, möglichst ganz oft da zu sein. Dann fahre ich lieber morgens ganz früh los, und noch am Abend [zurück]. Also das schon. Also das, das äh... Und das liegt daran, dass man, glaube ich, immer einen Ort braucht. Also jedenfalls bei mir ist das so. Und ich kann mir vorstellen, dass das bei allen so ist.'
} 
stress, it's also tiring to be here and there. Because well hotels for example, I don't like to go to hotels any longer. Even when they are five-star hotels. I think, well, it's just a room of a hotel, with a code' (Henriqua; lines 416-428).

This example shows that travelling can lose the character of something exciting after a while, it becomes a routine. However, it is a routine which equals efforts. In particular, if it is considered in the light of social ties it can thus be a burden of being far from the people who one wants to have around. Even though Henriqua's quote seems to stress the rather negative aspects, she also admits that she would feel bored only living a monolocal life without travelling. Therefore, both aspects the negative and the positive - are mainly reflected within one lifeworld, and they can become conflicting. One cannot live without travelling even when it interferes with the sought after stability in life. Juliana, who has two small children in Berlin, also describes the problems associated with travelling and the multilocal lifestyle. Whereas she works in Mainz, her husband holds a position in Stockholm, Sweden. And this multilocal family situation is stressful in terms of organising parental presence in Berlin. Sometimes this leads to the situation that both parents are outside of Berlin, and third persons have to take care of their children. Juliana actually does not like these situations, and this feeling of dislike makes her perceive travelling as a constraint to her family life. She and her husband therefore take care in coordinating their absence times from Berlin. Juliana says:

'But we try not to... mainly not to have these situations uhm that we are both out of town, because I... and that we leave the kids to third persons, because for us that's somehow... we do not like to do that, and it's also not good to do that regularly. For the kids, I think, it's also not good' (Juliana; lines $\left.88-92^{115}\right)$.

Thus, as far as family context with small children is affected by physical travel, the negative aspects are more strongly evaluated. Furthermore, there is also another negative aspect of physical travel which is described by my interview partners: physical travel always includes the fact that one has to leave one place. I have described in section 7.1 that my interviewees actually imagine their places in order of their functional complementarity and preferential hierarchies. Despite this, they still mainly like all of their places. As a consequence, they are often upset about the fact that they often have to leave one of 'their places' for another one, and they would prefer to have some more time to spend there and be able to live better in that specific place. For example, Emil regularly circulates between Leipzig, Bayreuth and Basel. Whereas Bayreuth does not have so much importance to him - he describes it as a workplace - Leipzig and Basel have a high emotional value for him. Leipzig is his historic place, where he grew up. He had lived in Basel for some time, and his Italian partner is working and living there, so Emil basically loves to spend time in both places. He explains:

'Yeah, it is an effort, an effort of coordination. And then, of course it is also, yeah, sometimes quite enjoyable that one can leave. To go somewhere else. So that one does not feel so provincial. In the sense of only being attached to the one [place]. You can escape sometimes. And on the other hand, it is also the moment of arriving everywhere else, because... yeah, somehow I am happy to arrive elsewhere. And I have difficulties leaving again [laughs]. So, often I would prefer to stay in a place,

\footnotetext{
115 Translated by the author from the German text: 'Aber wir versuchen das nicht... möglichst wenig solche Situationen zu haben, ähm dass wir beide nicht in der Stadt sind, weil ich... und dass wir an dritte Personen die Kinder übergeben, weil das ist für uns irgendwie... wir finden das beide nicht schön. Und das ist auch nicht gut, wenn man das oft macht. Für die Kinder, glaube ich, ist das auch nicht unbedingt gut dann.'
} 
because I think: well, it would be nice, if you would not have to leave already again. [...] And being on the move is also valuable in itself, which somehow belongs to oneself, which one cannot think away. [...] Also because I am a relatively unsteady and mobile character, the continuous travelling suits me, I think. Uhm when you can run away from... from the tranquillity' (Emil; lines 139$\left.158^{116}\right)$.

Two things show up in this quote by Emil: first, the negative aspect of being forced to leave and not being able to stay a bit longer, and second, the positive aspect of being able to escape sometimes from 'provinciality' and 'tranquillity'. The last aspect also includes the counter-piece to the former aspect, because leaving always includes arriving somewhere else. In this sense, Pia also describes her relationship to travel. Pia's historic, current and future place is Berlin. She was born and grew up there, and she loves the city, but she runs her own architecture firm in Copenhagen. Additionally, she is engaged in many architecture projects in China, and she recently negotiated on being more involved in a university in Riga, Latvia. Thus, she is travelling quite a lot and she says:

'First of all I am always delighted in arriving [to Berlin] [laughs], if I touch down and I can see the Fernsehturm [television tower]. I then come, I enjoy coming home. Actually I travel, I don't know how to formulate that, actually I don't like travelling very much. Actually I love the most to be at home. But I approve being off and away once in a while. Because I think, then one has, it opens another perspective again, and I appreciate that' (Pia; lines 494-499 ${ }^{117}$ ).

In this quote we find again the aspect of 'arriving' to places at the end of physical travel as something which is evaluated positively, while my interview partners often perceive as more negative the initial moment of physical travel, the leaving. Another point is interesting in Pia's quote: as in Emil's description, physical travel is associated with the possibility to escape from the stability, rigidity and routine in one place. It allows for changing perspectives through a temporary re-location to another place. This aspect is also highlighted by Xaverio, who seems to acknowledge the fact that physical travel means making full use of his multilocal arrangement:

'It's like I need, you know... I just have to change and to move. I mean, it's not that you don't have a... a normal rhythm or... I mean, I have my, my routine. Because it's impossible finally to not have some [regularity, or everyday life] [...] Yeah, even because, yeah, you go crazy, I mean. You need a... okay, I know that this is the place where I stay in Milan. In Berlin I stay there. But then you have the possibility to change everything when you want. I mean, you don't have the... you know the... the schedule really strict, that you have in the, in the normal life, where you stay in the office,

\footnotetext{
${ }^{116}$ Translated by the author from the German text: 'Das ist natürlich einmal Aufwand und Anstrengung, Koordinationsanstrengung. Und dann natürlich eben auch, ja, manchmal ganz angenehm, dass man weg kann. Woanders hin. Sich dadurch auch nicht so provinziell fühlt. So, so, du bist jetzt nur verhaftet dem [einen Ort]. Du kannst auch mal raus. Und andererseits ist es dann eben auch wieder immer überall anzukommen, weil man... Also irgendwie bin ich überall froh, wenn ich ankomme. Und komme eigentlich fast schwer überall wieder weg [laughs]. Also, oftmals möchte ich dann eigentlich lieber irgendwo bleiben, weil ich denke: ah, es wär schön, wenn du jetzt nicht schon wieder weg musst. [...] Und dieses Unterwegssein an sich, das ist eben auch schon irgendwie so'n, so ein Wert, der irgendwie, irgendwie dann zu einem dazugehört, den man sich gar nicht mehr wegdenken kann. [...] Also weil ich halt auch so'n, so ein ziemlich unruhiger, motorischer Mensch bin, kommt mir glaube ich dieses Unterwegssein ganz entgegen. Ähm wo man dann da so vor den... vor der Ruhe einfach auch mal weglaufen kann.'

${ }^{117}$ Translated by the author from the German text: 'Erst mal freu ich mich immer wieder, wenn ich ankomme [laughing], wenn ich einfliege und ich sehe den Fernsehturm. Ich komme dann, ich komm gern nach Hause. Ich reise eigentlich, ich weiß gar nicht wie ich das sagen soll, eigentlich reise ich nicht gerne, eigentlich bin ich am liebsten zu Hause. Aber ich find es gut, ab und an mal weg zu sein. Weil ich denke, dann hat man, dann eröffnet sich eben wieder ein anderer Blick, und ich kann es wertschätzen.'
} 
in a... just in a city, and stop. So, you can, you can manage a lot. If I'm just fucked up about Milan, I just take... and go take a plane and go to Berlin for a while. I mean, if I need just, you know, to change, to just to relax, I can go there, and just because I know that there I can have some stuff. If I want... or more... I don't know, more business, I can come here, and everything. So, it's, it's a way to create even more opportunity. Yeah" (lines 805-817).

A multilocal life could be understood as a continuous sequence of switches between movement and being in places. Multilocality means having a set of places and their assets at one's disposal, and physical travel is the key to the exploitation of these places' resources. Additionally, physical travel not only allows one to go somewhere else to retrieve something, but it also allows one to get away and avoid other things. In this sense, some of my interview partners report that they use the physical travel to escape from social stress and problems with people in their places. They simply take the next train or plane and they go to another of 'their places' where they are far from these problems. This motive of 'small escapes' also shows up in an interview sequence with Gunnar, who describes that he lived for certain moments in a small apartment with his Spanish girlfriend. Whereas before they had lived for some months in a long-distance relationship, he was quite overwhelmed when his girlfriend suddenly lived in his room. By then he appreciated short trips related to his job as a stem cell courier for the Leipzig University Hospital, a job which made him travel on a monthly basis, for two or three days throughout Europe:

'At that time, I flew once a month somewhere else, and I picked up stem cells from a foreign hospital and brought them here to Leipzig. This has also contributed to my internationalisation [laughs], because once a year... once a month I went to completely new places. I liked it, because we shared a room while Ana Lucía lived here. And after a while, it became very, very tight, you know. I mean, I also need a lot of 'me-time', in which I can think about myself. And I did not have that anymore in the year when Ana Lucía was here in Leipzig for Global Studies. So I appreciated that I uh... could get away for two days each month. Mostly I read very much then, I listened to music and I took time for myself' (Gunnar; lines 434-443 ${ }^{118}$ ).

My interview partners can easily relax from such stressful situations simply by going to the other places. Isabel also uses the alternation between places and travelling as a way to leave stressful situations for a moment. Isabel is a career-oriented woman who meets high-rank politicians and business managers throughout the entire world. She travels a lot and she focuses strongly on her business as an interpreter. She does not have a stable relationship, and her trial-and-error in respect to men also forces her into 'small escapes' once in a while. Isabel mentions:

'Well, I also used it often in order to get away from some problems. It is quite practical if I messed up something, that... when something went wrong in one place, normally with men, I can always go to the other place. I can forget about the problem there, and that's fantastic. And when I come back,

\footnotetext{
118 Translated by the author from the German text: '[Ich] bin dann halt einmal im Monat irgendwie in der Weltgeschichte rumgeflogen und habe aus 'nem anderen Krankenhaus Stammzellen abgeholt, und hier nach Leipzig gebracht. Was auch enorm zu meiner Internationalisierung beigetragen hat [laughs], weil ich wirklich einmal im Jahr... einmal im Monat nochmal komplett woanders war. Das war mir ganz lieb, solange Ana Lucía hier war, weil wir hatten uns ein Zimmer geteilt. Und das wurde immer sehr, sehr eng, ne. Also, ich brauch auch viel so ,Ich-Zeit‘, wo ich so mich mit mir selbst auseinander setzen kann. Und das hatt“ich überhaupt nicht mehr in dem Jahr, in dem Ana Lucía Global Studies hier in Leipzig gemacht hat. Und da war mir das total lieb, dass ich äh... mal so raus konnte einmal im Monat für zwei Tage. Ich hab dann meistens ganz, ganz viel gelesen, Musik gehört und mich so mit mir selbst beschäftigt.’
} 
everything has calmed down, and I got rid of the problem. [...] Apart from that, travelling also means adventure to me. I mean, every time, if I have a new assignment, this includes new people, a new topic, a new city. Uhm... everything new. Even though it is always a bit hard to pack my bags and get started, because it's always exhausting and I like it a lot both in Berlin and Barcelona, and normally I do not want to leave from these places. But I know, if I overcome this first instinct uh... of inertia, I will be excited. If I get on the airplane or the train, then it's like: I feel like a Viking, I'm going to conquer something, I get an adrenaline rush from my work and I'm excited about the people and I really love my job' (Isabel; lines 105-122 ${ }^{119}$ ).

In addition to the related burden of always having to leave again, this quote by Isabel also reveals the other positive aspects of physical travel. It is associated with adventure, conquest, excitement and challenges. This motif shows up in various narrative sequences of different interview partners. The very positive aspect of physical travel is that it brings inspiration, it leads to new experiences and perspectives, it allows one to approach the exotic and unknown. As such, travelling means engaging in adventure and challenges, and my interview partners acknowledge this fact of diversion from ordinary everyday life. However, as physical travel itself is often described as a part of everyday life routine, I would interpret these descriptions as emphasis of the episodic character of a multilocal life. Such a life is to be seen as a consequence of small episodes of presence in the different involved places, and episodes of physical travel. These last episodes of physical travel are a central aspect of combining the individual places, and it is also important to maintain the social ties in places by engaging in face-to-face interaction. Despite this, as everyday life does not continuously happen physically in one place, my interview partners perceive physical travel as being dragged out and diverted from the stable continuity of everyday life in these places. While my interview partners have to leave place A to go to place B, most of the people with whom they engage continue their stable life in place A. So from the perspective of my interview partners, leaving place A equals temporarily leaving the continuity of physical everyday life in this place, and entering into a relation of absence in place A, while switching from absence to presence in place B. Physical travel is the mediating moment here. They switch between their places; even though travel is part of everyday life, it means entering something new, e.g. the other place's everyday life.

This alternation is also evaluated positively by my interview partners as it allows them to combine different lives within their lifeworld. Physical travel means alternating, and alternation can be translated into the idea of circumventing decision-taking for one of the places. It means that one sees the opportunities of two or more places and does not want to exclude the options for future life

\footnotetext{
${ }^{119}$ Translated by the author from the German text: 'Gut, also, ich hab`s auch oft eingesetzt, und um von irgendwelchen Problemen wegzukommen. Das ist ganz praktisch, wenn ich irgendwas, was... irgendwann was vermiest habe, mit Männern normalerweise, an dem einen Ort, kann ich immer flüchten in den anderen Ort. Und damit vergesse ich völlig das Problem, das ist schon ganz toll. Und wenn ich wieder zurückkomme, hat sich alles ein bisschen beruhigt, und ich bin davon gekommen. [...] Und sonst ist das Reisen für mich ähm... ein Abenteuer immer. Ich meine, jedes Mal, wenn ich einen Einsatz habe, heißt es neue Leute, neues Thema, neue Stadt. Ähm... alles neu. Und obwohl es mir immer ein bisschen schwer fällt, zu packen und irgendwie mich in Gang zu setzen, weil es ist immer ein bisschen anstrengend und mir gefällt es sowohl in Berlin als auch in Barcelona sehr gut, normalerweise will ich mich dann nicht trennen von dem Ort. Aber ich weiß, wenn ich diesen ersten Instinkt äh... der Trägheit überwunden habe, bin ich begeistert. Also wenn ich ins Flugzeug steige oder mich in den Zug setze, dann: auf zu neuen Ufern, dann fühle ich mich wie ein Wikinger, ich geh irgendwas erobern, also Adrenalinschub von der Arbeit und bin gespannt auf die Leute und ich mag meine Arbeit sehr.'
} 
by deciding against one of these places. In this sense, Stephan, when reflecting about the meaning of physical travel in his life, remarked:

'Of course it's also a bit that one does not really have to decide. Well, that one has this feeling of being everywhere a bit. One does not settle. I mean the... this must be somehow... one has to have this as a prerequisite. If I were someone who insisted on sleeping in my own bed and being at home each night, then I would not have chosen this way' (Stephan; lines 260-276 ${ }^{120}$ ).

A final observation which seems a bit detached from the aspects which I have presented so far, concerns the ecological impact that physical travel has. Some of my interview partners also reflect about the ecological consequences of their behaviour. Ronja currently lives with her husband in Berlin, they have recently become parents, and Stephan works in Brunswick, whereas Ronja at the moment takes care of their baby in Berlin. However, as much as she can, she takes smaller jobs in Copenhagen. Her German is still not good enough to work as a journalist in Germany, so she needs to rely on her jobs in Denmark. She generally does not like this frequent travelling, and she also discussed the contradictory attitudes of many mobile people. She argues:

'Unfortunately travelling has lost something, because it has become so ordinary and cheap and fast. So I think uhm... previously it was more of a luxury. And also it is not as though flying is very ecological. Uhm there is a type of replacement, that one tries to buy all organic products in life and so on. But then with flying, which actually is the worst, one does not abstain from' (Ronja; lines $\left.396-403^{121}\right)$.

This doubt about the ecological aspect is also reflected by Federico, who frequently has to travel between Leipzig, Prague, Ljubljana and Trieste. In his reflection about physical travel, he started from the moment when he first came to Leipzig for a year for an Erasmus exchange. At that time the low-cost airlines did not yet exist in the European market, so he relied on European bus lines. However, with time he has become more demanding in terms of travel comfort. As he has to travel often, choosing the right mode of transportation is finding a balance between travel time and a relaxing atmosphere. The ecological perspective also comes into play when he evaluates his travel options:

'I mainly travel by train, or by, by... or I fly. But for ecological reasons I try, uh if I go to Prague or Ljubljana, this is still feasible by train, you know. It also depends on, how, how often and so on. Uh... Flying, I fly rather to England, if I have something to do over there. Or I have recently been to the USA, of course, these things, yes' (Federico; lines 219-224 ${ }^{122}$ ).

\footnotetext{
${ }^{120}$ Translated by the author from the German text: 'Es ist natürlich auch das, dass man sich nicht so richtig entscheiden muss. Also dass man so dieses Gefühl hat, man ist so ein bisschen überall. Man legt sich nicht so fest. Also ich meine, die... das muss ja irgendwie... das muss man so als Grundvoraussetzung haben. Also wenn ich jetzt jemand wär, der einfach darauf besteht jeden Abend im eigenen Bett zu liegen und zu Hause zu sein, dann hätte ich das natürlich so nicht gemacht.'

${ }^{121}$ Translated by the author from the German text: 'Das Reisen hat wohl traurigerweise was verloren, indem dass das so gewöhnlich und billig und schnell geworden ist. Also ich denke ähm... früher war das wohl so eine Luxus. Und es ist auch nicht, also das ganze Fliegen ist ja auch nicht umweltfreundlich. Ähm da gibt es eigentlich so eine Verdrängung, dass man probiert so im Leben ansonsten biologische Sachen zu kaufen und so. Und gerade mit dem Fliegen, was das äh schlimmste für die Umwelt überhaupt ist, ähm verzichtet man dann nicht.'

${ }^{122}$ Translated by the author from the German text: 'Ich reise meistens entweder mit dem Zug, oder mit dem, mit... oder ich fliege. Aber aus ökologischen Gründen versuche ich, äh wenn ich nach Prag oder Ljubljana fahre, das ist noch machbar mit dem Zug,
} 
So we can summarise that physical travel is a central dimension in the lifeworlds of my interview partners. They are also identified by others as very mobile people, and this type of identification through others is taken up in their reflections about their own life. As such, my interview partners reveal an ambivalent relationship towards the continuous circulation between their places. They balance the positive against the negative aspects and then often conclude that the negative things are outweighed by the positive ones. Continuing with their mobile life, one could probably best follow Kate's quote, in which she explains how her friends characterise her:

'Round and round and round she goes, where she stops, nobody knows' (Kate; line 60).

\subsubsection{Virtual and imaginative travel}

Travel and mobility for my interview partners must not necessarily have a physical character. In addition to the actual physical travel my interview partners also travel in imaginative ways between their places. This form of imaginative travel is related to their individual mental maps. The places in their lifeworlds form coordinate systems in which my interview partners imagine their lifeworld. One could understand this as a mental geographic projection.

My interview partners use a type of mental geography which is a projection of their own territory, an awareness of where their own life actually takes place. The other people of their lifeworld to which my interview partners relate, are located somewhere in concrete geographic places. However, as they do not move across space with my interview partners, they do relate to them most of the time, and to many of these other people, by remembering them in mental ways. Here, the metaphor of having parts of oneself distributed to several places is an important issue. It symbolises the fact that my interview partners feel attached to people in different places, and in order to think of them, they travel in mental ways to these people in distant places.

This awareness helps to achieve emotional stability. Emotional stability is a category which is expressing the balance between mobility and belonging for my interview partners. Emotional stability depends on the relation to the surrounding people in any given place of physical presence, and it is also influenced by the degree to which the individual person is conscious about their personal long-term goals in life. Long-term projects, like property, in one place provide a mental stability through a clearer imagination of the future. Finally, also the self-confidence of a person has an effect on emotional stability. The last two points are extended by the personal maturity, which means the life experience and tranquillity that a person has in dealing with new situations and places. This maturity also positively affects emotional stability. Emotional stability then has an impact on the size of one's own territory, or action space. The stronger the emotional stability is, the larger the extension of the personal territory and spatial range becomes. Emotional stability is not stable and changes over time. As such, the action space of a person is also subject to change. This also means that mobility can better be handled in psychological terms: if a person is emotionally stable, in other words, if they can retreat from the mobility-induced stress through stable and satisfying social relations to other people, by being self-confident and knowing what they want in life, and through personal maturity and experience.

weisst du. Es kommt auch darauf an, wie, wie oft und so weiter. Äh... Fliegen, ich fliege eher nach England, wenn ich dort etwas zu tun habe beruflich. Oder ich war jetzt in den USA, natürlich klar diese Dinge, ja.' 
The personal environment, or lifeworld is not necessarily defined in geographic terms ('one's own places'), it also has other dimensions (e.g. social sphere, 'one’s own people’). However, one’s own lifeworld is always perceived of as embedded in geographic space. Given that this includes the distribution of the important people (family, friends, colleagues) to different places, travelling between these places is also considered an act of maintaining social ties. Travelling and mobility between places is a means of visiting the people that are important, and it helps to maintain emotional stability (I have mentioned that in section 7.2.1). However, in the everyday life of my multilocal interview partners, it is not always possible to be physically present in the places where their important persons live, or where they feel attached more strongly. Sometimes it is not even possible to immediately travel there in physical terms. Therefore, imaginative travel is a cognitive means to come closer to these places and people.

Tjark e.g. describes what his mental map looks like: we can find Copenhagen and Aarhus in Denmark, where he grew up and where he studied; there is also Tyrol in Austria, where his partner is from; then there is Rotterdam in the Netherlands and New York, where he had worked for some time; and finally there is Berlin in Germany. He feels most attached to Berlin and Copenhagen, and whereas he spends most of his time in Berlin, he keeps Copenhagen in mind:

'I also love reading the Danish newspapers and so on. So, in my mind there is always something... the weather forecast on my mobile phone, there also I see both the weather in Berlin and the weather in Copenhagen, for instance. [laughs] This means I am completely aware of what's going on there. [...] I also have in my mind that, I think, in a pinch... well, on the other side of Berlin's suburbs there is Copenhagen somehow. I orientate a bit towards the north, and then, you know, if you think about the people over there and so on, then you do not think anymore about the fact that it is 700 kilometres' (Tjark; lines 32-76 ${ }^{123}$ ).

These imaginative ways of travelling are also an issue in Kate's description of the sometimes paradoxical situation that she enjoys physically travelling between her main places of Berlin, Barcelona and Paris, but at the same time she imagines the other places upon arrival. She has a certain co-presence of other places in her head:

'If I am here, and I see that we are sitting here, then I think: but in Barcelona, there, they are currently doing this or that thing. So, I am always conscious about what is happening elsewhere at this moment' (Kate; lines 161-163 ${ }^{124}$ ).

Aurélien, who frequently travels between France and Germany and who still feels like a stranger in Leipzig, often thinks about his individual concept of belonging to places and of what is his own territory. He describes physical space as important for that, but he also thinks that physical space can have importance in an imaginative or mental way. Aurélien describes in a passage of our interview that having three current places in his lifeworld allows him to travel mentally between

\footnotetext{
${ }^{123}$ Translated by the author from the German text: 'Ich lese auch gern dänische Zeitungen und so weiter. Also in meinem Kopf ist immer was... das Wetter auf meine Telefon, sehe ich auch das Wetter in Berlin und das Wetter in Kopenhagen, zum Beispiel. [laughs] Also das heißt, ich bin so bewusst, mit was passiert dort. [...] Also ich habe auch so in meinem Kopf, glaube ich, dass also sobald zur Not... also auf der andere Seite die Vorstädte von Berlin kommt Kopenhagen irgendwie. Also ich orientiere mich ein bisschen so in den Norden, und dann, also weißt du, wenn man ab und zu so denkt über Leute da und so weiter, dann denkt man nicht darüber, dass es einfach 700 Kilometer ist.'

${ }^{124}$ Translated by the author from the German text: 'Wenn ich hier bin, und ich sehe, dass wir hier sitzen, und denke: aber in Barcelona, da sind die gerade dabei irgendwas zu machen. Also ich bin mir immer bewusst, was da irgendwo anders passiert.'
} 
them without actually moving in a physical sense. In his mind he has a map of his own territory, which is mainly composed of the triangle Leipzig, Brittany, and Nice, which provides him with emotional stability:

'But it's even more: there we enter actually uh... into a domain which I would call mental, of mental geography. This means that uh... this is a projection of my territory. [...] I am here, but I know that a part of me is there. You know. At the same time I can have a part of here, or we can mark also there and there, and perhaps here, too. And actually, this, this mental projection, if we call it like that, quote unquote, mental projection, geographic mental projection allows for stability. [...] This means that when I am in Leipzig, in my mind, I have the possibility to travel to Brittany or to Nice. If I feel like going there immedietaly.' (Aurélien; lines 81-101 ${ }^{125}$ )

This quote illustrates that imaginative travel, equally to physical travel, allows for 'small escapes'. If my interview partners feel emotionally dissatisfied in one place, they can imaginatively travel elsewhere without actually having to move physically. This is already helpful to stabilise in their multilocal lives in emotional terms. In a similar way, we can find this relevance of imaginative travel in narration by Emil. He speaks about how places are mentally present to him, even though he might not be there, or might currently not go there on a regular basis. This is caused by social ties to friends as well as his biographical background and places where he had lived before. The list of 'his places' where he travels to imaginatively is long: there is San Francisco, where he lived and worked for some time; there is Italy, where his partner is from; there is Lima, Peru, where he has a close friend, etc. So he emphasises that he applies techniques of imaginative travel:

'This plays a role then, also these mental places, where you are perhaps not permanently, but to which you pay more attention. So when I read something about Italy in the newspapers, of course I read that more attentively and I read about it more often. Similarly, I read about Peru, since I have this friend in Lima, which I have done less, for sure less often before. This becomes exhausting sometimes, when you know too many people [laughs], because then you have so many places' (Emil; lines 39-45 ${ }^{126}$ ).

Next to imaginative travel - which I would understand as a cognitive process of remembering and imagining distant people and places - there is also the possibility of virtual travel. Virtual travel is an important element in the lifeworlds of my interview partners, too. Whereas imaginative travel is first of all a process through which the multilocal person themself can travel alone to other places, virtual travel is an interactive form of non-physical travel. Virtual travel is dependent on the

\footnotetext{
${ }^{125}$ Translated by the author from the French text: 'Mais c'est plus: là, en fait, on rentre euh... dans un domaine que j'appellerais mentale, de géographie mentale. C'est-à-dire que euh... c'est un, une projection de mon territoire. [...] Je suis ici, mais je sais que j'ai une partie de moi là. Tu vois. Et en meme temps je peux avoir une partie de moi ici, ou à marquer là et là, et en fait peut-être ici aussi. Et en fait, cette, cette projection mentale, donc si on l'appelle, là est entre guillemets, projection mentale, projection géographique mentale nous permet de stabilité. [...] C'est-à-dire que, si je suis à Leipzig, dans ma tête, j'ai la possibilité d'aller en Bretagne ou à Nice. Si j’ai envie d'y aller toute de suite.'

${ }^{126}$ Translated by the author from the German text: 'Das spielt dann halt eine Rolle, auch solche mentalen Orte, wo man vielleicht nicht permanent ist, aber die man dann mehr beachtet. Also wenn ich irgendwas zu Italien in der Zeitung lese, lese ich das natürlich sehr viel intensiver und sehr viel öfter. Genauso wie ich halt jetzt, seit ich einen Freund in Lima habe, halt auch was über Peru lese, was ich vorher weniger, sicher weniger getan habe. Das wird dann schon anstrengend, wenn man zu viele Leute kennt [laughs], weil man dann so viele Orte hat.'
} 
technological opportunities provided by the information and communication technologies. In my interviews it became clear that telephoning, emailing, chatting, and video conferencing are very important ways of travelling virtually to other places and to distant people.

For example, Birgit considers her computer, her mobile phone and her analogue style agenda including pictures of her mother, her sister and her partner as essential things which she takes on her physical travel. The latter things are important to construct an everyday life atmosphere and remain connected in cognitive ways while travelling physically. She takes pictures of her loved ones to have them with her. These photos represent a form of tool for imaginative travel back home to Leipzig while Birgit is elsewhere. Her partner is more strongly based in Leipzig, he takes care of their apartment, he engages in keeping up with their neighbours, chatting around, and frequenting public places such as restaurants and cafés in the city. Birgit frequently has to leave this monolocal context, so her mobile phone is an important means of maintaining her relationship and the necessary everyday life communication through this type of virtual travel. Birgit describes:

'Well, you have to say good night and good morning to the person with whom you are living together, no matter where you are. And this is cultivated, uh independent of where one is. You do this in Shanghai, you do that in Kassel. This is part of a certain balance. And we have gotten used to this, I think, we live this as a part of our relationship' (Birgit; lines 192-196 ${ }^{127}$ ).

For Gunnar, this virtual and imaginative travel caused by the wish to maintain his relationship with his Spanish girlfriend is also an important issue in life. Gunnar describes that he is very much focused on his partnership and the place where his girlfriend is, which is most often not the same as where he is. His strong focus on his relationship made it a consequence that he engaged very much in virtual and imaginative travel. Even though he was physically present in one place, in a mental sense, he was mainly where she was. This meant that he perceived his life in Leipzig as surreal and did not participate in the local everyday life very much. Cognitively, he rather lived in his imagined place:

'After this year in Montana, I had the impression: I uh... for me there was always a type of real life, somehow, and then... and then the other life, you know. My three years that I spent in Leipzig after I came back from Montana, I did not really take them seriously. I didn't go out at night and I didn't meet so many people and so on. And uh, well my real life was basically the one I had with Ana Lucía. So, all the moments when I went to Spain, you know, and uhm... it was like that for about six years. And then things changed, with time this constellation became strange, in the summer of 2010, when my real life started to be here, and the life with Ana Lucía had become the other life. It had become a burden in a way, because I entered into a phase in which I thought that I am happy in Leipzig and uh... Ana Lucía is in my way, you know. This sounds horrible now, but it might have been related to this emotional and physical exhaustion [from living in several places and travelling between them], that I started to see it this way' (Gunnar; lines 130-144 ${ }^{128}$ ).

\footnotetext{
${ }^{127}$ Translated by the author from the German text: 'Also man muss ,Gute Nacht' sagen und ,Guten Morgen' sagen, mit der Person, mit der man zusammenlebt. Egal wo man ist. Und das kultiviert man, äh egal wo man ist. Das macht man in Shanghai, das macht man auch in Kassel. Also das gehört irgendwie zu so einem Gleichgewicht. Und da haben wir uns, glaube ich, auch dran gewöhnt, das auch als Teil von Beziehung zu leben.'

${ }^{128}$ Translated by the author from the German text: 'Den Eindruck, den ich hatte nach dem Montana-Jahr, war folgender: ich, äh... für mich gab`s immer irgendwie so `ne Art so `n echtes Leben irgendwie und dann... und dann so das andere, ne. Also meine drei
} 
Gunnar's narration illustrates that also imaginative and virtual forms of travel can have a negative and exhausting connotation. In Gunnar's case, the intensive imaginative and virtual travel to the places where his girlfriend lived, in combination with the frequent physical travel, caused a mental absence in his place of physical presence. He did not participate in everyday life in Leipzig anymore, and at a certain moment he realised the paradoxical situation that this was. Ugo also describes that virtual travel has a limited effectiveness. His case is a bit different from Gunnar's in the sense that Ugo's main problem is the geographic distance between his places. Whereas he currently lives in Weimar, Germany, his own apartment is in Rome, Italy. His home is in Chile, where his main places are Santiago and Concepcion, yet he cannot afford to travel between Europe and South America in a short-term rhythm, and he also underlines that the travel all around the globe is physically exhausting. So, for him maintaining ties to Chile mainly works through virtual travel. At the same time, Ugo explains that it is his character not to engage much in imaginative travel. He rather is very present mentally in the places where he is also physically present, so he does not follow the news and everyday life back in Chile very much. He says:

'Now, in my case, I am, I hardly look back, I am a bit like the Chileans with their cities, I rather look forward, and I adapt to the things that are happening around me, let's say, I am also quite patient, but creating relations to places is a complex issue, I stopped seeing my family [in Chile], you never return because it is difficult, there is internet, Skype. I regularly speak to my father in Skype, but never with other people. And it's like if one would move in two distinct times. So, when I come back to Chile I need a certain time to adapt because it's a very strange thing when you return. For example I left in 2007, and I went back in 2009, and it was mentally as if I came back in 2007. But they had already passed two years over there, so what actually happened in these two years I had not known. People had grown up, were speaking about different topics, they had other codes, you miss a large part. So in this case you never achieve being part of this new place and you lose your part in the old place, you remain there as if, so it's very strange. Because it's different, I know people in Italy who also live in various places, they move between, or they live in Paris and they live in Rome, Paris-Rome, but they can go there seven times a year, so they have a certain periodicity which allows to maintain relations. [...] I speak to my father at least once a week, once a month with my mother, and not much more basically, with the family very little. But if not, there is a level of relationship which gets lost with the time, my friends for example, where contact is very sporadic, and then it happens, that when I return, there are my topics, I mean it's normal, my life is here [in Europe], the people here, and the situations here, that over there no one knows. Then you

\footnotetext{
Jahre, die ich in Leipzig nach Montana verbracht habe, hab ich irgendwie nie ernst genommen. Ich war kaum aus und hab nicht so viel Leute getroffen und so und äh, so mein richtiges Leben war quasi das, was ich mit Ana Lucía hatte. Also immer, wenn ich denn irgendwie nach Spanien gefahren bin, ne, und ähm... so war das dann für sechs Jahre, ne. Und dann kam irgendwie der Wandel, in der Zeit, wo es denn irgendwie so komisch wurde auch, im Sommer 2010, dass mein echtes Leben auf einmal hier war und das Leben, was ich mit Ana Lucía hatte, war auf einmal irgendwie das andere geworden ist, also diese... das ist mir irgendwann zum Ballast geworden irgendwie, weil ich so, so 'ne Phase dann mitgemacht habe, wo ich hier dachte, ich bin hier glücklich in Leipzig und, äh... Ana Lucía ist mir irgendwie im Weg, ne. Das hört sich jetzt irgendwie furchtbar an. Aber das mag mit dieser Erschöpfung, irgendwie mit dieser emotional-körperlichen Erschöpfung zusammenhängen, dass ich das auf einmal dann angefangen habe, so zu sehen.'
} 
go and you do not have many topics to speak about because you will not bother them with people they do not know, or things that they do not understand' (Ugo; lines 9-28/116-122 ${ }^{129}$ ).

As a solution, Ugo underlines the role of personal visits, people coming from Chile to see him in Rome and understand his life in Europe. Here, his child was very important to 're-tie' him, as people in Chile are interested in their family life and the growing child. The interesting aspect in Ugo's description is that virtual travel alone is not sufficient to maintain relationships between places. Physical travel and imaginative travel are important, too. These three forms actually mutually reinforce each other in their function of binding places together. Ugo loses his ties to Chile because he is not able to travel physically, and he is not interested in travelling imaginatively.

Both these types of mobility: imaginative and virtual travel form elements of the multilocal lifeworlds of my interview partners. They make it possible that my interview partners feel more comfortable with physical mobility: virtual and imaginative travel allow for strengthening emotional stability and for maintaining social relationships to people at a distance. As an outcome, we can conclude that both forms of travel increase the awareness for other places. In the current place of physical presence they help to make co-present the current places of absence.

\subsubsection{Polyperspectivity as an outcome of travel and the possibility to compare}

Comparison is an important issue in multilocal lives. This comparison is based on the intellectual involvement in several places and the alternation between them, and the certain consequences for the attitudes of my interview partners towards foreign places and travelling. For example, Nikolina says that travelling does not automatically make people more open-minded. She tells me about her idea of being humbled when one travels abroad. This means that one is subordinating one's own perspective to that of the foreign place where one is. That helps both to orientate in any new environment and to connect in social terms. It is also a way to show respect towards local cultures and not being arrogant as a foreigner, imposing one's own culture and attitudes to local people in foreign places. Nikolina uses humbleness as a state of mind to find out and learn about different perspectives. She mentions that she likes travelling for the fact of having contact with new ideas:

\footnotetext{
${ }^{129}$ Translated by the author from the Spanish text: 'Ahora en mi caso yo soy, miro poco hacia atrás, soy un poco como los chilenos con sus ciudades, miro mucho hacia delante y me adapto a lo que viene pasando, digamos, soy también bastante paciente, pero crear relaciones con el lugar es complejo, la familia la dejas de ver, no vuelves nunca porque es difícil, está internet, Skype. Tengo regularidad con mi padre con Skype, pero con otras personas nunca y es como que uno se moviera en dos tiempos distintos, o sea cuando yo vuelvo a Chile necesito un periodo de adaptación porque es una cosa muy extraña, cuando uno vuelve, por ejemplo yo salí el 2007, volví el 2009, yo volví el 2007 mentalmente, pero ellos ya habían pasado 2 años ahí, o sea lo que pasó ahí en dos años yo nunca lo supe, entonces la gente es más grande, hablan de otros temas, tienen otro códigos, uno se pierde un pedazo, o sea en este caso uno no alcaza nunca a ser parte del nuevo lugar y pierde parte del antíguo lugar, te quedas ahí como, o sea una cosa bien extraña, porque es distinto, yo conozco gente en Italia que viven en ciudades distintas, se mueven o que viven en Paris y viven en Roma, Paris-Roma, pero vienen siete veces al año, o sea tienen esa periosidad que te permite tener relaciones. [...] Converso al menos una vez a la semana con mi padre, una vez al mes con mi madre, y no mucho más que eso en realidad, con la familia un poco. Pero sí no, hay un nivel de relaciones que se pierden en el tiempo, los amigos por ejemplo, es muy esporádico, luego sucede, que las veces que he vuelto, que hay temas que es normal, mi vida cotidiana está ahí [Europe], con gente de ahí, con situaciones de ahí, que acá [in Chile] nadie las conoce, luego tu vas y no tienes muchos temas de conversación porque no vas a empezar a hablarles de gente que nadie conoce, o sea dices cosas que nadie entiende.'
} 
'I don't know what's like pushing me to go to other places. I think I just like to learn new languages and to... uhm... yeah, just to like see new things and new ideas. Even though it's getting kind of old... I think sometimes that I probably get old' (Nikolina; lines 519-521).

Federico also speaks about the aspect that travelling and being transnational makes you more tolerant than immobile people, as you have the experience of being a stranger, and comparing systems, which leads to the insight that there are different systems, but they all work in their own way, none is better than the other:

'I don't think, for example, that scientists who actually travel a lot are necessarily better... uhm... more open-minded... no, that they are better than others [...]I am also not quite sure, but it's just an impression... the people who travel a lot, they are a bit more tolerant, because they know that they do not... they themselves... I have problems in Italy, large problems, because they... to me it is... well, to me the working conditions are often too vague. [...] On the other hand, I know that things work like that in Italy. And I don't know... with me they are a bit... I can... probably that's the only thing that one can say... well, each country has different rules. [...] I only started reflecting this once I was confronted with the two systems. That's why I also think 'live and let live'. Leave me with my pleasures, this is something I have had in me since I was a small child, and I will leave you with yours, but people who only know one system, they'd rather think: no, that's not how one does things, because that's how we like it here. But other people might not like it like that, but there might be other better ways of doing things. Nonsense! Nonesense' (Federico; lines 601-640 ${ }^{130}$ ).

In this quote we can see how my interview partners perceive comparison between places, which is made possible through travel and mobility. It leads to a perception of differences between places, and it relativises former convictions. Henriqua, who travels between Vigo, Spain and Wageningen (in the Netherlands), describes this in a reflection about the fact that the frequent travelling allows for such comparison:

'I think uh... you gather a different... a different perspective always, because you get influenced by what, what is happening, by the view the others have from you also, from your country, or from your place. And it's impossible to get... to not get influenced by that, I think' (Henriqua; lines 368390).

Yari lives in Mexico City, in New York and in San Francisco. He frequently travels between these places and other places in South America and the Caribbean as well. As a hip hop artist, he also passes through Europe and Africa when he goes on concert tours, so he has a wide range of experiences from all over the world. Yari thinks about travelling:

\footnotetext{
${ }^{130}$ Translated by the author from the German text: 'Ich glaube nicht, zum Beispiel, dass Wissenschaftler, die viel unterwegs waren, unbedingt besser ähm... offener... nee, unbedingt besser sind als andere [...] aber ich bin mir auch nicht ganz sicher, aber jetzt so als Eindruck... die Leute, die viel unterwegs waren, sind ein bisschen toleranter. Weil sie wissen, dass sie in keine, sie selber... ich habe Probleme in Italien, große Probleme, weil die... es ist mir... also die Arbeitsbedingungen sind mir immer ein bisschen zu unklar. [...] Andererseits weiß ich, dass die Sachen dort so funktionieren. Und keine Ahnung... so die, die sind mit mir ein bisschen... ich kann... das ist vielleicht das Einzige, dass man sagt, okay... jedes Land, andere Regeln. [...] das habe ich erst reflektiert, indem ich die zwei Systeme konfrontiert habe, ne. Und deswegen denke ich auch, leben und leben lassen. Lass, lass mir meinen Genuss. Das, das habe ich sowieso seitdem ich ein Kind bin, so. Und ich lasse dir deinen. Aber Leute, die nur ein System kennen, denken eher: nee, also macht man so nicht, weil es uns gefällt, aber anderen kann es auch nicht so gut gefallen, sondern weil es richtig ist, oder besser. Quatsch! Quatsch.'
} 
'It's that travelling allows you to see things from other perspectives, in each city, in each place, in each community, every individual has a different perspective. Exposing yourself to different reference frames gives you the opportunity to see things from different angles, and this provides the opportunity to be clearer, more critical and more objective' (Yari; lines 70-73 ${ }^{131}$ ).

Yari's main topic in his artistic production, mainly in his hip hop lyrics, is the social injustice which has evolved in the world of capitalism. He has an understanding of the local community as the key entity to fighting this globalisation induced injustice. To him, the ideas of local community and a critical analysis of the local context in the frame of wider global trends is a central part of his everyday work. In his places, he works with local communities and assists them in selfempowering, so travel is essential to generate knowledge which allows him to compare local situations in geographically distanced places. Similarly, Marta says that travel is allowing her to become more objective and see things more clearly and in more global and complex contexts. Marta lives in Slovenia, but she is currently working as a researcher in Lithuania. For her, the travelling is also part of her professional everyday life. She says that mobility makes a difference in terms of offering access to various perspectives:

'It makes a difference, and mainly because, I think I was pointing... before the same point. Mainly because I was doing my own job. And I was extending my... you know, my view. I'm seeing in the other country the same things going on, and I am comparing every day. And I see more, so I know more, so it makes sense, but only because I'm doing the, the job according to my profession. Otherwise, if I would wash the floors this would not happen. I would not be reading something, at least not for profession. So, it does. [...] It gives a wider view to the same thing. So, and you see the same thing from different angles, from more perspectives, so it can be more objective, I guess' (Marta; lines 234-242).

As Marta and Yari, many of my interview partners actually think of physical travel as a facilitator for this 'polyperspectivity' which helps to relativise local specificities. Valentin also acknowledges this. When he goes on his trips, he looks for uncertainty and is very aware of local contexts. He is open to new experiences and later elaborates on these experiences in order to become more relaxed about local issues back at home in Germany. Valentin says:

'Travelling clears your mind. [laughs] I have read that somewhere, but it's true. Honestly, I mean, there is no need to, I do not have to circumscribe it a lot, but it's really uhm [...] in any case, when I travel, I want to have a certain degree of adventure, some vagueness. And this is what it's all about, which finally relativises your life here [in Germany], your normal everyday life, not to become superficial or arrogant towards other people... so, [a type of prevention] against incredibly overemphasising certain problems, which actually aren't any. Insofar it is a very, very good way of not exaggerating, somehow exercising modesty... I think' (Valentin; lines 1077-1103 ${ }^{132}$ ).

\footnotetext{
${ }^{131}$ Translated by the author from the Spanish text: ,Es que viajar te permite ver las cosas desde otras realidades, en cada ciudad, en cada espacio, cada comunidad, cada individuo tiene realidades diferentes. Al exponerse a marcos de referencia diferentes te da la posibilidad de ver las cosas de diferentes ángulos y ésto te otorga una posibilidad de ser mas clara, de ser mas critico y más objetivo.'

132 Translated by the author from the German text: ,Reisen macht den Kopf frei, [laughs] das habe ich mal irgendwo gelesen. Das ist aber echt so. Also wirklich, also das braucht man gar nicht, das brauche ich jetzt gar nicht so groß zu umschreiben, aber es ist wirklich ähm [...] wenn ich reise, will ich auf jeden Fall irgendwie ein, einen gewissen Grad an, an, an schon irgendwie an Abenteuer haben, also so Unbestimmtheit. Und das ist halt das, was es ausmacht, dass dir sozusagen dann, dann dein Leben hier
} 
In this quote, Valentin touches upon the idea of 'humbleness' as expressed by Nikolina. Taking different perspectives in different places thus seems to help one become less egocentric and more open to other people's worldviews. I think we can use the terminology of 'polyperspectivity' to circumscribe this phenomenon of which my interview partners become aware when travelling. The frequent transition between different cities and their very specific social composites make it possible for my interview partners practice to flexibly enter and leave exactly these specific environments. For the creative knowledge workers I interviewed, this alternation between two or more socio-spatial contexts lets them collect different perspectives of how to see and evaluate certain issues in life. Polyperspectivity is part of the personality and the individual character of my interview partners. It helps them observe the wider societal change and to become more objective and knowledgeable.

My interview partners also mention that this possibility of comparing which comes along with travel can finally lead to an increase in self-confidence in terms of knowing one could get along in different and unknown contexts and territories. Dirk, who lives in Sofia, Bulgaria whereas his employment is in Leipzig, perceived his capacity to handle the everyday life both in Germany and Bulgaria as an enrichment of his personality:

'That's one thing which I had previously, and which was important to me... uhm... or a positive and very empowering experience, to come back from Bulgaria for instance, and to arrive here [in Germany] at the airport, and then uhm... I felt much stronger. Simply because I knew, I get along equally well in both these cultures. And uh in both languages, too. Uhm in a way this is basically a duplication of the personality. And you particularly realise that during the transitional phase, specifically upon return, if you still carry something completely different, and then you arrive here and this is also something of your own. So, this duplication in fact, of the power or the personality or something alike, this was an important experience' (Dirk; lines 180-189 ${ }^{133}$ ).

There is a mental shift during travel where the spatial focus shifts from the place just having left, to the place soon to be arrived at. Physical travel is experienced as the moment of transition between two places which are comparably different. In the next section I will have a closer look at this moment of transition and what it could mean within the lives of my interview partners.

\subsubsection{Transit spaces: Travel as place?}

For this section, we must imagine travelling as a transition from place A to place B on a specific route that passes through space, and let us call that a transit space. An important aspect of the

wieder relativiert, deinen normalen Alltag, also nicht irgendwie oberflächlich zu werden, arrogant gegenüber irgendwelchen Anderen... also gegen irgendwelche Probleme so unglaublich in den Vordergrund zu rücken, die eigentlich gar keine sind. Von daher ist es eine sehr, sehr gute Art sich so 'n bisschen flach zu halten, Bescheidenheit irgendwie zu üben, in Bescheidenheit zu üben... glaube ich.'

${ }^{133}$ Translated by the author from the German text: ,Das ist eine, eine Sache, die mir früher immer so ging, da war das für mich total wichtig... ähm... oder halt eine positive, sehr bestärkende Erfahrung zum Beispiel aus Bulgarien zurückzukommen, und hier wieder am Flughafen, und dann ähm... fühlte ich mich enorm viel stärker. Einfach weil ich wusste, ich komme in diesen beiden Kulturen gleich gut zurecht. Und äh in beiden Sprachräumen eigentlich auch. Ähm das ist dann sozusagen so eine Verdoppelung der Persönlichkeit im Grunde. Und das merkt man halt immer an den Übergangsphasen, also speziell beim Zurückkommen, wenn man was völlig Anderes noch mit sich trägt und dann aber hierher kommt und das aber auch das Eigene ist. Also diese Verdoppelung eigentlich, der Kräfte oder der Persönlichkeit oder so, das war schon irgendwo eine wichtige Erfahrung. ‘ 
multilocality of my interview partners is that they indeed have certain regularity in their travel routes. This means that they become used to handling their trips on these routes: routes become 'routines'. As an outcome of this routinised travelling, my interview partners describe certain moments of travel and time spent in transit spaces (such as train stations and airports) as having a character of 'familiar places' within the transit space (or corridor). While transit space is something that my interview partners travel through, a place is something they are in. If travelling is routine on ever same routes, then travel itself is not so much perceived as travel anymore, it is not perceived as the transgression of a space that lies between two places, rather travel itself becomes perceived as a place. In this place, which could also be a place in motion, a train or an aeroplane, everyday life takes 'place'. It is not anything particular anymore to travel on such routes; it is an ordinary activity in their everyday life.

Birgit travels on a weekly basis. She speaks of the train stations (Frankfurt, Eisenach, Erfurt, Berlin) in which she has to change trains as places that show up in her mental map (cf. section 7.1). Additionally, the trains themselves have obtained the character of a place to her. She uses them actively as part of her everyday life:

'And in-between [Kassel and Leipzig] there is this room. That is the ICE [German high-speed train], which I know by heart. Meanwhile, it is basically about this specific seat in the restaurant car, outward bound, where there is a table and you can order a coffee, and where you have two hours between Leipzig and Eisenach before having to change trains, and where you prepare the lectures, and where you can really smoothly work and where you are uninterrupted. It's like this... and then you can read all this pile of letters which have to do with this place [Kassel]. This is also part of the outward journey. It belongs also to that [to Kassel]. And on the return trip, which is mostly in the evening, Wednesday evenings, after these two days in Kassel, which are extremely exhausting, uh in this 'think space', then I buy a cup of cocoa and a women's magazine [laughs] and I sit, and I sit down and do, actually during this return passage I do the same what one would do at home, if you lie down on the sofa' (Birgit; lines 89-100 ${ }^{134}$ ).

Birgit's seat in the train becomes equated with a type of office desk on the weekly outward run, and it changes to become a sofa just like at home on the way back. It has a connotation of everyday life activities. To a certain degree, it loses its particularity as a space for travel. The train and the train stations become related to a homely feeling. We also find such an illustration in a quote by Gunnar, who frequently travelled between Valencia, Spain and Leipzig, Germany. During these trips he mostly changed aeroplanes on the Balearic Island of Majorca, so his description circulates around the airports as transit places:

\footnotetext{
${ }^{134}$ Translated by the author from the German text: 'Und dazwischen [zwischen Kassel und Leipzig] gibt es noch so einen Raum. Das ist dieser ICE, den man auswendig kennt. Eigentlich inzwischen so ein spezieller Platz im Speisewagen, wenn man hinfährt, wo ein Tisch ist, wo man sich noch einen Kaffee bestellt, und wo man zwei Stunden Zeit hat zwischen Leipzig und Eisenach, bevor man umsteigen muss, und wo man diese Vorlesungen bastelt. Und wo man wirklich gut arbeiten kann und auch wirklich ganz ungestört ist. Und das ist so... und dann auch nochmal diesen ganzen Stapel an Post durchliest, der was mit dem Ort zu tun hat [with Kassel]. Also das gehört auch noch dazu. Also zu dem da [Kassel]. Und auf der Rückfahrt, was ja dann auch immer irgendwann abends ist, Mittwoch abend, nach diesen zwei Tagen Kassel, die extrem anstrengend sind, äh in diesem Denkraum, dann kaufe ich mir einen Kakao und 'ne Frauenzeitung, [laughs] und setz mich, und setz mich da so hin und mache, mache auf dieser Rückfahrt eigentlich das, was man vielleicht zu Hause machen würde, irgendwie wenn man sich auf’s Sofa legt.'
} 
'Dealing with airports and so on, that does not surprise me anymore. Well, I also have... in this respect I have become an old hand through all this travel effort. Uhm... I know where, where I can find the free newspapers in the airports, in Leipzig for example... uh gate one. [...] I knew exactly, [...] where I could find a power outlet. This means I then always went to my place in the airport [in Palma de Mallorca], where there was the only freely accessible outlet, and I could sit down with my laptop and do anything, watching any series or anything else. So I developed my routines and $u h m . .$. it did not challenge me intellectually' (Gunnar; lines 668-711 ${ }^{135}$ ).

Here we can see that this idea of 'routes becoming routines' does not only apply to the vehicles in which my interview partners move, but also to the transit places in which they have to change between different vehicles, such as aeroplanes, train stations, etc. What is also interesting is the fact that this routinisation of the routes comes along with a de-emotionalisation towards travelling. Travel, with rising routine and everyday character, loses its exotic connotation and my interview partners have seen their excitement about travelling disappear. This also means that their own feelings towards travelling enter the self-concepts of my interview partners, as we can see when we follow Gunnar's narration:

'I get along quite well, I mean I uh... there is nothing which throws me off track. Sometimes I even become too casual. Then I have phases in which I do not take it sufficiently serious, the travelling. And then uh... then after all problems arise in the airports. But ones which do not make me nervous, which I can solve somehow. If I observe my parents, oh my gosh, if they travel somewhere, and they do not, already if they do not immediately find the parking area, my father gets giddy with excitement. But meanwhile I am completely left cold by these things' (Gunnar; lines 691-711 ${ }^{136}$ ).

In this quote Gunnar also stresses that he probably feels more at home in these transit places as his parents, who work as an example for less mobile people here. He doesn't get nervous anymore if unplanned changes occur. He is simply used to moving and operating in the environment of these transit places. This is also stated by Willi, who travels between Gent, Belgium and Aachen, Germany in a weekly rhythm. Willi calls the travel places 'second home'. When speaking about his perception of travel he explains:

'Uhm to me travelling has become nearly, the train, it's nearly like a second home [laughs]. By now, somehow it is so natural and I always use the travel time to work and as said before, uhm... I cannot say that I do not really enjoy it, generally speaking. It has something of... you... It has somehow, it represents something, it causes a separation, basically this travelling, between the places, and it has... it has therefore an important role. Even if I use it differently in functional

\footnotetext{
${ }^{135}$ Translated by the author from the German text: 'Auch so mit Flughäfen umzugehen und so dergleichen, das wundert mich nicht. Also, und ich habe auch so... bin schon so``n alter Hase mittlerweile geworden durch diesen ganzen Aufwand. Ähm... ich weiß, wo es, wo die Gratis-Zeitungen rumliegen im Flughafen, in Leipzig zum Beispiel... äh Gate 1. [...] Ich wusste genau, [...] wo `ne Steckdose ist. Das heißt ich bin dann immer so zu meinem Ort dann im Flughafen hingegangen, wo die einzige Steckdose war, die man so hätte benutzen können, und konnte mich da mit meinem Laptop dann hinsetzen und irgendwas machen, irgendeine Serie oder irgendwas gucken. Also ich hab schon so meine Routinen und das ähm... intellektuell fordert mich das nicht.'

${ }^{136}$ Translated by the author from the German text: 'Ich komme ganz gut zurecht, also ich äh... es gibt nichts, was mich da irgendwie aus der Bahn wirft. Manchmal werde ich zu, zu, zu arglos. Also dann hab ich so, so Phasen, wo ich das nicht mehr ernst genug nehme, das Reisen. Und dann halt äh... dann doch mal auf Probleme gerate auf dem Flughafen. Die mich dann aber nicht nervös machen, sondern die ich dann irgendwie löse. Wenn ich immer so meine Eltern sehe, Gottchen, wenn die irgendwo hinfahren oder nicht, wenn die schon irgendwie 'n Parkplatz nicht irgendwie sofort finden, da ist mein Vater schon ganz aus dem Häuschen. Aber mich lassen solche Sachen mittlerweile vollkommen kalt.'
} 
terms, it has a strong symbolic value, I don't know how to say that. Yeah, it has a high importance' (lines 641-657 ${ }^{137}$ ).

Therefore, also to Willi travelling has a transitional character between his places in which he actually feels at home to a certain degree. He can enjoy it and is important in his life. When we continued the discussion about the role of travelling, he also revealed that he had his routines in using the train. He describes his relevant knowledge to make himself comfortable during his weekly train rides:

'Yeah, the power outlet anyway. But then also, if you want to work calmly, then you perhaps keep away from the four-seaters and you look for a single seat. Then there are also different types of hooks for the jackets. There is this one seat where you have the window a bit in front of yourself, and where the jacket then always dangles in your way, [laughs] and then there is another one where the jacket hangs nicely aside, where the hook is perfectly positioned. And then, then this is, if it is far enough from the four-seaters, this is mostly the ideal seat. And I directly look for this one when I get on the train' (Willi; lines 677-685 ${ }^{138}$ ).

This last description is exemplary for the routines my interview partners develop in handling their daily travel (I will come back to this in section 7.7). The physical travel is conceived of as a transition between their places. This transition itself is not a-geographic: it happens through geographic space, actually through a transit space, and during travel there is a sequence of time spent in mobile as well as immobile transit places. The former are the vehicles that are used to get around. The trains and the aeroplanes, or any other modes to overcome geographic distance, have become a familiar aspect of their own lifeworld. They simply belong to it. The same accounts for the immobile transit places which are composed of these stations where my interview partners have to change between different vehicles. Therefore, we can understand that to a certain degree, travel itself is a 'place' in which my interview partners feel at home. They get used to these environments and they pursue everyday life activities within them which are sometimes not so different from those done in their other places.

\subsection{People: On the relevance of social ties}

A second dimension which is central in the lifeworlds of my interview partners consists in other people. The totality of their social ties is spread across their different places. Most often places do

\footnotetext{
${ }^{137}$ Translated by the author from the German text: 'Aber ähm an sich ist für mich das Reisen fast so, die Bahn ist so fast so `n zweites Zuhause [laughs] geworden. Also irgendwie, das ist so natürlich mittlerweile und ich nutze dann auch immer die Zeit dann da zum Arbeiten wie gesagt, ähm... ich kann nicht sagen, dass ich es wirklich nicht mag, im Allgemeinen. Das hat schon irgendwie... man... [thinking]... Es hat, das hat ja irgendwie, das hat ja irgendwie, das ist ja, das hat ja irgendwie, das schafft ja diese Trennung, das Reisen im Prinzip, zwischen diesen Orten, und es hat... es hat deswegen schon einen hohen Stellenwert. Und auch wenn ich es funktional vielleicht anders nutze, hat es schon irgendwie eine symbolische Bedeutung, ich weiß nicht, wie ich es anders sagen soll. Es hat schon eine hohe Bedeutung.'

${ }^{138}$ Translated by the author from the German text: 'Ja, Steckdose sowieso. Aber dann auch, wenn man in Ruhe arbeiten will, dass man sich eventuell von den Vierersitzgruppen dann doch ein bisschen entfernt und versucht einen Einzelplatz zu finden. Dann gibt`s aber auch wieder verschiedene Hänger für die Jacken. Es gibt da so einen Platz, wo das Fenster vorne ist, wo die Jacke einem dann immer irgendwie so im Weg rumbaumelt, und dann [laughs] gibt's einen anderen, wo die dann schön so an der Seite hängt, wo der Hänger dann richtig positioniert ist. Und dann, das ist dann, wenn der dann noch gut weit entfernt ist von diesen Vierergruppen, dann ist das meistens immer so der ideale Platz. Und den suche ich dann immer auch direkt, wenn ich dann in so einen Waggon gehe.'
} 
not become a part of their lifeworld just for themselves, but because there are certain people who have a relevance in the lives of my interview partners. During my interviews, these people were also part of my interviewees' narrations. Basically, my interview partners separate their social contacts according to the professional, the wider public and the private sphere of life. Furthermore, I observed that social networks are clearly distinguished by places. In combination with these two dimensions for separation, the places of my interview partners also receive different meanings in their lifeworld. Some places rather refer to private life networks whereas others draw relevance from professional networks.

\subsubsection{Family and household}

Parents have a very special function in the lifeworlds of my interview partners. They are related to the 'historic places' (cf. section 7.1) that are considered Heimat. First, parents mostly did not move after my interview partners had left their hometowns. Thus, regular trips to visit the parents make the home places of the parents a currently important place to my interview partners. On the other hand, these places are also important for being the places of their childhood, a time in which one did not autonomously decide about where to go and live. However, my interview partners have lived in the places where today only their parents live for quite a long time. Therefore, parents directly give meaning to one of the several places that form part of my interview partners' lives. Vice versa, places can lose their importance once parents leave them or pass away.

Another important type of persons are also the partners and children who define very strongly where my interview partners locate their 'current places' and where they feel their home is, or their central place, or their bases. Partners are also important in terms of how my interviewees reflect about their own multilocal lifeworld. If partners are equally multilocal or if they have experience with migration, they provide mainly more understanding for the multilocal situation of my interview partners. This in turn allows for a more positive evaluation of multilocality through my interviewees. Partners and children also become important when my interview partners reflect about their 'future places'. Deciding about a future perspective in life, in terms of geographic decisions, is nothing my interview partners do individually. It happens in dialogue with their partners and children. Less important but also relevant are the places where my interview partners met, and also from where their partners originate. The latter places have a more 'historic relevance' in their current lifeworld as well.

Willi is a good example of how partners and one's own family influence the perception of one's own multilocal lifeworld. He tells me about the conflicts that arise in family organisation caused by his weekly commuting from Gent to Aachen:

'Yes, this also causes situations of conflict. For example, I would say, this month, September: um we had one week of holiday in the meantime where we also went for a trip all together, the whole family. But then I had a situation when I actually could not come home on any of the Wednesdays of September because um, something was always scheduled. First there was something from the ILL, like for instance this week, the PhD students' day, then I had an alumni meeting in Frankfurt at the $D A A D$. All these things somehow. Then it was even on a Saturday, so that I couldn't come home. So, this month was actually a bit stressful, and you can feel that, you know. Because then she was really sick of doing everything alone, you know. But basically, if everything goes normal, then it 
works well. Because we have this rule that I am away for two and a half or three days' (Willi; lines 161-173 ${ }^{139}$ ).

The rule which Willi mentions is a rule between him and his girlfriend, and it says that after the three days of his absence he has to completely take care of the children and the household the rest of the week, so that his girlfriend can go to work. This rule seems to maintain their partnership and a family life that is in harmony. Birgit also mentioned that she has the rule to say good night and good morning to her partner every day, independent from where they both are. Juliana and her husband have the regulation to align their schedules so that always at least one parent is with the children in Berlin. Kate and her new, equally mobile partner have the agreement to not plan meetings in advance, but to wait for moments when their ways cross coincidentally somewhere in Europe. Basically, partners have a great influence on how the multilocality is lived and perceived. Within partnerships it does not work without negotiation and consensus about practices during absence and presence times.

\subsubsection{The wider social environment}

As I mentioned, there is a distinction between different social networks in different places. Therefore, my interview partners also mention that they become aware of various social roles which make up their personality and which are spread to different places. In other words, they inhabit different social roles in their different places. They can be different characters (cf. also section 7.6). Here, the social environment in the places also defines to a certain degree how close these social roles reflect one's own personality. If the surrounding environment is not open to all kinds of characters, my interview partners feel they have to play more artificial roles. Christian e.g. mentioned that he likes to be surrounded by people, and he had the impression that it was easy to find friends and get connected in Leipzig. He describes that he tries to also invite professional colleagues and artist friends outside of the artist social world (like galleries, events such as vernissages) in order to get to know them and spend time with them without their professional mask, in a relaxed atmosphere, where everyone can be themself, in the frame of non-professional situations like e.g. on a Sunday morning brunch. Christian mentions that the possibility to be oneself is a condition for feeling at ease with a place. Whereas he feels this in Leipzig, he perceived his time in Dresden quite differently. There people were rather narrow-minded and he had to adapt his personality to the local context. In this sense, Marta also mentions that actually the psychological state of being oneself to her equals a definition of home:

\footnotetext{
${ }^{139}$ Translated by the author from the German text: 'Ja, das erzeugt dann auch schon mal Konfliktsituationen. Also ich sage mal, zum Beispiel diesen Monat, also September: ähm wir hatten jetzt noch eine Woche Urlaub zwischendurch, wo wir dann also wirklich auch weg waren zusammen mit der ganzen Familie. Aber ich hatte dann `ne Situation, wo ich eigentlich den ganzen September nicht mittwochs nach Hause fahren konnte, weil ähm immer irgendwie was war. Also es war mal irgendwas vom ILL, also wie jetzt zum Beispiel diese Woche, also Doktoranden-Tag, dann hatte ich mal ein Alumni-Treffen in Frankfurt vom DAAD. Also solche Sachen irgendwie. Das war dann am Wochenende, also samstags dann auch, wo ich dann nicht nach Hause komme. Also dieser, dieser Monat war schon 'n bisschen stressig, und das merkt man dann auch schon, ne. Weil dann hat sie echt auch irgendwann die Nase voll, alles alleine zu machen, ne. Aber in der Regel, wenn das normal läuft, dann klappt das eigentlich ganz gut. Weil wir haben halt jetzt nun diese Regelung getroffen, dass ich dann halt diese zweieinhalb oder fast drei Tage weg bin.'
} 
'Home, we have a saying: My home is my fortress. That's the place where I used to be safe, where you uhm... [feel] understood. Where you can really be yourself. When you don't have to perform a certain role or whatsoever' (Marta; lines 199-206).

Open-mindedness of the surrounding others is thus important to develop any feelings of deeper attachment to places. If the local social climate is narrow-minded and exclusive, then the place itself can hardly deploy a binding function. Juliana expresses that she felt the narrow-mindedness and egocentrism of the Lithuanian professors and academia to be too boring, so she went to Copenhagen and later moved on to Germany, where she still lives. Therefore, the degree of open-mindedness of the wider local community in a place influences how well these creative knowledge workers feel, and how strongly the might relate to that place.

\section{Problems maintaining ties and managing social networks}

Another empirical observation concerns the interplay of mobility and social ties. While I have mentioned that mobility can be considered a means to maintain social relations (cf. section 7.2), my interview partners also mention that it is at the same time a factor that stresses these relations. Here, the duality of absence and presence is central: when my interview partners go to one place to meet up with someone, they have to leave someone else in the place from where they depart. This leads to the problem that people with more monolocal lifestyles often consider it difficult to maintain ongoing and stable relations to the multilocals. In this respect, Isabel reports difficulties with surrounding people who cannot understand her lifestyle and which makes it difficult for her to keep relationships with them:

'For me it is not at all traumatic, but rather for the people around me. They do not understand why I am here today and tomorrow I am away. I mean, I try to protect them and to explain it to them, that I simply want to be in Barcelona and in Berlin, and once in a while in Ibiza. And it is the personal relations are the most complicated issue in this context. In my mind everything is fine. I am such a moving person, it doesn't bother me' (Isabel; lines 34-39 ${ }^{140}$ ).

Kate also mentions the problem that other people tell her one cannot meet her, as she is like a 'moving target', always in motion and never there. People say about her:

'One can not meet up with you, because you are always in another place' (Kate; lines 62-63 ${ }^{141}$ ).

Both these quotes highlight that a multilocal life is often perceived by others as something extraordinary and different from how the majority of people live. As such, my interview partners often have to legitimate their behaviour and they feel that the duality of absence and presence stresses their social relations. Being absent is often regarded negatively by monolocal others, as shared time cannot be planned and is never a secure promise. In this sense, monolocal people might also withdraw to a certain extent from engaging in social relations with my multilocal interview

\footnotetext{
${ }^{140}$ Translated by the author from the German text: 'Das ist für mich gar nicht traumatisch, für die Leute um mich herum eher. Die verstehen nicht, warum ich heute da bin und morgen weg. Also, ich versuche sie zu verschonen und es ihnen verständlich zu machen, dass ich einfach in Barcelona sein will, und in Berlin, und ab und zu mal auf Ibiza. Und es ist also, es sind die persönlichen Beziehungen, die am schwierigsten sind bei der ganzen Sache. Also bei mir im Kopf ist alles klar. Ich bin so `n wandernder Mensch, mir macht's nichts aus.'

${ }^{141}$ Translated by the author from the German text: 'Man kann dich nicht treffen, weil du immer einfach an einem anderen Ort bist.'
} 
partners. This is illustrated in a narration by Emil, who continuously moves around between Bayreuth, Leipzig and Basel:

'The clear disadvantage is that I have the feeling that the other people react differently to me, and somehow they always think you are not there anyway. In Basel people think I am not there. In Leipzig people think I am not there. And in Bayreuth. Therefore, no one asks you if you want to join some activities; I have this impression. Perhaps they don't ask me because they don't like me, or they simply don't want to, but I think partly this has to do with the fact that they... if they have tried to reach me twice or three times, and in these moments I have always said: no, I am currently there or elsewhere. Um... and then they only ask friends from whom they know that they are in Leipzig, and that they are available. And sometimes I have the feeling I don't have any friends. And it is a large effort to keep up with them, and to make appointments. [...] Therefore I sometimes have the impression that people keep you less in mind, as they think you are not there. And then no one calls, never, saying: Yeah, what are we going to do at the weekend?' (Emil; lines 329-349 ${ }^{142}$ ).

Emil's quote shows how the perception of other people around him causes a type of disappointment with how the surrounding people react to the way he lives. Yet, this tension in respect to the maintenance of social ties can also be felt the opposite way around. Other interview partners tell me that they feel it as a type of social stress to keep up with all the people in all the places. Gunnar says that he has developed as a personal 'psychological survival strategy', which consists of paying attention mostly to the people in the places where he is at the moment, in the place of physical presence:

'Somehow it develop for me that I deploy a great empathy for the people in my direct environment, and I block out those who are at a distance. I keep contact with all of them. But like really um... paying attention, I only give this to my immediate surroundings, and if this changes, I also leave it behind quickly, meaning no harm. I still like the people and I am happy to see them again, but somehow... through the frequent relocations and living continuously in different places, I think this has become a sort of psychological survival strategy that I mainly take care of what happens immediately around me, and I leave out the rest. Otherwise I would always leave people in different places and I would have to write emails and postcards and letters, and I don't do that' (Gunnar; lines $\left.183-194^{143}\right)$.

\footnotetext{
${ }^{142}$ Translated by the author from the German text: 'Aber der ganz eindeutige Nachteil ist halt, dass ich irgendwie das Gefühl habe, dass dann die Leute anders auf dich reagieren, und halt immer irgendwie denken, du bist ja eh nicht da. Also in Basel denken die Leute, du bist ja nicht da. In Leipzig denken die Leute, du bist nicht da. Und in Bayreuth. Deshalb fragt dich auch nie jemand, ob du irgendwas mit machst, habe ich so das Gefühl. Vielleicht fragen sie mich nicht, auch weil sie mich nicht mögen, oder das nicht machen wollen. Aber ich glaube, das liegt zum Teil eben daran, dass sie... wenn sie zwei, dreimal versucht haben [mich zu erreichen], ich dann immer irgendwie gerade sage: nee, ich bin jetzt da oder dort. Ähm... und sie dann halt nur Freunde fragen, wo sie wissen, ja, die sind in Leipzig und die sind halt erreichbarer. Und dass man dann manchmal so das Gefühl hat, dass man eigentlich gar keine Freunde hat. Und dass man da wahnsinnig viel Aufwand machen muss, um, um mit denen irgendwie was zu vereinbaren. [...] Und da habe ich manchmal so den Eindruck, ja, dass die Leute einen weniger wahrnehmen, weil sie denken, man ist nicht da. Und dass dann eben auch nie jemand anruft und sagt: Ja, was machen wir denn am Wochenende?'

${ }^{143}$ Translated by the author from the German text: 'Und irgendwie hat sich das bei mir so entwickelt, dass ich irgendwie so große Empathie irgendwie entwickele für die Leute, die dort in meiner direkten Umgebung sind und die, die irgendwie weg sind, irgendwie so 'n bisschen ausblende. Ich bin zwar so in Kontakt mit allen. Aber so richtig, ähm... Aufmerksamkeit irgendwie gebe ich dann irgendwie immer meiner direkten Umgebung, und wenn die dann sich halt ändert, dann lass ich das auch ganz schnell wieder hinter mir, ohne das jetzt böse zu meinen. Ich mag die Leute natürlich immer noch und freu mich, wenn ich die wieder sehe, aber irgendwie... dadurch, dass man so oft umzieht und so oft irgendwie an anderen Orten wohnt, scheint mir das so eine Art
} 
Equally, Federico thinks that his own social network which is distributed all over the place already causes enough effort to keep contact with everybody. Thus there he describes a certain tiredness of making new friends:

'I already know enough people. And as they are everywhere in the world, it is difficult enough. Therefore I think, I do not have any energy left. Clearly, I like getting to know new people in Leipzig. New people and... um... doing something with them. But it's not that I think I do not have enough friends' (Federico; lines 738-741 ${ }^{144}$ ).

These examples show that my interview partners sometimes have difficulties managing the social relations in their networks. On the one hand, they perceive that their absence-presence duality causes problems with maintaining relationships, even if they would like to do so. Other monolocal people feel that the regular absence of my interview partners leads to unreliability in terms of organising shared time. On the other hand, my interview partners also find it stressful to keep contact in equal ways to all of their contacts in different places, therefore they develop specific routines of handling the totality of their social contacts (e.g. not adding too many new people, only focusing on the places of presence and avoiding too much imaginative and virtual travel).

Comparison between wider local communities in places

The frequent alternation between places allows my interview partners to compare the social environments, mentalities and people in their places. Comparison as such not only refers to the places themselves (cf. section 7.1), but also includes the people in these places. In this sense, Dirk observes e.g. that Bulgarian society is much rougher than the German. In Bulgaria social polarisation is stronger. Marta observes cultural differences between Slovenes and Lithuanians:

'We are, okay if we would compare how we are different, we are more balanced. We are more Nordic, this type of people. Slovenians are more emotional, more expressed. They are um, getting annoyed faster and showing their emotions and so on. So, that's actually what's the difficulty for me. For us it's not allowed this kind of behavior, so it took time for me to get used to that. But then it's not a problem at all. And for them it's also... I've heard comments like they were hearing that we are not emotional, that we are not showing... expressing emotions and that we are cold. That's not the case, of course. Then we are emotional, but we are not showing emotions the same way. So, it's different a bit' (Marta; lines 160-172).

Xaverio also compares peoples’ attitudes in Rome, Milan and Berlin. In particular in Germany he is sometimes astonished about the way how things actually work between people:

'German people sometimes are too much organized. So, like uh... I don't know, it's Monday, and you already know that Friday you go to, you go to the cinema. Ohh! [mimic of being bored by that]. And for me it's so impossible to plan. I mean, if you want to go to the cinema tonight, I just decide at seven o'clock: 'Okay, I want to go to see this'. And they are, you know, in this kind of planning

psychologische Überlebensstrategie geworden zu sein, dass ich mich immer ganz dolle um das kümmere, was direkt um mich rum passiert und den Rest so rauslasse. Also, ansonsten würd ich halt dann an verschiedensten Orten immer irgendwie Leute zurücklassen, denen ich irgendwie ständig E-Mails und Karten und Briefe schreiben müsste, und das mach ich nicht.'

${ }^{144}$ Translated by the author from the German text: 'Ich kenne schon genug Leute. Und da sie überall sind auf der Welt, ist es schon genug schwer. Also ich glaube, ich habe nicht viel Energie übrig. Also klar, ich kann gerne Leute hier in Leipzig kennenlernen. Neue Leute und... ähm... etwas mit ihnen unternehmen. Aber es ist nicht so, dass ich denke, ich habe nicht genügend Freunde.' 
world that I don't like it so much. Even because if they have already, you know, decided that they are going, I don't know, with a... two other friends, and you ask him: 'Okay, uhm Rike what are you going to do Friday?', 'Oh, I'm going to the cinema with uh...'. They don't tell you: 'Okay, do you want to come with us?' No, because for them it's already done. [...] You cannot, you cannot change the plan of a German person. I don't know what you feel about that? But it's really like: 'Okay, Friday, cinema, stop!' (Xaverio; lines 450-464).

And Federico explains how he perceives the mental differences between Germans and Italians in everyday life situations e.g. at work, where Germans always criticise to make things better, while Italians rather emphasise positive things to make atmospheres nice and pleasant. Here, he sometimes feels misunderstood or too much criticised, and would like more positive feedback from Germans. Then again, he admits that Italians are also 'figli di mammone', as he says, mummy's boys, who are continuously fishing for compliments, and sometimes he is embarrassed by his own reactions in specific situations in his German work life. He concludes that:

'Ultimately, these differences are small differences. It is basically the narcism which makes up this small difference, and if you overemphasize them, you make them bigger than they are' (Federico; lines $668-670^{145}$ ).

In these quotes we can see that comparison of different social contexts is an important issue in the life of the transnational creative knowledge workers. They actually become aware of the social particularities of each of their places, and in the end this feeds into their polyperspective state of mind (cf. section 7.2). They can actually evaluate their own character in respect to the character of the surrounding society in each place. Travelling between places allows them to compare people and mentalities. As a consequence, both the rejection and validation of stereotypes and clichés emerges. Mobility allows one to learn the specific local mentalities and they are then used to adjust one's own behaviour and then being able to quasi intuitively act in these places.

\section{Group belonging and 'othering'}

Through this comparison and developing polyperspectivity in respect to the social specificities of places, my interview partners also become aware of the opportunities that come along with being connected to these very different social environments. The comparison makes them aware of their 'otherness' or 'strangeness', but it also allows them to perceive the enriching effect of participating in different groups and different places; their own personality can widen its scope. Kate e.g. mentions that she receives a certain feedback to her lifestyle from her more monolocal brother and sister, which makes her compare herself to them and their lives. Her brother and sister still live in Philadelphia, where she grew up. Both her siblings have their own families, with children and a house, and a monolocal lifestyle. Thus Kate becomes very aware of her 'otherness':

'I think: Ha, that's great, it's interesting, it's nice. They have a nice life here, that's wonderful. But I have a more stimulating life. They might have a nicer life because it is simply so... very... the city is very aesthetic and beautiful. It's very green. And the houses are large and spacious, and the world is very pleasant there, where I grew up. But I think, well, I come home and I have a bit of

\footnotetext{
145 Translated by the author from the German text: 'Diese Unterschiede sind im Grunde kleine Unterschiede. Es ist wirklich der Narzissmus, der kleine Unterschied. Und wenn man sie zu stark thematisiert, macht man sie auch größer als sie sind.'
} 
that. My brother and my sister are fed up with that, and they come to see me, and they have... they can share a bit of my life' (Kate; lines $165-172^{146}$ ).

This awareness about the individual 'otherness' translates into personal preferences in terms of group belonging. My interview partners observe the different social contexts and they can choose where they would like to participate. Two things are to be observed here. First, my interview partners try to avoid becoming part of social groups that are similar to their other places once they arrive in a new place and rather go looking for new social environments and want to be part of more typical local groups. Ugo e.g. describes that he rejected sharing an apartment with other Latin American people in Rome. He observed that in particular people from Ecuador and Peru form ethnic networks and stick together; Ugo preferred to take part in Italian society, and he intended to share an apartment with Italians only:

'I started to live with, in an apartment with only Italians. And through them, in a certain way they introduced me, because I didn't have any network, unlike other groups of foreigners who already have their supporting network upon arrival and who then tend to group amongst themselves' (Ugo; lines $\left.199-202^{147}\right)$.

While Ugo was actually forced to a certain extent as there was no network of Chilean people available in Rome, he also decided to live with Italians to become integrated into the local Italian community. Today, he has a large circle of friends and every week they meet up to play soccer in the evening. Gunnar describes how - being abroad - he avoids being around other Germans, as he does not find that companionship abroad is desirable:

'If I am abroad, for instance, then I seldom tend to look for Germans; I don't like that. Often I feel embarrassed for them. Only if I am here [in Germany], I realise that I actually get along quite well with my compatriots, but if I am abroad, neither do I look for that, nor do I need to have German people around me. Also because they are strangers, and I don't have the feeling that I get along well with them, just because they are coincidentally from the same country as me' (Gunnar; lines $\left.334-341^{148}\right)$.

Gunnar also tries to connect with people from other nationalities or from the local context. Both of these quotes indicate my interview partners' awareness about their individuality as a multilocal person between worlds. They do actually not perceive of themselves as migrants who then group in

\footnotetext{
${ }^{146}$ Translated by the author from the German text: 'Ich denke: Ha, das ist toll, ist interessant, ist schön. Die haben ein schönes Leben hier, das ist wunderbar. Aber ich habe ein anregenderes Leben. Die haben dann vielleicht ein schöneres Leben, weil es so einfach... sehr... die Stadt ist sehr ästhetisch, und sehr schön. Und es ist sehr grün. Und die Häuser sind groß und großzügig. Und die Welt ist sehr angenehm da, wo ich aufgewachsen bin. Aber ich denke, ja: dafür komme ich dann nach Hause und habe ein bißchen davon. Mein Bruder und meine Schwester haben das satt und kommen mich besuchen, und haben... können ein bisschen von meinem Leben etwas abhaben.'

${ }^{147}$ Translated by the author from the Spanish text: 'Me fui a vivir con, en un departamento con puros italianos. Y a través de ellos, de alguna manera me introdujen, porque no tenía ninguna otra red, a diferencia de otros grupos de extranjeros que cuando llegan tienen una red de apoyo y después tienden a concentrarse.'

148 Translated by the author from the German text: 'Wenn ich im Ausland bin zum Beispiel, dann halte ich mich selten daran, irgendwie Deutsche irgendwie zu finden, das mache ich ungern. Also mir sind die, mir sind die oft unangenehm. Nur wenn ich halt hier bin irgendwie, merk ich irgendwie, dass ich wirklich ganz gut mit meinen Landsleuten auch zurechtkomme. Aber wenn ich so im Ausland bin, weder suche ich das, noch brauche ich das wirklich, dass ich da irgendwie Deutsche so um mich habe. Also, weil das auch wirklich komische Leute sind, und ich hab nicht das Gefühl, dass ich mit denen irgendwie gut zurechtkomme, nur weil die zufällig aus demselben Land kommen wie ich.'
} 
ethnic networks in the traditional sense; they rather want to go and discover a society on their own. This results in the second observation in respect to belonging. My interview partners appreciate being part of several groups at the same time. Avoiding their own ethnic network abroad complements the pattern that my interview partners more generally try to bypass being only part of one group with one dominant mentality, culture and value system. They prefer to switch between groups just as they appreciate switching between places. Christian e.g. describes that he is a person who needs to have different social groups in order to have a variety of inputs and inspiration:

'I am not that kind of person who only has one group. Uh... I do not feel good if I always stay with the same people. Uh, it is important to me, to have stable groups, but several ones, because I always feel like discovering new things. And I like hanging out with sportsmen, with engineers, biologists, artists etc. If I only stayed with artists, this would simply make the whole thing boring, because I often have the impression that it's, we remain with the same topic, or attitude. And there I don't feel so... well, I don't feel uncomfortable, but I always feel like getting to know something different, actually' (Christian; lines 321-328 ${ }^{149}$ ).

Federico says that he does not have one specific group of friends or acquaintances either. He is not engaged in regular group activities during leisure time - apart from a volleyball group - and thus he does not meet the same people very often. He considers himself part of different groups. Besides his volleyball group, there are some students from the local arts school, which he knows through his roommate. Then there are his colleagues, and in his private life his really close friends are rather individual people instead of groups:

'As I do not engage in this city, I am, I don't know, socially not active or... well okay, I play volleyball. That's one group of people which I meet on Friday when we play volleyball. But apart from that, I have... yes, with colleagues I can sometimes do something. But one specific group... well, I think, what you mean is a group in which you do not only meet regularly, but in which you also have very tight relations to the people. Both these dimension together. In my case they are... um... they are divided. The people to whom I have a tight relationship are not part of a group. [...] Even when I was still studying and I had a clique, from a certain moment I was tired of them, of this clique, because actually I do not like this regularity' (Federico; lines 709-733 ${ }^{150}$ ).

Federico's quote equals Christian's statement in the sense that they both express a certain mobility not only in spatial terms but in social terms as well. They like to switch between different groups

\footnotetext{
${ }^{149}$ Translated by the author from the German text: 'Und ich bin auch keine Person, der hat eine Gruppe. Äh... Ich fühle mich auch nicht so gut, wenn ich immer bei den gleichen Leuten bleibe. Äh es ist mir wichtig, so stabile Gruppen zu haben. Aber mehrere. Weil ich habe eigentlich immer Lust, etwas anderes zu entdecken. Und ich mag gern da mit Sportlern zu sein, Ingenieuren, Biologen, Künstlern, et cetera. Wenn ich nur mit Künstlern bleiben würde, das, das macht die Sachen einfach langweilig. Weil ich habe oft diesen Eindruck, das ist, wir bleiben bei dem gleichen Thema, oder Haltung. Und da fühle ich mich nicht so... also ich fühle mich nicht schlecht, aber ich habe Lust dann immer etwas anderes zu kennenzulernen, eigentlich.'

${ }^{150}$ Translated by the author from the German text: 'Da ich mich in der Stadt nicht engagiere, ich bin, ich weiß nicht, sozial nicht aktiv oder... ja okay, ich spiele Volleyball. Da ist eine Gruppe von Leuten, die ich am Freitag sehe, wenn ich Volleyball spiele. Aber ansonsten, ich habe... ja mit den Kollegen kann ich etwas manchmal unternehmen. Aber jetzt irgend eine enge Gruppe... also es gibt, was du meinst, glaube ich, ist so eine Gruppe, in der nicht nur man sich regelmäßig trifft zu einer Aktivität, sondern auch so eine enge Beziehung zu den Leuten hat. Diese zwei Dimensionen zusammen. Bei mir sind sie... ähm... sind sie getrennt. Die Leute, zu denen ich eine enge Beziehung habe, gehören zu keiner Gruppe. [...] Selbst als ich da noch studiert habe, und eine Clique hatte, war ich irgendwann müde, von dieser Clique. Weil ich so diese Regelmäßigkeit nicht besonders mag.'
} 
and they actually refuse to be part of one individual group only. My other interview partners also appreciate the opportunity which actually arises with the multilocal lifestyle. Furthermore, they also try to keep these different social environments separated; they do not aim at mixing them. As such, they become wanderers between their different social realities. Gunnar illustrates this in his interview. He has many different networks from different periods in his life and from different places. There is a network from his youth in Magdeburg, one from his early years at the university in Halle, one from his exchange year in Montana, and another from his time at the University in Leipzig. Only in Spain, he would not consider the people he is around to be 'his' people, for they are mostly friends of his Spanish girlfriend. Gunnar says that he always liked to invite all his people to big parties, but as he had different social groups, it was always difficult to moderate between these groups during his parties. As his networks have extended all over the world now, from Japanese over Turkish to Mexican people, he does not organise such large mixed parties anymore. He finds this to be too stressful, and he prefers to keep the lines of separation between his different social networks:

'One thing I stopped doing one day is, previously I had a lot of parties for birthdays, moving-ins, moving-outs and all that stuff, and I had different groups of friends who I never really wanted to bring together, but I did it. But that's something I have completely ceased doing in the last two, three years. This was somehow too much bustle; they were so many different people from many different contexts, so that I quit trying to bring them together. Since I do not organise birthday parties anymore, neither moving-out parties and moving-in parties, really nothing anymore. I meet up with people only in smaller groups. Within these small groups I actually bring people together, but these large events and so on, I really don't do this anymore. I don't really fancy doing that. That's become too much. For things like that, everything is too spread out' (Gunnar; lines 371$\left.383^{151}\right)$.

Still, Gunnar later mentions that most of his people are now located around him in Leipzig, where his network is the largest. He also stresses that nearly all these people are not originally from Leipzig, but from other cities or even countries. He only knows one person who is originally from Leipzig.

This section introduced the specific dimension of 'people', which is relevant in multilocal lifeworlds. I argued that my interview partners mainly distinguish social contexts between places. On the one hand there is a close social environment which is composed of parents and one's own family, and then there is a wider network of social ties, which includes friends, colleagues, other acquaintances and finally the local/national society as a whole. The family becomes particularly

\footnotetext{
${ }^{151}$ Translated by the author from the German text: 'Ne Sache, die ich aufgehört habe zu machen irgendwann ist, ich hab früher mal ganz viel so Geburtstagsfeiern und Einzugsfeiern und Auszugsfeiern und dergleichen gemacht, und da hatte ich schon so verschiedene Gruppen von Freunden, die ich nie so richtig zusammenbringen wollte, habe das aber trotzdem gemacht. Aber das ist irgendwas, was ich in den letzten zwei, drei Jahren komplett aufgegeben habe. Das wurde mir dann zu viel Wusel irgendwie, das waren so viel verschiedene Leute aus so vielen verschiedenen Kontexten, dass ich insgesamt aufgehört habe, die zu probieren zusammenzubringen. Seitdem mach ich keine Geburtstagsfeiern mehr und keine Auszugs- und Einzugsfeiern und nichts mehr, echt nicht mehr. Ich, äh treffe die Leute nur in kleineren Gruppen. Ich bring die auch in kleineren Gruppen... bringe ich auch wieder verschiedene Leute zusammen. Aber so diese großen Events und sowas mache ich echt nicht mehr, habe ich auch keine Lust mehr drauf, also ist mir zuviel geworden. Dafür ist das jetzt alles viel zu verstreut.'
} 
relevant in defining the 'own' places of my interview partners. Here, the parents mainly are referred to the 'historic places' and origin, and the individual's own family (partner and children) characterise the 'current places' and where they feel at home. The wider social community and different social contexts rather enter the perception of the lifeworld as related to the aspect of constant comparison and polyperspectivity. My interview partners compare the different local social contexts to each other and they position themselves, with their personality, in reference to these social contexts. The dominant pattern here is that they try to become part of different social groups in different places. Furthermore, maintaining these different groups by separating and ordering them according to places, they appreciate the opportunities in terms of various social stimuli and inspirations. Switching between their groups and comparing their social environments, they also become aware of their individuality and otherness as well as the problems in maintaining the social ties which come along with a multilocal lifestyle.

\subsection{Biography: The role of personal history}

The personal history of my interview partners, their own biography, points to several issues that are related to their current multilocal lifeworld. The way in which my interview partners reflect on it reveals certain aspects that can influence the current attitudes towards mobility and multilocality.

A first aspect is obviously the location where a person was born. In Henriqua's case, we have learnt that she was born to Spanish immigrants who worked in Germany, but they moved back to Spain when Henriqua was four years old. So, even weak today, she feels attached to Germany to a certain degree, and she developed an interest for the German language and culture, which also included that she start to travel around the country:

'Well, now, it's not anymore so important now. But, well, I was born there, I lived there four years, my parents lived there ten years. So they got you a bit to know a bit how Germany was, or they have their perspective of course. They were immigrants. No, I think it's important also in this respect. But let's say, nowadays, I don't think so much I am German, for example. Because I am not! Well, I was born there. I didn't grow up there' (Henriqua; lines 84-88).

The last sentence of her quote points to the second aspect which is relevant: namely where or with which places a person has grown up. Aurélien was born in Nice and moved to Brittany with his parents when he was a small child. As his parents had a home in Nice and also other family members lived there; he has frequently travelled between the places since his early childhood. Today, he argues that this led to an incorporation of a certain nomadism in his self-understanding. Christian reflects that he was born in Paris, but his parents moved around during his childhood for professional reasons, so he does not know where he should consider being from in the sense of his roots:

'In any case this is part of my personality, I think. It's harder, yeah, in a certain period when you have grown up, and after adolescence, there is a period in which you frequently go back to where you are from, finally because it is a resource. And then, I remember, when I was between eighteen and twenty years old, I had a bit that feeling that I didn't have a place where I was from, where I could return to feel at home, and this was a bit difficult. Afterwards I integrated that into my 
dynamic and finally each place where I have gone is a small piece of me' (Christian; lines 104$\left.110^{152}\right)$.

The time of adolescence was also an important moment when Henriqua was further influenced towards becoming a more curious and open-minded person who has a deeper interest in foreign culture. Henriqua explains that she started travelling to Zurich early on, where an aunt of hers lived. In Spain a cousin, who was the son of that aunt in Zurich, lived with her and her parents, so each summer she accompanied her 'brother' cousin to Switzerland for some weeks. Since then she has made travelling an important part of her life in more general terms:

'When I was 13 I also started to travel to Zurich to visit my aunt with my 'brother', you know. So, so he could visit his mother. But he didn't like it [laughs]. The one who liked it was me, so I always went with him. No, probably because I like it, and I like to see other cultures. And I also like to learn other languages, and... and I don't know if it's the same my personality or it is... I don't know how you construct that, if it is something that you are born with, or it's something that comes from experience, or... or both. I don't know. I am not a psychologist' (Henriqua; lines 496-505).

This example indicates that the wider family context could also affect the childhood experience of mobility and when defining my interview partners' affinity for a multilocal life. Birgit for example tells me the story of her mother. When her grandfather died in his family house in Liberec during WWII, her mother had to escape by the end of the war, and only took the belongings that fit into one suitcase. This is what she taught her daughters, Birgit and her sisters, later. Birgit today similarly has a certain affinity to mobility and a healthy distance to material belongings:

'And to me it is also, I have also thought about that intensively, my mother was a resettler after the war, a Sudeten-German. She quasi had to leave her home within overnight. With all the bells and whistles. My grandfather was an architect. And there was this house in Liberec. And then there was this cut, he died some years before the end of the war. And my grandmother and my mother had to leave house and farm overnight and they have arrived to Weimar with only a few suitcases. And my mother, in a subtle way, taught us a certain distance to place. The suitcases, or what was in there, was important. But where this actually was, uh that was not so relevant. So, she did not always completely identify with it [with place]. And that's why I didn't have difficulties living in Weimar then, and then in 1980s I had a job in Berlin, and still I maintained the room in my shared apartment. And when I moved from Berlin to Leipzig, I also started only with one suitcase. And now, practically, it's the same again. So, uh, this pattern exists. And my mother, when she had to move here to Leipzig from her place of residence in Thuringia because she couldn't live alone anymore, she also said, anyway one cannot take much on move. What you really possess is what you have in your head... and I think that's true' (Birgit; lines 143-159 ${ }^{153}$ ).

\footnotetext{
${ }^{152}$ Translated by the author from the French text: 'En tous cas ça fait partie de ma personnalité, je pense. C'est plus dur, si, qu'il y a une période où on grandit, et après l'adolescence il y a quand même une période où souvent on revient euh là d'où on vient, en fin parce que c'est un ressource. Et puis, je me souviens, quand entre dix-huit et vingt ans, j'ai eu un peu ce sentiment que j'ai pas d'endroit d'où je venais, où je pourrais retourner me sentir chez moi. Et c'était un peu difficile. Après j'ai intégré ca dans ma dynamique. Et finalement chaque endroit où je suis allé est un petit morceau de chez moi.'

${ }^{153}$ Translated by the author from the German text: 'Und bei mir ist das noch so, da drüber habe ich auch wirklich mal intensiv nachgedacht, meine Mutter ist Umsiedlerin gewesen nach dem Krieg, Sudetendeutsche. Die hat quasi ihre Heimat verlassen müssen. Mit allem Drum und Dran. Also mein Großvater war Architekt. Es gab da ein Haus in Liberec. Und es gab so einen Cut, der ist ein paar Jahre vor dem Ende des Krieges gestorben. Und meine Großmutter und meine Mutter mussten quasi über Nacht Haus und Hof
} 
Therefore, Birgit underlines that not only her personal history but also the wider family history influenced her attitudes towards multilocal living and place attachment. We can find a similar narration in Federico's interview. He describes that Trieste has become part of his life through his family background, and also that Trieste is a very particular place as it represents the encounter of eastern and western European culture. In this city, Italians mix with Slovenes and Croats and a particular transcultural micro-cosmos arises:

'And Trieste is... um Trieste is... also a part of my family comes from that region. My grandmother was born there [points to his map], on the peninsula of Istria. By that time it was still AustroHungarian, when she was born. Then it became Italy, then, then Yugoslavia. And she is, she moved to Italy. She did not want to stay in Yugoslavia. And I still have a part of my family close to Trieste. In Trieste we also have a family tomb. When I was a small child, I heard many narrations and many stories about Trieste. And about the Italians, and the Slovenes, and the Croats. And I think this is really relevant. Today I love the literature from Trieste. If I compare to Prague, I think, there, Trieste, is more of an autobiographic connection, a relationship, quite important' (Federico; lines $\left.77-86^{154}\right)$.

In Federico's narration we find again the relevance of the family background and history, and the interesting thing is that we find the same pattern also if we change the temporal perspective towards the future and the way in which my interview partners actually influence and raise their own children. Willi e.g. told me that his girlfriend has an Argentinean mother and a Belgian father, and she appreciated that her parents were a bi-national, multilingual couple who taught her that belonging to several places and countries is a value added in terms of personality. Having said this, Willi and his girlfriend also pay attention to bring up their own children with the same knowledge. He describes:

'Well me German, I speak only German to them. Anna only speaks French to them. In school as well as in the kindergarten they learn Dutch, because it's actually a Flemish city. And Celine, the older one is perfectly trilingual, and English passively, because we lived in England for a while,

verlassen und sind dann in Weimar gelandet mit ein paar Koffern. Und meine Mutter hat uns glaube ich auf eine subtile Weise so eine gewisse Distanz zu dem Ort vermittelt. Also die Koffer oder was da drin war, war entscheidend. Aber wo das eigentlich war, äh das war nicht ganz so wichtig. Also damit hat sie sich nicht immer so richtig identifiziert. Und mir ist das deswegen auch nicht so schwer gefallen, dann da in Weimar zu leben, dann habe ich da ja in Berlin in den 80er Jahren einen Job gehabt, und hatte immer gleichzeitig noch mein WG-Zimmer. Und als ich dann von Berlin nach Leipzig gegangen bin, hatte ich da auch erstmal nur so einen Koffer. Und jetzt ist das praktisch wieder so. Also dieses Muster äh gibt es schon. Und die Erfahrung ist eigentlich auch... Also das hat meine Mutter übrigens, als sie dann aus dem Wohnort in Thüringen hier nach Leipzig gezogen ist, weil das gar nicht mehr anders ging, sie meint, man kann sowieso nicht viel mitnehmen. Was man wirklich hat, hat man im Kopf. Also... und das ist auch, glaube ich, so.'

${ }^{154}$ Translated by the author from the German text: 'Und Triest ist... Ähm Triest ist... also ein Teil meiner Familie kommt aus der Umgebung. Meine Großmutter ist hier geboren [points to his map], auf dieser Halbinsel Istrien. Damals war es noch ÖsterreichUngarn, als sie geboren ist. Dann ist es Italien geworden, dann, dann Jugoslawien. Und sie ist, sie ist umgezogen nach Italien. Sie wollte in Jugoslawien nicht bleiben. Und ich habe noch einen Teil der Familie in der Nähe von Triest. In Triest haben wir ein Familiengrab. Als Kind habe ich viele Erzählungen, viele Geschichten über Triest gehört. Und die Italiener, und die Slowenen, und die Kroaten. Und ich glaube das macht ganz viel aus. Ich mag auch die Triester Literatur. Aber im Vergleich zu Prag ist, glaube ich, dort in Triest eher diese autobiographische Verbindung, Beziehung, so wichtig.' 
and she had been in the English kindergarten. And Anna and me, we speak a lot of English to each other, I must say. We met in London' (Willi; lines 249-255 ${ }^{155}$ ).

Willi's family is actually the prototype of a transnational family. They use four languages in everyday life, and they incorporate five countries in their own family story. So the point I would like to stress here is that for many of my interview partners, the affinity to their current multilocal life is deeply rooted in their personal history, and in a biographic moment which lies far before they started to decide on their own if they wanted this kind of life. They have often grown into it. As such there is also a strong relation between this biographic dimension and the 'historic places' (cf. section 7.1). As I have mentioned, the 'historic places' were also not chosen independently by my interview partners, but rather by their parents and their family context during their own childhood. If we look at the examples, we can see that the own migrational experience of parents, the binational composition of parents and a related double citizenship of the children can all influence the mobility pattern in the later adult life. Furthermore, growing up in a multilingual household is also an influential aspect. Not uncommonly, my interview partners then engage themselves in binational partnerships, move around with their own families, and raise their children drawing on various cultures and languages; there is a cumulative effect of the biographic dimension: once an initial experience with mobility has happened, it is more probable that another one will follow.

\subsection{Profession: The role of multilocality in the creative industries}

In this section I wish to have a look at the relationship between creative industries and transnational multilocality in the lifeworlds of my interview partners. Basically, I found four issues that showed up across the interviews. First, my interview partners often have different jobs or projects in different places. Second, they use travel between these places in order to transfer knowledge and skills between these places. Third, they are aware of the advantages that their multilocality offers in the labour market. Fourth, they perceive multilocality and mobility partly as a constraint caused by the market logics of creative industries.

\subsubsection{Multiple job-holding}

As I have discovered, many of my interview partners use their multilocal lifeworld as an opportunity to do several things in parallel which they would not be able to do in only one place. Multilocality itself might even be an outcome of this wish to do an additional job or project in another city and country. My interview partners' life stories provide rich examples of this. Relatively independent of the formal type of employment - employment vs selfemployment/freelancing - my interview partners combine the professional opportunities of several places.

\footnotetext{
155 Translated by the author from the German text: 'Also ich Deutsch, ich spreche nur Deutsch mit denen. Anna spricht nur Französisch mit denen. Und in der Schule beziehungsweise in der Kinderbetreuung haben sie Niederländisch, weil es eine flämische Stadt ist. Und Celine, die Ältere ist perfekt dreisprachig, und Englisch passiv, weil wir halt in England eine Zeit lang gewohnt haben, und sie war da im englischen Kindergarten. Und Anna und ich sprechen auch viel Englisch miteinander, muss man sagen. Wir haben uns in London kennengelernt.'
} 
Aurélien works as a freelance artist in different projects in Brittany, Nice, Paris and Leipzig. Additionally, he manages a cultural association in Leipzig. Birgit works both as manager and owner of her planning office in Leipzig and as a professor in Kassel. Christian is a freelance artist who engages in a broad variety of non-artistic jobs to finance his artistic endeavours and to draw inspiration from these completely different professional fields. When Christian spoke about his professional life, he explained:

'Basically each new job which I have done will always influence my art somehow. First in my poetry, always. And... because it opens new situations, new contexts, new thoughts. And then I write about it. And mostly there is also... For example, I worked a lot in construction zones in the last years. Not so much anymore in the last two years, but before really a lot. And a large part of my artwork was also uh... actually about it. I mean, not about what a construction zone is and so on. But I used these structures, mentalities, organisation and materials, which are part of construction zones, in order to do my art. And then uh something... I have mixed it. I also like to do things like that uh... I like trying to build such bridges between different worlds, I would say. And then the interesting thing is that people in the construction zones develop an interest for contemporary art. Because the [construction worker] will recognise the materials. And at the same time, people from contemporary arts will like or have an interest in the forms uh and thereby they uh will ask themselves in the direction of the construction zone - like what is going on here? What is that?... And so on. While the topics are relatively independent, one can experiment with new materials from the construction zones. [...] And then I always try to open doors for myself, to look into new rooms, and to discover why things are working like they do. And I do not want to sa, that this is good. But perhaps sometimes one should think of things in a different way and so on. Actually I do not want so say that. It is also... I, I do not tell this to myself. But let's say I try to point to new questions' (Christian; lines 400-426 ${ }^{156}$ ).

In Christian's quote we can see clearly how he makes use of different job environments in order to improve his arts. At the same time he is interested in making construction workers passionate about contemporary art. He takes the position of a joint element between his different professional environments. Dirk, on the one hand works in his research position which links him to Leipzig, where his employer is located, and to Bulgaria, where he does his fieldwork. On the other hand, he pursues his freelance jobs as a consultant in adult education on the Balkans and in Belarus. In order to finance his $\mathrm{PhD}$ project, Gunnar worked both as a tutor at the university and as a stem cell

\footnotetext{
${ }^{156}$ Translated by the author from the German text: 'Also jeder neue Werk [Arbeit], ich habe getan, wird immer in meiner Kunst irgendwie äh ankommen, also. Als erstes beim Schreiben, immer. Und... weil das macht neue Situationen, neue Kontexte, neue Gedanken. Und dann schreibe ich darüber. Und am meisten gibt es auch... Also ich habe zum Beispiel viel auf Baustellen gearbeitet die letzten Jahren. Also nicht mehr so viel seit zwei Jahren, aber vorher ziemlich viel. Und ein Großteil meiner Kunstwerke waren auch äh... darüber eigentlich. Also ich meine nicht darüber, was ist Baustelle und so. Aber da habe ich dann diese Baustellenstruktur und -mentalität, -organisation, und -material benutzt, Kunst zu machen. Und dann äh so wie... eine Mischung getan. Ich mag auch gern etwas so wie das äh... also ich mag auch gern probieren so Brücken zu bauen zwischen verschiedenen Welten, würde ich sagen. Und dann war interessant, dass man in der Baustelle wird ein Interesse für zeitgenössische Kunst haben. Weil der [Bauarbeiter] wird einfach das Material erkennen. Und gleichzeitig die Leute aus der zeitgenössischen Kunst werden die Formen äh dann gut finden oder Interesse dafür haben und dann dadurch äh in die Richtung Baustelle sich fragen - über, was passiert? was ist das dann?... und so. Obwohl die Themen können ziemlich selbstständig sein, man kann auch Material aus dem Baustelle probieren. [...] Und dann versuche ich immer, für mich selber Türen zu öffnen, in neuen Räumen zu gucken, und festzustellen wieso das läuft so. Ich will auch nicht sagen, das ist gut. Aber vielleicht man muss anders denken und so. Ich will das nicht sagen. Das ist auch... ich, ich, ich sag mich das nicht selber. Aber ich versuche einfach die, die Fragen zu öffnen.'
} 
courier for university hospital for some time. Furthermore, he worked as a German and English language trainer while he was living in Madrid, and before he worked in the cafeteria of the university in Montana. Henriqua is employed at a university in the Netherlands, but she also takes small freelance jobs in the field regional planning and as a German language trainer in Spain, which allow her to remain connected in the local Gallician networks. Kate and Isabel's freelance activities are per se structured according to individual projects which are implemented in different places across Europe. Additionally, Isabel writes novels in her leisure time, which she also publishes with small publishing companies. Nikolina studies in Weimar, but she works in a research project in Leipzig which actually deals with issues in Bulgaria. Oskar is the manager of a small firm for cultural mediation in Zurich, but he then also holds freelance positions in this field in Austria and several places in Germany. Pia holds her teaching position in a Berlin-based university, while at the same time being the owner and manager of an architecture firm in Copenhagen. Qamar runs his own artist atelier in Leipzig, where he produces commissioned artwork of any kind, ranging from silhouettes to painting. He also engages in media productions, partaking in TV shows, or acting in music video productions of Russian-American musicians. In Chechnya he is a celebrity and has to do a lot of representative events with e.g. high-ranking politicians. Ronja works as a freelance journalist. While mainly based in Berlin, she actually gets more jobs in Copenhagen, yet she is trying to become more integrated in Berlin projects as well. Stephan holds a teaching position at the university in Brunswick, but at the same time he works as a freelance architect for offices in Hamburg and Copenhagen. Tjark teaches at a university in Berlin, but at the same time he has opened his own interior design firm and produces furniture made of concrete and engages in sustainable product development and local value chains in Berlin. Once in a while he also assists in architecture projects in Copenhagen. Valentin does all kinds of jobs in order to finance his large passion for travelling and climbing. He works for film productions, he offers services in the field of graphic design and photography, but he also helps to manage the start-up company of a friend in the field of pedagogy and juggling. Willi is working on his own $\mathrm{PhD}$ project at the university in Weimar while at the same time holding a position as a researcher in regional development in Aachen. Xaverio works as a photographer in the field of fashion design in Milan, and he then follows his personal artistic undertakings in Rome, where he exhibits and cooperates with galleries. At the same time he works as a teacher of photography at a Rome-based school of design. Finally, Yari works as a freelance hip hop artist, mainly across North America, Middle America, and South America. His various projects dealing with self-empowerment in local communities bring him to all kind of places, but his main bases are San Francisco, Mexico City and New York, where he works with children and adolescents in deprived neighbourhoods.

You can see in this description of the individual cases that the creative knowledge workers who I have interviewed include of broad variety of activities in their everyday life. Most often their different projects are located in one of their different places, and frequently they deal with different topics. Nonetheless, my interview partners integrate each of these activities in all of these places into their own lifeworld. They autonomously construct their own professional identities and they seek the links between their individual jobs and projects which make sense as an overarching story. The variety of different jobs and projects is appreciated by my interview partners for the fact that it allows them to get in touch with ever new contexts and be continuously provided with challenges. Furthermore, this type of multiple job-holding broadens their perspective and intellectual range and 
it provides inspiration for further activities. As such it becomes part of the overall pattern in the lifeworld of these creative knowledge workers. They basically combine and compare things that others would say do not fit. They combine different forms of work organisation (self-employment with employment); they mix different professional fields; they also add short-term projects to longterm activities. Basically they experiment with combinations by stitching together different contrasting but complementary elements in a very unique and individual professional career. Again, we can see the two motifs of comparison and complementarity emerging just as in the dimension of 'places' (cf. section 7.2).

\subsubsection{Multilocality and knowledge transfer}

Multilocality can also become relevant in creative industries when it comes to transferring professional knowledge, skills and experiences between places and countries. The creative knowledge workers have a bridging function between different places and these places' knowledge stocks. Thereby, linking their own places in the professional sphere also gives a deeper sense to their own mobility.

Birgit speaks of her knowledge transfer in terms of the things which she perceived as working different in New York and Zurich as compared to Leipzig, e.g. the techniques to deal with cultural diversity in an urban community: that is what she always brings to Leipzig and Kassel upon return from these other places. Furthermore, she considers the global stage as something important where she could update her individual professional priorities according to current topics and knowledge from the global level, and in form of a mutual dialogue with people from all over the world. For example, she realised in China that she was quite right to think of climate change and energy supply as the main issues for the future. In global meetings she can reorder her preferential topics for her local activities. Thus, there is a knowledge transfer between the global and the local scale.

Christian actually does not so much understand himself as a carrier of knowledge between places. He rather speaks about how he networks people from different places who face similar problems so that they can learn from each other. This is not a direct form of knowledge transfer but a mediated one in which Christian is rather the mediator. He translates and compares between local knowledge systems and brings people in contact so that they can learn to translate and compare themselves.

Dirk refers mainly to the transfer of sociality: social interaction from Bulgaria to Germany, and to political decision making including civic participation and governance from Germany to Bulgaria. In addition, his work for the EU is also directly a form of knowledge transfer from West to East in the field of adult education. Dirks says:

'Of course I can offer a broad variety of social models from Germany to Bulgaria. Simply how you... how democracy might work. How civic participation works. Or how it should work, or what possibilities exist for citizens to impact these things. [...] Vice versa there are also things, rather in the sense of attitudes towards other people, or friendliness, or the readiness to help others, or service mentality, which to us [in Germany] often... or they [people in Bulgaria] are a bit ahead of 
the German culture in terms of being a bit more personal uhm... personal empathy' (Dirk; lines $\left.565-573^{157}\right)$.

Dirk can actually profit from knowing how things work in terms of social organisation in both countries and he can transfer knowledge between them. In a similar way, Federico speaks about the social skills side of professional environments, and how social interaction and working culture (Essayismus) is different between countries. He has experienced that in professional environments the interaction between colleagues is very different in Italy and Germany. Whereas in Italy colleagues stress achievements and praise other for what that they have done, in Germany the focus is rather put on the needs of improvement. In German work environments the good things are taken for granted, and one focuses on becoming better through criticising the negative aspects. Having experienced this, Federico has learned that these social differences can be overcome, and that is something he transports to people in Trieste, where he engages in projects who would like to mediate in cultural terms between the often conflicting positions of the Italian and the Croatian communities.

We can also find this aspect of knowledge transfer in Gunnar's descriptions. Gunnar is specialised in American Studies. His current life mainly takes place between England, Germany and Spain. In this context, he thinks that it is difficult to earn a living with the speciality in American Studies, because North America actually plays a sub-ordinate role in his current lifeworld. As such, he tries to find scientific topics within the field of American Studies which are easily transferable to other specialities, which might work better in his geographic triangle. He thus chose the broader topic of 'political populism' as a topic for his $\mathrm{PhD}$ thesis. He expects that this can also be applied in Germany, England or Spain. He further describes his professional alternatives:

'In terms of career building it is definitely not an intelligent move to go to Spain. Unless I would be the one who established American Studies in Spain. Therefore, I would need a good PhD graduation and I would need an entry point into the Spanish university system, which is actually not easy. But if successful, then it would have huge potential. I would be able to organise American Studies conferences and so on, which actually do not yet exist in Spain. This means that there is some, probably there is a market for things like that, it is not saturated. Insofar, if I decently finish my PhD, and engage in networking, I could for sure make a difference' (Gunnar; lines 585-594 ${ }^{158}$ ).

Willi thinks about his own future and how he could profit from his current multilocal experience. So far, his main places in terms of professional activity have been Birmingham and Aachen, but

\footnotetext{
${ }^{157}$ Translated by the author from the German text: 'Natürlich kann ich unheimlich viel an gesellschaftlichen Modellen aus Deutschland in Bulgarien anbieten. Schlichtweg wie man... wie Demokratie funktioniert. Wie Bürgerbeteiligung funktioniert. Oder funktionieren sollte, oder welche Möglichkeiten es wohl gäbe für den Bürger irgendwo was zu beeinflussen. [...] Und zurück auch, gibt es auch Sachen, mehr so im Sinne von so einer gewissen Auffassung gegenüber Leuten, oder Freundlichkeit, oder Hilfsbereitschaft, oder Service, die uns oft... oder der deutschen Kultur so ein bißchen voraus, so ein bißchen mehr persönlicher ähm... persönliches Einfühlungsvermögen.'

${ }^{158}$ Translated by the author from the German text: 'Karrieremäßig ist es sicherlich kein so ein intelligenter Move irgendwie nach Spanien zu gehen. Es sei denn, ich wäre der, der Amerikanistik ganz groß raus bringt in Spanien. Dafür müsste ich aber natürlich erstmal 'n guten Abschluss als Doktor schaffen und irgendwie ins Uni-System reinkommen, was nicht so leicht ist. Aber wenn, dann ist da natürlich ein riesiges Potential, ne. Ich könnte dann Amerikanistik-Konferenzen oder dergleichen irgendwie ins Leben rufen, die es ja per se eigentlich gar nicht so richtig gibt in Spanien bisher. Das heißt, es gibt da irgendwie so ein, wahrscheinlich noch `n Markt für so was, das ist nicht übersättigt. Von daher, wenn ich meinen Abschluss gut mache als Doktor, und 'n bisschen mir Netzwerke aufbaue, könnte ich da echt was bewegen, ne.'
} 
since he considers Gent in Belgium the centre of his private life, he is planning to get in contact with local professional networks there. He says:

'Well, for example, I try, through the university now, I, last year I lectured at the university, uh... in the faculty of architecture, on a topic which actually stems from the ILL, but it does not matter. And this year I want to do that again. So I'm trying to get connected to the academic field there, also with a professor there. Funnily, there is also a joint project with the ILL, where they also partake. So, these are the contacts which... which result somehow' (Willi; lines 515-521 ${ }^{159}$ ).

In these examples we find an indication for the fact that my interview partners are using their capacity to transfer knowledge in strategic ways as well. This also becomes visible in the description that Liam provides. Liam worked in the environment of British academia for many years before taking his current position in Sweden. Today he regularly travels between Stockholm and Aberdeen in Scotland, and he observes:

'The UK is quite a big academic community, I mean, whatever discipline you're in. And uh you can become inward-looking within your national academic community, and this [working in Sweden] has made me a little bit more outward-looking, I hope. And a little bit less sure that the UK way of doing things is the only way of doing things, you know. [...] I probably brought more European contacts to them. Because I, when I was working in Scotland, I... I was working on European projects, and I got fairly good networks already, and I've taken those with me. Uhm... I think one of the things I end up doing is editing their English language documents, because the uh... the suppletives of the English language are sometimes not... they don't, they're not aware of them' (Liam; lines 302-314).

Liam, as most of my other interview partners, can profit from the double or multiple experience in different work environments. He transfers knowledge and skills to from the UK to Sweden, mainly the professional contacts he has on a European level as well as his English language expertise, and vice versa he learns about other perspectives and methods for doing things in the academic environment of Sweden which he then can take to the UK to question and relativise procedures there. The same motif shows up in the interview with Oskar, who lives and works mainly in Zurich, Linz and Berlin. Based on living in three different cities and countries, he can better understand the local and national particularities:

'It is very helpful, let's say, my interest in cities, and urban realities, in... also from a professional side, in development projects, and also in the dynamics within cities. This is continuously stimulated by the fact that I... Well, I must probably formulate it like this: I live in that, in the reality of these cities. [...] Also, I do not only live in these cities, but I also work there. [...] And out of it, I think, a type of constant attention arises, which is not self-evident, but which is taken for self-evident, if one does not expose oneself to such distinct realities' (Oskar; lines 364-398 ${ }^{160}$ ).

\footnotetext{
${ }^{159}$ Translated by the author from the German text: 'Wobei ich versuche, zum Beispiel über die Uni jetzt, also ich hab, ich hab letztes Jahr eine Vorlesung gehalten an der Uni, äh... in der Architekturfakultät zu einem Thema, gut, was vom ILL war, aber das ist egal. Und dieses Jahr will ich das auch wieder machen. Also ich versuche im akademischen Bereich so `n bisschen Anknüpfpunkte da zu finden, mit `nem Professor dort auch. Da gibt es lustigerweise jetzt auch `n Projekt mit dem ILL, wo die auch mit bei sind. Also das sind dann so Kontakte, die... die sich dadurch irgendwie ergeben.'

${ }^{160}$ Translated by the author from the German text: 'Es hilft natürlich enorm, sagen wir jetzt, mein Interesse an Städten, an Stadtwirklichkeiten, an... auch beruflich an, an Entwicklungsprojekten, also auch Dynamiken von Städten. Das wird natürlich
} 
Similarly, Yari uses the terminology of different realities when he describes what being multilocal means to him:

'It means that... well, the capacity and the privilege to be able to expose myself to different realities and to be able to do a job which is legitimised and useful in different areas, not only in one place, right. Hence, this is really good for me, to have this opportunity to travel and expose my work, to share and exchange experiences with people in different places' (Yari; lines 123-126 ${ }^{161}$ ).

Yari and Oskar both use the same terminology of 'realities' to circumscribe the local contexts in which they are active, and between which they transfer knowledge and experiences. They think of this process as 'exposure' to the foreign and the unknown. This exposure to new experiences leads to a cognitive process in which, through comparison, my interview partners become aware of the manifold utility of this multilocal knowledge. We can find this also in descriptions of other interview partners. Pia e.g. mentions that she transfers German virtues such as punctuality and orderliness into her Danish work environment, whereas she brings back a certain nonchalance in professional terms and a pronounced appreciation for design and style in everyday life environments from Denmark to Germany. At least once a year Pia also goes to Delft in the Netherlands to inform herself about the latest trends in European architecture and to scout potential employees for her Copenhagen based architecture office. Ronja transfers the knowledge about dealing with gentrification from Berlin to Copenhagen, where public debates about the problem are less established. Qamar has brought profound knowledge and skills in the field of realistic painting from his Russian socialisation in the field of painting which he thinks western Europeans do not learn in arts schools. In contrast, he transfers a sense for abstract styles in paintings from the USA and western Europe to Russia and Chechnya. Ugo has learned a lot about how to deal with the phenomenon of shrinking cities in his scientific positions in Rome and Weimar. He wants to take this knowledge and all the scientific contacts which he has been able to establish to Chile, where there are also shrinking cities. However, no one cares about this phenomenon.

What my interview partners report in their narrations is a profound awareness for the types of knowledge and skills which they have learnt in their different places. They strategically think about the transfer to and application in 'their' other places. As such, each of my interview partners can be considered a very individual knowledge bridge between different cities, networks and countries. They acquire very different types of professionally relevant knowledge. They learn about specific business culture and ways of interaction in professional environments. They also learn about certain project ideas and how things can be organised and managed. Finally, they also gather knowledge about precise contents and topics. All these things might not be available and partly unknown in their other contexts and they can use the transfer to be early adopters of this knowledge in other

laufend stimuliert dadurch, dass ich... Also ich muss es vielleicht so sagen: also, ich leb in dieser, in der Wirklichkeit dieser Städte. [...] Auch weil ich halt nun in diesen Städten nicht nur wohne, sondern auch arbeite. [...] Und daraus entwickelt sich, glaub ich, so eine Art ständige Aufmerksamkeit für Dinge, die nicht selbstverständlich sind, die man aber natürlich für selbstverständlich hält, wenn man sich nicht so anderen Wirklichkeiten aussetzt.'

161 Translated by the author from the Spanish text: 'Significa que... pues la capacidad y el privilegio de poder exponerme a realidades diferentes y de poder hacer trabajo que sea legitimado y útil, en diferentes partes, no sólo en un lugar, no. Entonces esto es muy bueno para mi, tener la capacidad de viajar y exponer mi trabajo, compartir e intercambiar experiencias con gente en diferentes espacios.' 
places. The multilocality allows my interview partners to access new stocks of knowledge and to develop new insights from hybridising local knowledge stocks into new knowledge.

The professional sector, namely the work in creative industries, is particular in this context. As my interview partners work on the topic of how their surrounding places are, and how they have come to be like that, they have a specific perspective about local specificities and how processes and structures differ between places. This is most evident for the interviewees who are engaged in urbanism, architecture and planning, and whose work has to do with defining the 'look and feel' of places. To them, going to other places and studying how things can be done elsewhere, is quasi a professional imperative in order to provide innovative thinking and solutions in their places. Finally, they are also less expected to follow the traditional ways of thinking about things in a place. With their outsider position they are able to introduce new ways of thinking to places, which would probably be more difficult for local people who are socially expected to be loyal to local traditions.

\subsubsection{Effects of multilocality on the workers' 'market values'}

My interview partners revealed that multilocality can become a competitive advantage in creative industries. For example, in the field of fine arts, being multilocal means that one can play with professional identities. Buyers of artwork in one place often look for the exotic, the unknown, and something coming from elsewhere. As such, it is sometimes helpful for multilocal artists to identify as coming from the other place in order to increase sales. Aurélien explained me that his gallerist in Nice, France knows about the fact that he had studied at the École des Beaux Arts in Nice, yet he labels Aurélien’s artwork as being from Leipzig, Germany. Aurélien remarks:

'Today, in the field of arts, in particular in contemporary art, I know that it is a professional need. Uh, because the fact of going elsewhere allows you to better, better work where you actually are. Uh, in the sense of that there is this appetite for the other territory. In other words, uh, because now, uh, I have, because I have done my studies in Nice, I have this gallery in Nice. But my gallery in Nice, even that I have studied in Nice, it is written that I live and work in Leipzig. This causes a greater interest in a buyer to buy my artwork than if I lived and worked in Nice. It is a bit in this constellation, too. This constellation of appetite for the exotic, the, the other, for that which one does not know, from a place which one does not know' (Aurélien; lines 122-130 ${ }^{162}$ ).

Similarly, Qamar describes how his status as a Chechen artist in western Europe makes him more famous in Chechnya than in Germany. Expectations are high that he brings in money and helps Chechen artists back home, something he cannot fulfil as he is struggling to survive in Leipzig. He says:

'There, somehow I am uh also more famous than here in Leipzig. Probably, because I live in the West. The locals also find this interesting: our compatriot is an artist in Germany. And I am

\footnotetext{
${ }^{162}$ Translated by the author from the French text: 'Aujourd'hui, au niveau de l'art, en tout cas au niveau de l'art contemporain, je sais que c'est un besoin professionnelle. Euh parce que le fait d'aller ailleurs permet aussi de mieux, mieux travailler là où tu es. Euh dans le sens où il y a ce rapport de l'envie du territoire de l'autre. À dire que euh parce que maintenant euh j'ai, parce que j'ai fait mes études à Nice, j'ai une gallérie à Nice. Mais que ma gallérie à Nice, même si j'ai fait mes études à Nice, il y a marqué je vis et travaille à Leipzig. Ça donne plus d'un certain intérêt à un acheteur de m'acheter que si je vivais à Nice y travaillais à Nice. Il y a un peu ce rapport là aussi. De ce rapport de l'envie de l'exotique, de, de l'autre, de celui qu'on ne connaît pas, de, du lieu qu'on ne connaît pas.'
} 
supported by them. I have done some exhibitions there, and I am welcomed by the minister and up to the president. But that's only a bit... it is perhaps good for my publicity, shaking hands with them and shooting some pictures. But it does not get me anything else' (Qamar; lines 111-117 ${ }^{163}$ ).

In particular, the last words indicate that Qamar is a bit disappointed about the fact that it does not help his economic success as an artist, but he must also acknowledge that his multilocality is a reason for his fame in Chechnya and it helps his publicity, as he says. Vice versa, he first says that it has not much influence in Germany to be multilocal between Leipzig and Grozny. However, reflecting more deeply, Qamar remarks that out of his Eastern European migrant status, he gets contracted by German associations that deal with the integration of foreigners, and eastern European immigrants in particular. Here, he participates in workshops with youngsters and children with a migration background.

We can see that multilocality can become an aspect which helps to increase the individual market value in the creative industries. Multilocality across cities and countries has caused my interview partners to all be confronted with formally being and feeling like a foreigner in at least one of their places. Whereas this feeling and formal status can have disadvantages in several respects, it is also used by interview partners as a motif for their work. They have to personally deal with the meaning of being a foreigner, and where it fits, they make use of it.

Apart from the competitive advantage that arises from access to distant knowledge pools in other places (cf. section 7.5.2), multilocality itself is like a label which makes my interview partners more interesting for potential customers and clients. Liam also observed that his decision to take the position in Sweden and become a multilocal between the UK and Sweden has increased his attractiveness in professional terms. Liam says that it is an opportunity to work for a Swedish employer, because he was working for the same employing institution in Scotland for seventeen years, which does not look good on his Curriculum Vitae:

'I mean, it was, it was an opportunity because uhm... it was a good thing for my CV. It was good to learn about the Nordic countries and uhm... good to, I mean, I previously had basically uh seventeen years working for the same employer. And, and it was good to see a different environment, and it being... I mean, it's, it is quite an unusual arrangement. So if I go to a meeting and people ask, you know, you have around the table... it's usually, people are interested in the fact that I'm doing this. And that's good for you, I think it's good. It opens doors and... and also, you know, you learn quite a lot about the different culture' (Liam; lines 291-297).

This more general aspect of opening doors also translates to better chances in terms of job opportunities. In the case of Liam, international experience and multilocality can be advantageous not only in academia, but this is also a central prerequisite in architecture. In the field of architecture, there is also something like a 'multilocality bonus'. Here, certain locations and countries are particularly prestigious, and being identified as being related to them can have positive impacts on one's own professional situation, as Stephan explains:

\footnotetext{
${ }^{163}$ Translated by the author from the German text: 'Irgendwie bin ich dort auch äh bekannter als hier in Leipzig. Dadurch, dass ich wahrscheinlich im Westen lebe. Das finden auch die Einheimischen interessant: unser Landsmann ist in Deutschland ein Künstler. Und ich werde dort unterstützt. Ich hab dort ein paar Ausstellungen gemacht und ich werde vom Minister gut empfangen bis zum Präsident. Aber das ist nur ein bisschen so... ist gut für mein Publicity vielleicht mit denen mal ,Guten Tag'zu sagen und ein paar Fotos zu schießen. Aber das bringt dir weiter nichts.'
} 
'Well, the Danish architecture, and it is of course the same for Dutch architecture, but the Danish architecture is also uhm... a marketing factor, which is used by certain offices to put themselves in place. [...] Ah, Danish design, Danish architecture, Scandinavian architecture, that's something which we... with which we can identify well, it fits us, or uh we would like to present ourselves with it. Then it is suddenly a competitive advantage. If you can really see that in the architecture, I would... there I would uh put a large question mark. But it is definitely used as a marketing tool, this national property. [...] And uhm this is a large competitive advantage, simply to say... I mean first of all in terms of language skills, and on the other hand uhm also when applying for jobs. The job in Brunswick, I think, I only got it for this reason [of having worked in Denmark]. It was... or probably one cannot reduce it to that. But it is surely a large advantage, I think so. [...] When I started to work in the second office [in Denmark], it was simply... they have explicitly looked for a German architect. In this case the German language, a certain expertise in the German context were... were the basic conditions' (Stephan; lines 615-645 ${ }^{164}$ ).

Along the same line, Berlin-based Danish interior designer and architect Tjark argues that there is a mutual advantage in his multilocal constellation of Berlin-Copenhagen, with people in Germany acknowledging his supposedly 'given' Scandinavian talent for design, and with Danish people imagining Berlin as a current hotspot for arts and culture in Europe. So, Tjark can profit from a defined mutual interest of the wider local and national societies in both of these places. When he comes to Copenhagen, people are more interested in him than in other designers due to the fact that he lives in Berlin, and the same happens to him in Berlin with his Danishness. Even though he has developed things which are actually 100 percent 'made in Berlin', the local journalists applaud these design objects as Scandinavian design. Tjark is amused by that irony, but he also uses it in order to sell his objects and making his little design office more known in the competitive Berlin market.

A similar increase in personal attention was observed by Xaverio: once he was in New York to improve his photography skills, he became aware of that mechanism, and he now uses it both in Milan and in Berlin. He explains:

'Yeah, in certain sense it's always like that. I mean, I, I think it's not a... just the connection of these two cities, but it's always, if I'm, if I go to... I don't know, to New York, and I say, I'm Italian, I'm from Milan, they look at you in a certain way. If a New Yorker came here in Milan... So, I think it's true, I mean, you have like something more [in] respect [to] the other people. So, when I'm here, and I say I'm living also in Berlin, I know very well Berlin, yeah people are more interested, you

\footnotetext{
${ }^{164}$ Translated by the author from the German text: 'Also die dänische Architektur, bei der holländischen Architektur ist das natürlich genauso, aber die dänische Architektur ist natürlich auch ein ähm... Marketingfaktor, also wo sich dann die entsprechenden Büros einfach international auch platzieren. [...] 'Ach, dänisches Design, dänische Architektur, skandinavische Architektur, das ist irgendwas, was wir ... mit was wir uns gut identifizieren können, was irgendwie zu uns passt, oder äh was wir uns gern so anheften wollen auch so. ' Das ist dann in dem Fall plötzlich auch ein Marktvorteil. Ob man das wirklich in der Architektur sehen kann, das würde ich mal... da würde ich mal ein großes Fragezeichen äh stellen. Aber es wird auf jeden Fall als Marketinginstrument genutzt, so diese nationale Eigenheit. [...] Also das ist ähm bei uns in der Branche ein Riesenvorteil, so einfach zu sagen... also ich meine, einmal natürlich sprachlich, und andererseits ähm auch beim Bewerben. Also den Job in Braunschweig habe ich, glaube ich, nur darüber gekriegt. Das war... oder vielleicht ist das, kann man's jetzt auch nicht darauf reduzieren. Aber das ist auf jeden Fall ein Riesenvorteil, das, das denk ich. [...] als ich in dem zweiten Büro [in Dänemark] angefangen habe, war das einfach `ne... die haben explizit einen deutschen Architekten gesucht. Also da war einfach dann die deutsche Sprache, und eine gewisse Expertise aus dem deutschen Umfeld, war... war einfach die Grundvoraussetzung.'
} 
know. Because even now, I mean, in the... now in this situation, Berlin is like, cooler than Milan. Now, I mean, like a global vision of the city. Because it's more, yeah more clubs, and more culture, more people, more young people. It's... I mean, in the Italian magazines, people have this kind of uh feedback, you know. It's like the 'place to be', now it's Berlin. And the place to see. Because uh things happen there, and not for sure here in Milan, that it's everything more static. So, if you're... if you're here [in Milan], and you talk about Berlin, they are... people are more uh... uhm interested in you, even uh in a kind of way, in uh... in a working... workly speaking, I mean. And I think also when you go to Berlin. Because Italian, it's always uh yeah it's, yeah you are Italian, so you are something different from the, from the normal life. But I think that in Berlin, yeah it's okay, but it... you... I can feel it more here in Milan now. [...]And it happens to me more to speak about Berlin than speak about Milan' (Xaverio; lines 226-251).

Willi has also observed that he won the competition for his current position in the research institute because he had his foreign work experience from Birmingham. He explains:

'Obviously it brings advantages if you have been abroad for a time, and you apply somewhere. I think this is important. And uh... I also do not think I would have got my job at the ILL if I hadn't done a certain job in Birmingham beforehand, which exactly fit to the job description of the vacant position. This, yeah, that's clear!' (Willi; lines 621-630 ${ }^{165}$ )

This transport of ideas is also relevant in other fields of creative industries. On the other hand, obvious competitive disadvantages also become apparent in my interviews. Ronja works as a journalist and as such her central working material is 'language'. Now that she is based in Berlin, and no longer in Copenhagen, she says that she rather sees her Danishness in Germany as a competitive disadvantage as her language skills are not on a German mother tongue level. Compared to her husband's professional field, architecture, she remarks:

'Well, as an architect you can work anywhere in the world. I have a lot with, with language... well I uhm... I often mediate. This means uhm I write texts, I edit and so on. And of course this is difficult in German, because I don't [know] German perfectly... which I will actually never be able to offer. Unfortunately! Then... yeah, you need to hope that it... I can still edit. But I could never do final proofreading for instance. This is now a disadvantage' (Ronja; lines 325-331 ${ }^{166}$ ).

On the other hand, Ronja can also draw competitive an advantage from her multilocality in the opposite direction: when she applies in Denmark it is advantageous for her that she is based in Berlin. Berlin is suspected to offer interesting content-wise input for working as a journalist in Denmark. Therefore, Ronja is still more often engaged in professional projects in Copenhagen than in Berlin.

\footnotetext{
${ }^{165}$ Translated by the author from the German text: ,Also natürlich bringt es dir Vorteile, wenn du dich bewirbst irgendwo, wenn du mal im Ausland gearbeitet hast oder so. Das denke ich, ist schon wichtig. Und äh... also ich denk auch nicht, dass ich den Job im ILL bekommen hätte, wenn ich nicht 'ne gewisse Tätigkeit in Birmingham gemacht hätte vorher, die jetzt genau irgendwie auf die Stelle jetzt, die ausgeschrieben war, zutraf. Also das schon natürlich, klar!‘

${ }^{166}$ Translated by the author from the German text: ,Also man kann eigentlich überall in der Welt als Architekt arbeiten. Ich habe mich viel mit, mit Sprache... also ich ähm... ich vermittle oft. Also ähm schreibe Texte, mach Redaktion und so. Und das ist natürlich schwierig auf Deutsch, weil ich kein perfektes Deutsch... was ich eigentlich jemals ähm anbieten werde können. Leider! Dann... ja, man muss dann hoffen, dass es... ich kann ja trotzdem Redaktion machen. Aber ich könnte zum Beispiel nie End-, Endkorrektur machen. Das ist schon ein Nachteil.'
} 
What I would like to point out here is that my multilocal creative knowledge workers can draw a specific competitive advantage in their professional fields as they are experienced in particular national and local contexts. I think that this type of foreign work experience might also occur as a competitive advantage for any other person who is possibly more monolocal. However, my multilocal interview partners can add on this competitive advantage as they are still integrated into these 'other' distant contexts. They keep their knowledge updated (cf. previous section on knowledge transfer). Thus, they make use of their double or multiple place affiliation and their alternation between countries and cities. Being identified or self-identifying with several places, with very particular places, or with the foreign character of these places increases the attention that my interview partners receive in their professional environments. And often this includes enhanced access to jobs and interesting new projects. Here it makes a difference that they are multilocal.

\subsubsection{Labour market constraints and the forced nature of multilocality}

Whereas so far the positive aspects of multilocality in creative industries have been mentioned, I also found that the coin has a second side. My interview partners also stated that multilocality is also a reaction to the demand for labour flexibility in creative industries. As my interview partners are multilocal, they actually have decided to play this game of flexibility and mobility. In the interviews they also express their disapprobation with these circumstances, and as I mentioned in section 7.2, my interview partners perceive also the negative and stressful aspects of frequent physical travel, and this also stresses their social relations (cf. section 7.3). In their reflection about their multilocal lifeworlds they often find the reason for this in the labour market demands in their professional fields.

For example, Federico reflects on the fact that he could imagine living monolocally in Leipzig, but he recognises the labour market constraints in the scientific field of humanities, where mobility is an imperative and most jobs are temporary:

'I could imagine spending my whole life in Leipzig if I got a job, but everything depends on work. Because this work... uh... that I do, has now become precarious work. I mean external funding and so on... uhm... I actually try to apply for permanent positions, but this means that I do this across Germany, and across the German speaking area, including Austria and sometimes Switzerland. I also tried in England, in Belgium. Anyhow, I have to be prepared for moving on' (Federico; lines $\left.748-754^{167}\right)$.

According to Federico, this also causes problems for the organisation of everyday life in a stable frame. Federico lives in a shared apartment where he and his roommate would like to move out in order to find a more comfortable apartment, but they want to remain roommates since they get on quite well. Given that he cannot really predict where he will be working in a few years, Federico has problems convincing his roommate to move and engage in long-term apartment sharing with

\footnotetext{
${ }^{167}$ Translated by the author from the German text: 'Ich kann mir vorstellen, ich kann auch mein ganzes Leben in Leipzig verbringen, wenn ich eine Arbeit kriege. Aber ich mache alles von der Arbeit abhängig. Weil diese Arbeit... äh... die ich mache, ist jetzt eine prekäre Arbeit geworden. Also Drittmittelprojekte und so weiter... ähm... ich versuche mich natürlich, um feste Stellen zu bewerben. Aber das bedeutet, dass ich das deutschlandweit oder im deutschsprachigen Raum mache, auch in Österreich und in der Schweiz manchmal. Ich habe auch mit England probiert, mit Belgien. Also sowieso muss ich bereit sein, umzuziehen.'
} 
him as there is always the risk that he might leave for another job. Gunnar also identifies the disadvantages of working in a scientific job:

'I do not long for any precise place, where I want to be. But stabilisation would be good, you know. Uhm... I would like this. Yet, interestingly it is not possible to really predict this. Because I have two options, probably two basic ones: I could somehow go on with Ana Lucía, or without her, you know. If I go on without her, then I will be most probably back here, and do my PhD, and then I am in this academic environment. And this means also to move, if necessary. And this could also happen quite spontaneously, that I go somewhere else. And if I stay with Ana Lucía, then I have different things I could do, and different places, where I could stay. But it will always be that we are not at the same time at home. [...] Consequently, I cannot save myself from this to and fro, if I were to go on without Ana Lucía. Because then I will be part of this academic jet-set, which is, in many respects, basically not very different from the relationship jet-set, you know' (Gunnar; lines 865$\left.882^{168}\right)$.

In Gunnar's quote we can see again the longing for a more steady and monolocal life, and the perception that his professional field does not allow for this stability. Just as Federico, Gunnar also wishes to have a precise future perspective in one place with his girlfriend, but somehow things in his life would not seem to fit into one place if he decided to stay in his professional field. Stephan also thinks of his professional field, architecture, as one of the most internationalised labour market segments in Europe, and explaining the impacts of this internationalisation for the individual worker, he also expresses that he dislikes it:

'I think there is no other sector in which this [internationality] is so deeply implemented as for architects. I mean, I have the impression that there are no students who have not at least spent one semester abroad. There are so many who do that. [...] I mean, they must know how to draw, they must know how to conceptualise... and with these skills they can work right away in every office in Europe. Provided they speak English. [...] I also do not want to idealise that. I mean, the labour market demands... is simply so flexibilised and demands that the labour crosses borders, so that this is somehow expected from us, you know, the young architects, if there is nothing to do at the moment in Berlin, then you should go somewhere else. This is also brutal. But in certain years one can also consider this as an advantage and as uhm... as something nice, as a chance somehow, that you get around' (Stephan; lines 157-180 ${ }^{169}$ ).

\footnotetext{
${ }^{168}$ Translated by the author from the German text: 'Ich hab keinen speziellen Ort im Auge, wo ich jetzt unbedingt hin will. Aber Verstetigung wäre gut, ne. Ähm... würde mir gefallen. Aber interessanterweise ist das nicht zu sagen, so richtig. Weil zwei Wege habe ich halt so, vielleicht so zwei generellere: ich könnte irgendwie mit Ana Lucía weiter machen, oder auch ohne, ne. Wenn ich ohne weiter mache, dann bin ich halt wahrscheinlich wieder hier, mache meinen Doktor, und dann bin ich halt irgendwie in diesem Akademiker-Umfeld, ne. Und das heißt dann schon irgendwie auch Umziehen, wenn`s sein muss, ne. Und das kann auch relativ fix gehen, dass ich dann irgendwo anders hinkomme. Und wenn ich mit Ana Lucía weiter mache, dann habe ich ja irgendwie verschiedene Sachen, die ich machen könnte, und verschiedene Orte, an denen ich bleiben könnte. Das wird aber immer so sein, dass wir nicht zur selben Zeit wirklich zu Hause sind. [...] Also ich kann mich nicht irgendwie vor diesem Hin- und Hergetingle irgendwie retten, indem ich jetzt irgendwie, irgendwie ohne Ana Lucía weiter mache. Weil dann bin ich halt in diesem Akademiker-Jetset, der im Prinzip dem Beziehungs-Jetset gar nicht so unähnlich ist, in mancherlei Hinsicht, ne.'

${ }^{169}$ Translated by the author from the German text: 'Das ist in kaum einer Branche, glaube ich, so umgesetzt wie bei den Architekten. Also ich habe den Eindruck, es gibt keine Studenten, die nicht mindestens ein Semester mal ins Ausland gehen, oder so. Das machen ganz viele auf jeden Fall. [...] ich meine, sie müssen zeichnen können, sie müssen konzeptionell denken können... damit können sie von Tag 1 in jedem Büro in Europa sofort mitarbeiten. Sofern sie englisch sprechen. [...] ich will das auch gar nicht idealisieren. Ich meine, der Arbeitsmarkt fragt halt... ist halt einfach so flexibilisiert und fragt das nach, dass einfach die Arbeitskraft über die
} 
In this quote we can see that Stephan is trying to balance the positive and the negative sides of flexibility demands in the architecture sector. Stephan has recently become a father, and whereas he accepted flexibility earlier, even against his personal convictions, he immediately adds to the formerly said:

'I mean, certain things, a, a certain flexibility, uh which I had so far, has ceased to exist. This is not possible anymore. I mean, I cannot anymore uhm... well, before I could have said without any problem, I'll leave for two weeks, going somewhere and working for a competition, and I am completely away for two weeks. Or, the whole time I have, it was not easy, it was also quite difficult... but I could commute between Copenhagen and Germany. Now I cannot do this anymore. It's simply, this time is over' (Stephan; lines 182-188 ${ }^{170}$ ).

In this additional comment, we see that problems with multilocal and mobile arrangements in creative industries become evident when it touches upon family structures and the organization of everyday life with small children. In this direction, Juliana's argumentation about her multilocal arrangements between Mainz and Berlin can also be interpreted as something less than perfect. She mentions:

'I perceive that not as a compulsion but as a necessity. [...] Especially with the family, for us it is of course much more complicated, the whole thing, you know. My second child is now already two and a half years old. But when I took the position in Mainz for example, she was not even two years old. And yeah, this is very exhausting. And also it is unclear, for the children it is perhaps not particularly good, if the parents are somehow continuously absent. [laughs] I don't know. I mean, it is... so far it is okay. I do not think that my kids are traumatised by that. But it's simply... we long for a certain tranquillity. [...] Sometimes I am like, often that I am desperate, because I find this exhausting or not so good, this... and I think: okay, we need to do something, and then whatever, I mean, it is not Berlin, and the moving. But my family, they don't want to. [...] Well, honestly I don't want this either. I understand them. [...] Because if they would come there, and be unhappy, or they would feel isolated, this would not be the correct solution. [...] And then again... for example I think, okay, I have this position in Mainz limited for three years. [...] If we moved, we would have to find a kindergarten, a school, an apartment. We would pay so much more for rent, that it would be the same as if I paid the whole travelling... Then it would probably take half a year until everyone would feel comfortable. My husband would still have to commute. And then probably something else comes along, and practically one would already need to go again' (Juliana; lines $\left.216-262^{171}\right)$.

\footnotetext{
Grenzen hin- und herwechseln, dass irgendwie auch fast erwartet wird von uns, ne, also dass wir als junge Architekten, wenn es halt in Berlin gerade nix zu tun gibt, dann soll man halt woanders hingehen. Das ist ja auch ziemlich brutal. Aber in gewissen Jahren kann man das ja auch als Vorteil sehen und als ähm... als was Schönes, als 'ne Chance irgendwie, dass man mal ein bisschen rumkommt.'

${ }^{170}$ Translated by the author from the German text: 'Also ich meine, ähm... so gewisse Sachen, eine, eine gewisse Flexibilität, äh die ich bisher hatte, die fällt ja jetzt einfach weg. Die fällt komplett weg. Also ich kann gar nicht mehr so diesen ähm... also früher habe ich ganz locker äh sagen können, ich gehe zwei Wochen irgendwohin, arbeite an einem Wettbewerb, und bin dann zwei Wochen komplett weg. Oder ich habe die ganze Zeit, das war zwar nicht locker, das war auch schon schwierig... aber ich konnte dieses Pendeln zwischen Kopenhagen und Deutschland machen. Das kann ich jetzt nicht mehr. Also das ist einfach, die Zeit ist vorbei.'
}

\footnotetext{
${ }^{171}$ Translated by the author from the German text: 'Ich empfinde es also nicht als Drang sondern eher Zwang. [...] Also das ist für uns natürlich so, gerade mit Familie, ist das dann immer sehr viel komplizierter, diese ganzen Sachen, ne. Mein zweites Kind ist jetzt
} 
Juliana knows that her professional field of ethnology is also subject to high labour market flexibility. And her multilocality could be understood as a strategy to avoid that her family would have to follow her to her jobs all the time. In this sense, she defends the central base of her family, which is their apartment in Berlin, and all their friends and wider family they have there. Balancing the positive and negative aspects of working in such a flexibilised professional environment, she concludes:

'It also has pleasant aspects. But it is often not that you sit down with a map, and you say, I want to go there and there, and then there. Basically it's the opposite way around: I must!' (Juliana; lines $750-752^{172}$ )

Given the mentioned examples, we can highlight that my interview partners are also aware of the negative impacts of the flexible labour market conditions in creative industries. In particular, when my interview partners have small children and they are seeking to stabilise their own life, they perceive the mobility demands as constraints to their lives and the personal multilocality becomes connotated with a forced nature. The main strategy that my interview partners apply in these moments of dissatisfaction and exhaustion is to try to construct discourses about the advantages and to emphasise the positive aspects of a multilocal work life in creative industries. Here, we can look at the example of Birgit, who says that it is necessary to develop a positive attitude towards this mobile life, which in itself is already exhausting. Not liking it would make it unsupportable. She concludes:

'I think it is not necessarily a life one chooses in this way. And I think the only thing one has to mention is that it is easier and easier to be organised, and to be sustained if one lets oneself in for it. And one rather underlines the positive side, and looks less at the sacrifices' (Birgit; lines 557$\left.560^{173}\right)$.

\subsection{Identity \& belonging}

Identity is composed of different elements. First, there is a personal aspect such as one's own character and perceived individuality. Second, the professional dimension comes into play. Third,

schon zweieinhalb Jahre. Aber als ich diese Stelle in Mainz zum Beispiel angefangen habe, mein Kind war noch nicht einmal zwei Jahre alt. Und ja, das ist sehr anstrengend. Und es ist auch noch nicht klar, für das Kind ist das vielleicht auch nicht besonders gut, dass die Eltern ständig irgendwie weg sind [laughs]. Ich weiß nicht. Naja, es ist schon... bis hierher ist es okay. Ich glaube jetzt nicht, dass meine Kinder dadurch irgendwie traumatisiert sind. Aber es ist einfach... also wir sehnen uns nach so einer Ruhe. [...] ich bin dann eher so schon, dass ich dann öfters verzweifelt bin, weil ich das anstrengend, oder irgendwie nicht so gut finde, dieses... und denke: okay, wir müssten was machen, und dann egal, ich meine, dass es irgendwie nicht Berlin ist, und dann umziehen. Aber meine Familie, die wollen das nicht. [...] Na, ich will das sozusagen auch nicht. Ich verstehe sie. [...] Weil wenn sie dann dahinkommen und unglücklich sind, oder sich isoliert fühlen irgendwie, dann ist das auch nicht die richtige Lösung. [...] und wieder... zum Beispiel denke ich mir, okay, dann ich habe da in Mainz die Stelle, ich glaube, drei Jahre. [...] Dann ziehen wir dahin, dann müssen wir Kita, Schule, Wohnung suchen. Für die Wohnung bezahlen wir so viel mehr, dass es wahrscheinlich genauso ist, als ob ich jetzt diese Reiserei bezahle, oder ja... Dann dauert es vielleicht ein halbes Jahr bis alle sich da eingewöhnt haben. Mein Mann müsste trotzdem noch pendeln. Und dann ergibt sich was anderes, und dann muss man schon praktisch wieder, wieder woanders hin.'

${ }^{172}$ Translated by the author from the German text: 'Es hat ja auch schöne Aspekte. Aber das ist ja oft nicht so, dass man so sich an die Karte setzt und sagt, ich will dahin, und dahin, und dann dahin. Das ist ja eher andersherum, ich muss!'

${ }^{173}$ Translated by the author from the German text: 'Es ist glaube ich nicht unbedingt ein Leben, was man sich so aussucht. Das einzige, was man glaube ich sagen muss, dass es leichter ist und einfacher zu organisieren ist, und besser auszuhalten ist, wenn man sich drauf einlässt. Und eher äh die Habenseite betont, und weniger den Verlust aufrechnet. ‘ 
the individual mobility is a constituting element (cf. also section 7.2) and leads to such things as perceiving oneself as a 'nomad'. Furthermore, the multilocality itself has consequences for the place-based identification in terms of confounding the clarity of belonging, but also allowing for variation.

\subsubsection{Place-based Identity: Being based vs being from}

Place-based identification is a tricky issue in transnational multilocal lifeworlds. There are not only different places, but different scales as well, which are all available as reference objects of placebased identification. I will discuss the scale issue in section 7.6.5. Here, I will focus on another problem. My interview partners are actually quite often confronted with the question for their identity. In their everyday life they are asked by others: where are you from? Where's your home? These situations always put them in a certain situation where they have to explain themselves and their geographic pattern in a more detailed way. Some of my interview partners actually do not like to here that anymore, and they react with a certain irony.

Dirk e.g. uses his possible identification alternatives in a playful way in everyday life situations. When he is in Sofia and goes shopping for food at the market, people realise that he is not Bulgarian. Even though his Bulgarian is quite decent, his dialect reveals that he comes from elsewhere. In his self-identification, Dirk feels at home in Sofia and Bulgaria, so for him it is paradoxical that people tell him that he should not be from there. As an answer he develops invented identities: he explains to Bulgarian people at the market that he comes from Poland or from other countries in the East. And he plays around with the people in order to find out if they can tell him that he is actually a German.

Some of my interview partners cannot clearly express where they feel at home. One strategy to tackle this problem is provided by anchoring one's own belonging in an imaginary space, basically in one's own mental map. This imaginary space, which can also be thought of as a mental projection of one's own geographic space, helps to support the continuous alternation between places. As such, they feel at home in their whole lifeworld. Christian e.g. uses this method to identify and explain his belonging. He explains:

'I think I do not have a Heimat [home]. I think, the collectivity of these different places, this is a bit my Heimat, you know. But there are also other places where I have spent some months, sometimes I worked there, and which stay very present in my mind. I am not a person who functions with a temporal-linear structure. Uh, it's more a psychic time. This means I do not have the impression that I have done this, and thereafter that, and thereafter that. Everything is nearly in the same moment. There are more important and less important things. It depends also on what I am actually living in the moment, where I am living. Sometimes certain things move and come to the front. At the moment, I can say that I feel at home when I come to Leipzig. [...] I have done quite a lot of things here, I have met many people. And I also work quite a lot here. Both for earning money and for my exhibitions. Hence, in the last time, I have rather installed myself here. If I come here, that's where I feel a bit at home' (Christian; lines 246-259 $9^{174}$ ).

\footnotetext{
${ }^{174}$ Translated by the author from the French text: 'Je pense, je n'ai pas de Heimat. Je pense, l'ensemble des ces différentes places, c'est un peu mon Heimat, quoi. Mais il y a aussi d'autres endroits où j'ai passé que quelques mois, parfois travaillé, qui restent très
} 
This quote of Christian indicates that it sometimes difficult for my interview partners to precisely name a place or a geographic reference to identify. Still, he distinguishes between places according to their relevance in his current everyday life. Leipzig draws relevance from the people around and from the professional dimension, so place-based identity interferes with these lifeworld dimensions when it comes to defining a current place where one feels at home. Whereas Christian cannot define where he is from in terms of roots and origin, he has constructed a temporary home, a base in Leipzig, or as he says, he has installed himself there.

Others of my interview partners also distinguish between places of origin, where they feel to 'be from' and places where they are at home, where they are currently 'based'. This first category seems to reflect the ideas of the notion of Heimat (cf. chapter 3). They link the places to their own 'roots' as an important term in this respect. Roots can refer to a feeling of originating from somewhere. They refer mainly to historic places of origin, combined with cultural and family roots, and which provide 'resources' to recover from mobility and their imagined homelessness. As such, these places rather have a historic and infinitely temporal character. They are fixed in life; they do not change anymore, and will most probably remain part of their lifeworld's geography. On the other hand, the second notion of 'being based' is used in the vocabulary of ' $z$ u Hause' in my German interviews. These places rather define a present temporal state, they are current places which are important in everyday life, where important people live, and being close to these people, and in these familiar places, my interview partners feel that they belong there in the sense of having their current base in these places. However, this term is not an infinite one either. The bases can be relocated in time.

We can illustrate this differentiation with the case of Emil. He distinguishes between Leipzig and Basel to describe where he is from and where he feels at home. Being from somewhere, having roots to him means the place where he grew up: Leipzig. Whereas Leipzig is both Heimat and $z u$ Hause, he says that Basel is also a zu Hause to him. The differentiation stems from - not only growing up - but also having lived through all aspects of everyday life: e.g. dealing with bureaucracy, knowing how social interaction in professional environments works, serving in the military and participating in elections. When thinking about his places, Emil describes in detail the relation between Leipzig and Basel in his place pattern:

'And then secondly I would also name Basel, where I am at least equally feeling at home, because I am there since twenty years now. But I have not grown up there, I have not gone to school there, I do not have any friends from school or others there. And hence I am less rooted there, because I also worked there for a short time. And of course, I don't know certain aspects of life in this country. I mean, you quickly think you know a country, but you haven't experienced a lot of things

présents dans ma tète. Je ne suis pas quelqu'un qui fonctionne avec une structure temporel-linéaire. Euh c'est plutôt un temps psychique. Ça veut dire que je n'ai pas une impression que j'ai fait ça, et ensuite ça, et ensuite ça. Toute est presque toujours sur le même moment. Il y a des choses plus importantes et moins importantes. Ça dépend aussi de ce que je vis au moment, où je vis. De fois certaines choses se déplacent, et passent devant. En ce moment, je peux dire que je me sens chez moi quand je viens à Leipzig. [...] J'ai fait pas mal des choses ici, j'ai rencontré beaucoup des gens. Et je travaille pas mal aussi ici. Aussi pour gagner de l'argent, et pour les expositions. Donc, le dernier temps, j'ai plutôt installé ma personne ici. Quand je viens ici, c'est là où je me sens un peu chez moi.' 
there which you have lived through in the place where you have grown up, or where you lived for a longer time' (Emil; lines 68-106 ${ }^{175}$ ).

'The thing with Heimat is you cannot so quickly clear that away. That is so complex; you cannot get rid of that. And also you cannot so quickly... I mean, I always ask myself that in respect to Basel. I have not only commuted there, I also lived there for two years, and I have studied there. Even more than two years. To me this is very complex. You cannot get that out of the way. I cannot put that aside, with this, with this Leipzig here' (Emil; lines 210-214 ${ }^{176}$ ).

'So if you would really call that Heimat, I think this has more connotated as if I'd say: I am at home. I am at home in Basel. I go home to Basel. And then I go back home to Leipzig. But Heimat, this is even another term, which according to my view involves much, much more. Which you also hardly [thinking]... even if you live somewhere for a long time, you cannot create that' (Emil; lines 352-371 ${ }^{177}$ ).

These three quotes from different sections in the interview highlight that Heimat, in the sense of 'being from' in the sense of origin, is distinguished from zu Hause, 'being based' somewhere, where one currently feels at home. Emil clearly links childhood, school time and growing up to Leipzig, which to him is his Heimat. This has a historic value, which is rooted in Emil's own biography. In contrast, Basel has become an additional zu Hause, where he also feels to be based. These bases work in the sense that they anchor the current multilocal life. They are related to everyday life activities in the spheres of profession, people or private leisure activities at the current moment. These bases can be added to and removed from the place pattern, while the Heimat is a stable item which one cannot get rid of. We can also look at the example of Federico, who describes the different meanings of Leipzig and Bergamo in his life:

'If one asked me where I am at home, I would say that this is in Leipzig. Leipzig is the city where I have lived for the most time of my adult life, because in Bologna I spent five years, but in Leipzig meanwhile more than six years. Uh and... okay, of course I have lived for eighteen years in Bergamo, but to me Bergamo is the city of my childhood and of my parents. As such it is not mine' (Federico; lines 57-62 ${ }^{178}$ ).

\footnotetext{
175 Translated by the author from the German text: 'Und als zweites würde ich dann, dann schon Basel auch nennen, wo ich also mindestens genauso zu Hause bin. Weil ich da seit zwanzig Jahren bin. Aber ich bin da halt nicht aufgewachsen, ich bin da nicht zur Schule gegangen, ich habe da keine Schulfreunde oder irgendwas. Und bin dann natürlich auch weniger verankert, weil ich da nur mal kurz gearbeitet habe. Und natürlich, bestimmte Bereiche des Lebens auch überhaupt nicht kenne in dem Land. Ich meine, man meint dann schon, man kenne ein Land. Aber man hat eben viele Dinge dort gar nicht mitgemacht, die man in, in dem Ort, wo man aufgewachsen ist, oder wo man schon länger gelebt hat, halt dann mitgemacht hat.'

176 Translated by the author from the German text: 'Das kann man nicht so schnell weg machen, Heimat. Das ist so komplex, das kriegt man nicht weg. Das kann man auch nicht so schnell... also ich frag mich das immer, in, in Basel. Ich bin ja auch nicht bloß jetzt der Pendler, sondern ich habe ja auch zwei Jahre in Basel fest gewohnt, und habe da studiert. Sogar über zwei Jahre. Das ist für mich so komplex. Das kriegst du nicht weg. Das kann ich nicht weglegen, mit diesem, mit diesem Leipzig hier.'

177 Translated by the author from the German text: 'Also wenn du das wirklich dann so mit dem Wort Heimat [verbindest], das ist glaube ich noch mal belegter als wenn ich sage: ich bin zu Hause. Ich bin schon in Basel zu Hause. Ich fahre nach Hause, nach Basel. Und ich fahre dann auch wieder zurück nach Hause, nach Leipzig. Aber Heimat, das ist nochmal ein anderer Begriff, der meiner Ansicht nach sehr viel mehr involviert. Was du auch kaum [thinking]... selbst wenn du irgendwo lange wohnst, so einfach kriegst.'

${ }^{178}$ Translated by the author from the German text: 'Wenn man mich fragt, wo mein zu Hause ist, würde ich sagen, dass es in Leipzig ist. Leipzig ist die Stadt, in der ich am längsten als erwachsener Mensch gewohnt habe. Weil ich in Bologna fünf Jahre verbracht
} 
Federico says that he has his zu Hause in Leipzig, where he has spent most of his time as an independent adult. In contrast, he lived longer in Bergamo, where he grew up, but this to him is a historic place of his childhood and related to parental decisions, and not his own. He further explains:

'I would say that Leipzig is where I feel at home [zu Hause]. But I would not say that Leipzig is my home [Heimat], because Heimat has this connotation of where your roots are. [...] Uh Leipzig is where I feel at home. I think, the time that I spent there was a long time. Um, I like the city. And I like my life here. I can live better here than in any other city' (Federico; lines 177-185 ${ }^{179}$ ).

In these descriptions we can see that place-based identification is solved in different ways. Yet, a common pattern is to combine different geographic references in the personal identity. The two main components are the 'place of origin', where one comes from, and the 'places where one lives', where one actually is based at the moment. An interesting fact is that the current 'bases' will have a higher prospected temporal stability and thus a stronger binding function if property is owned there by my interview partners. Examples among my interview partners are Birgit and her apartment in Leipzig, Liam and his house in Aberdeenshire, Aurélien and his house in Leipzig, Kate and her apartment in Barcelona. All these interview partners explain since owning their own homes in these cities their attachment to them has increased, and these places have become more central in their place patterns. They are thus stabilising elements in the core action spaces, and they become anchor points for place-based identification. In the same way, family foundation works as stabilising. Those of my interview partners who have small children have a clear idea about where their family home is. Henriqua thinks of Vigo as her family home, where her Dutch husband and her live in a family-owned apartment; Juliana knows that she and her family do not want to leave Berlin; Liam and his family will stay in Aberdeenshire; Qamar, even though divorced, lets one apartment in his house in Leipzig to his ex-wife so he is close to her and his son; Ronja and Stephan know that they want to stay with their small daughter in Berlin; Willi is already orientating in professional terms towards Gent, where his girlfriend and his two daughters live with him. In this sense, Henriqua explains her idea of the rather mobile 'bases' and the stable 'Heimat':

'Look, I had this idea, always, before I had a child: it doesn't matter where you are, as long as you have a job and friends, you can be happy. Now that I have the child, I start to give more importance to family and even give more credit and more... I mean, not that I love them more, but I, I just understand there are my parents also. So, but still I think the idea... You, your... both is true... your home you can take with you, everywhere, you know, if you consider... If I consider my child, my husband, and we are happy together. Well, with my child, you know, we'll be happy. [...] Yeah, and then Heimat, yeah, it's more like the roots maybe, no. This idea where... what gives you a picture, what helps you to form your character also. Or your, I don't know... or your personality, I don't know. Äh character is something maybe that is innate, you know. But of course character you can

\footnotetext{
habe. Aber in Leipzig mittlerweile mehr als sechs. Äh und... Okay, natürlich habe ich achtzehn Jahre in Bergamo verbracht, aber für mich ist Bergamo die Stadt meiner Kindheit und meiner Eltern. Also nicht, nicht meine.'

${ }^{179}$ Translated by the author from the German text: 'Ich würde sagen, Leipzig ist mein zu Hause. Ich würde aber nicht sagen, dass Leipzig eine Heimat ist. Weil Heimat eben diese Konnotation hat, wo deine Ursprünge sind. [...] Äh Leipzig ist mein zu Hause. Ich glaube, die Zeit, die ich da verbracht habe, das war ein lange Zeit. Ähm die Stadt gefällt mir sehr. Also ich mag mein Leben hier. Ich mache mein Leben hier besser als mein Leben in jeder anderen Stadt.'
} 
mold, and you can... you know... and it can get influenced or... I don't know. I don't know. It helps you' (Henriqua; lines 261-281).

Again we can see the differentiation between places that are Heimat, related to roots and socialisation, and which actually strongly influences cultural and place-based identity, and on the other hand, places which form temporary 'home bases' which may be taken elsewhere. And Henriqua reflects on the fact the place-based identification in the sense of home feelings can be detached from place in the sense that she might feel at home everywhere as long as her husband and daughter are there, too (cf. also section 7.6.3). On the other hand, she argues that the proximity to her parents would also speak for stabilising their home base in Vigo, and keeping it the central family place.

We can conclude that place-based identity is concerned with the two distinct elements of current 'home bases', which can have a temporary and mobile character, and the places which are a home in terms of biographical roots and cultural socialisation. The places of one's own home bases can change with time. Places can become additional home bases once enough time is spent there and a connection to the other central dimension of everyday life takes place (e.g. profession and people). Places of origin are fixed historically, they are not changed, and my interview partners do not add new places of such character in the course of their lives. Both these types of places are reference objects of place-based identification, and they are creatively mixed in multilocal arrangements to express identity (see also section 7.6.5).

\subsubsection{Geographic scales in identification: What about Europe?}

I also found that my interview partners refer to very distinct geographic scales when speaking about their places. This ranges from the very local level of the neighbourhood, going through the city, the region, the nation, up to the European level. As these creative knowledge workers are acting in a transnational manner, I was interested in finding out about the role of the European scale and its role in place-based identification.

What is obvious in the place-based identification patterns of my interview partners is the mixture of scales (national, regional, local) and places (integration of several places). They develop hybrid forms which always expose them to situations in which they have to explain their life's story. Federico e.g. tells me that he says he is an Italian from Leipzig. The regional level is also important to him: he distinguishes within Italy that he originally comes from northern Italy. The same is reported by Juliana, who says the she is a Lithuanian from Berlin and Mainz; or Ugo, who feels that he is a Chilean in Rome. For Birgit, only the local scale is important; she identifies as being from Kassel and Leipzig, while rather neglecting her other places, as this would confuse people even more.

It became clear that differentiation in scale reference is related to the difference of situations in a transnational multilocal life. One such difference is between being in the person's own home country or not. While being in their country, my interview partners mainly use the regional or local scale as a reference for place-based identity, while abroad they are identified and identify rather with the national scale. Thereby, I found that small places such as rural towns are mainly named along with their surrounding region or with the next larger cities in order to explain where a small 
place is located. However, I was astonished about the minor role of the European scale. Mobility clearly enhances the perception of the supra-national scale of Europe, but it does not automatically lead to a deeper identification with this scale.

For example, Aurélien refers to very different geographic scales when speaking about his own lifeworld. The local sphere is important to him, speaking about Leipzig and Nice, but even breaking it down to his own neighbourhood within Leipzig as an important territory. When he speaks about his exchange projects, he refers mainly to the regional level, speaking about Saxony and Brittany, and that he feels to be part of both. If it comes to being new to Leipzig, where he feels to be a stranger, he refers to the national level, as he perceives himself to be a Frenchman in Germany, in a different language and culture. Finally, he also draws back on the supra-national European level when he speaks about the professional work of his association, seeking for a European network of artists. In this respect, he also makes the diminishing importance of nation-states an issue in which he sees the emergence of a stronger European and regional level. However, he does not feel as though he is a European, even when he engages in European topics. Similarly, Christian perceives himself to be a European, but he thinks that this scale of identification is not practical in everyday life. The problem arises from the fact that most people identify him with a national scale, so even if he felt himself to be a European, others will always consider him to be French. Additionally, he also becomes aware of his Frenchness in small situations of everyday life, e.g. sitting in a German restaurant and not having a decanter of water on the table, or bread coming with the plates. Therefore, his national cultural socialisation always shines through. The fact that the national scale is relevant abroad also shows up when Emil speaks about identifying with Switzerland when he is in Leipzig. If people around him mention something about Switzerland, or if there are things on the news about it, or if there are even any Swiss people he meets by chance in Leipzig, he loves to highlight that he is also attached to Basel and that he feels certain empathy for Switzerland.

In other circumstances, the national scale also has different meaning. If we look at the case of Yari, who mainly circulates between the Americas, he presents a completely different view of the national scale than my European interview partners have. Yari says that local community is far more important than the national level:

'Well historically our people, the indigenous communities and the black communities have been massacred by colonialism and after the rise of the independent nation-states, quote unquote, our countries were shaped into new models of internal colonialism in which the people which keep the power in their hands are direct descendants of the colonists, now in the ex-colonies. Hence, the states and the nations, in most of the cases, have never acknowledged uh... have never made us officially a part of their national project other than merely that of a labour force, which is like a new part of, like a system of slavery. Therefore it is uh... I do not feel responsible to support these national projects which have never supported me. As a consequence I rather believe in the local communities, I rather believe in the people, in the communities, in the neighbourhoods, without supporting the national projects as such' (Yari; lines $46-55^{180}$ ).

\footnotetext{
${ }^{180}$ Translated by the author from the Spanish text: 'Bueno históricamente nuestra gente, los pueblos índios y los pueblos negros han sido masacrados por el colonialismo y después del surgimiento de los estados independientes, entre comillas, de nuestros países se crearon modelos de colonialismo interno, donde las personas que se quedaron en el poder eran los descendientes directos de los colonizadores, ahora en las ex-colonias. Entonces los estados y naciones en la mayor parte de los casos nunca han reconocido, eh...
} 
Yari passionately argues against any form of national identification as he rejects the national projects of Latina American political elites, and therefore he even draws back on the local scale. While this case is somewhat individualised for the specific background of the American history of nation building, we can still see that nearly all of my interview partners prefer to self-identify with the local scale, but they are often enough identified by others mainly on national scales. Even though they might be reflecting that the national scale is not that with which they want to identify, they are reverberated to that scale by others. This means that the European scale is actually irrelevant. Federico argues that this is a general social phenomenon: the European scale is not important to most people. He mentioned that he conceives of the creative knowledge workers, and scientists in particular, as a type of pioneer group with an increased awareness for the European dimension, as they have to travel a lot and as they work in highly internationalised labour markets. Even there people tend to not identify with Europe.

The overall pattern for my interview partners in terms of scale relevance is that the local level is most relevant when self-identifying within the country of origin. In the other places abroad the national scale becomes more relevant because my interview partners often are identified by the locals by using national references. Abroad my interview partners then trickle down through the regional scale to the local scale if the people with whom they are speaking are interested and seek for further precision. Henriqua e.g. explains that within Spain she would prefer a subnational level of Galicia, abroad she starts with Spain, then she would make it more precise upon request that she comes from Vigo. She says:

'Well, if I am abroad, I say I come from Vigo, Spain. Or from Spain. Well, if they subsequently ask where from, then you say Vigo, Galicia. If you are in Spain, you say you come from Galicia. If you are abroad, you say you come from Spain' (Henriqua; lines 71-73).

However, while Henriqua might use it, the regional level also plays a minor role for most of my interview partners. Finally, the European scale is hardly used in terms of place-based identification. The European scale is rather abstract and formal, and it has less relevance in the lifeworlds of most people. Even though my interview partners become more aware of what the European Union and Europe means through their travel, they do not refer to that scale in their identification patterns. For example Oskar, who lives in Zurich, Switzerland, Linz, Austria, and Berlin, Germany, says that a European scale is difficult to think of in practical terms:

'I think you cannot feel as a European. This is a, a difficult exercise. It is easily said. I mean, we have to deal now a lot with this European capital of culture things. And I observe, let's say, these whole European stories around not only, but also with the Euro more intensively as if I would only follow that from Switzerland. And of the endless stories is now probably, to have a European identity in a Europe of nation-states. One has a different kind of identity. For example, my daughter, if she travels around, then she already has a different identity from the one I had as a Swiss at her age, but I would doubt that this is a European one. It is perhaps a polyperspectivist one. [...] This has rather to do with the interplay between different identities. For example, I start 
to think, what's German in this situation, which I am in now? Or what is Austrian, or what is Swiss? And me myself, I am a bit outside of that, I am not German, I am not Austrian, I can understand Switzerland, but I feel... of course I say, I'm Swiss and I have the red passport... but mentally, and in several respects, I have dismissed from that country. On the other hand, I feel... I am not an expatriate. I am this other kind of traveller, who you are probably looking for in your PhD research. This is a different kind of existence. And I would have never imagined that in the past' (Oskar; lines 545-567 ${ }^{181}$ ).

The Oskar's main idea that Europe cannot be imagined for identification is also directly formulated by Valentin. When Valentin thought about a European identity, he recalled the USA as a comparative construction. He highlights the internal difference which actually prohibits identification:

'This is, because, simply because it is such a large, large country, with such huge differences, and it's the same for Europe. Somehow it would sound completely nuts to say: 'I come from Europe" (Valentin; lines 897-899182).

Finally, it can also be stated that the multilocal arrangements and the accompanying travel frequency between countries do not necessarily lead to positive opinions about the European idea. Loyal to the wider EU scepticism in the UK, Liam, for example, mentions that he acknowledges the benefits he had from the European labour market - he could improve his CV through an easily found job in Sweden, which actually resulted from EU research projects. On the other hand, he adds that he does not feel like identifying with a European scale:

'No, I wouldn't actually. No, I'm... I'm quite um loyal to my cultural roots, actually. Um... and uh, you know, even in the sort of food I like to eat and so on. I... I mean, when I'm in Stockholm I, I eat what's available. So I eat meatballs and all that stuff. Uh... but um... No, I'm not a citizen of the world. No. Or a European. And um although... uh although my career has benefited a lot from the EU, I'm not particularly pro-EU either. So, I can see lots of criticisms there' (Liam; lines 391-396).

One should also not forget that the European Union itself can be a very exclusionary project when it comes to people from the countries outside of the Union. Being derogatorily called citizens of 'third countries', these non-EU citizens feel that they are actually not identified as part of the European Union. For example, when Ugo has to deal with bureaucracy in Rome, he is continuously made

\footnotetext{
${ }^{181}$ Translated by the author from the German text: 'Ich glaube als Europäer kann man sich nicht fühlen. Das ist eine, eine schwierige Übung. Das sagt sich so leicht. Oder wir haben ja viel jetzt auch mit Kulturhauptstadt Europas. Oder ich verfolge, sagen wir, diese ganze Europageschichte nicht nur um, aber auch mit dem Euro, intensiver, als wenn ich das nur von der Schweiz aus verfolgen würde. Und eine der endlosen Geschichten ist jetzt wahrscheinlich wirklich, in einem Europa der Nationalstaaten eine europäische Identität zu haben. Man hat eine andere Identität. Oder meine Tochter, wenn die rumreist, dann hat die eine andere Identität, als ich das in ihrem Alter gehabt hätte als Schweizer. Aber ob das eine europäische ist, wag ich zu bezweifeln. Es ist vielleicht eine polyperspektivische. [...] Das hat viel mehr wiederum mit dem Wechselspiel der verschiedenen Identitäten zu tun. Also ich überlege mir dann, was ist jetzt hier deutsch, an dieser Lage, die ich vorfinde? Oder was ist österreichisch, oder was ist schweizerisch? Und ich selbst bin da wie außen vor, ich bin nicht Deutscher, ich bin nicht Österreicher, ich versteh die Schweiz, aber ich fühle mich... natürlich sag ich, ich bin Schweizer und ich hab einen roten Pass... aber geistig, oder in einigen Belangen, habe ich mich verabschiedet vom Land. Ich fühl mich aber... ich bin auch kein Expat. Ich bin eben dieser andere Typus von Reisender, der Sie dann wahrscheinlich bis in Ihre Dissertation hinein verfolgt oder ausmacht. Und das ist schon eine andere Existenz. Und ich hätte mir das auch als Entwurf nie vorstellen können.'

${ }^{182}$ Translated by the author from the German text: 'Und das ist, weil 's halt, weil's halt einfach so ein riesiges, riesiges Land ist, mit so krassen Unterschieden, und genauso ist Europa. Es wäre irgendwie total blöd zu sagen: ,Ich komme aus Europa'.’
} 
aware of the fact that he actually does not belong. As such, he explains to me that he had more difficulties in finding an apartment than EU nationals, he was not allowed to open a bank account, and currently he is not covered by health insurance, as the Italian system only covers EU nationals. While he can actually circulate freely like any other EU national, being able to pursue his work in Germany as well, he still knows and is made aware of the fact that he is a foreigner in Europe. As such, he thinks of himself as a Chilean in Rome, and he explains:

' $M e$ as a resident in Europe, I ask myself the same question, my answer is basically that I do not feel to be part of Europe, but definitely of Rome [...] yeah because I know how it works, I know the rhythm of the city, more or less I understand the mentality of the people, I have my routines, my space, I have appropriated this city' (Ugo; lines 45-50 ${ }^{183}$ ).

Looking at my interview partners' scale combinations when it comes to their place-based identification, we can see that Europe as a scale is reflected by these transnational creative knowledge workers. However, the idea of a European identity seems far from applicable even for these border-crossing people who should actually benefit from the European labour market integration and the right to free movement. The point is that the European scale is too abstract and transnational lifeworlds are still confronted with bureaucratic problems (I will go into this in detail in section 7.7.7). Furthermore, the everyday value of a European identity is not visible for my interview partners. Most other people in their places cannot deal with Europe as a scale of identification. They mainly speak of place-based identification in terms of the local and national scale. For my interview partners, it is obvious that they develop a stronger distance to the national scale, and they prefer to identify with concrete places, with the local scale; they want distance from national stereotyping. Yet, others repeatedly refer them to this context - and as such the local and the national scale seem most important.

\subsubsection{People and identification}

So far, we have looked at the role of places for identification, and this has a specific reason: during my research I was particularly interested in place-based identification, and my interviews rather focussed on that. However, in the narrations about places, the role people play also showed up several times. Actually, places not only work as reference objects in a direct way; they also become relevant in indirect ways through the people that are associated with these places.

For example, Christian explains that places become a home to him more quickly if the people in these places are open-minded and easy to access for social interaction. In this sense, he had the feeling that this works easier in Leipzig than in Dresden, where people were more reserved and it needed more effort to make contact. Therefore, he considers Leipzig home today. He finds that people there provide a social context in which he feels at ease, and this makes the place more relevant in terms of identity and belonging.

Not only the wider social community in a place works in a binding way, but also the closer social networks or the family itself define which places are more relevant than other for my interview

\footnotetext{
${ }^{183}$ Translated by the author from the Spanish text: 'Yo como residente en Europa, me hace la misma pregunta, mi respuesta es basicamente que yo no me siento perteneciente a Europa pero sí a Roma [...] que nada más que conozco como funciona, conozco el ritmo de la ciudad, entiendo más o menos la psicología de la gente, tengo mi rutina, mi espacio, me he apropiado de esa ciudad.'
} 
partners' place-based identification. Juliana e.g. says that Berlin is where she is based and where she feels at home because it is related to her husband and her children:

'But meanwhile I have actually not much nostalgia for Lithuania. Even though I have friends and family there, and I like going there. And of course there are emotions, which are linked to it. And some kind of atmospheres, which remind me of and which attract me. But my children are in Berlin. And I now have... I mean, previously I felt a stronger need to go there' (Juliana; lines 402-407 ${ }^{184}$ ).

This quote actually illustrates two things. First, it includes the aspect of temporality. Lithuania becomes less relevant with the passing of time and the changing of social ties. Second, as long as social ties exist to a place, the place remains important, although less important. Still, Juliana has family and friends in Lithuania, and this makes Lithuania remains a relevant reference for her belonging. Isabel also explains that feeling at home is not related to places themselves, but more to people. Thus Berlin, Barcelona, Saarbrücken and Ibiza are all a type of home to her as she links these places to loved ones:

'I think that this is dependent on the people. I mean, there, where the people are who give me love. If I go back to Saarbrücken, just like two weekends ago, clearly, after having lived there for twelve years, I have left a lot of people who I really like a lot, and who are very close to me. So, I try and go to pass by once in a while, in Saarbrücken, to see them again. And they give me the feeling of being at home there. And similarly, my brother in Barcelona, my mother and my father in Ibiza. [...] I have realised that it is very important for me that they remember me; that they give the feeling to love me; that they miss me, even if it hurts me if I am not there. If something important happens, I am often not there, and I disappoint people constantly, because they get used to me, and suddenly I have left. I make an appointment, and then a new job interferes and I have to leave. That happens frequently and no one likes that. Nonetheless, I intensively try to cultivate these relationships and that gives me the feeling of home. That's why I am at home in different places, because it's actually not about the places' (Isabel; lines 185-207 ${ }^{185}$ ).

Nikolina also mentions that it's more the people in places than places themselves that influence her feeling of attachment:

\footnotetext{
${ }^{184}$ Translated by the author from the German text: 'Aber mittlerweile habe ich eigentlich nicht wirklich Sehnsucht nach Litauen mehr. Obwohl, natürlich habe ich da Freunde und Eltern, und fahre sehr gerne dahin. Und da sind natürlich da Gefühle, die ich da habe. Und irgendwelche Atmosphären, die mich daran noch so erinnern und da ziehen. Aber meine Kinder sind in Berlin. Und ich habe jetzt... früher hatte ich bestimmt mehr Bedürfnis dahin zu fahren.'

${ }^{185}$ Translated by the author from the German text: 'Ich denke, das mache ich mehr von den Menschen abhängig. Also, dort, wo die Leute sind, die mich lieben. Wenn ich zurück nach Saarbrücken geh, wie jetzt vor zwei Wochenenden, klar, nach zwölf Jahren hab ich sehr viele Leute da gelassen, die ich sehr gerne mag und die mir sehr nahe stehen. Also gehe ich, versuche ich ab und zu mal nach Saarbrücken zurückzukommen, um sie alle zu sehen. Und die geben mir das Gefühl, dass ich dort zu Hause bin. Und mein Bruder in Barcelona genauso, und meine Mutter und mein Vater auf Ibiza auch. [...] Also, ich habe gemerkt, für mich ist das sehr wichtig, dass sie sich an mich erinnern, dass sie mir das Gefühl geben, dass sie mich lieben, dass sie mich vermissen, auch wenn es mir weh tut, wenn ich nicht da bin. Wenn irgendwas Wichtiges passiert, bin ich oft nicht da, und ich enttäusche die Leute am laufenden Band, weil die gewöhnen sich an mich, und plötzlich bin ich nicht mehr da. Ich mach was aus und dann kommt ein Job dazwischen und ich muss verschwinden. Das passiert sehr oft und das gefällt natürlich niemandem. Aber ich versuch trotzdem irgendwie sehr, also sehr intensiv diese Beziehungen zu pflegen und das gibt mir das Gefühl der Heimat. Deshalb bin ich ein bisschen an verschiedenen Orten zu Hause, weil: das hängt nicht mehr mit dem Ort zusammen.'
} 
'I think it really impacts me like the places... like the people that are there. And I think that these three places like I really connect with some people there, like really on an... on a deeper level. And that's like a really important function, I guess' (Nikolina; lines 626-629).

Dirk also says that the most important person now is his girlfriend and he needs her proximity. Additionally, there is also a great circle of friends in Sofia and Bulgaria now, which is mainly due to the fact that he spends most his time there. If he spentmore time in Germany, it could be the same there. In this situation, Sofia and Bulgaria are the stronger references for Dirk even though his employment formally takes place in largely Germany, the Balkans and Belarus. Thus, physical presence in places seems to interfere with the strength of social relations, and tight social relations seem to make places more important in terms of identification. On the other hand, social relations might also be maintained for the person and not for the places, as in the case of Juliana and her family and friends in Lithuania. In professional terms, Juliana identifies with Germany; in her current private life, Berlin is the most important reference. Yet Lithuania remains important for the connection to her family and friends. These examples illustrate the interference of people and places when it comes to place-based identification.

\subsubsection{Nomadic identity: Mobility as part of identification}

Mobility is also translated into the term 'nomadism'. Some of my interview partners used that term in reference to the continuous alternation between and return to their own places. The places might be those of their personal origin, the places where their parents live, the places they lived before, or the other familiar places of their current multilocal lifeworld. My interview partners come back to each of these places just like a 'nomad' who always frequents the same territories. Yet, nomadism, for my interview partners, also includes the aspect of discovery, of going to new places in order to explore. In a metaphoric sense, mobility becomes the appropriation of the unknown, which is driven by a profound curiosity for things and places which so far have only existed in their own imagination and are based on mediated stereotypes. My interview partners want to see how foreign and unknown places are in reality. Curiosity is thus a central element of the mobile lifeworlds. My interview partners want to see 'what's over there'. This curiosity does not only exist in my interview partners. In this sense, Aurélien describes what this curiosity means to him. He integrates travelling and being multilocal into his nomadic self-conception and self-understanding:

'Now, in my artistic work, I take into account this idea of nomadism which I have lived since my childhood. [...] But there one can also add another thing, finally, which is finally... which will be that uh... of someone who goes to discover new territories, you know. And I will come back to the animal term which is that of a conquest, you know, going back in the history of the human being, which means to always go and look what's going on a bit farther, in a sense of curiosity. This is always also imaginary, because as long as you have not seen the things, your imagination continues' (Aurélien; lines 106-118 ${ }^{186}$ ).

\footnotetext{
${ }^{186}$ Translated by the author from the French text: 'Maintenant, dans mon travail artistique, je prends en compte cette idée de nomadisme que finalement j'ai vécu dans mon enfance. [...] Dans cette chose là en fait tu pourrais ajouter une autre, finalement, qui est que finalement... qui va être celui que euh...qui va à des territoires à découvrir, hein. Et je vais revenir au terme animal qui est celui de voir même de, des conquêtes, tu vois, revenir en arrière dans l'histoire de l'homme, qui est celle de aller toujours voir qui
} 
To Aurélien, the fundamental curiosity in the new and unknown equals a type of nomadism just like in the beginning of mankind. This metaphor of nomadism also shows up in Kate's self-description. Kate considers herself a nomadic character, too. When she explains to me how she dwelled in her different places, she told me proudly that for a certain time she did not have her own apartment, and she commented on that:

'Well yeah, I am gypsy girl. I cannot do anything against that. A nomad. And this suits me; I like that, having no keys. [...] Stimulation, exploring, I am a very kinetic person. I am absolutely not static, just like Isabel. And she is the only person, who does something similar to what I do. Even though I probably do that a bit more extremely. Nonetheless, we have our orbits, and once in a while they overlap, and we meet in Barcelona and in Berlin, and nobody understands us' (Kate; lines $\left.113-121^{187}\right)$.

In Kate's quote we also find a hint to the fact that considering herself a nomad also means being different from others. Kate mentions that Isabel is the only person who can completely understand her, and both are regarded by others as having an extraordinary lifestyle. Thus, there is a mutual interdependence on the self-perception and feedback from other when it comes to the mobile moments in their own lives. Kate later tells me an anecdote about such moment of selfidentification. She once was asked to describe herself in five words, and she replied as follows:

'And then I have said: 'let's see what's over there!' Let's have a look what can we find there. That's what I would say, simple curiosity, yeah. And this, I think so, that I... um... it does not frighten me, or I do not feel overwhelmed. Quite the contrary; it turns me on' (Kate; Lines 309-312 ${ }^{188}$ ).

This incorporation of mobility into one's own identity through the metaphor of the 'nomad' can also become part of a discourse of distinction as the example of Valentin shows. Valentin, who travels a lot, told me in one section of his interview about a moment when he had to move somewhere again, and here he stresses the nomadic moment which is related to having few material belongings which can be taken everywhere without large effort:

'I think, I have never been... well, this somewhat unpractical for your study now, in the last three months I travelled so little, like now in the last three months. And before, I think, I have actually, just like a nomad, about one year I did not have an apartment. And this is really cool, if you, because you don't actually need anything. Basically, you only have a backpack and you can... well, you don't have books, which you all store somewhere, no furniture, relocations. In one moment, I moved from Leipzig to Berlin, and I rented such a cheap car from Wientje \& Robben's, the low cost car rental, such a Fiat Panda model, and I could transport all of my stuff. [...] And I even took two guys, car sharing. So really no, yeah it was really cool. This is definitely an aspect, this... so this

\footnotetext{
est ce qui se passe plus loin, dans un rapport de curiosité, quoi. Et de l'imaginaire, toujours. Parce-que en tant que t'as pas äh vu les choses, ton imaginaire continue.'

${ }^{187}$ Translated by the author from the German text: 'Und ja, ich bin Zigeunerin. Ich kann nichts dagegen tun. Nomadin. Und mir liegt das, mir liegt das keine Schlüssel zu haben. Ja. [...] Anregung, Exploring, ich bin ein sehr kinetischer Mensch. Ich bin überhaupt nicht statisch. Wie Isabel auch. Und das ist die Einzige, die ich kenne, die ungefähr das macht, was ich mache. Obwohl ich das vielleicht etwas extremer mache. Aber trotzdem, wir haben Orbits, die sich überschneiden, ab und zu mal, wir sehen uns in Barcelona und in Berlin und keiner versteht uns.'

${ }^{188}$ Translated by the author from the German text: 'Dann habe ich gemeint: 'let's see what's over there! 'Mal gucken, was da drüben ist! Das würde ich sagen, einfach Neugierde, ja. Und das ja, ich glaube schon, dass ich... ähm... es macht mir keine Angst, oder ich fühle mich nicht überfordert. Es ist im Gegenteil, es macht mich einfach an!'
} 
nomadic life is really funny, because you are always in new places, and... because you continuously get new input. But from a certain moment it also becomes um... exhausting, because you only live out of your bags. So it is, I think, what I tell you here, it's nothing completely extraordinary, everybody knows about that' (Valentin; lines 241-255 ${ }^{189}$ ).

For Valentin, the nomadic element is 'cool' and in his last sentence he stresses that to him it is nothing extraordinary, 'everybody knows about that'. We can see how his discourse also describes an element of distinction: being a nomad is cool, you can transport everything with you in a Fiat Panda, but yeah... it's normal, everyone does it. Valentin is quite aware of the distinctiveness of his lifestyle and the mobile element in his nomadic identity. This becomes obvious when he comes back to the negative aspects of it, in which he sees that such a life is not so normal to everyone:

'I think, through this nomadic life, you are then less caring about some relationships to friends and so on. This can, this is a bit of the disadvantage in it. You do not get involved with anything, that's obvious, because you know: 'Hey, well, in four weeks I will be somewhere else for work and...'. [...] Travelling is awesome, except for this continuous off \& on thing' (Valentin; lines 375-402 ${ }^{190}$ ).

The examples I have presented here are extreme cases and with the metaphor of 'nomadism' they overdraw to a certain extent. However, they serve to illustrate that my interview partners all deal with their mobility when thinking about their own identity. Granted, not all my interview partners would perceive themselves as nomads, but they all know that they are somehow different from a large majority of their social environment as they are far more mobile.

\subsubsection{Playing with multiple identities and social roles}

As I have already mentioned, many of my interview partners develop playful ways of identification; they show a flexible way of dealing with their identities according to situations and places. When I asked my interview partners for the way how they perceive themselves and how they would describe their identity, they quickly came up with something, most often a place or a country. This quick answer also stimulated reflection which then revealed how they strategically make use of their multiple ways of identification. Depending on the context in any given moment, these creative knowledge workers use different place-based identities in order to introduce themselves, or to be identified by others. Therefore, it can be of advantage in professional terms to use a specific identity in a certain situation. My interview partners actually let their different place-based identities work

\footnotetext{
${ }^{189}$ Translated by the author from the German text: 'Ich glaube, ich bin noch nie so... das ist halt ein bisschen blöd jetzt gerade für deine Sache, in den letzten drei Monaten so wenig unterwegs gewesen, wie jetzt in den letzten drei Monaten. Und vorher, glaube ich, habe ich halt echt, wie ein Nomade halt, fast ein Jahr lang keine Wohnung gehabt. Und es ist echt cool, wenn man, weil man halt nix braucht. Man hat halt echt nur einen Rucksack und du kannst... du hast halt keine Bücher, die man alle irgendwo lagert, keine Möbel, Umzug. Ich hab mal einen Umzug gemacht von Leipzig nach Berlin. Da hab ich mir von Wientje \& Robben's, hier so eine Billigauto-Firma da, da hat ich mir so `n Fiat Panda ausgeliehen, und damit habe ich halt alles weggekriegt [...] Und ich hab sogar noch zwei mitgenommen, Mitfahrgelegenheit. Also wirklich nicht, es war echt krass. Also das ist auf jeden Fall eine Sache so, dieses Nomaden-Leben ist ziemlich witzig, weil man halt immer woanders ist, und... halt ständig neuen Input bekommt. Aber irgendwann ist es natürlich auch ähm... anstrengend, weil du halt immer nur aus dem Rucksack lebst. Also es sind eigentlich, ich glaube, was ich jetzt so sage, das ist eigentlich nix komplett Abgefahrenes, das weiß ja jeder.'

${ }^{190}$ Translated by the author from the German text: 'Ich glaube, durch das Nomadenleben, da bist du dann irgendwann nicht mehr so intensiv bei irgendwelchen freundschaftlichen Beziehungen und so. Das kann, das ist ein bisschen der Nachteil daran. Du lässt dich halt nicht mehr drauf ein, ist ja klar. Weil du ja eh weißt: ,Hey, na gut, in vier Wochen bin ich wieder woanders arbeiten und... ' [...] Reisen ist geil, aber das ständige Off \& On-Ding.'
} 
for them. They are quite aware of the advantages and disadvantages that come along with certain places in respect to certain contexts. Playing with their multiple identities gives them control over the impression that they give to others.

Willi, for example, describes that he was quite aware of the difficulties that arise from being German in UK. Even if WWII was long ago, he sometimes had the impression that in particular the elderly looked at him in a different way when he presented himself as a German. However, knowing this, he also knew what to expect when introducing himself, and he dealt with his Germanness in the UK in a self-conscious way. To himself, his Germanness is not a big issue, because of having grown up on the outskirts of the Benelux area, he rather thinks that national identity makes less sense. Yet, in the UK he is always confronted with it:

'As a German, you always have a problem abroad, I mean you always encounter hostility, you know. Which still stems from the war, from the Nazi time and so on. That's always... it's in particular in Great Britain, where you can still find that. One can feel that there is a certain threshold, but I always tried to deal with that in an offensive way. I did not try to, because I do not feel like a Belgian, even though I spend a lot of time there, but I still feel like a German and this is my origin' (Willi; lines 297-305 ${ }^{191}$ ).

Willi knows about the problems with German identity and he tries to face them, but we can also observe a different strategy. Valentin similarly perceives being German abroad as problematic in some contexts, as he experienced in France. In the specific case of a trip through Israel he even tried to hide his Germanness, and he tried to cover himself with a French identity:

'Perhaps it is different if you are abroad, and you say: 'I'm from Berlin'. [laughs] It's funnier than saying 'I'm from Germany' or something alike, you know? So, the way how people react to that, I think. I don't know, in France sometimes it's still, in particular elder people, they still react a bit odd to that. And also in, well, I think, in Israel, there I have also... I hesitated to, for example while hitch-hiking I have told a guy that I am from France. The problem was that this guy came from France, too. [laughing] And then I have, I had to make up something quickly. And so I said: 'Well, my mother is French and my father is German and I have spent more time in Germany, so my French is a bit rubbish' [laughs]' (Valentin; lines 860-876 ${ }^{192}$ ).

These examples show how specific place-based identities are perceived and what different strategies there are to deal with this perception of the effects. On the other hand, places are

\footnotetext{
${ }^{191}$ Translated by the author from the German text: 'Als Deutscher hat man ja immer ein Problem im Ausland, also man, man stößt manchmal auf Feindseligkeiten, ja. Also die immer noch aus der Kriegszeit, aus der Nazizeit und so weiter, stammen. Das ist immer... ist ja gerade so in Großbritannien, manchmal trifft man das noch an. Also zumindest merkt man, da ist so eine Schwelle. Aber ich habe immer versucht, das trotzdem sehr offensiv anzugehen. Ich hab das jetzt nicht versucht, weil ich mich nicht als Belgier jetzt fühl oder so, auch wenn ich da sehr viel bin, aber ich fühl mich trotzdem immer noch als Deutscher und das ist halt meine Herkunft.'

${ }^{192}$ Translated by the author from the German text: 'Vielleicht ist es was anderes, wenn man jetzt im Ausland sagt, und man sagt so: ,ich komme aus Berlin'. [laughs] Es ist witziger, als wenn du sagst: ,I'm from Germany' oder so, weißt du? So, wie die Leute halt so reagieren darauf, glaube ich. Ich weiß es nicht, in Frankreich ist es manchmal noch immer, halt so ältere Leute, die reagieren da trotzdem noch manchmal ein bisschen komisch drauf. Und auch in, also, ich glaube, in Israel so, habe ich auch ein bisschen... manchmal mit mir gehadert so, da habe ich zum Beispiel beim Trampen habe ich Einem gesagt, dass ich aus Frankreich komme. Und die Scheiße war aber, dass der Typ selber aus Frankreich war. [both laughing] Und ich hab dann, da musste ich mir halt schnell mal was ausdenken, und da habe ich so gesagt: ,ich bin halt, meine Mutter ist halt Französin und mein Vater Deutscher und ich hab halt länger in Deutschland gelebt, deshalb kann ich nicht so gut französisch', [laughs].'
} 
functionally complementary (as I outlined in section 7.1). This means that they also refer to different activities and different social roles. These social roles and activities are also reflected in the playful identification patterns. My interview partners also incorporate these roles into the way of they switch between identities. Birgit e.g. uses her two locations - Leipzig and Kassel - in terms of playing with her identities, when she is introduced to other people in the professional realm:

'Certainly I play with it. I, I... normally I'd say both [Leipzig and Kassel]. Because I consider it important. Sometimes then I think about the order, for tactical reasons. Um... Yeah, at least for my self-understanding it is important that I am not only the professor [from Kassel]. And I also stress that Leipzig... I mean, I then also emphasise Leipzig for various reasons, as it is the more prominent place. If it is about a situation such as a conference, and people ask where is she from? Then I prefer to say Leipzig than Kassel' (Birgit; lines 381-386 ${ }^{193}$ ).

Birgit also differentiates her social roles according to her places. In Leipzig, she is a business woman, managing her planning office with a hierarchy in which she is the boss. In Leipzig she is also the caring daughter for her old mother and she is the significant other to her partner. She is also part of the local architecture and planning scene. In contrast, in Kassel she is mainly a colleague amongst others within the university, and she tries to avoid being involved in hierarchies. She also keeps a rather equal contact to her students, avoiding hierarchy. Similarly, Emil differentiates, according to how he is asked and in which situation, how strongly he emphasises that he also lives in Switzerland:

'There [in Basel], there I also say: Yes, I actually come from Leipzig, but I have also lived here for quite a while. And then this is always... if I am elsewhere, this is always a part of my identity which I express. I mean, here, I think I quite quickly make clear that I also live in Switzerland, or that I am well informed about Switzerland, that I am knowledgeable in this respect. [...] This is quickly a part of my self-understanding, so to say' (Emil; lines 384-399 ${ }^{194}$ ).

The way in which Emil plays with his identity only concerns nuances. Basically, he knows that he is from Leipzig and Basel at the same time. Depending on where he is, he also adds that he is from the other place, too, thereby expressing a certain expertise. However, playing with multiple identities can also have a more ironic character. Nikolina also plays with her identities if someone asks her where she is from. She actually does not like the question, as she does not have a straightforward answer to it:

'I hate that because like I basically... okay, it's really looming, because I have to like just decide basically at that time, like I am just, like, whatever. Like, I mean, sometimes I am, like, America, sometimes I am Bulgaria. And then people are like: Oh, so do you do that? Like, based on the

\footnotetext{
${ }^{193}$ Translated by the author from the German text: 'Natürlich spiele ich damit. Ich, ich... In der Regel sage ich beides. Weil mir das wichtig ist. Manchmal überlege ich die Reihenfolge. Aus taktischen Gründen. Ähm... Ja, also mindestens zu meinem Selbstverständnis gehört schon, dass ich nicht nur die Professorin bin. Und mir ist auch wichtig, dass Leipzig... also ich betone dann schon Leipzig aus vielen Gründen, weil es der prominentere Ort ist. Wenn es jetzt gerade um so eine Situation wie eine Tagung geht, wo kommt denn die her? Da sage ich lieber Leipzig als Kassel.'

194 Translated by the author from the German text: 'Da, da sage ich dann schon: ja, ich komme eigentlich aus Leipzig, aber ich wohne halt hier schon lange. Und das ist dann immer im... jeweils wenn ich woanders bin, auch Teil meiner Identität, die ich artikuliere. Also hier mach ich dann glaube ich schon relativ schnell deutlich, dass ich auch in der Schweiz wohne. Oder dass ich von der Schweiz irgendwas weiss. Dass ich da Ahnung habe. [...] Das ist dann schon sehr viel schneller ein Teil auch meines Selbstverständnisses sozusagen.’
} 
person, or like, what they wanna think about you. And it's like, really not that. Like, I just... honestly, I sometimes just try to say the thing that I think will, like. have the least questions. Like associated with it. [laughs] I really think that's what it is sometimes. Unless it's like someone that I can connect with, you know. Um... like someone from the midwest, or... or from, like, that area, from Ann Arbor, really from that area, not the whole midwest, because that's a really big area. [...] Or someone from, like, eastern Europe, I guess. Then... then I would like say I am from there, too. But if it's just, like, some German person, or whatever-like person' (Nikolina; lines 404-415).

In this quote it is obvious that playing with multiple identities can also be a tool to avoid annoying explanations of one's own multilocal or transnational situation. Federico also changes his identity according to situations. Within Italy, he pronounces that he is from northern Italy, yet when he is in Germany, he is often fed up with being identified as a foreigner, and continuously being asked where he is from. He has developed different fictional biographies that he applies in a playful way during evenings out and at parties:

'If I go to parties and if I am a bit drunk, I often invent other identities. Probably, you know that too, there is this community of foreigners around the university: Where are you from? And then I am annoyed, and I say for example that I was born in Prague, but my parents are Italians. Somehow I always have to explain my strong Italian accent. Therefore I have some [laughs], some alternative autobiographies. Or I come from Trieste, but I am a Slovene. In Trieste there is this Slovene minority. And they speak Slovenian, but they have a strong Itali... one would say, such an Italian accent' (Federico; lines 161-169 ${ }^{195}$ ).

Whereas this is an example of a more playful situation, Federico also remarks that in more serious contexts he definitely does not play as ironically, but he still plays. When asked in any professional environment for his identity, he would always stress that he is from Leipzig, and as such from Germany. Federico says that it might be a comparative advantage to be identified with a professional affiliation to science in Germany. According to him, the German academia has a better international reputation than the Italian; it indicates more money and a more serious work attitude. Thus, he consciously explicates that he works in Germany and not in Italy. He uses this German affiliation in order to make himself more respected in professional terms. The interesting thing is that his circumscription of 'alternative autobiographies' fits both these situations, the party at night and the professional environment; he grabs from his place pattern what might be opportune in any given situation. Juliana also plays with her spatial identities in her professional environment. Depending on the context and her situational mood, she highlights Berlin or Lithuania or Mainz, but in a professional context she mostly mentions that she is socialised scientifically in Germany and not in Lithuania:

\footnotetext{
${ }^{195}$ Translated by the author from the German text: 'Wenn ich aber auf Parties bin, und ein bißchen betrunken bin, erfinde ich sehr oft eine andere Identität. So, weisst du, vielleicht auch, weil da ist ein bißchen diese Ausländercommunity, die um die Uni herum ist: Wo kommst du her? Und dann bin ich genervt, und ich sage zum Beispiel, dass ich in Prag geboren bin, aber meine Eltern Italiener sind. Irgendwie muss ich immer meinen sehr starken italienischen Akzent rechtfertigen. Deswegen habe ich einige, [laughs] so einige alternative Autobiographien. Oder ich komme aus Triest, aber ich bin ein Slowene. In Triest gibt es eine slowenische Minderheit. Und die reden slowenisch, aber sie haben einen ziemlich starken itali... man würde sagen, so italienischen Akzent.'
} 
'Surely, if it is in a professional context, then it is more importan, that I come from Berlin or Mainz, you know, because this reflects my institutional affiliation, and also I was socialised as a scientist in Germany. This has only little to do with Lithuania, for instance, but if it is more about origin, then... or if I feel like disclosing it, then I must say Lithuania' (Juliana; lines 356-375 ${ }^{196}$ ).

Playing with multiple identities is not only relevant in a professional context; it is also a pattern of the private sphere and the manner of conduct with people. Isabel describes how she consciously pays attention that her Berlin and Barcelona micro cosmoses stay separated and do not become confused, as she loves to switch between these two distinctive worlds. This includes that she finds it disturbing if people come with her from one place to the other, because she feels like she has different personalities in these two places and she feels uncomfortable if people from one place see how she acts in the other place.

'Um, I feel like a girl from Ibiza, but I think, uh, in Ibiza I am a different person. Well... and in Barcelona and in Berlin I am also not the same person, I said that already. My ex-boyfriend, until some months ago, came to see me in Barcelona, and he was disgusted by me. He could not handle how I behaved over there. And I also did not want to let him into my world of Barcelona. And he said, 'You are completely different here'. And I already assumed that, I mean that I behave differently there, that I have a different character, that I am a bit a different person there. Also because I do different things and, uh, I have to do with other people. And then you become a different person, to a certain extent. And somehow unconsciously, I change my identity. I do not tell stories that are false. But I act a bit different. That's for sure' (Isabel; lines 238-248 ${ }^{197}$ ).

In this respect, Isabel also mentions that she plays the role of a substitute mother to the children of her divorced brother in Barcelona, and that she also is a more outgoing and playful person there. In contrast, she is more reflective and focussed on work in Berlin. Her places, social surrounding and identities are actually intertwined in the sense of functional complementarity. I think this section has shown that my interview partners develop various strategies to express and explain their specific and dynamic place-based identity. Whereas they are quite aware of their own place pattern, it is often difficult to make this clear to others, so they have prepared playful and flexible ways of telling others where they are from. We could also say, in the words of Federico, that my interview partners dispose of a set of 'alternative autobiographies'.

\footnotetext{
196 Translated by the author from the German text: 'Natürlich, wenn das irgendwie in so einem beruflichen Kontext ist, dann ist es relevanter, dass ich dann aus Berlin oder Mainz komme, ne. Weil es dort ja meine institutionelle Anbindung ist. Und ich bin sowieso als Wissenschaftlerin sozusagen hier in Deutschland sozialisiert. Das hat wenig mit Litauen zu tun, zum Beispiel. Aber wenn es mehr so ja nach Herkunft, dann... oder wenn ich das verraten will, dann muss ich Litauen sagen.'

${ }^{197}$ Translated by the author from the German text: 'Ähm ich fühle mich als Ibizanerin, aber ich denke, äh auf Ibiza bin ich schon unterschiedlich, also... Und in Barcelona und in Berlin bin ich auch nicht die gleiche Person, das hat man mir auch gesagt. Also mein Freund bis vor ein paar Monaten kam mich besuchen nach Barcelona, und der fand mich dort ganz eklig. Der kam nicht damit zurecht, wie ich dort war. Und ich wollte ihm meine Welt dort auch nicht eröffnen. Und der hat gesagt: Du bist anders hier, du bist echt anders. Und das habe ich schon vermutet, also dass ich mich dort anders benehme, dass ich anders drauf bin, dass ich ein bisschen ein anderer Mensch bin dort. Und eben weil ich dort andere Dinge mache und mich mit anderen Leuten äh beschäftige. Und dann ist man ein anderer Mensch, ein bisschen. Und also unbewusst schon, verändere ich die Identität. Ich erzähle nichts, was nicht stimmt. Aber ich benehme mich ein bisschen anders. Das auf jeden Fall.'
} 


\subsection{Resources \& Action: Doing multilocality in creative industries}

The central dimensions in multilocal lifeworld's perception also translate into concrete action and in particular the use of specific resources, or infrastructures, which are part of my interview partners' mobile lifestyles. I will use this section to briefly give an overview about the specific action patterns and relevant resources that have shown up in my interviews.

\subsubsection{Travel infrastructure \& routines}

My interview partners mainly use aeroplanes and trains to travel between their places. This means that the emergence of low-cost airlines and the increasing cross-border connectivity with high-speed trains are the main infrastructure that allow for mobility. As such, nearly all of my interview partners dispose of certain member cards to transport companies, such as mileage clubs and special tariff cards. Let us have a look at one such tool which was frequently mentioned: the Bahncard for the largest train company in Germany. The Bahncard exists in several forms. The entry level allows for a reduction of $25 \%$ of the normal ticket prices, then one can upgrade to $50 \%$ off the normal price of train tickets, and then there is also a version which allows a $100 \%$ reduction on normal prices. This Bahncard 100, which is valid for a year, costs about 4,090 Euros, but then it allows unlimited access to the entire national train network, including local public transport in major German cities. Seeing this amount of money, one normally would think of it as an expensive product, but counterbalancing it to the mobility demand of my interview partners, this offer is a useful tool to access an important infrastructure. Birgit, who frequently travels between Leipzig, Kassel, Berlin and Zurich, explains how she feels about her Bahncard 100:

'I have such a Bahncard 100, which I deem an extreme luxury. Uh, and what also... what also, where one actually lives this multilocality, because you simply go to the next train station and get on the next train, and you do not have to take care of anything. Uh, and this is of course uh related to a certain budget, which you have to deploy for it, and honestly speaking, I do not calculate, I would never calculate if it pays off; I simply do it' (Birgit; lines 230-235 ${ }^{198}$ ).

This example also shows that these infrastructures and tools are considered something special to my interview partners. They are often related to an increase in expenses, but they are a part of the mobile life, thus they also receive a latent distinctive function, which brings into the awareness the otherness compared to monolocal people who travel less. The unlimited and flexible access to mobility here can quickly become a symbol of distinction. Birgit emphasises that this tool is costly, but at the same time she says that she does not calculate whether or not it pays off. She underlines that she can afford it and that she is part of the mobile group of insiders. Emil also holds a Bahncard 100. He also uses this tool as a metaphor for individual mobility and he wishes he had something similar for flying. That such a wish is known to air carriers can be seen in Lufthansa's new offer of

\footnotetext{
198 Translated by the author from the German text: 'Ich habe so eine Bahncard 100, was ich auch ausgesprochen luxuriös finde. Äh und was auch so... was auch, also wo man auch diese Multilokalität wirklich lebt. Man geht nämlich einfach irgendwie nur zum Bahnhof und steigt in den nächsten Zug, und muss sich um gar nix kümmern. Äh und das ist natürlich äh mit so einem Budget verbunden, was man dafür einsetzt. Und ehrlich gesagt, ich rechne das auch nicht, ich würde niemals nachrechnen, ob sich das lohnt. Ich mache das einfach.'
} 
an hourly flight connection between large German cities for which ex ante reservation is not as important anymore. If one airplane's seats are sold completely, or if tickets are too expensive, one could simply take the next flight. This brings us to another point in infrastructures: they have to be simple and flexible at once. My interview partners want to use the infrastructures without having to think about it too much. Travel has become so ordinary in their everyday life that it should also be easy to use and fit into their everyday life; the travel planning should not require much effort.

As my interview partners all travel a lot, they have all developed their own ways of making travel more efficient and comfortable. Here, a first aspect is that my interview partners look for time efficiency. Even though they have to organise their travel on their own, also including that they have to pay it mainly from their private financial resources, they still prefer to invest more money in order to arrive quicker and to buy more comfortable passages. For example, Marta has to fly between Ljubljana and Kovno. Even though actual flight time is short, she has to change and loses time in airports. She tries to seize the travel time for reading and for working, but she tries to have quick connection in order to not spend much time in travelling:

'I have to change in the middle of Europe somewhere. Reacting to the price. It's not big difference here because it's just price, and time is important for me. So, you don't spend so many time' (Marta; lines 360-362).

Whereas Marta has to respect the capacity of her individual financial resources she does not necessarily try to save money on tickets, but she rather tries to look for the flights which allow for quick connection somewhere in Europe, even if this means spending more money. Another aspect of time saving is to develop practices which allow for reducing the time spent for individual steps within a trip. For example, Willi explains to me how he tries to save on check-in time at the airports:

'I have such a small case; I have this with me for one night. For one night I never take some of these large hard protective cases. This is one of these cases which are standardised for aeroplanes so that you can take it as hand luggage. This is quite practical, you can... so you do not have to check in luggage at the counter. You can put this directly above... this was very important to me, because coming from Birmingham, I often flew to Brussels. I mainly took the Eurostar train, but sometimes flights were so cheap that I flew, because there were direct connections between Brussels and Birmingham. And then from Brussels to Gent, that's not far, but then it was important to me that I don't lose time at the check-in; then you can check-in half an hour before instead of one and a half hours' (Willi; lines 756-766 ${ }^{199}$ ).

Tjark told me the same thing about time management in relation to flights; to make it more fun, Tjark played a game of coming as late as possible to the check-ins in airports, but after he misseda flight by a few minutes, he also lost the fun in that game:

\footnotetext{
199 Translated by the author from the German text: 'Aber ich hab so einen kleinen Koffer, den hab ich jetzt für eine Nacht hier dabei. Für eine Nacht nehme ich nie einen irgendwie so einen Hartschalenkoffer. Das ist so ein Koffer, der ist auf Flugzeuggröße genormt, dass du den als Handgepäck mitnehmen kannst. Das ist ganz praktisch, da kannst du halt... da musst du also nicht Gepäck einchecken. Den kannst du oben direkt... das war für mich ganz wichtig, weil ich bin aus Birmingham, bin ich oft über Brüssel dann geflogen. Ich hab meistens den Eurostar genommen, aber ab und zu oder so waren die Flüge so günstig, dann bin ich geflogen, weil es gab Direktflüge Brüssel-Birmingham. Und dann von Brüssel nach Gent ist es nicht mehr weit. Und dann war es mir aber wichtig, dass ich keine Zeit verliere mit Einchecken und so; und dann konnte man eine halbe Stunde vorher ganz normal einchecken, ohne jetzt anderthalb Stunden vorher.'
} 
'I had this battle for years, and it was a personal one, in which I always wanted to lose as little time as possible in the airport. This means I always tried to go to the airport at the last second' (Tjark; lines $289-292^{200}$ ).

Federico equally prefers to pay a bit more in order to travel faster and particularly more comfortably, as he uses travel time to work:

'I have changed in this respect. Ten years ago, I took far more things with me. But these were... mainly I always took a lot of books. More books than underwear. But now I also take fewer books. I think I have grown older; I want to travel more comfortably. For example, as you have asked me about trains... When I was an Erasmus student and I stayed in Leipzig, I was still studying at two universities in parallel. [...] By then, when I had to go to Italy for my exams, I often went by bus. I would not do this anymore today, no way. This is too exhausting. Compared to previous times, I also spend less time um... um, for example... I mean, I travel low-cost, but then also not so super [low-cost]. Also this thing with car sharing, no. Also because I work in the train, very often' (Federico; lines 284-296 ${ }^{201}$ ).

Next to the aspect of time, my interview partners also develop routines to increase the comfort of travel and to reduce stress during their trips. An important practice in this respect is to take as few things as necessary. Willi's and Tjark's examples already point to that: they only take hand luggage. This has also the functional aspect that one cannot get confused by where things are located between one's own places, and one cannot lose or forget that many things. Xaverio explains this as follows:

'Even because I'm really good in losing stuff. But a lot, really. Like sunglasses, keys, wallet, and stuff for dress. I really learned to be minimal for everything. Because otherwise you [thinking]... you... the risk is to, to lose everything. And then even if you miss something: 'Yeah okay, no, it's in Berlin, wow'. And so, I pay lot of attention in this stuff. I mean, I know that I have to remember every time, yeah the recharge for, the recharge for the laptop, and recharge camera' (Xaverio; lines 371-376).

Xaverio tries to minimise the things he takes with him to the most important, such as chargers for his electronic devices. This practice of taking few things and saving time on travel is also visible in the way my interview partners prepare their trips. They try to spend little time on that in order to have more time in their places and to be less involved in the actual physical travel. Gunnar e.g. explains that he always observes how much time others need to prepare travel if compared to his preparation routines:

\footnotetext{
${ }^{200}$ Translated by the author from the German text: 'Ich hatte auch einen jahrelangen Kampf, und das war ein persönlicher Kampf, wo ich immer so wenig Zeit wie möglich in die Flughafen verlieren wollte. Das heißt, ich versuchte immer in letzter Sekunde in die Flughafen rein[zugehen].’

${ }^{201}$ Translated by the author from the German text: 'Ich habe mich geändert in der Hinsicht. Vor zehn Jahren habe ich viel mehr mitgenommen. Aber waren... vor allem habe ich viele Bücher immer mitgehabt. Mehr Bücher als Unterhosen. Aber jetzt nehme ich auch weniger Bücher mit. Ich glaube, ich bin auch älter geworden, ich will gemütlicher reisen. Also zum Beispiel du hast mich gefragt, Zug und... Als ich Erasmus gemacht habe, und dann in Leipzig geblieben bin, aber trotzdem ich habe an zwei Unis gleichzeitig studiert. [...] Aber damals als ich nach Italien musste, um meine Prüfungen abzulegen und so, bin ich oft mit dem Bus dahin gefahren. Das würde ich jetzt auf keinen Fall machen. Das ist zu anstrengend. Also ich verbringe auch ein bißchen, im Vergleich zu früher, weniger Zeit ähm... äh um zum Beispiel... also ich, ich fahre schon billig, aber jetzt nicht so super[billig]... auch die Mitfahrt[zentrale] und so, das nee. Auch weil ich im Zug arbeite, eigentlich oft.'
} 
'Since I am a stem cell courier and so on, I have a certain pattern somehow, such a routine which is executed. I uh, pack my bag only the day immediately before or even the morning when I have to go on a trip, and thereby I know exactly what I will need. And because... I mean this does not make me nervous anymore if I see other people who have already packed their bags two days before departure, I wonder, you know' (Gunnar; lines 668-677 ${ }^{202}$ ).

Besides to these routines in organising and managing their travel, my interview partners also use the travel time itself. Once they have planned and prepared a trip in a time saving modus and made the trip as comfortable and less stressful as possible, the trip itself is the next stage in the mobile life. I mentioned in section 7.2 that my interview partners then even develop homely feelings in respect to the time they spend in mobile and immobile transit places. Train, aeroplanes, train stations and airports are all part of their everyday lifeworld. Being on a trip is nothing extraordinary. As such, travelling becomes less exciting, and my interview partners focus on other everyday activities during travel. As they mainly do not share the travel time with beloved ones and persons from the private sphere, and as they mostly travel alone, they use a large share of that travel time for concentrated work. And here they develop also routines concerning the individual segments of their trips. I had already mentioned in section 7.2 how Birgit uses her trips to Kassel to prepare her professional things for the university, while on her ways back, she rather tends to relax and enter into her Leipzig part of the week. Liam similarly describes his passages between Stockholm and Aberdeen:

'I tend to uh, work on the way out, on the Mondays. And then I'll work as far as... because I usually come through London... I work as far as London. And then I sleep or listen to music or whatever on the London-Aberdeen because it's usually eight o'clock at night by the time I leave London' (Liam; lines 330-333).

Willi uses his travel time as work time as well. He even knows in detail the different timetables of the trains he has to take and he knows where he has to change:

'That is pure work time for me. I have, because I do this same passage every week, I exactly know in which car I have to get in, for that it... that it is fairly empty, and at which time. I have memorised all the schedules. I know... I mean, it's complete routine. And I sit down with my laptop, doing... I get on the train, switch the laptop on and I work. I have to change once, that's not so nice, but yeah...' (Willi; lines 182-187 ${ }^{203}$ ).

\footnotetext{
${ }^{202}$ Translated by the author from the German text: 'Aber dadurch, dass ich Stammzellkurier bin, und dergleichen, habe ich irgendwie schon so `n Muster, irgendwie so Routinen, die so ablaufen. Ich äh packe meinen Koffer irgendwie immer erst am Tag davor oder am Morgen, an dem ich abreise, wobei ich sowieso haargenau weiß, was ich brauchen werde. Und durch... mich macht das also nicht mehr nervös irgendwie, wenn ich so Leute sehe, die irgendwie zwei Tage vor ihrer Abfahrt irgendwie schon ihren Koffer fertig haben, wundere ich mich, ja.'
}

\footnotetext{
${ }^{203}$ Translated by the author from the German text: 'Das ist für mich echt Arbeitszeit. Ich habe, weil ich die Strecke ja wöchentlich fahr, ich weiß ganz genau, in welchem Wagon ich sitzen muss, um das... damit der einigermaßen leer ist, zu welcher Zeit. Ich habe die Fahrpläne im Kopf. Ich weiß... Also das ist so totale Routine. Und ich setz mich echt dahin mit dem Laptop, mache... ich steige ein in den Zug, mache den Laptop an und arbeite. Ich muss auch einmal umsteigen zwischendurch, das ist nicht so ganz schön, aber ja...'
} 
Also Juliana describes that she use her weekly train rides between Berlin and Mainz for work, as the train is a silent transit place in between her busy family life in Berlin and her busy work life at the university in Mainz. Therefore, Juliana describes:

'Well, if I got up early in the morning, perhaps I sleep a bit, but otherwise I work. Also because I say, I... as mentioned before, at the university it is sometimes so stressful, but for other reasons, that you have to organise something, and you have to do the administrative things. And back home, as I said, with the family. And then the university... um, in the train it is like... yeah five hours, I cannot afford to simply sit around [laughs] for ten hours a week. Then, meanwhile I got used to it, and I realise that I can handle a lot there, but I am often tired, of course. But I force myself to work in the train, always' (Juliana; lines 324-331 ${ }^{204}$ ).

Juliana's quote also indicates the necessity to use the travel time as efficiently as possible. As time spent for travel takes a larger share in everyday life than for many monolocal people, my interview partners develop their individual strategies to seize that time. The most important thing here is indeed that the preparation takes only little. The trip itself must be comfortable and quick, and the means of travel have to provide an atmosphere which allows easy orientation and workflow. Therefore, my interview partners also are willing to pay a bit more money than for the cheapest but more uncomfortable ways of doing the same trip. They also try to reduce their luggage to the necessary minimum in order to travel less stressful and not to lose things during their trips. As such, my interview partners represent a type of nomadism, yet without their tent on the back. While they mainly travel on the same routes, which actually become routines, they do not take all their belongings. This brings us to the second specific type of practice, which I call 'ubiquity'.

\subsubsection{Ubiquity}

With ubiquity I would like to point out that my interview partners have developed practices of distributing their material belongings to their different places. Here, in particular smaller things which are necessary to handle everyday life in several places, are bought in double or multiple ways, and these things are then left in the individual places. This means that my interview partners actually have to take less and can travel more comfortable. They also cannot forget important things when they are already available in ubiquitary form in all places. Emil describes having things doubled in his apartments: toothbrushes, pyjamas, underwear, books and music CDs. He also has goggles and shorts for swimming in all three cities (Leipzig, Basel and Bayreuth), as he loves to go swimming wherever he is. Isabel has equipped her two apartments with business clothes so that she can use both Barcelona and Berlin as a basis for her professional trips as an interpreter across Europe. Marta describes a specific type of helpful ubiquity, which she does not actually prepare: her sister's clothes in Lithuania are one of the resources that she uses. As she earlier took a lot of things

\footnotetext{
${ }^{204}$ Translated by the author from the German text: 'Also, na gut, wenn ich jetzt früh fahre, vielleicht schlafe ich ein bisschen. Aber sonst arbeite ich. Auch weil ich mir sage, ich... wie gesagt, in der Uni ist es manchmal so hektisch, aber wegen anderen Sachen, dass man irgendwelche, ja, irgendwas organisieren muss, und irgendwelche administrativen Sachen da erledigen muss. Zu Hause, wie gesagt, mit Familie. Und in der Uni... ähm, im Zug ist das so... ja fünf Stunden, ich kann mir nicht leisten einfach da rumzusitzen. [laughs] zehn Stunden in der Woche. Dann, mittlerweile bin ich daran gewöhnt, und merke, dass ich da auch schon viel schaffen kann. Aber oft natürlich bin ich müde, oder so. Aber ich zwinge mich zu arbeiten, immer, im Zug.'
} 
to travel, she also lost a lot of things in different places. Today she only flies with hand luggage, only taking her laptop and some work documents. She relies on her sister's wardrobe while she is in Lithuania. Oskar describes that it is important for him to have three furnished apartments with equipped bathrooms. He explains:

'Currently I circulate between Zurich and Linz and Berlin, and to me, circulating here means that I have an apartment in all of these cities, so I am not, uh... I have ceased going to hotels or subletting, and now it's really three places, where - if you might take like that - the indicator is for me to have three places where I can find the same things on the shelf in the bathrooms. I always travel without luggage, and I try to live in each of these places as if I was autonomous there' (Oskar; lines 62-67 ${ }^{205}$ ).

Additionally, Oskar mentions that he also keeps clothes available in all these three places. And the most important items are his business suits. He therefore observes very precisely how much and which type of suits he needs in which city, and he also rearranges this distribution. Furthermore, he also bought running trainers for each of the cities, as he likes jogging. Equally, Kate distributes her belongings to her places, but Kate is very specific case; she mentioned having no apartment at all for several years. She was proud to have no keys. Nonetheless, Kate belongs - in her case, to hotels. Other than Oskar, she did not cease 'hotelling'. She actually appreciates the full service and that she does not have to take care of anything, but she also wants to give these stays in hotels a type of homely and personal atmosphere. Whereas other people around her cannot understand this practice of having no place of her own, she actually appreciates a lot the comfort of hotel service:

'Well, I don't suffer from my travelling. I often hear: 'Oh, always staying in hotels and so on.' Here I have, I have my two suitcases down here. Basically down here, one over there [pointing to the suitcase in the corner of the room]. This means I always leave two or three suitcases in each hotel, where I stay frequently. I do not need to prepare my bed. I don't have to cook. What's wrong with that? I don't get it' (Kate; lines 147-151 ${ }^{206}$ ).

Kate regularly has to work in Berlin, Barcelona, and Paris. She decided to always go to the same hotels, and as she is known meanwhile, she stores her personal belongings in the hotels. Shortly before arrival, the staff of the respective hotel prepares her suite and brings up her suitcases, which contain her cleaned clothes. For Kate this makes more sense than hiring apartments in all these cities as she cannot predict presence and she does not want to care for an apartment (cleaning up, cooking, laundry, etc.). Leaving her suitcases is a similar type of ubiquity as Oskar's bathroom shelves. Both examples, as well as the others above, show that my interview partners equip their places with their things for everyday life so that travelling is free of carrying everything around.

\footnotetext{
205 Translated by the author from the German text: 'Jetzt beweg ich mich praktisch zwischen Zürich und Linz und Berlin. Und bewegen heißt für mich jetzt, also ich hab in allen drei Orten eine Wohnung, also ich bin nicht, äh... ich hab das mit den Hotels und so aufgegeben und mit Untermiete, und in Wirklichkeit sind`s jetzt drei Orte, wo - also wenn's so wollen - der Indikator für mich ist dann immer drei Orte, wo im Badezimmer auf dem Tablar dasselbe steht. Also ich reise ohne Gepäck, und versuche an jedem Ort dann so zu leben, wie wenn ich autonom wäre dort.'

${ }^{206}$ Translated by the author from the German text: 'Also ich leide nicht unter meinen Reisen. Ich höre sehr oft: ,Oh, immer in Hotels bleiben und so.' Ich habe hier, ich habe zwei Koffer hier unten. Eigentlich hier unten, eins da [pointing to the suitcase in the corner of the room]. So, ich lasse immer zwei oder drei Koffer in jedem Hotel, wo ich öfters bin. Ich muss mein Bett nicht machen. Ich muss nicht kochen. Was ist schlecht daran? Ich verstehe das nicht.'
} 
This ubiquity does not only concern the small things such as clothes or toiletries; it can also be applied to the actual tools for doing one's work. Aurélien uses a specific type of having things in a double manner; he has implemented completely equipped ateliers in both Leipzig and Brittany, in which he has all his tools and materials for doing his artwork. This allows for the flexibility to travel spontaneously without having to carry things with him, or without having to transport these tools in a long car ride:

'In fact, the more time goes by, the more I know how to shorten the travel time. I will probably spend more money, but I prefer it, because it equals a gain of energy. Still sometimes if it is about mobility of materials, not only of me as a person, I choose to go by car, but I avoid it as much as I can, more and more. Because now for example, I just installed a workshop in the garage of my parents' in Brittany and I decided to buy everything a second time. All my working tools are in Brittany and in Leipzig, because this makes it possible for me to travel without carrying everything around with me' (Aurélien; lines 256-263 ${ }^{207}$ ).

Maintaining items and tools for work in a double way can also have another functional value. It helps my interview partners to imitate everyday life routines and time structure from one place in the other. Liam has an office space in Stockholm, but for his telework hours he also has an office room in his private house in Scotland where he tries to structure time as in the office:

'I basically work um... I try to work a 9-5 day. Uh, and have lunch at a set time and coffee breaks at a set time. Because um I think you have to have structure if you're working at home. Otherwise there's too many temptations' (Liam; lines 230-233).

Another aspect of ubiquity concerns the use and consumption of services. There is a set of things that I found to be mentioned as relevant by my interview partners. The most important service was access to telecommunications. Nearly all of my interview partners had several SIM cards from different countries. Some changed these SIM cards when crossing the border; others used several mobile phones. This ubiquity in terms of telecommunication services is necessary to maintain relations to local networks in different countries. Then there are bank accounts: many of my interview partners maintain bank accounts in different countries, as employers and clients often still expect to get national bank details. Furthermore, bank accounts are also important when looking for apartments. Bank accounts indicate stable income and they signal to landlords that the rent will most probably be paid. Then local public transport subscriptions for various places are important to maintain access to flexible mobility.

I think this section has highlighted how ubiquity is used by my interview partners to actually allow for their frequent travel. It is a practice of distributing things in a multiple way to all relevant places. As such, it also allows my interview partners to feel at home to a certain extent, because they find their personal belongings upon arrival. And most importantly: they can leave their personal belongings upon departure, and they know that 'their things' are in 'their places'.

\footnotetext{
${ }^{207}$ Translated by the author from the French text: 'En fait, plus le temps passe, plus je sais de, de raccourcir le temps de voyage. Je vais peut-être mettre plus d'argent, mais je préfère, parce que c'est un gain d'énergie. Mais de temps en temps, quand il y a mobilité, pas que de moi-même, mais aussi matériels, je optais de prendre ma voiture. Et j'évite au maximum en fait, de plus en plus. Parce que le fait que par exemple là je viens d'installer un atelier de travail dans le garage de chez mes parents en Bretagne, et j'ai décidé de tout acheter en double. Tous mes outils de travail sont en Bretagne et à Leipzig. Parce qu'il fait que j'ai plus besoin de les trimballer avec moi, de les prendre avec moi.'
} 


\subsubsection{Dwelling resources \& practices}

Whereas the section before described how ubiquity is used to produce stability and comfort in a mobile life by equipping the regularly visited places with their own belongings, there are also places which are less regularly and less frequently visited. To my interview partners it does not make sense to include all their places into the ubiquity pattern. Concerning some places, physical presence is less predictable or also seldom enough to use another important action pattern: flexible dwelling practices. This practice refers to the different ways in which my interview partners use to stay overnight in places where they pass by less often. Whereas Kate's example above showed that hotels are one possible example of such practice, my interviews revealed a larger variety of lived forms of staying overnight. Aurélien lists Paris as one of his places. When I asked him how he organises his stays in all these places, he explained me, that for example in Paris he always stays at friends' places. He doesn't maintain his own apartments in Brittany and Nice; his parents have their own apartments at which he can stay once he is in both of these places. He only maintains his own apartment in his apartment building in Leipzig. To Aurélien, the question of artist residencies has become a personal professional issue; he considers residency programmes one of the important artist specific infrastructures that allow for mobility. He believes that artist residencies are too bureaucratic as they exist today. Applying for a room takes a long time, and thus can even become hindering for mobility. His solution to this problem is to offer rooms in his building for incoming artists who spontaneously need a place to stay and work. As he also stays at friends' places in Paris, he considers this exchange system of giving and taking a room to stay overnight as a new form of economy, which takes place outside of traditional markets:

'It is about always having a free room for someone who needs to come spontaneously. [...] To work in Leipzig, for example. And like this establishing different places across Europe, where this possibility is provided, too. And why not in a sense of uh... how to say that, also of professional assistance. You know, having this kind of relationship. That's what I am starting to do in Paris. I have some artist friends who will house me for the time of an exhibition, because I will probably organise something in Paris, well, because someone also lets me use his apartment. Or there is a kind of exchange, of apartments which can be organised. This is also a completely new form of economy which one can set up' (Aurélien; lines 296-305 ${ }^{208}$ ).

This quote illustrates that the multilocal creative knowledge workers try to create new and flexible models to provide accommodation in different cities independently from the market. Besides traditional artist residencies, more informal practices like staying at friends’ places or exchanging apartments with people from other places is also practiced. Artist residencies are also used by Christian if he wants to work one some new projects. Christian also mentions staying at friends' places if possible, and he tries to invite these friends to his home as well so that a certain mutuality is established. This practice of staying with friends, colleagues or other acquaintances also has a

\footnotetext{
${ }^{208}$ Translated by the author from the French text: 'C'est d'avoir toujours une chambre de libre, pour quelqu'un qui a besoin de venir en dernière minute. [...] Travailler à Leipzig par exemple. Et ainsi de mettre en place des différents lieux en toute l'Europe, où né aussi ce, cette possibilité là. Et pourquoi pas dans un rapport äh... de, comment dire, de, d'entraide professionnelle aussi. Tu vois, avoir ce rapport là aussi. C'est ça que je commence à faire sur Paris. J'ai des amis artistes qui me vont héberger le temps d'une exposition. Parce-que j'organise quelque chose à Paris, bon, parce-que quelqu'un me prête son appartement, ou il y a un échange de, d'appartements qui se peut faire. Ça c'est aussi toute une nouvelle économie que on peut mettre en place.'
} 
social value, as it provides easy access to local everyday life. If my interview partners arrive in new places and they do not want to feel like a complete outsider, like a tourist, they prefer to stay at local people's places. Federico also perceives that his type of travelling is different from the one of his older colleagues, who prefer to go to hotels and who would not host other colleagues in their own apartment:

'Even though I work now, I am employed, I still use this way of travelling, which is a bit like a student. Uh, this means, I expect that, if I uh, need accommodation, my colleagues have to shelter me more or less. I don't even expect that they have much time for me. If they are stressed, uh, I actually do not want to be their guest. Um, but I need a bed. Yeah. Uh, and vice versa; I am always ready to host people at my place' (Federico; lines 315-320209).

In this sense Xaverio also reports that upon arrival in Berlin he decided to share an apartment with Germans only. He did not want to share with people from abroad, but he sought contacts to Germans in order to learn German and get to know the city and the country in a more profound way.

My interview partners also mention that furnished accommodation plays an important role. For example, Birgit always stay at a hotel in Kassel, where she always gets the same room and where she says to feel at home meanwhile without having the burden of her own household. Gunnar also chose to live in a furnished room in Leipzig, as he always expected to leave again after a while and he did not want to furnish a complete apartment. The same accounts for Emil and his furnished apartment in Bayreuth, where he only has left few personal things such as his toothbrush, some clothes and music CDs.

In addition the use of hotels, residency programmes, furnished apartments and staying at family and friends', I also found that some of my interview partners actually share rooms and apartments with other. This does not refer to the type of room-sharing where different rooms of an apartment are inhabited by different people. From the perspective of my interview partners sharing refers to actually sharing the same room with another multilocal person through a mutual coordination of presences and absences. If one person is not there, the other can use the apartment and vice versa. In this sense Xaverio, organised his dwelling in Rome as well as in Berlin. His sharing partner is a multilocal historian who also circulates between Rome and Berlin:

'I had to, to pass just a period here in Milan, so I decided: Okay, I left... I left the apartment [in Berlin] and then I would go back looking for something new. After me, went like, a French uh student, and that left immediately. And then went a very good friend of mine, just for case, you know. He took my room. And he is doing like a PhD in History. He was here to visit me three days ago. And uh the funny thing is that he is living between Berlin and Rome. So, normally when I'm going there, just we are dividing the flat, with him, and the, the German guys. Because it's quite big. And uh it was quite the same with him in Rome. Because he has like a flat of a friend of him,

\footnotetext{
${ }^{209}$ Translated by the author from the German text: 'Obwohl ich jetzt arbeite, also angestellt bin, habe ich noch eine Art zu reisen, die ein bißchen studentisch ist. Äh das heißt, ich gehe davon aus, wenn ich äh Unterkunft brauche, müssen mich meine, müssen mehr oder weniger mich meine Kollegen aufnehmen. Also ich verlange nicht, dass sie Zeit mit mir verbringen. Wenn sie gestresst sind, äh ich will gar nicht der Gast sein. Ähm aber ich brauche ein Bett. Genau. Äh und umgekehrt bin ich jederzeit bereit, Leute bei mir äh zu unterbringen, oder unterzubringen.'
} 
that uh now he is not anymore in Rome, but sometimes he has to go back, so there is a room for him. And then we were like sharing the room there. [...] Even because it's really difficult to manage like three places. And uh... uh I mean in this case, it's uh the cheapest way to, to go on. Because I cannot permit to pay three normal rents' (Xaverio; lines 273-293).

Finally, some of my interview partners even told me that they used very exceptional practices to bridge certain periods in which they have difficulties organising other forms of dwelling. Valentin spent some summer months working in Berlin. As he did not have any money to rent a room or an apartment, he also stayed at friends' places. Some of his friends did not have large apartments, so Valentin actually slept on their balcony using his camping equipment. This camping equipment was also helpful to him in Brisbane. While he worked in a small company for some months, he looked for a pragmatic solution to dwelling. Through a friend he heard about a woman who offered to let him put his tent in her garden, so he actually lived in this lady's garden for his time in Brisbane. Qamar mentioned that when he arrived in Germany, he did not have the money to rent his own dwelling, so he stayed for some months in vacant apartment buildings in Leipzig. While these two examples have a rather anecdotic character, they still serve to illustrate that my interview partners all decided to apply dwelling practices which are different from a traditional understanding of dwelling. My interview partners do not need their own rented apartment or property where they 'nest'. They apply a broad range of different practices.

An important issue in dwelling consists in finding the right balance between flexibility and stability. My interview partners seek to have their 'own' places in order to achieve an everyday life character and a homely feeling, but they are willing to accept staying at other people's places if it fits into the situation better. In this respect, one should not forget that the maintenance of one's own household also comes along with a lot of effort, and with the necessity for relatively regular presence. As such, renting or buying homes is not always the most suitable option. Coping with the organisational effort of running homes is often impossible for my interview partners. For example, Isabel has two apartments, one in Berlin and one in Barcelona. Equally as Birgit remarked, Isabel also mentions that having an apartment is work, and an apartment needs care, which she is not able to always do well. In one passage of the interview she explains the situation in her Berlin-based apartment when she arrives:

'I first have to throw away or save all the plants, provided they are still alive. I always have forgotten some oranges or apples, and it smells somewhere. I forget some food in the fridge, so that it has fur on it, and I always have to check everything. Or a handyman came by, and I had forgotten the appointment. Now that I was absent, the chimney sweeper came to Berlin, and I wasn't there. Uh, or the telephone broke, because lightning struck. This means I always have to check everything that has happened, and I have to clarify issues, and somehow these apartments need a bit of caretaking' (Isabel; lines 250-258 ${ }^{210}$ ).

\footnotetext{
${ }^{210}$ Translated by the author from the German text: 'Ich muss erst mal die ganzen Pflanzen wegschmeißen oder retten, wenn sie noch leben. Immer hab ich irgendwo Orangen vergessen, und Äpfel, und es riecht schlecht irgendwo. Das Essen, im Kühlschrank habe ich irgendwas Komisches vergessen, dass ein Leben sich da entwickelt hat. Und ich muss immer alles überprüfen. Oder ein Handwerker war da, und ich habe einen Termin vergessen. Jetzt, während ich weg war, kam der Schornsteinfeger nach Berlin, und ich war nicht da. Also das passiert immer oft. Äh oder das Telefon ist kaputt gegangen, weil da irgendwo ein Blitz eingeschlagen ist, also ich muss immer überprüfen, was da alles passiert ist, und Sachen richtig stellen. Und irgendwie Wohnungen brauchen schon ein bisschen Pflege.'
} 
Given all these dwelling practices and the different resources which my interview partners use, there is certain irony in the data on residential registrations. A good example for this is provided by the case of Dirk. He primarily lives in Sofia, in the apartment he rented for himself and his Bulgarian girlfriend and which is the centre of his everyday life and his family life. On the other hand, he is not registered there, and his Leipzig residential situation differs quite strongly as well:

'I mean in terms of residence I am registered in Munich, and I also have an apartment there which I rent out currently as I don't use it. Um, and in Leipzig I have an apartment, or I should say I use an apartment. This happened because first, the institute of course wanted the employees to be installed somehow in a place. Second, I also wanted that I can come as I want. Without... no matter how often I am here, but something which is simply there, where I can come. And when I started the job, I looked for what I could find in terms of sharing an apartment, and the solution that it has become is that I can use an apartment from a colleague of mine, a journalist actually, who has gone to Berlin for work but who did not want to abandon her apartment. She looked for someone who might live there, or who wanted to use it with her, and meanwhile she has come back to Leipzig, but she spends more time at her boyfriend's place. So basically I use this apartment when I am here' (Dirk; lines 88-99 ${ }^{211}$ ).

Dirk's quote highlights the dynamic that exists in dwelling practices. Things change quickly and are organised in flexible ways which do not necessarily pass through market institutions nor will they become visible in bureaucratic and official registration. From the perspective of the German administration, Dirk lives in Munich and commutes to Leipzig, but this does absolutely not reflect his everyday life practice, in which he actually lives in Sofia, spends some short periods in Leipzig, but works most of the time across eastern Europe. The same accounts for Willi, who is registered in Aachen in a single household, but his empirical reality actually consists in a multilocal situation in which his main place is his family home in Gent.

This section has shown that dwelling practices are flexible as well. They can take different forms and they are differentiated according to the relevance of places. However, as the place pattern of my interview partners is dynamic, the dwelling practices also change with time. Sometimes they even change quickly so that it is actually difficult to use 'dwelling' as criterion of definition for multilocality. Dwelling does not necessarily consist of renting an apartment; there are various other forms of staying overnight. Furthermore, the variety of dwelling practices of these multilocal creative knowledge workers hardly shows up in official statistics and registries.

\footnotetext{
${ }^{211}$ Translated by the author from the German text: 'Also ich bin wohnsitzmäßig gemeldet in München, und habe da auch eine Wohnung, die ich dann halt jetzt vermiete, weil ich sie nicht selber nutze. Ähm und in Leipzig habe ich eine Wohnung, da bewohne ich eine Wohnung mit. Das war so, ja, erstens wollte das Institut das natürlich, dass der Mitarbeiter hier auch irgendwie installiert ist. Zweitens wollte ich das auch, dass ich einfach kommen kann. Ohne... also egal wieviel ich hier bin, aber das da einfach was ist, wo ich kommen kann. Und dann hatte ich so mit Dienstantritt hier gesucht, was gibt es, so im Sinne von WG. Und die Lösung, die es jetzt halt geworden ist, ist äh eine Mitnutzung einer Wohnung von einer Journalistenkollegin eigentlich, die damals nach Berlin ging, um da zu arbeiten. Und die aber ihre Wohnung hier auch nicht aufgeben wollte. Und dann hat die jemanden gesucht, der da wohnt, oder mitwohnt. Und die ist zwar inzwischen schon wieder in Leipzig, aber ist mehr bei ihrem Freund. Und so im Prinzip nutze ich die Wohnung, wenn ich hier bin.'
} 


\subsubsection{Workplaces and working practices}

The way in which my interview partners organise their work environments in their different places also reflects the flexible use of different resources. As most of my interview partners have no obligation to spend a complete working week in one and the same office, they can work from nearly any place where they have a table on which to put their laptop and which offers access to the internet. I have already mentioned that for many of my interview partners, their mobile transit places provide such an environment, although there are also other locations which equal these possibilities to work.

A repeated infrastructure consists of public libraries. Libraries mainly offer access to the internet once one has obtained membership. They often have a small restaurant, and they also allow for concentrated working. Therefore, Willi mentions that he goes to work on his $\mathrm{PhD}$ thesis in the city library of Gent, because he only has his own office in Aachen. Emil similarly uses the libraries of Basel, Leipzig, Berlin and Bayreuth for work, and Federico mainly works in the National Libraries in Prague and Trieste. Federico also explains that he would consider libraries the co-working spaces of scientists in the field of humanities:

'Our co-working space is the library, you know. The national libraries all have internet now. They are very comfortable. Yeah, I think, it's like that. Because if I think of Prague, I always go to the National Library. If I am in Ljubljana and Trieste, I do the same. If I am in London, I always go... or I can go the British Library' (Federico; lines 348-355 ${ }^{212}$ ).

Therefore, libraries not only provide valuable books for humanities' scientists, they are also a type of flexibly accessible workplace. Aurélien, rather representing the perspective of artists, stresses the role of co-working spaces that are currently developing and emerging in European cities. He likes the philosophy of having standardised infrastructures in different cities, which, by providing internet access, allow for easy access to the personal virtual network. On the other hand, they permit networking on a local level, within the micro-cosmos of the individual co-working space. Aurélien is starting to collaborate with one such co-working space in Leipzig. He wants to link his cultural association with a recently opened co-working space in order to allow incoming artists who partake in his flexible residency programme to access a flexible office infrastructure which he does not offer in the frame of the residency programme. Pia also went to work in co-working spaces in Berlin, but she had some negative experience with people who stole ideas and concepts from her. Despite this, she thinks that co-working spaces are a good idea for organising workplaces in creative industries. Her architecture firm is actually based in Copenhagen, where a large office space is available, but Pia spends most of her time in Berlin. She has access to office space in the university as she is currently employed there as well. On the other hand, she would like to establish her architecture firm in Germany, so she is currently re-organising her workplace in Berlin. She plans to share a rented office with a friend, just like a private co-working space:

\footnotetext{
${ }^{212}$ Translated by the author from the German text: 'Unser Coworking Space ist die Bibliothek, weisst du. Die Nationalbibliotheken haben alle mittlerweile Internet. Die sind sehr gemütlich. Ja, ich glaube, das ist so. Weil wenn ich daran denke, wenn ich in Prag bin, gehe ich in die Nationalbibliothek. Wenn ich in Ljubliana und Triest bin, mache ich dasselbe. Wenn ich in London bin, gehe ich immer... oder kann ich in die British Library gehen.'
} 
'The way how we plan that is simply that we want to hire a larger office where we can sit together. Um because we think that it is quite good to have some come and go, and mainly because Ronald, the renovator, um has other competences than I have, and we already advise each other in our projects. And then we can directly sit together, and probably also develop projects together in Berlin, by throwing together what we both can offer, so to say. And this has... I can imagine that this works well on the personal level if we share an office' (Pia; lines 632-639213).

Isabel says that she rented an office space for some years, but she quit it again, as she never went there to work. Now she organises all her business administration in a mobile way, e.g. faxes that arrive to her number are also sent to her email account. She has also developed the habit of not caring about reading the arriving post in one place right away, but letting this reading wait until she is present at the respective place. For her work, interpreting, she does not need office infrastructure or a desk anywhere. The office she had rented was also a type of co-working space infrastructure, because it was part of a nationwide chain of office spaces called Ihr Büro, where one can buy the service of renting in different German cities. She already used that service during her time in Saarbrücken; now she uses cafés as workplaces, where she says that she is more inspired being outside on the street and seeing other people:

'I go to a café and I work there, I drink tea ad nauseam, and I enjoy it. Here cafés are so comfortable, much nicer than in Barcelona actually, and I love that. It inspires me. I also write, and many funny novels have their origins in cafés, because I enter into this small world where I observe the people, and then it's fun to write and work there. Yeah, I don't need an office, I think. I am far too curious and unsteady to sit in an office. And I don't meet clients in my office either; I arrange everything by telephone' (Isabel; lines $445-452^{214}$ ).

Kate tells me about the infrastructure which is provided by the film companies for which she works: she uses the dubbing studios as office space. She sometimes really has her own office there, sometimes she only has a desk somewhere in the building. However, she does not need more than that. In the same way, Federico mentions that he also has access to temporary office space in the research institute abroad which is collaborating with his Leipzig-based employer or where he has personal contacts to other researchers.

These examples show that my interview partners have also developed practices to secure efficient and stimulating work environments in their places. As they are mainly employed by local employers, their professional environment has a different character than e.g. in the case of expatriates who are sent abroad to a branch office by their employer. My interview partners mainly

\footnotetext{
${ }^{213}$ Translated by the author from the German text: 'Wie wir das planen, ist einfach, dass wir ein etwas größeres Büro mieten, wo wir eben zusammen sitzen können. Ähm weil wir auch glauben, dass es ganz gut ist, also `n bisschen Publikumsverkehr zu haben. Und vor allem einfach weil also Ronald, der Sanierer, ähm hat andere Kompetenzen als ich, und wir beraten uns eh schon oft mit unseren Projekten. Und da können wir uns auch gleich zusammensetzen, und vielleicht auch zusammen was entwickeln in Berlin, einfach in dem wir zusammenwerfen, was wir haben sozusagen. Und das hat... das kann ich mir aber einfach auf so 'ner persönlichen Ebene gut vorstellen, dass wir uns zusammensetzen irgendwie.'

${ }^{214}$ Translated by the author from the German text: 'Ich setze mich in ein Café und arbeite von dort aus, trinke Tee bis zum Abwinken, genieße es. Hier sind die Cafés so gemütlich, viel schöner als in Barcelona eigentlich. Und ich mag das. Es inspiriert mich. Ich schreibe auch, und viele lustige Geschichten sind einfach so in Cafés entstanden, weil ich tauche in eine kleine Welt ein, und beobachte die Leute, und dann macht's sehr viel Spaß dort zu schreiben und zu arbeiten. Ja, und ich brauche kein Büro, ich denke, ich bin viel zu unbeständig und neugierig und unstetig, um in einem Büroraum zu sitzen. Und ich treffe mich ja nicht mit meinen Kunden in einem Büro. Das wird alles übers Telefon geregelt.'
} 
work for employers who are monolocal and who do not possess office space elsewhere. This means that my interview partners have to organise their workplaces on their own. These workplaces have to provide flexible use and they should be easy to access. Furthermore, the access to internet is relevant in order to maintain connection to their other places at a distance. Finally, in respect to workplaces, my interview partners develop individual solutions which, to a certain extent, bypass pure market transaction (e.g. the private co-working space of Pia; cafés and libraries as workplaces).

\subsubsection{Place appropriation and orientation}

A basic problem in a multilocal life is the integration of new places into the individual place pattern. Here, my interview partners describe the situation of arriving at new places and how they deal with that. The main problem consists in the fact that all places work differently, as my interview partners experience. This is mainly due to the fact that local people have developed a type of common local culture which always has to be understood and learned upon arrival. So, although my interview partners might have routines during travelling, they cannot apply this routinised behaviour to all of their places. They actually have to adapt to new places when they first arrive. Adaptation is a key to appropriation, which I understand as the process of making any place one's 'own place'. In this sense, Christian describes what he feels when he comes to new places for the first time. When I asked Christian if moving around has become routine to him, he responded that it has not; it is always rather difficult to be new again. On the other hand, it is an important topic for his artwork:

'Each time that you move, it's always a new difficulty. I don't think that one can say that you can have a habit of relocating. There is always a... every new place is always a new pitfall, one has to re-install one's own personality, one has to find purchase again. One is always a different kind of person, because this depends on what others reflect. Uh, therefore, yes, I don't think... When I came to Germany, I didn't speak any German, and I couldn't be who I was elsewhere. And even if I moved around in France, when you arrive to a new location, the mentalities are different, the systems are different, people already know each other. You are always a stranger again. You always have to re-install. Therefore, the question of the stranger is that of the strangeness which comes along, to use Albert Camus, for instance. That's a question which I am highly interested in and which is part of my artistic work, mainly the poetry that I write' (Christian; lines 114-125 ${ }^{215}$ ).

Mainly appropriation consists of a step of social orientation and a subsequent step of constructing new social ties. In the transnational context, this first step involves things such as language learning and dealing with institutions. It is similar on a local level, even in mono-national contexts one has to learn the local culture and rhythm of life. Whereas Christian makes this type of appropriation an

\footnotetext{
${ }^{215}$ Translated by the author from the French text: 'Chaque fois qu'on déménage c'est toujours une nouvelle difficulté. Je pense pas que on peut dire quand on a l'habitude de déménager. Il y a toujours un... chaque nouvel endroit est toujours une nouvelle difficulté, on doit réinstaller sa personnalité, on doit retrouver ses appuis. On est toujours un peu une personne différente, parce qu'on dépend de miroir des autres. Euh donc, oui, je pense pas... Quand je suis venu en Allemagne, je ne parlais pas du tout allemand, et je ne pouvais pas être ce que je suis ailleurs. Et même si je déménageais en France, quand on arrive dans un nouvel endroit, les mentalités sont différentes, les systèmes sont différents, les gens se connaissent. On est toujours un étranger, à nouveau. Et on doit toujours se réinstaller. Du coup la question de l'étranger est de l'étrangeté qui va avec, pour peut-être prendre Albert Camus. C'est une question qui m'intéresse et qu'est assez prédominante dans un partie de mon travail, notamment dans la poésie que j'écris en fait.'
} 
artistic theme, namely that of a hut that he always builds anew, my other interview partners do not make this issue similarly central in their professional life. Nonetheless, they are also affected by the necessity to orientate and appropriate. A section in Henriqua's interview also reveals that this problem not only affects new places, but also ties to former places can weaken, and one would have to re-install them:

'We emigrate, in a way we emigrate. I mean you can say you move temporarily, but you emigrate. When you emigrate, you go through this process, where is that you go to another country, you can get used to it or not. I mean this depends on the person. I am a person that always got used quite easily, but I am flexible in this respect, also moving from one house to another. So, this depends on your personality. But I think if you move, you, you get in this country, then you go through this process that you reject things, because you think: oh my god, look these people, they are like square, and how do they think, and they have so many topics. And you start to think: oh yeah, but we have also topics about them, and you know this kind of things. And then, when you go back, things were not any more like when you left them. And, and, and then you go through another process that you have to re-accommodate yourself. You thought, maybe it was easier to come back, and everything will stay like it was. But it's never like that. And I met many emigrants in my family, friends, and it's something they always have been through. One difference, for me personally, is that when I go to Vigo, I can uh, adapt faster, you know' (Henriqua; lines 373-386).

This moment of arriving in a place and not feeling at home causes a specific kind of mental state; a person has physically arrived, but psychologically does not yet feel as though they are in their own place. In terms of perception, the place is a intermediary place. It is not yet their own because one does not yet know how everyday life works and one is not yet integrated in the local community. However, the place is neither a completely foreign place, in the sense of a place on visits as a tourist. The arrival has a certain long-term perspective. The multilocal creative knowledge workers, when arriving in a new place, enter into a passage through which the unknown place is transformed into their own place. Various things are helpful for this passage. First, people often arrive for professional projects. These projects are immediately a starting point for social insertion and integration in a new place. Once such nucleus of social integration is activated, a further social integration outside of the professional sphere might start. Second, buying property can enhance appropriation as it directly forces someone to engage with local institutions as well as the neighbours or the neighbourhood in general. Third, existing social ties can be an anchor for place appropriation. If a person already knows someone in the place, this someone can equally work as a catalyst for social integration and orientation.

Throughout all this passage from an unknown to one's own place, the multilocal people continuously look for all kinds of things to relate to the new place. A fundamental curiosity and open-mindedness goes along with humbleness. People want to discover how social life works in this new place, and this means they do not want to impose their own mentality on local people as it might inhibit integration. This means that multilocal people have learnt to defer their own personality and cultural background in order to be able to enter a new cultural and social environment. During this time, the formerly appropriated 'own' places work as a psychological anchor point. Imaginative and virtual travel play an important role in maintaining emotional stability. Upon arrival in new places, the loved ones are often far away, and the new place of physical presence is more characterised by a feeling of being alone. Thus, keeping contact to the 
loved ones elsewhere is an important source of the energy which is necessary to establish one's own action space in the new environment.

Practically, this establishment of one's own action space takes often place by walking around. Many of my interview partners explained me that they loved to walk around their new environment. Some even mention that 'getting lost' is the key to discovering a new place. Getting lost means that one explores a spatial surrounding, but one also has to interact with the social environment. One actually has to start communicating with the local people, asking for directions, and as such starts to feel this new place. As time goes by, my interview partners then explain that the passage of making an unknown new place and their own place comes to an end when they develop place-specific new activities. When my interview partners have developed their everyday life routines for a new place, the unknown place has finally become their 'own' place. Many of my interview partners then name examples for such place-specific activities which makes places 'their' places. Birgit goes to lunch and dinner in the cafeteria in the Kassel university, whereas she never does so in Leipzig; Dirk uses his bicycle to get to his workplace in Leipzig, whereas he only walks in Sofia; Emil goes to theatres in Basel whereas he rather attends classical concerts in Leipzig; Isabel goes out to party in Barcelona and she rather reads and writes in Berlin. This list could be continued. The main point here is that once one of my interview partners has discovered which place-specific activities fit into their preferences and interests, the place's specificity is also incorporated into their own lifeworld, and the place as such is appropriated to a certain degree.

On the other hand, my interview partners also develop a type of protective distance against too strong of a pressure of place appropriation, in particular when travelling only for a short term to places. They always bring habits with them to new places, and they cultivate these habits. Tjark e.g. mentions that the more he travels, the less he experiences it as an exotic thing. There comes a routine of leaving aside the place-specificities, and bringing with him his individual lifestyle and needs:

'Sometimes I absolutely don't care if I am in Barcelona or elsewhere, then I only want... I don't know, to eat dumplings or a soup [laughs]... then I simply order that. I mean, that you do not so uh... well, you gradually bring more and more from yourself when you travel. Previously, where you have been, this was more of a theatre play or something like that, one played a role: 'And now I live in Barcelona, because I am here for a weekend, and I try to live Spanish, for one week or something.' I think I don't do this anymore' (Tjark; lines 315-321 ${ }^{216}$ ).

Tjark's quote highlights that with the growing experience in travelling he develops a stronger sensitivity also for his own personality and how he can satisfy his needs. What I would like to highlight here is there is always a certain balance between humbleness and respecting one's own personality and personal needs in the way in which my interview partners approach new places.

\footnotetext{
${ }^{216}$ Translated by the author from the German text: 'Ab und zu ist es mir vollständig egal, ob ich in Barcelona oder so was bin, dann will ich einfach... ich weiß nicht, Knödel essen oder Suppen [laughs]... dann nehme ich das. Also dass man nicht so äh... also, man bringt langsam mehr und mehr von sich mit, wenn man reist. Also wo man vorher war, so das war mehr so ein Theater oder so was, das spielte man: ,Aber jetzt wohne ich in Barcelona, weil ich hier bin für ein Wochenende, dann versuchte man Spanisch zu leben, für eine Woche und so was. 'Ich glaube, das mache ich nicht so wirklich mehr.'
} 


\subsubsection{Local civic engagement}

I found that my interview partners are part of local groups that engage in their places. Aurélien is part of a local artists' network in Leipzig and has founded his own association which works in France and Germany; Birgit engages in local groups of architects' and planners' association; Christian is part of a local artists' network in Leipzig; Dirk engages with the local Green party in Sofia, fighting building projects, and he is a member of a folkloristic dance group in Sofia; Emil is part of a cultural association in Leipzig; Federico organises intercultural encounters between Italians and Slovenes in Trieste; Gunnar is a member of the local Green party in Leipzig; Henriqua was part of a climbing group in Vigo; Isabel went to a dance school in Berlin; Juliana is member of the local church group in Berlin, and she used to be part of a cultural group of Lithuanians in Berlin; Kate is a member of the US Democrats of Barcelona; Liam is part of the church community in Aberdeen; Marta was part of a folkloristic group of Lithuanians in Ljubljana; Nikolina was part of an environmental protection group in Ann Arbor, fighting a mining project; Oskar is engaged in local development through his professional projects, and then he is also voluntary member of boards of associations in urban planning; Pia sang in a choir; Qamar tried to organise a partner city between Leipzig and Grozny, and he partakes in integration projects for immigrant children in Leipzig; Ronja participates in neighbourhood seminars on gentrification; Tjark experiments with local value chains, offering 'keep-it local design furniture'; Ugo plays football with some university colleagues in Rome; Valentin helped in an urban gardening association in Leipzig; Willi was engaged with the local Green Party of Aachen, and now he participates in the parents' board at his daughter's school; Xaverio organised photography workshops for children in his Berlin neighbourhood and is about to start a voluntary project about urban public space in Rome, and he helped re-organise the archive of a public library in his neighbourhood in Milan; Yari organises hip hop workshops with children in deprived neighbourhoods in large cities in the Americas.

Obviously, my interview partners engage in a broad variety of activities in their places. Yet, this engagement is massively influenced by the travel that they have to follow. My interview partners, in their current life situations, often participate less in civic engagement or voluntary work outside of their professional world than they would have done earlier in life. Many of my interview partners were more active when they were less mobile, or less involved in the multilocal professional life. Therefore, currently they are less active in voluntary work or associations as a result of their multilocality. One reason for the lack of engagement and leisure activities is the lack of time. My interview partners face a lack of leisure time because travelling takes so much time that they simply have none left to engage somewhere.

There is also another issue which is related to time: the rhythm of alternation influences the ability of engagement. My interview partners face problems in organising their lives and in particular their travel in regular rhythms. This irregularity of travel results in an unpredictable absence-presence dialectic. Many civic engagement groups and associations, however, need regular and predictable participation in order to produce visible results and progress. Thus, if civic engagement is done by my interview partners, the only way possible remains to become a passive or pay-only member of a group. Isabel e.g. describes that she does not stay much longer than three or four days in one place, but her travel routes are also not predictable. Thus, she cannot guarantee to local groups when she will be available: 
'This jars with my life. Once I have... I really wanted to engage with the Red Cross, for any NGO, doing some volunteer work. I had the feeling to be fortunate in life, and wanted to give something back, and I could do something, accompany elder people, to go shopping for them, these small things. And then I went to the Red Cross, and they told me: Okay. When are you available for us, we have to schedule you. And I said: I don't know, I don't know where I will be next week. I can be here today and tomorrow probably not anymore. And of course they said: We must be able to rely on you. What do you mean, you don't have time? Either you want to work with us, and then you take the time... Yeah, and the I have to explain to him that I work all across Europe and very irregularly. I mean, that's always a conflict. People ask me: When do you have time? Yeah, I don't know' (Isabel; lines 317-388 ${ }^{217}$ ).

The basic problem of my interview partners is that they cannot make long-term commitments which are based on physical presence. Gunnar also explains that due to his demanding work life and the circumstances of frequent travel between Leipzig and Valencia, he does not feel like he has the energy and time to engage actively:

'I was a member of C33, the cultural association in Lindenau, but I was only a paying member, and I am only a paying member uh, in the Green Party, too. But I [...] perceive my life always as too exhausting for going somewhere every week and taking a stand for something, apart from paying' (Gunnar; lines 806-809 ${ }^{218}$ ).

Another point which showed up in my interviews was that my interview partners sometimes have a fundamental aversion for stable and small local groups. My interview partners appreciate the fact that their multilocality allows them to switch periodically between local groups in different locales (cf. section 7.3). However, this also causes the awareness about the differences between the ways of how things work and are done in different places (cf. 'polyperspectivity' in section 7.2). My interview partners then become bored by being in one place and one local social environment for too long. Henriqua told me about her earlier membership in a group of rock climbers in Vigo, where she felt annoyed after communication and interaction always turned out to be the same:

'I was many years belonging to a club. I, I was a climber, for many, many years. But you know, I thought I got so bored of always hearing the same stories about how to climb this or how to climb that. And it happened to me always when I [laughs]... you know when I am in this sort of clubs or... I get bored, I don't know. I just can't stand it. It becomes like a sect, you know. And if you don't fit in, if you don't speak about those topics, and if you don't... So, I have my friends. And my friends

\footnotetext{
${ }^{217}$ Translated by the author from the German text: 'Das verträgt sich mit meinem Leben nicht. Ich hab mal... ich wollte unbedingt etwas für das Rote Kreuz machen, für irgendeine NGO irgendeine freiwillige Arbeit machen. Ich hatte das Gefühl, ich habe Glück gehabt im Leben, ich gebe nichts zurück, und ich könnte was machen, irgendwelche alten Menschen begleiten, für sie einkaufen gehen, diese kleinen Sachen. Und dann habe ich mich gemeldet beim Roten Kreuz, und die haben mir gesagt: Okay. Was für Zeiten hast du zur Verfügung, wir müssen dich einteilen. Und ich sagte: Ich weiß es nicht, ich weiß nicht, wo ich nächste Woche bin. Ich kann heute da sein und morgen nicht. Und sie meinten natürlich: Wir müssen uns auf dich verlassen können. Was heißt, du hast keine Zeit? Entweder willst du das machen, und dann nimmst du dir die Zeit... Ja, und dann muss ich ihm erklären, ich arbeite über ganz, in ganz Europa und ganz unregelmäßig. Also das ist immer mein Konflikt. Die Leute fragen mich: Wann hast du Zeit? Ja, keine Ahnung.'

${ }^{218}$ Translated by the author from the German text: 'Ich war Mitglied im C33, dem Kunstverein in Lindenau. Aber nur zahlendes Mitglied, und ich bin auch nur zahlendes Mitglied äh bei den Grünen. Aber ich [...] empfinde mein Leben immer als zu anstrengend, um über das Bezahlen hinaus irgendwie so einmal in der Woche irgendwo hinzugehen und mich irgendwie einzusetzen oder sowas.'
} 
come from all kind of uh... of uh... how do you say?... jobs and uh disciplines, and no studies even, and you know, and I just [thinking]... And I just have people where I can speak a bit more a level above' (Henriqua; lines 477-484).

This is also obvious in the way in which my interview partners talk about their circle(s) of friends who are distributed in different places. Along with this hesitation to become involved too strongly in one local context, my interview partners also express a small interest in local issues in some of their places. While they are basically aware of such local issues, and sometimes follow the local news, they still do not translate this awareness into a passionate personal interest which would be the basis for civic engagement.

A further observation I made is related to the way in which my interview partners speak about their jobs. Many of my interview partners would consider their jobs part of local engagement. If local issues are part of their professional work, my interview partners say they actually engage in the places in which they are located. Indeed, there is a certain connotation of creative knowledge work as affecting place.

I also found that local engagement differs according to places. As mentioned above, my interview partners prefer some places to others (cf. section 7.1.5), even though they might basically like all of their places. However, this preference also translates into the fact that if they try to engage somewhere, this engagement is not spread equally to all places. For example, Birgit considers Leipzig the centre of her lifeworld and Kassel is rather a satellite. Her weekly trips to Kassel lead her directly from the train station to the office. She spends nearly all time with work, and as a consequence she does not have much to do with the wider urban community in Kassel. This type of 'social isolation' in specific places which are more part of the work life, is also a strategy to relax from the stressful multilocal life. My interview partners decide very consciously when they want to be around people and when they want to be alone. This includes that in places where they appreciate the silence and the distance to people, they also do not engage locally. Finally, I observed that being less engaged, or not at all, provokes a regretful feeling in my interview partners. My interview partners somehow know that local engagement is socially important, and also that it has an honourable connotation, thus they look for explanations that could excuse that they are currently less available for such activities, something which becomes evident in the interviews through expression of pity about the current situation, or the wish to do more in the future.

\subsubsection{EU and its legal framework as resource?}

Finally, the European Union and the legal framework it provides have for too long been mentioned as an important aspect of everyday life. In particular the freedom to go and work in each of the EU's countries is an important condition to enhance mobility. As my interview partners are transnationally multilocal, they also perceive the advantages and disadvantages of living in several countries at the same time. Tjark stresses the advantages and speaks about becoming more European through the experience of a life abroad in the context of the European Union:

'I am more pro-EU now that I have also lived in three different European countries. This makes me much more pro-EU. Also this whole Erasmus exchange program, I think, uh... which actually is financed by the EU, this is really good propaganda. I think, nearly all Erasmus students are more 
pro-EU afterwards, because someone from Paris lived in Barcelona or in Berlin and so on. Then one thinks: 'Ah okay, the difference is not that big. Of course we can work together, blablabla.' And then in the end you have friends all over Europe. [...] As EU citizen you have many advantages. Practically it was not very complicated' (Tjark; lines 488-506 ${ }^{219}$ ).

However, not all my interview partners express these advantages like Tjark does. Many also perceive the difficulty of living in several countries when problems with bureaucratic details arise. For example, problems with paying taxes or the transferability of pension schemes are related to the EU, which makes the theoretical free labour market still partly a political image and less a practical reality. Gunnar describes how he arrived in Spain, thinking he could start working right away. He thought that this was what the EU labour market was about. Yet, in the end he had to deal with many Spanish authorities, and he had to organize a N.I.E - Nummero identidad extranjero, which is also a prerequisite for EU nationals who want to work in Spain. Juliana also mentioned having problems with bureaucracy across borders. Whereas she is a Lithuanian working in Germany, her German husband works in Sweden, thus they continuously have problems with social security systems. In particular, the authorities do not yet have experience with the very individual lifeworld realities of my multilocal interview partners. Those who work in these authorities mostly only know their own national and local reference frame and thus cannot competently provide information to people who cross borders in a regular rhythm. This is also reflected by Oskar, who continuously has problems with financial authorities in Switzerland, Austria and Germany, where he explains his situation, but officers cannot understand his realities. Oskar describes that he even developed strategies to avoid bureaucratic issues. His registered main residence is in Zurich, but German authorities continuously trouble him in the sense of registering a company branch in Germany:

'Yes, sometimes there are such absurdities, and one gets very sensitive for the national particularities, I mean that I have to explain to German revenue authorities that my main residence is here [in Zurich], this has also to do with the fact that I can tell them I have a mobile phone number from Switzerland, and I am here. Otherwise they would suspect me to operate an office in the Schiller street [in Berlin], which is really not the case. And these are things, which you... here [Linz] I am not even registered anymore; I live there in a lawless status' (Oskar; lines 313-320220).

Looking at these examples, it becomes obvious that the European Union actually provides the legal framework for my interview partners' transnationality. On the other hand, practically the EU regulations seem to suffer from little practical utility in the everyday life of my interview partners.

\footnotetext{
${ }^{219}$ Translated by the author from the German text: 'Ich bin viel mehr pro-EU jetzt als ich auch also in drei verschiedene europäische Länder gewohnt hab. Das macht mich viel, viel mehr pro-EU. Ich glaube auch die ganze Erasmus-Austauschstudentenprogramm, das äh... was ja auch von EU sponsiert ist, das ist unglaublich gute Propaganda. Ich glaube, fast alle Erasmus-Studenten wäre danach dann pro-EU, weil jemand von Paris hat in Barcelona gewohnt oder in Berlin und so. Dann denkt man: „Ah, okay, der Unterschied ist nicht so groß. Wir können natürlich zusammenarbeiten, blablabla. " Und dann hat man am Schluss die Freunden überall in Europa. [...] So als EU-Bürger hat man viele Fortschritte. Das war eigentlich nicht besonders kompliziert.’
}

\footnotetext{
${ }^{220}$ Translated by the author from the German text: 'Ja, manchmal gibt es so Absurditäten, man wird natürlich sehr sensibel für die nationalen Besonderheiten, also dass ich den deutschen Steuerbehörden erklären muss, dass mein Arbeitslebensmittelpunkt hier [in Zurich] ist, das hat dann auch damit zu tun, dass ich dann sagen kann, ich hab eine Handynummer aus der Schweiz, und ich bin hier. Weil sonst verdächtigen sie mich, dass ich in der Schillerstraße [in Berlin] eine... auch ein Büro habe, was nicht stimmt. Und das sind so solche Dinge, die man... hier [Linz] bin ich nicht einmal mehr angemeldet, da lebe ich im gesetzlosen Zustand.'
} 
They are aware of the value of the EU as a background frame for cross-border mobility, but they are actually confronted with a lot of persisting bureaucratic problems.

\subsubsection{Changing role of holiday trips}

A central theme that shows up in the narration of my interview partners is that they feel astonished about the way their relation to going on holiday has changed. Due to the fact that they are continuously alternating between different places, they do not wish to travel when they have holiday; they prefer to stay at one place and not move around. This has to do with different things. First, my interview partners feel stressed by travelling. They want to relax from being on the move. When I had already finished my interview with Birgit, she finally added this one thing that she found relevant:

'Through this type of life I do not miss holiday trips, no vacation and no city trip, nothing, never. In the last three years, I spent my holidays in Leipzig, at the lake. This has to do with the family situation [her elder mother who needs care]. But it was actually for the peace of mind, also in the household, where I would say, this is the most important. I probably would have chosen one alternative, and I would have gone to the Baltic Sea. But I do not have the feeling, I need to do another trip to Paris with Thomas, or that I need to spend my holiday somehow like this and that. I actually do not have this need' (Birgit; lines 561-568 ${ }^{221}$ ).

She also says that her work life takes her to so many places and this is so enriching that she prefers tranquillity in her sparse free time. A similar theme shows up in Dirk's description of his ideal holiday. Dirk describes that this would be in nature, without internet, which he necessarily uses everyday for work:

'My perfect holiday trip would be to go down the Danube with a foldboat or something like that. Or just camping on a small island in the Danube. And with the rush of the water. There you actually do not need internet, but if it is for work, then of course I need it' (Dirk; lines 347-350 ${ }^{222}$ ).

Dirk also reflects on how his personal relations to his places change with time. Whereas he has been attracted by eastern Europe since his youth, he now - living there - realises the disadvantages, e.g. the strong pollution and the environmental awareness. He, therefore is engaging with the Green Party of Sofia, but then he also perceives how the relation to his place of adolescence is changing, which now becomes a holiday destination to him, and where he more than previously perceives the environmental advantages and the nice landscape.

\footnotetext{
${ }^{221}$ Translated by the author from the German text: 'Durch diese Art Leben vermisse ich keine Ferienreise, keine Urlaubsreise und keinen Städtetourismus, nix, niemals. Ich habe in den letzten drei Jahren meine Ferien in Leipzig verbracht, am Cospudener See. Das liegt an der Familiensituation. Aber es war für den eigentlichen Seelenfrieden, im Haushalt, wo ich wirklich sagen würde, das absolut wichtigste. Ich hätte noch eine Alternative gewählt, und wäre an die Ostsee gefahren. Aber ich habe nicht das Gefühl, ich muss jetzt nochmal eine Reise nach Paris machen mit Thomas, oder ich muss jetzt unbedingt nochmal meine Ferien irgendwie so und so verbringen. Also diese, dieses Bedürfnis habe ich überhaupt nicht.'

${ }^{222}$ Translated by the author from the German text: 'Mein Idealurlaub ist immer mit dem Faltboot so die Donau da runter oder so. Und solche Sachen, oder nur auf einer Donauinsel dann übernachten. Und dann so das Rauschen dabei. Da brauchst du kein Internet natürlich. Aber so für, wenn es irgendwie in einem Kontext ist, wo man arbeitet, dann brauche ich das.'
} 
Both descriptions reveal that vacation is clearly opposed to and distinguished from everyday life. It has to have a specific, different character from work and everyday life. Work and everyday life are related to travel and the intensive use of ICT. Vacation should then be taking a break from that, going back to nature and becoming slow and immobile again. Gunnar as well as Birgit says that his multilocality is the reason for that he does not feel like travelling when he is on holiday. He also prefers to 'be there' in one place:

'The whole thing which occurred somehow through this to and fro and these visiting trips, is that holidays, for example, actually do not happen anymore, because our travel load was already high enough due to the fact that we have constantly been in different places and that we visited our families. That's why our inclination to travel elsewhere, to a completely different place, that has decreased with the years, you know. Generally, my pleasure in travelling has gone away. I mean, I was quite happy if I could spend some time here' (Gunnar; lines 92-100 223 ).

Gunnar also mentions that he would love to live more monolocally again. When I asked him if he would miss travelling then, he answered that it would only change character, so that travelling has the character of holiday again, and not of everyday life, and he would appreciate that. Also, this narration by Gunnar highlights the opposition between everyday life and the role of holiday as something particular and opposed to everyday life situations. Whereas for many monolocal people the opposition would consist of travelling on holiday versus relative spatial stasis in everyday life, it is rather the opposite way around for my interview partners. For my interview partners, spending holiday time at one place and waiving further travel is not only an issue to relax from the everyday and professional trips. Spending time in one place is also important in the sense that it allows for time to be spent with local loved ones. It means not feeling stressed by the coming departure which introduces the next (business) trip. Thus, monolocal and immobile ways of spending holiday time compensate for the psycho-social and physical stress that comes along with living a multilocal life.

\subsubsection{Post-materialism}

A final observation during the analysis of my interviews concerns my interview partners' attitude towards material belongings and consumption. Based on their experience of travel and mobility they have developed a specific distance to material consumption. They are aware of the fact that their multilocal place pattern can change quickly and that moving with a lot of things is a difficult and costly part of multilocal everyday life. Furthermore, as they have distributed their material belongings to different places, they also know that they can never use all their belongings whenever they want. As a consequence, my interview partners show a latent orientation to post-material attitudes. For example, Birgit compares her situation with her professional colleague, who is also multilocal: he has more property and responsibilities for his possessions. Birgit thinks that in a

\footnotetext{
${ }^{223}$ Translated by the author from the German text: 'Das Ganze, was sich irgendwie jetzt irgendwie so getan hat, durch diese ganze Hin- und Herfahrerei und Besucherei, ist, dass irgendwie Urlaub zum Beispiel jetzt nicht mehr so richtig stattfand. Weil irgendwie unser Reisepensum schon dadurch ausgeschöpft war, dass wir irgendwie ständig an verschiedenen Orten waren, und dass wir gegenseitig unsere Familien besucht haben; deshalb war irgendwie unsere Lust, irgendwie nochmal so wegzufahren, irgendwo an 'nen komplett anderen Ort, das ist dann irgendwie so Jahr für Jahr gesunken, ne. Insgesamt meine, meine Reiselust ist so `n bisschen, ein bisschen dahin. Also ich war ganz froh, wenn ich jetzt mal hier war.'
} 
demanding mobile life physical things can become a burden. As mobility increases, this will lead to an overall change in value systems towards more post-materialist patterns, as she reflects:

'Perhaps priorities will change in respect to the how one's own life is equipped. We will probably spend more resources on mobility. And we will have fewer resources for the presence in particular places. This has already changed. You rather buy three decent suitcases for all cases and you spend a lot of money. That's more important than having three more tablecloths' (Birgit; lines 495$\left.500^{224}\right)$.

Tjark also remarks that he got rid of many things which he actually did not need or use. He always stored his personal belongings at his parents' place. While spending time abroad, he made a list of things really missed, and all the other things he threw away or sold upon return to Denmark. In this sense he says:

'This is my idea about the good life: having few things. Then I am always more flexible, and I can travel. And always when I... all the three times, when I uh went abroad, from Denmark to another country, I have um... that was always within one week actually. I made an interview. And then they said: 'Okay, can you start on Monday?' And then I actually moved to another country. It was always like that. [...] And then you keep moving. And that's why I did not want to have so much furniture and stuff. But now, slowly, in the last three years here... I have even bought a sofa. Gradually, I have more things again [laughs]' (Tjark; lines 190-201 ${ }^{225}$ ).

Tjark's quote also indicates that this post-material attitude is subject to change. Now that he has become a bit less mobile, and as he is currently focussing on getting his business started in Berlin, he has also become a bit more settled and has started to furnish his apartment. Furthermore, the expression of post-material attitudes can also be a tool for distinction. Valentin told me about one of his relocations which he was able to do with a rented compact car. Whereas he had already put all his belongings in to the car, there was even space enough to take some more people who wanted to share travel costs. Later on he mentions that he observes other people who spend a lot of money on the consumption of clothes and cars, and he thinks that these people also have to work a lot for this. He rather prefers to have fewer things, but to have the freedom of being mobile, freed from physical belongings, and having time to travel than having more leisure time. Here, post-materialism becomes a metaphor for freedom and mobility.

\footnotetext{
${ }^{224}$ Translated by the author from the German text: 'Vielleicht werden sich dann eher auch die Prioritäten in Bezug auf die Ausstattung des eigenen Lebens nochmal verändern. Wir werden wahrscheinlich mehr Ressourcen reingeben in diese Mobilität. Und nicht so sehr viele Resourcen mehr haben für diese Anwesenheit an bestimmten Orten. Das hat sich schon verändert. Da kaufst du dir lieber drei vernünftige, ordentliche Koffer, für alle Fälle. Und da gibst du richtig viel Geld aus. Das ist wichtiger als noch drei Tischdecken.'

${ }^{225}$ Tranlated by the author from the German text: 'Weil das war so meine Idee von das gute Leben, wenig zu haben. Dann bin ich immer mehr flexibel, und konnte, konnte reisen. Und immer, wenn ich... also alle drei Mal, wo ich äh nach in das Ausland, also von Dänemark nach ein andere Land umgezogen hab, war ähm... da war's immer so innerhalb einer Woche eigentlich. Also ich habe ein Interview gemacht. Und dann haben sie gesagt: ,Okay, kannst du nächsten Montag anfangen?`Und dann hatte ich eigentlich umgezogen zu einem anderen Land. Das war immer so. [...] Und dann zieht man wieder weiter so um. Und deswegen wollte ich auch nicht so viele Möbeln und so weiter haben. Aber jetzt langsam, jetzt habe ich hier in drei Jahren... ich habe sogar ein Sofa hier gekauft. So langsam habe ich wieder mehr Sachen [laughs].'
} 


\subsection{Transnational multilocal lifeworlds in creative industries}

To conclude my empirical observations, I will focus on how we can grasp the creative knowledge multilocal lifeworlds of my interview partners. The figure below shows a scheme including the main dimensions that I have found to influence the individual perception of the multilocal lifeworlds.

Figure 23: Five dimensions of lifeworld perception

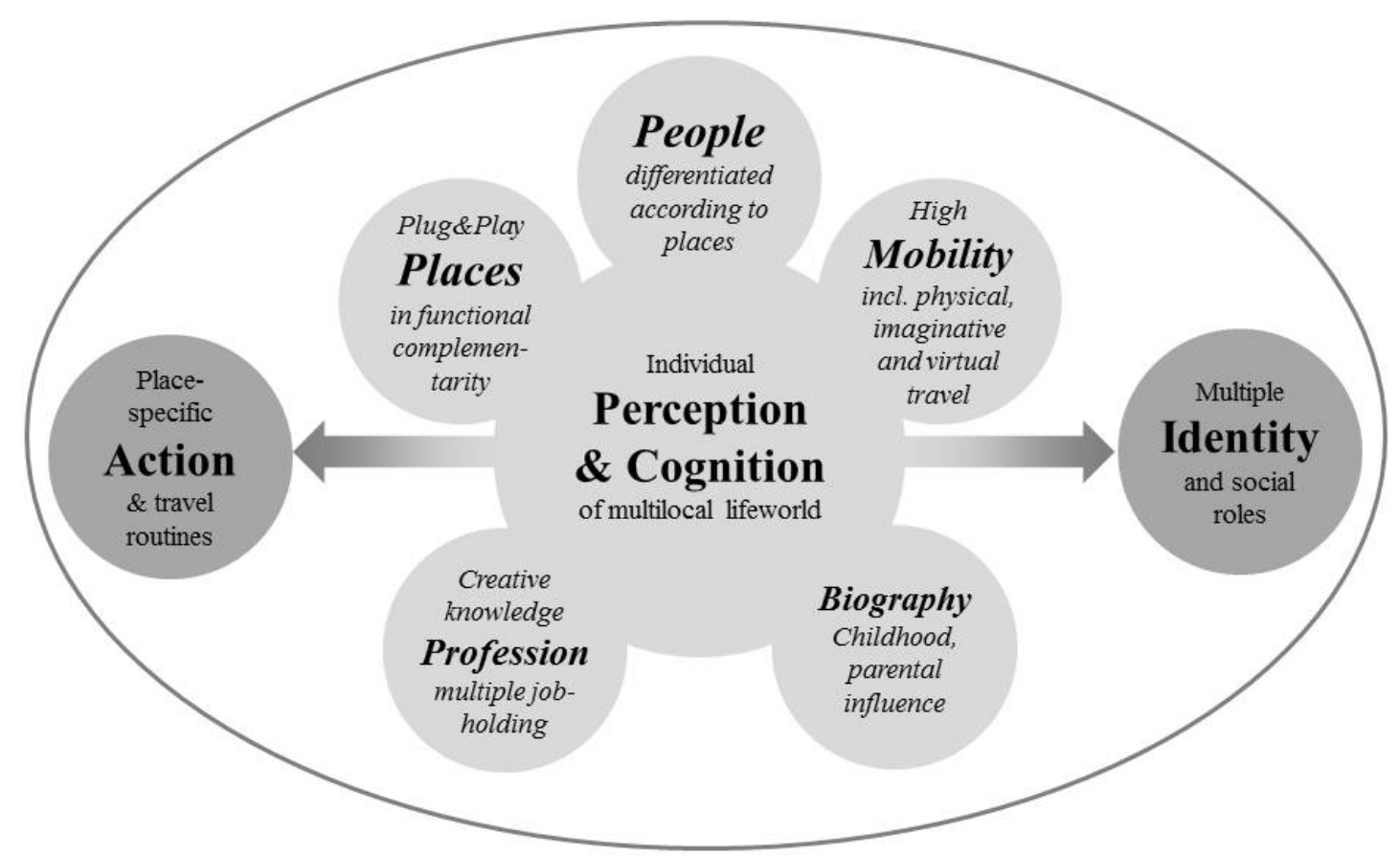

First, the various places which are related to each other in functional complementarity affect the individual perception of one's own multilocal lifeworld. As seen so far, my interview partners line up a set of places throughout their lives. The interplay of professional and private as well as historic, current and future places forms a coherent pattern equal to a puzzle which, put together, allows one to view a coherent picture. The difference to more monolocal people consists of the number as well as the importance of different places. Even though my interview partners also might name a most important or central location, a basis, of their current lifeworld, the pattern would lose coherence if a place was to fall out. Then their lifeworld would need to be re-ordered, and functional relations between places would need to be adjusted. This does not mean that the pattern is stable; in fact it is subject to continuous change. However, in a given moment, the pattern of places makes sense to my interview partners. The mental maps revealed that the perception of this geographic dimension of the lifeworld includes different techniques of imagination and various carriers of content. The perception of place is heavily related to time. Furthermore, places are constantly compared to each other. This means that the creative knowledge multilocals can evaluate 
them and they can develop preferences for places, and for what they want to do in these places. However, the composition of the overall place pattern is not completely in the hands of these multilocals. The place pattern is interrelated and influenced by the other dimensions. The labour markets, and for the presented cases the creative knowledge ones, influence adding and removing places. Biography also heavily interferes in the place patterns, namely when it comes to defining the historic places of a person. Then, the place pattern is influenced by the other people who are important in one's life. For example, the partners and one's own children become relevant when place-related decisions have to be taken. Mobility then also defines what place pattern is plausible in terms of having the time, money and mental disposition to travel between the places in a given periodicity. Finally, coincidence also plays a partial role. Therefore, place patterns are only within limits the result of autonomous decision-taking. The most important thing we should keep in mind is that places are ordered according to functional complementarity. This means that my interview partners perceive of the place-specific resources and opportunities that each place can offer, then they balance in which place they can better satisfy their needs. The output is a certain logical order within the place pattern which describes the mental map of the multilocals. Here, we find information about which places are more central and more important than others, we can see which places are rather oriented to private life and which ones serve professional needs, and we can also see which places become emotional anchor points in terms of providing a home base where one can recover from mobility.

Second, there is the dimension of personal mobility, which opposes the rather rooting aspects of people and places. Mobility contains different aspects. There is actual physical travel: my interview partners spend hours in trains and aeroplanes on their way from place A to place B, yet this is not the only way in which we should think of travel in the lives of my multilocal interview partners; imaginative travel is also an important issue here. Imaginative travel consists of the mental capacity to consciously think oneself into the other places of one's own lifeworld. This means that the multilocal creative knowledge workers dispose of a technique of remembering the distant places. While they are physically present in place A, they also can be mentally present in place B through cognitively bridging the geographic distance. In addition there is virtual travel, which is mediated through ICT, e.g. regular phone calls, emails, videoconferences or chats with people in the other places. Mobility might be voluntary or rather forced by market constraints. I have also highlighted that actually the different forms of travel within this mobility dimension have a bridging function between the places. Only travelling allows one to live one's own life in different places, and as such travel is the basis for the comparison of places to each other. As an outcome of this travel facilitated comparison, I have described that the creative knowledge multilocals have developed a state of mind which has been termed polyperspectivity. Through switching between places in a physical way, they also become knowledgeable enough to switch in mental ways. As such, they are able to relativise and reclassify local issues in their places. Furthermore, I have also underlined the fact that there are places that are related to travel and mobility, and which are less perceived as actual places but rather as components of a journey. These transit places can be mobile (trains, aeroplanes) or immobile (train stations, airports), and they also provide a form of home for multilocal creative knowledge workers.

Third, there is the dimension which consists of the people who are important 'others' in the lifeworlds of my interview partners. Their people are mostly spread around in different places. 
Fitting into the functional complementarity of places, the character and composition of social networks in places also varies. The multilocal creative knowledge workers also order their social contacts according to their places, and they appreciate the fact that their multilocality allows them to switch between different social networks. Private places are often linked to partners, children, other family members and friends. Professional places are linked to colleagues and business partners. Furthermore, people also play an important role in terms of social orientation and feeling at ease with places. The wider local community of a place can be welcoming, open-minded and international, or it can also be exclusive and narrow-minded. We have learned that foreign social environments can also lead to a feeling of social pressure. Social pressure means that a person, upon arrival in a new or foreign place, needs to adapt step by step to local the culture and language, to the ways in which people interact with each other, to local codes and norms. This process of adaptation is often perceived as challenging and stressful, and it can affect the emotional stability of the multilocal persons. Yet, given that these multilocal persons have the possibility of comparing places and the local people in these places; they also can handle this social pressure by relativising the demands on them during the adaptation process. As an outcome of this comparison in social terms, my interview partners feel more or less rooted in their places. Often they also intentionally refuse to become member of any local social group. The multilocal people love some places for the fact that they are alone in these places, and they enjoy being alone if they are strongly involved in social networks in other places. In these constellations the functional complementarity of places shows up again. In line with this, my interview partners are identified as foreigners or people who live a strange or extra-ordinary lifestyle, but they also engage in active 'othering' as well. They often think of themselves as different from others and this difference becomes a constituting factor of their personality.

Fourth, there is the long-term temporal aspect of their biography and personal history. Nearly all my interview partners' biographies contain facts that were helpful for the development of a multilocal lifeworld. The earliest aspect is the place of birth and the place(s) where an interview partner grew up. For a lot of my interview partners these two moments in life - birth and childhood - did not happen in the same location. Often their parents were mobile, too, so they had to move with their parents since early childhood. This means that already in the earliest moments in life the mental setup for living a mobile and multilocal life were prepared. Then there are other aspects such as being the child of a bi-national couple, or having a dual citizenship, which allows for easy life in different places. This point is also related to language competencies. For example, having several mother tongues is a facilitator for a multilocal and transnational lifeworld. Being part of a binational partnership is also an influence on the personal multilocality. This dimension is strongly linked to the historic places of a multilocal person. Childhood, the lifespan in which one does not yet take autonomous decisions, can influence this basis of a mobile capital. Obviously, I have to add that not all my interview partners really had these experiences during their early years. Still, they have become multilocal today. What I wish to stress with this dimension is: if a person has had any of these experiences of mobility as a child or adolescent, it is highly probable that it will positively affect the disposition of becoming mobile. This dimension is nothing a person can actively influence today; it is rather a distant dimension affecting the current life from the past, from the personal history. 
Fifth, the professional dimension also appears in the perception of the multilocal lifeworld. In fact, the creative industries as a labour market are very demanding in terms of mobility and geographic flexibility, as my interview partners stated. However, the multilocal creative knowledge workers also appreciate the flexibility and make use of it. Creative knowledge work is in fact a professional field in which my interview partners are able to work nearly everywhere as long as they dispose of an internet connection and their laptop. Therefore, creative industries can indeed be considered an economic branch which not only demands flexibility and mobility, but it also is organised and structured in a way that allows for mobility. Many of my interview partners engage in different professional environments and hold multiple jobs. Often these different jobs are also related to different places and different social networks. Being involved in several jobs and projects in different locations can be understood as both a necessity in order to earn a living and an opportunity to broaden the individual horizon and multiply one's own knowledge and skills. Travelling between different jobs and places, these creative knowledge multilocals also work as supporters of knowledge transfer between places. They actively try to apply the things that they learnt in one place in any of their other places, not in an altruistic way to help all local professional environments become more competitive; they use their multilocality and their multiple professional affiliations in order to raise their own market value. The knowledge and skills from other places as well as their attachment to these other places gives them a competitive advantage against more monolocal competitors. On the other hand, there is also the negative aspect that they might become excluded from local networks as other more monolocal network members consider the multilocals' unpredictable absence/presence duality as a problem for continuous collaboration. Local professional networks often work in a way that membership is granted for engaging in the network. However, exactly this engagement frequently cannot be guaranteed to my interview partners, as they often are elsewhere. Therefore, with time they become excluded step-by-step from the advantages of local network membership and lose access to important local information flow.

These five dimensions influence the individual perception of one's own multilocality and one's own multilocal lifeworld. My interview partners perceive clearly that they are different from a lot of other people. They can exemplify this with concrete things that are part of their lifeworld, e.g. the fact that their monolocal friends signal that they do not understand the multilocality of my interview partners. The individual perception of the five dimensions is cognitively processed in the minds of these multilocal people. They link these individual dimensions in order to construct a coherent multilocal lifeworld, including a set of attitudes and preferences. The next step is then that this perception-cognition sequence results in concrete action and identification patterns.

As I have already stated, my interview partners are aware of the fact that their lives and the way in which they move between places is different from the geographic behaviour of most other people around them. They all told me that they e.g. have more difficulties than monolocal people in explaining where they are from or where their home is. However, they have developed creative ways to deal with this issue. On the one hand, they playfully use different identities in different contexts. Depending on the concrete situation, they might identify as a person from place A, while in another situation they introduce themselves as from place B. In a third situation they might even explain that they stem from place A and place B. They know that they most often confuse their conversation partners, and they somehow take delight in it. This is reflected in the playful character of switching between their different identities. These multilocal creative knowledge workers 
dispose of a wide set of identities that can be used according to individual utility and enjoyment. These multiple identities are not only fun and games, they also stem from the place-specific differentiation between social environments, therefore they are also linked to concrete social roles that vary according to places. Whereas a multilocal might be a family father in one place with a more private character, he might be more of a colleague and artist in any of his other more professional places. My interview partners' social contacts, social roles and social identities vary according to their places. Furthermore, my interviews have shown that these individual components of identification not only make sense as single identities, but they also can be merged into a new and hybrid type of identity. Here, I observed that my interview partners often used terms like nomad or nomadism, so they identify as something other than the monolocally rooted people. They are moving continuously in space and consider themselves nomads if compared to the immobile people. However, the use of the term 'nomad' is ambivalent. Actually, my interview partners are not really nomads in the sense that they move through space taking all their belongings with them; they have clearly defined places which work as base stations of their movement across space. This ambivalence is also reflected by my interview partners; it comes out when they think about the place-based aspects of identification. The creative knowledge multilocals place-based identity is evident, and therefore they are actually not nomadic in the sense that their home is merely a mobile one. My interview partners relate to and identify with concrete places and they include placespecific aspects in their identity. Thereby, the identification pattern is characterised by a difference between two feelings. First, there is a feeling of where one comes from (Heimat). This feeling mainly relates to the historic places, places of birth, where one spent the childhood, or where the parents live. These are the environments in which my interview partners were primarily and secondarily socialised. Second, there is a feeling of where one is based at the moment (zu Hause). This feeling points to the place where one feels to be living at the moment. Here, my interview partners frequently tell that they are actually living in several or all of their current places. Whereas the first place strongly relies on biography, the second one is related to the current everyday life and its locations. We can therefore say that my interview partners identification patterns are not detached from geographic space. They feel that they belong to and are attached to places. I also looked at geographic scales and their interrelation in this sense of providing an anchor for placebased identification. Here, the dominant role of the local became visible. The multilocal creative knowledge workers draw mainly on local scales, concrete cities, to identify in geographic terms. This is also related to the professional environment in which the individual competitive advantage can result from being associated with a specific place. A prominent example for this in my empirical sample was the relevance of Rotterdam and having worked in Rotterdam for architects in Europe. The national scale can also work in this direction, but it is rather important in terms of national culture and language. Being multilocal across national borders, my interview partners say that they actually do not want to identify with their national background. However, as soon as they arrive abroad they become identified as being from a certain country, and not from a certain city. Therefore, they included their national cultural socialisation and background into their identity. Finally, the European scale is the least relevant to my interview partners. Cross-border multilocality allows them to understand better what Europe actually means, e.g. in terms of the freedom of movement and the integrated labour market, or the value of a common currency. Yet, this is insufficient to work as anchor point for identification. Europe still seems to be a fairly abstract scale. Additionally, the promised advantages of European integration still have 'teething problems'. 
In their everyday life, the multilocal creative knowledge workers constantly have to struggle with bureaucratic issues and incompatibility of nation-state institutions such as health insurance, tax regimes, unemployment protection, pension schemes, legal systems etc. All these problems are counterproductive for European identity.

Another outcome of the five dimensions and their cognitive processing are specific action patterns or concrete everyday life practices which reflect the interplay of being rooted in different places and of being mobile. My interview partners have developed place-specific practices which vary across 'their places'. This means that they develop routines which they do in one place, but that they would not do in other places. As such, they translate the perception of what a place offers into concrete action, which is adapted to this place-specific offer. These things can be of minor importance, such as a practice of jogging in place A and a practice of using bicycles in place B. They can be related to leisure time or to things that aim at providing stimulation and inspiration. They can also relate to more important aspects. In the professional dimension, my interview partners reline their multiple job-holding and the accompanying professional identities with placespecific professional activities. While they might interact with many colleagues in one place, they might work in a relatively isolated manner in other places. Therefore, there is differentiation between more communicative and more reflexive parts. This is also visible in the ways in which they use the available infrastructures that allow working from everywhere. As mentioned before, these creative knowledge workers often only need an internet connection and their laptop, so they develop routines in frequenting certain locales within their places which provide internet access and a table to work. Furthermore, I highlighted the central role of flexible dwelling practices. My interview partners have developed strategies to ensure flexible and low-cost possibilities of overnight staying. We found a broad range of things mentioned here: sporadic stays at friends' or family members' places, shared apartments, shared rooms, hotels, company apartments, colleagues' places, and even camping tents. Another concrete practice at the intersection between places and mobility is keeping things which are necessary in everyday life, available in ubiquitary form in several places at once. This starts with small things such as leaving the toothbrushes and other hygiene products in all their places and could later lead to the installation of something like completely equipped ateliers. The frequent travel does not allow them to carry everything around, so important things are purchased in a double or multiple way. Interestingly, this finding goes hand in hand with the observation that the multilocal interviewees develop post-material attitudes. While providing important things for everyday life in ubiquity, they consciously renounce material consumption in several other spheres. For example, investing in long-term furnishings for apartments is something they do to a smaller extent than perhaps monolocal people would. In contrast, they put more money and time into travelling and making travel time more comfortable (e.g. buying more expensive tickets in order to have better seats, or buying small and costly ICT devices that allow connection and workflow while travelling). In respect to place, my interview partners have also developed precise routines to appropriate the places for their own lifeworld, and they have developed techniques to translate between experiences of different places. As such, they can also orientate in new environments without much irritation. On the other hand, they have also developed routines and practices for dealing with the frequent travelling. This includes the mentioned communication practices to keep in touch with the people in different places, or practices of organising and seizing their trips. Concerning communication and the use of ICT, my 
interview partners mention that this is very important to stay in touch. However, as it is so central in their lifeworlds, they sometimes get annoyed by always being available, and they also switch off their communication devices. When it comes to travel organisation, I presented the finding that my interview partners often plan their journeys as multi-purpose trips in order to efficiently satisfy their private and professional needs. Then, the multilocal creative knowledge workers also reported that private holiday trips have changed their character. Due to the professional mobility which dominates their everyday life, they often seek immobility during their holidays. Here, they often prefer to stay in one of their places where they want to spend time with loved ones, whom they do not seem to be able to see in everyday life.

I think this five-dimensional model of multilocal lifeworld perception helps us to reflect about the nature of a multilocal life. Until now, the discussion on multilocality has mainly circulated around defining it by using criteria that originates from monolocal lifeworlds. For example, much of the discussion on multilocality focuses on residential issues. Multilocality is illustrated as having multiple places in which to dwell, yet the definition of dwelling follows the logic of monolocal people. Taking into account the five dimensions as outlined above, we can better understand how such multilocal lifeworlds actually work in the life of my multilocal interview partners, and hypothetically for multilocals in general. In particular, the influences of biography and personal history have been neglected so far. The role of specific economic sectors, such as the creative industries in my sample, might also be helpful to elucidate the composition of multilocal lifeworlds. Having said this, I would like to focus on two overarching aspects of the creative knowledge lifeworlds which I have studied: first, the everyday life character, and second the temporality of multilocal lifeworlds. Both these overarching aspects relate to time, and I think reflecting on them can point out their relevance for defining multilocality in general.

\subsubsection{Everyday life character}

There is a meta-level aspect that characterises the overall lifeworld of my interview partners. They actively try to make the alternation between places, the coming and going, the subsequence of travelling and maintaining an everyday life. The aspect of making something, which seems extraordinary to the wider society, normal in one's own life is something that is a repeated theme in my interviews. We can see that in the way in which routines are reflected, and building routines, and having routines in for example travelling and orientation, which for the others has nothing to do with routine, become a self-evident component of the own everyday lifeworld. My interview partners do not want to live in a circumstance of continuous exceptionality; they want a common life, and in light of the perceived difference to monolocal people, the multilocals actively construct the everyday life character of their multilocality.

\subsubsection{Temporality and future plans}

Another important observation during my interviews is that mobility is not a definite decision in life. While in individual cases (e.g. Kate or Valentin) mobility might be a type of leitmotif for life, in most of my other interview partners reflect about the temporality of their current lifeworld. The relation towards mobility is often ambivalent (cf. section 7.2), in which the advantages as well as the disadvantages are counterbalanced. Whereras my interview partners do not perceive mobility as 
something negative per se, they often wish to be able to decide to stay in a place a bit longer. Often the moment of having to leave 'right now' is perceived as a frustrating aspect of a multilocal lifeworld. Within limits, nearly all of my interview partners reflected on the alternatives, and in particular about living a monolocal and less mobile life again. The multilocal people I interviewed rather tend to exclude monolocality as they have realised the advantages of multilocality and mobility. However, they long for more autonomous decision power about their place and mobility patterns. A gradual reduction of mobility is a possible alternative which all my interview partners consider, and which becomes particularly powerful as an idea in the moments when frustration during physical travel is peaking again. As a conclusive observation we should keep in mind is that multilocal lifeworlds and the related place and mobility patterns have to be considered dynamic. They are subject to frequent minor and major changes. They can be related to specific biographical episodes with a predictable end, or they can be entered without knowing about the temporal end, yet with the knowledge about their temporary and finite character. They are not stable in time; flexibility is expected from these people. Then again, these people also expect flexible social and institutional environments which allow them to define how multilocal and mobile they would like to be at certain moments in life. 


\section{Interlude G: Tjark - Valentin}

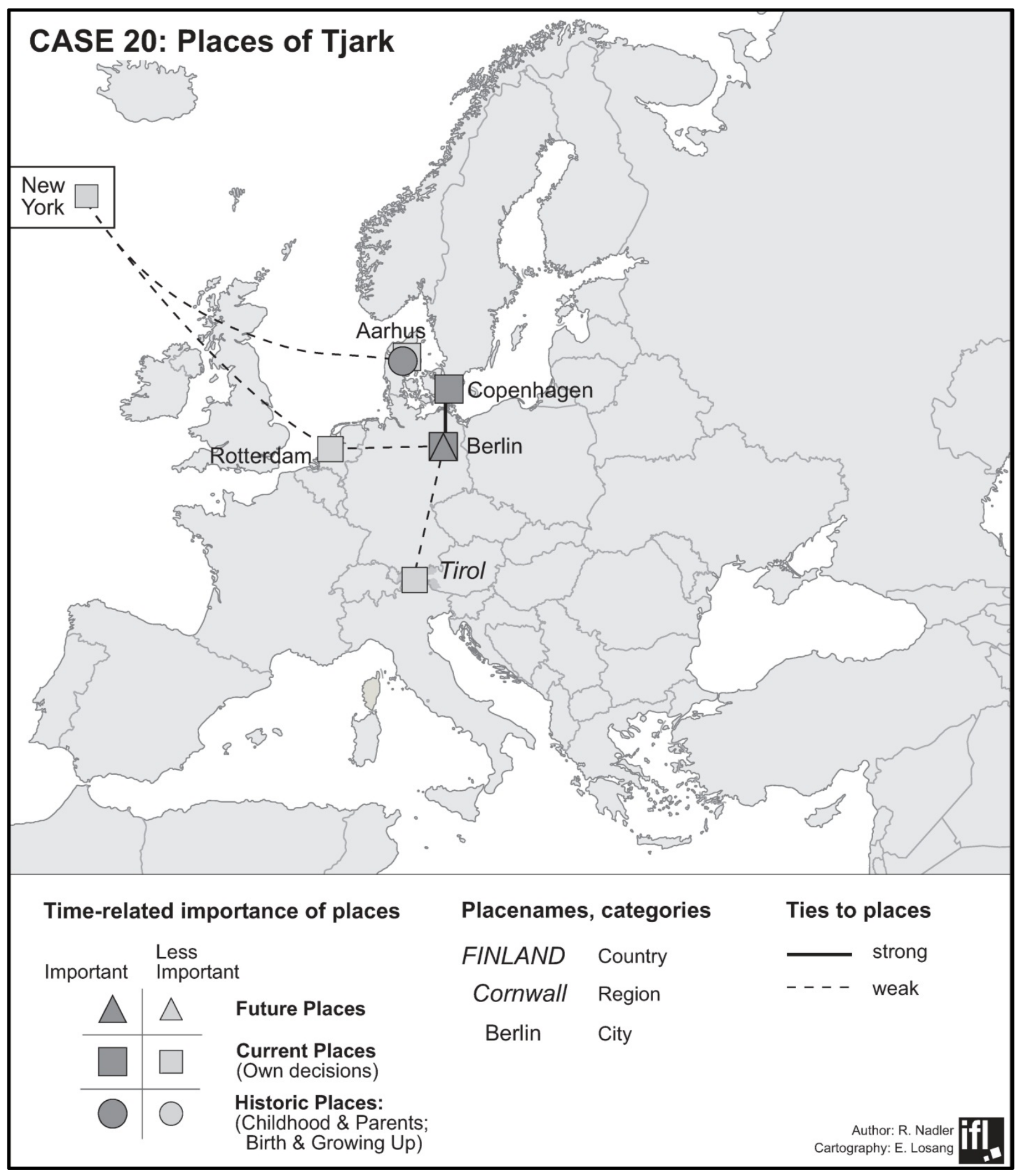




\section{Meet Tjark}

Tjark is a Danish architect and interior designer in his 30s. He grew up in the small Danish town of Aarhus and then moved to Copenhagen, where he studied architecture. During his years at the university, he went to live in Rotterdam and New York, where he did internships in architecture firms. After finishing his university degree, Tjark was interested in the city of Berlin, and while he is still registered with his main residence in Copenhagen, he has now been living in Berlin for two years. Tjark describes his mental map as a mixture of Berlin and Copenhagen neighbourhoods and the process of moving to Berlin also took a whole two years since he arrived to Berlin. On his regular trips to Copenhagen, he continuously brought more things to Berlin, but he is keeping his Copenhagen apartment for which he still has the keys and which he rents to friends. He still stays there when he is in Copenhagen.

Tjark also described how he got rid of everything superfluous. When he moved to Rotterdam and New York, he put all his belongings into the cellar of his father's house. All the things he did not miss while being abroad, he simply threw away. The gained material freedom was one of the aspects that allowed him to relocate to Berlin without many problems. He loves having only few things to be able to move quickly elsewhere. Interestingly, Tjark designs furniture and he has equipped his own apartment in Berlin with a concrete table which cannot be moved. The frequent travelling between the places is something which Tjark actually does not like. In his childhood, travelling for vacation had something exotic and exciting, but with his frequent trips today, this exotic character has disappeared. Travelling is something boring to Tjark, and in order to make it more exciting he plays a personal game: spending as little time as possible in airports. This has already resulted in missed flights and costly new tickets, so he now accepts that travelling is a necessity in his life which has a functional character.

Getting along in new places is nothing which makes him nervous, as Tjark has moved frequently around Denmark with his parents. Today, in order to explore new places, Tjark provokes getting lost in cities. He mostly does this while walking through a city. This technique of actively getting lost is something he had learned in his field trips with his architecture mates in university, and he still applies this method with his own students at the Berlin University of Arts, where he holds a position as assistant professor in architecture. Tjark also stresses the advantages of a transnational life between Copenhagen and Berlin: in Denmark people have a very positive image of Berlin as the cultural hotspot of Europe. Being based there brings more attention to Tjark. On the other hand, German people love Scandinavian architecture and interior design, so Tjark's Danish background is helpful in generating more attention in Berlin.

In terms of civic engagement in his places, Tjark does not like engaging in any groups as he thinks it was more of a social model of the hippie generation of his parents, who more aggressively expressed their political opinion. He thinks that today people of his generation rather look for compromise and less for confrontation, including bottom-up projects on a small scale. He thus has also done some projects in the field of 'keep-it-local' design, working with local farmers and carpenters in Berlin. From a professional perspective as an architect and urbanist, he also follows the news about local developments in Copenhagen and Berlin. However, he is not a member of any association or regular activities.

In the future, Tjark wants to stay in Berlin, where he met his Austrian boyfriend two years ago, and where he has built up a sound professional network of freelancers with whom he can flexibly work. Tjark's story is that of a designer who profits from belonging to two cities of international kudos in his professional field. 


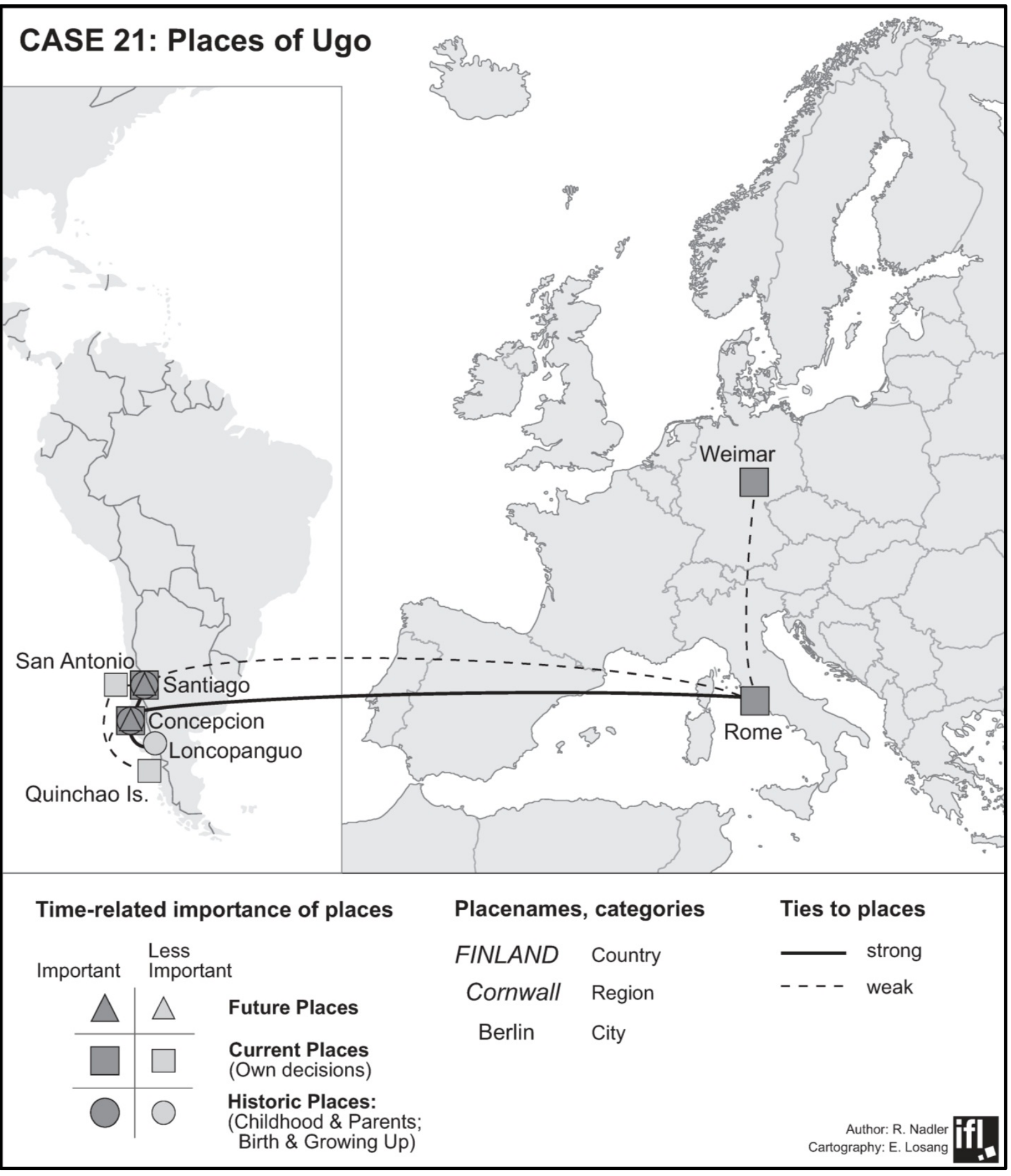




\section{Meet Ugo}

Ugo is a Chilean urban sociologist in his 30s who is currently living in Weimar, Germany, where he participates as a fellow in the frame of a bilateral agreement of his PhD school in Rome and the Bauhaus University. His main base in Europe is Rome, where he and his Chilean wife have an apartment and where their child was born. Ugo still thinks of Concepcion in Chile as 'his place', his home. He was born in Santiago de Chile, and his parents and brothers are still living there. Concepcion is more important to him as it was the place where he moved to study, where he started his work life, and where he met his wife. Each time he goes to Concepcion, he feels like he has come home, even though he no longer has an apartment there. Additionally, he considers the Islands of Quinchao and Chiloe and the island village of Achao important places in his life. Living in this archipelago in Patagonia, he spent some time doing fieldwork and writing his master thesis there. After finishing his thesis he returned to Concepcion, and later on he found a job in the port city of San Antonio, near Santiago. After some time, he founded a company with his cousin in Santiago. The company also had clients in Concepcion, so Ugo commuted frequently the 500 kilometres between the two cities. He perceived this commuting as stressful, and he then took a position in research on public policies in a Santiago based institute. At this time he also applied for his doctorate in Rome.

Ugo thinks of himself as a person who can easily adapt to new places, make new friends and build social relationships. Therefore, the local level is the most relevant. Whereas he would not identify as a European, he indeed expresses a feeling of belonging to Rome. However, an intercontinental transnational life also brings problems in maintaining relationships. Although Ugo describes his everyday life as taking place in Rome and Weimar, his Chilean family and friends do not know very much about it, and he increasingly loses joint topics to talk about. Furthermore, he describes the seldom moments of returning to Chile as a feeling of a time gap. Ugo comes back with memories of how life was in Chile the last time he was there - often one or more years. Meanwhile life and topics have changed and he feels like a stranger. In order to maintain relations on at least a minimum level, he follows the news about Chile on the internet, and he speaks with his parents and brothers by using Skype. In terms of civic engagement for his places, Ugo mentions that he is somewhat restricted by Italian law to engage in political initiatives in Rome. Foreigners with a citizenship other than EU are not allowed to become active in Italy, therefore he is not engaged in any official association which deal with local issues. In contrast, he is part of university initiatives which have a project-based and non-political character, and he has a stable group of Italian friends with whom he plays football twice a week. Ugo appreciates this last type of social activity, as he prefers to be with Italians. Unlike other foreigners in Rome, he avoids becoming part of ethnic networks and groups. His wife, who is a photographer, volunteers in the Italian Association of Photographers.

Ugo also explains that he has a lot of problems with bureaucracy in his two countries. At the moment, he is not covered by any health insurance. In Chile health insurance is only available with a Chilean income, but he is paid in Italy. And in Italy health insurance is dependent on the status of a resident, which is not granted to him as he comes from a so-called 'Third Country'. Ironically, he mentions that his insurance is his good health. Furthermore, he cannot open a bank account in Italy, nor can he bring his child to public daycare. In the future, Ugo could imagine returning to Chile with his wife and his baby, where he would love to engage in establishing a transatlantic scientific network, using the professional contacts he has in Italy and Chile. His scientific topic of 'Planning in the Context of Shrinking Cities' is fairly unknown in Latin America, and he thinks he would be able to make use of it back home. Ugo's story is that of intercontinental multi-locality in which the geographic distance is still an obstacle. 


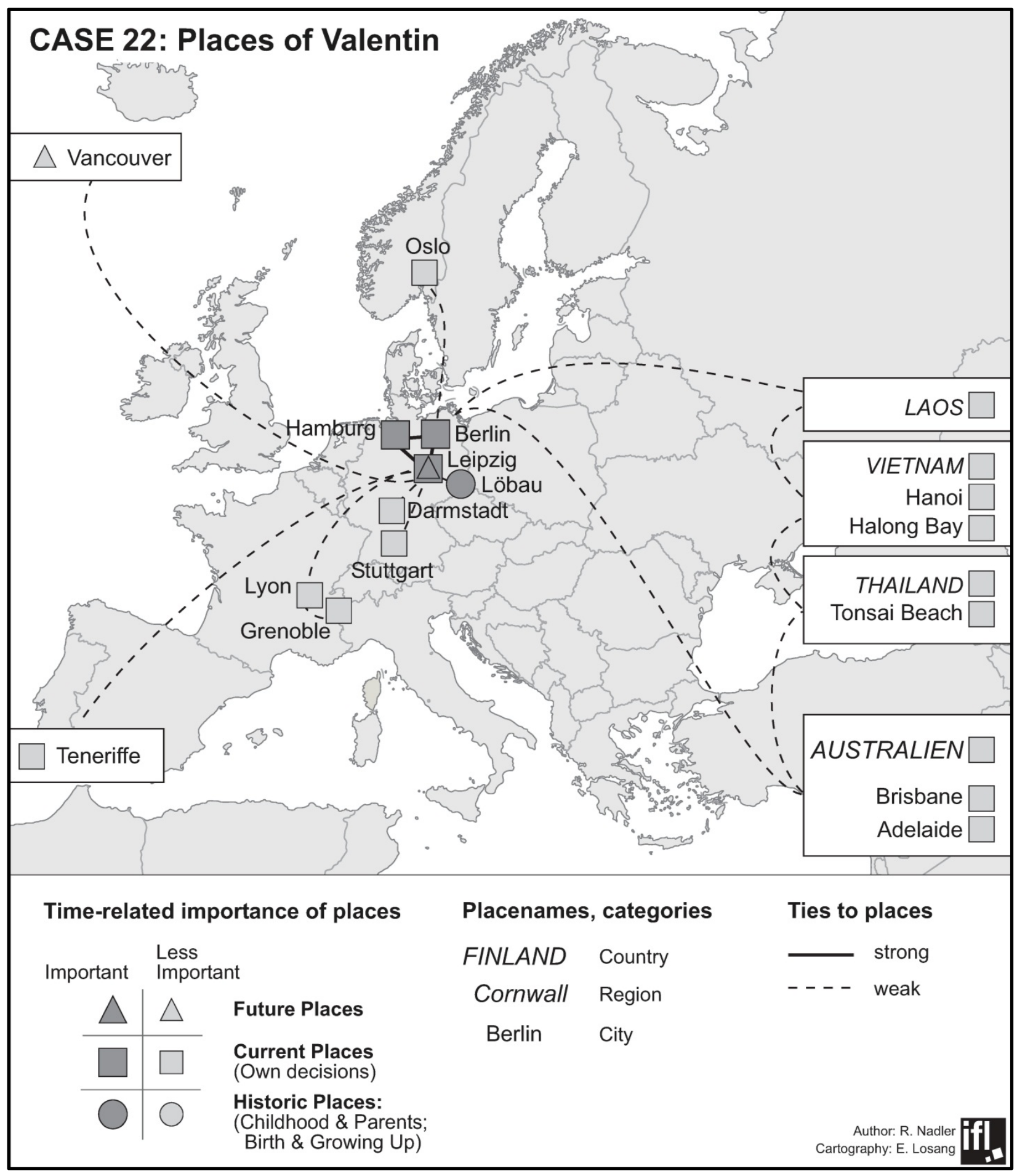




\section{Meet Valentin}

Valentin is a German freelance juggler and pedagogue in his 30s. He was born in a small rural town in a mountainous region in eastern Saxony, close to the German-Czech border. After graduation from school, Valentin first moved to Dresden and later to Leipzig, where he started studying theatre and film. His mobile life began with an exchange year at the university in Lyon, France. After a year in Lyon, he came back to Leipzig, where he continued his studies. Then he did an internship in a film production company in Berlin, which was specialised in dubbing documentary films. Upon finishing the internship, he went back to Leipzig, before he then moved on to Hamburg. In Hamburg, he helped a friend to finish his recent film. Subsequently, Valentin returned to Leipzig and started his own thesis. However, as it was winter, he headed towards the Canarian island of Tenerife; he preferred to write his thesis in a sunny place. He returned to Leipzig for a few months, from where he also commuted to Hamburg again for another film project. After a short trip through Israel, he went to Berlin as a freelance film producer, assisting a larger company with some film projects. After a while of working long hours, he realised that he preferred travelling to career-building. He remembered his Australian roommate from Leipzig who had always invited him for a visit to Australia, so he decided to leave Germany again. He travelled through Australia, Thailand, Vietnam and Laos. Once he ran out of money, he came back to Berlin and worked in several jobs (e.g. in trade fairs) to earn some more money. During that time he stayed at Berlin-based friends' places, to save money on rent. He mentions having slept on balconies during summertime, if his friends did not have an extra room for him. He went to Hamburg again, and assisted a film production. After finishing the project in Hamburg, Valentin travelled through Europe for several weeks with his bicycle. Back in Hamburg, he worked for a documentary production about a group of people jogging all the way across Germany. He travelled with them and helped with the filming. Finally, after some smaller jobs in Spain and in Berlin, he returned to Leipzig due to the cheap rent. Coincidentally, he was asked to join a start-up company of a friend. Having joined this company, he now works as a graphic designer, juggler and pedagogue with school children.

Valentin is a passionate climber. In his rural home town, Valentin began climbing at an early age, and it remains his hobby today. Climbing is also one of the reasons for travelling and which guided his mobility. He stayed at world-famous climbing locations on Tenerife and in Thailand, and he also says that he decided to go to Australia and leave his job in Germany to be able to climb in a sunny climate. Actually, Valentin was just about to leave for Canada to join a Swedish-Australian friend he had met in Thailand. Unfortunately he had just broken his foot while climbing, so he was forced to remain immobile in Leipzig. In the future, Valentin wants to go abroad again, but he is currently in a conflict as he realises the advantages of making money in his current Leipzig-based job. However, he does not really feel at home in the city. He says that his roots, his origin is a stable fact - the small rural town where he grew up and where his parents own a house. There he stores his belongings (important papers in particular) while abroad. On the other hand, he cannot really say where he feels at home. Valentin's travel routine and his short-term and unpredictable stays in places also detain him from engaging more profoundly with his places, or with the local community. Travel means adventure and exploration to him. Most often he does not know how long he will stay in a place once he arrives. However, he always knows that it has temporary character, and thus he does not feel like engaging in local initiatives or associations. He only assisted an urban gardening initiative with their PR material. He took photos for them, and prepared flyers and designs. Valentin's story is mainly that of someone who travels with curiosity to explore insiders' tips. He is mainly orientated to travelling far from backpacker routes, close to nature and solitude. His sporadic jobs in creative projects mainly serve the funding of his journeys. Valentin uses travelling in order to express his individuality and to distance himself from others. 


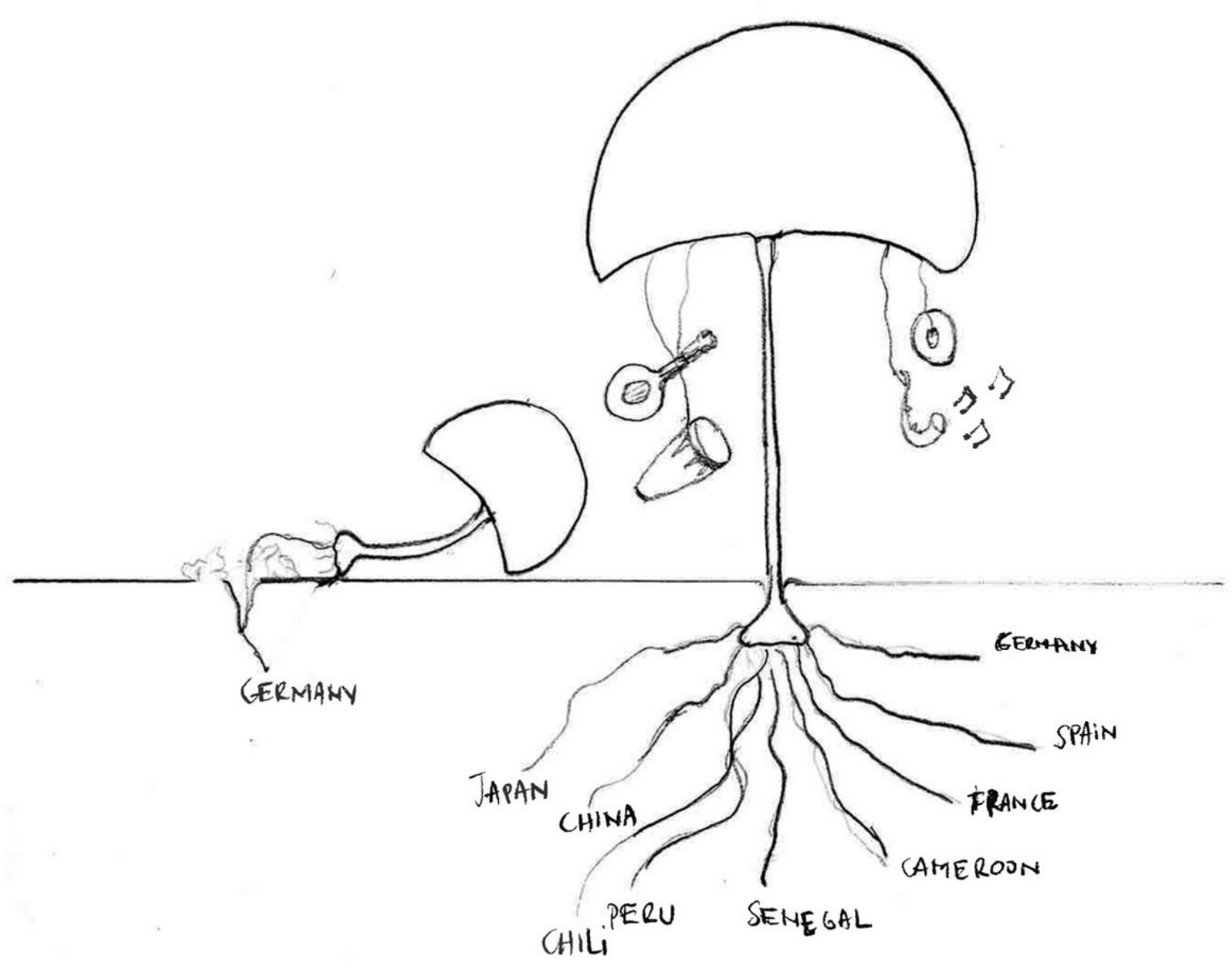




\section{Creative industries, roots, and routes: Discussion of the findings}

After having presented the main empirical findings, I will now return to my initial questions for this thesis. In chapter 5 I outlined three different and, as I believe, paradoxical theoretical situations which have arisen from my literature review. Based on these observations, I formulated sets of research questions which I considered as 'fields of interests' to guide my empirical research. In this chapter I will recall these questions and discuss to what extent I can provide answers to them with empirical material from chapter 7. Furthermore, I will also relate these findings to the theoretical propositions as outlined in chapters 2-4. This chapter is structured similarly to chapter 5, thus I will begin with discussing the field of interest which evolved around the ideas of rootedness and place attachment in multilocalmultilocal and mobile contexts. Thereafter, I will look at the findings in terms of the role of mobility in the lifeworlds of my interviewees. Third, the empirical outcome concerning the transnational dimension will be reflected.

In section 5.4 I mentioned that I would like to use the term 'place' not as a theoretically defined concept, but as an empirical term in the sense of my interview partners. I have presented the empirical insights on the role and meaning of places in section 7.1. Therefore, I will elaborate a bit further on 'places' in multilocal lifeworlds in the last section this chapter. Here, I will introduce a first draft for the heuristic concept of the 'plug \& play places' which is an abstraction of the empirical findings.

8.1 Roots and multilocality: Implications for our understanding of belonging in the light of multilocal creative knowledge lifeworlds

In section 5.1 I presented my first theoretical paradox, which I called the 'multilocality paradox'. I will briefly repeat what I found to be paradoxical here: rootedness in place is strongly dependent on the physical presence and experience of places in everyday life. Out of this physical experience of the place and its social environment, a person can develop a feeling of specific attachment, which can be described as place-based identity and as a feeling of being at home or sedentary. For creative industries, local integration and participation in professional networks and milieus is also described as something important in order to have access to market-relevant knowledge and information as well as projects and jobs. In the light of multilocality literature, place attachment and being rooted, which seem so important for mental well-being and professional success, somehow stand opposed to the duality of absence and presence which results from multilocal alternation between places. Consequently, I posed the following question, which I will try to answer now:

On a very descriptive level, which places actually constitute the action spaces of multilocal creative knowledge workers?

As outlined in section 7.1 I have found that the creative knowledge workers' action spaces consist of mainly more than two places. The multilocality which my interview partners describe includes far more places when it comes to imaging their own mental map. I also said that there is a core action space which is surrounded by a set of satellite places. The core action space mainly consists of larger cities and these cities have a strong importance in the person's current everyday life. The 
satellite places are less important and often are part of the action space as they have a historic importance in the life of my interview partners. The latter are often located in rural areas and are smaller than the pillar places of the core action spaces. The biographies of my interview partners follow the latent logic of urbanisation. After school graduation they had to leave their rural home towns and go to larger cities which offered a university education. Once in a larger city, they tend to stay there instead of going back to their smaller places.

This finding supports the propositions in the literature on creative industries which suggest that creative industries tend to be located in large agglomerations (Lange 2005). Indeed, my interview partners tend to orientate towards larger cities in order to work in these professional fields of creative industries. Meusburger (2008) pointed to the fact that spatial disparities will persist in the knowledge economy, and other authors pointed out that creative knowledge workers are drivers of re-urbanisation in the sense that they tend to move to the city centres of large agglomerations (Wiest 2008; Kujath 2012; Gornig \& Mundelies 2012). Looking at the geographies of my interview partners' lifeworlds, I would support this argument; however, I would put a small condition on it. My interview partners do not completely lose the ties to their historic and often rural places. Given that they also spend minor parts of their time in these places and that they also work temporarily from there, there is no automatism which excludes creative knowledge activities from rural areas. In this regard I would also support Ermann (2011) in his argument that rural places can also host creative activities. The geographic pattern of my interview partners suggests a preference for urban agglomerations, but it is not to be understood as evidence for the exclusion of rural places from participating in the new creative knowledge multilocality. I would prefer to pick up Dienel's (2004) idea of spatial partnerships which might evolve between urban and rural places. I think that my empirical evidence shows that my interview partners actually live their individual spatial partnerships. In their everyday life they combine rural and urban areas and they link them. Nonetheless, it remains an open question if critical masses can develop in order to found institutional spatial partnerships on the basis of collective action patterns, as Dienel suggests. The patterns which I have observed are definitely individual ones. Perhaps reducing the individual place patterns to a bi-polar relationship between two places might help to identify collective relations. Based on my sample, I could imagine that e.g. the bi-polar relationships of Berlin-Copenhagen or Berlin-Leipzig might provide potential for more collective spatial partnerships, but in other cases significant collectivity can seriously be questioned.

Furthermore, I have found that geographic distance continues to matter. Multilocality actually means that my interview partners seek to be physically present as far as they could in all of their places, therefore physical travel is necessary. Other than monolocalmonolocal people who might also have to include several places in their life but who do so mainly in terms of virtual travel, my interview partners really have to bridge geographic distance by physical travel. This means it is an effort of time and money, and it represents a precise strain. As such, geographic distance and connectivity between places decides on the feasibility of multilocal arrangements. This is visible in the geography of these multilocal lifeworlds: the places are mainly located in Europe. Intercontinental multilocality is less of an issue for my interview partners. Whereas there are some who combine places in different continents and over long distance, they can less often bridge this geographic distance. As such, geographic distance affects the rhythm of alternation and the feasibility of arrangements. This finding supports the arguments which recall that the internet and 
the enhanced travel infrastructures do not necessarily lead to a 'flattening of the world' or the 'death of distance' (as was suggested by Cairncross 2001). According to my observations, the world remains 'spiky', to use Florida's term (2008). Places are perceived as different from each other, and combining them in a multilocal life necessarily causes the perception of geographic distance through physical travel.

Are places equally important, or can we observe an unequal distribution of meaning and importance between places within one multilocal lifeworld? What relation exists between different places in the perception of multilocal creative knowledge workers?

Based on my empirical findings, I would answer this question according to the second suggestion. Indeed, the places have neither equal importance nor equal meaning in the lifeworlds of my interview partners. The main pattern that I have found is that places are ordered, often hierarchically, according to a time axis (historic, current, and future places) as well as to their functional utility in the person's current everyday life. This means they have varying importance in the life of my interview partners. The main pattern that I have found in my study is the 'functional complementarity' between places; the places fulfil different roles in the lifeworlds of the creative knowledge workers. This finding I think is equal to Stock's idea of Polytopizität, where he argues that there is a 'specific attribution of place and practice' (2009: 111). Some places are more frequently visited than others, some are part of the private and personal aspects of the lifeworld, and others rather refer to the professional dimension. Places are also related to different persons who are unequally important in life. As such, the importance of a place itself also mutually interferes with the importance of certain people who live there.

Given these findings, I would say that the first insights of multilocality research can be underlined. For example, Weiske, Petzold et al. (2009) differentiate in their study of multilocal households between places that refer to the professional dimension and places which are the home of the household and have a private connotation. This is a pattern that I have also found among my interview partners and the way in which they organise their households. Furthermore, Stock (2009) describes that people differentiate between familiar places (Eigenorte) and foreign places (Fremdorte). For Stock, familiar places are places where everyday life takes place, whereas foreign places have a character of being extraordinary. I also found circumscriptions of that kind in my interviews. Stock argues that in the context of multilocality, foreign places might be close by and familiar places in geographic distance. In some of my cases I found this differentiation between familiar places and foreign places in this geographic logic, too. However, what my interview partners describe as foreign places are also places in their everyday life. When speaking about their less important places, or about places that were recently added to their place pattern, my interview partners then also used descriptions that express foreignity and familiarity.

How does place-based identification work in the context of multilocal creative knowledge work? How much of the identification of creative knowledge multilocals is actually based on place? Are any other categories of identification emerging among creative knowledge multilocals (social groups, profession, or mobility instead of rootedness)?

Here, I would like to mention that, yes, place still plays a very important role in the identification of multilocal and transnational creative knowledge workers. However, place only becomes indirectly relevant as a reference frame when it comes to emotional attachment and belonging. Here, mainly it 
is about people to whom my interviewees develop an emotional relationship. Place is then simply the location of the person. On the other hand, places can directly work as reference objects of identification. This might be related to the personality and the lifeworld as a whole, but I rather observed this in relation to the professional environment in creative industries. Places can become a marketing factor here, as they can have symbolic value within certain sub-sectors of creative industries; think of Rotterdam for architecture, Berlin for sub-culture and nightlife, Leipzig for alternative urban planning, or Milan for the fashion industry. Based on my interviews, I would argue that place-based identity remains important. Yet, as my interview partners realise that different places could be anchor points of identification, they also identify in multiple and hybrid ways. This includes any other kinds of 'reference objects' than place. The wider social community of a place plays a role, but it is also about the places themselves in terms of their built environment and architecture, their usability in everyday life, and their value in satisfying needs connected to leisure time.

Looking back at the literature, I would agree with Weichhart's (1990) idea that place-based identity can be considered a 'symbolic community' which does not necessarily have an emotional character; sometimes place-based identity can even have an economic character. This is visible in the way in which my interview partners systematically make use of their different place affiliations in order to influence how they are identified by others. 'Being identified' by others was described by Graumann (1983) as one of the components influencing personal identity. In terms of 'place', my interview partners have learned to subvert this component. They actively influence how they are identified by applying 'alternative autobiographies'. They also use this to distinguish themselves from others. Here, the limits of Mead's (1934) idea of a sequence of 'appearance' and 'discourse' become problematic. Weichhart mentioned that Mead's appearance referred to the initial moment of encounter between two persons in which first identification of the partners of this interaction takes place without verbal communication. Secondly, discourse starts between the two persons which leads to identification with the other, in the sense of finding sameness. Yet, my interview partners sometimes rather tend to enter into a discourse of finding difference and marking distinction through their multilocality and the multiple attachments of which they dispose. They often tend to stress their individuality and otherness. As such, they mainly root in their own multilocality and in their mobility instead of in one place and one place-based identity. In this sense, I would remember Petzold's (2009) idea of multilocal identification which suggests that multilocal people can develop emotional and cognitive attachment to several places. I also found this in my data. This pattern finally also underlines the relevance of Jung's (2012) idea of 'psycho-social sedentariness' according to which people first of all have to root in themselves and in their lifeworld. None of my interview partners expressed feeling uprooted by multilocality and mobility, even though they perceive negative consequences of such a life. They are rooted in their lifeworld and as such they are psycho-socially sedentary.

What impacts does their multilocality have on their potential to actively shape their cities? Are creative knowledge multilocals still 'citizens' of a particular place who engage in local civic engagement, neighbourhood initiatives and governance activities?

Based on my empirical findings, I would suggest that multilocal creative knowledge workers are actually less probable to shape their places and participate in public local life than their monolocalmonolocal counterparts. I have mentioned that they all try to be involved in local social 
life, both in the private and the professional realm, yet there are three main inhibiting factors. First, my interview partners do not have much time left to engage in activities outside of family life and their professional duties. A large share of their time is actually used for travelling, and this time is then lacking for other activities. Second, my interview partners are confronted with the unpredictable absence-presence duality; they have very irregular schedules. This translates into the impossibility of engaging in long-term commitments which need regular presence. Third, many of my interview partners have realised which benefits there are to be part of different social contexts, and through comparison between these they developed a type of polyperspectivity, therefore they dislike local groups which only focus on their own perspective. They actually get bored by such social environments and tend to avoid spending too much time in these environments. They prefer to switch back and forth.

Having said this, I think that the temporal disposition as well as the willingness to engage and commit to an individual place's development is dramatically limited by multilocal lifestyles and I would agree with Petzold (2010: $254^{226}$ ) who guessed: 'It can be assumed that particularly for local structures of sustainability, which include ecological as well as social and economic place properties, translocal multi-location might have a positive impact. Yet, it is of the highest interest which backlash this has for activities within the context of a multilocal life in the place of origin. To what extent do translocal people change their relations to this place?' In order to understand Petzold's quote, we have to recall that he considers those multilocal people to be translocals who actually develop similar emotional attachment to both or all of their places. By contrast, he explained that interlocal people actually do not link equivalently to all places, but they have one central place and other emotionally and cognitively less relevant places. Insofar, translocality can have a positive impact to the recently added place if the translocal person develops a new attachment to it. Knowing this, we can understand Petzold's suggestion that in a translocal life the recently added place might benefit from the translocal person's presence, but it is unclear what happens to the former places of the lifeworld. Will the aspect of 'absence' become more dominant there? I would agree with Petzold's scepticism and I would even go a bit further: I would say that interlocal people will also have to retract from their engagement in their one central place. Not only the absence-presence duality becomes a problem here, but also the time which is spent on travel. As such, I would suggest that all multilocal people - meaning translocals as well as interlocals - will be less available for sustaining a place's social vitality.

8.2 Routes: Implications for our understanding of mobility in the light of locally embedded creative knowledge lifeworlds

A second paradox that I have observed in the literature is the mobility paradox. The main curiosity I had in this respect was in what manner do mobility and belonging actually go together? I observed that the metaphors for belonging and rootedness in the literature, namely that Heimat, place-based identity, dwelling and sedentariness all suggested that one needs to be fixed in a place to become

\footnotetext{
${ }^{226}$ Translated by the author from the German text: 'Zu vermuten ist, dass insbesondere auf lokale Nachhaltigkeitsstrukturen, die neben ökologischen auch soziale und ökonomische Ortsmerkmale betreffen, translokale Multilokationen einen positiven Einfluß haben dürften. In hohem Maße von Interesse ist jedoch auch die Frage der Rückwirkung auf Aktivitäten eines multilokalen Lebens am Ausgangsort. Inwiefern verändern translokale Personen ihr Verhältnis zu diesem Ort?'
} 
rooted and to feel a sense of belonging. On the other hand, there was a large strand of literature in the field of mobility studies, transnationalism and multilocality which all argued that people are increasingly on the move and as such spend their life on routes. This literature tries to capture the way in which belonging and attachment can work under the influence of continuous mobility, but the answers were not satisfying; some scholars here even suggested that some sort of uprootedness from places will develop. In particular, in the light of creative industries research, which suggests a strong involvement in local creative milieus and in creative cities in general to be an important aspect for the successful operation in this market, I found the idea of a continuous border-crossing mobile person to be an antagonism. Given these somehow contradictory positions, I asked myself the following questions:

What does mobility mean in the lives of mobile creative knowledge workers? Is mobility eroding the stability of the lifeworlds and professional situations of mobile creative knowledge workers? How do mobility and belonging interrelate in terms of personal meaning and professional symbolic value in the context of creative knowledge work? Can mobility become a constituting element of identity and belonging for creative knowledge workers? Can mobility even become a professional advantage?

In my interviews I found that mobility is a constituting element of multilocal lifeworlds. I have described how physical travel, imaginative travel and virtual travel actually interrelate. The functions that I described in chapter 7 clearly indicate that mobility has a strong relevance in my interview partners' lives. It signifies separation and departure, but it also means arrival and connection. It allows for constant comparison between different social and physical environments and leads to polyperspectivity which itself supports multiple identities. As such, I can confirm much of what is said in the literature on multilocality. I can confirm Rolshoven \& Winkler's (2009) idea of travel (or mobility) to represent places within the everyday lifeworld. In section 7.2.4 I outlined how we can think of transit spaces as mobile and immobile places: herein the mobile multilocals are actually moved through a transit space and as such, they move, but at the same time they do not move themselves. They sit in a train, in a train station, in an aeroplane, etc. This means a large part of travel time is actually spent not moving their own body with their own energy. Hence, this time can be used for other everyday activities, and as such travel can take the form of a place, too. Duchêne-Lacroix' (2009) also commented that the intensity and regularity of presence in the individual places is important to find a foothold in the rather monolocalmonolocal surroundings can be stated through my empirical findings. Here, mobility actually becomes the facilitating and binding element between the places, which allows for maintaining ties and switching according to presence needs in the individual places. I would also agree with Weichhart's standpoint, in which he argues that 'being on the move' - which is actually mobility - allows for the exploitation of place-specific offers (Standortofferten; as formulated by Weichhart 2009). This is also a recurrent theme for my interview partners. As such, mobility should not be thought of as contradictory to belonging and attachment.

How can the absence/presence duality be understood?

Now that we know what mobility might mean and that it works bindingly, we still do not know what cognitive implications the absence-presence duality can have. The main argument in the literature on 'roots' (cf. chapter 3) is that belonging and attachment emerge from physical presence 
in a given social and built environment. We have heard about the notion of 'home' which suggested that nearly all aspects of the lifeworld are located in the place called home (cf. Scheffel 2012: the 'six dimensions of home'). Similarly, the traditional understanding of dwelling refers to the idea of having one's own stable place in life to which one knows to belong and where one can go to in order to recover from public social life (remember Goffmans' idea of the 'back region'). Dwelling as a practice was also initially thought of as deeply rooted in the neighbourhood (cf. Herlyn 2003). It was about spending time in the neighbourhood and engaging in interaction with the neighbours and the local community. How should this work if people are temporarily absent from their places? Here, I will come back to the ideas of social phenomenology as outlined in chapter 6 in which I introduced the concept of appresentation/Appräsentation according to Schütz \& Luckmann (2003). Based on my empirical findings, I would like to argue that we could actually use ideas from 'appresentation' in order to understand how people deal cognitively with the absence-presence duality. Petzold (2010: 241) had already begun to consider this idea, but he did not further elaborate on it. I will briefly recall what appresentation means. Husserl developed this concept in order to understand why human perception does not end at the limits of physiological senses. Physiological perception has temporal and spatial limits; it is limited to the present moment and the present spatial environment. If we are limited to the present by our physiological senses, then making things appresent becomes an important cognitive effort to embed situations into the entirety of our lifeworld. That is basically what happens in appresentation. Appresentation can be described as the cognitive effort to connect a currently lived and perceived situation (the impression) to a recently lived experience (the retention) and to the immediately following situation (the protention). Appresentation thus helps us to connect these three parts in a frame of sense. It helps us to interpret and give sense to any given situation; we can only understand the entirety of our lifeworld in this manner.

If we now think of the fact that a multilocal person is always absent and present in their places, then I believe that we can understand belonging in the multilocal lifeworld as a state of 'appresence'. The 'other places' where the multilocal person currently cannot be physically are made aware through appresentation. As such, multilocal belonging receives sense. The multilocal lifeworld makes sense. If we apply this idea to the core item of spatial belonging, namely the 'home', we can speak of an 'appresentative home'. What do I mean by that? In monolocalmonolocal lifeworlds home is mostly one place and one can think of it as the place of presence. As such the monolocalmonolocal person can continuously perceive large parts of this home with their own physiological senses. MonolocalMonolocal people are present at home. By contrast, multilocal people constantly alternate between places. In their cognitive awareness there is always one place of absence that they have experienced directly before (retention), there is always the current location (impression), and there is the place which will come next (protention). Here, one could use the image of a journey between two places in which the traveller's place of departure equals retention, the train itself equals impression, and the place of destination equals protention. On the other hand, this could also be used to imagine how a person perceives being in one place while distant from the others. Then the current places would again represent the impression while the last visited place of the multilocal place pattern represents a form of retention. Furthermore, the place to which this person goes next is cognitively a protention, thereby continuously a former place of presence, which is currently a place of absence and is included into the current situation in the place of presence. In 
the same way, the following journey cognitively points to a future place of presence, which is still a place of absence. This in fact makes action, identity and sense structure possible in multilocal lifeworlds. Multilocal people are at home in a cognitive way. I think there is parallelism to Jung's idea of psycho-social sedentariness, yet Jung does actually make places a topic. However, if we apply appresentation to the place pattern of multilocal lifeworlds, we can also understand how people are at home in their places - namely in appresentative ways. In this sense, we have to rethink rootedness in space. My interviews have revealed that my interview partners distinguish between different types of belonging: being based (zu Hause) and being from (Heimat). This finding correlates with the results of Mühler \& Opp (2006), who similarly found that Heimat can be differentiated from other items of belonging because it represents something stable in life and refers more to the roots and childhood. Equally, they say that Heimat can have a lower relevance for current place-based identification than the current socio-spatial bases of life. This is exactly the pattern that I found in my data. In multilocal lifeworlds, this implies that we should think of Heimat and zu Hause as distinct things which are spread to different places. As all places - those related to Heimat (the 'historic places' in my case maps) and those that form zu Hause ('current places' in my case maps) - are spread across space but relevant in my interview partners' lives, they somehow must relate and maintain ties to them through appresentation. Things such as physical, imaginative and virtual travel are thus all part of the practice of 'appresentative home-making'.

\subsection{Individual transnationalism in creative industries}

There has been another intriguing point in the comparison of literature on transnationalism and creative industries which I call the transnationalism paradox. As mentioned above, creative industries literature suggests that these industries produce mainly immaterial symbolic goods. The production of these goods as well as their consumption is heavily embedded in national and sometimes also regional and local cultures. In respect to the national scale, we could easily think of the importance of language in the production and consumption of immaterial goods and service. However, once an individual creative knowledge worker becomes transnationally multilocal, and here I would like to stress the circulation between countries, what does this actually mean for their ambitions in the creative industries? In this respect I would like to answer the following questions:

How are the national and local scales actually interrelated in creative knowledge workers' transnationality? Do reference frames become multi-scalar? Do creative knowledge workers also relate to the supra-national scale (e.g. Europe, the European Union)?

I asked this question because I had doubts about the increasing relevance of the national scale as based on the literature on transnationalism; it is suggested that the local remains an important scale in lifeworlds, while the national reference frame becomes less important in favour of supra-national or transnational reference frames (cf. section 4.1). Looking at the lifeworlds of my interview partners, I found two empirical results. First, my interview partners actually become more aware of the supra-national scale. Given the geographies of my interviewees' lifeworld, this is mainly the European Union or Europe as a continent. However, the awareness of the supra-national scale does not automatically lead to the establishment of deeper relations to that scale; I have found very few incidences of European identity. Furthermore, my interview partners also express their disdain for the persisting international bureaucratic problems which occur in their transnational life. Therefore, 
they actually say that in everyday practice, the supra-national scale is actually less effective. As such, I would definitely support what the European Commission (2010: 20) says: 'Every day businesses and citizens are faced with the reality that bottlenecks to cross-border activity remain despite the legal existence of the single market. They realise that networks are not sufficiently interconnected and that the enforcement of single market rules remains uneven. Often, businesses and citizens still need to deal with 27 different legal systems for one and the same transaction.' In this statement there is some irony; it has the character of self-description of the European Commission and its own work.

Furthermore, I surely found evidence for post-national attitudes; my interview partners expressed this when speaking about their identity and the way in which they perceive themselves. They actually do not want to be related to a national frame of reference. Due to their transnational lifestyle, they actually think of themselves as wanderers between the nations and they stress their individuality, which does not fit into the national constraints. On the other hand, my interview partners cannot completely detach their personal identity from national references, as they are most often identified by others with exactly this national frame. Specifically when they are in one of their places which is not located in their country of origin or nationality, they are primarily identified as foreigners and the question then arises of which country they come from. As multilocal lifeworlds across national borders are even more seldom than intra-national multilocality, my interview partners then try to avoid the difficult explanations to monolocalmonolocals by accepting the national identity.

Third, I think that my empirical results must be reflected in the light of transnationalism. Transnationalism literature suggests that transnational social spaces and transnational social fields might evolve (Faist 2000; Pries 2008) in which transnational migrants (Glick Schiller, Basch et al. 1992, 1997) unfold their action spaces. Considering my interview data, I see two problems in this respect. On one hand, my interviewees actually could not be thought of as migrants, and as such not as transnational migrants. This has to do with the fact that they live multilocally; this means they actually do not migrate in a traditional sense of migration. This traditional idea of migration explains that a migrant actually move his usual place of residence across an administrative border into a different geographic territory (cf. Ambrosini 2005; Demuth 2000; Franz 1984; Han 2005; Palidda 2008; Plane \& Rogerson 1994). The point is that my interview partners do not actually relocate any 'usual place of residence' from location A to location B. They rather open a new 'branch of their lifeworld'. They add a new place instead of substituting one place for another one. Whereas transnationalism studies are quite close to multilocality studies in the manner in which they stress the role of alternation between locales and the importance of the everyday life character of this alternation, they do not reflect the terms 'migration' and 'migrant'. These terms are used with legitimate reason - in other fields of migration studies (e.g. diaspora research, asylum seekers, refugees) where regular alternation between places is not as central as in migration research. Yet, I think that in terms of everyday practice the transnational migrants, who are described in the relevant literature (cf. section 4.1.2) are closer to multilocal people than to other migrants. As often described in the transnational migration literature, these migrants maintain links to their home countries; this often includes even the maintenance of households and owning property. As such, one should probably rather speak of border-crossing (or transnational) multilocals instead of transnational migrants. 
The second problem which I see in relation to the transnationalism literature results from the dominant assumption that through the practice of alternation across borders some kind of transnational collectivity emerges. The two concepts of transnational social field and transnational social space suggest that people with a shared experience of border-crossing group together or form spaces or fields whose joint is a lived transnationalism. Looking at the narrations of my interview partners I can hardly see such collectivities in their lives. My interview partners rather live in very individual lifeworlds in which they often separate places in practice even when they bridge them in cognitive terms. For my interview partners, separation takes place through the distinction between social contexts (cf. section 7.3.2), multiple identities and social roles (cf. section 7.6), or by using places according to their functional complementarity (cf. section 7.1.4). Whereas I found that my interview partners also talked about aspects of hybridity, the dominant pattern of lifeworld perception and action is an alternation which keeps places separated. Even though my interview partners might be perceived as transnational migrants if one took the literature as a basis of definition, the empirical results speak against such a characterisation. My interview partners do not live in any shared transnational social space or field. They live in their very own lifeworld, and perceive themselves as wanderers between the worlds. At this point I am unable to answer how far this 'individual transnationality' has any wider societal effects; this would call for deeper analysis, but my overall impression is that there is no such thing as collective transnationality in my interview partners' lifeworlds. In this sense I would agree to Favell (2003) who questions the 'transnational social power' of individuals who circulate across borders in Europe.

Do transnational creative knowledge workers develop transcultural products and services, or do they rather stick to the international exchange of national ones?

Studying the literature on creative industries and transnational entrepreneurship, I found it to be intriguing how these two things should go together. Let's look at the similarities first: both strands of literature suggest that transnational migrants as well as creative knowledge workers are selfreliant and autonomous individuals with a strong capacity to recognise professional opportunities which arise around them. However, there is not much more than this that I can see as a commonality. The transnational entrepreneurs make use of the advantage in access to relevant business knowledge as they are part of different countries' markets and business networks (cf. Faist 2000; Henn 2012). This means that the transnational entrepreneur focuses on the border-crossing and bridging aspects in his professional life. The creative knowledge worker also exploits such an advantage of access to business-relevant knowledge and information. However, the relevant knowledge and information for the creative knowledge worker stems from a strong integration into a local network which is deeply rooted in one place (cf. Storper \& Venables 2004; d'Ovidio 2010). Creative knowledge workers need to focus on the local business networks in order to take part in the market. They need to be aware and have expertise in manoeuvring in the specific local culture and mentality. As such, their focus is local instead of transnational. If a higher scale comes into play, then it will be the national one which becomes effective through its influence on language and culture. We have also learned that culture can be interpreted as something very individual and that it can dynamically develop collective forms of 'transculture', so I was actually interested in how far the transnational creative knowledge workers can actually draw on several national/local cultural systems, or even transcultural resources in order to pursue their professional goals if local embeddedness is supposedly so important. 
I have said that they live an individual transnationality, and this includes that they actually also develop forms of 'individual transculturality'. They speak several languages, they navigate through different cultural environments, they are partly engaged in bi-national partnerships, or they even have dual citizenship. In terms of everyday life practice, we can also see that they perform transculturality in that they develop professional knowledge stocks which contain hybridised knowledge gathered from all of their places and countries. They also produce transcultural symbolic goods. For example, we can see this in the paintings of Qamar, who merges Russian realism with western European abstractionism. It is also visible in Christian's artistic topic of the hut which is moved around and rebuild in different places. Oskar's services in the field of cultural mediation are influenced by his Swiss, Austrian and German affiliations. Tjark designs furniture with local materials from around Berlin, but gives it a spirit of Scandinavian design. Federico applies a way of Italian essayistic scientific writing mixed with German rationality. Therefore, my interview partners in fact soak up knowledge and information in all their places and elaborate it into creative products and services. However, as far as I have found in my interviews, the strong embeddedness in local networks plays a subordinate role. Perhaps my interview partners can be more characterised as transnational entrepreneurs who construct successful professional paths by using their multilocalmultilocality and transnationality, but they see themselves as individuals who float between their places and thereby find inspiration and ideas. While linking to local networks is important in terms of connecting to a place, it is not the most important thing for my interview partners. As such, I would say that the literature on creative industries fairly neglects the role of transnational links and overemphasises the importance of local embeddedness. My interview partners are not equally embedded in all their places, and - as I mentioned in section 8.1 concerning local civic engagement and their potential to shape places - they have to retract to a certain degree from local embeddedness in order to circulate between their places. Nonetheless, they develop successful products and perform well in the (labour) market.

\subsection{The ‘plug\&play places’}

In this section, I would like to elaborate a bit more on the character of places in the multilocal lifeworlds of my interview partners. In section 5.4, I said that I aim at deriving an empirical understanding of 'place' in my interview partners' multilocal lifeworlds and I will use this section to sketch out some ideas. As mentioned in chapter 2, in the literature on creative industries we can find the concept of the 'creative city', which includes ideas such as the importance of local networks and milieus for creative industries' production and reputation systems. Therefore, I believe that it is worthwhile to provide a bit of reflection on 'places' in the lifeworlds of multilocal creative knowledge workers.

\subsubsection{What is 'plug\&play'?}

Therefore, I will first switch the perspective to the technical term 'plug\&play'. With the technological innovation in the field of computer technology and software as well as the simplification of home user applications, 'plug\&play' appeared as a philosophy for making individual technological items compatible with each other. Individual gadgets (e.g. a computer and a mouse) should be able to communicate and exchange data and information without the user 
having to install any software (a driver), which should help translation between the gadgets' languages. The individual gadgets should be programmed to communicate automatically. For the user, this software solution is often not even visible. The only thing we can see is the physical plug. Everyone knows about USB plugs, for example. Nowadays, this philosophy has been developed into a technological standard. We can look at our latest vacation pictures on our flat screen television without even 'plugging in' our photo camera in a physical sense. Plug\&play has long become wireless, too. We plug in only in a metaphorical sense by using the Bluetooth connection. The latest developments tend to leave the computer environment and extend their aims to the complete networked home. In such a networked home, the television might be used to access a storage device where personal photos are kept, or it might access the music player device and transfer data to the audio system of the apartment. Therefore, 'plug\&play' has become a synonym for an easy-to-use component integration into existing systems.

However, let us take a step backwards. What do formal definitions of 'plug\&play' tell us about this concept? When the idea of 'plug\&play' arose in the early 1990s, several books were published by IT specialists and software engineers who aimed at distributing the idea of standardising software and physical connections between devices. Among these books, we can find titles like 'Programming Plug and Play' by Kelsey \& Kelsey (1995), 'Plug and Play System Architecture' by Shanley (1995), or 'The Plug \& Play Book' by Bigelow (1999). All of these books tried to engage in defining what plug\&play refers to. Shanley (1995: 11) e.g. writes: 'When a perfect card is first installed in the perfect machine, the machine detects its presence and interrogates the device to determine its vendor and device ID and resource requirements. These requirements are compared to the resources already assigned to previously installed devices. A non-conflicting set of resources are allocated to the new device by writing the appropriate values to its configuration registers. In addition, the list of resources allocated to the new card are stored in the system's configuration memory for use in configuring the device each time that the machine is restarted. Conversely, when a device is removed from the machine, the system detects its absence and deallocates the resources that had been assigned to it. [...] The event is reported to the configuration software. The configuration software deallocates its resources (if a device had been removed), or, if a new device is installed, configures the new device with non-conflicting resources and activates it (and load its device driver into the memory).' The terminology is very reminiscent of computer sciences. Shanley speaks about 'cards' and 'machines', and we can still remember the times when one had to install sound cards in order to be able to listen to music with the PC systems of the 1990s.

The technology and terminology have developed further nowadays. For example, we can look at UPnP Implementers Corporation's definition of 'plug\&play' from 2006. Under the headline of 'The Ideal Network' they formulate (2006: 3): 'Usage of UPnP network devices offers numerous benefits and advantages to the end user. UPnP enabled devices create seamless interoperability and unprecedented simplicity, reliability and ease-of use.' And later they go on (2006: 7): 'UPnP enabled devices seamlessly and transparently locate each other. UPnP technology defines a standard way of controlling devices using extensible markup language (XML) simple object access protocol (SOAP), which is the standard for Web messaging. The UPnP architecture supports zeroconfiguration networking and automatic discovery whereby a device can dynamically join a network, obtain an IP address, announce its name, convey its capabilities upon request, and learn about the presence and capabilities of other devices. DHCP (Dynamic Host Configuration 
Protocol) and DNS (Domain Name System) servers are optional and are only used if available on the network. A device can smoothly and automatically leave or be removed from a network without leaving behind any unwanted state information.' UPnP stands for Universal Plug and Play. This type of 'plug\&play' enhances the compatibility between different types of systems. The UPnP Implementers Corporation is a non-profit organisation composed of more than 800 companies which lobby for the implementation of a single UPnP standard. In this corporation's definition, we can see the changing terminology as compared to the 1990s. It is about adding 'devices' to 'networks'. Whereas Shanley still spoke of 'configuration' in the machine, here the 'zeroconfiguration' character of the network is stressed. Both definitions share the idea of a component being added to a system and then removed again without major problems. It is about the possibility to easily extend a given system by another component and thus increasing the system's capabilities.

If one browses the internet for 'plug\&play' today, one can find several definitions most often offered on IT expert websites ${ }^{227}$. All of these definitions share the idea that there is a computer system to which one can 'plug' in a new device 'and' it will automatically be usable: it is ready to 'play'. There is no need to worry about any configurations, because configuring happens automatically when connecting the device to a system. The former problem of distribution of resources in the system has been replaced by a standardised and shared language (a protocol) to manage resources automatically and ad hoc. There is no need for time-consuming manual setups and driver installation, and devices can be used immediately upon demand as there are routines for automatic recognition.

The idea of plug\&play is not only famous in computer sciences; it has also been applied to business administration and economic studies. The economist Richard Veryard (2001) used the plug\&play concept in a textbook for business planning. According to him, successful businesses depend on the capacity to flexibly include business modules into their total enterprise. For this purpose, Veryard draws on the plug\&play concept as used in computer sciences (2001: 4): 'In many domains, we expect components from different manufacturers to work properly together. For example, a consumer with no electronics expertise can easily plug a Panasonic VCR into a Sony television, or even an HP printer into a Compaq computer. And I can send faxes anywhere in the world from my ancient Canon fax machine, without my needing to know anything about the fax machine at the other end. The notion of plug-and-play implies instant connection and instant operation - ideally, you shouldn't need to restart your computer, or go through an elaborate installation routine. It also implies that you can unplug and you're back to where you started - ideally your computer shouldn't remain littered with memories of devices you have no intention of using again.'

Therefore, Veryard also uses the idea of a flexibly composed system of components which can enhance the capabilities of a system. I think the idea of 'plug\&play' might also help to understand action patterns in spheres other than business administration. However, so far I am not aware of any application of this concept in geographic or sociological studies.

\footnotetext{
${ }^{227}$ You might have a look at:

http://www.webopedia.com/TERM/P/plug_and_play.html (accessed 31 October 2012)

http://www.pcguide.com/ref/mbsys/res/pnp-c.html (accessed 31 October 2012)

http://www.techterms.com/definition/plugandplay (accessed 31 October 2012)

http://www.pcmag.com/encyclopedia_term/0,2542,t=plug+and+play\&i=49389,00.asp (accessed 31 October 2012)
} 


\subsubsection{Analogies between the lifeworld and a computer system}

If we now remember what I found out about 'places' in the lifeworlds of my multilocal interview partners, I would argue for conceptualising 'place', and the way in which multilocal people use it, as 'plug\&play places'. I will briefly explain what I mean by starting from three basic analogies:

- The 'lifeworld' of my interview partners can actually be thought of as a system as well. Just like a computer system, it is composed of different elements that form a coherent functional structure to allow my interview partners to live their mobile lives.

- We can think of the above mentioned dimensions which 'emerged' from the analysis of my interviews as the 'components' of the 'lifeworld system'. My interview partners perceive their lifeworlds as composed of these components. In a computer system, we could think of components as input devices, output devices, processing units, etc. In a lifeworld, analogously these components are places, mobility, people, biography and profession. The specific furnishing of my interview partners' lifeworlds then defines how they identify and how they behave.

- Furthermore, we can then assume that these components include specific devices - such as individual places, individual persons, specific biographical aspects, or particular jobs - that are added to the system, and which are configured according to the setup of the system of one's 'own lifeworld'. The individual devices - just like mice, screens, keyboards, printers in a computer system - then define the capabilities of the person in the frame of their lifeworld.

As I am interested in the relation that my interview partners develop towards 'their places' in the light of all the other components of their lifeworld, I will now focus on the individual places, and I understand them as 'plug\&play devices' in the system of the lifeworld. From the definitions of 'plug\&play', we know that there is a process of automatised configuration to make a device compatible and useable in a system. We thus have to look for these modes of configuration in relation to places. Second, we have learned that devices can be played immediately after being plugged in. Therefore, flexibility and ease of use are important characteristics in 'plug\&play' contexts.

\subsubsection{Configuration through place appropriation and orientation techniques}

Just like in a computer system, places are devices that are plugged into the individual lifeworld. This process of plugging a place into one's own lifeworld basically consists of the action of orientation and appropriation that I have described in section 7.7.5. My interview partners have developed techniques, or one could also say routines, which help to make a new place compatible to their own lifeworld. Basically, this consists of comparing a new place to the experiences from other places (cf. also section 7.1.3). According to this comparison, my interview partners are conscious of similarity and difference between this new place and their older places; they can translate between former and current experience of place. Through this translation they become aware of the specificity of the new place. Knowing in which sense the new and recently plugged in place is different from the other places, they can also develop appropriation strategies. These appropriation strategies allow them to make the place useable according to their own needs and preferences. They 
make it compatible by means of place appropriation. A computer enters into an automatic communication with a new device. The computer's processing unit asks a new device through standardised languages (protocols), what resources the new device needs from the system. Vice versa, the new device explains not only what it needs from the system, but it also declares what it has to offer. In a similar way, we could think of the moment when a person plugs a new place to their lifeworld. The system's processing unit, the person itself, asks what can this place offer them, and what I have to offer (or what resources do I have to provide) in order to be able to exploit the offered opportunities that are accessible in this new place. Using standardised place appropriation techniques (comparable to the standardised communication protocol for computer devices) a person enters into a communication process with a new place.

Figure 24: Playful imagination of the 'plug\&play places'; source: own picture

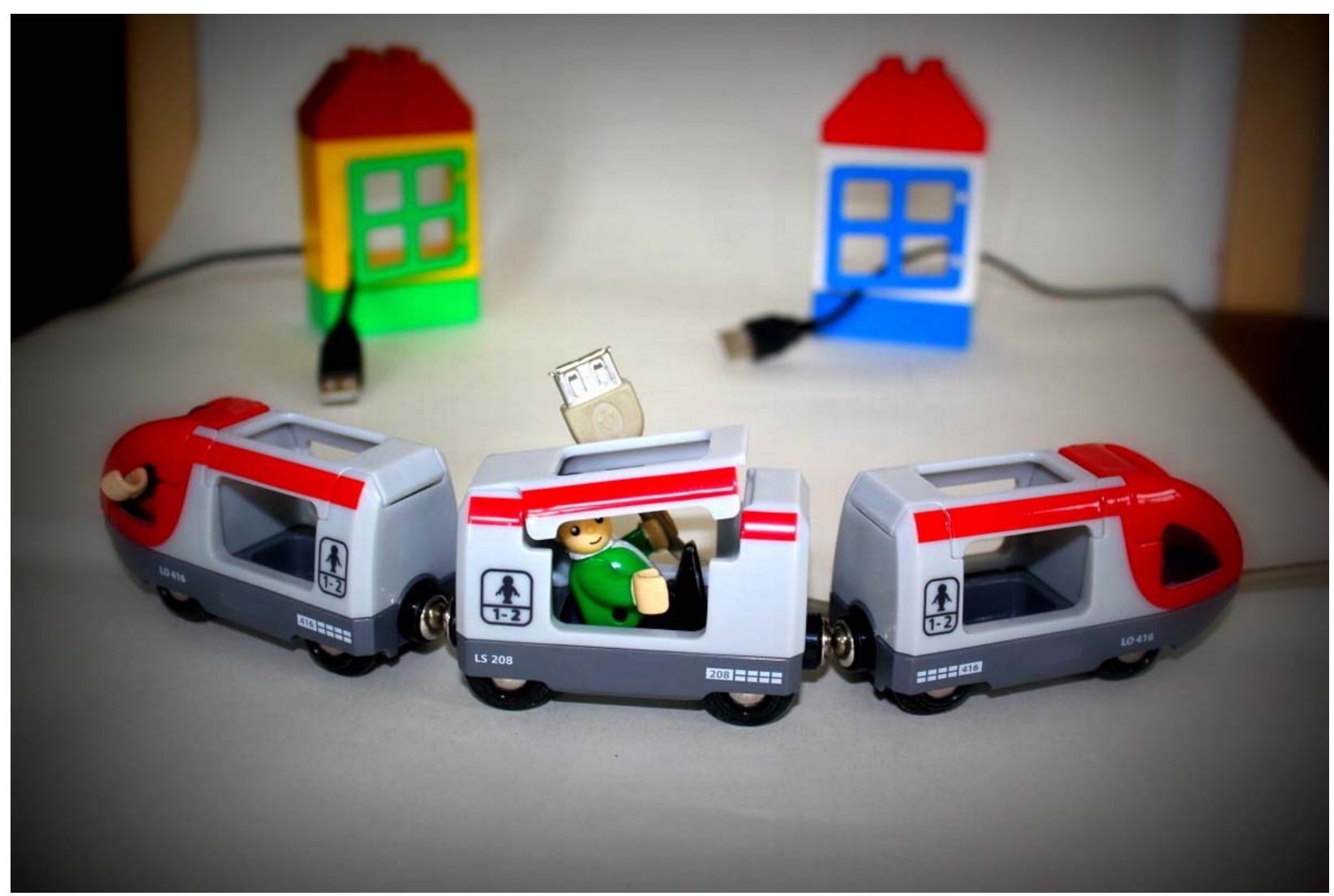

When a new device is plugged into a computer, the system's resources - here e.g. in terms of processing capacity of the chip - are checked against the needs of the new device. For example, if a new printer is plugged into the computer, the hardware management automatically looks for how much processing capacity is provided by the hardware, how much processing capacity is needed by the printer for its operation, and how much capacity to all other devices need. Then, the reallocation of processing capacity is balanced between the individual devices. Similarly, a person, when adding a new place to the own lifeworld, is checking for how many resources they dispose of in total. We could think of these resources in several ways: they could be time, money, means to travel, the capacity to cognitively handle a number of places in their own lifeworld, the willingness to include 
a new place, etc. In a second step, this person will look for how many of these resources will be needed to integrate the new place into their own lifeworld. In a third step, the person will balance the new place's needs with the needs of all other 'own places'. This means they will integrate the new place in a way that the system's total capabilities are best enhanced, and the resources allocated to the new place are not disturbing the functioning of the total lifeworld. Their lifeworld will only maintain its coherence if there is a satisfying balance between the places.

To make this more illustrative, we can pick out any of my interview partners. Let's take Xaverio, who circulates between Berlin, Milan and Rome. When plugging Berlin into his lifeworld, he configured Berlin through the evaluation of what he can get from it, and what he needs to give in order to receive what he expected. From the interview transcript, we can see that Xaverio expected to receive inspiration for his artistic work, he expected a rich cultural milieu and a vivid nightlife, and he expected low living costs. Then he looked at what he had in total as available resources, and how much he could give to make Berlin useable on an everyday basis. He calculated how much money he had to spend for renting in Berlin, in addition to the apartment he had in Milan. He also looked at how much time he actually had for spending in Berlin, balancing it with the time he needed for fulfilling his professional duties in Milan. Knowing that he would make more money with photography jobs in Milan, and expecting fewer opportunities in this respect in Berlin, he decided to rent only a room in a shared apartment where he could share certain fixed costs, and where he would not need to furnish the whole apartment. He decided to use Berlin as his source of inspiration and to use it rather in a private sphere, while Milan remains his professional centre. We know that later on Xaverio also plugged Rome into his lifeworld. Again he started to balance the give-and-take. He expected Rome to allow him additional money from a teaching job at the European Design Institute, yet he also perceived Rome as the cultural capital in Italy, so he expected to be able to do his artwork and exhibit it in an environment from which he expected more comprehension and acknowledgment than from his Milan environment. On the other hand, he calculated the travel time he needed to get there. Balancing all of these aspects, he decided to configure Rome in a way that he would not have to pay very much, and he opted for sharing one room with another multilocal person who is not regularly in Rome. The travel distance of three hours in a high-speed train to Milan also allows plugging in Rome on a one-day basis.

\subsubsection{Coordination of the system and mediation between devices}

In computer sciences the complex computer system is illustrated and made perceivable through the scheme of the system architecture. I would argue that we can think of my interview partners' mental maps as their system architecture scheme of their lifeworld in geographic terms (cf. section 7.1.1). A person becomes aware of which places are located where, and how they are related to one another. More widely, the mental map does not only contain geographic information, but also information on functional aspects of these places. Thereby, it describes a relation between 'place devices' in the lifeworld and other devices such as jobs, persons, temporal aspects and biographical moments. In a computer, the processing unit has the job to mediate between the individual devices' resource needs, and it has to guide the communication between them. Basically, it translates between the devices. Equivalently, we can imagine the individual person as a mediator between the single places of their lifeworld. As the phenomenologists have mentioned, a person seeks for a 
coherent lifeworld, in which they feel at home and feel like they can be themself. In this sense, my multilocal people use the different forms of travel - physical, imaginative and virtual travel - to connect their places to each other. The derived polyperspectivity, which I have described in section 7.2.3, can be understood as the resource which is used to translate between different places.

Here we can recall the example of Dirk, who has developed a polyperspectivity on different topics of his lifeworld while travelling between Bulgaria and Germany. One topic he mentioned concerned the way in which one can organise internet connections for one's own apartment. He described that in Bulgaria a technician would have no problem in connecting the apartment within two hours after calling by laying a cable along the building's façade, yet in Germany it would need several weeks and a lot of bureaucracy. Furthermore, he observed that in Germany ecological awareness is much higher than in Bulgaria, as well as that social inequalities make the Bulgarian society rougher in public space and on the streets. Knowing all these different aspects and being able to take the German as well as the Bulgarian perspective, Dirk can mediate between his different places. His mental map works like the scheme of system architecture; he immediately knows which place offers a more service-oriented internet provision, or where environmental issues are respected more seriously. This means he can manage in everyday life which place to plug in for which purpose. Knowing that things might work differently - sometimes better, sometimes worse - in other places, my interview partners can switch between the different perspectives ofone and the same issue. That is what I would think of as mediation between their 'place devices'. My interviewees become brokers of information on problem solutions in everyday life between their places. As such they are able to relativise problems and find different solutions from their intermediary role.

\subsubsection{Flexible use of places}

My interview partners select individual places and they add them to their lifeworld in order to enhance their capabilities; the places are functionally complementary (cf. 7.1.4). Devices on a computer also allow the system as a whole to do something new. If you plug a printer into a computer, it provides the additional capability of printing on paper what you see on the screen. Similarly, a place plugged into one's own lifeworld allows access to new resources and therefore enhanced opportunities in life. If we draw on the example of Xaverio, by adding Berlin and Rome, he extended the capabilities of his lifeworld. He added a place which provides inspiration, and he added a place which allows for artistic expression (going to galleries, teaching his artistic philosophy to others). Additionally, there is his productive place of Milan, where he makes his money in the fashion industry, and with which he enhanced the diversity of opportunities to selfactuate in his lifeworld. As such, his lifeworld is even more his own, it allows being himself, and as such it makes him happier.

This idea of the extended capabilities might basically also be true in monolocal migratory contexts. If a monolocal person decides to move from place A to place B without continuing to rotate in short-term rhythm between these places, they might enhance the opportunities to satisfy her individual needs, and thus to become happier. However, in monolocal environments, when a person moves their daily action space, places do not have this plug\&play character. A place is configured in more stable and less flexible ways. The speciality of multilocal arrangements lies in the fact that places are configured for flexible use. The continuous and irregular alternation between absence and 
presence calls for a configuration which allows for both being there and being away in unplanned ways. As such, the places must be able to be left, and temporarily 'unplugged' in a way. At the same time, they must remain configured and it must be possible to quickly plug them in again. Here, we can recall what I mentioned in section 7.7.2 concerning the empirical finding that my interview partners use the ubiquity as a standard setup of their places. Ubiquity is described as the way in which my interview partners hold available the things they need in their everyday life in a double or even more manifold way. We can switch to another example here. Aurélien described in his interview that he had recently installed a completely equipped atelier in the garage of his parents' property in Brittany, while he also has an atelier with all necessary tools available in Leipzig. This ubiquity allows him to alternate in a more flexible way between the two places. He can take fewer things with him and more flexibly chose the mode of travelling (in an aeroplane he might not have been able to carry large tools all the time). He now has configured his places so that they can be easily plugged in and played in at any time he needs.

Ubiquity can also be found in relation to the working spaces that my interview partners frequently use. Their main tools for doing their creative knowledge jobs consist of laptops and internet access. The first can easily be taken in hand luggage during travel between places. In order to access the second, my interview partners have developed routines in using their places. For example, many of my interview partners described that they use university and public libraries in their places as workspaces. They often organise memberships to libraries across Europe,so that they have their preconfigured access to workspaces. Arriving in a city then equals plugging it in, including the access to all forms of 'flexible offices' (cf. section 7.7.4).

Another plug\&play aspect which improves flexibility and ease-of-use is the post-material attitude and behaviour which I observed among my respondents. They abstain from consuming a lot of things (cf. 7.7.9) and they develop routines of travelling which are characterised by taking only a few things (cf. 7.7.1). Consuming a lot of things does not make sense in a multilocal life for several reasons. First, renting dwellings in different places does necessarily lead to the fact that the dwellings in the different places are smaller, so they can only store fewer things. Furthermore, we have observed that there are also very flexible ways of dwelling (7.7.3); some of my interview partners live in their 'own dwellings', but they might also live at friends' places, in a hotel, or share a place with others. Therefore, they cannot store things there either. Second, there is the fact that even having many things in one place would not allow them to always be used, because my interview partners are continuously on the move and would be separated from their material belongings most of the time anyways. A reaction to this is not to buy so many things. Finally, my interview partners also spend much of their financial and temporal resources on travel, and this directly translates to a lack of time and money for extensive shopping. Combined to these postmaterial attitudes and behaviour, I have found that my interview partners try to 'travel light'. They do not want to take many things in order to make travel faster and more comfortable. Check-in at airports is then less stressful if one must not queue to check-in luggage. Similarly, a long train ride is easier when one does not have to carry around a large suitcase when switching trains. All of these things are also aspects which allow for plugging in and playing. Finally, to make a place pluggable \& playable, the local mentalities must also be learnt. Multilocals then dispose of a catalogue of different local mentalities that they can switch on or off upon arrival (cf. section 7.6.5 \& 7.7.5). 


\subsubsection{Is standardization of place subjective or objective?}

If we think of Marc Augé's ‘non-places' (1995) or Duyvendak’s 'generic places’ (2011), we find an implicit suggestion that places become increasingly similar and standardised through the process of globalisation and global mobility. This idea orientates much at Ritzer's (1993, 2010) McDonaldisation thesis, which suggests that consumption will become standardised through globalisation. Using the examples of McDonald's and IKEA stores, Ritzer (2010: 10-20) highlights four aspects of such standardisation. First, these standardised consumption models are based on 'efficiency'. There is not much emphasis on quality but on delivered or sold quantity. For both the producer as well as the consumer efficiency in terms of quick consumption for low budget is important. The interaction of consumption should be as quick as possible, without losing any time. The second aspect is 'calculability'. It refers to the property of these consumption models that customers (can) know exactly that they can buy many for little money. Most often the offered products are low-quality products which are quickly produced, using cheap materials and inputs. So, the prices of sale are low in the end. Customers can easily calculate what they would get for their money. Additionally, there is not much variation in prices between geographic locations. This includes a third aspect, which is 'predictability'. Production steps and products have to be simple, easy to understand, and as such standardised mass products. Things and services which one can buy are the same in all locations around the world, and they remain the same for a long time. So, for customers it is highly predictable what they get for their money. They can rely on standardised offers independent of where they buy the product or service. This includes workers in the companies to offer their services and products in standardised behavior, too. They are not allowed to deviate from corporate modes of conduct with the clients. The fourth aspect is thus 'control'. Customers as well as workers are controlled to not leave the predefined paths of how the interaction of consumption should take place. Workers are trained to do few simple tasks that lead to a quick dispatch of customers. Customers are also manipulated through several forms of guidance, without that they necessarily become aware of that. Duyvendak's (2011) 'generic places' - e.g. the global hotel chains - have a similar character as a burger in a McDonald's restaurants or the Billy bookshelves in the IKEA stores. Duyvendak's highly-mobile people know exactly what service they will get, everywhere in the world and for how much money. These 'generic places' are standardised in that they are highly predictable, calculable, efficient and controlled. The same accounts for Augé's ‘non-places' which actually become 'non'-places as they do not have a specific and individual history. Moving and orientating in them works according to a standardised scheme. People can calculate with a high probability that their expectations about these places will be fulfilled. As such there is a standardisation of place: places become compatible for customers.

Also the 'plug\&play' philosophy also draws on standardisation as a means of allowing immediate compatibility without manual translation and configuration. Taking these two strands of thinking as they are, I would logically derive the assumption that the places of my interview partners are probably 'plug\&play', because they are already standardised and interchangeable. This would equal the suggestion that places are generally pluggable \& playable. As a consequence, one would have to formulate that the plug\&play character is an objective property.

However, based on my interviews, I have doubts about this suggestion. Rather, I would think of standardisation of places as subjective. It is about a standardisation of places from the viewpoint of 
the multilocal individual. While 'McDonaldisation', 'non-places’ or 'generic places' refer to single and less complex environments such as a shop, a highway, or a hotel, I would use the term 'place' to refer to the more complex environment of a whole city, or as my interview partners' also have done, to whole regions. And here I think that a whole city cannot so easily be standardised as there is a variety of different actors involved in shaping them, also with conflicting interests. Nonetheless, my interview partners individually standardise complex and unique places with each other in order to make them work in their everyday life. Places' resources or offers must be available in flexible and accessible ways. They must be predictable, efficient to use, calculable, and controllable, too. People must be able to come spontaneously and everything must work according to their needs, and not to the needs of any other person. People adapt places to their needs, but this does not necessarily mean that they really change these places efficiently by this adaptation. The process of adaptation (consisting of comparison with other places, appropriation, orientation, etc.) can also be a merely cognitive process. The individual person subjectively perceives a place and makes it fit into the geographic pattern of their lifeworld through cognition. Therefore, I would rather argue that the pluggability \& playability of the places of my interview partners is rather a subjective property. The same place might not be equally pluggable \& playable to other people. It is a very subjective configuration. The plug\&play place, in this specific configuration, fits functionally into the lifeworld of one interview partner, but pluggability \& playability of the same place might mean something completely different for another multilocal person.

Having said this, I would like to emphasise that I do not wish to exclude the fact that there is a parallel process of objective standardisation of space and places. There might be external macroprocesses which lead to standardisation. It might also be conceivable that a large group of multilocals with similar needs to places might lead to a collective instead of individual standardisation of selected places (cf. the idea of the institutionalised spatial partnership according to Dienel 2004). Clearly, it is also possible that multilocal people intend to change and re-design their places through concrete action. I have had a look at the ways in which my interview partners engage in civic activities for their places, and I have found out that they hardly do so (cf. 7.7.6), yet I could imagine that in a different sample, the outcome might be different as well.

\subsubsection{What's the value of knowing about the 'plug\&play' character of places in multilocal lifeworlds?}

I understand this idea of the 'plug\&play places' as a heuristic concept which allows a better understanding of how multilocal people relate to places, and how they make these places work in their multilocal lifeworlds. Transferring the idea of 'plug\&play' from computer science into sociological and geographic research might help to reconstruct the mechanism of belonging and attachment in the context of ever more mobile lifeworlds.

The concept allows us to perceive mobile people not as uprooted and nomadic people. Plug\&play places suggest that specific and regularly visited places are combined into a logical pattern, within a coherent system of the person's individual lifeworld. They are functionally complementary and can be used in flexible ways. The individual can quickly connect and disconnect from them without having to invest energy in orientation and appropriation efforts. These plug\&play places exist permanently in the mental map of the multilocal people, but they are not permanently plugged in. 
According to personal demand, a multilocal individual will pass by or touch down in these plug\&play places, and they expect immediate playability. However, as individual needs are very distinctive, we have to conceive of 'pluggability \& playability' as a subjectively perceived and individually constructed property of places in the context of multilocal lifeworlds.

By applying this heuristic concept, we can understand that people develop techniques to relate to very different and specific local contexts. This technique consists of translating the local specificity into the logics of one's own lifeworld. It produces a systemic order of place-related experiences. The 'plug\&play places' idea therefore helps us to understand how people re-root themselves in a context of increasing mobility.

I also believe that the 'plug\&play places' serve as an example for the precise applicability of the phenomenological concept of appresentation. Appresentation was described as a cognitive process of ordering and relating former and present experiences into logical and coherent order (cf. chapter 6, in particular sections in 'retention', 'impression', and 'protention'). From this combination, an individual starts to prospect into the future. The 'plug\&play places' incorporate all of these three dimensions of appresentation. A multilocal individual, when configuring a new place (impression), draws on former experiences with their own older places (retention), and once a place is configured for their own lifeworld, there is a certain expectation (protention) in respect to this place: namely that is useable immediately upon return.

Furthermore, I would like to stress the limits of the above mentioned plug\&play places. The coherencies that I have sketched out in section 7.8 have a hypothetical and preliminary character. They are derived from my 25 qualitative interviews and mental maps. As such this idea of the 'plug \& play places' must be considered the result of an empirical exploration and it cannot be more than a draft version. It would be important to further elaborate on that; one could probably test the individual hypotheses that are underlying this concept.

Finally, I also think that it is worthwhile comparing the idea of 'plug\&play places' to definitions of place in sociology and human geography. Sociologist Knut Petzold says (2010: $242^{228}$ ): 'In this regard, a place is a dynamic set of individually perceived physical, social and economic conditions of action.' Petzold here referes to a sociological understanding of place, which is close to the idea of Esser (1996) who prefers to think of places as 'situations'. Human geographer Peter Weichhart formulates (2008: $170^{229}$ ): 'Place refers to the material substrate of spatial constructs, which contain sense. Therefore, one can understand concrete locations and territories as 'place', which are embedded into an emotionally defined and mainly by a feeling of attachment characterized context of sense for specific individuals and social groups.' In these two definitions place is actually related to specific offers for action and emotional belonging which are provided by a geographic location. Using these offers and relating emotionally to these locations makes sense to people in the context of their lifeworld. I believe that the idea of 'plug\&play places' is actually fitting into these

\footnotetext{
${ }^{228}$ Translated by the author from the German text: 'Ein Ort ist in dieser Auffassung daher ein dynamisches Set individuell wahrgenommener physischer, sozialer und ökonomischer Handlungsbedingungen. '

229 Translated by the author from the German text: "„Place“ bezieht sich gleichsam auf das materielle Substrat sinnbehafteter räumlicher Konstrukte. Unter „Place“ versteht man also konkrete Orte oder Gebiete, die für ein bestimmtes Individuum oder eine bestimmte soziale Gruppe in einem emotional definierbaren und meist durch ein Gefühl der Zuneigung charakterisierten Sinnkontext stehen.'
} 
definitions. 'Plug\&play places are actually resources in the lives of my interview partners and they also provide anchors for emotional attachment and belonging. The additional value of integrating the philosophy of 'plug\&play' lies in the enhanced understanding that 'place' is not one unit in the lifeworld of multilocal people. They use several places; and by using several places they have access to more resources. Plug\&play then describes how these places are configured and integrated into a coherent place pattern. Furthermore, plug\&play helps to understand how multilocal people actually switch between their places and thus make use of them. 


\section{Interlude H: Willi - Yari}

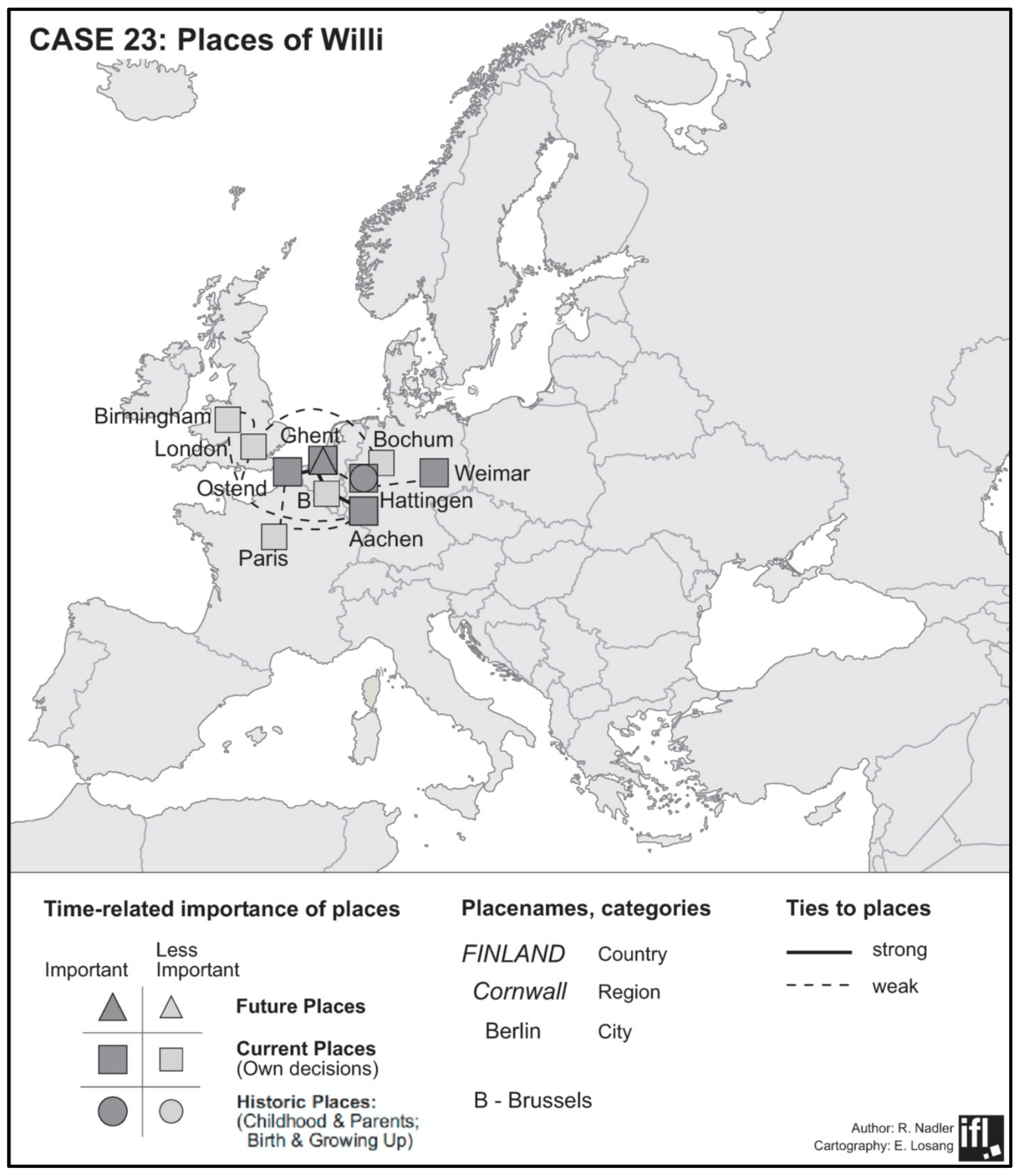




\section{Meet Willi}

Willi is a German regional planner in his 30s who currently works as a researcher in the field of regional development. He grew up in the rural town of Hattingen, and after school he went to study in Bochum. After he finished his studies, he went to London and added a Master degree. After graduating with his Master's, he went back to Bochum and worked as a freelance urban planner. He then changed to a job in an architecture office in the small town of Erkelenz, close to the city of Aachen, where he dwelled. From Aachen he went back to the UK and took a position at the University of Birmingham, which aimed at knowledge transfer into a Birmingham based architecture office in the field of urban planning. Finally, he went back to his current research position in Aachen. While in London, he met his girlfriend, who is a Belgian-Argentinian freelance journalist and photographer. His girlfriend moved from London to Gent, Belgium, and later on to Paris for a PhD course. Therefore, Willi also frequently travelled to Belgium and France. Since his time in Birmingham, he and his girlfriend had their first daughter. So, Willi was commuting between his job-related places and his family base in Gent. For one year they all moved together to Birmingham, but his girlfriend and his daughter went back to Gent. Later his second daughter was born, and both have a double citizenship - Belgian and German. Willi thinks that this is important; even though he has already experienced that the EU works as a liberalised supra-national labour market. Actually, it does not matter which country's citizenship it is as long as it is an EU citizenship, he says. This is also visible in his family: he mainly speaks English with his girlfriend, but German to his daughters. His girlfriend speaks French to his daughters, and in school they are taught in Flemish. So, they use four languages in their everyday life. Thus, Willi also tends to identify as a European citizen, yet he cannot hide his German identity. While abroad he always becomes aware of his national identity. Yet, he does not feel that he knows Germany very well, as he travelled more abroad than within the country. Sometimes he is astonished at how poorly he feels to be in his country from a cultural perspective when travelling to other German cities. Culturally, he feels more at home in-between England, Belgium and the Ruhr Region. He also thinks that travelling leads to polyperspectivity, and one becomes more open to other opinions and ways of thinking. The travelling between his places is a routinised activity for Willi; he mainly works during his train rides. The frequent travelling between Aachen, his job-related place, and Gent, his family-related place, makes him feel at home in both cities. However, his home in the sense of his biographic roots remains in Hattingen. Finally, travelling is also a burden to Willi. In his job he has to travel a lot, and in combination with his regular commuting between Gent and Aachen, during some weeks he has to travel every day. In terms of civic engagement, Willi was an active member of the local Green Party in Aachen, but the weekly meetings take place on Thursdays, and he is only in town Monday through Wednesday, so he cannot participate anymore. He even asked for flexible activities, but the board of the local branch did not understand his situation. He also had to quit his regular swimming activities, and he goes to cultural activities less often. The combination of family, job, $\mathrm{PhD}$ project and commuting takes up all of his time; he does not have any leisure time to spend on civic engagement. However, to a certain degree, his children make sure that he becomes more integrated in social life when it comes to accompanying them to their activities and meeting other parents. In the future, Willi could imagine living a more monolocal life again if a place would offer satisfaction in terms of professional and family related needs, and if this place generally provides a high quality of life. Willi as well as his girlfriend work in jobs with flexible and unpredictable labour market conditions, so even if he preferred to be more monolocal, he does not consider this realistic at the moment. Willi's story is mainly that of a commuter between the worlds of work life and family life. His two main places are separating these two spheres of his lifeworld, and the weekly travel between them has a symbolic meaning of switching between these worlds and his roles the single professional on the one hand, and the caring family father, on the other hand. 


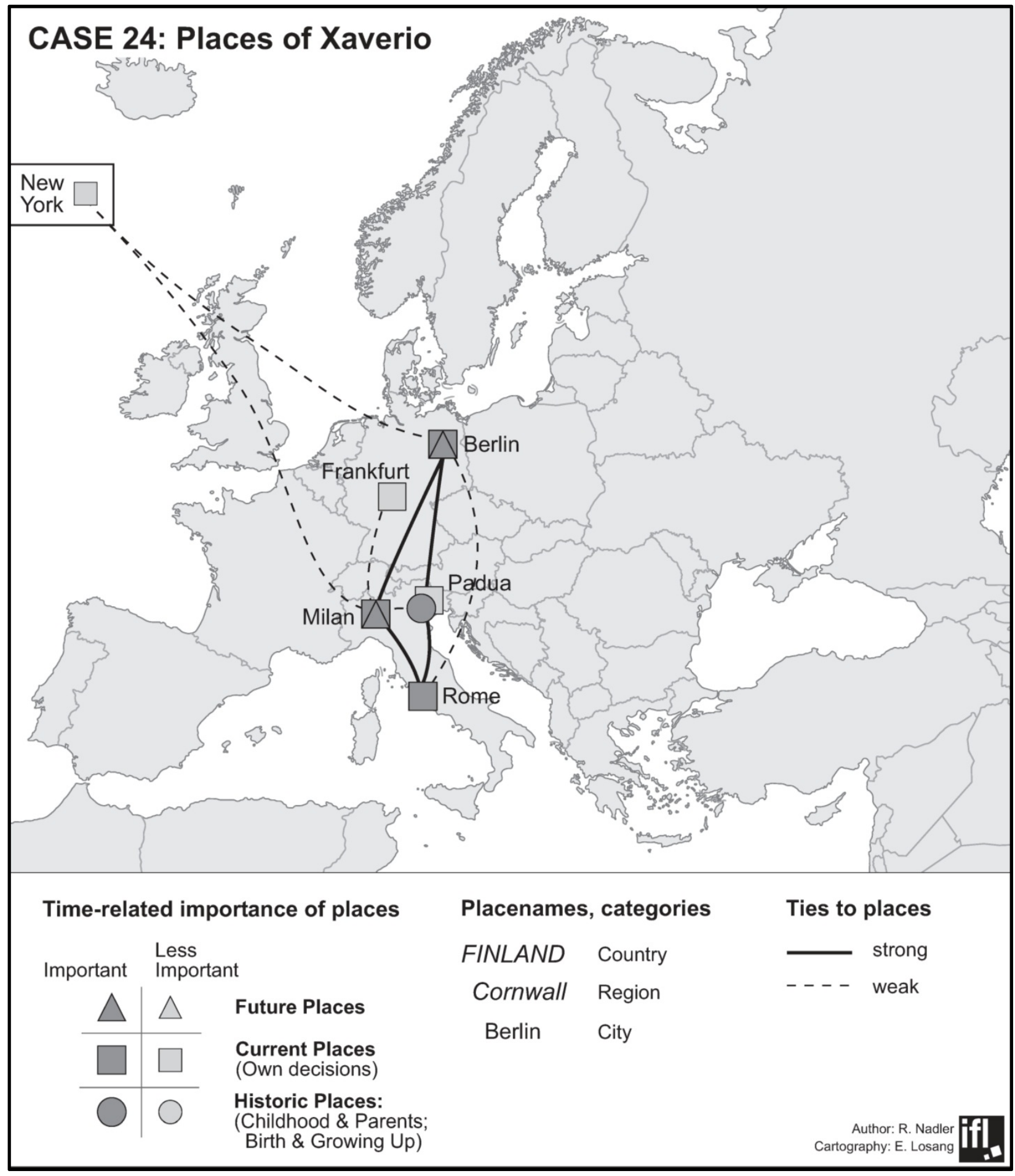




\section{Meet Xaverio}

Xaverio is an Italian freelance photographer and photography teacher in his late 20s. He was born in Padua, where he also grew up. After finishing school, he started studying sociology and communications in the local university, and he finished his studies at the university in Milan after having moved there. His final thesis dealt with Berlin, so he started to go there and did some field work in Berlin. He then returned for a while to Milan to finish university in administrative ways. During his university years in Milan, he also took classes in photography at an art school. He then went back to Berlin for half a year before subsequently moving to New York for a few months, where he resumed studying photography. In New York, he received a call from a German company in the field of international trade fairs which was looking for an event manager for its Milan branch. He took this job and returned from New York to Milan. As the company was German, he frequently had to travel to the Frankfurt-based headquarters.

After a while Xaverio became bored by this job and wanted to foster his professional career in photography, about which he had always been more passionate. He started as an assistant photographer and then developed his own projects. He says that it is very important to be well connected in Milan's local scene in order to get jobs - even more during the recent crisis, but he appreciates the freedom he has in defining what he wants to do.

Recently, he also started working as a photography professor at an arts school in Rome. There he also organises exhibitions with his own artwork. Currently he is circulating between Rome, Milan, and Berlin. The cities all fulfil different functions in his everyday life: Rome is the place to exhibit and live his individual creativity and artwork; Milan is the place to do commercial photography jobs in the fashion industry in order to finance his life; and Berlin is a place he visits for inspiration and during leisure time.

Xaverio's residential situation is quite interesting, as he lives in shared apartments in Berlin and Rome, where he then shares his room with another Italian friend, who is multi-locally circulating between Rome and Berlin as well. Xaverio has only rented his own loft apartment in an old factory in Milan, where he is surrounded by other artists and designers. In this constellation, Xaverio would still consider Padua his home, his roots, and where his parents and old friends still live. Additionally, he says that he currently lives in and feels at home in Berlin and Milan; he feels connected to the place, its people, and the state of mind. Rome is still not a home as he only recently started his job there.

Xaverio is very active in civil society as he sees engagement as a form of developing a more profound relationship to a place, something non-market based, with money exchange, where you pay for experience. In Berlin, he was involved in a neighbourhood initiative which taught children about photography, in Milan he helped the public library to organise the archive and survive threats of closure, and in Rome he partakes in an initiative to make public space more beautiful and clean through changing trash into art with young artists. Such engagement is also a way to connect with local people, which he finds important.

In the future, Xaverio does not want to become a monolocal person again. He can even imagine integrating further places into his lifeworld, because it is in his character to quickly become bored of spending too much time in one place and environment. 


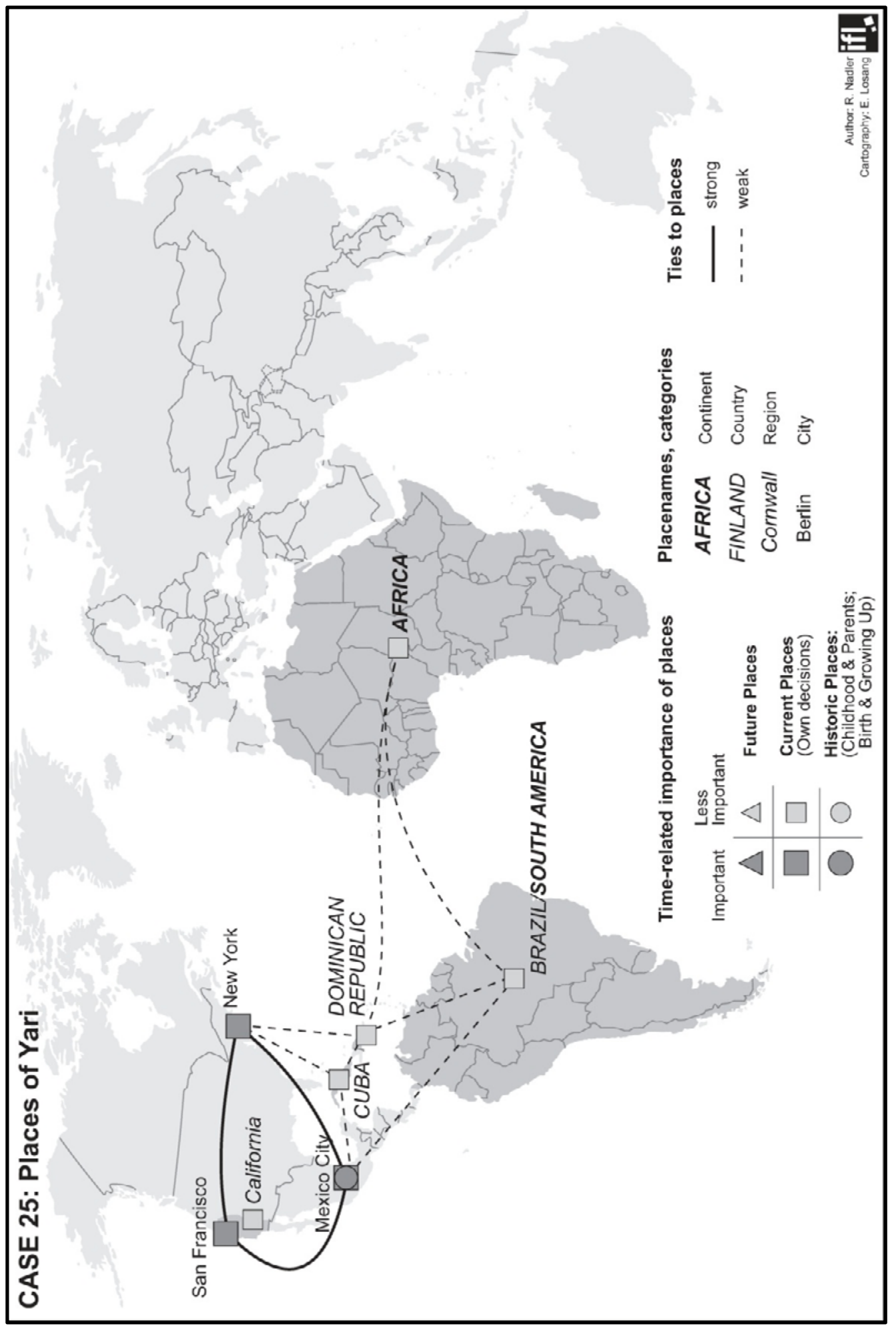




\section{Meet Yari}

Yari is a Mexican hip hop artist in his 30s who was born in Mexico City. He was influenced by the growing success of the US American hip hop scene during the 1990s. He started to do his own Mexican hip hop with other young Mexicans,, which was among the first autonomous productions of hip hop in Latin America. Yari also made social inequality and injustice an issue in his songs. Different from the Afro-American hip hop in the US, the hip hop scene to which Yari belongs originates from an educated middle-class background in Mexico City. Yari grew up in the bohemian Coyoacán neighbourhood, where Frida Kahlo and Leon Trotsky also lived. Thus, Yari's hip hop is intellectual and political. In the tradition of the Latin American independence spirit, he criticizes the neo-colonial behaviour of the nation-states in Latin America which oppress local communities and racially discriminate against minority groups.

With his early success, Yari started to travel around the Latin American countries, where problems of native minorities are omnipresent. He travelled a lot in the Caribbean Islands and Brazil, where he got in touch with communities of Afro-Americans. This experience showed him that there is a lot of similarity between the USA and Latin America in terms of racism against Afro-Americans.

He started to travel frequently to the USA because of his music as well. The Spanish-speaking audience was large enough to secure his commercial success, and he then rented an apartment in New York after having received an official residence permit. Since then, he has frequently travelled between the USA, Mexico and other Latin American countries. Recently, he also extended his range to Europe and is looking for connections to Africa.

Yari has made music his profession. He founded an association called Mocambo, which takes up the old word for the fugitive black slaves during colonial times in the Americas. This association is organized as a network, which is active in several countries (the USA, Mexico, Brazil, Cuba, the Dominican Republic etc.). Its aim is to empower local communities and marginalized ethnic groups through the ways that hip hop culture offers, but also through educational programmes. Yari therefore travels between the involved places in the different countries in order to support the association's local groups. He has just finished a project with native communities in California.

In addition he is continuing to foster his musical career and he uses the travel that comes with it in order to get in contact with other ethnic groups. During the time of our interview, he was just about to get in touch with hip hop artists in Germany. He stayed in Berlin with some Turkish communities and he could observe the similarities to e.g. Latin American groups in US cities.

Yari's main conviction is that the nation-states oppress the local level communities. The latter should develop a more healthy self-confidence to defend their rights and autonomy. Yari therefore also identifies with all of the communities that face such problems throughout the entire world. He refuses to be identified with any one nationality. He says his Mexican passport and his US Residence Permit are only tools to communicate the necessary issues with the bureaucratic systems, but he does not feel any loyalty for any kind of home country. If he goes home, he might go anywhere in the world where there is a self-dependent local community. He has recently given up his Mexico City apartment and considers his New York apartment as a technical tool for organizing his life, but he basically prefers staying with other local people wherever he goes in order to become part of the local everyday life and not end up like a tourist. In the future Yari wants to travel even more as he is interested in getting to know more local situations of communities around the world. Yari's metatopic is that of the transnational awareness for vivid and autonomous communities that result from nation-state oppression. 


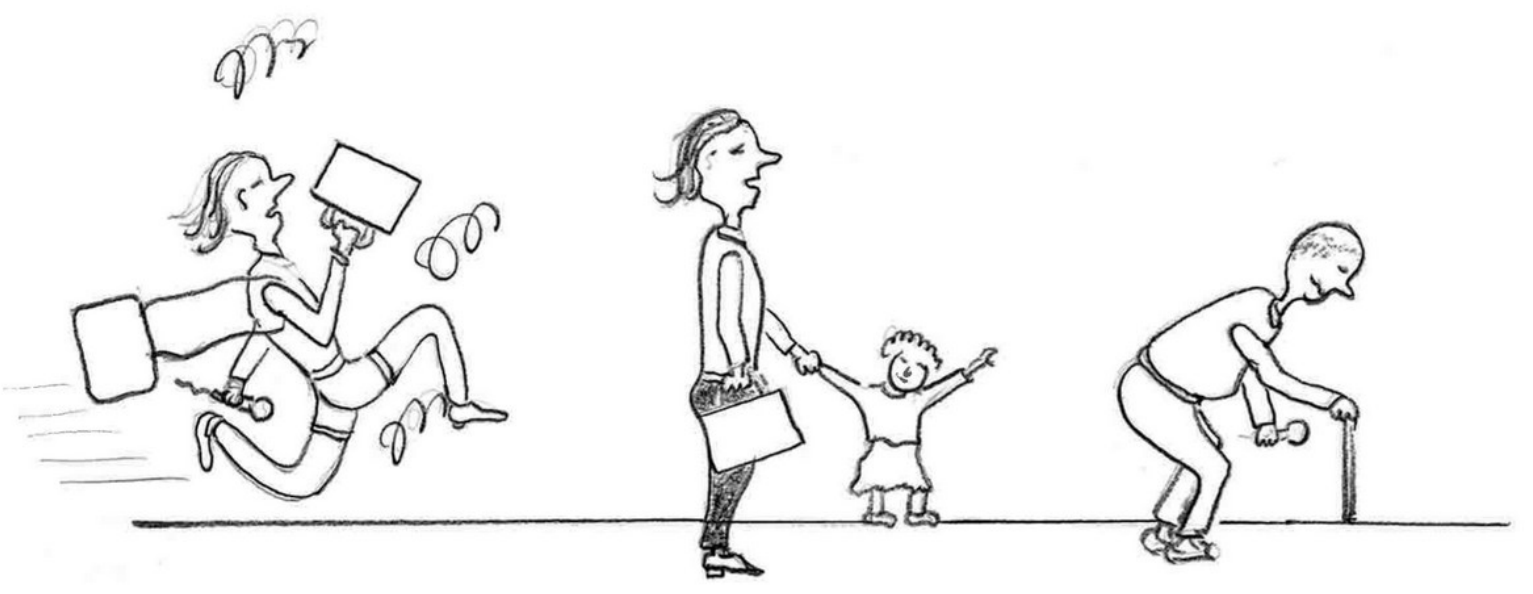




\section{Conclusions}

This thesis has revealed exploratory insights into the lifeworlds of multilocal creative knowledge workers who circulate across national borders. The set of 25 cases has been used to explore similarities in their geographical patterns and I have highlighted the relation between multilocality, transnationalism and creative industries. In doing so, I hope this thesis has helped to develop a first understanding of the place relations that creative knowledge workers have developed in circumstances of multilocality. As we have witnessed, these people frequently circulate between different places and develop certain routines in dealing with these places and the mobility that come along with multilocality. I think that the metaphor of the 'plug\&play places' appropriately describes what relation the mobile creative knowledge workers develop to their places. The idea of 'plug\&play' is known to all of us, for example, when we use a USB device on our computers. Regardless of the computer you use, the USB device always works with that new hardware and software environment. I think this is an excellent metaphor for the relation of my interview partners and their places: after an initial moment of quasi routinised configuration, a place is ready to be 'played' for the multilocal creative knowledge workers; they are then able to make use of that place.

My interview partners 'touch down' temporarily in the different places that they consider to be theirs. As they do so in certain regularity, although always for a limited time, they need these places to work for them directly upon arrival. They cannot begin to reflect about how to use these places once they arrive. These places must work, like switching on the light in the living room when you come home late at night - you don't want to think about installing the cable, putting in a lamp, or checking the securities, it should simply make light. The same accounts for a USB stick that you bring to a friend to show your latest holiday photos: after plugging in the USB to the computer, you do not want to bother with installing a driver, fitting it into the specific local software environment of your friend's computer - you just want to plug it in, and it should work. My interview partners have developed exactly such a relation to their places. They have developed routines within the places they visit frequently. They know their ways to come home or to the hotels, from airports and train stations, they know how local public transport works. They know what to expect at home or in the hotel; they also no what not to expect. They have made these places work by using techniques of place appropriation, and the experience that they have with their more regular places also translates into routines within orientation in completely new environments where they feel less lost than people who seldom travel.

On the other hand, these places themselves have to provide a certain character in order to become plug\&play places. They can be designed to offer easy orientation, e.g. through a standardised system of navigating people. A good example for such standardisation of places is an international airport, as we have witnessed in Gunnar's description of getting along in Mallorca's airport, where he frequently had to change planes between Valencia and Leipzig. This thesis could not shed light on this objective type of place standardisation, but the narrations of my interview partners point in the direction that it would help them to get along. So far, they rather accept places with all their individuality and specialities, and they develop subjective ways of standardising 'their places' within their own lifeworld. 
This plug\&play metaphor also includes a connotation of superficiality. My interview partners often do not take a closer look at all of their own places. While there is often a central place, a Lebensmittelpunkt, other involved places are only appropriated as functionally necessary. There is no deeper interest in the local specificities. Many places have less of an emotional character to which the creative knowledge workers relate, but is has a mere functional use. Places are order in functional complementarity. This again highlights the plug\&play character of how people relate to their places. Functionality and easy usability go hand in hand.

Nonetheless, the geography of their lifeworlds makes sense to them. The creative knowledge workers also relate to the places which form their individual and dynamic place pattern. They incorporate these places into their identity. This was obvious in the way in which my interview partners played with multiple identities which are composed of different places and geographic scales. They developed these identity components into 'alternative autobiographies', which they employ as fitting best in any given situation. Furthermore, the multilocals emotionally root in their geographic pattern using a kind of 'appresentative home-making practice', which consists of making all their places mentally present at the same time. Here, imaginative, virtual and physical travel work in combination with place appropriation techniques and travel routines. As a result, the multilocal individuals can feel at home in several places at the same time, but they are also at home in their multilocal lifeworld in general, including the aspect of travel and mobility. Mobile (e.g. trains and aeroplanes) and immobile (e.g. train stations and airports) transit places also become part of this appresentative home-making practice. As such, we should think of 'roots' and 'routes' not as an opposed and contradictory dimension. Terms of rootedness such as home, place-based identity or dwelling should be seen in a wider context of the lifeworld and not only as limited to individual geographic places. Multilocal people include both 'routes' and 'roots' in their personality and they are at home in the totality of their lifeworld. As such, Jung's concept of the psycho-social sedentariness is indeed helpful to understand these forms belonging (cf. chapter 3).

\section{Multilocality in a mobile world}

I have empirically studied a group which is more mobile than the majority of the total population, and where multilocality is obvious. However, I think there is certain evidence for the fact that multilocality affects an ever increasing part of our population, not referring only to those who travel themselves. Werlen (2000) e.g. pointed out that the action spaces of people in general have grown throughout the last hundreds of years. Picking up Dickens' idea of a compression of time-space through technology and Thrift's idea of an extension of action spaces, Werlen describes that people use ever larger territories within their everyday life. These territories have grown so far that daily commuting is often no longer possible. People have to move around, stay overnight, and start dwelling in several places. This means that basing one's own life in a stable way in only one monolocal context seems illusionary for more and more people. Even when an individual might be based monolocally in one place, their social network will contain people who will be forced into, or who will decide voluntarily at a certain moment in life to move somewhere else or to begin a multilocal life. In this case, the monolocal individual will at least become multilocal in a mental way. Even if they refuse to visit the friends who have moved in their new environment, ICT mediated communication - telephoning, emailing, Skyping etc. - will make it possible for the monolocal individual to be virtually multilocal to a certain degree. Furthermore, remembering the loved friends or family members who recently moved away to other places, this person might 
engage in imaginative forms of multilocality. These phenomena are also described in the mobility studies literature. Larsen, Urry et al. (2006: 1) introduce their book 'Mobilities, Networks, Geographies' with the observation that: 'Increasingly, people who are near 'emotionally' may be 'geographically' far apart; yet they are only a journey, email or a phone call away.' Thus, the virtual and imaginative aspects of mobility have mainly been understudied until now - that's what Larsen, Urry et al. summarise and what I would underline.

The debates circulating around the neat definition of multilocality - an example of a mobile lifestyle - are necessary in the context of distinct research projects, e.g. if you want to compare multilocals to monolocals. However, I would suggest that the multilocality concept is also appropriate to understand the general mobility patterns that arise in our society and that include the virtual and imaginative aspects of mobility between places. Florida also remarks that 'The old forms don't work, because they no longer fit the people we've become' (2002: xxx). In this way, we have to ask ourselves to what extent people have become multilocal in general, throughout all social groups or strata? I think multilocality is helpful to understand how the everyday lives of all of us have at least been mentally spread to different places. I would therefore argue for a shift of focus in multilocality studies. Instead of focusing on Wohnen/dwelling and dwelling practices, I would prefer to think of everyday life character of the place involvement as the main criterion. Dwelling practices are so manifold that it is nearly impossible to find a good definition between multilocal or not. However, using the idea that multilocality is evident when everyday life is spread to different places, and when the distance between these places makes overnight stay in different places necessary, is more appropriate to study the phenomenon. Overnighters do not necessarily perceive themselves as dwellers in these places, yet they live in them as their activities in these places have an everyday life character. Without becoming a catch-all term, we should use such a revised approach to study the multilocality phenomena in more detail.

\section{Multilocality and creative industries}

This study has also revealed interesting insights into the relationship between creative industries and multilocality. So far, creative industries have been regarded as an emerging economic sector which might be the future 'growth machine' of the highly-industrialised, or probably post-industrial countries. However, the discussion has always worked with concepts which are oriented backwards. Whereas it is argued that 'creative industries' are a sign of deep transformations of our society, towards more self-actualisation, independence and autonomy of the individual, this debate is most often nationally embedded. It uses the national cultural context as a reference frame, it looks for national creative industries' policies and it compares the position of creative industries to other sectors in the national economy. Additionally, it stresses the role of specific local milieus and networks. A general image arises of creative industries as locally isolated clusters which are somehow influenced by the national scale.

I hope I could shed a bit light on the transnational and transcultural links which are actually connecting creative industries across the European Union and beyond. Creative knowledge workers are also participating in the 'mobilisation' of society; they cross borders and become transnational. They hybridise local and national cultural products and services. On an individual scale, this can even be a competitive advantage, as they can chose between offering products for market $\mathrm{A}$ in cultural context A, for market B in cultural context B, or offering a new and hybrid product for both 
markets and cultural contexts. Perhaps the role of the local context for creative industries is overemphasised, while at the same time transnational links - and here I refer to links on the microlevel of the individual - are fairly neglected in studies on creative industries. This study has shown that transnational and multilocal ties allow for polyperspectivity and inspiration, which can both be helpful to cultural innovation. Being a cross-border multilocal creative knowledge worker can have a variety of competitive advantages. In particular, in the light of increasing mobility of wider parts of society, it can be questioned if people remain rooted in national and local culture. Perhaps transculturality will also become a wider social phenomenon in the future, and creative knowledge workers with personal experience of mobility can profit from it. Hence, these observations also point to a further field of interesting research.

A comment on mental maps as a research method

As mentioned in chapters 6 and 7, I believe in the potential of mental map research techniques. I have had positive experience with this method in the course of this research project. Mental maps provide an additional source of information for those who want to study lifeworlds on an individual level. They are helpful to understand how the world is ordered and structured in the mind. However, I want to argue for openness in defining what mental maps are. Their methodological value should not be limited by considering them cartographic illustrations. Whereas they can take this form, they might also come along in different forms such as narrations, drawn schemes, textual fragments, or sketches of any other kind which resemble a cartographic representation. I think they are still mental maps and they transport valuable information. Actually, this means that we should continue to refine the method for appropriately 'from the outside looking in at the inside looking out', as Stea \& Downs formulated (1970).

\section{Limits of the study}

The research project of this thesis was designed to be qualitative and explorative. I have interviewed 25 creative knowledge workers. Obviously, this limits the generalisability of the findings. Whereas I applied techniques to increase variety and heterogeneity within this sample of the 25 interview partners, this provides only a limited perspective on the social reality. I cannot even say that I have sufficiently analysed the broad range of different activities and sectors within the creative industries. I have also mainly studied the phenomenon within the European Union. As such, I understand my results as preliminary, to have a hypothetical character. In particular, the idea of the 'plug\&play places', which are characterised by functional complementarity and which are related to each other in a sense-making cognitive process of constant comparison, as well as the resultant polyperspectivity - all of these things could be proven wrong by a subsequent study. However, this also points to potential for further research.

\section{Open questions and prospects for future research}

While being limited to a small extent of the empirical world, this exploration of the thematic field 'multilocality and creative industries' has opened up interesting starting points for future research. First of all, I think that long-term panel studies have value per se, but their implementation would be particularly interesting in this dynamic empirical reality. My results point to the temporality of multilocal arrangements. People understand their geographic patterns as temporary constellations. It would thus be very highly interesting to study a group of multilocals over a longer period. The 
patterns of change might only become visible by following the life course for a certain period. Perhaps one could also identify certain groups of mobile creative knowledge who remain multilocal and develop strategies to stabilise in their multilocal arrangements. The interview partners to whom I have talked rather considered stability something which should come after their multilocal episode in life. Therefore, multilocality and stability were thought of as opposing items, yet this does not mean that there are not any groups of multilocals who actually want to stabilise their multilocality itself.

Furthermore, I am convinced that multi-sited ethnography (cf. Marcus 1995; Falzon 2012) could enrich the empirical study of multilocality in the frame of creative industries. There are critical voices that think that multi-sited ethnography represents a misleading perspective on mobile lifestyles as it suggests the existence of several sites in parallel, while social realities actually must be understood as one site (cf. Hage 2005). However, I think my research has shown that the lifeworld of my interview partners happens in distinct places or 'sites', so I think it is valuable to spend some time in these places with the interviewees. Hage (2005) also doubts that e.g. migrants are mobile, as they spend most of the time in one place. Here, I also would say that the empirical field of multilocality research is indeed defined by mobility. Therefore, applying moving methods might also be helpful to study the actual physical travel (cf. Watts \& Urry 2008).

A third point I have left open here is the precise functioning of knowledge transfer. While I have found that my interviewees transfer knowledge in different forms, I was not able to investigate in detail how this transferred knowledge is then applied in new contexts and what consequences this has for a local environment. Whereas knowledge transfer is studied relatively well in the field of technology transfer and technological innovation, there are nearly no insights in the field of transnational knowledge transfer in the creative industries. I am also less sure what method would be appropriate to capture this phenomenon, as the implementation of transferred knowledge takes time as well. This could probably be combined with panel studies or long-term case study research and drawing on theoretical insights from evolutionary economic geography (cf. Nelson \& Winter 1984; Bathelt \& Glückler 2003: 194-226).

As this research has concentrated on the elaboration of a comprehensive overview of multilocal lifeworlds, I was only able to work out first ideas on individual relations between categories and dimensions in such lifeworlds. More profound research is necessary for the future. Several conceptual ideas were mentioned in chapter 7: ubiquity, playing with multiple identities, alternative autobiographies, functional complementarity, polyperspectivity, flexible and non-market based dwelling practices etc. All these ideas were pointed to in a rather superficial way. It would now be the moment to break the surface and study individual themes more in detail. 


\section{Epilogue}

This PhD project has accompanied me for the last four and a half years, and this time is quite a lot for a single project in the dynamic environment of social sciences. I meanwhile assisted in my third research project during this period, and as such I can consider this $\mathrm{PhD}$ the most stable thing in my professional life; all projects which happened around and in parallel were of much shorter duration. This highlights the rapidly changing contexts in which we work today. My interview partners have experienced that their lifeworlds have changed since I carried out the interviews with them, so it would actually be time to do a second set of interviews with them. I am very interested in how their lives have evolved. I know, for example, that Pia has meanwhile reached her aim to become a fulltime professor in architecture. She finally took such a position at a German university, not in Riga and not in China. However, she has still not reached her second goal of a professorship in Berlin. I also know that Aurélien has separated from his German girlfriend who he considered an anchor point and a source of emotional stability. I wonder how his place pattern has changed due to that fact. Willi is about to leave his job in Aachen; he will move to New Zealand and become a lecturer in the university. Taking his family, he will also become more monolocal again, merely and simply on the other side of globe. Furthermore, I was quite happy to hear that Gunnar has achieved his goal of stabilising his long-distance relationship with his Spanish girlfriend. They have married and recently became parents of a small daughter. In this ever changing world, it seems that everything is possible, and one cannot really predict what comes next. However, something is about to arrive. For me, this $\mathrm{PhD}$ thesis means the end of my own four and a half years of multilocality which has been located between Milan and Leipzig. I will now get into a more monolocal stage again. I have configured Milan as my own 'plug\&play place' which is ready to use in everyday life and to where I can always come back if a new opportunity arises. During this $\mathrm{PhD}$ project I also became a father, got married and moved into a larger family apartment in Leipzig. I will now be allowed to profit from the moments of monolocal tranquillity - but who knows, perhaps life will once again become more multilocal. 


\section{References}

Abel, G. (2008). Forms of Knowledge: Problems, Projects, Perspectives. In P. Meusburger, M. Welker \& E. Wunder (Eds.), Clashes of Knowledge: Orthodoxies and Heterodoxies in Science and Religion. (Vol. 1, pp. 11-33). Dordrecht: Springer Science + Business Media.

Albrow, M. (1997). Travelling beyond Local Cultures. Socioscapes in a global city. In J. Eade (Ed.), Living the Global City. Globalization as a local process. (pp. 37-55). London, New York: Routledge.

Ambrosini, M. (2005). Sociologia delle migrazioni. Bologna: Il Mulino.

Anderson, B. (1983). Imagined Communities: Reflections on the Origin and Spread of Nationalism. London, New York: Verso.

Andreotti, A., Le Galès, P. \& Moreno Fuentes, F. J. (2010). Not barbarians: Transnational mobility and Rootedness of Upper Middle Classes in European Cities. Working Papers du Programme Villes \& Territoires, Sciences Po Paris.

Andrews, R. B. (1953). Mechanics of the Urban Economic Base: Historical Development of the Base Concept. Land Economics, 29(2), 161-167.

Apitzsch, U. (2003). Migrationsbiographien als Orte transnationaler Räume. In U. Apitzsch \& M. Jansen (Eds.), Migration, Biographie und Geschlechterverhältnisse (pp. 65-81). Münster: Westfälisches Dampfboot.

Applegate, C. (1990). A nation of provincials: The German idea of Heimat. Berkley, Los Angeles: University of California Press.

Augé, M. (1995). Non-places: An Introduction to an Anthropology of Supermodernity. London; New York: Verso.

Bakhshi, H., McVittie, E. \& Simmie, J. (2008). Creating Innovation. Do the creative industries support innovation in the wider economy? In NESTA Reserach Report. London: NESTA.

Balducci, E. (1990). L'Uomo planetario. San Domenico di Fiesole: ECP.

Bathelt, H. \& Glückler, J. (2003). Wirtschaftsgeographie: Ökonomische Beziehungen in räumlicher Perspektive. Stuttgart: Verlag Eugen Ulmer.

Bathelt, H., Malmberg, A. \& Maskell, P. (2004). Clusters and Knowledge. Local Buzz, Global Pipelines and The Process of Knowledge Creation. Progress in human geography., 28(1), 31-56.

Bauman, Z. (2000). Liquid modernity. Oxford: Blackwell.

Becattini, G. (1991). The Industrial District as a Creative Milieu. In J. Benko \& M. Dunford (Eds.), Industrial Change and Regional Development: The Transformation of New Industrial Spaces (pp. 102-116). London, New York: Routledge.

Beck, U. (1992). Risk society: towards a new modernity. Thousand Oaks: Sage.

Beck, U. (2002). The Cosmopolitan Society and its Enemies. Theory, Culture \& Society, 19(1-2), $17-44$.

Beck, U. (2007). Weltrisikogesellschaft. Frankfurt/Main: Suhrkamp. 
Beck, U., Bonß, W. \& Lau, C. (2004). Entgrenzung erzwingt Entscheidung: Was ist neu an der Theorie reflexiver Modernisierung? In U. Beck \& C. Lau (Eds.), Entgrenzung und Entscheidung: Was ist neu an der Theorie reflexiver Modernisierung? (pp. 13-62). Frankfurt/Main: Suhrkamp.

Beck, U. \& Grande, E. (2010). Varieties of Second Modernity: The Cosmopolitan Turn in Social and Political Theory and Research. The British Journal of Sociology, 61(3), 409-443.

Bell, D. (1976). The Coming of Post-Industrial Society. A Venture in Social Forecasting. (2nd ed.). New York: Basic Books.

Bell, D. (1980). The Social Framework of the Information Society. In T. Forester (Ed.), The Microelectronics Revolution (pp. 500-549). Oxford: Basil Blackwell.

Berger, P. L., Berger, B. \& Kellner, H. (1973). The Homeless Mind: Modernization and Consciousness. New York: Random House.

Betzelt, S. (2006). Flexible Wissensarbeit: AlleindienstleisterInnen zwischen Privileg und Prekarität. Zes-Arbeitspapier (Vol. 3/2006), Zentrum für Sozialpolitik, Universität Bremen.

Bhushan, B. (2003). Anmol's Dictionary of Sociology. New Delhi: Anmol.

Bichi, R. (2002). L'intervista biografica: Una proposta metodologica. Milan: V\&P.

Bigelow, S. J. (1999). The Plug \& Play Book. New York: McGraw-Hill.

Blackler, F. (1995). Knowledge, Knowledge Work and Organizations: An Overview and Interpretation. Organization studies, 16(6), 1021-1046.

Blanke, J. \& Lopez-Claros, A. (2004). The Lisbon Review 2004: An Assessment of Policies and Reforms in Europe. Geneva: World Economic Forum.

(https://members.weforum.org/pdf/Gcr/LisbonReview/Lisbon_Review_2004.pdf, accessed 31 October 2012)

Bommes, M. (2002). Migration, Raum und Netzwerke. Über den Bedarf einer gesellschaftstheoretischen Einbettung der transnationalen Migrationsforschung. In J. Oltmer (Ed.), Migrationsforschung und Interkulturelle Studien: Zehn Jahre IMIS. (Vol. 11, pp. 91108). Osnabrück: Universitätsverlag Rasch.

Bonin, H., Eichhorst, W., Florman, C., Hansen, M. O., Skiöld, L., Stuhler, J., Tatsiramos, K., Thomasen, H. \& Zimmermann, K. F. (2008). Geographic Mobility in the European Union: Optimising its Economic and Social Benefits. IZA Research Report Series (No. 19), Forschungsinstitut zur Zukunft der Arbeit (IZA), Bonn.

(http://www.iza.org/en/webcontent/publications/reports/report_pdfs/iza_report_19.pdf, accessed 31 October 2012)

Bonß, W., Kesselring, S. \& Weiß, A. (2004). "Society on the move". Mobilitätspioniere in der zweiten Moderne. In U. Beck \& C. Lau (Eds.), Entgrenzung und Entscheidung: Was ist neu an der Theorie reflexiver Modernisierung? (pp. 258-280). Frankfurt/Main: Suhrkamp.

Braidotti, R. (2011). Nomadic Subjects: Emobodiment and Sexual Difference in Contemporary Feminist Theory. (2nd ed.). New York: Columbia University Press.

Brenner, N. (1998). Between fixity and motion: Accumulation, territorial organization and the historical geography of spatial scales. Environment and Planning D - Society and Space, 16(4), 459-481.

Brickell, K. \& Datta, A. (2011a). Introduction: Translocal Geographies. In K. Brickell \& A. Datta (Eds.), Translocal Geographies: Spaces, Places, Connections. (pp. 3-20). London: Ashgate. 
Brickell, K. \& Datta, A. (2011b, Eds.). Translocal Geographies: Spaces, Places, Connections. London: Ashgate.

Bröckling, U. (2006). On Creativity: a brainstorming session. Educational Philosophy and Theory, 36(4), 513-522.

Bröckling, U. (2007). Das unternehmerische Selbst: Soziologie einer Subjektivierungsform. Frankfurt/Main: Suhrkamp.

Brown, J., Nadler, R. \& Meczinski, M. (2010). Working on the Edge? Creative Jobs in Birmingham, Leipzig and Poznan. In S. Musterd \& A. Murie (Eds.), Making Competitive Cities. (pp. 208-232). Oxford: Wiley-Blackwell.

Bürkner, H.-J. (2005). Transnational Migration. Cultural Turn and The Nomads of The World Market. Zeitschrift für Wirtschaftsgeographie, 49(2), 113-122

Buttimer, A. (1976). Grasping the Dynamism of Lifeworld. Annals of the Association of American Geographers, 66(2), 277-292.

Cairncross, F. (2001). The Death of Distance: How The Communications Revolution Will Change Our Lives. Boston: Harvard Business School Press.

Castells, M. (1997). The Power of Identity. (The Information Age: Economy, Society, and Culture Vol. 2). Massachusetts, Oxford: Blackwell.

Castells, M. (2000a). End of Millenium. (2nd ed. The Information Age: Economy, Society, and Culture Vol. 3). Oxford, Malden: Blackwell.

Castells, M. (2000b). The Rise of the Network Society. (2nd ed. The Information Age: Economy, Society, and Culture Vol. 1). Oxford, Malden: Blackwell.

Caves, R. E. (2000). Creative Industries: Contracts between Art and Commerce. Cambridge, London: Harvard University Press.

Chamaz, K. (2005). Grounded Theory in the 21st Century: Applications for advancing Social Justice Studies. In N. K. Denzin \& Y. S. Lincoln (Eds.), The Sage Handbook of Qualitative Research. (pp. 507-536). Thousand Oaks: Sage.

Charmaz, K. (2006). Constructing Grounded Theory. London: Sage.

Charmaz, K. (2008). Grounded Theory as an Emergent Method. In S. N. Hesse-Biber \& P. Leavy (Eds.), Handbook of Emergent Methods. (pp. 155-170). New York, London: The Guilford Press.

Chartrand, H. H. (1984). An Economic Impact Assessment of the Fine Arts. In The Third International Conference on Cultural Economics \& Planning. Akron, Ohio, USA. (http://www.compilerpress.ca/Cultural\%20Economics/Works/Econ\%20Impact\%20Ass\%20 1984.htm, accessed 31 October 2012)

Christopherson, S. (2008). Beyond the Self-expressive Creative Worker: An Industry Perspective on Entertainment Media. Theory, Culture and Society, 25(7-8), 73-95.

Clarke, A. E. (2007). Grounded Theory: Critiques, Debates, and Situational Analysis. In W. Outhwaite \& S. P. Turner (Eds.), The Sage Handbook of Social Science Methodology. (pp. 423-442). Thousand Oaks: Sage.

Clifford, J. (1994). Diasporas. Cultural anthropology, 9(3), 302-338.

Cloke, P., Milbourne, P. \& Widdowfield, R. (2002). Rural Homelessness: Issues, Experiences and Policy Responses. Bristol: The Policy Press. 
Conti, L. (2010). Vom interkulturellen zum transkulturellen Dialog: Ein Perspektivenwechsel. In M. Hühn, D. Lerp, K. Petzold \& M. Stock (Eds.), Transkulturalität, Transnationalität, Transstaatlichkeit, Translokalität. Theoretische und empirische Begriffsbestimmungen. (pp. 173-189). Münster, Hamburg, London: LIT Verlag.

Creswell, J. W. (2007). Qualitative Inquiry \& Research Design: Choosing among five Approaches. Thousand Oaks: Sage.

Currid, E. (2007). How Art and Culture Happen in New York. Journal of the American Planning Association, 73(4), 454-467.

d'Ovidio, M. (2010). Fashion and the City: Social Interaction and Creativity in London and Milan. In S. Vicari Haddock (Ed.), Brand-building: The Creative City. A Critical Look at Current Concepts and Practices. (pp. 113-136). Florence: Firenze University Press.

Dannenbeck, C. (2002). Selbst- und Fremdzuschreibungen als Aspekte kultureller Identitätsarbeit. Ein Beitrag zur Dekonstruktion kultureller Identität. Opladen: Leske + Budrich.

DCMS. (1998). A new cultural framework. London: DCMS (Department of Culture, Media, and Sport).

Demuth, A. (2000). Some conceptual Thoughts on Migration Research. In B. Agozino (Ed.), Theoretical and Methodological Issues in Migration Research: Interdisciplinary, Intergenerational and International Perspectives. (pp. 21-58). Aldershot: Ashgate Publishing.

Dienel, H.-L. (2004). Was sind Raumpartnerschaften? Begriffliche Angebote und Perspektiven. In H.-L. Dienel, H.-P. Meier-Dallach \& C. Schröder (Eds.), Die neue Nähe. Raumpartnerschaften verbinden Kontrasträume. (pp. 20-37). Stuttgart: Franz Steiner Verlag.

Dienel, H.-L. (2009). Multilokales Wohnen zwischen Kontrasträumen. Befunde und Konzepte zu individuellen und politischen Raumpartnerschaften. Informationen zur Raumentwicklung, 1/2.2009, 117-124.

Dienel, H.-L., Meier-Dallach, H.-P. \& Schröder, C. (2004, Eds.). Die neue Nähe: Raumpartnerschaften verbinden Kontrasträume. Stuttgart: Franz Steine Verlag.

Dirksmeier, P. (2007). Stadt und Habitus. Die Inkorporierung von Urbanität als Ursache der Urbanisierung. Die Beispiele Bodolz, Tegernsee/Stadt und München. Bremen: Universität Bremen, Fachbereich Sozialwissenschaften.

Dirksmeier, P. (2012). Multilokalität als Abwesenheit: eine Herausforderung für landschaftlich attraktive ländliche Räume - das Beispiel Tegernsee/Stadt. Europa Regional, 18/2010, 6070 .

Doise, W. (1998). Social Representations in Personal Identity. In S. Worchel, J. F. Morales, D. Paez \& J.-C. Deschamps (Eds.), Social Identities: International Perspectives. (pp. 13-23). London, Thousand Oaks, New Delhi: Sage.

Downs, R. M. \& Stea, D. (1977). Maps in Minds: Reflections on Cognitive Mapping. New York: Harper \& Row.

Downs, R. M. \& Stea, D. (1982). Kognititve Karten: Die Welt in unseren Köpfen. New York: Harper \& Row.

Downs, R. M. \& Stea, D. (1973, Eds.). Image and Environment. Cognitive Mapping and Spatial Behavior. Chicago: Aldine Publishing Company. 
Drori, I., Honig, B. \& Wright, M. (2009). Transnational Entrepreneurship: An Emergent Field of Study. Entrepreneurship Theory and Practice, 33(5), 1001-1022.

Drucker, P. (1969). The Age of Discontinuities. London: Heinemann.

Duchêne-Lacroix, C. (2009). Mit Abwesenheit umgehen. Kontinuität und Verankerung einer transnationalen Lebensführung jenseits territorialer Abgrenzungen. Informationen zur Raumentwicklung, 1/2.2009, 87-98.

Duclos, D. (1999). La Nascita dell'Iperborghesia. In G. Martinotti (Ed.), La Dimensione metropolitana: Sviluppo e Governo della Nuova Città. (pp. 175-187). Bologna: Il Mulino.

Duso, G. (2004). Regionale Identität: eine neue politische Bedeutung. In A. Pasinato (Ed.), Heimatsuche: Regionale Identität im österreichisch-italienischen Alpenraum. (pp. 21-34). Würzburg: Verlag Königshausen \& Neumann.

Duyvendak, J. W. (2011). The Politics of Home: Belonging and Nostalgia in Western Europe and the United States. Basingstoke, New York: Palgrave Macmillan.

Eberle, T. S. (2000). Lebensweltanalyse und Handlungstheorie: Beiträge zur Verstehenden Soziologie. Konstanz: UVK Universitätsverlag Konstanz.

Ellegård, K. \& Vilhelmson, B. (2004). Home as a Pocket of Local Order - Everyday Activities and the Friction of Distance. Geografiska Annaler, 86B(4), 281-296.

Ermann, U. (2011). Kunst und Kreativität als Faktor ländlicher Ökonomien: Vernachlässigbar oder unterschätzt? Geographische Rundschau, 63(2), 20-28.

ESPON 2013 Programme Coordination Unit (2011). Territorial Dynamics in Europe: The Creative Workforce. In ESPON 2013 Programme Coordination Unit (Ed.), Territorial Observation (No. 5). Luxembourg: ESPON 2013 Programme Coordination Unit.

(http://www.espon.eu/export/sites/default/Documents/Publications/TerritorialObservations/ TrendsInCreativeWorkforce/186759-ESPON-TO5-WEB.pdf, accessed 31 October 2012)

Esser, H. (1991). Alltagshandeln und Verstehen. Zum Verhältnis von erklärender und verstehender Soziologie am Beispiel von Alfred Schütz und "Rational Choice". Tübingen: J.C.B. Mohr (Paul Siebeck).

Esser, H. (1996). Die Definition der Situation. Kölner Zeitschrift für Soziologie und Sozialpsychologie, 48(1), 1-34.

European Commission. (2010a). EUROPE 2020: A strategy for smart, sustainable and inclusive growth. Brussels: European Commission.

(http://eur-lex.europa.eu/LexUriServ/LexUriServ.do?uri=COM:2010:2020:FIN:EN:PDF, accessed 31 October 2012)

European Commission. (2010b). European Competitiveness Report 2010 - Commission Staff Working Document. Brussels: European Commission.

(www.proinno-

europe.eu/sites/default/files/newsroom/2010/11/European_Digital_Competitiveness_report_ 2010(STAFF_WORKING_DOCUMENT).pdf, accessed 31 October 2012)

European Commission (2010c). Green Paper: Unlocking the potential of cultural and creative industries. Brussels: European Commission.

(http://ec.europa.eu/culture/documents/greenpaper_creative_industries_en.pdf, accessed 31 October 2012) 
European Council. (2000). Lisbon European Council 23 and 24 March 2000. Presidency Conclusions.

(http://www.europarl.europa.eu/summits/lis1_en.htm, accessed 31 October 2012)

Evans, G. (2009). Creative Cities, Creative Spaces and Urban Policy. Urban Studies, 46(5-6), 1003-1040.

Faist, T. (2000). The Volume and Dynamics of International Migration and Transnational Social Spaces. Oxford: Clarendon Press.

Falzon, M.-A. (2012). Introduction. Multi-sited Ethnography: Theory, Praxis and Locality in Contemporary Research. In M.-A. Falzon (Ed.), Multi-Sited Ethnography: Theory, Praxis and Locality in Contemporary Research. (pp. 1-23). Farnham: Ashgate.

Favell, A. (2003). Games without frontiers? Questioning the Transnational Social Power of Migrants in Europe. Archives Europeennes de Sociologie, 44(3), 397-427.

Favell, A. (2008). Eurostars and Eurocities: Free Movement and Mobility in an Integrating Europe. Oxford: Wiley-Blackwell.

Flick, U. (2006a). Analytic Induction. In V. Jupp (Ed.), The Sage Dictionary of Social Research Methods. (pp. 4-5). Thousand Oaks: Sage.

Flick, U. (2006b). Constant Comparative Method. In V. Jupp (Ed.), The Sage Dictionary of Social Research Methods. (pp. 37-38). Thousand Oaks: Sage.

Florida, R. (2002). The Rise of the Creative Class: And How It's Transforming Work, Leisure, Community \& Everyday Life. New York: Basic Books.

Florida, R. (2005). The Flight of the Creative Class: The New Global Competition for Talent. New York: Routledge.

Florida, R. (2008). Who's Your City?: How the Creative Economy Is Making Where to Live the Most Important Decision of Your Life. New York: Basic Books.

Franck, G. (1998). Ökonomie der Aufmerksamkeit: Ein Entwurf. Munich, Vienna: Carl Hanser Verlag.

Franz, P. (1984). Soziologie der räumlichen Mobilität: Eine Einführung. Frankfurt/Main, New York: Campus.

Friebe, H. \& Lobo, S. (2006). Wir nennen es Arbeit: Die digitale Boheme oder: Intelligentes Leben jenseits der Festanstellung. Munich: Wilhelm Heyne Verlag.

Friebe, H. \& Ramge, T. (2008). Marke Eigenbau: Der Aufstand der Massen gegen die Massenproduktion. Frankfurt/Main, New York: Campus.

Ganesan, S., Malter, A. J. \& Rindfleisch, A. (2005). Does Distance still matter? Geographic Proximity and New Product Development. Journal of Marketing, 69(10), 44-60.

Garnham, N. (2005). From Cultural to Creative Industries: An analysis of the implications of the "creative industries" approach to arts and media policy making in the United Kingdom. International Journal of Cultural Policy, 11(1), 15-29.

Gather, M., Kagermeier, A. \& Lanzendorf, M. (2008). Geographische Mobilitäts- und Verkehrsforschung. Berlin, Stuttgart: Gebrüder Borntraeger Verlagsbuchhandlung.

Giddens, A. (1985). Time, Space and Regionalisation. In D. Gregory \& J. Urry (Eds.), Social Relations and Spatial Structures. (pp. 265-295). Basingstoke, London: Macmillan. 
Gill, R. \& Pratt, A. (2008). In the Social Factory?: Immaterial Labour, Precariousness and Cultural Work. Theory, Culture and Society, 25(7-8), 1-30.

Glaser, B. G. (1978). Theoretical Sensitivity: Advances in the Methodology of Grounded Theory. Mill Valley: Sociology Press.

Glaser, B. G. (1992). Emergence vs. Forcing: Basics of Grounded Theory. Mill Valley: Sociology Press.

Glaser, B. G. (1998). Doing Grounded Theory. Issues and Discussions. Mill Valley: Sociology Press.

Glaser, B. G. (2004). Remodeling Grounded Theory. Forum Qualitative Sozialforschung/Forum Qualitative Social Research, 5(2), Article 4 [80 paragraphs].

(http://www.qualitative-research.net/index.php/fqs/article/view/607, accessed 31 October 2012)

Glaser, B. G. \& Strauss, A. L. (1967). The Discovery of Grounded Theory: Strategies for Qualitative Research. Chicago: Aldine.

Glick Schiller, N. (2005). Transborder Citizenship: An Outcome of Legal Pluralism within Transnational Social Fields. In F. von Benda-Beckmann, K. von Benda-Beckmann \& A. Griffiths (Eds.), Mobile people, mobile law: expanding legal relations in a contracting world. (pp. 27-49). Aldershot: Ashgate.

Glick Schiller, N., Basch, L. \& Blanc-Szanton, C. (1992). Transnationalism: A New Analytic Framework for Understanding Migration. In N. Glick Schiller, L. Basch \& C. BlancSzanton (Eds.), Towards a transnational perspective on migration: Race, class, ethnicity, and nationalism reconsidered. (pp. 1-24). New York: New York Academy of Sciences.

Glick Schiller, N., Basch, L. \& Szanton Blanc, C. (1997). From Immigrant to Transmigrant: Theorizing Transnational Migration. In L. Pries (Ed.), Transnationale Migration. (pp. 121140). Baden-Baden: Nomos Verlagsgesellschaft.

Goeke, P. (2004). Transnational Migratory Identities between Nuremberg, Serbia, Croatia and Bosnia-Herzegovina. In A. Böcker, B. de Hart \& I. Michalowski (Eds.), Migration and the Regulation of Social Integration. (pp. 191-201). Osnabrück: Institut für Migrationsforschung und Interkulturelle Studien (IMIS).

Goffman, E. (1959). The Presentation of Self in Everyday Life. New York: Anchor Books.

Gornig, M. \& Mundelius, M. (2012). Reurbanisierung und wissensbasierte Ökonomie. In K. Brake \& G. Herfert (Eds.), Reurbanisierung: Materialität und Diskurs in Deutschland. (pp. 130150). Wiesbaden: Springer VS.

Grabher, G. (1993). The Weakness of Strong Ties: The Lock-in of Regional Development in the Ruhr Area. In G. Grabher (Ed.), The Embedded Firm: On the Socioeconomics of Industrial Networks. (pp. 255-277). London, New York: Routledge.

Grathoff, R. (1995). Milieu und Lebenswelt: Einführung in die phänomenologische Soziologie und die sozial-phänomenologische Forschung. Frankfurt/Main: Suhrkamp.

Graumann, C. F. (1983). On Multiple Identities. International Social Science Journal, 35(2), 309321.

Gustafson, P. (2001). Roots and routes: exploring the relationship between place attachment and mobility. Environment and Behaviour, 33(5), 667-686. 
Hage, G. (2005). A not so multi-sited ethnography of a not so imagined community. Anthropological Theory, 5(4), 463-475

Hägerstrand, T. (1970). What about people in regional science? Papers of the Regional Science Association, 24(1), 7-21.

Hall, S. (1996). Who needs 'Identity'? In S. Hall \& P. du Gay (Eds.), Cultural identity. (pp. 1-17). London: Sage.

Hammersley, M. (2006). Induction. In V. Jupp (Ed.), The Sage Dictionary of Social Research Methods. (pp. 146-147). Thousand Oaks: Sage.

Han, P. (2005). Soziologie der Migration: Erklärungsmodelle, Fakten, Politische Konsequenzen, Perspektiven. Stuttgart: Lucius \& Lucius (UTB).

Hannerz, U. (1990). Cosmopolitans and Locals in World Culture. In M. Featherstone (Ed.), Global Culture: Nationalism, Globalization and Modernity. (pp. 237-252). London: Sage.

Hansen, A. L., Andersen, H. T. \& Clark, E. (2001). Creative Copenhagen: Globalization, Urban Governance and Social Change. European Planning Studies, 9(7), 851-870.

Hansen, K. P. (2009). Kultur, Kollektiv, Nation. Passau: Stutz.

Harding, J. (2006). Grounded Theory. In V. Jupp (Ed.), The Sage Dictionary of Social Research Methods. (pp. 131-132). Thousand Oaks: Sage.

Hardt, M. \& Negri, A. (2000). Empire. Cambridge: Harvard University Press.

Häußermann, H. (1987). Neue Urbanität. Frankfurt/Main: Campus Verlag.

Häußermann, H. \& Siebel, W. (1996). Soziologie des Wohnens. Eine Einführung in Wandel und Ausdifferenzierung des Wohnens. Weinheim, Munich: Juventa.

Häußermann, H. \& Siebel, W. (2004). Stadtsoziologie: Eine Einführung. Frankfurt/Main: Campus Verlag.

Heider, F. (1958). The Psychology of Interpersonal Relations. New York: Wiley.

Helbrecht, I. (1998). The Creative Metropolis. Services, Symbols, and Spaces. International Journal of Architectural Theory 3(1), 1-10.

(http://www.tu-cottbus.de/BTU/Fak2/TheoArch/wolke/X-

positionen/Helbrecht/helbrecht.html, accessed 31 October 2012)

Heller, M. (2007). Multiligualism and transnationalism. In P. Auer \& L. Wei (Eds.), Handbook of Multiligualism and Multilingual Communication. (pp. 539-553). Berlin, New York: Mouton de Gruyter.

Henn, S. (2012). Transnational entrepreneurs, global pipelines and shifting production patterns. The example of the Palanpuris in the diamond sector. Geoforum, 43(3), 497-506.

Henninger, A. \& Gottschall, K. (2007). Freelancers in Germany's Old and New Media Industry: Beyond Standard Patterns of Work and Life? Critical Sociology, 33(1), 43-72.

Herlyn, U. (2003). Stadt. In B. Schäfers (Ed.), Grundbegriffe der Soziologie. (pp. 374-378). Opladen: Leske + Budrich.

Hesmondhalgh, D. \& Baker, S. (2008). Creative Work and Emotional Labour in the Television Industry. Theory, Culture and Society, 25(7-8), 97-118.

Hilti, N. (2009). Multilokales Wohnen: Bewegungen und Verortungen. Informationen zur Raumentwicklung, 1/2.2009, 77-86. 
Hitzler, R. \& Eberle, T. S. (2008). Phänomenologische Lebensweltanalyse. In U. Flick, E. von Kardorff \& I. Steinke (Eds.), Qualitative Forschung: Ein Handbuch. (pp. 109-118). Reinbek bei Hamburg: Rowohlt Taschenbuch Verlag.

Hitzler, R. \& Honer, A. (1995). Qualitative Verfahren zur Lebensweltanalyse. In U. Flick, E. von Kardorff, H. Keupp, L. von Rosenstiel \& S. Wolff (Eds.), Handbuch Qualitative Sozialforschung: Grundlagen, Konzepte, Methoden und Anwendungen. (pp. 382-385). Weinheim: Psychologie Verlags Union.

Hitzler, R. \& Honer, A. (1997). Einleitung. Hermeneutik in der deutschsprachigen Soziologie heute. In R. Hitzler \& A. Honer (Eds.), Sozialwissenschaftliche Hermeneutik: Eine Einführung. (pp. 7-27). Opladen: Leske + Budrich.

Hopf, C. (1995). Qualitative Interviews in der Sozialforschung. Ein Überblick. In U. Flick, E. von Kardorff, H. Keupp, L. von Rosenstiel \& S. Wolff (Eds.), Handbuch Qualitative Sozialforschung: Grundlagen, Konzepte, Methoden und Anwendungen. (pp. 177-182). Weinheim: Psychologie Verlags Union.

Howkins, J. (2001). The Creative Economy: How People Make Money from Ideas. New York: The Penguin Press.

Huchler, N., Dietrich, N. \& Matuschek, I. (2009). Multilokale Arrangements im Luftverkehr. Voraussetzungen, Bedingungen und Folgen multilokalen Arbeitens und Wohnens. Informationen zur Raumentwicklung, 1/2.2009, 43-54.

Hühn, M. (2010). Die internationale Ruhesitzwanderung - ein transkulturelles Phänomen? In M. Hühn, D. Lerp, K. Petzold \& M. Stock (Eds.), Transkulturalität, Transnationalität, Transstaatlichkeit, Translokalität. Theoretische und empirische Begriffsbestimmungen. (pp. 191-215). Münster, Hamburg, London: LIT Verlag.

Hühn, M., Lerp, D., Petzold, K. \& Stock, M. (2010a). In neuen Dimensionen denken? Einführende Überlegungen zu Transkulturalität, Transnationalität, Transstaatlichkeit und Translokalität. In M. Hühn, D. Lerp, K. Petzold \& M. Stock (Eds.), Transkulturalität, Transnationalität, Transstaatlichkeit, Translokalität. Theoretische und empirische Begriffsbestimmungen. (pp. 11-46). Münster, Hamburg, London: LIT Verlag.

Hühn, M., Lerp, D., Petzold, K., \& Stock, M. (2010b, Eds.). Transkulturalität, Transnationalität, Transstaatlichkeit, Translokalität. In Region - Nation - Europa (Vol. 62). Münster, Hamburg, London: LIT Verlag.

Husserl, E. (1969 [1936]). Die Krisis der europäischen Wissenschaften und die transzendentale Phänomenologie. The Hague: Nijhoff.

Husserl, E. (1973). Zur Phänomenologie der Intersubjektivität. Texte aus dem Nachlass. Erster Teil: 1905-1920. The Hague: Nijhoff.

Itzigsohn, J., Dore Cabral, C., Hernández Medina, E. \& Vázquez, O. (1999). Mapping Dominican Transnationalism: Narrow and Broad Transnational Practices. Ethnic and Racial Studies, 22(2), 316-339.

Jacobs, J. (1970). The Economy of Cities. London: Jonathan Cape.

Jenkins, R. (2008). Social Identity (3rd ed.). New York: Routledge.

Jung, M. (2012). Ein Modell von Sesshaftigkeit als Grundlage einer Rekonstruktion der Implikationen von Multilokalität. In Ici et là: Ressources et vulnérabilités de la vie multilocale/Hier und dort: Ressourcen und Verwundbarkeiten in der multilokalen Lebenswelt. Basel. 
Kaiser, C. (2011). Transnationale Altersmigration in Europa: Sozialgeographische und gerontologische Perspektiven. Wiesbaden: VS Verlag für Sozialwissenschaften.

Kalwa, D. (2010). Teil II: Migration polnischer Frauen in Privathaushalte der BRD. In S. MetzGöckel, A. S. Münst \& D. Kalwa (Eds.), Migration als Ressource: Zur Pendelmigration polnischer Frauen in Privathaushalte der Bundesrepublik. (pp. 61-176). Opladen, Famington Hills: Verlag Barbara Budrich.

Kaufmann, V., Bergman, M. M. \& Joye, D. (2004). Motility: Mobility as Capital. International Journal of Urban and Regional Research, 28(4), 745-756.

Kelle, U. (2005). 'Emergence’ vs. 'Forcing' of Empirical Data? A crucial Problem of 'Grounded Theory' reconsidered. Forum Qualitative Sozialforschung/Forum Qualitative Social Research, 6(2), Article 27 [52 paragraphs].

(http://www.qualitative-research.net/index.php/fqs/article/view/467, accessed 31 October 2012)

Kelsey, J. \& Kelsey, J. (1995). Programming Plug and Play. Indianapolis: SAMS.

Kleining, G. \& Witt, H. (2000). The Qualitative Heuristic Approach: A Methodology for Discovery in Psychology and the Social Sciences. Rediscovering the Method of Introspection as an Example. Forum Qualitative Sozialforschung/Forum: Qualitative Social Research, 1(1), Article 13 [19 paragraphs].

(http://www.qualitative-research.net/index.php/fqs/article/view/1123, accessed 31 October 2012)

Kok, W. \& Group, H. L. (2004). Facing the Challenge: The Lisbon strategy for growth and employment. Report from the High Level Group chaired by Wim Kok. Luxembourg: Office for Official Publications of the European Communities.

Koopmans, R. (2004). Migrant mobilization and political opportunities: Variation among German cities and a comparison with the United Kingdom and the Netherlands. Journal of Ethnic and Migration Studies, 30(3), 449-470.

Körber, K. (2011). Nähe aus Distanz. Transnationale Familien in der Gegenwart. In G. MarinelliKönig \& A. Preisinger (Eds.), Zwischenräume der Migration. Über die Entgrenzung von Kulturen und Identitäten. (pp. 91-112). Bielefeld: Transcript.

Kramer, C. (2012). „Alles hat seine Zeit“ - die „Time Geography“ im Licht des „Material Turn“. In N. Weixlbaumer (Ed.), Anthologie zur Sozialgeographie. (pp. 83-105). Vienna: Institut für Geographie und Regionalforschung der Universität Wien.

Kujath, H. J. (2012). Reurbanisierung des Wissens - zur Herausbildung von Metropolregionen unter dem Einfluss der Wissensökonomie. In K. Brake \& G. Herfert (Eds.), Reurbanisierung: Materialität und Diskurs in Deutschland. (pp. 216-238). Wiesbaden: Springer VS.

Kunzmann, K. R. (2009). Kreativwirtschaft und strategische Stadtentwicklung. In B. Lange, A. Kalandides, B. Stöber \& I. Wellmann (Eds.), Governance der Kreativwirtschaft: Diagnosen und Handlungsoptionen. (pp. 33-45). Bielefeld: Transcript.

Kurt, R. (2002). Menschenbild und Methode der Sozialphänomenologie. Konstanz: UVK Universitätsverlag Konstanz.

Labour Party. (1997). Create the Future: A Strategy for Cultural Policy, Arts and the Creative Economy. London: Labour Party.

Landry, C. (2000). Editorial: The Creative City. Streetwise, 11(2), 2-7.

Landry, C. (2001). The Creative City. A Toolkit for Urban Innovators. London: Earthscan. 
Landry, C. (1996, Ed.). The Creative City in Britain and Germany. London: Anglo-German Foundation.

Lange, B. (2005). Sociospatial Strategies of Culturepreneurs. The Example of Berlin and its New Professional Scenes. Zeitschrift für Wirtschaftsgeographie, 49(2), 81-98

Lange, B. (2007). Die Räume der Kreativszenen: Culturepreneurs und ihre Orte in Berlin. Bielefeld: Transcript.

Lange, B. \& Bürkner, H.-J. (2010). Wertschöpfungsketten in der Kreativwirtschaft: Der Fall der elektronischen Clubmusik. Zeitschrift für Wirtschaftsgeographie, 54(1), 46-68.

Lange, B. \& Ehrlich, K. (2008). Geographien der Szenen - Begriffsklärungen und zwei Fallvergleiche im Feld der urbanen Kultur- und Kreativwirtschaft von Berlin und Leipzig. Sociologia internationalis, 46(2), 237-261.

Lange, B. \& Mieg, H. A. (2008). Professionalisierungswege und Konstituierungen von 'Märkten' in den Creative Industries. Geographische Zeitschrift, 94(4), 225-242.

Larsen, J., Urry, J. \& Axhausen, K. (2006). Mobilities, Networks, Geographies. Aldershot: Ashgate.

Legewie, H. (2005). Rezension: Jörg Strübing (2004). Grounded Theory. Zur sozialtheoretischen und epistemologischen Fundierung des Verfahrens der empirisch begründeten Theoriebildung. Forum Qualitative Sozialforschung/Forum Qualitative Social Research, 7(2), Article 1 [63 paragraphs].

(http://www.qualitative-research.net/index.php/fqs/article/view/105, accessed 31 October 2012)

Legewie, H. \& Schervier-Legewie, B. (1995). Im Gespräch: Anselm Strauss. Journal für Psychologie, 3(1), 64-75.

Lévy, J. (2003). Capital spatial. In J. Lévy \& M. Lussault (Eds.), Dictionnaire de la géographie et de l'espace des sociétés. (pp. 124). Paris: Belin.

Lutz, H. (2004). Transnationale Biographien in globalisierten Gesellschaften. In M. Ottersbach \& E. Yildiz (Eds.), Migration in der metropolitanen Gesellschaft: Zwischen Ethnisierung und globaler Neuorientierung. (pp. 207-216). Münster: LIT Verlag.

Lynch, K. (1960). The Image of the City. Cambridge: MIT Press.

Machlup, F. (1962). The Production and Distribution of Knowledge in the United States. Princeton: Princeton University Press.

Manske, A. (2007). Prekarisierung auf hohem Niveau: Eine Feldstudie über Alleinunternehmer in der IT-Branche. Munich: Hampp.

Marcus, G. E. (1995). Ethnography in/of the world system: The emergence of multi-sited ethnography. Annual Review of Anthropology, 24, 95-117.

Markusen, A. (2006). Urban development and the politics of a creative class. Environment and Planning, 38(10), 1921-1940.

Marlet, G. \& van Woerkens, C. (2007). The Dutch Creative Class and How it Fosters Urban Employment Growth. Urban Studies, 44(13), 2605-2626.

Marston, S. A. \& Smith, N. (2001). States, scales and households: Limits to scale thinking? A response to Brenner. Progress in Human Geography, 25(4), 615-619. 
Martin-Brelot, H., Grossetti, M., Eckert, D., Gritsai, O. \& Kovacs, Z. (2010). The Spatial Mobility of the 'Creative Class': A European Perspective. International Journal of Urban and Regional Research, 34(4), 854-870.

Mau, S., Mewes, J. \& Zimmermann, A. (2008). Cosmopolitan attitudes through transnational social practices? Global Networks, 8(1), 1-24.

Mayer, H. O. (2008). Interview und schriftliche Befragung - Entwicklung, Durchführung, Auswertung. Munich: Oldenbourg Wissenschaftsverlag.

Mayring, P. (1995). Qualitative Inhaltsanalyse: Grundlagen und Techniken. Weinheim: Deutscher Studien Verlag.

Mead, G. H. (1934). Mind, Self and Society: from the Standpoint of a Social Behaviorist. Chicago: University of Chicago.

Mecheril, P. (2011). Hybridität, kulturelle Differenz und Zugehörigkeit als pädagogische Herausforderung. In G. Marinelli-König \& A. Preisinger (Eds.), Zwischenräume der Migration. Über die Entgrenzung von Kulturen und Identitäten. (pp. 37-53). Bielefeld: Transcript.

Meusburger, P. (2008). Introduction to the Book Series "Knowledge and Space". In P. Meusburger, M. Welker \& E. Wunder (Eds.), Clashes of Knowledge: Orthodoxies and Heterodoxies in Science and Religion. (pp. 1-3). Dordrecht: Springer.

Meusburger, P., Welker, M. \& Wunder, E. (2008, Eds.). Clashes of Knowledge: Othodoxies and Heterodoxies in Science and Religion. In P. Meusburger (Ed.), Knowledge and Space (Vol. 1). Dordrecht: Springer.

Morley, D. \& Robins, K. (1995). Spaces of Identity: Global Media, Electronic Landscapes and Cultural Boundaries. London: Routledge.

Morris-Suzuki, T. (1988). Beyond Computopia. Information, Automation and Democracy in Japan. London, New York: Kegan Paul Int.

Mühler, K. \& Opp, K.-D. (2006). Region - Nation - Europa: Die Dynamik regionaler und überregionaler Identifikation. Wiesbaden: VS Verlag für Sozialwissenschaften.

Musterd, S., Bontje, M., Chapain, C., Kovács, Z. \& Murie, A. (2007). Accommodating Creative Knowledge: A Literature Review from a European Perspective (Vol. 1). Amsterdam: AMIDSt, University of Amsterdam.

Musterd, S. \& Deurloo, R. (2006). Amsterdam and the Preconditions for a Creative Knowledge City. Tijdschrift voor Economische en Sociale Geografie, 97(1), 80-94.

Musterd, S. \& Gritsai, O. (2010). Conditions for 'Creative Knowledge Cities': Findings from a comparison between 13 European metropolises. 'Going creative'- An option for all European cities? Amsterdam: AISSR, University of Amsterdam.

Nadler, R. (2010). Creative knowledge workers between free self-actualisation and precarity: A study on job satisfaction in creative knowledge industries in the European Union. In 8th European Urban and Regional Studies Conference 'Repositioning Europe in an Era of Global Transformation'. Vienna.

(http://www.dur.ac.uk/resources/geography/conferences/eursc/17-09-10/RobertNadler.pdf, accessed 31 October 2012) 
Nadler, R. (2011a). Identity between places and peers? Insights from a study about multilocal creative knowledge workers in the European Union. In Annual RC21 Conference 2011: 'The struggle to belong. Dealing with diversity in 21st century urban settings. '. Amsterdam. (http://www.rc21.org/conferences/amsterdam2011/prog-19.php; accessed 31 October 2012)

Nadler, R. (2011b). Multilocalità: Un Concetto emergente fra Mobilità e Migrazione. Sociologia urbana e rurale, no. 94, 119-134.

Nadler, R. (2012). Transnational und multilokal. Über die Rahmenbedingungen von grenzüberschreitender Mehrfachverortung bei kreativen Wissensarbeiter_innen. In Ici et là: Ressources et vulnérabilités de la vie multilocale/Hier und dort: Ressourcen und Verwundbarkeiten in der multilokalen Lebenswelt. Basel.

Nadler, R. \& Montanari, G. (2012). Multilokalität und die Bedeutung von Orten: Die Beispielgruppen Großeltern in Nachtrennungsfamilien und kreative Wissensarbeiter/-innen. In Dortmunder Konferenz 2012 „Mobilitäten und Immobilitäten“. Dortmund.

Neilson, B. \& Rossiter, N. (2005). From Precarity to Precariousness and Back Again: Labour, Life and Unstable Networks. In The Fibreculture Journal, FCJ-022.

(http://five.fibreculturejournal.org/fcj-022-from-precarity-to-precariousness-and-back-againlabour-life-and-unstable-networks/, accessed 31 October 2012)

Nelson, R. R. \& Winter, S. G. (1982). An Evolutionary Theory of Economic Change. Cambridge: Belknap Press.

Nowicka, M. (2005). Transnational Professionals and their Cosmopolitan Universes. Munich: Campus.

Oldenbourg, R. (1999). The Great Good Place: Cafes, Coffee Shops, Bookstores, Bars, Hair Salons, and Other Hangouts at the Heart of a Community. New York: Marlowe.

Oßenbrügge, J. (2004). Transstaatliche, plurilokale und glokale soziale Räume. Grundbegriffe zur Untersuchung transnationaler Beziehungen und Praktiken. In J. Oßenbrügge \& M. Reh (Eds.), Social Spaces of African Societies: Applications and Critique of Concepts about "Transnational Social Spaces". (pp. 15-34). Münster: LIT Verlag.

Ossewarde, M. (2007). Cosmopolitanism and the Society of Strangers. Current Sociology, 55(3), 367-388.

Paivia, A. (1971). Imagery and verbal processes. New York: Holt, Rinehart, and Winston.

Paivia, A. (2006). Mind and Its Evolution: A Dual Coding Theoretical Approach. East Sussex: Psychology Press.

Palidda, S. (2008). Mobilità umane: Introduzione alla Sociologia delle Migrazioni. Milan: Raffaello Cortina Editore.

Pasonati, A. (2004). Einführung. In A. Pasinato (Ed.), Heimatsuche: Regionale Identität im österreichisch-italienischen Alpenraum. (pp. 13-20). Würzburg: Verlag Königshausen \& Neumann.

Pethe, H. (2007). Un-restricted agents? International migration of the highly skilled revisited. Social Geography Discussions, 3, 211-236.

(http://core.kmi.open.ac.uk/display/710067, accessed 31 October 2012)

Pethe, H., Hafner, S. \& Lawton, P. (2010). Transnational Migrants in the Creative Knowledge Industries: Amsterdam, Barcelona, Dublin and Munich. In S. Musterd \& A. Murie (Eds.), Making Competitive Cities. (pp. 163-191). Oxford: Wiley-Blackwell. 
Petzold, K. (2009). Multilokale Identifikation: Ein theoretisches Modell zur Entstehung kognitivemotionaler Bindungen an mehrere Orte. In W. Andexlinger, S. Obkircher \& K. Saurwein (Eds.), Dokonara 2008: 2. Internationales DoktorandInnenkolleg Nachhaltige Raumentwicklung. (pp. 157-172). Innsbruck: Innsbruck University Press.

Petzold, K. (2010). Wenn sich alles um den Locus dreht: Multilokalität, Multilokation, multilokales Wohnen, Inter- und Translokalität als Begriffe der Mehrfachverortung. In M. Hühn, D. Lerp, K. Petzold \& M. Stock (Eds.), Transkulturalität, Transnationalität, Transstaatlichkeit, Translokalität. Theoretische und empirische Begriffsbestimmungen. (pp. 235-257). Münster, Hamburg, London: LIT Verlag.

Phillips, T. (2002). Imagined Communities and Self-identity: An Exploratory Quantitative Analysis. Sociology, 36(3), 597-617.

Plane, D. A. \& Rogerson, P. A. (1994). The Geographical Analysis of Population: With Applications to Planning and Business. New York: John Wiley \& Sons.

Ploch, B. (1995). Eignen sich Mental Maps zur Erforschung des Stadtraumes? Möglichkeiten der Methode. kea. Zeitschrift für Kulturwissenschaften, 8 (Stadtdschungel), 23-41.

Ponzini, D. \& Rossi, U. (2010). Becoming a Creative City: The Entrepreneurial Mayor, Network Politics and the Promise of an Urban Renaissance. Urban Studies, 47(5), 1037-1057.

Porat, M. \& Rubin, M. R. (1977). The Information Economy: Definition and Measurement. Washington: Government Printing Office.

Prato, G. B. (2009). Introduction - Beyond Multiculturalism: Anthropology at the Intersections Between the Local, the National and the Global. In G. B. Prato (Ed.), Beyond Multiculturalism: Views from Anthropoloy. (pp. 1-19). Farnham, Burlington: Ashgate.

Pries, L. (1997). Neue Migration im transnationalen Raum. In L. Pries (Ed.), Transnationale Migration (Vol. 12, pp. 15-44). Baden-Baden: Nomos Verlagsgesellschaft.

Pries, L. (2008). Die Transnationalisierung der sozialen Welt: Sozialräume jenseits von Nationalgesellschaften. Frankfurt/Main: Suhrkamp.

Pries, L. (2009). Transnationalism and the challenge of differentiated concepts of space. Tijdschrift voor Economische en Sociale Geografie, 100(5), 587-597.

Pries, L. (2010). Transnationalisierung. Theorie und Empirie grenzüberschreitender Vergesellschaftung. Wiesbaden: VS Verlag.

Putnam, R. D. (2000). Bowling alone: The Collapse and Revival of American Community. New York: Simon \& Schuster Paperbacks.

Putz, R. (2003). Culture and entrepreneurship - Remarks on transculturality as practice. Royal Dutch Geographic Society, 94(5), 554-563.

Rapport, N. \& Dawson, A. (1998). Migrants of Identity: Perceptions of Home in a World of Movement. Oxford: Berg.

Reinders, H. (2005). Qualitative Interviews mit Jugendlichen führen. Munich, Vienna: R. Oldenbourg Verlag.

Reuschke, D. (2009). Raum-zeitliche Muster und Bedingungen beruflich motivierter multilokaler Haushaltsstrukturen. Informationen zur Raumentwicklung, 1/2.2009, 32-42.

Ritzer, G. (1993). The Mcdonaldization of Society: An Investigation into the Changing Character of Contemporary Social Life. Thousand Oaks: Pine Forge Press. 
Ritzer, G. (2010, Ed.). McDonaldization: The Reader. Thousand Oaks: Pine Forge Press.

Rolshoven, J. (2006). Woanders daheim. Kulturwissenschaftliche Ansätze zur multilokalen Lebensweise in der Spätmoderne. Zeitschrift für Volkskunde, 102(2), 179-194.

Rolshoven, J., \& Winkler, J. (2009). Multilokalität und Mobilität. Informationen zur Raumentwicklung, 1/2.2009, 99-106.

Ross, A. (2008). The New Geography of Work. Theory, Culture and Society, 25(7-8), 31-49.

Roudometof, V. (2005). Transnationalism, Cosmopolitanism and Glocalization. Current Sociology, 53(1), 113-135.

Rumford, C. (2008). Cosmopolitan Spaces: Europe, Globalization, Theory. New York, Oxon: Routledge.

Said, E. (1979). Zionism from the standpoint of its victims. Social Text, no. 1, 7-58.

Samers, M. (2010). Migration. Abingdon, New York: Routledge.

Sassen, S. (2002, Ed.). Global Networks, Linked Cities. New York: Routledge.

Schäfer, C. (2010). Die Stadt ist unsere Fabrik/The City is Our Factory. Leipzig: Spector Books.

Schapp, W. (1976). In Geschichten verstrickt. Zum Sein von Mensch und Ding. Wiesbaden: Verlag B. Heymann.

Scheffel, C. (2012). Rückwanderung nach Ostdeutschland. Eine Analyse von strukturellen Ursachen und individuellen Beweggründen auf Mikroebene - dargestellt am Fallbeispiel Thüringen. Munich: GRIN - Verlag für akademische Texte.

Scherke, K. (2011). Transnationalität als Herausforderung für die soziologische Migrationsforschung. In G. Marinelli-König \& A. Preisinger (Eds.), Zwischenräume der Migration. Über die Entgrenzung von Kulturen und Identitäten. (pp. 79-90). Bielefeld: Transcript.

Schier, M. (2009). Räumliche Entgrenzung von Arbeit und Familie. Die Herstellung von Familie unter Bedingungen von Multilokalität. Informationen zur Raumentwicklung, 1/2.2009, 5566.

Schier, M. \& Jurczyk, K. (2007). Familie als Herstellungsleistung in Zeiten der Entgrenzung. Aus Politik und Zeitgeschichte, 34, 10-17.

Schmiz, A. (2011). Transnationalität als Ressource? Netzwerke vietnamesischer Migrantinnen und Migranten zwischen Berlin und Vietnam. Bielefeld: Transcript.

Scholz, G. (2011). Der SaarLorLux-Raum zwischen Realität, Illusion und Vision: Wahrnehmung und aktionsräumliches Verhalten aus der Sicht von Bewohnern einer europäischen Grenzregion. Saarbrücken: Institut für Landeskunde im Saarland e.V.

Schütz, A. (1932). Der sinnhafte Aufbau der sozialen Welt. Eine Einleitung in die verstehende Soziologie. Vienna: Springer.

Schütz, A. (1944). The Stranger: An Essay in Social Psychology. The American Journal of Sociology, 49(6), 499-507.

Schütz, A. \& Luckmann, T. (2003). Strukturen der Lebenswelt. Konstanz: UVK Verlagsgesellschaft.

Scott, A. J. (2006). Creative Cities: Conceptual Issues and Policy Questions. Journal of Urban Affairs, 28(1), 1-17. 
Sennett, R. (2006). The Culture of New Capitalism. New Haven: Yale University Press.

Sennett, R. (2008). The Craftsman. Yale: Yale University Press.

Shanley, T. (1995). Plug and Play System Architecture. Colorado Springs: MindShare, Inc.

Siedentop, S. (2008). Die Rückkehr der Städte? Zur Plausibilität der Reurbanisierungshypothese. Informationen zur Raumentwicklung, 3/4.2008, 193-207.

Simmel, G. (1903). Soziologie des Raumes. Jahrbuch für Gesetzgebung, Verwaltung und Volkswirtschaft im Deutschen Reich, 27(1), 27-71.

Sinan, A., Brynjolfsson, E. \& Van Alstyne, M. (2012). Information, Technology, and Information Worker Productivity. Information Systems Research, 23(3), 849-867

Smith, C. (1998). Creative Britain. London: Faber.

Smith, M. P. (2001). Transnational urbanism: Locating globalization. Oxford: Blackwell.

SMWA/SMWK. (2009). Erster Kulturwirtschaftsbericht für den Freistaat Sachsen 2008. Dresden: SMWA/SMWK.

Social Platform. (2004). The goals of "more and better job" and "greater social cohesion" are as important as "competitiveness" and "growth": A Social NGOs' review of the Lisbon strategy.

(http://www.socialplatform.org/module/FileLib/ENSPEvaluationLisbon2005final.pdf, accessed 31 October 2012)

Soja, E. (1996). Thirdspace: Journeys to Los Angeles and Other Real-And-Imagined Places. Cambridge, Oxford: Blackwell.

Söndermann, M., Backes, C., Arndt, O. \& Brünink, D. (2009). Gesamtwirtschaftliche Perspektiven der Kultur- und Kreativwirtschaft in Deutschland. Berlin: Bundesministerium für Wirtschaft und Technologie.

(http://www.kultur-kreativ-wirtschaft.de/Dateien/KuK/PDF/doku-577gesamtwirtschaftliche-perspektiven-kultur-und-kreativwirtschaftkurzfassung,property=pdf,bereich=kuk,sprache=de,rwb=true.pdf, accessed 31 October 2012)

Spellerberg, A. \& Wilbert, K. (2008). Wohnwünsche von IT-Beschäftigten als Motor der Reurbanisierung? Ergebnisse zweier empirischer Untersuchungen in Bonn und Kaiserslautern. In G. Schmitt \& K. Selle (Eds.), Bestand? Perspektiven für das Wohnen in der Stadt. (pp. 124-134). Dortmund: Rohn.

Stea, D. \& Downs, R. M. (1970). From the Outside Looking in at the Inside Looking out. Environment and Behavior, 2(1), 3-12.

Steinbicker, J. (2001). Zur Theorie der Informationsgesellschaft: Ein Vergleich der Ansätze von Peter Drucker, Daniel Bell und Manuel Castells. Opladen: Leske+Budrich.

Steinbrink, M. (2009). Leben zwischen Land und Stadt. Migration, Translokalität und Verwundbarkeit in Südafrika. Wiesbaden: VS Verlag.

Stock, M. (2009). Polytopisches Wohnen - ein phänomenologisch-prozessorientierter Zugang. Informationen zur Raumentwicklung, 1/2.2009, 107-116.

Stock, M. (2010). Transkulturalität als lokale Eigenart. Zur kulinarischen Geschmackslandschaft Berlins. In M. Hühn, D. Lerp, K. Petzold \& M. Stock (Eds.), Transkulturalität, Transnationalität, Transstaatlichkeit, Translokalität. Theoretische und empirische Begriffsbestimmungen. (pp. 277-291). Münster, Hamburg, London: LIT Verlag. 
Stone, G. P. (1962). Appearance and the Self. In A. M. Rose (Ed.), Human Bevhavior and Social Processes. An Interactionist Approach. (pp. 86-118). London: International Library of Sociology and Social Reconstruction.

Stone, G. P. (2005). Appearance and the Self: A slightly revised version. In D. Brissett \& C. Edgley (Eds.), Life as Theater: A Dramaturgical Sourcebook (2nd ed., pp. 141-162). New Jersey: Transaction.

Storper, M. \& Venables, A. J. (2004). Buzz: face-to-face contact and the urban economy. Journal of Economic Geography, 4(4), 351-370.

Storr, A. (1988). Churchill's Black Dog, Kafka's Mice: And Other Phenomena of the Human Mind. New York: Grove Press.

Stracke-Mertes, A. (2003). Soziologie. Der Blick auf soziale Beziehungen. (3rd ed.). Hannover: Vincentz Verlag.

Strauss, A. L. (1987). Qualitative Analysis for Social Scientists. Cambridge: Cambridge University Press.

Strauss, A. L. \& Corbin, J. (1990a). Basics of Qualitative Research: Grounded Theory procedures and techniques. Newbury: Sage.

Strauss, A. L. \& Corbin, J. (1990b). Grounded Theory Research: Procedures, Canons and Evaluative Criteria. Qualitative Sociology, 13(1), 3-21.

Sturm, G. \& Meyer, K. (2009). Was können Melderegister deutscher Großstädte zur Analyse residenzieller Multilokalität beitragen? Informationen zur Raumentwicklung, 1/2.2009, 1530 .

Tausch, A. (2006). The Lisbon process, re-visited. A reality check of the European social model. Munich Personal RePEc Archive Paper, no. 310.

(http://mpra.ub.uni-muenchen.de/310/1/MPRA_paper_310.pdf, accessed 31 October 2012)

Thomas, G. \& James, D. (2006). Reinventing Grounded Theory: Some Questions about Theory, Ground and Discovery. British Educational Research Journal, 32(6), 767-795.

Toplak, K. (2011). Zwischen den Kunstwelten von Buenos Aires und Ljubljana. Die Pluralisierung von Ideen, kulturellen Praktiken und Kunstformen. In G. Marinelli-König \& A. Preisinger (Eds.), Zwischenräume der Migration. Über die Entgrenzung von Kulturen und Identitäten. (pp. 249-268). Bielefeld: Transcript.

Tversky, B. (1992). Descriptions and depictions of environments. Memory \& Cognition, 20(5), 483496.

UPnP Implementers Corporation (2006). UPnP ${ }^{\mathrm{TM}}$ Technology - The Simple, Seamless Home Network.

(http://upnp.org/resources/whitepapers/UPnP\%20Technology_The\%20Simple,\%20Seamles s\%20Home\%20Network_whitepaper.pdf., accessed 31 October 2012)

Urry, J. (2000). Sociology beyond Societies - Mobilities for the twenty-first Century. London, New York: Routledge.

Vandenbrande, T., Coppin, L., van der Hallen, P., Ester, P., Fourage, D., Fasang, A., Geerdes, S. \& Schömann, K. (2006). Mobility in Europe: Analysis of the 2005 Eurobarometer survey on geographical and labour market mobility. Luxembourg: Office for Official Publications of the European Communities.

(http://www.eurofound.europa.eu/pubdocs/2006/59/en/1/ef0659en.pdf, accessed 31 October 2012) 
Vasileva, K. (2012). Nearly two-thirds of the foreigners living in EU Member States are citizens of countries outside the EU-27. In Statistics in focus (Vol. 31/2012). Luxembourg: Eurostat. (http://www.eds-destatis.de/de/downloads/sif/KS-SF-12-031-EN-N.pdf, $\quad$ accessed 31 October 2012)

Vertovec, S. (2001). Transnationalism and identity. Journal of Ethnic and Racial Studies, 27(4), 573-582.

Veryard, R. (2001). The Component-Based Business: Plug and Play. London, Berlin, Heidelberg: Springer Verlag.

von Moltke, J. (2005). No Place like Home: Locations of Heimat in German Cincema. Berkley, Los Angeles, London: University of California Press.

von Streit, A. (2010). Entgrenzter Alltag - Arbeiten ohne Grenzen? Das Internet und die raumzeitlichen Organisationsstrategien von Wissensarbeitern. Bielefeld: Transcript.

Vonderach, G. (1997). Geschichtenhermeneutik. In R. Hitzler \& A. Honer (Eds.), Sozialwissenschaftliche Hermeneutik: Eine Einführung. (pp. 165-189). Opladen: Leske + Budrich.

Walker, I. (2011). Hybridity, Belonging, and Mobilities: the Intercontinental Peripatetics of a Transnational Community. Population, Space and Place, 17(2), 167-178.

Watts, L. \& Urry, J. (2008). Moving methods, travelling times. Environment and Planning D: Society and Space, 26(5), 860-874.

Weckerle, C., Gerig, M. \& Söndermann, M. (2008). Creative Industries Switzerland: Facts, Models, Culture. Basel, Boston, Berlin: Birkhäuser.

Wedemeier, J. (2010). The Impact of the Creative Sector on Growth in German Regions. European Planning Studies, 18(4), 505-520.

Weichhart, P. (1990). Raumbezogene Identität: Bausteine zu einer Theorie räumlich-sozialer Kognition und Identifikation. Stuttgart: Franz Steiner Verlag.

Weichhart, P. (2008). Entwicklungslinien der Sozialgeographie: Von Hans Bobek bis Benno Werlen. (Vol. 1). Stuttgart: Franz Steiner Verlag.

Weichhart, P. (2009). Multilokalität - Konzepte, Theoriebezüge und Forschungsfragen. Informationen zur Raumentwicklung, 1/2.2009, 1-14.

Weichhart, P. (2010). Das "Trans-Syndrom". Wenn die Welt durch das Netz unserer Begriffe fällt. In M. Hühn, D. Lerp, K. Petzold \& M. Stock (Eds.), Transkulturalität, Transnationalität, Transstaatlichkeit, Translokalität: Theoretische und empirische Begriffsbestimmungen. (pp. 47-70). Münster, Hamburg, London: LIT Verlag.

Weichhart, P., Weiske, C. \& Werlen, B. (2006). Place Identity und Images: Das Beispiel Eisenhüttenstadt. Vienna: Institut für Geographie und Regionalforschung der Universität Wien.

Weiske, C., Petzold, K. \& Zierold, D. (2009). Multilokale Haushaltstypen. Bericht aus dem DFGProjekt "Neue multilokale Haushaltstypen" (2006-2008). Informationen zur Raumentwicklung, 1/2.2009, 67-76.

Welsch, W. (2000). Transkulturalität. Zwischen Globalisierung und Partikularisierung. Jahrbuch Deutsch als Fremdsprache, no. 26, 327-351.

Werlen, B. (1997). Sozialgeographie alltäglicher Regionalisierungen. Band 2: Globalisierung, Region und Regionalisierung. Stuttgart: Franz Steiner Verlag. 
Werlen, B. (2000). Sozialgeographie: Eine Einführung. Bern, Stuttgart, Vienna: Haupt Verlag/UTB.

Werner, T. P. (2009). The Well Role Ideal Type Theory: A Companion to Parsons Sick Role Ideal Type in the Social System. Ann Arbor: ProQuest.

Wessel, K. (1996). Empirisches Arbeiten in der Wirtschafts- und Sozialgeographie: Eine Einführung. Paderborn, Munich, Vienna, Zurich: Ferdinand Schöningh.

Wiest, K. (2008). Reurbanisierungs- und Revitalisierungstendenzen in der ostdeutschen Stadtentwicklung. In G. Schmitt \& K. Selle (Eds.), Bestand? Perspektiven für das Wohnen in der Stadt. (pp. 264-275). Dortmund: Rohn.

Wiest, K. \& Zischner, R. (2006). Aufwertung innerstädtischer Altbaugebiete in den neuen Bundesländern - Prozesse und Entwicklungspfade. Deutsche Zeitschrift für Kommunalwissenschaften, 45(1), 99-121.

Wimmer, A. \& Glick Schiller, N. (2002). Methodological Nationalism and Beyond: Nation-State Building, Migration and the Social Sciences. Global Networks, 2(4), 301-334. 


\section{Appendices}

Appendix A: Statistical definitions of creative industries

\begin{tabular}{|c|c|}
\hline \multicolumn{2}{|r|}{ ESPON 2013 (2011: 19) } \\
\hline ISCO-88 4D codes & \\
\hline 2131 & Computer systems designers and analysts \\
\hline 2132 & Computer programmers \\
\hline 2139 & Computing professionals not elsewhere classified \\
\hline 2141 & Architects, town and traffic planners \\
\hline 2310 & College, university and higher education teaching professionals \\
\hline 2320 & Secondary education teaching professionals \\
\hline 2431 & Archivists and curators \\
\hline 2432 & Librarians and related information professionals \\
\hline 2442 & Sociologists, anthropologists and related professionals \\
\hline 2443 & Philosophers, historians and political scientists \\
\hline 2444 & Philologists, translators \\
\hline 2451 & Authors, journalists and other writers \\
\hline 2452 & Sculptors, painters and related artists \\
\hline 2453 & Composers, musicians and singers \\
\hline 2454 & Choreographers and dancers \\
\hline 2455 & Film, stage and related actors and directors \\
\hline 3131 & Photographers and image and sound equipment operators \\
\hline 3429 & Business service agents \& trade brokers not elsewhere classified \\
\hline 3460 & Social work associate professionals \\
\hline 3471 & Decorators and commercial designers \\
\hline 3472 & Radio, television and other announcers \\
\hline 3473 & Street, night club and related musicians, singers and dancers \\
\hline 3474 & Clowns, magicians, acrobats and related associate professionals \\
\hline 3475 & Athletes, sportspersons and related associate professionals \\
\hline 3480 & Religious associate professionals \\
\hline 5113 & Travel guides \\
\hline 5210 & Fashion and other models \\
\hline 7311 & Precision-instrument makers and repairers \\
\hline 7312 & Musical instrument makers and tuners \\
\hline 7313 & Jewellery and precious-metal workers \\
\hline
\end{tabular}




\begin{tabular}{|c|c|}
\hline 7321 & Abrasive wheel formers, potters and related workers \\
\hline 7322 & Glass makers, cutters, grinders and finishers \\
\hline 7323 & Glass engravers and etchers \\
\hline 7324 & Glass, ceramics and related decorative painters \\
\hline 7331 & Handicraft workers in wood and related materials \\
\hline 7332 & Handicraft workers in textile, leather and related materials \\
\hline 7341 & Compositors, typesetters and related workers \\
\hline 7342 & Stereotypes and electrotypers \\
\hline 7343 & Printing engravers and etchers \\
\hline 7344 & Photographic and related workers \\
\hline 7345 & Bookbinders and related workers \\
\hline 7346 & Silk-screen, block and textile printers \\
\hline \multicolumn{2}{|r|}{ Howkins (2001: 116) } \\
\hline & $\begin{array}{l}\text { Research and development } \\
\text { Publishing } \\
\text { Software } \\
\text { TV and radio } \\
\text { Design } \\
\text { Music } \\
\text { Film } \\
\text { Toys and Games } \\
\text { Advertising } \\
\text { Architecture } \\
\text { Performin Arts } \\
\text { Crafts } \\
\text { Video Games } \\
\text { Fashion } \\
\text { Art }\end{array}$ \\
\hline \multicolumn{2}{|r|}{ European Commission (2010a: 194) } \\
\hline \multicolumn{2}{|l|}{ NACE Rev. 2} \\
\hline \multicolumn{2}{|r|}{ Information Services } \\
\hline J58 & $\begin{array}{l}\text { Publishing activities (publishing of books, periodicals and software } \\
\text { publishing), motion picture, video and television programme production, } \\
\text { Sound recording and music publishing activities }\end{array}$ \\
\hline $\mathrm{J} 60$ & Programming and broadcasting activities \\
\hline J62 & Computer programming, consultancy and related activities \\
\hline \multicolumn{2}{|r|}{ Business Services } \\
\hline
\end{tabular}




\begin{tabular}{|c|c|}
\hline M711 & $\begin{array}{l}\text { Architectural and engineering activities and related technical consultancy } \\
(25 \%)\end{array}$ \\
\hline M731 & Advertising \\
\hline M741 & Specialised design activities \\
\hline M742 & Photographic activities (25\%) \\
\hline M743 & Translatation and interpretation activities \\
\hline \multicolumn{2}{|r|}{ Art and Entertainment } \\
\hline R90 & Creative, arts and entertainment activities \\
\hline \multicolumn{2}{|r|}{ European Commission (2010a: 198) } \\
\hline \multicolumn{2}{|l|}{ ISCO-88 3D codes } \\
\hline 211 & Physicists, chemists and related professionals \\
\hline 212 & Mathematicians, statisticians and related professionals \\
\hline 213 & Computing professionals \\
\hline 214 & Architects, engineers \& related professionals \\
\hline 221 & Life science professionals \\
\hline 222 & Health professionals \\
\hline 243 & Archivists, librarians \& related professionals \\
\hline 244 & Social science \& related professionals \\
\hline 245 & Writers and creative or performing artists \\
\hline $347+521$ & Artistic, entertainment \& sports associations \\
\hline \multicolumn{2}{|r|}{ European Commission (2010a: 238) } \\
\hline \multicolumn{2}{|l|}{ NACE Rev. 1.1} \\
\hline \multicolumn{2}{|r|}{ Advertising } \\
\hline 74.4 & Advertising \\
\hline \multicolumn{2}{|r|}{ Architecture } \\
\hline 74.2 & $\begin{array}{l}\text { Architecture and engineering activities and related technical consultancy } \\
(25 \%)\end{array}$ \\
\hline \multicolumn{2}{|r|}{ Arts \& Antiques } \\
\hline 52.48 & Other retail sale in specialized stores (5\%) \\
\hline 52.5 & Retail sale of second-hand goods in stores (5\%) \\
\hline \multicolumn{2}{|r|}{ Crafts } \\
\hline & $\begin{array}{l}\text { No data available: Majority of businesses too small to be picked up in } \\
\text { business surveys }\end{array}$ \\
\hline \multicolumn{2}{|r|}{ Design } \\
\hline & No codes match this sector \\
\hline 17.7 & Manufacture of knitted and crocheted articles (0.5\%) \\
\hline \multicolumn{2}{|r|}{ Fashion Design } \\
\hline
\end{tabular}




\begin{tabular}{|c|c|}
\hline 18 & Wearing apparel; dressing and dyeing of fur (0.5\%) \\
\hline 19.3 & Manufacture of footwear (0.5\%) \\
\hline 74.87 & Other Business activities n.e.c. (2.5\%) \\
\hline \multicolumn{2}{|r|}{ Video, Film \& Photography } \\
\hline 22.32 & Reproduction of video recording (25\%) \\
\hline 74.81 & Photographic activities (25\%) \\
\hline 92.1 & Motion picture and video activities \\
\hline \multicolumn{2}{|r|}{ Music and the Visual \& Performing Arts } \\
\hline 22.14 & Publishing of sound recordings \\
\hline 22.31 & Reproduction of sound recording (25\%) \\
\hline 92.31 & Artistic and literary creation and interpretation \\
\hline 92.32 & Operation of arts facilities \\
\hline 92.34 & Other entertainment activities n.e.c. (50\%) \\
\hline 92.72 & Other recreational activities n.e.c. (25\%) \\
\hline 22.11 & Publishing of books \\
\hline 22.12 & Publishing of newspapers \\
\hline \multicolumn{2}{|r|}{ Publishing } \\
\hline 22.13 & Publishing of journals and periodicals \\
\hline 22.15 & Other publishing (50\%) \\
\hline 92.4 & News agency activities \\
\hline \multicolumn{2}{|r|}{ Software, Computer Games \& Electronic Publishing } \\
\hline 22.33 & Reproduction of computer media (25\%) \\
\hline 72.2 & Software consultancy and supply \\
\hline \multicolumn{2}{|r|}{ Radio \& TV } \\
\hline 92.2 & Radio and television activities \\
\hline \multicolumn{2}{|r|}{ Florida (2002: 328) } \\
\hline SOC 1998 & \\
\hline \multicolumn{2}{|r|}{ Super-Creative Core } \\
\hline & Computer and mathematical occupations \\
\hline & Architecture and engineering occupations \\
\hline & Life, physical, and social science occupations \\
\hline & Education, training, and library occupations \\
\hline & Arts, design, entertainment, sports, and media occupations \\
\hline \multicolumn{2}{|r|}{ Creative Professionals } \\
\hline & Management occupations \\
\hline & Business and financial operations occupations \\
\hline
\end{tabular}




\begin{tabular}{|l|l|}
\hline & Legal occupations \\
\hline & Healthcare practitioners and technical occupations \\
\hline & High-end sales and sales management \\
\hline
\end{tabular}


Appendix B: Background information on interview partners, interview situations and mental maps by individual cases

\begin{tabular}{|c|c|}
\hline & Interview No. 1 \\
\hline & Who is the interview partner? \\
\hline Person & $\begin{array}{l}\text { Aurélien, French, in his 30s, freelance artist, head of cultural association, no } \\
\text { children, German girlfriend (opera singer) }\end{array}$ \\
\hline $\begin{array}{l}\text { Personal } \\
\text { places }\end{array}$ & $\begin{array}{l}\text { Leipzig (DE), Nice (FR), Brittany (FR), Berlin (DE), Paris (FR), Corsica (FR), } \\
\text { Italy }\end{array}$ \\
\hline $\begin{array}{l}\text { Tools for } \\
\text { multilocality }\end{array}$ & $\begin{array}{l}\text { Artist's tools redundant in Leipzig \& Brittany | internet (communication) | air } \\
\text { planes | car | computer | artist residences | own house in Leipzig | parents' } \\
\text { apartments in Nice and Brittany | friends' places in Berlin and Paris | speaks } \\
\text { French, English and German }\end{array}$ \\
\hline & What was the interview situation like? \\
\hline Where? & Interviewer's private home, Leipzig (DE) \\
\hline When? & 11 May 2010, 21h45 \\
\hline Length? & $00: 53: 48$ \\
\hline Language? & French \\
\hline Atmosphere & $\begin{array}{l}\text { I knew Aurélien already from another interview I had done with him for an } \\
\text { earlier research project in 2008. So, I knew his current situation and thought he } \\
\text { would be the perfect 'test case' for this PhD project. Feeling a bit bad for using } \\
\text { him for another interview, I invited him at least to my place, I ordered pizza and } \\
\text { organised something to drink. Doing the interview in my place had the advantage } \\
\text { to be in a silent and known environment. I wanted to test my interview style, and } \\
\text { thus any unpredictable disturbance was to be avoided. Also, doing the interview } \\
\text { in French, as Aurélien prefered, gave the interview a certain complexity. I did not } \\
\text { use a fixed and structured guideline with formulated questions. On the other hand } \\
\text { it was good like that, as it allowed for a very open and flexible conversation. }\end{array}$ \\
\hline
\end{tabular}




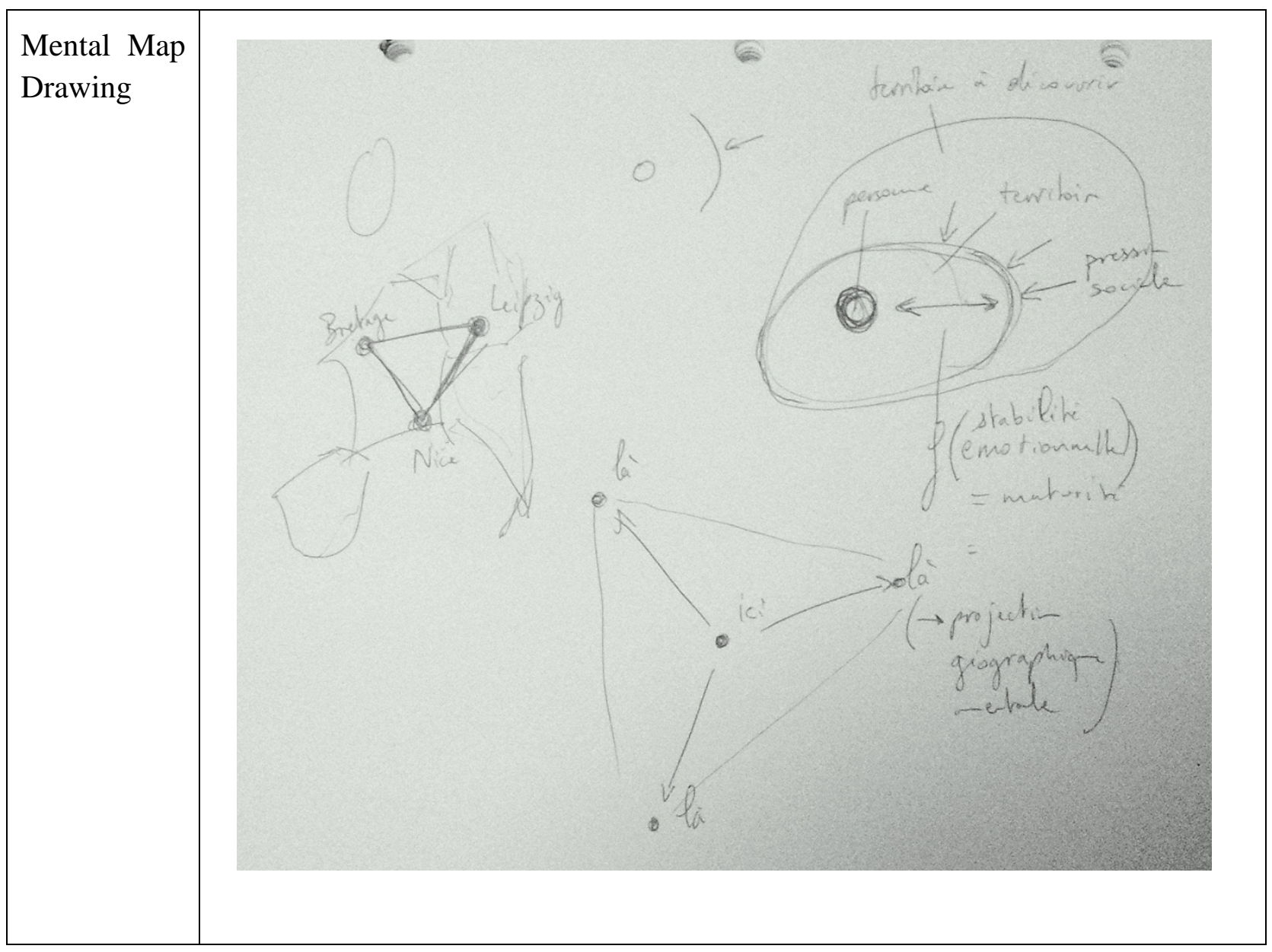

\begin{tabular}{|l|l|}
\hline \multicolumn{2}{|c|}{ Who is the interview partner? } \\
\hline \multicolumn{1}{|l|}{} \\
\hline Person & $\begin{array}{l}\text { Birgit, German, in her 50s, architect, owner and manager of urban planning } \\
\text { office, professor for regional planning, freelance planning consultant, no } \\
\text { children, German partner (freelance photographer) }\end{array}$ \\
\hline Personal places & $\begin{array}{l}\text { Leipzig (DE), Kassel (DE), Zurich (CH), Berlin (DE), New York (US), } \\
\text { Shanghai (China) }\end{array}$ \\
\hline $\begin{array}{l}\text { Tools for } \\
\text { multilocality }\end{array}$ & $\begin{array}{l}\text { Bahncard 100 | mobile phone | computer | traditional agenda | hygiene bag } \\
\text { with personal cosmetics | own car | own bank account | own apartment in } \\
\text { Leipzig I personal hotel room in Kassel (special fare for university staff) | } \\
\text { speaks German, English and Russian }\end{array}$ \\
\hline \multicolumn{1}{|c|}{ What was the interview situation like? } \\
\hline Where? & \begin{tabular}{l} 
Birgit's office room, Leipzig (DE) \\
\hline
\end{tabular}
\end{tabular}




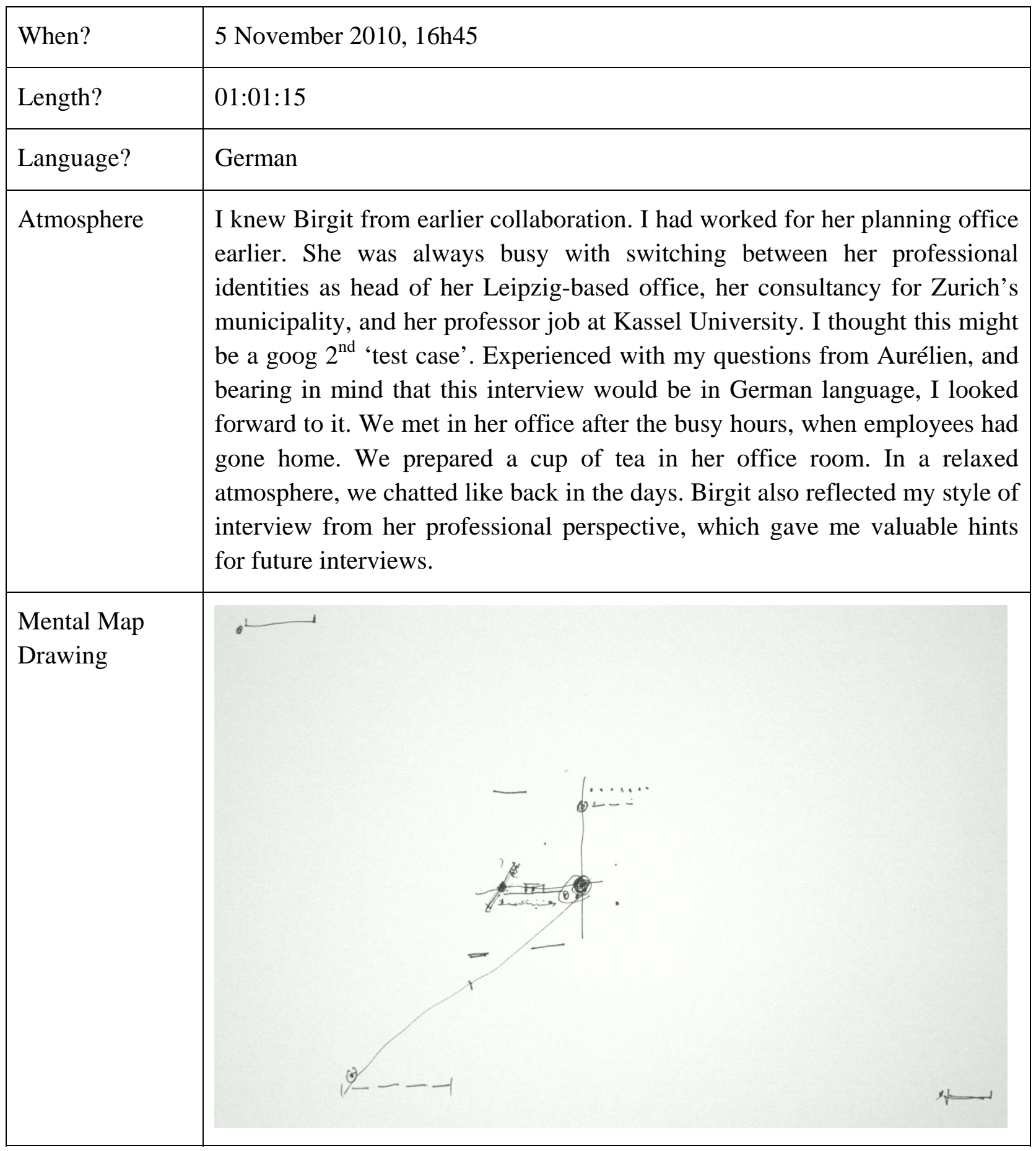

Interview No. 3

Who is the interview partner?

Person

Christian, French, in his 30s, freelance artist, poet, model, translator, construction worker, no children, single (recently broke up with German exgirlfriend) 


\begin{tabular}{|c|c|}
\hline Personal places & $\begin{array}{l}\text { Leipzig (DE), Dresden (DE), Marseille (FR), Berlin (DE), Paris (FR), } \\
\text { Genève (CH), Nice (FR), Lyon (FR), Amiens (FR), Avignon (FR) }\end{array}$ \\
\hline $\begin{array}{l}\text { Tools for } \\
\text { multilocality }\end{array}$ & $\begin{array}{l}\text { Student ID card (access to low cost health insurance; university ateliers) | } \\
\text { Travel scholarship Promobil | Apartment in Leipzig in the house of French } \\
\text { friend Aurélien | Pocket book for idea sketches | mobile phone | emails | } \\
\text { friends' places and residence projects in other places | speaks French, English } \\
\text { and German }\end{array}$ \\
\hline \multicolumn{2}{|r|}{ What was the interview situation like? } \\
\hline Where? & Christian’s apartment, Leipzig (DE) \\
\hline When? & 16 November 2010, $13 \mathrm{~h} 30$ \\
\hline Length? & $00: 52: 27$ \\
\hline Language? & French \& German \\
\hline Atmosphere & $\begin{array}{l}\text { I didn't know Christian before. Aurélien advised me to speak to him and gave } \\
\text { me his number. Both were friends, and Aurélien told me he would inform } \\
\text { Christian about my project. When I called Christian, he invited me } \\
\text { immediately to come over to his place, which is an apartment in the building } \\
\text { that Aurélien bought in Leipzig. The apartment was still a building site, and } \\
\text { we took place on a large working table in a room full of tools. Christian told } \\
\text { me that he only had one hour, as he had to prepare a trip to Berlin the day } \\
\text { after. We started in the interview in French, but he offered me to switch to } \\
\text { German if I would find it easier. The atmosphere was very relaxed and open. } \\
\text { Two minor interruptions occurred when Aurélien passed by for a moment, } \\
\text { looking for some tools, and when a friend called on his phone. }\end{array}$ \\
\hline
\end{tabular}




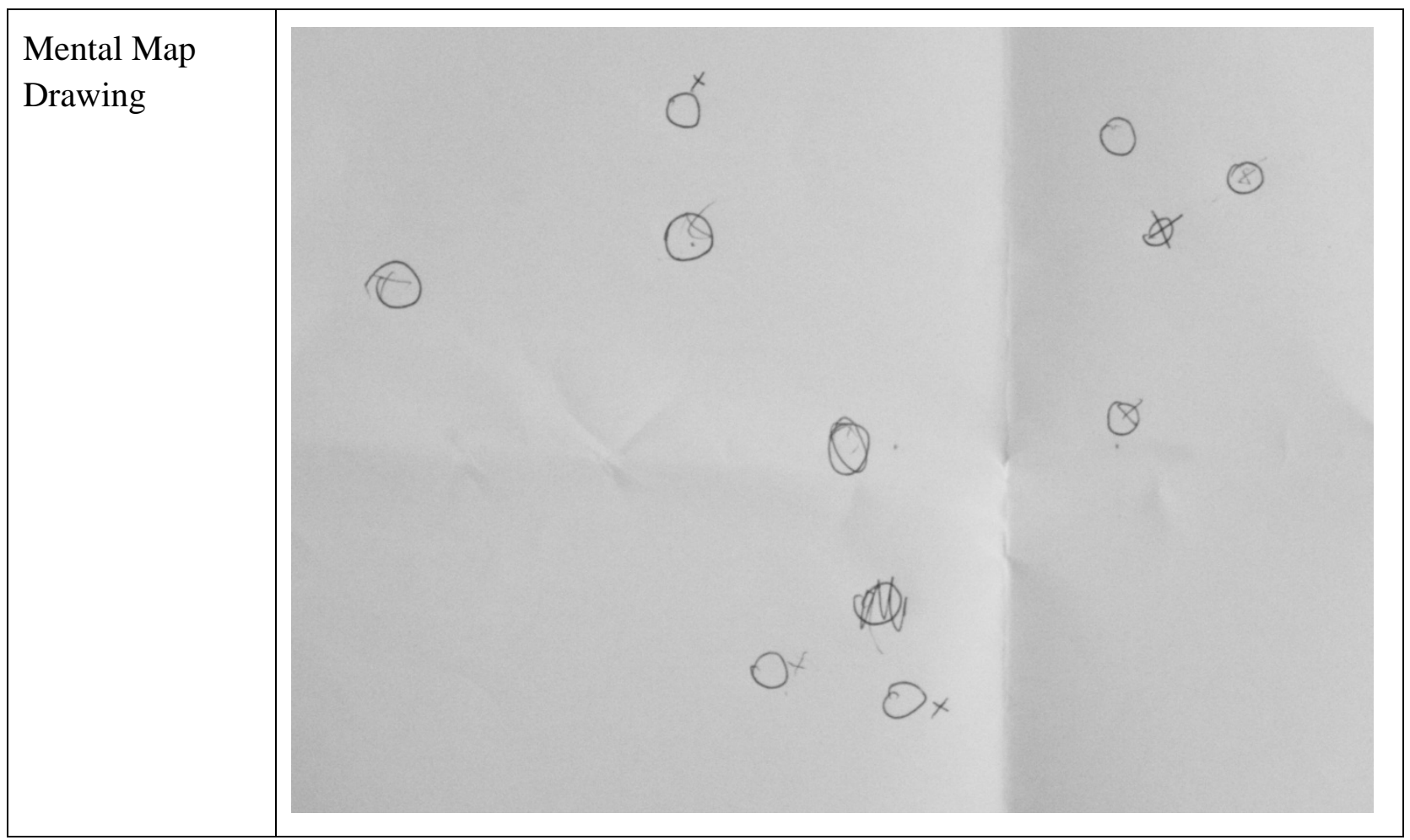

\begin{tabular}{|c|c|}
\hline & Interview No. 4 \\
\hline & Who is the interview partner? \\
\hline Person & $\begin{array}{l}\text { Dirk, German, in his 30s, slavicist \& journalist, researcher working on his } \\
\text { PhD thesis and in a part-time position as researcher in Leipzig based research } \\
\text { institute, freelance consultant in the field of adult education, no children, } \\
\text { Bulgarian girlfriend }\end{array}$ \\
\hline Personal places & $\begin{array}{l}\text { Sofia (BG), Leipzig (DE), Munich (DE), Biberach (DE), Minsk (Belarus), } \\
\text { Belgrade (Serbia), Pristina (Kosovo), Podgorica (Montenegro) }\end{array}$ \\
\hline $\begin{array}{l}\text { Tools for } \\
\text { multilocality }\end{array}$ & $\begin{array}{l}\text { Magnetic compass | dictionary | Email | Skype | status as freelance journalist } \\
\text { in Bulgaria | own apartment in Munich (currently let) | own apartment in } \\
\text { Sofia | a friend's apartment for use in Leipzig | speaks German, English, } \\
\text { Bulgarian, Russian and French }\end{array}$ \\
\hline & What was the interview situation like? \\
\hline Where? & Dirk’s work place, Leipzig (DE) \\
\hline When? & 24 November 2010, 19 h30 \\
\hline Length? & $00: 56: 37$ \\
\hline
\end{tabular}




\begin{tabular}{|l|l|}
\hline Language? & German \\
\hline Atmosphere & $\begin{array}{l}\text { I met Dirk in my professional environment when he started to work for a } \\
\text { research project with some colleagues. I knew that this project was based on } \\
\text { field work in Bulgaria, and I knew that Dirk was travelling frequently } \\
\text { between Germany and Bulgaria, having an apartment abroad. So, I asked him } \\
\text { for an interview, while he was passing some days in Germany. He said that } \\
\text { he would take the time, but we should do it in the evening hours in his } \\
\text { workplace. When he is in Germany, he spends most of the time working in } \\
\text { the office. The evening of the interview was the last chance to talk to him } \\
\text { before he was about to go back to Bulgaria for some time. So, I decided to } \\
\text { organize some drinks and we met in a sofa corner of the institute. All } \\
\text { colleagues of his floor had already gone home, and we could talk in a relaxed } \\
\text { atmosphere. Dirk was a rather critical interview partner, asking for } \\
\text { clarification of my questions and pointing to my mistakes in interviewing. }\end{array}$ \\
\hline Mental Map \\
Drawing
\end{tabular}

Interview No. 5

\section{Who is the interview partner?}

\begin{tabular}{|l|l|}
\hline Person & $\begin{array}{l}\text { Emil, German, in his 40s, political scientist, researcher working on his PhD } \\
\text { thesis on copyrights, active in cultural association, no children, Italian } \\
\text { boyfriend }\end{array}$ \\
\hline Personal places & $\begin{array}{l}\text { Leipzig (DE), Bayreuth (DE), Basel (CH), Berlin (DE), Genoa/Liguria (IT), } \\
\text { Rostock/Stralsund/Baltic Sea (DE), San Francisco/California (US), Lima }\end{array}$ \\
\hline
\end{tabular}




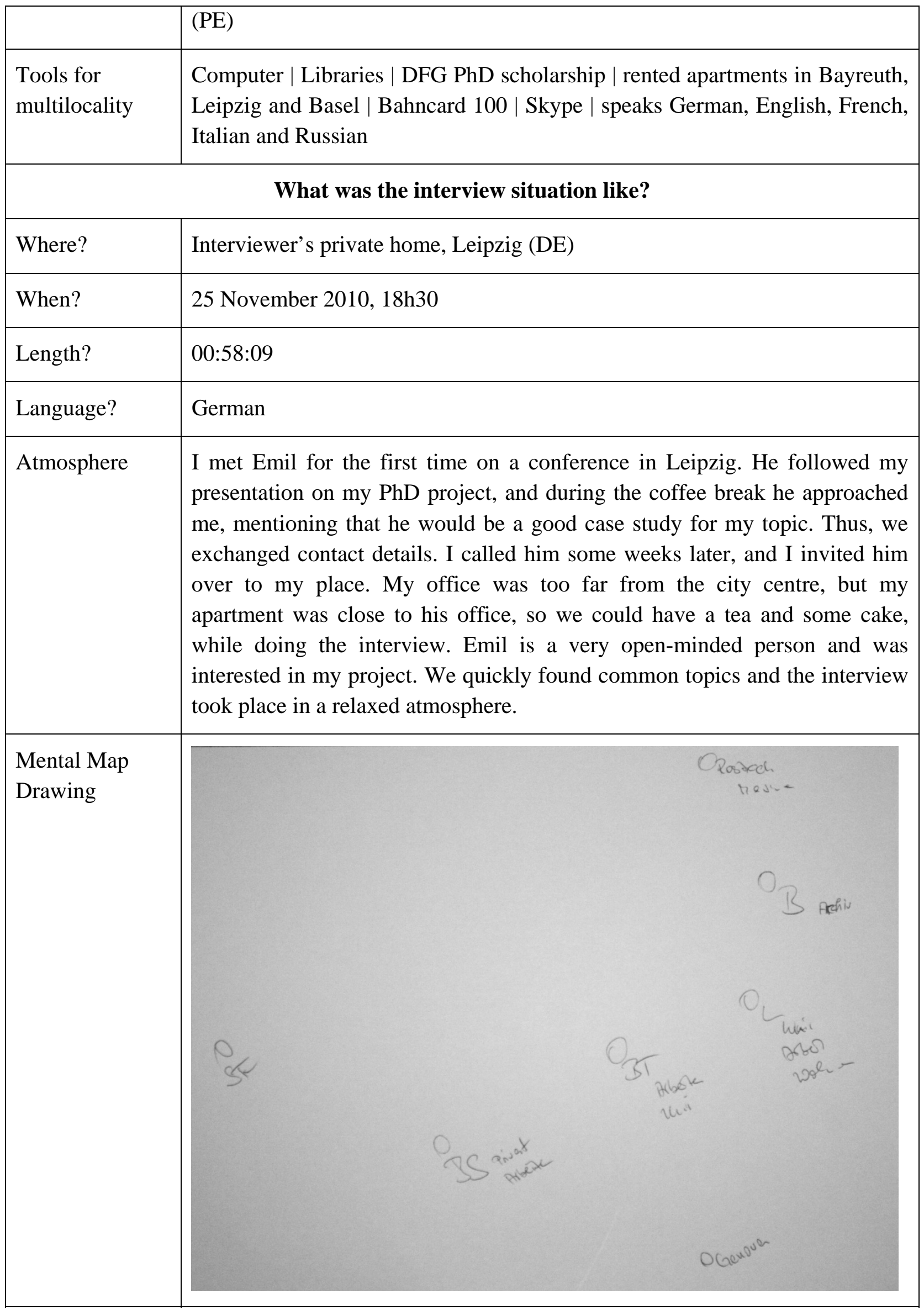


Interview No. 6

\section{Who is the interview partner?}

\begin{tabular}{|l|l|}
\hline Person & $\begin{array}{l}\text { Federico, Italian, in his 30s, cultural scientist, researcher working in a post- } \\
\text { doc position in Leipzig, with focus on Eastern European transnational } \\
\text { literature, active in cultural association dealing with movies from Balkans, no } \\
\text { children, single }\end{array}$ \\
\hline Personal places & $\begin{array}{l}\text { Leipzig (DE), Prague (CZ), Ljubljana (SI), Trieste (IT), Bergamo (IT), } \\
\text { L'Aquila (IT), Bologna (IT), Curitiba (Brazil) }\end{array}$ \\
\hline $\begin{array}{l}\text { Tools } \\
\text { multilocality }\end{array}$ & $\begin{array}{l}\text { Computer | Shared apartment in Leipzig | parents' house in Bergamo | } \\
\text { friends' places | temporary renting of rooms and apartments | libraries | office } \\
\text { provided by host institutions | Bahncard 50 | travel websites | German and } \\
\text { Italian bank accounts and credit cards | 5 different SIM cards | speaks } \\
\text { German, English, Czech, Italian, Slovenian, French, Portuguese, Spanish and } \\
\text { Polish }\end{array}$
\end{tabular}

What was the interview situation like?

\begin{tabular}{|l|l|}
\hline Where? & Federico's work place, Leipzig (DE) \\
\hline When? & 1 December 2010, 13h00 \\
\hline Length? & $01: 21: 54$ \\
\hline Language? & German \\
\hline Atmosphere & $\begin{array}{l}\text { I was offered the contact details by a colleague who is friend with Federico. } \\
\text { My colleague thought, Federico would be an interesting case for my research. } \\
\text { He told Federico that I might call or email him. When I did so, Federico had } \\
\text { just returned from a longer trip to the United States and he agreed to have an } \\
\text { interview. But he will only be available in Leipzig during the following } \\
\text { week, after he would be out of town for some weeks again. So we fixed a } \\
\text { date at his workplace during the lunch break. When I arrived, we left his } \\
\text { office room because he shares it with a colleague, who needed silence for } \\
\text { work. Instead we went to the library on the roof floor of his research institute. } \\
\text { As there was nearly no one in the library, our conversation did not disturb } \\
\text { anyone. We sat down on sofas in the corner of the room. And the interview } \\
\text { took place in a smooth and relaxed atmosphere. As we did not have } \\
\text { something to drink or eat, I invited him to a close by bar for lunch, when we } \\
\text { finished the interview. }\end{array}$ \\
\hline
\end{tabular}




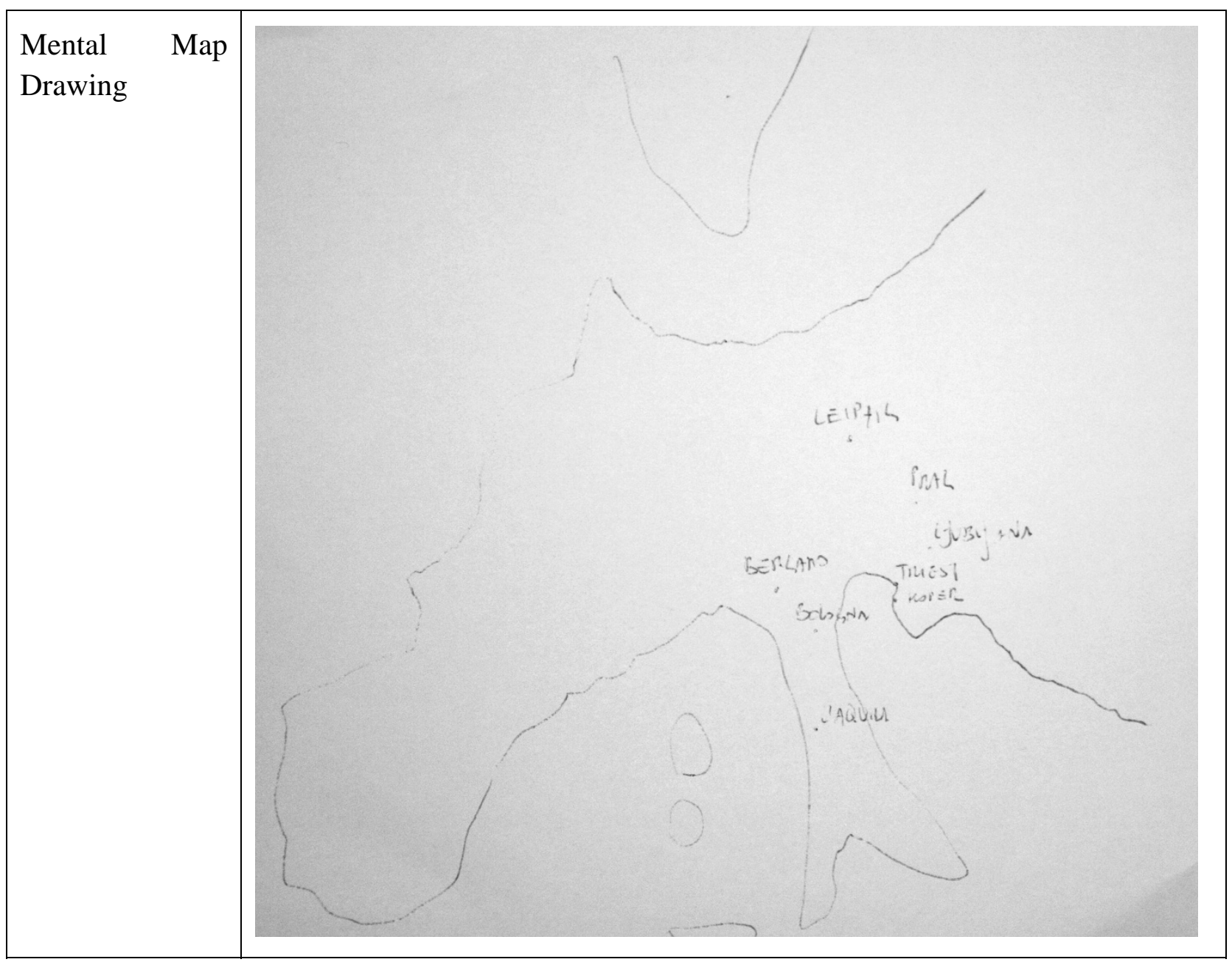

Interview No. 7

\section{Who is the interview partner?}

\begin{tabular}{|l|l|}
\hline Person & $\begin{array}{l}\text { Gunnar, German, in his late 20s, teaches American Studies at Leipzig } \\
\text { University, jobbed around earlier (cafeteria job in Montana, international } \\
\text { stem cell courier in Leipzig, language teacher in Madrid) and starting his own } \\
\text { PhD project, no children, engaged to his Spanish girlfriend }\end{array}$ \\
\hline Personal places & $\begin{array}{l}\text { Leipzig (DE), Valencia \& Caraixent (ES), Nottingham (UK), Montana (US), } \\
\text { Halle/Saale (DE), Ecuador, South Africa, Magdeburg \& Osterweddingen } \\
\text { (DE), Cologne (DE) }\end{array}$ \\
\hline $\begin{array}{l}\text { Tools } \\
\text { multilocality for }\end{array}$ & $\begin{array}{l}\text { Furnished room in Leipzig | Room of his girlfriend | Apartments of his } \\
\text { girlfriend's family in Carcaixent and Valencia | financial support by parents | } \\
\text { two bank account (Germany and Spain) | Skype | Facebook | low cost airlines } \\
\text { | two SIM cards (Spanish and German) | Lufthansa Silver Card (Frequent } \\
\text { Traveler Status) | speaks German, English and Spanish }\end{array}$ \\
\hline
\end{tabular}




\begin{tabular}{|l|l|}
\hline \multicolumn{2}{|c|}{ What was the interview situation like? } \\
\hline Where? & Gunnar's office room at Leipzig University, Leipzig (DE) \\
\hline When? & 19 March 2011, 13h45 \\
\hline Length? & $01: 19: 24$ \\
\hline Language? & German \\
\hline Atmosphere & $\begin{array}{l}\text { When I went to a friend's birthday party, I met Gunnar, who was also invited. } \\
\text { During dinner we spoke about our professional environments, and when he } \\
\text { heard about my PhD project, he became interested in it, and started talking } \\
\text { about his own life, which fits into my sample, too. So, I told him that we } \\
\text { should stick to beer and dancing this night, and speak in detail about his life } \\
\text { another day. He agreed. Comparing our agendas, we met some weeks later in } \\
\text { his office. We fixed the date for a Saturday afternoon, as he shared his office. } \\
\text { But on a Saturday all his colleagues were at home, while he regularly comes } \\
\text { to work for the silent atmosphere. I brought some cake and we prepared a } \\
\text { coffee before starting the interview. The interview itself was a pleasant } \\
\text { conversation. }\end{array}$ \\
\hline
\end{tabular}




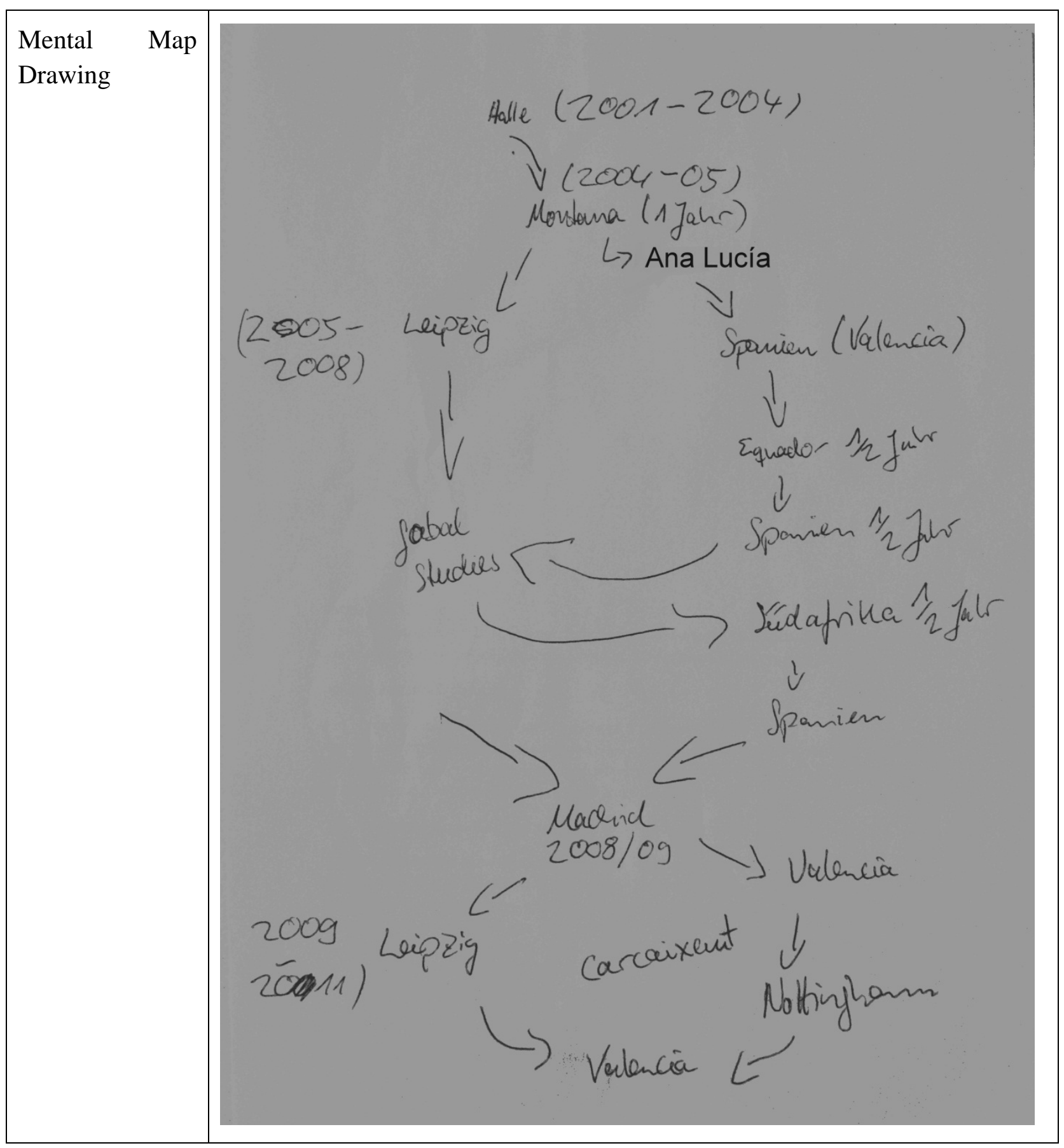

Interview No. 8

Who is the interview partner?

Person

Henriqua, Spanish, born in Germany, in her late 30s, works as post-doc researcher (regional development) in Vigo (ES) and Wageningen (NL), 1 daughter, married to her Dutch husband 


\begin{tabular}{|c|c|}
\hline Personal places & $\begin{array}{l}\text { Verin (ES), Gehrden/Hanover (DE), Zurich (CH), Wageningen (NL), Vigo } \\
\text { (ES), Groningen (NL), Apeldoorn (NL), Madrid (ES) }\end{array}$ \\
\hline $\begin{array}{l}\text { Tools for } \\
\text { multilocality }\end{array}$ & $\begin{array}{l}\text { Aunt's apartment in Vigo | Own apartment in Wageningen | House of } \\
\text { parents-in-law in Groningen | speaks Spanish, Gallician, Potuguese, Italian, } \\
\text { French, German, English and Dutch }\end{array}$ \\
\hline \multicolumn{2}{|r|}{ What was the interview situation like? } \\
\hline Where? & Café in the historic centre of Brussels (BE) \\
\hline When? & 31 March 2011, 18h30 \\
\hline Length? & $0: 56: 20$ \\
\hline Language? & English / (Explanations in Spanish \& German) \\
\hline Atmosphere & $\begin{array}{l}\text { I met Henriqua in } 2010 \text { in the context of a European research project, that we } \\
\text { both worked on. When she was introduced to me, I was wondering what her } \\
\text { story was, being Spanish and working in Wageningen in the Netherlands. } \\
\text { Wageningen is not the global city in which international migration tends to } \\
\text { flow. So, I wanted to know in detail. After we had chatted a while during a } \\
\text { project meeting and I had explained my PhD project, I asked her for an } \\
\text { interview. She was too busy that time. But she promised me to have time for } \\
\text { me during our next project meeting. So, three months later, we met again in } \\
\text { Brussels at the EU Commission. As these meetings are scheduled quite } \\
\text { densely, I always took my voice recorder in order to be ready to seize the } \\
\text { silent moment. One of the days in Brussels, we had a joint dinner with } \\
\text { colleagues in a downtown café. So, I convinced Henriqua to meet at this café } \\
\text { one hour before the dinner was about to start. The place was quite crowded, } \\
\text { and we chose a table in the corner, which was isolated from the project } \\
\text { partners' dining room. Henriqua is a quite busy person, working as much as } \\
\text { she can during such project meetings. So I had to get her mentally out of that } \\
\text { project's environment. We first ordered some drinks and started some small } \\
\text { talk on how our families were going. When I found Henriqua to be more } \\
\text { relxed, we started the interview. The interview situation was suboptimal, as } \\
\text { music was playing loud, noise of other people interfered, and finally the } \\
\text { waitress and arriving project partners passed by. Yet, Henriqua was a patient } \\
\text { and informative interview partner. At some points she criticized my style of } \\
\text { interviewing from her perspective as a researcher. }\end{array}$ \\
\hline
\end{tabular}




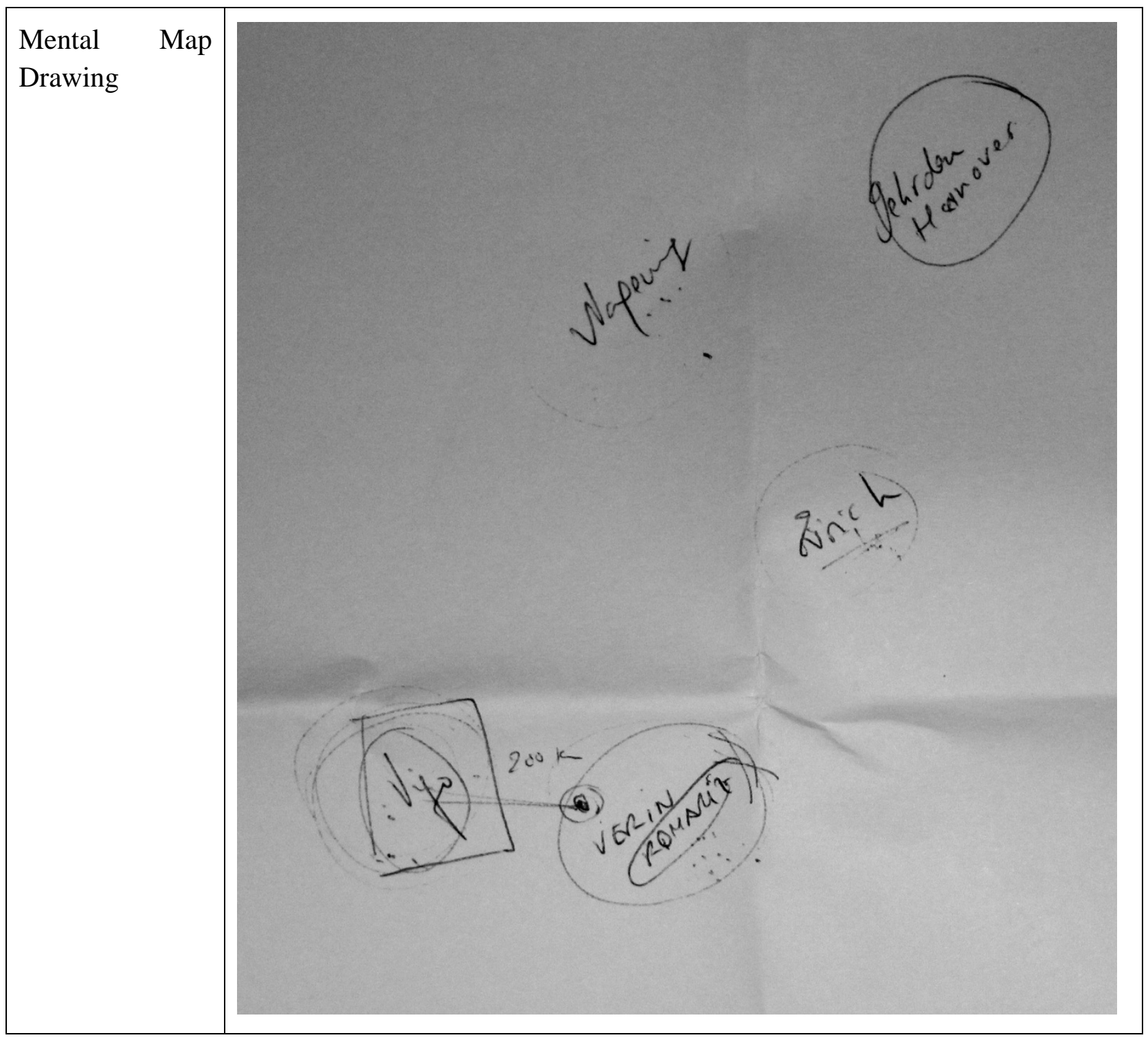

Interview No. 9

Who is the interview partner?

\begin{tabular}{|l|l|}
\hline Person & $\begin{array}{l}\text { Isabel, Spanish, in her 40s, works as an interpreter and translator for high- } \\
\text { rank politicians, based in Berlin and Barcelona, no children, single }\end{array}$ \\
\hline Personal places & Barcelona (ES), Berlin (DE), Ibiza (ES), Saarbrücken (DE) \\
\hline $\begin{array}{l}\text { Tools } \\
\text { multilocality for }\end{array}$ & $\begin{array}{l}\text { Own apartment in Berlin | own apartment in Barcelona | house of parents in } \\
\text { Ibiza | airports | mobile phones, credit card, passport | small laptop \& Wifi } \\
\text { access | charging cables | swim suit | fits-to-all clothes | hotel laundry services } \\
\text { | cafés as work space | two SIM cards (Spanish and German) | speaks Catalan, } \\
\text { Spanish, Italian, German and English }\end{array}$ \\
\hline
\end{tabular}




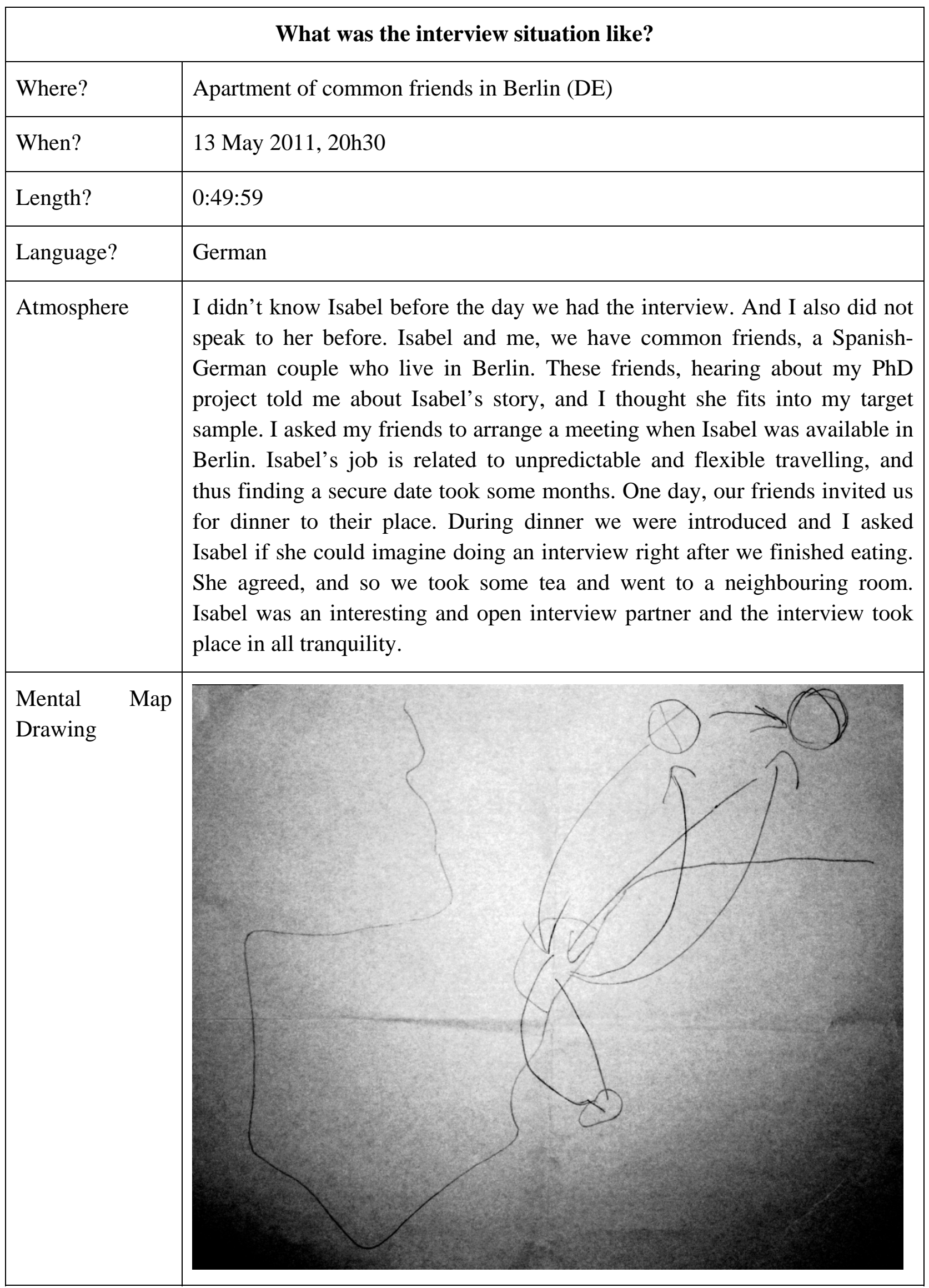




\begin{tabular}{|c|c|}
\hline & Interview No. 10 \\
\hline & Who is the interview partner? \\
\hline Person & $\begin{array}{l}\text { Juliana, Lithuanian, in her 30s, works as a junior professor in the field of } \\
\text { ethnology in a German university, lives in Berlin, two children, married to a } \\
\text { German husband working in Sweden }\end{array}$ \\
\hline Personal places & Berlin (DE), Mainz (DE), Vilnius (LT), Stockholm (SE), Copenhagen (DK) \\
\hline $\begin{array}{l}\text { Tools for } \\
\text { multilocality }\end{array}$ & $\begin{array}{l}\text { Own apartment in Berlin | own apartment in Mainz | parents-in-law in Berlin } \\
\text { | Bahncard } 50 \text { | trains | parents and their house in Vilnius | speaks Lithuanian, } \\
\text { Danish, Russian, German, Swedish, Norwegian, Latvian and English }\end{array}$ \\
\hline & What was the interview situation like? \\
\hline Where? & At a café in Berlin's Wilmersdorf neighbourhood (DE) \\
\hline When? & 16 May 2011, $14 \mathrm{~h} 00$ \\
\hline Length? & 01:04:17 \\
\hline Language? & German \\
\hline Atmosphere & $\begin{array}{l}\text { I met Juliana during a presentation in Leipzig, where she spoke about her } \\
\text { latest research findings on Lithuanian consumption habits in the post-Soviet } \\
\text { era. A colleague of mine, who was affiliated with the Humboldt University in } \\
\text { Berlin, where Juliana also worked before as a PhD student, told me a bit } \\
\text { more about her and the mobile life she lives. So, I decided to use her Leipzig } \\
\text { presentation as an ice-breaker and contacted her through email. I explained } \\
\text { my concern and asked her for an interview. Juliana agreed to have an } \\
\text { interview in Berlin, but as she spends most of the week at her employer's } \\
\text { location, we needed to plan the date well in advance. We finally met some } \\
\text { weeks after our email conversation. Juliana wanted to meet in one of her } \\
\text { neighbourhood's cafés, where she was not far from her home and from the } \\
\text { kindergarten of her children. We met at lunch time, but she told me right } \\
\text { away that she only had one hour before she would have to leave to pick up } \\
\text { her kids from the day care. So, a bit under time pressure, we had a smooth } \\
\text { conversation in the café, in which it turned out that she also was scientifically } \\
\text { interested in my PhD research topic. She also knew a lot about it, and } \\
\text { sometimes I had the impression she answered more from her perspective as a } \\
\text { researcher instead of a private person. The atmosphere in the café was silent; } \\
\text { we had a table in the corner, where music was shallow. I invited her for some } \\
\text { tea and cake. }\end{array}$ \\
\hline
\end{tabular}




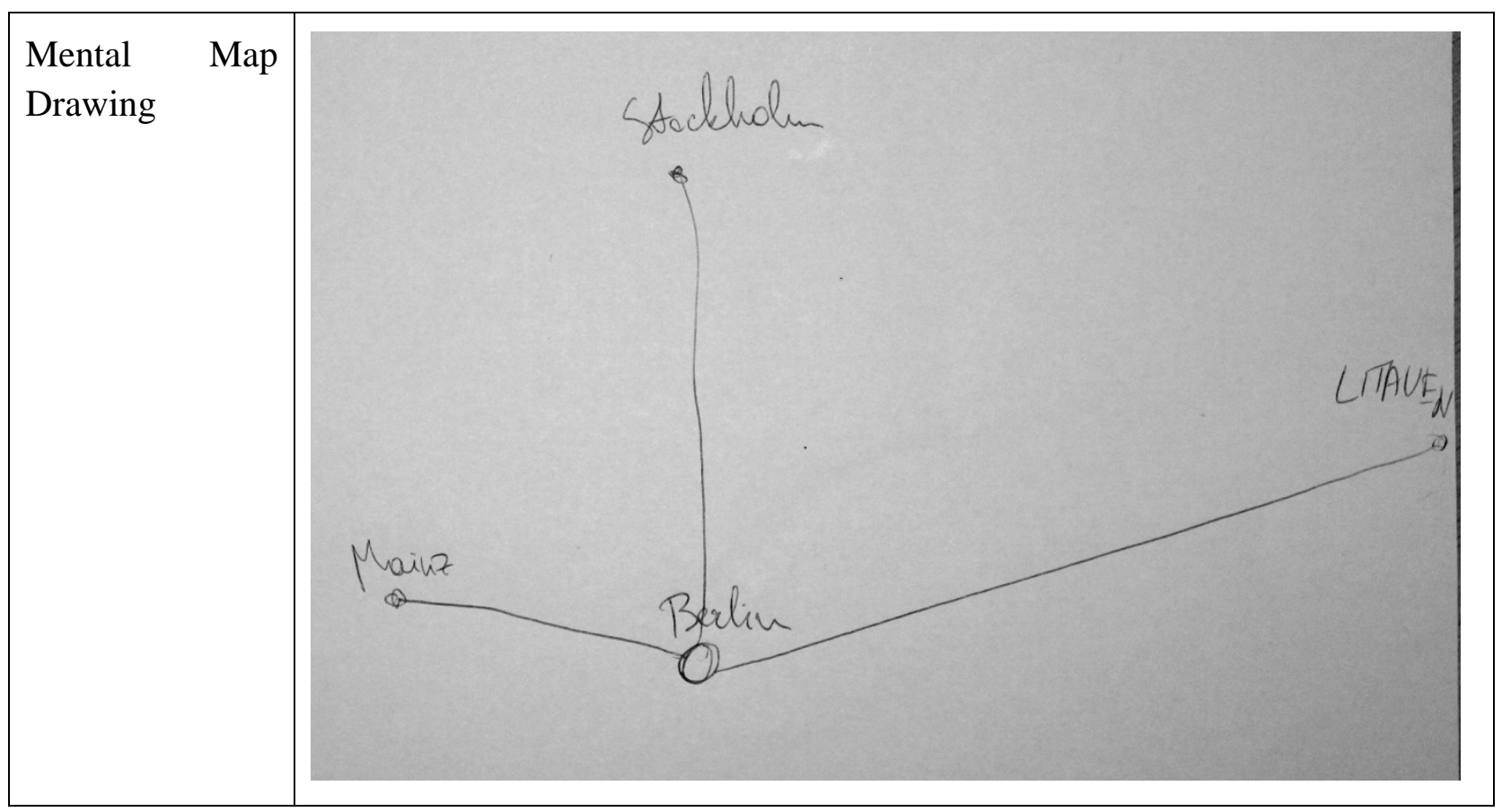

\begin{tabular}{|c|c|}
\hline & Interview No. 11 \\
\hline & Who is the interview partner? \\
\hline Person & $\begin{array}{l}\text { Kate, US American, in her 60s, artistic director for European dubbing of } \\
\text { Hollywood movies \& formerly professor for linguistics at German university, } \\
\text { circulates between hotels in Berlin, Barcelona, Rome and Paris, no children, } \\
\text { divorced from German husband }\end{array}$ \\
\hline Personal places & $\begin{array}{l}\text { Berlin (DE), Saarbrücken (DE), Barcelona (ES), Madrid (ES), Rome (IT), } \\
\text { New York (US), Philadelphia (US), Paris (FR), London (UK), Buenos Aires } \\
\text { (ARG), Trier (DE), Chicago (US), Vienna (AT), Munich (DE), Cologne } \\
\text { (DE) }\end{array}$ \\
\hline $\begin{array}{l}\text { Tools for } \\
\text { multilocality }\end{array}$ & $\begin{array}{l}\text { Computer | Skype | telephone | emails | } 5 \text { SIM cards (USA, France, Italy, } \\
\text { Spain, Germany) | Bahncard } 50 \text { | suitcases deposited in different hotels | } \\
\text { speaks English, German, French, Spanish, Italian and Catalan }\end{array}$ \\
\hline & What was the interview situation like? \\
\hline Where? & $\begin{array}{l}\text { In her penthouse suite with roof terrace in a five star hotel located on } \\
\text { Kuh'damm, West-Berlin's famous avenue (DE) }\end{array}$ \\
\hline When? & 16 May 2011, $18 \mathrm{~h} 00$ \\
\hline Length? & $01: 17: 18$ \\
\hline
\end{tabular}




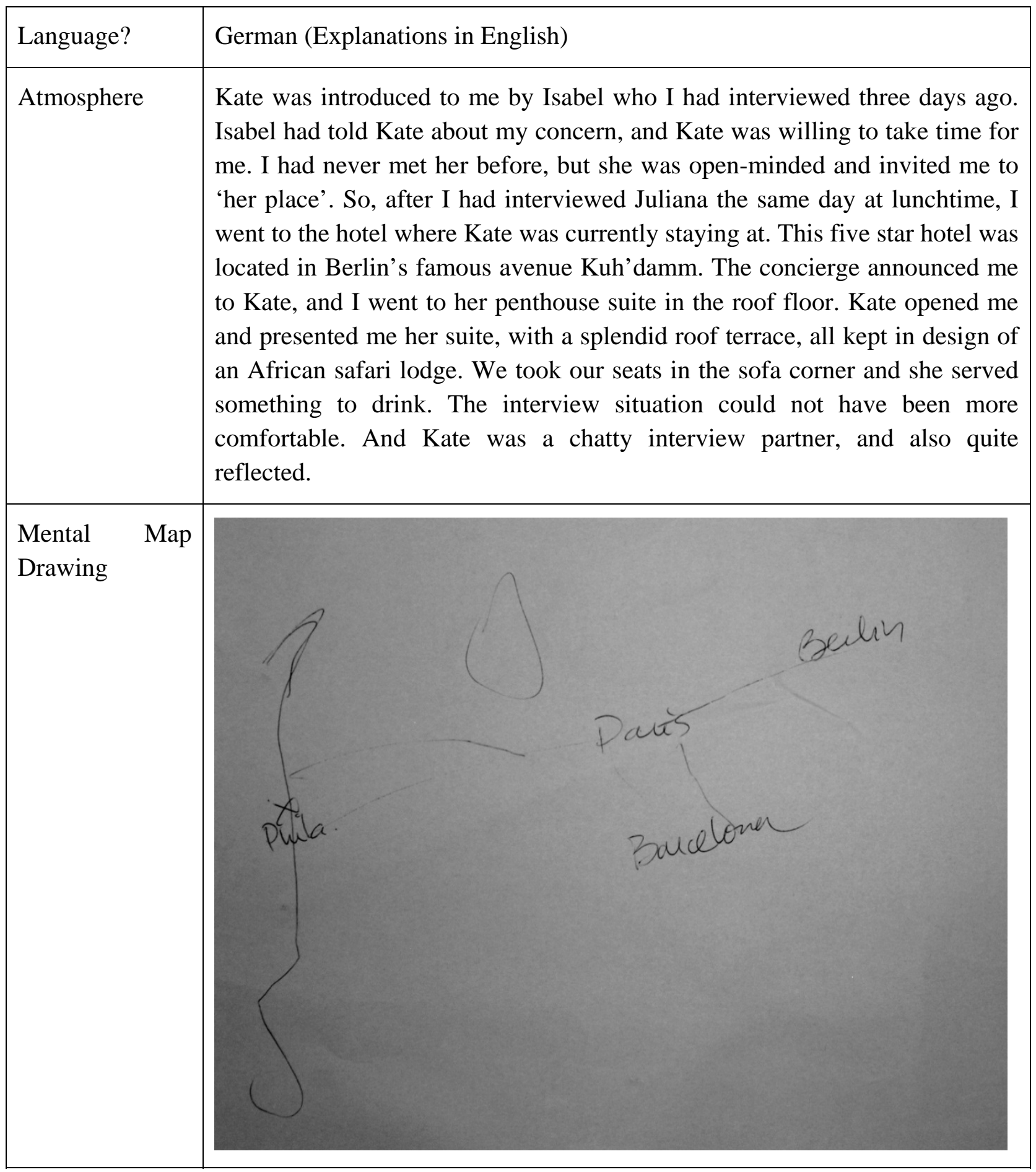

Interview No. 12

\section{Who is the interview partner?}

Person

Liam, English, in his 60s, senior researcher in the field of regional development, living in Aberdeen and working in Stockholm, three children, married to a Scottish wife 


\begin{tabular}{|c|c|}
\hline Personal places & $\begin{array}{l}\text { Aberdeen/Aberdeenshire (Scotland), London (England), Aberystwyth } \\
\text { (Wales), Cranleigh/Hampshire (England), Stockholm (SE), Luton (England), } \\
\text { Glasgow (Scotland); Edinburgh (Scotland) }\end{array}$ \\
\hline $\begin{array}{l}\text { Tools for } \\
\text { multilocality }\end{array}$ & $\begin{array}{l}\text { Access to British Airways Executive Lounges | Own house in Aberdeenshire } \\
\text { with office room | Company apartment in Stockholm | Computer | mobile } \\
\text { phone | speaks English, Scottish and Swedish }\end{array}$ \\
\hline \multicolumn{2}{|r|}{ What was the interview situation like? } \\
\hline Where? & In interviewer's hotel room in Aberystwyth/Wales (UK) \\
\hline When? & 29 June 2011, 17h45 \\
\hline Length? & 00:59:17 \\
\hline Language? & English \\
\hline Atmosphere & $\begin{array}{l}\text { I met Liam in the frame of a European research project, in which we } \\
\text { collaborated. He was part of the Swedish team, and during several meetings I } \\
\text { learnt more about his story and how he had come to Sweden. When he told } \\
\text { me he was still living in the UK, I thought he is also a person who fits into } \\
\text { my sampling strategy. During one of our project workshops, I explained him } \\
\text { my PhD project and asked him for an interview. He agreed to do the } \\
\text { interview during the current meeting and we decided to speak in one of the } \\
\text { work breaks, right before dinner. We all stayed in the same hotel in the small } \\
\text { university town Aberystwyth in Wales. So, it was an easy thing to organize } \\
\text { the interview. Liam just showed up in my room, and as we just had seen } \\
\text { 17h00 passing by, and I thought he is an Englishman, I offered him tea. But } \\
\text { he preferred a glass of water. Then we opened the window with direct view } \\
\text { over the beach, and we took our seats in the sofa corner of my hotel room. } \\
\text { Liam at first seemed a bit astonished that I found his story so interesting, but } \\
\text { while time went on, he became quite chatty. In a nice atmosphere we had an } \\
\text { interesting conversation without interruptions. }\end{array}$ \\
\hline
\end{tabular}




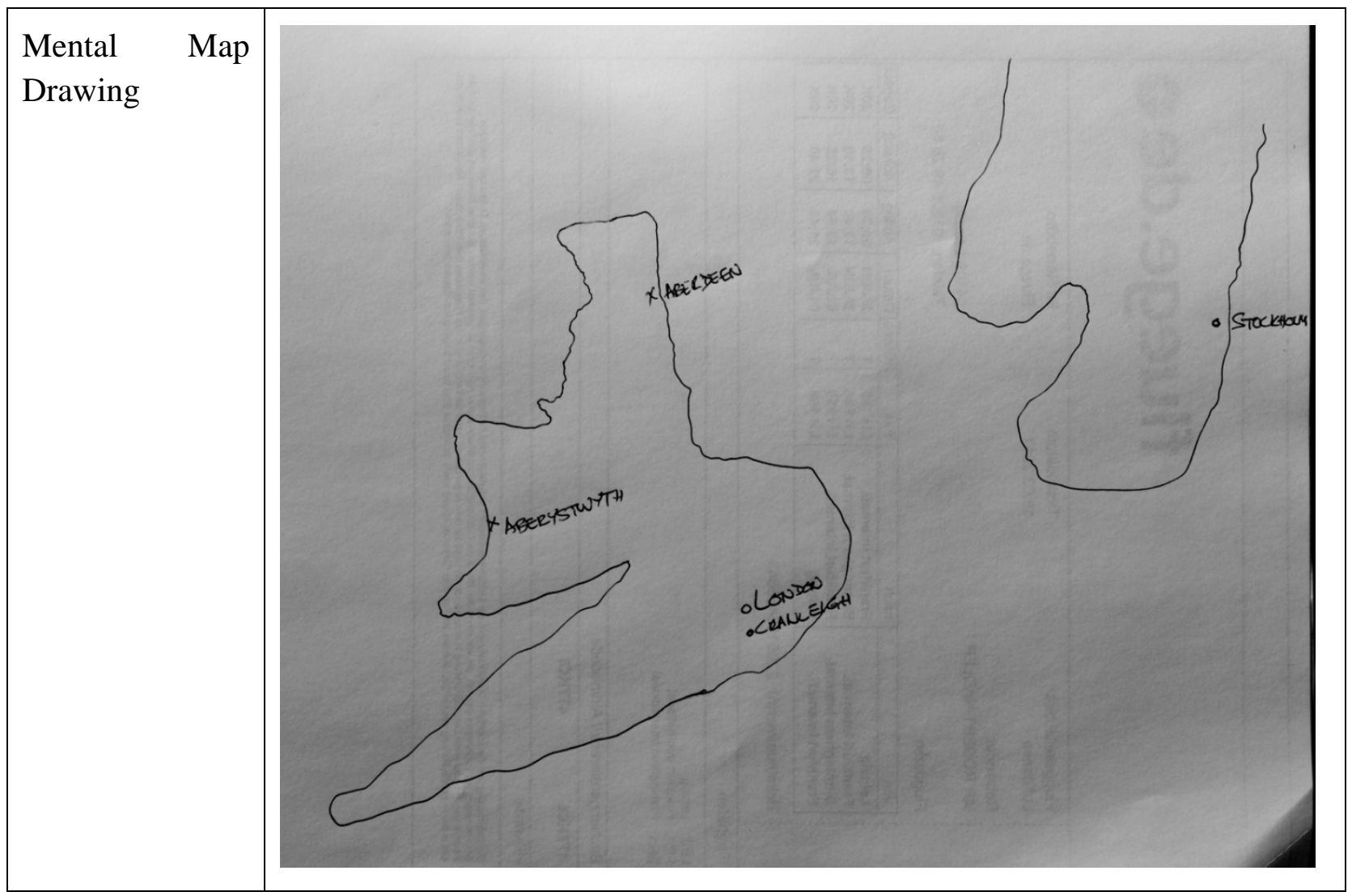

Interview No. 13

Who is the interview partner?

\begin{tabular}{|l|l|}
\hline Person & $\begin{array}{l}\text { Marta, Lithuanian, in her 30s, freelance researcher in the field of regional } \\
\text { development, living in Ljubljana and working in Alytus, no children, } \\
\text { Slovenian partner }\end{array}$ \\
\hline Personal places & $\begin{array}{l}\text { Alytus (LT), Ljubljana (SI), Finland, Kaunas (LT), Vilnius (LT), United } \\
\text { Kingdom }\end{array}$ \\
\hline $\begin{array}{l}\text { Tools } \\
\text { multilocality for }\end{array}$ & $\begin{array}{l}\text { Sister's place in Kaunas | airport Kaunas | airplanes | her sister's clothes in } \\
\text { Lithuania | laptop | speaks Lithuanian, Russian, English and Slovenian }\end{array}$ \\
\hline \multicolumn{1}{|c|}{ What was the interview situation like? } \\
\hline Where? & In interviewer's hotel room in Aberystwyth/Wales (UK) \\
\hline When? & 29 June 2011, 23h30 \\
\hline Length? & $00: 43: 47$ \\
\hline
\end{tabular}




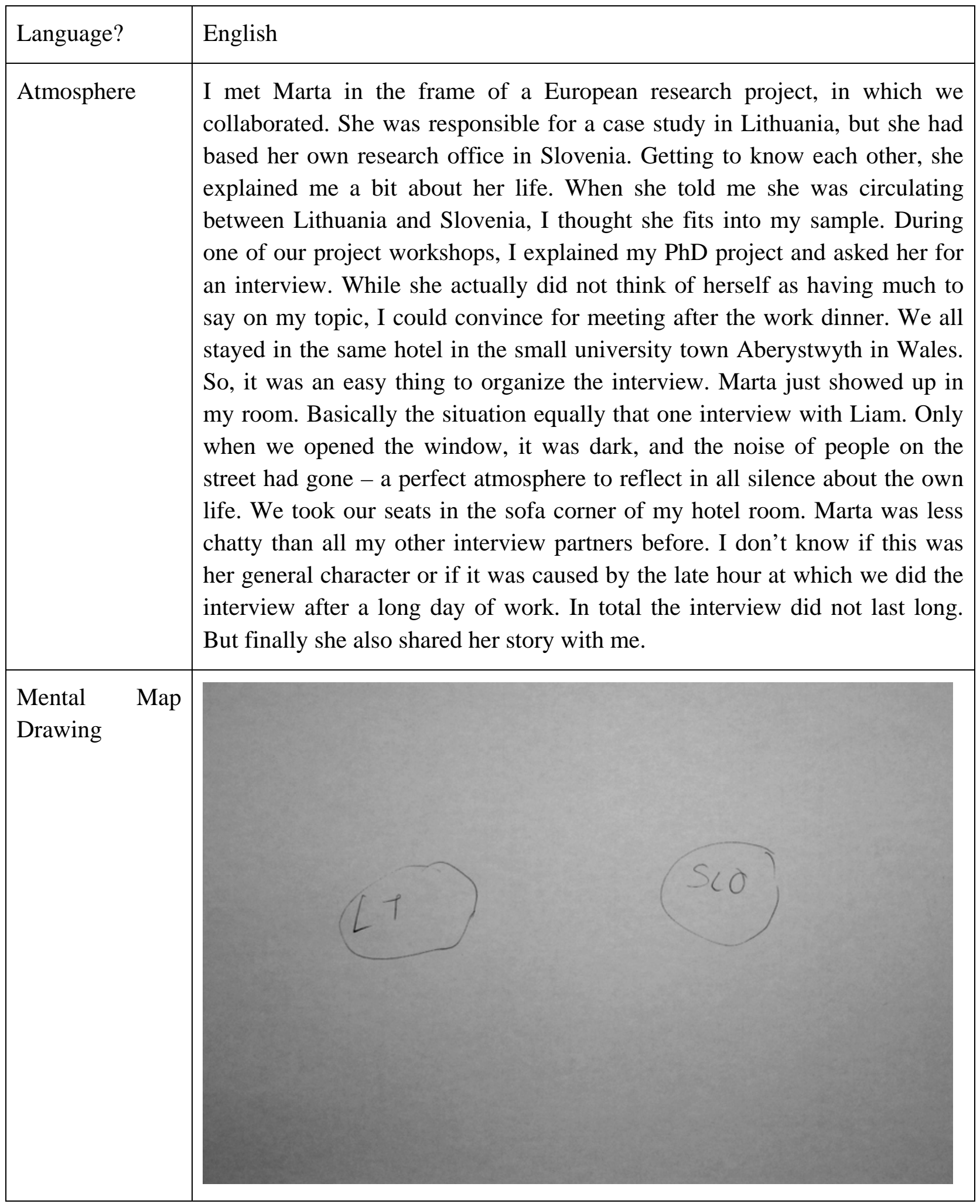

Interview No. 14

Who is the interview partner? 


\begin{tabular}{|c|c|}
\hline Person & $\begin{array}{l}\text { Nikolina, Bulgarian-American, in her 20s, student research assistant in the } \\
\text { field of geography, living in Leipzig, studying in Weimar and working on } \\
\text { Bulgaria, no children, single }\end{array}$ \\
\hline Personal places & $\begin{array}{l}\text { Sofia (BG), Ann Arbour (US), Blacksburg (US), Weimar (DE), Leipzig } \\
\text { (DE), Toledo (US), Holland (US), Berlin (DE), Magdeburg (DE), } \\
\text { Manchester (UK) }\end{array}$ \\
\hline $\begin{array}{l}\text { Tools for } \\
\text { multilocality }\end{array}$ & $\begin{array}{l}\text { Double citizenship (US \& Bulgarian) | apartment in Leipzig | grandma’s } \\
\text { house in Sofia | parents' house in Blacksburg | friends' places in Berlin, } \\
\text { Manchester, Ann Arbor | health insurance in EU and US | speaks Bulgarian, } \\
\text { English, Russian, Spanish, Macedonian, Serbian, Croatian, Bosnian and } \\
\text { German }\end{array}$ \\
\hline \multicolumn{2}{|r|}{ What was the interview situation like? } \\
\hline Where? & In interviewer's office room in Leipzig (DE) \\
\hline When? & 29 July 2011, $12 \mathrm{~h} 00$ \\
\hline Length? & 01:52:45 \\
\hline Language? & German \& English \\
\hline Atmosphere & $\begin{array}{l}\text { I met Nikolina when she started working in my institute. When Nikolina was } \\
\text { introduced to her colleagues, I did not immediately understand her } \\
\text { geographical pattern. And thus, I became interested in knowing more about } \\
\text { her life trajectory. While some busy weeks passed since this first curiosity, } \\
\text { we met on a summer evening for a barbecue in the garden of a Czech } \\
\text { colleague who was a visiting scholar at our Leipzig institute. There, I chatted } \\
\text { with Nikolina about scientific interests and their relation to our lives, and } \\
\text { while I was telling about my PhD, I asked if she would be available for an } \\
\text { interview. She said that she would be only be in Leipzig the next day, then } \\
\text { she would leave for some time to Weimar, then to Bulgaria. So, we decided } \\
\text { that she would come to see me in my office the next morning. The morning } \\
\text { finally turned out to be lunch time as we stayed for quite a while at our } \\
\text { colleague’s place during the night before. But as it was a Friday, there } \\
\text { weren't many people to join us for lunch. We simply order some pizza to the } \\
\text { office and decided to do the interview during the lunch break. }\end{array}$ \\
\hline
\end{tabular}




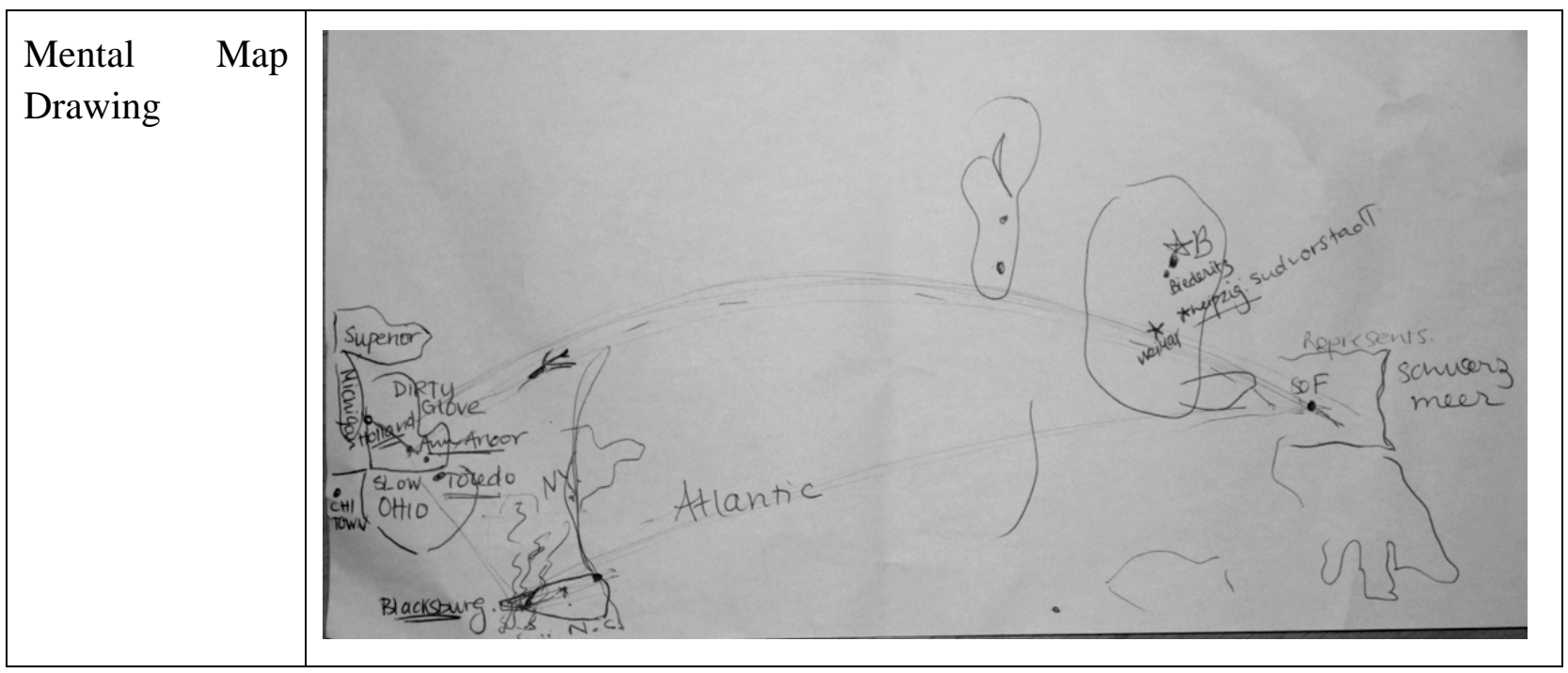

\begin{tabular}{|c|c|}
\hline & Interview No. 15 \\
\hline & Who is the interview partner? \\
\hline Person & $\begin{array}{l}\text { Oskar, Swiss, in his late 50s, freelance cultural mediator, living in Linz, } \\
\text { Berlin and Zurich, divorced from Swiss wife, one daughter, new Austrian } \\
\text { girlfriend (curator) }\end{array}$ \\
\hline Personal places & $\begin{array}{l}\text { Zurich }(\mathrm{CH}) \text {, Basel }(\mathrm{CH}) \text {, Berlin (DE), Linz (AT), Hamburg (DE), Bremen } \\
(\mathrm{DE}) \text {, Paris (FR), Vienna (AT), Salzburg (AT), Kassel (DE), Neuenburg } \\
(\mathrm{CH}) \text {, Frauenfeld (CH) }\end{array}$ \\
\hline $\begin{array}{l}\text { Tools for } \\
\text { multilocality }\end{array}$ & $\begin{array}{l}\text { Senator Card of Star Alliance | Bahncard } 50 \text { | Carsharing | special tariff ticket } \\
\text { for urban public transport | } 3 \text { apartments in Berlin, Zurich and Linz | clothes, } \\
\text { shoes and sanitary products in each of the places | airplanes and night trains | } \\
\text { laptop | mobile phone | Skype | W-LAN | his secretary and back office | } \\
\text { speaks German, English and French }\end{array}$ \\
\hline & What was the interview situation like? \\
\hline Where? & $\begin{array}{l}\text { In the temporary office, rented by his current project, in an old-industrial co- } \\
\text { working space in Berlin-Kreuzberg (DE) }\end{array}$ \\
\hline When? & 10 August 2011, $12 \mathrm{~h} 00$ \\
\hline Length? & $00: 57: 15$ \\
\hline Language? & German \\
\hline Atmosphere & A colleague of mine, knowing about my PhD project, told me that he had \\
\hline
\end{tabular}




\begin{tabular}{|c|c|}
\hline & $\begin{array}{l}\text { collaborated with Oskar in another project. He explained me that Oskar } \\
\text { would perfectly fit into my sample. I replied that then I am interested in } \\
\text { meeting Oskar. My colleague informed Oskar about my concern. Even } \\
\text { though we did not know us, Oskar let his details to my colleague, who } \\
\text { forwarded them to me. So, some days after this idea of meeting Oskar had } \\
\text { developed, I wrote Oskar an email, to know when and where he would } \\
\text { available. He briefly explained his schedule, and we fixed a date in Berlin } \\
\text { some weeks after our first email conversation. As Oskar is already a senior } \\
\text { level worker, he is quite busy. He reserved exactly one hour for my } \\
\text { interview, and he invited me to his office in a former factory in Berlin's } \\
\text { upcoming Kreuzberg neighbourhood. The office looked like used by an } \\
\text { architecture firm. It was one huge room, with a large meeting table in the } \\
\text { middle, and some individual desks distributed across the room. When I } \\
\text { arrived we introduced, and took our seats on the one corner of the large } \\
\text { conference table. As Oskar did not have much time, we immediately started } \\
\text { the interview without much chatting. In the end Oskar seemed to like the } \\
\text { reflection about his own life, which I initiated through my questions. So, } \\
\text { finally we even took more than one hour for the interview. }\end{array}$ \\
\hline $\begin{array}{l}\text { Mental } \quad \text { Map } \\
\text { Drawing }\end{array}$ & $4952 \quad+22-76 \quad 8$ \\
\hline
\end{tabular}

Interview No. 16

\section{Who is the interview partner?}

Person

Pia, German, in her 30s, head of architecture office in Copenhagen and professor for architecture in Berlin, writing a PhD thesis at Royal Danish 


\begin{tabular}{|c|c|}
\hline & Academy of Fine Arts, single, no children \\
\hline Personal places & $\begin{array}{l}\text { Copenhagen (DK), Berlin (DE), Rotterdam (NL), Delft (NL), Riga (LV), } \\
\text { Tianjin and other cities (China), Munich/Western Germany (DE), USA }\end{array}$ \\
\hline $\begin{array}{l}\text { Tools for } \\
\text { multilocality }\end{array}$ & $\begin{array}{l}2 \text { SIM cards (DK, DE) | Skype | Emails | Airports | Bus connections between } \\
\text { Copenhagen and Berlin | Hostels in Copenhagen | small laptop | Bahncard | } \\
\text { sanitary bag in Copenhagen office | speaks German, Russian, English, Danish } \\
\text { and Dutch }\end{array}$ \\
\hline \multicolumn{2}{|r|}{ What was the interview situation like? } \\
\hline Where? & In interviewee's office room at Technical University in Berlin (DE) \\
\hline When? & 10 August 2011, $14 \mathrm{~h} 00$ \\
\hline Length? & $00: 50: 13$ \\
\hline Language? & German \\
\hline Atmosphere & $\begin{array}{l}\text { I did not know Pia in advance. It was a colleague who had worked with her in } \\
\text { another project, who advised me to speak to Pia. He informed Pia about my } \\
\text { concern, and I then wrote her an email. She was very open-minded and } \\
\text { invited me to her office room some days after. Her university office room is } \\
\text { located in a tower building in Berlin-Charlottenburg. From her office room } \\
\text { she had an amazing view over the city and could look to the lakes at the } \\
\text { outskirts of Berlin. Her office actually is a shared office, but as it was a hot } \\
\text { summer day, her colleagues had left. So, the office was quiet and there were } \\
\text { no interruptions. Pia organized some coffee and apples. We opened the large } \\
\text { windows and looked at the cars passing by. Up in her office the traffic noise } \\
\text { seemed distant. We talked in a relaxed and friendly atmosphere. }\end{array}$ \\
\hline
\end{tabular}




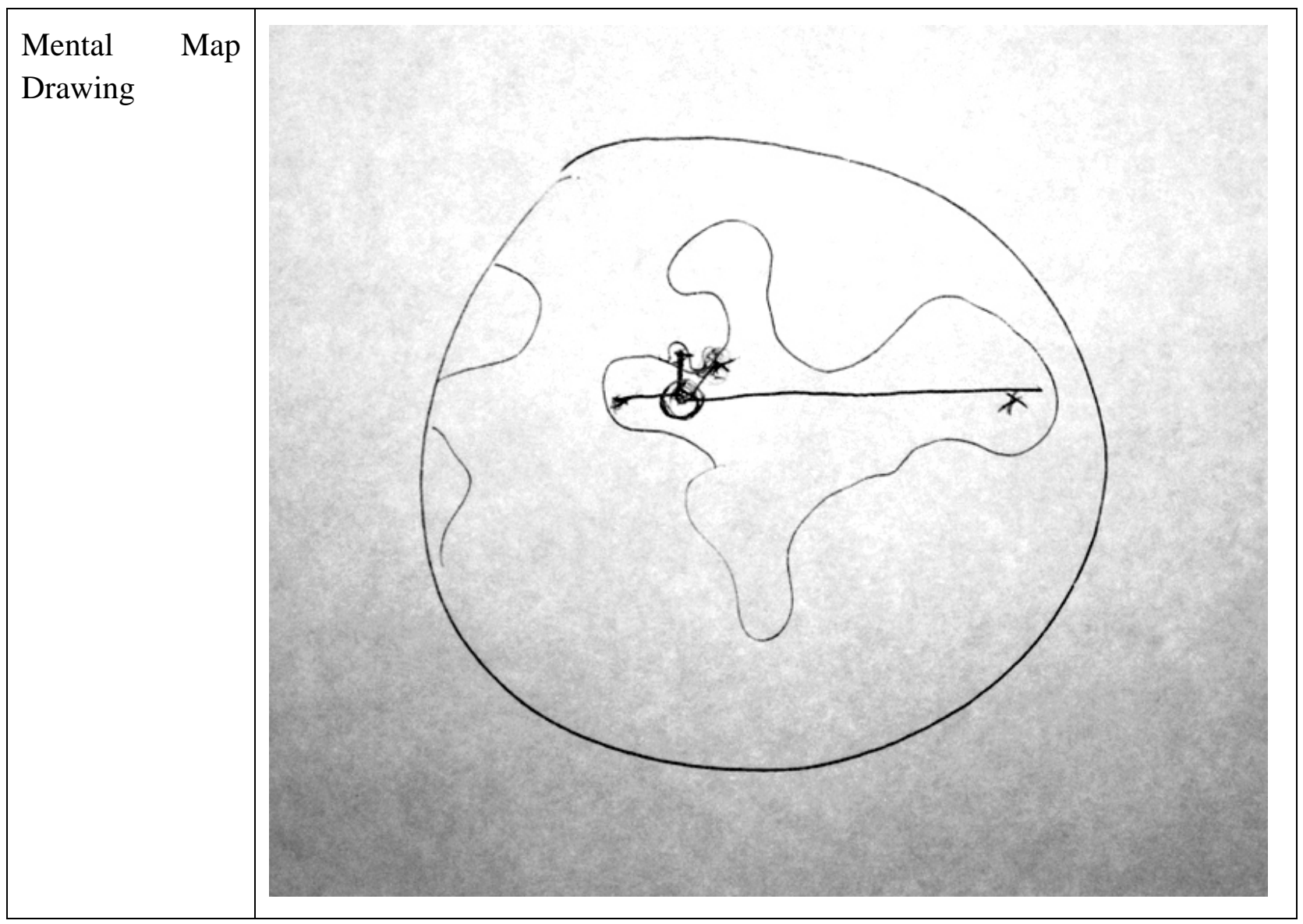

Interview No. 17

\begin{tabular}{|l|l|}
\hline \multicolumn{2}{|c|}{ Who is the interview partner? } \\
\hline Person & $\begin{array}{l}\text { Qamar, Chechen-German, in his 40s, freelance artist, living in Leipzig, but } \\
\text { doing projects in Grozny and Los Angeles, widower of a Dagestanian wife, } \\
\text { divorced from German wife (mother of first son), then separated from a } \\
\text { Russian resettler girlfriend of German ethnicity (mother of second son) }\end{array}$ \\
\hline Personal places & $\begin{array}{l}\text { Leipzig (DE), Grozny (Chechnya), Los Angeles (US), Moscow (RU), St. } \\
\text { Petersburg (RU), Paris (FR), Prague (CZ), Belgium }\end{array}$ \\
\hline $\begin{array}{l}\text { Tools } \\
\text { multilocality for }\end{array}$ & $\begin{array}{l}\text { Own house in Leipzig | brother's house in Dagestan | painting sets in Leipzig } \\
\text { and in Chechnya | low cost airlines | Russian and German SIM cards } \\
\text { friends' places to stay at | speaks Chechen, German, English and Russian }\end{array}$ \\
\hline \multicolumn{1}{|c|}{ What was the interview situation like? } \\
\hline Where? & $\begin{array}{l}\text { In the interviewee's atelier in an old apartment block in an Eastern } \\
\text { neighbourhood of Leipzig (DE) }\end{array}$ \\
\hline
\end{tabular}




\begin{tabular}{|l|l|}
\hline When? & 29 August 2011, 11h00 \\
\hline Length? & $01: 04: 23$ \\
\hline Language? & German \\
\hline Atmosphere & $\begin{array}{l}\text { On my way between my apartment in Leipzig's city centre and my office } \\
\text { room at the outskirts of Leipzig, I passed Qamar's atelier. The atelier is a } \\
\text { former shop floor in a non-renovated Wilhelminian apartment block. It is } \\
\text { located in a rather deprived neighbourhood, and I always wondered why an } \\
\text { artist locates in this area, while on the opposite side of the city the creative } \\
\text { industries flourished and a vivid cultural scene was about to develop. One } \\
\text { day I stopped by and decided to ask. The atelier was closed, but I found an } \\
\text { internet address. Browsing the website, I found that Qamar was a Chechen, } \\
\text { who worked and lived in Leipzig, and who frequently flies to the USA, to } \\
\text { meet a bunch of creative people. So, I decided to interview him. Passing his } \\
\text { atelier another day, I found him working on a large painting. I introduced } \\
\text { myself and my concern, and Qamar agreed for an interview. Some days later } \\
\text { I arrived at his atelier in the morning hours. We took place on a large table in } \\
\text { the atelier, he served some coffee, and then he continued painting throughout } \\
\text { the whole interview. The interview was only interrupted by an incoming } \\
\text { phone call. In general, the interview situation was relaxed and we could talk } \\
\text { without time pressure. }\end{array}$ \\
\hline
\end{tabular}




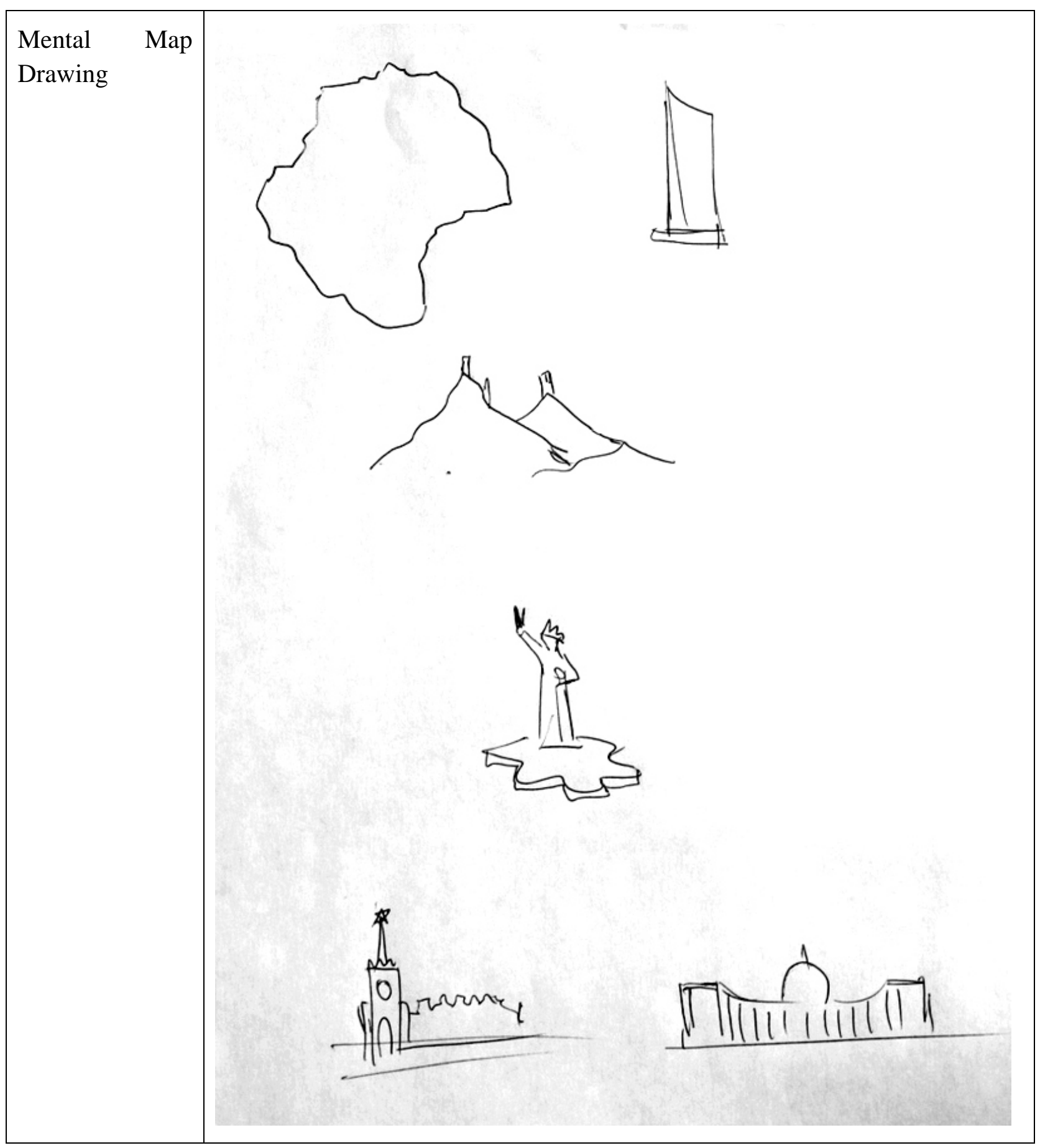

Interview No. 18

Who is the interview partner?

\begin{tabular}{|l|l|}
\hline Person & $\begin{array}{l}\text { Ronja, Danish, in her 30s, freelance journalist, living between Berlin and } \\
\text { Copenhagen, German husband (architect; Stephan), one daughter }\end{array}$ \\
\hline Personal places & Aarhus (DK), Copenhagen (DK), Berlin (DE), Rotterdam (NL),
\end{tabular}




\begin{tabular}{|c|c|}
\hline & Klingenmünster/Palatinate (DE) \\
\hline $\begin{array}{l}\text { Tools for } \\
\text { multilocality }\end{array}$ & $\begin{array}{l}\text { SIM cards (DK and DE) | Bahncard } 25 \mid \text { low cost airlines | laptop | Email | } \\
\text { Skype | speaks German, English and Danish }\end{array}$ \\
\hline \multicolumn{2}{|r|}{ What was the interview situation like? } \\
\hline Where? & In recently opened Café Leuchtstoff in Berlin-Friedrichshain (DE) \\
\hline When? & 7 September 2011, 14h00 \\
\hline Length? & 00:58:06 \\
\hline Language? & German \\
\hline Atmosphere & $\begin{array}{l}\text { I was advised to talk to Ronja by Pia. Pia gave me Ronja's details after I had } \\
\text { interviewed her. I didn't know Ronja before. I contacted Ronja via email } \\
\text { first, the we spoke on the phone. Ronja agreed to do an interview, and she } \\
\text { also suggested me to talk to her husband, too. Ronja said that her } \\
\text { multilocality has a lot to do with her husband. So, I thought it would be a } \\
\text { good option to interview a couple and to look at similarities and differences } \\
\text { between the two partners. I wanted to interview both of them on the same } \\
\text { day. It was not so easy to find a good date. Ronja gave birth to her daughter } \\
\text { some months ago, so we would need to find a date when her husband was in } \\
\text { town, too, so that I could interview both one after the other while they just } \\
\text { handed over the buggy. Furthermore, they did not want to leave their } \\
\text { neighbourhood. We finally agreed to meet at lunch time in Café Leuchtstoff, } \\
\text { a recently opened young and hip bar in Friedrichshain. We met there at lunch } \\
\text { time. I first interviewed her, then her husband. The bar was small, only one } \\
\text { room, and it was crowded with people. In addition a DJ already played loud } \\
\text { music for the lunch time hang around. This made the interview situation a bit } \\
\text { tensed, because acoustically the situation was difficult. Also we were not } \\
\text { really separated from other guests. Thus, the interview situation did not really } \\
\text { fit into that environment, or vice versa. }\end{array}$ \\
\hline
\end{tabular}




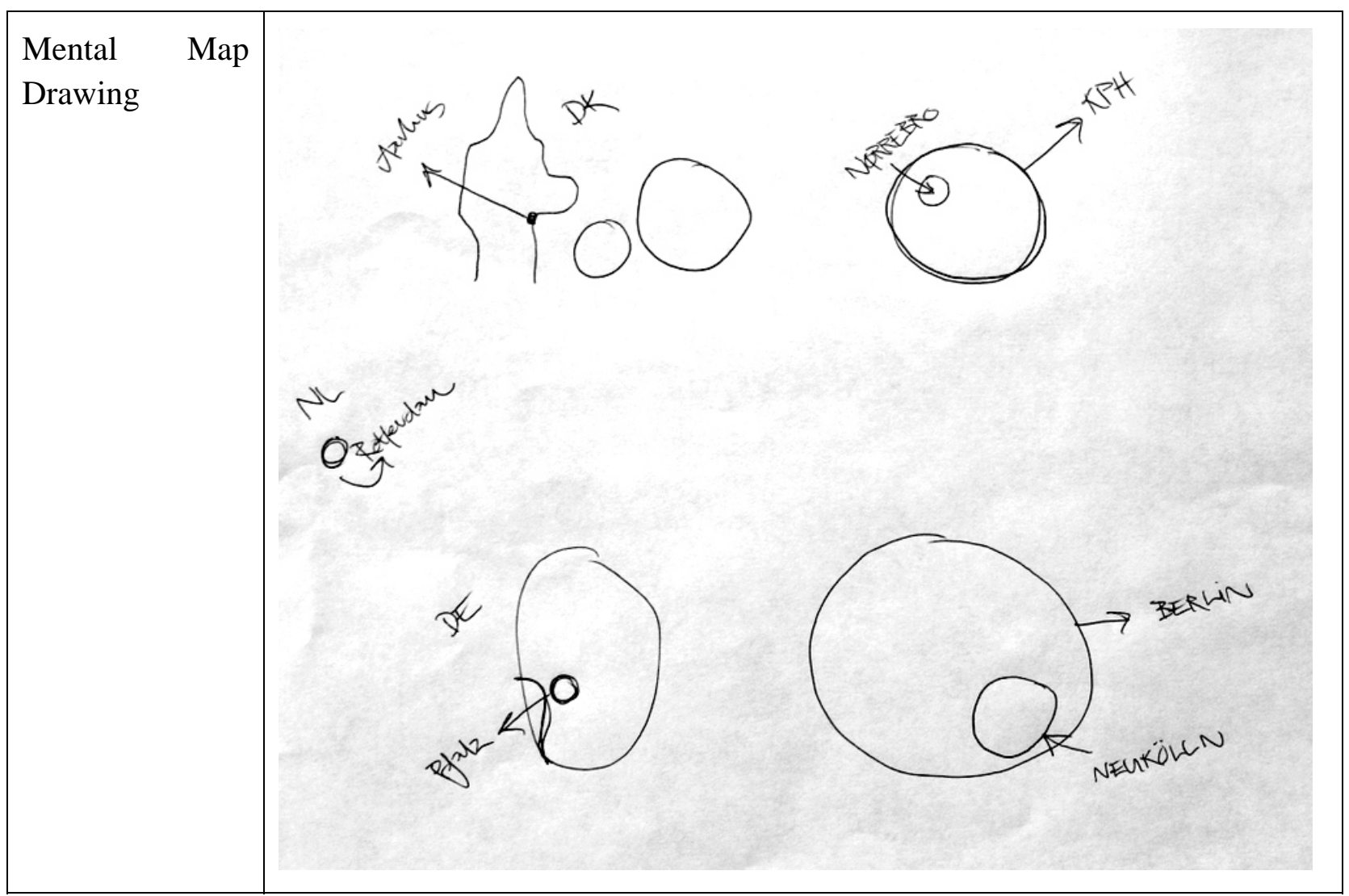

Interview No. 19

Who is the interview partner?

\begin{tabular}{l|l} 
Person & Stephan, German, in his 30s, architect, currently working as assistant
\end{tabular} professor in university, married to a Danish wife (journalist; Ronja), one daughter

Personal places $\quad$ Berlin (DE), Copenhagen (DK), Brunswick (DE), Aarhus (DK), Rotterdam (NL), Klingenmünster/Palatinate (DE), London (UK), Hamburg (DE)

Tools for Bahncard 100 | low cost airlines | shared apartment rooms | two mobile multilocality phones (DK and DE) $\mid$ hand luggage sized case $\mid$ frequent traveler lounges in German railway stations | BahnComfort services | speaks German, English, Dutch, Danish, a little French, Latin and Spanish

\section{What was the interview situation like?}

Where?

In recently opened Café Leuchtstoff in Berlin-Friedrichshain (DE)

When?

7 September 2011, $15 h 00$ 


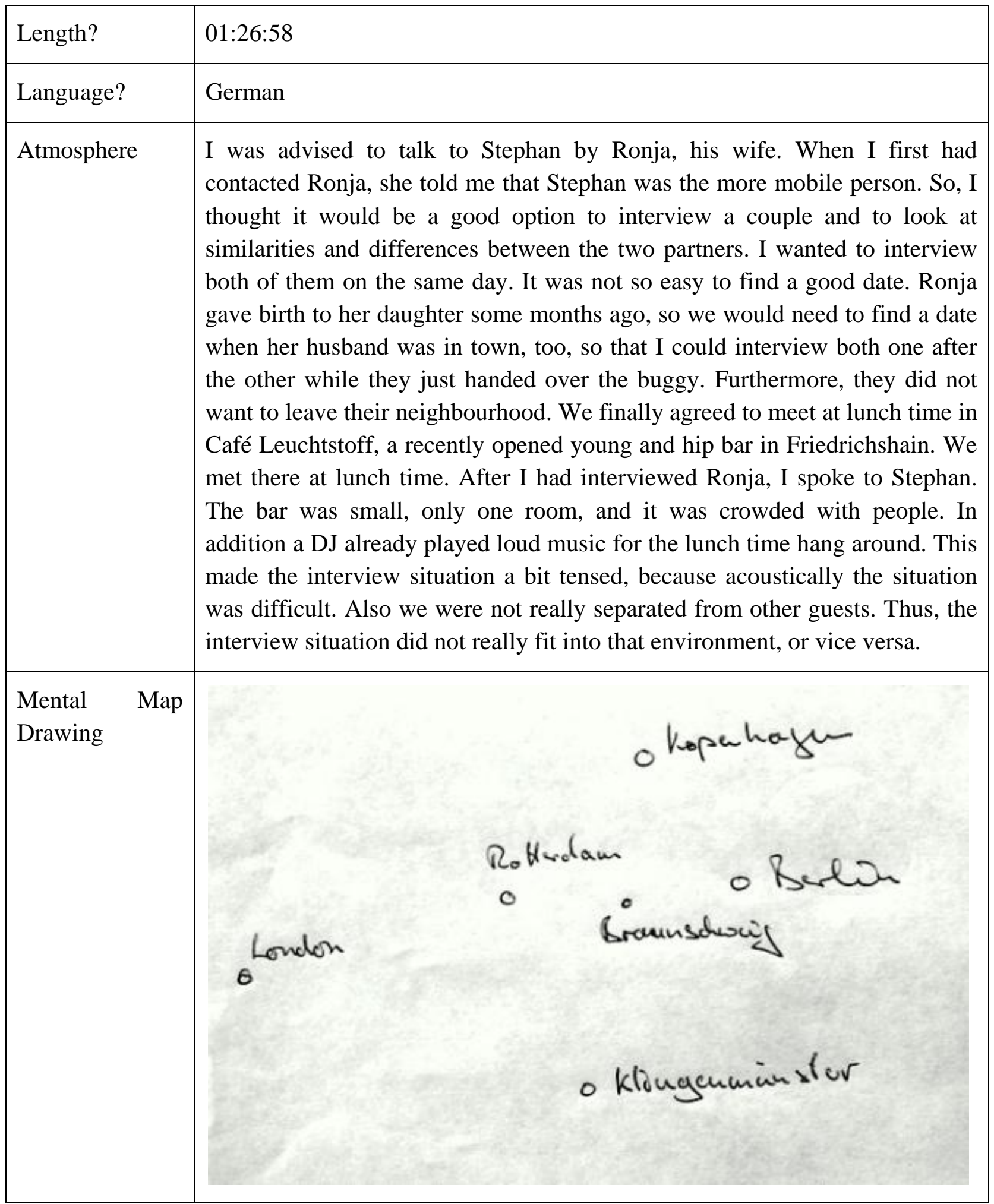

Interview No. 20

Who is the interview partner? 


\begin{tabular}{|c|c|}
\hline Person & $\begin{array}{l}\text { Tjark, Danish, in his 30s, freelance architect and interior designer, also } \\
\text { teaches in university, Austrian boyfriend, no children }\end{array}$ \\
\hline Personal places & $\begin{array}{l}\text { Berlin (DE), Copenhagen (DK), Aarhus (DK), Rotterdam (NL), Tyrol (AT), } \\
\text { New York (US) }\end{array}$ \\
\hline $\begin{array}{l}\text { Tools for } \\
\text { multilocality }\end{array}$ & $\begin{array}{l}\text { Several bank accounts (DK, DE, NL, US) | two SIM cards (DK, DE) | } \\
\text { apartments in Berlin and Copenhagen | Skype | low cost airlines | shared } \\
\text { office space | two health insurances (in DE and in DK) | web-based social } \\
\text { networks | laptop and laptop desktop as virtual mobile home | speaks Danish, } \\
\text { English and German }\end{array}$ \\
\hline \multicolumn{2}{|r|}{ What was the interview situation like? } \\
\hline Where? & In Café Eckert in Berlin-Neukölln (DE) \\
\hline When? & 7 September 2011, 17h00 \\
\hline Length? & 01:31:49 \\
\hline Language? & German \\
\hline Atmosphere & $\begin{array}{l}\text { After my interview with Pia, she gave me the details of Tjark, with whom she } \\
\text { had worked before. I did not know Tjark, but Pia told him about my PhD } \\
\text { project. When I called Tjark, he agreed to do an interview. But I would have } \\
\text { to meet him in Berlin. As Tjark was currently more involved in projects in } \\
\text { Berlin, he was flexibly available there. So, I arranged a date on the same day } \\
\text { I spoke to Ronja and Stephan. I met Tjark in a calm bar in Berlin's Neukölln } \\
\text { district. At that time the bar was nearly empty, silent salsa music was playing. } \\
\text { We sat at a table in a corner and order something to eat, an early dinner. } \\
\text { Tjark brought enough time and he was a good-humoured and chatty } \\
\text { interview partner. The bar was a calm setting for the interview and fitted } \\
\text { perfect as location. }\end{array}$ \\
\hline
\end{tabular}




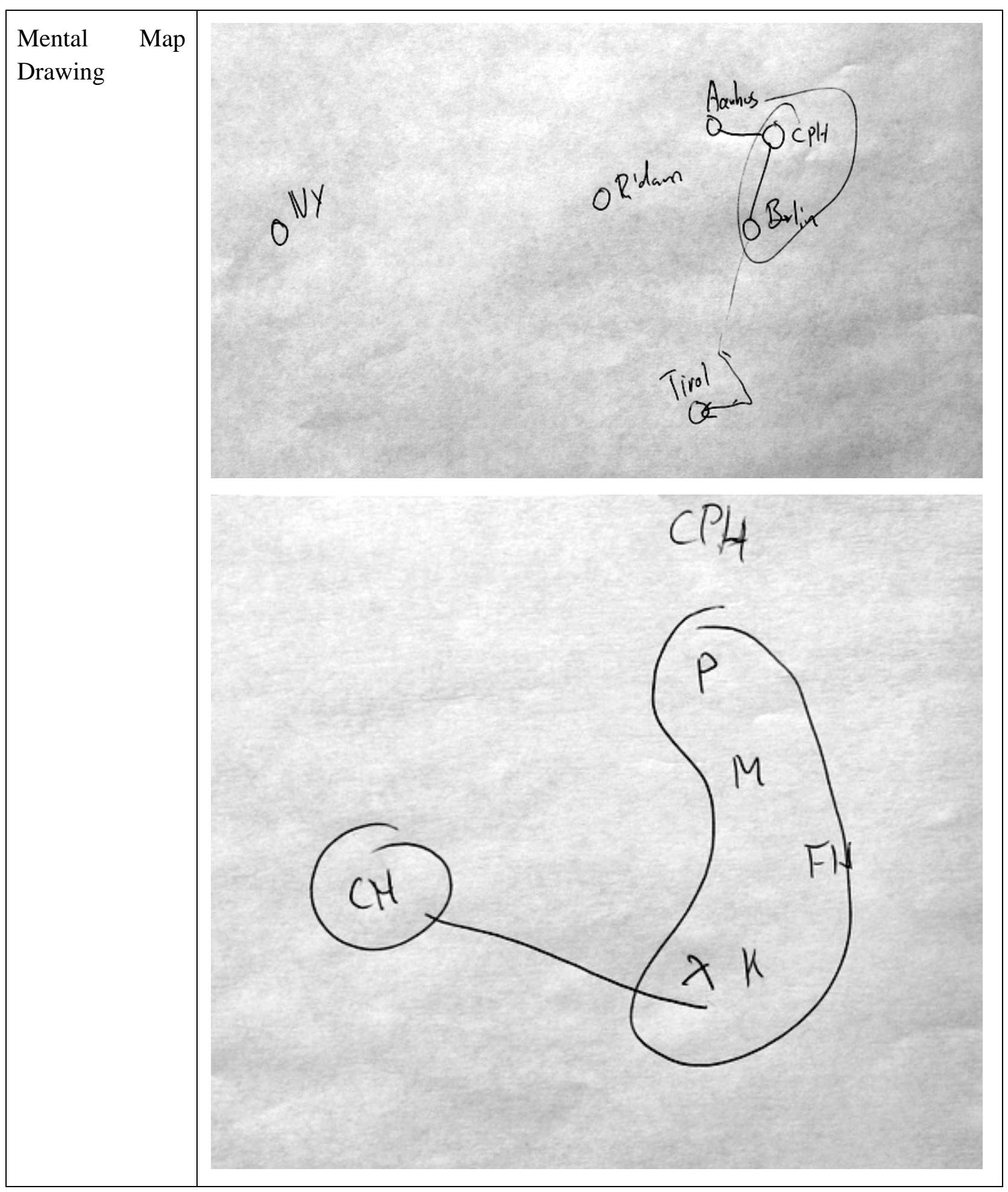




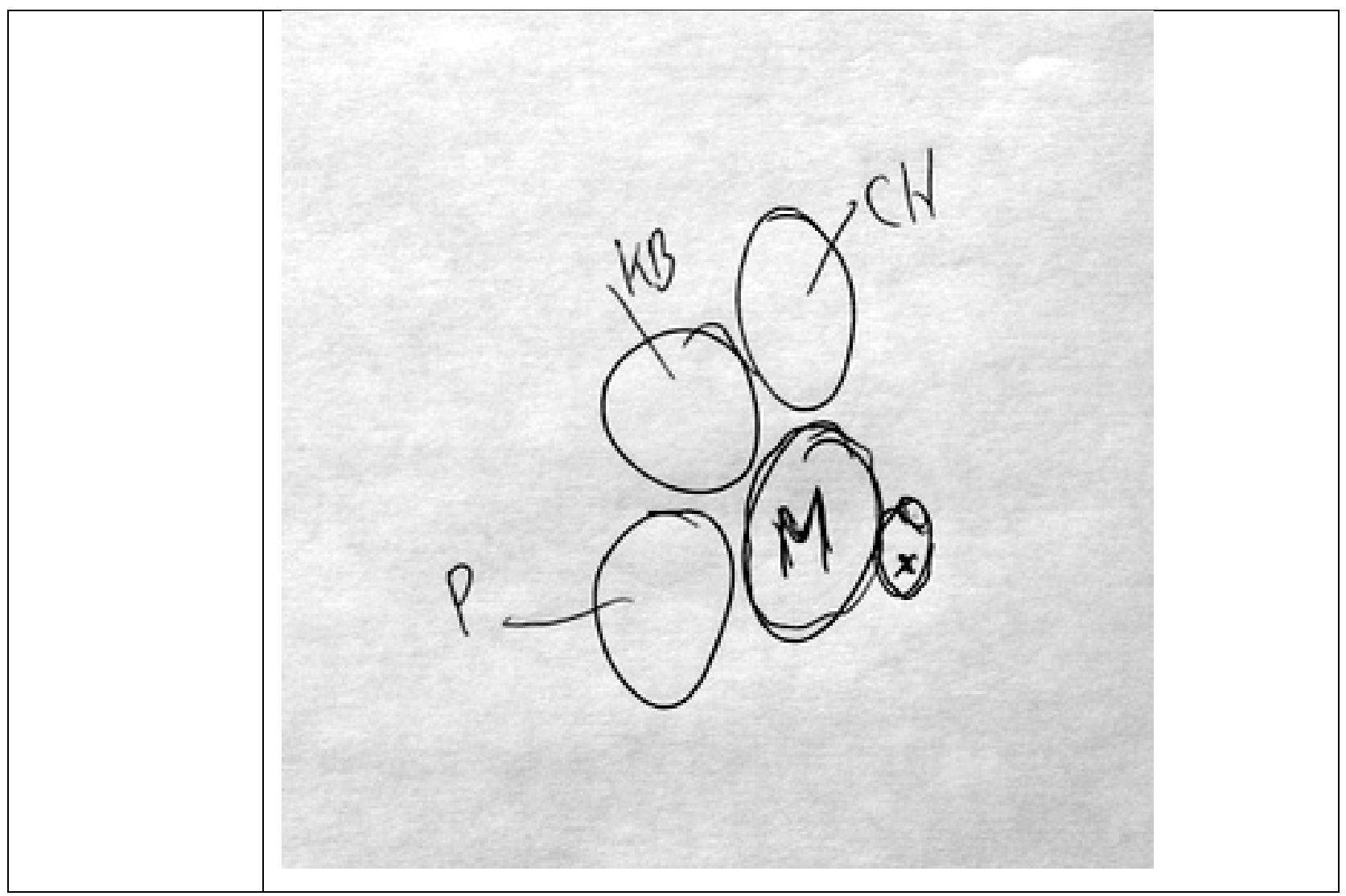

\begin{tabular}{|c|c|}
\hline & Interview No. 21 \\
\hline & Who is the interview partner? \\
\hline Person & $\begin{array}{l}\text { Ugo, Chilean, in his 30s, urban sociologist, living in Rome and Weimar, one } \\
\text { small daughter, married to a Chilean wife (freelance photographer) }\end{array}$ \\
\hline Personal places & $\begin{array}{l}\text { Santiago de Chile (CL), Concepcion (CL), Loncopanguo (CL), Chiloe Island } \\
\text { (CL), Achao/Quinchao Island (CL), San Antonio (CL), Rome (IT), Weimar } \\
\text { (DE) }\end{array}$ \\
\hline $\begin{array}{l}\text { Tools for } \\
\text { multilocality }\end{array}$ & $\begin{array}{l}\text { Airplanes | Skype | Websites of Chilean newspapers | apartment in Rome, and } \\
\text { a house in the suburbs of Santiago de Chile | speaks Spanish, English, French } \\
\text { and Italian }\end{array}$ \\
\hline & What was the interview situation like? \\
\hline Where? & In interviewer’s office room in Leipzig (DE) \\
\hline When? & 20 September 2011, 14h30 \\
\hline Length? & $01: 28: 52$ \\
\hline
\end{tabular}




\begin{tabular}{|l|l|}
\hline Language? & Spanish \\
\hline Atmosphere & $\begin{array}{l}\text { I was introduced to Ugo through an Italian colleague. Ugo had recently } \\
\text { started a fellowship at Bauhaus-University in Weimar. His original scientific } \\
\text { affiliation was with the University of Rome. His Italian professor in Rome } \\
\text { had visited Leipzig beforehand, and I had shown him around the city. This } \\
\text { same professor one day wrote me an email, asking if he could tell Ugo to } \\
\text { pass by in our institute in Leipzig in order to explain the research program on } \\
\text { shrinking cities. That's what Ugo was interested in. I agreed and the Italian } \\
\text { professor gave my details to Ugo. We fixed a date through email, and Ugo } \\
\text { came over on the day of the interview. As we had not met before, we } \\
\text { introduced ourselves and I showed him around the institute, introducing him } \\
\text { to colleagues and then we went for lunch. During lunch I explained him my } \\
\text { PhD project, and he started talking about his life. We rapidly figured out that } \\
\text { Ugo would fit into my sample, and I asked him right away how much time he } \\
\text { had this afternoon. He said, he might have to catch a train back to Weimar, } \\
\text { but there is some time left. So, after the lunch break we went to my office } \\
\text { room, prepared some coffee and started with the interview. Ugo was really } \\
\text { interested in my PhD topic and he also was a very chatty interview partner. } \\
\text { As the interview was in my office room it was quiet and no disturbance } \\
\text { occurred. }\end{array}$ \\
\hline
\end{tabular}




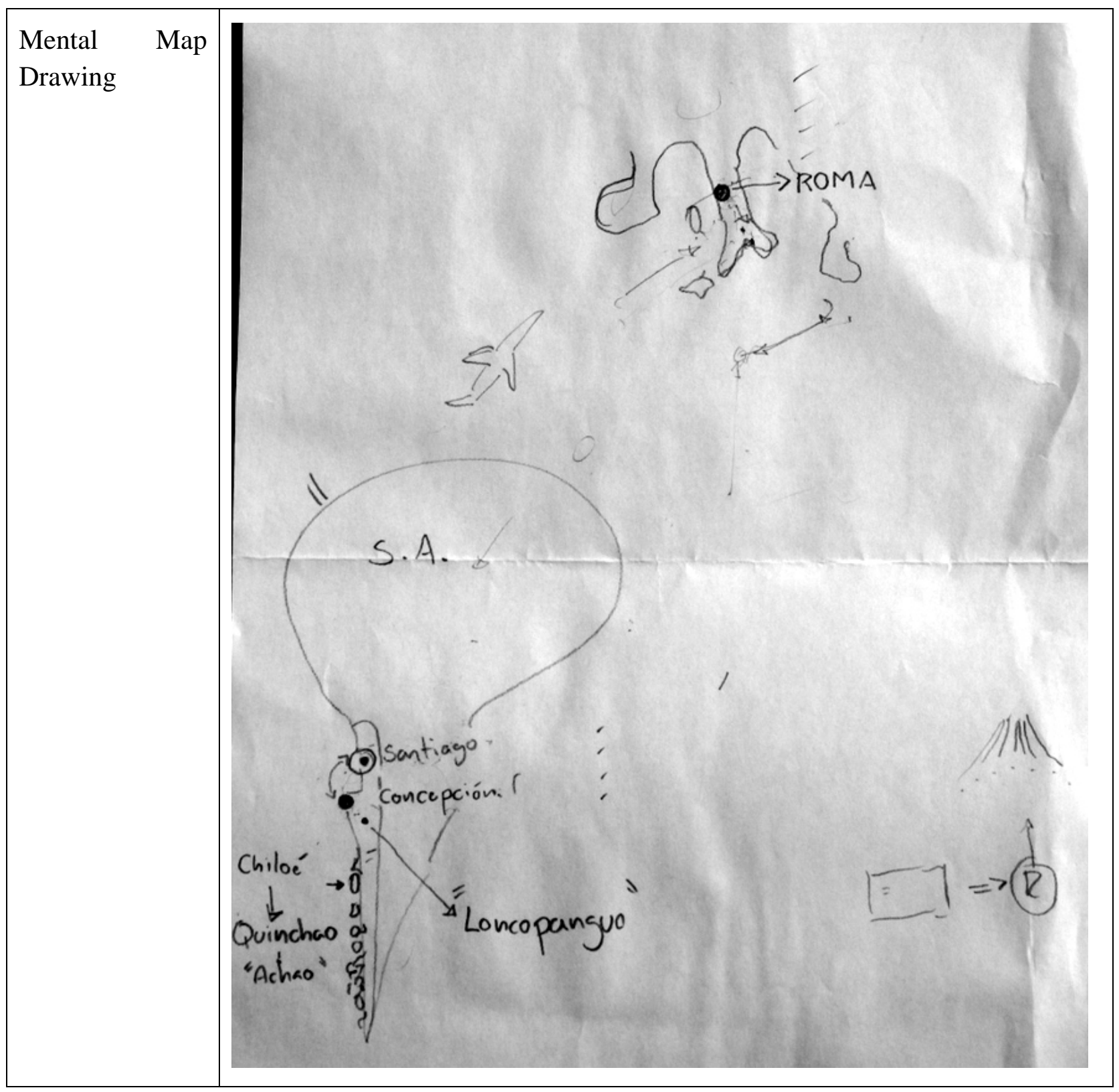

\begin{tabular}{|c|c|}
\hline & Interview No. 22 \\
\hline \multicolumn{2}{|r|}{ Who is the interview partner? } \\
\hline Person & $\begin{array}{l}\text { Valentin, German, in his 30s, studied theatre and film, freelance juggler and } \\
\text { pedagogue, German girlfriend, no children }\end{array}$ \\
\hline Personal places & $\begin{array}{l}\text { Leipzig (DE), Berlin (DE), Hamburg (DE), Löbau (DE), Lyon (FR), } \\
\text { Grenoble (FR), Brisbane (AUS), Adelaide (AUS), Laos, Hanoi (VN), Halong } \\
\text { Bay (VN), Tonsai Beach (TH) }\end{array}$ \\
\hline Tools & Tent as mobile home | global network of friends, who offer places to sleep | \\
\hline
\end{tabular}




\begin{tabular}{|c|c|}
\hline multilocality & $\begin{array}{l}\text { his parents and their house for storage of his belongings and clearing his } \\
\text { bureaucratic issues while abroad | photo camera | climbing equipment | credit } \\
\text { card | hitchhiking | speaks German, English, French, Italian, Spanish and } \\
\text { Norwegian }\end{array}$ \\
\hline & What was the interview situation like? \\
\hline Where? & $\begin{array}{l}\text { On a park bench in Leipzig's upcoming creative Plagwitz neighbourhood } \\
\text { (DE) }\end{array}$ \\
\hline When? & 26 September 2011, $18 \mathrm{~h} 30$ \\
\hline Length? & 01:33:56 \\
\hline Language? & German \\
\hline Atmosphere & $\begin{array}{l}\text { Christian told me about Valentin and his frequent stays abroad. I thought I } \\
\text { should meet him for an interview in order to figure out how his multilocality } \\
\text { might be situated. Christian had told Valentin about my PhD project, but } \\
\text { since several months had passed. When I called Valentin, he immediately } \\
\text { remembered that Christian had mentioned my concern. So, he agreed to meet } \\
\text { some days later. It was still warm outside, the last warm evenings of the year. } \\
\text { So, Valentin preferred to do the interview in a neighbourhood park. We met } \\
\text { in front of the park and sat down on a park bench. I had brought some drinks } \\
\text { and we started with the interview. Actually, the park was really quiet, } \\
\text { surrounded by apartment blocks, residents seemed to pay attention that it } \\
\text { remained calm. The interview took place in a smooth atmosphere. It was only } \\
\text { interrupted when Valentin went to the corner shop to buy new cigarettes. }\end{array}$ \\
\hline $\begin{array}{l}\text { Mental Map } \\
\text { Drawing }\end{array}$ & 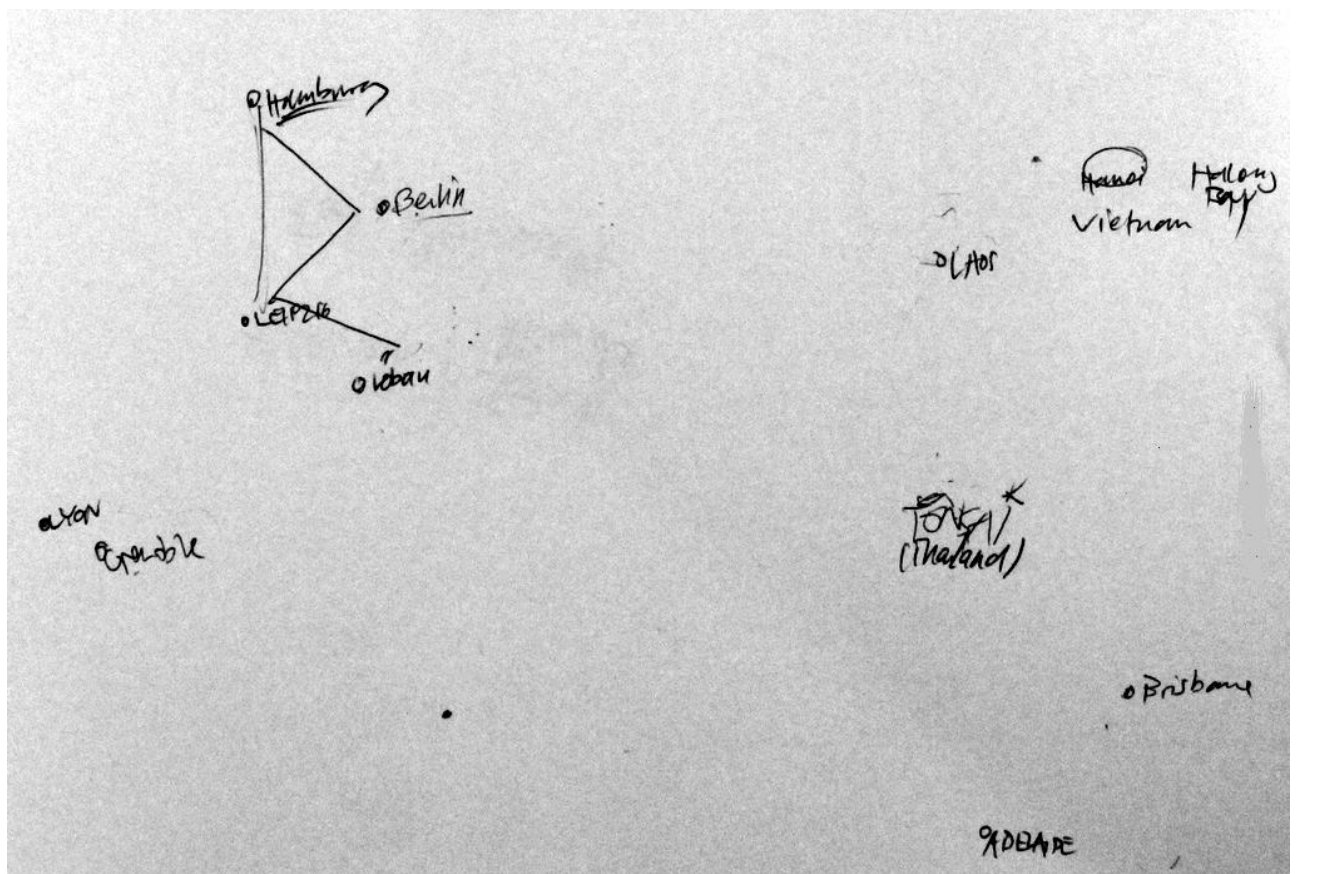 \\
\hline
\end{tabular}




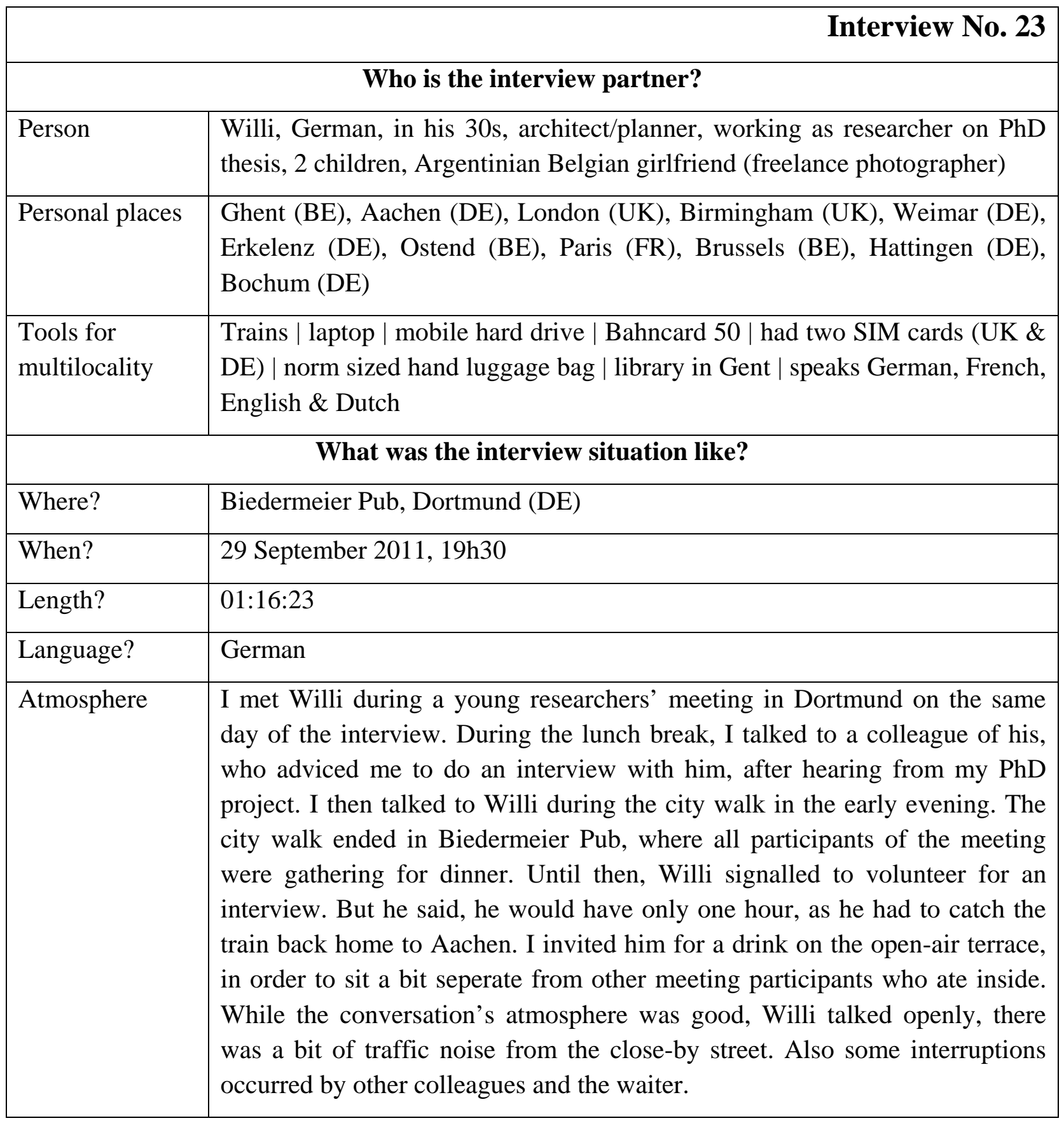




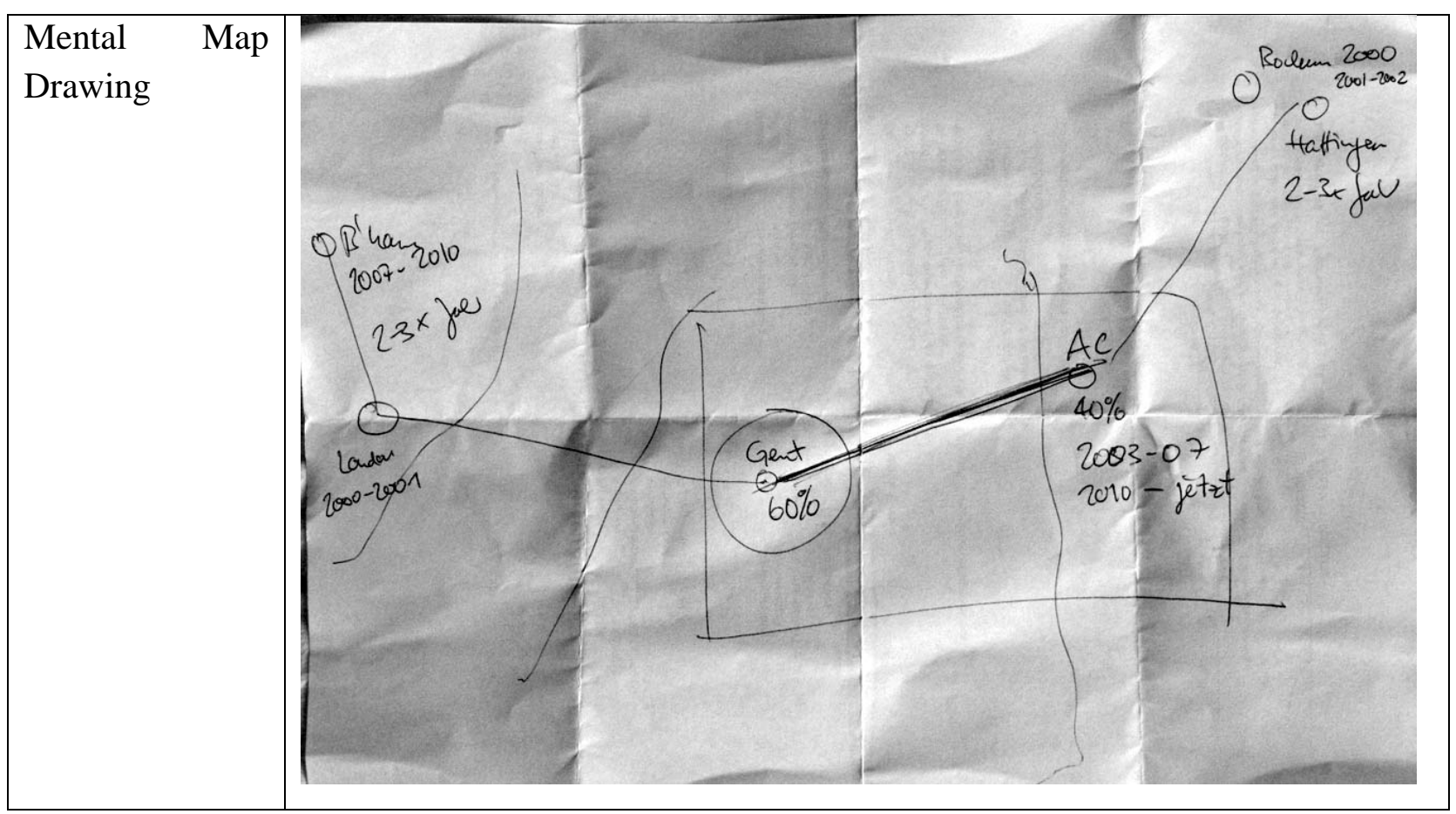

\begin{tabular}{|c|c|}
\hline & Interview No. 24 \\
\hline & Who is the interview partner? \\
\hline Person & $\begin{array}{l}\text { Xaverio, Italian, in his 20s, studied communication and sociology, works as } \\
\text { freelance photographer for fashion industry and tutor at university, single } \\
\text { (ex-girlfriend German), no children }\end{array}$ \\
\hline Personal places & Milan (IT), Berlin (DE), Rome (IT), Padua (IT), New York (US) \\
\hline $\begin{array}{l}\text { Tools for } \\
\text { multilocality }\end{array}$ & $\begin{array}{l}\text { Internet | low cost airlines | trains | hand luggage | two shared rooms (Berlin } \\
\text { and Rome), and a loft in Milan | laptop | rechargers for electronic equipment | } \\
\text { agenda | photo cameras in Berlin and Milan | email | two SIM cards (DE \& } \\
\text { IT) | speaks Italian, English, German and Spanish }\end{array}$ \\
\hline & What was the interview situation like? \\
\hline Where? & In interviewee’s loft apartment in Milan’s Romolo neighbourhood \\
\hline When? & 3 October 2011, 17h00 \\
\hline Length? & 01:44:13 \\
\hline Language? & English (partly Italian) \\
\hline Atmosphere & I read about Xaverio's photographic work on an artists' internet blog which \\
\hline
\end{tabular}




\begin{tabular}{|c|c|c|}
\hline & & $\begin{array}{l}\text { featured latest Milan based photographers. Xaverio was introduced as living } \\
\text { in Berlin and Milan, and I thought that's a good opportunity for an interview } \\
\text { partner, too. On the weblog there was only an email address available and } \\
\text { looking him up on the internet I couldn't find more information. So, I wrote } \\
\text { him an email. Some days passed, and he replied that he was available for an } \\
\text { interview. I had to go to Milan anyway, so I decided to meet him in Milan, } \\
\text { instead of waiting until he would find time in Berlin. Xaverio offered me } \\
\text { some time slots during my stay in Milan. We finally met one afternoon in his } \\
\text { loft apartment, which is part of an old factory estate in the southern part of } \\
\text { Milan, close to the creative district of Porta Genova. In this factory estate } \\
\text { there were several lofts and ateliers all occupied by creative people, who } \\
\text { managed the whole estate in a collective way. He picked me up in the court, } \\
\text { and we went upstairs in one of the buildings. His two-floor loft was sunny } \\
\text { and the large windows allowed a view over the whole court. I had never met } \\
\text { Xaverio before, so we small-talked a while. He told me that he had an } \\
\text { appointment two hours later, and he seemed a bit tense by that. So, we started } \\
\text { the interview, taking a coffee and some glasses of water. }\end{array}$ \\
\hline $\begin{array}{l}\text { Mental } \\
\text { Drawing }\end{array}$ & Map & 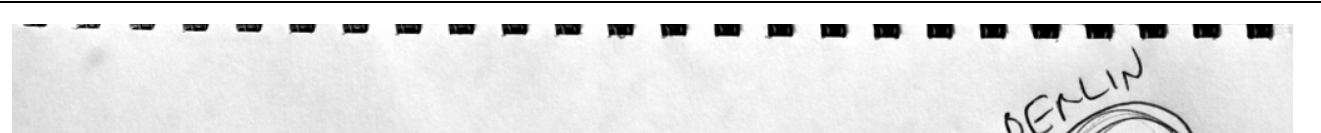 \\
\hline
\end{tabular}

\begin{tabular}{|l|l|}
\hline \multicolumn{2}{|l|}{ Who is the interview partner? } \\
\hline \multicolumn{1}{|c|}{ Interview No. 25} \\
\hline Person & $\begin{array}{l}\text { Yari, Mexican, in his 30s, works as a freelance hip hop artist in the Americas, } \\
\text { Europe and Africa }\end{array}$ \\
\hline
\end{tabular}




\begin{tabular}{|c|c|}
\hline Personal places & $\begin{array}{l}\text { Mexico City (MX), New York (US), San Francisco/California (US), } \\
\text { Dominican Republic, Africa, Brazil, Cuba, Caribbean Area, Latin America }\end{array}$ \\
\hline $\begin{array}{l}\text { Tools for } \\
\text { multilocality }\end{array}$ & $\begin{array}{l}\text { Computer | paper notebook | pencil | headphones | apartment in New York | } \\
\text { grandmother's or brother's place in Mexico City | money to finance his } \\
\text { community work | speaks Spanish, English and Potuguese }\end{array}$ \\
\hline \multicolumn{2}{|r|}{ What was the interview situation like? } \\
\hline Where? & $\begin{array}{l}\text { In front of a vacant apartment block in Leipzig, where Yari was about to have } \\
\text { a concert (DE) }\end{array}$ \\
\hline When? & 03 June 2012, 21h00 \\
\hline Length? & $00: 23: 38$ \\
\hline Language? & Spanish \\
\hline Atmosphere & $\begin{array}{l}\text { Some days before his concert, I found promotional posters for Yari's show in } \\
\text { my street. I then studied his website in order to know more about him, and } \\
\text { his presentation there made clear to me that he would perfectly fit into my } \\
\text { sample. But there were several problems: I did not have contact details of } \\
\text { Yari; I imagined that being on a live tour through Europe, he would not have } \\
\text { time; and finally I did not know if my Spanish was appropriate for an } \\
\text { interview. Anyway, I decided that there would only be one way to find out: I } \\
\text { went to his concert. I also took my wife who his Spanish mother tongue, and } \\
\text { she helped me with linguistic problems. When we arrived at the concert } \\
\text { room, there were few people. It was a rainy Sunday evening, and the concert } \\
\text { took place in the ground floor of a vacant apartment block in Leipzig's ex- } \\
\text { industrial neighbourhood. Entering the room, I found Yari, leaning to the } \\
\text { wall, behind a merchandise table, offering his latest albums. We went there } \\
\text { and introduced ourselves to him. He told us that the concert would start later, } \\
\text { as only few people had arrived so far - good news for me! I explained Yari } \\
\text { what my research is about, and that I wanted to interview him. He agreed to } \\
\text { do an interview right away. So, I suggested leaving the concert room (music } \\
\text { was playing loud) and going outside in front of the building. The interview } \\
\text { situation was anything but perfect: It was cold, started to rain, outside people } \\
\text { were chatting and wanted to talk to Yari, too. Then there was noise of a close } \\
\text { by street, with cars passing by. And finally, when we wanted to start the } \\
\text { interview, my voice recorder broke. So, I had to record with my mobile } \\
\text { phone, which only records } 5 \text { minutes at a stretch. This interview then was the } \\
\text { shortest one of my series. But given the circumstances, I decided not to } \\
\text { bother Yari for too much time. Anyway, we started our interview, and Yari } \\
\text { could provide some interesting insights into his mobile life. After the } \\
\text { interview we entered the room again, and listened to his concert. Yari left the }\end{array}$ \\
\hline
\end{tabular}




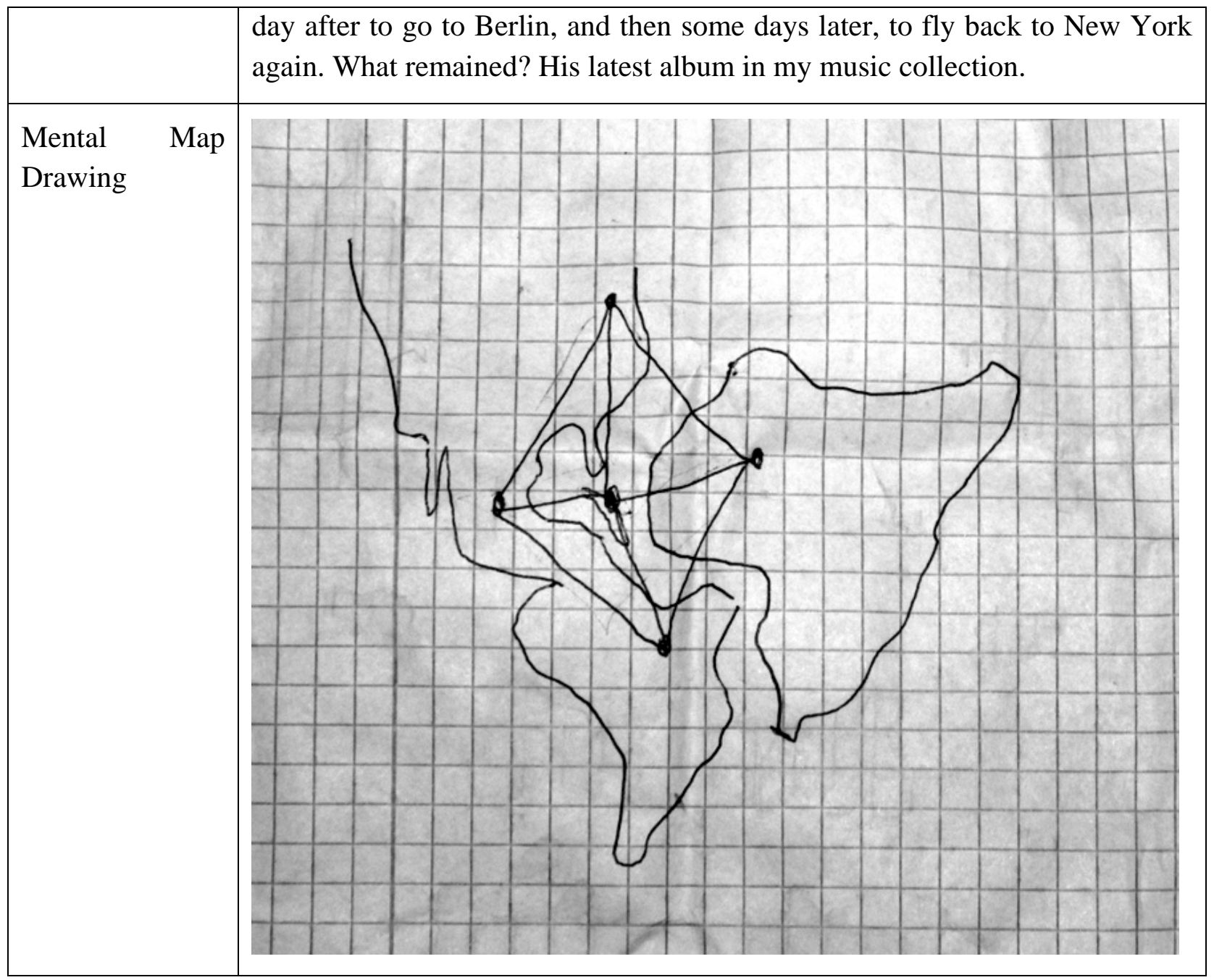


Appendix C: Exemplary interview transcript - Case of Xaverio

X: [RECORD_START]... in Berlin I've tried. But then, I mean, photography is so strange that you cannot uh...///

I: Predict where to be?

$\mathrm{X}$ : Yeah, exactly. [laughs, puts water in glasses]

I: Mm-mh.

X: And that's why now I'm also going to Rome. So, I'm like uh Milan, Rome, Berlin. It's like 7 uh...

I: Triad?

X: Yeah. And I'm from another city. So, it's like four.

I: [laughs] Excuse me, I, I want to record the interview. So I just don't have to put notes all 1 the time. It's just a bit annoying to write down things.

$2 \mathrm{X}$ : Yeah, yeah. [washing some fruits in his kitchen]

I: Does it mind you, if I switch off the radio?

4 X: Ah, no, no, you can do it.

I: It's just like background noise.

$6 \mathrm{X}$ : Yeah, yeah, yeah. It's uh...[designer radio where it's difficult to find the button]

7 I: Mute... ah okay here in the outputs, this one?

$8 \mathrm{X}$ : Yeah!

9 I: Alright, thank you. Now, it's exactly so, I... to explain you, I'm, I'm doing a PhD thesis 0 here in, in Milano, in Università Bicocca. And that's about people who live uh their everyday 1 life between different places. As you have mentioned I have seen that uh... I have come 2 across a website of Professore Sandro Balducci.

3 : Mm-mh [listening]

4 I: I don't know if you have heard it, but at least from this site uh I found a link to your profile 5 on Milan-based uh list, or a website of photography about the city. Which is, uh I don't know, 6 a monthly magazine, online, for photography.

7 X: Yeah, it’s Milano Città Aperta.

8 I: Si, esatto. This one.

9 X: It's a site about... yeah it... Milan... uh people from Milan, that uh work with 30 photography. 
I: Mm-mh. So, and I'm... uh I have studied geography before. So sociology is not my origin, where I come from. And as a geographer I'm always very interested in place and in space, and issues like that. And I start interviews with the people I talk to and ask them to uh draw me a mental map of the places which are important for them. So, could you design me...///

$\mathrm{X}$ : Each one? Each, each... uh...

I: Each place which has a certain significance in your life. Could you draw me a map of your places, that are important in your life?

$\mathrm{X}$ : Okay. But do you mean like uh the connection, or just the physical aspect, that I have in my mind?

I: It might be both. But the connection, I am interested more in.

$\mathrm{X}$ : Ah okay. So, I think it's something like that. It's [drawing] in the centre, there is uh Padova. Padova that is, where I was born. So, it's... after all it's my home town. So it started... Everything started from here. And then there is uh... we can say something like that... Here there is Milan... And here, there is Berlin. I mean for me it's really like an escalation. So it's uh... But then it's, it's quite uh... it's like that. Because then it's... and like that... and here, there is also Rome. So, there are... this kind of really stoned connection. Since today, I never had these two... I mean, they are like really parallel. Because uh... yeah Milan is really, that's really a strong connection because finally I made my final work in Milan at the university about Berlin. So, I really studied a lot about uh... the city and uh the place there. And then I came back to discuss this final work really quite big in Milan. So it's really uhm... a big connection. Otherwise instead, there is no connection, no, not at all till now between Berlin and Rome. But now I'm teaching in Rome as a photography professor at European Design Institute. So, I'm going also there. And uh... Yeah, and it's strange because, it's like only one, no six months, eight months that Rome came uh... came out like, like another chance of my working life. And uhm... so for me now, it's a little bit more difficult. Because there is not, not so much place for each city that I have to, really to, to match, how to organize everything. But they're... it's funny, because they are... three completely different cities. So, for me it's like if I, in every city I can find something. And I, I'm starting to know what I can find in each of them. So, I know that Milan has a certain type of a rhythm, connection, people, a way of life. That Berlin, it's more quiet, it's more easy to live there, the quality of life is higher, and uh there is more culture. Rome, it's amazing, because it's a place where the culture was born, I mean. But at the same time, it's a little bit more traditional, I mean, in respect to Berlin. But for me, Berlin is... it's the way to, to have new feedback. When I want to, to know something new, and to have new feedback, uh... normally I go to Berlin.

\section{I: Mm-mh.}

X: Even if I don't have to work, because actually Berlin, it's the more difficult part for me to work. Because there is not so much work in Berlin. I mean, there are so many artists, you know, and so many creative people. But finally there is no, no way to have a good job, especially in, in the arts' sector, in photography. So, uh... I'm doing something, I'm doing some projects. But for me, it's more even to be in the city, to be there, to, to stay there, and 
to... sometimes it's a good connection, even because I stay there, but sometimes even I, I don't work. But I have some good uh idea, inspiration, that maybe I can develop in Ber... Milan, or in Rome also. So, it's a, it's really a part of the, the... triad connection. But I'm trying to, to passion a little bit more Berlin, because uh I would like to stay there more time. And uh... and maybe the... I mean the ideal would be like stay there to live and then move in Milan to work. Like, you know, make an inversion. And now it's a little bit uh the opposite. I mean, yes I am here, I have like a place where to stay there. But I'm going there when I have uh yeah free time to live there and to have my personal project, to develop my stuff, and to... yeah even to work a little bit. But uh till now, it's not so strong.

I: Mm-mh. So, to, to understand, you, you were born in Padova, and then you moved to Milan to study?

\section{$\mathrm{X}$ : Yeah.}

\section{I: You studied photography?}

X: No. I studied communication. A lot of socie... sociology, really a lot [laughs] relating to this sector. And then uh... Actually I made three years of university in Padova. Because in Italy, there is uh...///

\section{I: Triennale?}

$\mathrm{X}$ : Three plus two, the... like...

\section{I: Bachelor and Masters?}

$\mathrm{X}$ : Yeah. Yeah, something like that. So, I made the two in Milan. But inside the two, I made one year in Berlin. No, more, actually it was like yeah one year. Then I came back to finish with final work. Then I went back again to Berlin, for a while. For like six months. And then I found... in parallel, I was studying photography at the, in Milan, at school of photography, during the university years. And then, I went to New York. Also to study. And uhm... then I went back, because uh... a German company, in Milan, Messe Frankfurt...

\section{I: Mm-mh.}

X: I don’t know, if you know. Uhm... was looking for people speaking uh even German quite well at work, like as an event marketing organization. So, I said: 'Okay, why not'. I mean, I was already into photography and everything. But just it was okay, I already finished university... I mean, I thought: 'Okay, it could be a good chance just to have an experience'. So, I went back here. And I stayed in the Messe Frankfurt for one year and half. And I was... I used also there in this period to go often to, to Germany, Frankfurt. It was nothing linked with Berlin. [laughs] Completely different. And then after that, just I realized that it was not my life. So, I decided to cut everything, and to start only doing uh photography.

\section{I: Mm-mh.}

\section{$\mathrm{X}$ : And so I started to... to work as photographer.}

I: Mm-mh. So, this... the thing with photography evolved somehow from a hobby or something that you did in leisure time? And then you professionalized it by doing some courses, studying it? 
$\mathrm{X}$ : Yeah! Yeah, I mean, I was uh... yeah, since a lot of time I was like taking pictures, yeah with... with cameras, and... But then when I started the scuola in... here in Milan, I realized that it was a... it has been really for me interesting to go more deep with this kind of uh... work sector. So, I was more and more and more involved. And then, when I went to New York at this really incredible school, like in two thousand and uh... eight [2008], I really decided, so four years ago, I decided really to... after that... to leave everything, the other part of my working life, and to... to go in more and more deep in photography. So, I started to be an assistant of photographer, doing shootings like... like uh...yeah like assistant. And then starting more and more with my stuff, and uh... I mean, it's still quite difficult, because uh... now with uh... yeah with the crisis and everything, it's... you have to be really well connected with people. You have to know the other actor, you have to be always in the right place in the right time. So, but it... I mean, for me it's uh... it's a good way to, to live. I mean, I can, I cannot anymore stay in an office, just uh working in front of a computer.

I: Mm-mh [listening]. What was it, Messe Frankfurt, you worked for them, but in Milan? You organized...

X: Yeah, in Milan. But I used to go like once a month like to Frankfurt.

I: Okay. Now, you said, you are, now I think you are more satisfied with your situation, that you don't have to be anymore in offices, and you can work as a photographer. So, is it also that you came here, into this place to live in uh in this area, in this old factory building, because you have free space to work and...?

$\mathrm{X}$ : Yeah, I mean this is a brand-new place. I was looking since long time for a place where to stay, to have a... and finally, now, really now in September, I was looking for, yeah, a loft that would be like really independent. And for me, it's, it's really funny, because it's like a little bit like a Berlin style, this place. I don’t know, if you can uh...///

\section{I: Yeah, yeah. I was, I was astonished to find something like that in Milan.}

$\mathrm{X}$ : Yeah, even me. So, for me, when I... when I discovered this, because a friend of mine... because here it's full of yeah photographers, designers, video makers, so it's like a creativity village... so, three, four years ago, a friend of mine was work... living here, like in a studio. And I was like: 'Okay, you have to go there, you have to go there'. But then time...///

\section{I: Passed by.}

$\mathrm{X}$ : Passed by, yeah. And then finally, I was living really close to, yeah, to this place. But uh in another, another flat. So, it was close here, but really noisy also. So, for me it's a completely a mess. And uhm... once I went... I came here inside. Uh in the backyard. And there was this uh advertisement, like 'loft free, call!', like that. So, immediately I called. And... and now, I mean, I'm here since... yeah, one month and a half, but it's part of the development of my working life. Because now, I'm really... I mean, it's... it's not... it not seems to be Milan, for me it's like really, really calm and more quiet... uh I am sure that a place can be really, I mean it's important for uh... your uh... daily life, to have a, even a kind of freedom. So, I was looking really for a place not noisy, not with the car under, full of house and really grey. And this is really lighty. So, it's sunny every day. There is not so much noise. Uh people inside 
here are really, have quite the same vision of me of the things. So, it's... it's a way to, not to be part of the Milan side that I don't like, that it's really business, business and nothing more.

\section{I: Mm-mh.}

\section{$\mathrm{X}$ : So, for me it's important, I mean.}

I: So, basically uh Milan is more the place that you connect to, to business and uh... to traffic, to stress, or noise?

$\mathrm{X}$ : Yeah.

I: And Rome is more a place which is uh historic culture? And Berlin is more the place where you find upcoming cultures?

$\mathrm{X}$ : Yeah. I mean I'm going to... I have an exhibition on Rome. In November. So, I mean... yeah it's like if my working life is really dividing. Because here [Milan] yes, I have like commercial work, more like, yeah, I don’t know, advertisements, something like that. In Rome, okay, I'm teaching. But uh really in a free way, like they said me: 'Okay, you can have a photography course. You can talk about, what uh what you want'. So, okay, I'm really free. And now I'm doing uh an exhibition in uh in an old palace in Rome, in Trastevere, that is a uh... yeah it's an area in the city centre, and old area. So, uhm... yeah, it's more like an exhibition. So, there... my artistic parts are more between Berlin and Rome. And Milan, it's more like a commercial, to... to be able to pay the rent at the end of the month, something like that. But uh... Not, but it's... I think, it's the only way, I mean for my job, to create different uh sectors. And uhm... I would like to be more stronger in the German sector, because I know that... I mean, in Germany, now it's, the economy, it's the strongest, I think, of the Europe. So, even if Berlin is not so... so strong, I would like to be to more there, even because they are more uh... serious in their way of... yeah to approach uh... I mean, for instance, I, I worked last week for the fashion week, at White, that is the fair of fashion, here in Milan.

\section{I: White?}

X: White, White Shop, it's called. So, and it's here in uh Superstudio, Via Tortona [a famous creative industries' neighbourhood in Milan]. They are a really big, big studio. And there is... all the fair developed there. And uh this is really like Italian style to work, that I don't like it, because they called me one day before the beginning: 'Oh please, we need a photographer. We like you. So, please come!’ Just an email. And even without any kind of uh specific uh... I said: 'Okay', I mean, I was free. So, it was not... it would be stupid to say: 'No. Please before, write me everything'. I said: 'Okay, I go'. And then I worked really... I mean, they told me: 'Yeah, we will give you at least...', they told me a price, but really generic. Then I worked more than I was expecting. So, like uh yeah twelve hours per day. For three, four days. So, it was really hard. And with... and I had to give back all the work post-producted like in one day. So, like I will 4,000 of images to post-product there. And it was they, they were like calling me every five minutes: 'Okay, are you ready with this kind of photo? Are you ready, could you send it?’ Okay, it was okay. I mean, I made that like really in a professional way, all the stuff. After the last part, I said: 'Okay, now I write you the payment. With the extra hours also'. Till now, it’s passed one week, I didn’t ever had an answer, you know. 
I: [laughs surprised and appalled] Yeah, yeah.

$\mathrm{X}$ : I mean... and this is really common. And it's really Italian style to work. They just call, call, call you. And then when you have finished the work: 'Okay, take it easy! We will pay you'. But you don't know never when. So...

196 I: Mm-mh.

X: It's... and German... I mean, the... it's not a cliché. Because sometimes there are clichés between Germany and Italy. But here it's really true, I mean. Even because I had one, one year and a half, working with Messe Frankfurt, with the German uh...///

\section{I: Company?}

$\mathrm{X}$ : Colleagues. So, they... because we were like the Italian uh division, the office. And then we were working with, with the German uh central studios. So, we were everyday calling and writing email, and everything. And then, I was also working yeah with Italian uh people, clients that wanted to take part of... to the...///

I: In the fairs, in the exhibitions?

X: Yeah, exactly. So, I really had the possibility to understand the difference a little bit between the Germany and Italy. And Germans, they are more... you know, more... sometimes too much uh strict, you know. But they work, they are more professional. Sometimes they don't have like the crea... the Italian creativity to... Italian explosion ideas, that sometimes really make the difference. But uh I mean, German people are more, in the long-term they are more uh... uh how do you say?... costante. Can you say costant? No?

214 I: In Italian?

215 X: Costante.

216 I: Costante? In un senso che, che vanno piu risponsabile, no?

$\mathrm{X}$ : Yeah, no, I mean in the sense of uhm... they are not like high, and they go high and down, high and down. They are like more [whistling sound; movement with hand: horizontal line indicating constancy].

I: Yeah, you can calculate better beforehand?

X: Yeah. Yeah.

I: How is it for you personally to be involved in Milan and in Berlin? Other people that I have talked to, they told me that uh working in two different countries, and in two different cities, uhm could have advantages - in whatever sense. For example that you come from Berlin, and in Berlin being from Milan gives you a certain significance, or it gives you an advantage. Do you, do you uh... experience things like that? 
and I say I'm Italian, I'm from Milan, they look at you in a certain way. If a New Yorker came here in Milan... So, I think it's true, I mean, you have like something more [in] respect [to] the other people. So, when I'm here, and I say I'm living also in Berlin, I know very well Berlin, yeah people are more interested, you know. Because even now, I mean, in the... now in this situation, Berlin is like cooler than Milan. Now, I mean, like a global vision of the city. Because it's more, yeah more clubs, and more culture, more people, more young people. It's... I mean, in the Italian magazines, people have this kind of uh feedback, you know. It's like the 'place to be', now it's Berlin. And the place to see. Because uh things happen there, and not for sure here in Milan, that it's everything more static. So, if you're... if you're here [in Milan], and you talk about Berlin, they are... people are more uh... uhm interested in you, even uh in a kind of way, in uh... in a working... workly speaking, I mean. And I think also when you go to Berlin. Because Italian, it's always uh yeah it's, yeah you are Italian, so you are something different from the, from the normal life. But I think that in Berlin, yeah it's okay, but it... you... I can feel it more here in Milan now. I mean Berlin, it's so... it's so common, you know. Lots of people go there, and there are lots of people from all over the world, it's really okay. You are not German, but uh I mean... Berlin it's not Germany. It's... it's like a big place, where a lot of people from all over the world are going there. So, it's... you can make the difference, but not so much, because there are a lot of other people, even Italian people going there. Uh here it's, now it's even more, I mean uh yeah you are from Berlin, so you are... have a completely different vision.

252 I: Mm-mh.

X: And Ber... uh Milan have not so much foreigners. So, not so much connection with Berlin, so, it's more strong the feeling that I have, normally. And it happens to me more to speak about Berlin than speak about Milan.

I: Really?

$\mathrm{X}$ : Yeah.

I: Why that? Because you feel personally more attached to Berlin, or...?

I: That's funny.

$\mathrm{X}$ : For a kind of uh yeah mental distortion, my mind... Even because of people that are every time asking me about Berlin. Because they know that I live in some period there, I'm living there, so I know very well the city. And it happens really often to speak about Berlin. Really, really often. respect [to] last year. Because of this student project.

\section{I: Professor?}


$\mathrm{X}$ : And a little bit less in Berlin. But uh I'm going to increase it for the autumn. I would like to go there, after, after October, yeah.

I: And how do you live in Berlin and in Rome? So now, I know how you live in Milan. But do you also have apartments there? Do you have something rented, or...?

$\mathrm{X}$ : Yeah, till now it was really comic, because uh... uhm... I had an apartment in Mitte [gentrified Berlin city centre neighbourhood]. And then living with a, with a German uhm guy. Because I decided just to live with Germans, and no Italians, and so. And it was perfect. Uhm and then, like it was of... just the case, a friend, a very good friend of mine, Italian, after some months, after maybe... because then arrived when I left the apartment. Because I had to do, to pass just a period here in Milan, so I decided: Okay, I left... I left the apartment and then I would go back looking for something new. After me, went like a French uh student, and that left immediately. And then went a very good friend of mine, just for case, you know. He took my room. And he is doing like a PhD in History. He was here to visit me three days ago. And uh the funny thing is that he is living between Berlin and Rome. So, normally when I'm going there, just we are dividing the flat, with him, and the, the German guys. Because it's quite big. And uh it was quite the same with him in Rome. Because he has like a flat of a friend of him, that uh now he is not anymore in Rome, but sometimes he has to go back, so there is a room for him. And then we were like sharing the room there.

$\mathrm{X}$ : Yeah, in Rome. So...

I: So, you both are sharing rooms in Rome and in Berlin?

$\mathrm{X}$ : Yeah.

I: That's funny. I have never heard such a construction. [laughs] That's good, that's efficient, no?

X: Yeah, yeah. No, it's... even because it's really difficult to manage like three places. And uh... uh I mean in this case, it's uh the cheapest way to, to go on. Because I cannot permit to pay three normal rents. And uh... and so now, it's something like that. Then, I... I mean I would like to, to create also an evolution about that. But with the time.

I: Mm-mh. So you always travel. Do you have things in a double way, in these places? Or in a triple way? For example like the toothbrush or something like that? Or your clothes? Or do you take it with you always [when] you travel?

X: Uh no, I have also some stuff divided. Yeah. Yeah, not everything, because then I really, I learned to go everywhere in every kind of weather with the bag, you know, just for like dressing with a... yeah from the t-shirt to the pullover, to the... so, just leaving, going and travelling with every kind of type, of stuff. analogic... the stuff for the development. For the black and white.
I: Mm-mh, ah yeah, yeah, yeah, for the photos? 
307

308

309

310

311

312

313

314

315

316

317

318

319

320

321

322

323

324

325

326

327

328

329

330

331

332

333

334

335

336

337

338

339

340

341

342

343

X: Yeah. All in Berlin. So, I know that when I'm there I can develop. Because uh...///

\section{I: Black and white?}

X: Yeah. Because I bought it there. And then it stayed there. It's... and also sometimes I take just a film here, I took the film here, to go there, you know, when I sometimes... every, I don't know, three months that I made some film, just to take it with me, go to develop there. And uhm yeah in Rome till now I don't have so much stuff. Because it's not so much time that I'm there. But uh... Yeah, and I normally, I just... even because I, I go by train. And by train it's like three hours. So, it's not so...

I: Not so far.

$\mathrm{X}$ : Yeah, not so far. It's not so long trip. It's uh... sometimes I just, for instance, I have to go to Rome uh yeah tomorrow, uh and I stay there three days. But then when I go there, I just travelling for a while, and uhm it happens even that I'm going just for one day. So, even I don't live there, because there are these special trains, that you...

I: La freccia rossa?

$\mathrm{X}$ : Yeah. You go there in... yeah actually you arrive at ten o'clock in the morning, and you can go back, uh be back in Milan at seven. Something like that. If I have like something the day after, here. Yeah, otherwise... yeah I can stay there.

I: Mm-mh. Do you also use certain infrastructures for travelling? For example, in Germany, you know, that the German train company, they offer you a special card, and you have a reduction on all the tickets. BahnCard.

X: Yeah.

I: Do you have something like that for Germany, or for, for Italy? Do you use these things? If you travel with the Freccia Rossa, I don’t know how it works in Italy?

$\mathrm{X}$ : In Italy it works that uh yeah you have like a card, and uh... I mean you don't have not so much discount. Uh but it depends how early you can find the ticket. For instance, I took this one for tomorrow, like uh 50 Euros. And the normal price is, it's 91. So, finally I paid like 100 instead 180s. Something like that.

I: And you find this on the internet, or how does it work?

$\mathrm{X}$ : Yeah.

I: Okay.

$\mathrm{X}$ : Yeah, on the, on the Trenitalia website.

I: Mm-mh. Because other people told me for example, if they have to fly frequently, then at certain mileage, you become uh a certain passenger with a golden card, or something like that. And then you can go to the lounges on the airport where you have internet access. You can work there, have something to eat and drink.

$\mathrm{X}$ : Yeah, yeah. But with which company?

I: That's all different countries... uh companies. All the air flight companies. 
$\mathrm{X}$ : Yeah cool.

I: Normally not the low cost ones.

$\mathrm{X}$ : Ah no. I'm just using low cost normally, yeah. Because now, because I know that the miles give you the... yeah some credits. But it's the Lufthansa for instance.

$$
\text { I: Yeah. }
$$

X: Yeah. But uh no, normally, I, I'm travelling with EasyJet from Milano-Malpensa to Schönefeld.

\section{I: Mm-mh.}

X: And yeah, it's... I, I know so, so well the travel that I made so much time, so many times. So, I just go out from here. I go to Cadorna, take the train, go to Malpensa. And then uh... yeah normally I don't take so many luggage with me, if I have to be there a short period. Otherwise I have luggage. Yeah, and then I arrive to Schönefeld, take the Bahn, and then go to Schlegelstrasse, yeah.

I: There is your apartment?

\section{$\mathrm{X}$ : Yeah.}

I: So, do you have also cameras uh in all these places? Or do you take your, your photo equipment with you?

$\mathrm{X}$ : I have a... no, beh, my digital photo equipment is always with me. The, the working normal one. I have some analogic camera in Berlin. Like yeah, Polaroid, and uh... yeah a Minolta, yeah. A normal old one. No, but normally I uh... the, the classic equipment uh is always with me. I cannot stay without [both laughing]... Yeah, for me it's important to, to have it with me, and always.

I: Are there other things that you have to take with you, when you travel? That you always take?

X: Uh uff... yeah laptop is always useful. I mean the, the small one. And then uh... uh... nothing... I mean, yeah, some books or a magazine. But I don't have a... some, yeah, specific stuff that I always take with me. I'm quite uh... uh... uh [thinking]... I'm quite able, easily to travel without a lot of stuff. I mean you have to, otherwise it starts to be really a confusion. [I laughs] Even because I'm really good in losing stuff. But a lot, really. Like sunglasses, keys, wallet, and stuff for dress. I really learned to be minimal for everything. Because otherwise you [thinking]... you... the risk is to, to lose everything. [I laughs again] And then even if you miss something: 'Yeah okay, no, it's in Berlin, wow'. And so, I pay lot of attention in this stuff. I mean, I know that I have to remember every time, yeah the recharge for, the recharge for the laptop, and recharge camera. I mean for me these are more important than dress, because if I forgot something, I can even go to H\&M and take a t-shirt and it's not important for me. It's more the... my documents. Yes, I have this uhm... agenda.

I: Un calendario? 
$\mathrm{X}$ : Yeah. That for me is important. To divide the period and the organizement, I'm just used to write there. And yeah, this is one of the things that I have always with me. And uh... yeah, for dress, it’s... [makes a mimic of indifference]

I: It... this sounds a bit like uh as if you started to develop certain routines in travelling?

$$
\mathrm{X} \text { : Yeah, yeah. }
$$

I: Do you... Have you had the feeling for yourself, to have become more relaxed in travelling?

\section{$\mathrm{X}$ : Yeah, yeah, yeah. For sure!}

I: That's what a lot of people tell me. If they frequently travel between places... Like you said before you start to know all the way, you go to - in your mind it's already - that you go to Cadorna, then you take the train to Malpensa. And so you already do it a bit like unconscious?

X: Yeah, no, no, for sure it's unconscious. I mean I can... I can already know everything about the even timing, and I start doing this kind of action without thinking so much. It's like a mechanical one. You know, I can do... I can read and work with the phone, and uh... I mean for me it's not travelling anymore. I mean, when I want to make a travel, I... I do completely different uh... things, I mean. Because there you have to uh... just to be smart as... as more as possible, to be quickly and not lose time and uh... uh yeah to do what you know that you have to do and stop. So, yeah, absolutely. I know that it's part of uh... and for me it's... I think for all people that are travelling so much... yeah for me they are so closer these three cities. And if I talk with someone who is living just in Milan, they say: 'Okay, well, you go like in Rome twice a month, and then you go to Berlin, and... and for me...'. I mean, I'm afraid of for, for instance... a friend of mine is going one time per month in New York. He's living here, he is a lawyer, and every fucking month he goes to New York. It's more difficult this, I mean, respect mine. Because you have jetlag, you have a really completely different uh... you are in the other part of the world. So... but for him it's normal. So, it's just a question of uh... be able to be part of this mechanical action. For him, it's okay. It's okay, just going ten days per month in New York.

\section{$\mathrm{I}: \mathrm{Mm}-\mathrm{mh}$, do you get used to it, he?}

\section{$\mathrm{X}$ : Sorry?}

\section{I: Do you get used to it, you become abituato?}

\section{$\mathrm{X}$ : Yeah, yeah.}

\section{I: Yeah, that's interesting. Where are the most important people of you living?}

X: Uh... it's quite difficult because... okay, my family, it's okay, it's in Padova. So sometimes I was to go back there. But I'm... and I have a good friend in Padova, also. So, my first friends, I mean of the teenage and everything, it's, are from there. And I am still in touch with them. So, they are quite important. Then I have a second sector of friends, living here in Milan, quite strong. And then I have a... in Berlin also for instance this friend of mine that just came here to visit me, that he is from Berlin. I mean, he is Italian, but he is living in Berlin since uh eight years, nine years. So, he's really living there. And then I have other 
friends living there [in Berlin]. And uh... and even some good friends in Rome. So it's... it's like if uh... I have even there [in Rome], I have different kinds of uh... relationships.

I: But, but you have like a social life in each of the city? So, it's not that one city is only a city where you are most of the time alone? That's also what some people tell me, that in, in their places, that they... the maps that they show me, that there is one place for example, which is only for working and being very concentrated, and not seeing anyone. It's like only being for themselves, working.

$\mathrm{X}$ : Ah, ah, ah [neglecting]. No, no, for me no. I mean I love go to Berlin having fun. I'm yeah living here in Milano with a lot of people. So, I'm enjoying Milano also in uh... yeah in a way that it's not... I mean I'm not going out uh... to... I'm not into fashion and the super-luxury world that Milan sometimes it's so awful. But I have a lot of friends here, and also in Rome. So, it's uh... for me... uh... there are no differences. I mean there are differences in the way of going out. Because I used to do different stuff, different things. But uh... in all the three cities. For instance in Berlin I love to go partying and clubbing. It's the best place to [do so]. In Milan, never, I never go in a club, never, never, never. I don't like it here. They are so much expensive, and people and the music. So, here I have a... yeah I go out with friends, I don't know, go to concerts, uh to... yeah to have a beer. To just so invite some friends here. It's, it's more like a... uh... yeah familiar. And uhm... and in Rome, yeah in Rome it's quite similar like Milan. And uh... but it's more excited because uh... it's better the city, how, how people are in uh...///

\section{I: The mentality of people?}

$\mathrm{X}$ : Yeah! Yeah, yeah, they are more open... not open-minded. Because that's not true, but more... more easy going, they like more having fun, relaxed. Here they are all stressed. Sometimes I call some friend of mine, they are so stressed, that I say: 'Okay, come with me in Rome for a while, because you are too much stressed. You cannot...', I mean, it’s not possible. And in Rome they are so... sometimes too much... they are like, yeah they go to have dinner at eleven o'clock. If you go out here at eleven o'clock, you... it's really difficult to have dinner. I mean, so it's... there are so, so differences between the city and the other. And then, there is Berlin that it's... there is everything, you know. It's a... but it's really cold, that there the weather it's really [I laughs]... you have to be... there in another way. And people there, they are not so... yeah they... they are so organized that sometimes you know, just uh... German people sometimes are too much organized. So, like uh... I don't know, it's Monday, and you already know that Friday you go to, you go to the cinema. Ohh! [mimic of being bored/annoyed by that]. [I laughs] And for me it's so impossible to plan. I mean, if you want to go to the cinema tonight, I just decide at seven o'clock: 'Okay, I want to go to see this'. And they are, you know, in this kind of planning world that I don't like it so much. Even because if they have already, you know, decided that they are going, I don't know, with a... two other friends, and you ask him: 'Okay, uhm Rike what are you going to do Friday?', 'Oh, I'm going to the cinema with uh...', They don't tell you: 'Okay, do you want to come with us?' No, because for them it's already done the... the...///

\section{I: The plan?}


461

462

463

464

465

466

467

468

469

470

471

472

473

474

475

476

477

478

479

480

481

482

483

484

485

486

489

491

492

$487 \quad \mathrm{X}$ : Come?

488 I: E quindi ho sentito bene che hai fatto la fotografia dei spazi vuoto, no?

490 I: Per Roma, hai fatto fotografia dei spazi vuoto o abbandonato?

493 X: E’ vicino, sì, è vicino à uhm... sempre Trastevere, questa zona in centro.

494 I: Ma à Roma, in centro di Roma?

$\mathrm{X}$ : The plan is that.

I: And you can't change it?

X: Yeah. [I laughs] But for me, so this... okay, I know because... and now after years, I know. But... you cannot, you cannot change the plan of a German person. I don't know what you feel about that? [I laughs] But it's really like: 'Okay, Friday cinema, stop!' [thinking]... It's uh... it's difference.
I: Mm-mh, but at least for clubbing, it works good in Berlin, to go out at night?

$\mathrm{X}$ : Yeah. Yeah, yeah, it’s... I love it, I mean.

I: I came... I, I've just spent the last days in the area of Ruhr, Ruhrgebiet, in Dortmund, in the city. Have you visited it a bit?

$\mathrm{X}$ : No.

I: I mean they, they, they are always also looking at Berlin. They are very much focused on Berlin. They say: 'Oh yeah! They are...', the City... you know that the Mayor of, of Berlin...

$\mathrm{X}$ : Sorry, un secondo. [interruption by phone call]

I: Yeah.

X: Pronto? Si. [listening on the phone] Eh si, è troppo tardi.

[break in recording]

X: Ah be, certo. Assolutamente. Ma lei si chiama, scusi? Colla P? Okay, Perrachi. Okay perfetto. Mm-mh, okay. No, no, okay. Sì. Okay. Okay. Okay.

[break in recording]

I: No pasa nada. [laughs]

$\mathrm{X}$ : I'm coming.

[longer break; $\mathrm{X}$ is writing dates into his agenda at desk upstairs in his little loft]

I: Hai nuovo lavoro?

X: Eh, più o meno. No, ma il... il... le foto che devo fare per questa esposizione a Roma.

I: Ah sì.

$\mathrm{X}$ : Che cosa?

X: Sì. Sì, sì, sì.

I: Dov'è? Interessante, he? 
X: Sì, sì. Sì, sì.

496 I: Ah, perché la cosa che me interesse sempre molto è qua in Italia che ci devono essere le 497 città fantasma.

$498 \quad$ X: Aha.

499 I: Come il luogo Cossono, o come se llama, come si chiama, che è vicino di Milano, nel nord, 500 verso la montagna.

$501 \quad$ X: Uh?

502 I: E’ un luogo che ha costruito il uhm... nella epoca di Mussolini, come un luna park.

503 X: Ah! Sì, sì, sì, sì. Ho capito. Capito, capito, capito. Ci sono anche stato.

504 I: Sì?

505 X: A fare delle foto, sì.

$506 \quad$ I: E’ buono para, per andare?

507 X: E’ bello, pero, sì, due anni fa avevano recintato dei pezzi. Si faccia fatica di andare. Pero 508 poiché c’è tutta la vegetazione, gli alberi, eh. No, pero è bello.

509 I: E si può arrivare con la macchina?

510 X: Sì̀, sì.

$511 \quad$ I: O come si fa?

512 X: Sì, sì, colla macchina.

513 I: Perché sto pensando di andarlà. [laughs]

514 X: Sì, sì. No, è bello di andare, sì.

515 I: Ah okay. Terminiamo in...

516 X: Sì.

517 I: Non è molto, he. Uhm... Pero una, una domanda che sempre m’interesse è come ti uh

518 identifica? Se una persona ti chiede: 'Da dove vieni tu?' Che cosa dici tu?

519 X: Uh... io dico che sono di Padova.

520 I: Veramente?

521 X: Sì.

522 I: Che è una relazione alla tua origine?

523 X: Sì.

524 I: O che, che significa?

525 X: Sì, sì. No, c’è, se mi dicono... tu intendi dove vivi, o di dove sei?

526 I: Queste due sono cose differenti per te?

527 X: Sì. 
I: Okay. Che cosa è la differenza?

I: Ah okay.

$\mathrm{X}$ : In this moment. E da dove vieni è... it's more where are you from. Like uh where is your home town, something like that, I mean. So, it's a little bit different for... I mean for me. So...///

I: So, your hometown is Padova?

$\mathrm{X}$ : Yeah.

I: And if you, if you have to answer where are you living, what do you say? quite new, so it's... And then it starts to be, you know, a lot, between Milan, Berlin and Rome. And so it's...///

I: It's more difficult [to name all three places] with Rome?

X: Yeah. Yeah, because then people start to... I mean, for me it's okay, but you have always to explain, you know. Because if you say more than one city, it's: 'Why? What are you doing there?' So, you have to say: Okay, Berlin or in Rome... No, but uh I mean now, for me, Rome it's not yet a place where I'm feeling home. In Berlin, yes. If I just walk around the city and uh... yeah spend some free time, and I'm going out. Even if I'm alone sometimes, I'm... I feel like home.

I: Mm-mh. What's necessary to feel home, for you?

$\mathrm{X}$ : To have enough uh... connection with the cities. I mean connection in the, in the meaning of relation with the place, with people, and with your state of mind.

I: Mm-mh.

X: Yeah, I think it's really a question of uh... uh... how do you say? [thinking]...

I: You can also say it in Italian, if you think...///

X: No, I don't even remember it in Italian [laughs]. I'm so tired, that sometimes I forget a word. And uhm... the state of mind is important. I mean, that it's something higher than the, the idea of be there just to work. I mean, I don't want to live in a place where just I have to work. I want to be there, because I am even living there, and having something there, and some connection. I mean, I'm... because I'm feeling good. So, it's a place not... it's not, just not uh a ball where you go there in, and just work, and go back in another place.

I: Also to spend some leisure time, go out? it's always the same in... maybe not the same, because it's impossible, because it's another city, other people, other culture, so... But it's uh... the, the approach you have with, with this place and to be able to create something, with the place where you go. So, the ability of uh... to have the same pleasure that you have in the, the other city, that maybe you know better. 
And... because of... yeah, I'm Italian and everything. But trying to take this kind of way of life.

\section{I: Also in the others? Mm-mh.}

$$
\mathrm{X} \text { : Yeah. }
$$

I: In the other city?

$\mathrm{X}$ : Yeah.

I: And uhm... what do you answer people then, if they say: 'Okay, you are living between these two cities. How do you identify yourself?' Do you feel like a Berliner, or do you feel like a Milanese? Or do you feel like an Italian? Or like European?

\section{$\mathrm{X}$ : They?}

I: No, you.

X: Ah... uff [thinking]... I don’t know. I'm really uhm not so proud, now, nowadays, to be Italian.

I: Mm-mh.

X: Because I don't like the Italian situation. It's really uh... completely a mess, a problem, and uh... Maybe that's even why I'm trying to escape sometimes, you know. It's just to feel better in another world. I mean, Germany that doesn’t have all these...

\section{I: Political?}

X: Political problems, that I... but that are not even political problems, because they are so stupid and so big in the same time, that sometimes I am so astonished, you know. And then I even have the feedback of the people not living here. Also they are like: 'Beh, how can you live there?' And I say: 'Okay, it's a good question, you know, for me. I don't know'. So, it's... Italy it's not my, now it's, I mean I love Italy for... because okay, it’s my country, and uh... and a lot of really good, good things happened here in the past, with yeah culture, scientists, artists. A lot of sectors, and everything, and even the places, the cities, and the people. But now it's uh... it's a really an horrible place, I think. And uh I don't know why there is not a revolution in Italy. Yet. Because the other countries, I mean, uh if you see France, if you touch something to the...///

I: The people?

$\mathrm{X}$ : To the people, they start really fighting like... England, the same. I mean, you have seen...///

I: All the strikes.

X: The strikes. Here, I mean it's like everything, it's okay. It's just possible, you can do it. What's the problem? And people, I mean, what's the problem? There is no problem, because people are the reflection of the political situation. That's the biggest problem. I'm afraid of that, really people make just the same. I mean, it's, it's a... and I think it's normal, because if you have a teacher that makes something in a certain way, the child made the same. So, if you have the prime minister, that is a bullshit without mind, completely crazy, people just make 
603 the same. They say: 'Okay, if he's going to do that, why not'. So, I, I think that's the reason

604 why people doesn't have any kind of react[ion]. Because, okay, there are... there is a... a

605 yeah... a sector of people that is really...///

$606 \quad$ I: Trying to change it?

$607 \quad \mathrm{X}$ : Trying. But uh... they are not enough.

608 I: They are alone. Yeah, yeah.

609 X: And it's not normal. Because uh... I mean it's so, it's so illegal what he's going to do. I 610 mean it's so... not normal that uh...

611 I: So do you prefer to say that you come from Milan to saying that you come from Italy?

612 X: Uh ufff... No, normally I say, okay, I’m from Milan.

613 I: Mm-mh.

$614 \quad \mathrm{X}$ : Yeah.

615 I: Mm-mh. Does it have a different connotation, or a different meaning?

616 X: Uhm... no. But I mean, if I say I'm from Milan, you're just more specife... uh...///

617 I: Precise?

618 X: Yeah, precise. So, if you are talking with someone maybe you just go quicklier with uh... 619 with cliché of people from Milan.

620 I: [laughs]

621 X: No, because then it's like that, I mean.

622 I: Yeah.

$623 \mathrm{X}$ : Because if you are... I mean, in a certain way it's true that people from Palermo are 624 completely different from people... the people from Milan, or from Torino. But... but uh it's 625 not always true. If I'm saying to you that you are not from Italy, I'm come from... I'm from 626 Milan, you maybe, in your mind, you just have a... it is okay to you. If I'm from uh...///

627 I: Berlin or Munich, whatever?

628 X: Catania. No, always Italy.

629 I: Ah, alright.

630 X: But another city. Maybe you can have a... another view of me.

631 I: Mm-mh. Another image.

$632 \quad \mathrm{X}$ : Yeah.

633 I: Ah okay. And what do you do? Do you have uh developed a certain way of approaching 634 new places? Now you have, you have drawn a map of places that you visit frequently. But has 635 the experience of making these three, or four places your own places uh... has it helped you 636 to develop a routine to approach any new place? If you come to a new place, what's the first 637 activity that you do? If you arrive in a new city, or in a new region? 
X: Uhm... I mean, a really good actress that I met last year told me a thing that I think it's completely true... I don't know abroad, but in Italy it's like that. When you arrive in a new city, if you go to see the stu... so if you go to the theatre and you see how is it the theatre, you can understand how is it the rest of the city. If it's good, really nice, the theatre, it's a city where culture passed by in the past age and it has really like a certain kind of sensibility. Otherwise, it's like a really boring one, you know. And uh... no, it's stupid. But in a certain way it's really true. You can uh... There are some uh... uh some connections with these things. And in fact normally, when I gonna go to a new place, I just try to understand a lot about the culture. And uh... and the places, and the relation between them.

\section{I: Mm-mh.}

X: So, it's... But I am really fascinated, also my... my photography project in a certain way talks about that, the relation between the people and the places. And so I really love to see what happens in a new place, starting a little bit 'people and places', the relation. So, and this fact of the theatre is really curious but it's true. When I go in a new place, in a new city, I just ask for the theatre.

\section{I: Mm-mh.}

$\mathrm{X}$ : Even to... even just to see where is, where is it inside the city. Is it in the city centre, or is it just outside. If it's here, maybe it's more important. It means that people care about this. Or maybe it's not true. Maybe just they built a new, but there was no space, so it's outside. And, so it's... there are, I mean, there are many reasons for that. But uh the collocation of places in a city are, are quite... I mean they are... they have always a reason.

I: Mm-mh, so they are an indication of, of the atmosphere in the city? $\mathrm{X}$ : Yeah.

I: Mm-mh, okay. And do you think that then culture and the people in a city, they reflect or they represent the city in a certain way?

$\mathrm{X}$ : Yeah, absolutely.

I: Mm-mh. Because other people, other people told me the first thing, they always do, is uh walk around the close-by to the hotel. If they are in a hotel in a new city, they just go outside and walk, walk through the close, close area, to see how it is, to experience it by walking. Others told me, if they arrive in a new place, they always look for the highest point, to go upstairs and have an overview from above, and to see how the city is structured in the whole, in the global perspectives. So, everyone has certain routines.

$\mathrm{X}$ : Yeah, it depends. When I went to New York, the first things we, I made with the friend, we just stopped a taxi. I said: 'Okay, I give you 100 Eu... Dollars, please take us around New York. We want to see it through your eyes’, you know. So, not like, like a tourist. So just... so normally, another thing, I just try to know someone from the place.

I: Mm-mh. 
$\mathrm{X}$ : And... and try to... in fact it's the same. Because in Berlin, I just tried to find a place where to live with a German, with a person living since fifteen years in Berlin. So, it's like some, something subconsci...///

\section{I: Subconscious.}

$\mathrm{X}$ : Yeah. But I try to... to have a relation with someone that is from the place. And start to view the... okay, with my eyes, but even with the... to understand his approach to stuff. Because for me, I mean, it could be an also touristic way. And so, so superficial, you know. It's completely different. But someone who is living there, that knows exactly the culture of the place, it's really important.

\section{I: Mm-mh.}

X: I made a travel in Israel. Three years ago. And just... it happened because a friend of mine that is from there just took us to make, you know, an incredible car trip from Tel Aviv till Haifa. So, we went with him, and people and things, and all changed completely. Before we made like two days alone, me and my brother and another friend of mine. Yeah, and we were like, you know, just stupid tourists. Okay, going out, with the map, to see the normal stuff. So, it's really... I, I don't like, it's really horrible, I mean. Its' the... It's the worst way. I mean, because even if you... if you... [noise of falling object]... yeah, don't worry... if you, yeah if you walk and walk, you can also have a whole the vision of the city. But if you don't have the connection with a person, with someone, you cannot go deeper... so.

I: Mm-mh. And uhm if you say that you want to do, you want to have contact with people who are living in a place quite long, then it is for the reason, you know that they know better the place than you might know it. Because they are living there for so long time. Do you also have, vice versa, uhm the experience that you, coming from different places, or living in different places, have uh a more cosmopolitan understanding of places?

\section{$\mathrm{X}$ : Uhm? [sound like finding the idea interesting]}

I: Because sometimes what people tell me, is, uhm if they get in touch, in the places that they are part of their lives, uhm that they have, have the feeling that people only living in this place are more, more egocentric in their worldview. They only know their own place, and they don’t understand how things might work in a different place.

\section{X: Horrible. [I laughs]}

[X walks to kitchen, sound from the back, is getting some fresh water to drink]

$\mathrm{X}$ : No, it's true. I mean... if you live in a... yeah first in a bigger city, and then, if you have connection between... with two or three cities, it's normal that you are more open-minded. I mean, there is nothing to do, it's like that. I mean, I can see the difference between me and my friends living in Padova, that just stay there. I mean, I lived two years in Berlin, five years in Milan, living also in Rome. Went to New York, three times, for quite a long time. I travel quite a lot. So, you... you have completely different feedback, and connection and uh... idea of the world. I mean, I think it's just normal. If you just live in a place uh... you cannot imagine what happens outside. You just have your vision, and it's like that and stop. And uh... so yeah, the thing is you have also to, to understand the... who with are you speaking, I 
mean. If you are with someone that never left the place where you are going to, or if these are people that just have travelled around the world and came back in his hometown to live, so has a completely different view of the world. I mean, it's always different. It's a different situation. But uh, I mean, for me, I can understand that uh... I have always started in a new place with uh... with the feedback I have all the experience that uh... I had in... in the last... in the cities where, where I lived. So, maybe I'm, I am more able to create a global uh... description of the place, I mean. Or maybe not. But maybe you're just able to see more, more things, more details, to understand more aspects of the different culture.

\section{I: Mm-mh.}

$\mathrm{X}$ : Just because you are more open-minded, more open to, to... to understand something different from you. Just that. Because if you are going to New York, where there is, I don't know, the big black uh... uhm...///

\section{I: Community?}

$\mathrm{X}$ : Community of people uh... respect of someone living here in Italy. Because I mean here just, you know, black people are just... if you are Italian... just seen like people coming from the... this, the colonial part, from the South, from Africa. They don't have money. They just are here to do... to try to do something. So, we don't have interracial uh... integration, till now. I think, and there it's so different. You know, just a, another way. So, you just learn to accept other things. And just to see that it's in other place, it's possible. And uh it's normal. So...

I: Did this change your mind also towards these people who are only living in one city? Do you... for example, what other people I talked to... they told me that they are... they feel more comfortable or more simpatico with other people, who have travelled a lot, than with people, who have only lived in one place?

\section{$\mathrm{X}$ : Mm-mh [trying to get the question]. If I can... uh...?}

I: Yeah, yeah. Do you feel, now that you have travelled a lot, and that you know different places, you develop a certain cosmopolitan attitude? If we can say it like that, then do you also feel more comfortable with other people, who are travelling a lot? Or doesn't it make any difference for you, if a person comes from one place only or if he travels a lot?

X: No, I mean, for me it's not so... so different. But I, I can feel... you can feel if someone is more... uh open-minded because of he has travelled a lot, or just, he's just from a place and he just stays there. Maybe you can have a feeling, but, I mean, for me it's not a, it's not a problem. Just uh, just you have to... uh yeah to create the right relation, I mean. Just speaking, and if you know that, just you want to be, you know, just... I don't... for instance I don't want to be, you know, when I'm in Padova, the one who talks: 'Okay, and in Rome I'm doing that, in Berlin or whatever'. Because it seems like if you are doing special stuff. And people living in Padova not. For these are not special stuff, for me it's routine. Because, because of the... what we said before. So, for me it's normal stuff. Like their normal stuff. I mean they just have to be parallel. And maybe then there are some differences... on the mind level, because I have more input about different cultures and different stuff. But it's not.../// 
$\mathrm{X}$ : No.

I: And uh finally I want to know, in these places, are you, are you doing anything for the places in terms of uh... engaging in civil society projects? During you leisure time, are you part of some, some urbanist projects for local development in a neighbourhood, some social initiatives?

X: Well uhm... Well in Berlin I used to... to help a friend of mine, that is an artist and works with children to organize some... some appointment about photography and art. So, just a way to... to make children more comfortable with this kind of sector. Just to play, you know, and just some meeting for free, for uh family and for the neighbourhood. And just to play with children. To give them some new input. So, that was a little bit more civic kind of work. And I mean it was not paid. It was just to work with these, with them. Just for the pleasure. And uh... here in Milan, right now, no. But I used to... uhm... I used to go for a while in a... uh... bibliotheque... just to help uh for re-organize uh archive and old stuff. They had to... to create this long, long list, I mean. There was like, the, the risk was to close the bibliotheque, if there were nobody doing this kind of work, for it was really, really long, long, long work. So, it was just for them. And in Rome, it's not... I mean they asked me to make uh... to be involved from, I think, December, to a project, an urbanistic project about uh... décor urbano. Décor urbano it's the urban uh... the urban beauty. I mean, how to... to make more, more clean and more beauty the city, where you live. So, the aspects like uh... okay then how divide the trash, clean the buildings, I don't know, people going out and making even art with uh recycled pieces of stuff that normally you just throw away. So, there is this big project going on, and uh... yeah, they asked me to be involved. So, we are just starting now to... to start organizing this uh.... this project, that I think we will call 'Scarti', that it's a, a tricky track, I mean. Because scarti it's the plural for uh something you scarto, something you throw away. But then if you... if you read like that [showing something] it's arti, so it's art. So it's uh...//I

I: Okay, from trash to arts somehow.

$\mathrm{X}$ : Yes. And this would be in Rome. From uh...

I: Mm-mh. So, you also try to... to be involved in these places through such uh non-paid or voluntary work?

$\mathrm{X}$ : Yeah. It's not easy because it's really sometimes hard to... to be. But if the possibility... there is a possibility, I like [it]. Because it's even a way to... to understand better a place. Not just on a commercial aspect. Where you just do something for money and stop. Or you pay just for eat and stop. You have a beer... But as some action, just uh... there are... they stand up by themselves. Just because they are there. And that's it.

I: Mm-mh.

$\mathrm{X}$ : Yeah, yeah. 
I: That sounds interesting. So, and for the future, what, what are your plans? Do you want to become a monolocal person again, living in only one place, or...?

X: No. No, no, I think for me it would be really difficult to go back. I mean it's... I'm okay like that. It's a... it's a good way to live. I mean for my... my [thinking]... I mean, I know myself, and if I just stay in a place too much time, in the same place, just I start to... not get bored... but in a certain way I need to change a lot. Even... my life in a certain way. So, I mean, photography gives me the chance to do this, because you can often... you can... yeah, you can do it everywhere. I mean you don't have any kind of uh... deadline or you can do it where you want, where you like to live. And uh... I mean I would like to... even to try new cities.

\section{I: So, it's also a type of, of your personal nature to... to be on the move, move around?}

$\mathrm{X}$ : Yeah. Yeah, yeah, and I think it started... everything started from that point. That it's uh something natural that I, I have, and it's like I need, you know... I just have to change and to move. I mean, it's not that you don't have a... a normal rhythm or... I mean, I have my, my routine. Because it's impossible finally to not have some...

I: Regularity, or everyday life?

$\mathrm{X}$ : Yeah, yeah. Yeah, even because, yeah, you go crazy, I mean. You need a... okay, I know that this is the place where I stay in Milan. In Berlin I stay there. But then you have the possibility to change everything when you want. I mean, you don't have the... you know the... the schedule really strict, that you have in the, in the normal life, where you stay in the office, in a... just in a city, and stop. So, you can, you can manage a lot. If I'm just fucked up about Milan, I just take... and go take a plane and go to Berlin for a while. I mean, if I need just, you know, to change, to just to relax, I can go there, and just because I know that there I can have some stuff. If I want... or more... I don’t know, more business, I can come here, and everything. So, it's, it's a way to create even more opportunity. Yeah.

I: Mm-mh, yeah, that sounds good. So, it has a certain significance, travelling?

$\mathrm{X}$ : Yeah.

I: It's a way of... to raise opportunities, as you say?

X: Yeah. Yeah, yeah. For me yeah, because if I'm, you know, just staying just in Milan, or just in Berlin, you just stop your style... I mean, I would do something new in Rome in November exhibition, in Milan doing stuff, and in Berlin the same. It's really difficult because you really have to be able to uh... to move really quickly, you know. Just okay, I don't know: 'Tomorrow I have to go to Berlin? Okay!' Just not, you know, there are people: 'Oh no!' Just to go crazy if you go and tell them that tomorrow you have to leave, to go. You just have to, to be mental...///

I: Prepared?

$\mathrm{X}$ : Prepared, and uh... stop. And every things follow you. 
I: Do you also have then different... what I found astonishing is that people have different uh SIM-cards, like different national SIM-cards in different mobile phones? Do you use that also? Do you have a German mobile phone? everything. Just uh... I mean, now I have one phone. I don't never stay with two phones uh... 24 hours. I just sometimes, it happens... then I, I lost the SIM-card. Now I have only the Italian one.

X: Yeah. Yeah, I had a German one because for instance I had some work uh... where I had to phone call and to organize myself. So, with the Italian I used to spend lots of money. So, it was impossible. But actually, the, the more used is the Italian one. And then I use email a lot. I mean, I prefer for instance to have internet than the phone.

I: Ah okay, mm-mh. And what about things like, like bank accounts, or health insurance, social insurance? Are you registered in Italian... in Italy, or in both countries? Or how does it work?

I: Hauptwohnsitz, the main residency?

$\mathrm{X}$ : Yeah. No, I have, I have the residence there [in Berlin]. But I don't have the... uh... [thinking]... asistenza sanitaria? $\mathrm{X}$ : Yeah.

I: Okay. And you only use a... do you have a German and an Italian bank account? Or you only use one?

X: No. Now I have only the Italian one.

859

I: Because that's also what people told me frequently, that they then start to install in each

860 country, like step by step, opening a second bank account, uh changing the health insurance, or getting like international ones.

X: No. I mean till now, no. I... I have... it has no sense, I mean. Because it starts to be, you know, too much complicated, everything. Two bank accounts, and then the PIN with the card, the... No! I mean, now I just have a... one, and I mean, even because with internet, I can do a lot of stuff. Just by... with my bank on internet. So, I can do it everything by there. So, it's quite easy.

I: Yeah. And what languages do you speak then, living in-between the countries?

$$
\mathrm{X} \text { : Where, there in Berlin? }
$$

I: No, no. In general. What, what kind of languages have you learnt so far? 
X: Uhm, but where?

$870 \quad$ I: In general. In your life.

871 X: Ah so, ah in general, okay. Yeah, English, German and uh... yeah Spanish. But it's, I don’t

872 speak so much Spanish. German, but now I'm losing a lot. I have to... to, to start to...///

873 I: Try again?

$874 \mathrm{X}$ : No, because now with, with photography, they normally speak everything... everyone

875

876

877

878

879

880

881

882

883

884

885

886

887

888

889

890

891

892

893

894

895

896

897

898 I: Okay, fine. Then we have spoken too much time.

899 X: Yeah.

900 I: You told me, you are busy [laughs]. And you are tired. Then I will leave you now.

901 X: No, okay, but it’s okay. I mean, what time is it? Okay, six. Yeah.

902 I: Okay, then thank you.

903 X: But you are living here in Milan? Till when? speaks English, you know. Just because if you have a... I don't know, to make a shooting, there is, I don't know, an art director from Germany, the photographer is a French, the... So, there are some sectors, where English it's like: okay, and stop. But it's okay, I can understand quite everything in German.

I: So, also during your time with Messe Frankfurt, you have spoken German in the professional environment?

$\mathrm{X}$ : Yeah.

I: Mm-mh, okay. And have you had any uh relationship to, to a German girl friend? Or have you been like in a binational partnership?

$\mathrm{X}$ : I had a relation with a, yeah, with a German girl.

I: That has also happened? [laughs]

$\mathrm{X}$ : Yeah. Yeah, and uh... but it was in the period where I was uh there [in Berlin].

I: When you did your Master?

$\mathrm{X}$ : Yeah, I stayed there like for one year and a half. So, I was... actually I was always there. So... Yeah.

I: Mm-mh. No, that's what I, what I also found, that people, who move around quite frequently, often they are in binational partnerships or things like that. That also helps to create a... a more cosmopolitan attitude.

X: Yeah. Yeah, that's true. Yeah. No, in fact, for me, I mean, it's not a problem, just to find a... I mean, when I know someone, I'm not uh... afraid about the nationality. Maybe someone could be: Okay, no it's from a... For me it's okay.

I: Mm-mh.

$\mathrm{X}$ : Yeah. 
904 I: Uh I'm here in Milan only for this week.

$905 \quad \mathrm{X}$ : Ah okay.

906 I: I have lived in Milan, the whole time, in two thousand... when did I come here? 2008 to 907 2010, no 2009. One year only.

$908 \quad$ X: Okay.

909 I: Then I went back home. To Leipzig.

910 X: But you're from there?

911 I: No, I'm not from there. Actually I'm also from a, from a smaller city which is closer to

912 Hanover.

913 X: Okay.

914 I: Then I moved, also for studying, to, to Leipzig. And I've lived in, in Corsica, and in, in the

915 French Alps for some time. And then I came to Milan. Now, I'm living a bit in Leipzig, 916 sometimes I'm here.

917 X: Ah okay.

918 I: So, I also... Now it's not so much anymore. Because now I'm having a small, a small child, 919 and I don't travel too much anymore. To be with the child and so. And... But before I 920 travelled frequently between Milan and Leipzig. So, it was my, my interest to talk to people 921 who do a bit a similar lifestyle.

922 X: Movement, yeah.

923 I: No, that sounds good.

924 X: Yeah, yeah, no, it's okay. We... I... it's uh... Sometimes I even forget, you know, just to, 925 to move so much. Just to say: Okay, it’s really like when you start to do, then you just...///

$926 \quad$ I: It becomes normal.

927 X: [It] takes part of you. Yeah. So, it's even now with Rome, it’s... okay. Okay, you have to 928 go to Rome. It's... I mean, you, you create a way to, to make the rhythm full regular, and it 929 goes. Yeah.

930 I: And the Rome Institute of European Design, it's the same they have in Milan?

$931 \quad \mathrm{X}$ : Yeah.

932 I: There is also something like that, he?

933 X: Yeah, yeah. Yeah, it’s Milan, Rome, Barcelona, and uh...///

934 I: Ah Barcelona?

935 X: San Paulo, Sao Paulo.

936 I: You can open one in uh Berlin.

937 X: [laughs] Yeah, why not?

938 I: If you have the idea from here. 
X: It would be great, yeah.

940 I: You told me you always take ideas from Berlin, and you put them into Milan. Now you can

941 bring something from Milan to Berlin.

942 X: Take it to Berlin, yeah. Yeah a good...///

$943 \quad$ I: Business opportunity.

$944 \quad \mathrm{X}$ : Yeah.

945 I: If you want to become more settled in, in Berlin, for a while.

$946 \quad \mathrm{X}$ : Yeah. No, Berlin is so strange, working speaking.

947 I: Yeah? It’s difficult to get contracts, I can imagine?

$948 \quad \mathrm{X}$ : Yeah.

949 I: It's a very international city, from... for, especially for creative people. There is a lot of $950 \quad$ Italians, and Spanish people.

951 X: Yeah, but I don't like the idea to have a lot of people living there. Because a lot of... even

952 a friend of mine... just stay there just because okay it’s Berlin. You don't pay rent so much,

953 you know. You just get money from the, the city. And then they start like to live uh like

954 parasites. A lot of people in Berlin do like that, you know.

955 I: Yeah, I know.

956 X: Or they just do like: Okay, I work in a restaurant, for three days of... Three days a week.

957 And then uh I go... yeah I work in a shop. But you are like 32 years old, I mean.

958 I: You have to do something with...?

959 X: For me, I mean, I am 28. And sometimes I say: 'Okay, I'm too late for some stuff', you 960 know. I have to be maybe... go quicklier, I don’t know. But a lot of people, 30 years old, that 961 are just living like teenagers, you know. They say: Okay, I'm doing that, doing... and I don't 962 like it. Because it's so... it's a... because even, because like for a certain time I had... it 963 happens even to me, you know. Just I went inside this kind of circle, just was living.

964 I: Yeah, yeah.

965 X: I was living in Berlin, yeah okay, with some money that I earned from Italy. And staying 966 there, just, you know, okay, going out, and then relaxing. Okay, some time working, but 967 nothing really special. Bur for me it was uh...

968 I: Yeah, yeah, yeah.

969 X: It was... I mean, it was killing me.

970 I: Yeah, this is... this is a bit dangerous in Berlin, and also in Leipzig. If you have a place...

971 when you come from places which are quite expensive, as for example Milan, you have to 972 work to pay your rent... there you don't have to have so much money. You can live very 973 cheap and.../// 
974

975

976

977

978

979

980

981

982

983

984

985

986

987

988

989

990

991

992

993

994

995

996

997

998

999

1000

1001

1002

1003

1004

1005

1006

1007

1008

1009

1010

1011

1012

X: Yeah, but then it's not... I mean, it's a [XY_parasitus], even the people don't have so much ambition.

I: Yeah.

X: And I hate this kind of feeling because you know, they are: Okay, just uffh... I have my life. It's okay, I'm doing some stuff. I mean, I was talking with a friend of mine, who told me: Okay, you can come here with your photos. I said: I don't want to make an exhibition in a place that, you know, was the day before an underground club, and tomorrow would be a bar. You know, this kind of change or anything. Just to put some photos and uh... and stop. I mean, to me it doesn't change anything. I don't want to be the, you know, the... the bad artist. Just to feel like, you know, an artist to be part of them. It doesn't work like that. I mean, I make a... I need some... sorry [cell phone rings]... Posso chiamarti fra poco? Sto faccendo una cosa. Ciao, ciao... And Berlin is really strange for uhm... Sorry [looking on his cell phone]... sorry [other incoming call].... Pronto. Sto bene. Ah okay. Ah, sei, sei partito. Eh, ma smettilà. Ascolta, faccio chiamarti fra poco che sto finendo questa cosa qua. Okay. Eh stai fermo però. Eh okay, ciao, ciao... Eh, there is no, you know, this kind of ambition. But not uh... in a bad way. You know, just, the... the way of a person to, you know, to involve himself to have an escalation to be better, and better, and better. Just to have... in Berlin, this is really difficult, you know. It takes so much time. And you don't have... there it's... there is so, so slowly the rhythm, you know. Just people really like are calmed. And it seems that, okay, you are doing some stuff. But it's okay.

I: But you don’t advance?

$\mathrm{X}$ : Yeah, you take it easy. It's: okay you're in Berlin, it doesn't care, you know. And there was a friend of mine, who was living between Berlin and New York. That, for him, was really a problem, because, you know, in Berlin it was like 'ooh' [making mimic summarizing his recent statements on Berlin]. Then New York was really just totally different...///

I: It's totally different.

$\mathrm{X}$ : And actually, this fatt.... this fact really influences you. Because like in Berlin, he was not able to do nothing. I mean, he is an architect, photographer, and he is doing, he is finishing a $\mathrm{PhD}$ in architecture. Actually doing really nothing. And in New York he was like uh... because he made a membership with the Columbia University. So, he was inside Columbia University, paying nothing, because uh he was just... it was like if he was from the University in Berlin. So, he was like a...///

I: Visting?

X: Visiting, visiting student. Uh... having like meeting with the best architects of all the US, having a studio, making some lecture paid, and having lots of work. You know, he was just really... a lot of stuff, really hard, and really good, it was a lot of feedback. People say: 'Okay, your work is really, really good. You have to go like that'. And then, when he was like passing some months in Berlin, he was like...///

I: Nothing? 
X: Nothing! Really. Nothing. Sometime, okay some little work. Bad paid. You know, people just: 'Okay'. He has to ask for the money from the, from the city. Because... And for him it was like also frustrating. Because it was... it's just a question of cities. Maybe not always. But it's true that sometimes cities can be really... can give you a good or bad influence. In relation of what you want to do.

\section{I: Mm-mh. And now...}

$\mathrm{X}$ : They can make some more good feedback or a bad one.

I: Yeah, they can push you, or they can just stop you on your way.

$$
\mathrm{X} \text { : Yeah, yeah. }
$$

I: And now he's living in New York? Only? Or is he still commuting between Berlin and New York?

X: Yeah, no. New York it's another world. It's uh... I mean, I, I'm still quite afraid about New York. [I laughs] Because it's really too much for me. It's... it's really too much. It's quickly and you have no time for nothing. But I would like maybe to pass some period there. Because you learn really a lot. You have really a lot of feedback, you learn, the best people are there. I mean photography speaking, it's there. But not only photography. I mean for a lot of sectors, I mean, things happen in New York. And... and it's a place amazing, because... because there is this mentality where, you know, that is really American style, that you can do everything, you know. Just okay, democracy, and uh... go on with your belief. It's really equal with everything. I mean... I mean that is really true, I mean, if you have ideas, and if you have capacity, you can really go on in the US, especially in New York. You could... but without... here it's like... you know, it's uh... you have to know the people who know you, that will... it's a shit, completely. Everybody knows that. In New York it's different, completely different. If you have just something to say, you are sure that you will go on.

$$
\text { I: Mm-mh, okay. [noise of cell phone] Well. }
$$

X: [responding cell phone] Adi, tutto bene? Bene, bene, bene. Ascolta, scusa che te ho un po' disposto ma sono stato in coinvolto in un po' di cose che mi ho dimenticato risponderti. Ma, a che ora vuoi passare tu di qua? Eh. Sì. Okay. Sì, io sono à casa. Eh, non lo so. Dimmi tu, se ti vene in mente qualcosa, non mi fa niente di particolare. Allora, io volevo fare un risotto di funghi, e una frittata di zucchine. Ecco sì, bell'idea. Okay. Okay, okay. Via Marbella, 4. Sì, 88 è il mio suono, che ti devo venire da prendere perché è un po’ un casino. Okay, ciao. Ciao, ciao.

I: Okay.

$\mathrm{X}$ : If you want to stay here for dinner?

I: No, I will go. I have to go to, to the place where I stay, when I'm in Milano right now. Because I don’t have any uh... any apartment anymore to live.

$\mathrm{X}$ : Okay, and where are you?

I: I stay at some family people. They are waiting with dinner as well. 
$1051 \quad$ X: Okay.

1052 I: Because one, it’s his birthday.

1053 X: Ah okay, so you have to go.

1054 I: I would appreciate, but today, it’s a bit...

1055 X: No, because I have to finish some stuff, but then at like at eight is coming here a friend of 1056 mine, and uh...

1057 I: You will cook.

1058 X: So, we will cook, yeah. So, if you were free, you were really...

1059 I: I would, but...

1060 X: No, no. It’s not a problem. I mean next time you come to Milan.

1061 I: Okay. [RECORD_STOP] 